然举

45. $\Rightarrow$ 


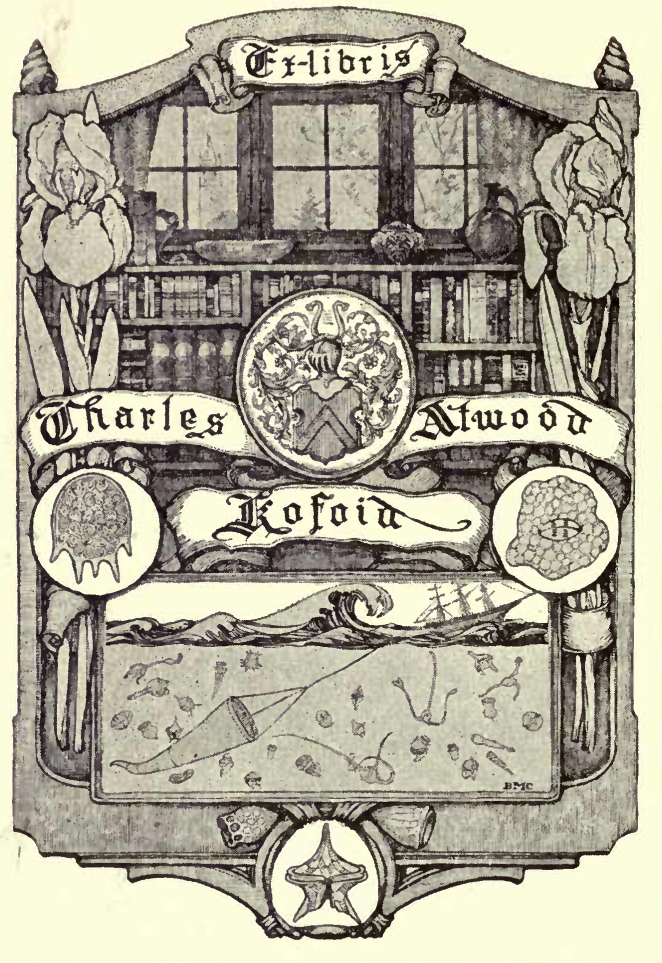



1.4
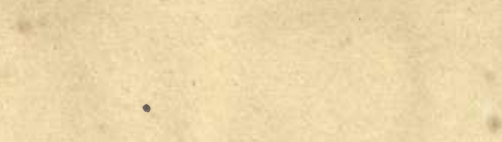

$x+2$

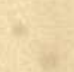

tat? 



\section{•}




\section{THE ESSENTIALS}

\section{OF \\ H I S T O L O G Y}

DESCRIPTIVE AND PRACTICAL

FOR THE USE OF STUDEN'TS

BY

E. A. SCH ̈̈̈FER, F.R.S.

JODRELL Y'ROFESSOR OF PHYSIOLOGY IN UNIVERSITY COLLKGE, LONDON EDITOR OF THE HISTOLOGICAL PORTION OF QUAIN's 'ANATOMY'

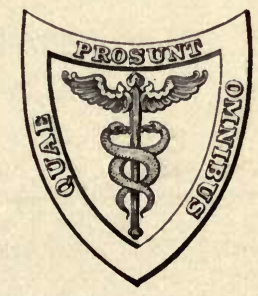

\section{PHILADELPHIA}

LEA BROTHERS \& CO. 


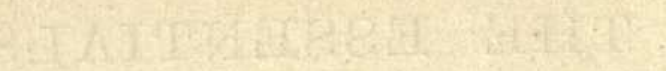

$$
\checkmark
$$

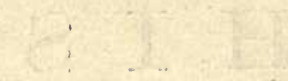




\section{PREFACE.}

THIs BооK is written with the object of supplying the student with directions for the microscopical examination of the tissues. At the same time it is intended to serve as an Elementary Textbook of Histology, comprising all the essential facts of the science, but omitting unimportant details, the discussion of which is only calculated to confuse the learner. For a similar reason references to authorities have also been omitted. Most of the illustrations are taken from the second volume of Quain's Anatomy, ninth edition, where their authorship will be found acknowledged. Of the remainder, those which have been selected from other authors are duly indicated; the rest have either been drawn expressly for this work, or have been transferred to it from the author's ' Course of Practical Histology.'

For conveniently accompanying the work of a class of medical students, the book is divided into forty-two lessons. Each of these may be supposed to occupy a class from one to three hours, according to the extent to which the preparations are made beforehand by the teacher or are prepared during the lesson by the students. A few of the preparations-e.g. some of those of the sense-organs-cannot well be made in a class, but it has been thought advisable not to injure the completeness of the work by omitting mention of them. 
Only those methods are recommended upon which long experience has proved that full dependence can be placed, but the directions given are for the most part capable of easy verbal modification in accordance with the ideas or experience of different teachers.

For other processes and more minute details than could suitably be given here the student is referred to manuals which are devoted to practical work, such as the 'Practical Physiology' of Prof. Foster and Mr. Langley, the 'Practical Histology' of Prof. Rutherford, the 'Textbook of Practical Histology' of Prof. Stirling, and the author's ' Course of Practical Histology.' 


\section{CONTENTS.}

INTRODUCTORY.

ENUMERATION OF THE TISSUES-GENERAL STRUCTURE OF ANIMAL CELLS

PAGB

LESSON I.

USE OF THE MICROSCOPE-EXAMINATION OF COMMON OBJECTS •

LESSON II.

STUDY OF THE HUMAN BLOOD-CORPUSCLES • • . • . . . 7

LESSON III.

ACTION OF REAGENTS UPON THE HUMAN BLOOD-CORPUSCLES • • • 12

LESSON IV.

STUDY OF THE BLOOD-CORPUSCLES OF AMPHIBIA . . . . . . . 14

LESSON V.

THE AMGEBOID PHENOMENA OF THE COLOURLESS BLOOD-CORPUSCLES - 16

LESSON VI.

EPITHELIUM .

LESSON VII.

COLUMNAR AND CILIATED EPITHELIUM AND TRANSITIONAL EPITHELIUM • 23 


\section{LESSON VIII.}

STUDY OF CILIA IN ACTION.

\section{LESSON IX.}

THE CONNECTIVE TISSUES: AREOLAR AND ADIPOSE TISSUE

\section{LESSON X.}

THE CONNECTIVE tissues (continued) : elastic tissue, Fibrods tisSUE, SPECIAL VARIETIES-DEVELOPMENT OF CONNECTIVE TISSUE . . • 35

\section{LESSON XI.}

THE CONNECtive tissues (continued): ARTICULAR CARTIlage

\section{LESSON XII.}

THE CONNECTIVE tissues (continued): costal cartilage, fibro-carTILAGE .

\section{LESSON XIII.}

THE CONNECTIVE TISSUES (continued): BONE AND MARROW

\section{LESSON XIV.}

THE CONNECTIVE TISSUES (continued) : DEVELOPMENT OF BONE. . 54

LESSONS XV. AND XVI.

STRUCTURE OF MUSCLE

\section{تESSON XVII.}

STRUCTURE OF NERVE-FIBRES

LESSON XVIII.

STRUCTURE OF NERVE-CELLS

\section{LESSON XIX.}

MODES OF TERMINATION OF NERVE-FIBRES .

\section{LESSON XX.}




\section{LESSON XXI.}

\section{SMALLER BLOOD-VESSELS, LYMPHATIC VESSELS, SEROUS MEMBRANES,} SYNOVIAL MEMBRANES

\section{LESSON XXII.}

LYMPHATIC GLANDS, TONSIL, THYMUS .

\section{LESSON XXIII.}

THE SKIN .

\section{LESSON XXIV.}

STRUCTURE OF THE HEART

\section{LESSON XXV.}

THE TRACHEA AND LUNGS

\section{LESSON XXVI.}

STRUCTURE OF THE TEETH, THE TONGUE, AND MUCOUS MEMBRANE OF THE MOUTH

\section{LESSON XXVII.}

THE SALIVARY GLANDS

\section{$\cdot+\cdot \cdot+\cdot$ \\ LESSON XXVIII.}

THE STRUCTURE OF THE STOMACH

\section{LESSONS XXIX. AND XXX.}

STRUCTURE OF SMALL AND LARGE INTESTINE $\quad . \quad \ldots . \quad . \quad . \quad . \quad . \quad . \quad . \quad 146$

\section{LESSON XXXI.}

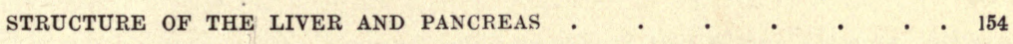

\section{LESSON XXXII.}

STRUCTURE OF THE SPLEEN, SUPRARENAL CAPSULE, AND THYROID BODY • 159

\section{LESSON XXXIII.}




\section{LESSON XXXIV.}

PAGE

STRUCTURE OF THE URETER, BLADDER, AND MALE GENERATIVE ORGANS • 171

\section{LESSON XXXV.}

GeNeRATIVE ORgaNS OF THE FEMALE, AND MAMMARY GLANDS .

\section{LESSON XXXVI.}

STRUCTURE OF THE SPINAL CORD

\section{LESSON XXXVII.}

THE MEDULLA OBLONGATA, PONS VAROLII, AND MESENCEPHALON

\section{LESSON XXXVIII.}

STRUCTURE OF THE CEREBELLUM AND CEREBRUM

LESSONS XXXIX. AND XL.

STRUCTURE OF THE EYELIDS AND OF THE PARTS OF THE EYEBALL . - 211

\section{LESSON XLI.}

STRUCTURE OF THE OLFACTORY MUCOUS MEMBRANE AND OF THE EXTERNAL AND MIDDLE EAR . . . . . . . . . . . 228

\section{LESSON XLII.}

STRUCTURE OF THE LABYRINTH 


\title{
THE ESSENTIALS OF HISTOLOGY.
}

\author{
INTRODUCTORY. \\ ENUMERATION OF THE TISSUES AND THE GENERAL \\ STRUCTURE OF ANIMAL CELLS.
}

Animal Histology ${ }^{1}$ is the science which treats of the minute structure of the tissues and organs of the animal body; it is studied with the aid of the microscope, and is therefore also termed Microscopical Anatomy.

Every part or organ of the body, when separated into minute fragments, or when examined in thin slices (sections), is found to consist of certain textures or tissues, which differ in their arrangement in different organs, but each of which exhibits characteristic structural features.

The following is a list of the principal tissues which compose the body :-

1. Epithelial.

$\begin{aligned} & \text { 2. Connective }\left\{\begin{array}{l}\text { Areolar } \\ \text { Fibrous } \\ \text { Elastic } \\ \text { Adipose } \\ \text { Lymphoid } \\ \text { Cartilage } \\ \text { Bone }\end{array}\right. \\ & \text { 3oluntary } \\ & \text { Involuntary or plain } \\ & \text { Cardiac }\end{aligned}$

4. Nervous.

Some organs are formed of several of the above tissues, others contain only one or two.

It is convenient to include such fluids as the blood and lymph amongst the tissues, because they are studied in the same manner and contain cellular elements similar to those met with in some of the other tissues.

The elements which compose the tissues are of the nature either

1 From i $\sigma \tau o ́ s$, a web or texture. 
of fibres or cells. Some tissues are composed almost entirely of fibres with relatively few cells interspersed amongst the fibres; this is the case with most of the connective tissues. Others are composed entirely of cells, which, however, may in some cases be prolonged so as to form fibres; but these are different from the fibres of the connective tissues in being formed directly from the cells of the tissue, whereas the fibres of the connective tissues are formed between the cells in an intercellular substance. Tissues which are entirely composed of cells are the epithelial tissues, whilst nervous and muscular tissue are formed of cells which are partly or wholly extended so as to form fibres.

Cells.-In the early embryo the whole body is an agglomeration of cells. These are minute portions of living substance or protoplasm, enclosing a vesicle which is known as the nucleus. The tissues are subsequently formed either by changes which occur in the intercellular substance, or by changes in the cells themselves; frequently by both these processes combined. The cells which are least altered from their embryonic condition are the white corpuscles of the blood, and these may be regarded therefore as typical cells.

The protoplasm of a cell (fig. 1, $p$ ) is composed of a soft albuminous substance, which is characterised in typical cells by possessing the property of spontaneous movement. When the cell is unenclosed by a membrane a change in the shape, or even in the position of the cell, may be thereby produced (amœboid movement). The protoplasm often contains granules of various kinds or droplets of watery fluid (vacuoles); the latter may be present in sufficient abundance to impart a reticular or sponge-like structure to the protoplasm. In some cells the protoplasm has a striated or fibrillar structure.

The nucleus of the cell (fig. 1, $n$ ) is a minute vesicle embedded in the protoplasm. It is bounded by a membrane which encloses a clear

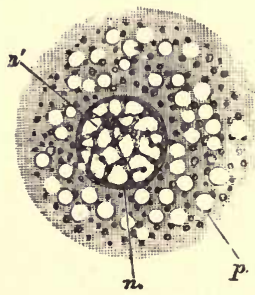

Fig. 1.-Diagram of a Cell.

$p$, protoplasm with racuoles and granules; $n$, nucleus with intranuclear network, $n$, and nucleonuclear
lus. substance (nuclear matrix), and the whole of this substance is generally pervaded by an irregular network of fibres, some coarser, others finer (intranuclear network, $n^{\prime}$ ). This intranuclear network often exhibits one or more enlargements, which are known as the nucleoli. The fibres within the nucleus have been observed to undergo spontaneous changes of form and arrangement, but this becomes much more evident when the cell is about to divide. The division of the protoplasm is always preceded by that of the nucleus, and the intranuclear fibres undergo during its division a series of remarkable changes in arrangement and position, which are known collectively by the term karyomitosis (karyokinesis). These changes may best be studied in the division of epithelium-cells (see Lesson VI.), but exactly similar phenomena have been shown to occur in cells belonging to the other tissues. In 
some cases it may be observed that the filaments of the intranuclear network are made up of fine juxtaposed particles, arranged either in a single or a double row.

All the embryonic cells are formed from the division of the ovum or egg-cell, which divides first into two cells, these again into two, and so on until a large number of cells (embryonic cells) are produced. Eventually these resultant cells arrange themselves in the form of a membrane (blastoderm) which is composed of three layers. These layers are known respectively as the ectoderm or epiblast, the mesoderm or mesoblast, and the entoderm or hypoblast. The ectoderm gives rise to most of the epithelial tissues and the tissues of the nervous system; the entoderm to the epithelium of the alimentary canal (except the mouth), and the glands in connection with it; and the mesoderm to the connective and muscular tissues.

The histogenetical relation between the three layers of the blastoderm and the several tissues and organs of the body is exhibited in the following table:-

The epithelium of the skin or epidermis, and its appendages viz., the hairs, nails, sebaceous and sweat glands.

The epithelium of the mouth, and of the salivary and other glands which open into the mouth. The enamel of the teeth. The gustatory organs.

Ectoderm The epithelium of the nasal passages, and the cavities and glands which open into them.

or

Epiblast

The epithelium covering the front of the eye. The crystalline lens. The retina.

The epithelium lining the membranous labyrinth of the ear.

The epithelium lining the central canal of the spinal cord and the fourth, third, and lateral ventricles of the brain.

The tissues of the nervous system.

The pituitary body. The pineal gland.

The connective tissues.

The blood and lymph corpuscles.

The epithelial lining of the heart, blood-vessels, lymphatics, and serous membranes.

Mesoderm The epithelium of the uriniferous tubules (in part).

or

Mesoblast

The epithelium of the generative organs, and the generative products in both sexes.

The muscular tissues, voluntary, involuntary and cardiac (except the muscular fibres of the sweat glands, which are epiblastic in origin).

The spleen and other lymphatic and vascular glands.

The epithelium of the alimentary canal (from the pharynx to the lower end of the rectum) and all the glands which open into it (including the liver and pancreas).

The epithelium of the Eustachian tube and cavity of the tym.

Entoderm

or

Hypoblast panum.

The epithelium of the larynx, trachea, and bronchi, and of all their ramifications. The epithelium of the pulmonary alveoli.

The epithelium of the thyroid body. Part of the thymus gland. The epithelium of the urinary bladder and ureters, and of part of the uriniferous tubules. 


\section{LESSON I.}

\section{USE OF THE MICROSCOPE: EXAMINATION OF COMMON OBJECTS.}

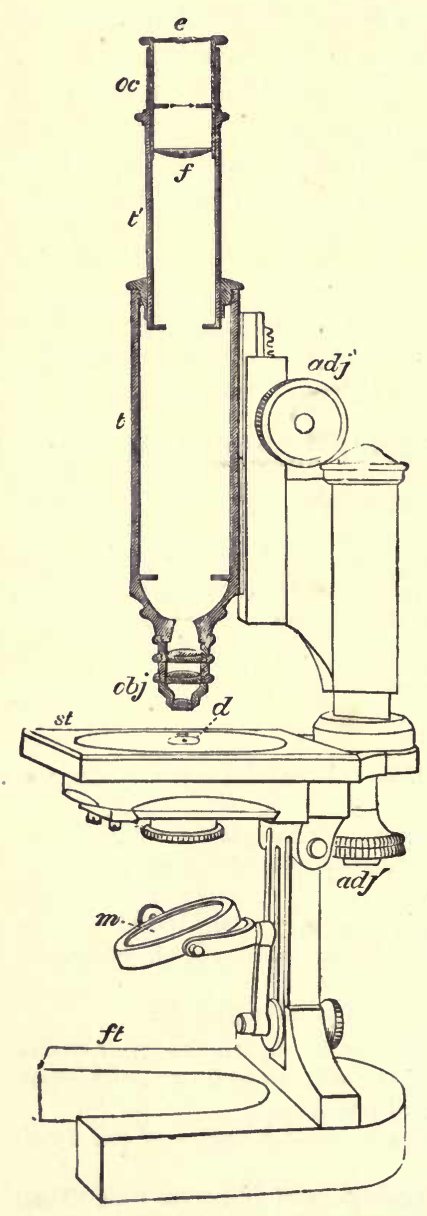

Fig. 2.-Diagram of Microscope.
THE requisites for practical Histology are a good compound microscope magnifying from about 50 to 400 linear; slips of glass technically known as 'slides,' upon which the preparations are made; small pieces of thin glass used as covers for the preparations; a few simple instruments, such as a razor, a scalpel, scissors, fine-pointed forceps, and needles mounted in wooden handles; and a set of fluid re. agents for mounting and staining microscopic preparations. ${ }^{1}$ A sketch-book and pencil are also requisite, and must be constantly employed.

Examine the microscope (fig. 2). It consists of a tube $\left(t t^{\prime}\right)$ having two systems of lenses, one at the upper end termed the 'eye-piece' or 'ocular' (oc), the other, at the lower end, termed the 'objective' $(o b j)$. There should be at least two objectives - a low power, working at about $\frac{1}{2}$ inch from the object, and a high power, having a focal distance of about $\frac{1}{6}$ inch. The focus is obtained by cautiously bringing the tube and lenses down towards the object by the coarse adjustment, which is either a telescopic or a rack-and-pinion movement (adj), and focussing exactly by the fine adjustment, which is always a finely cut screw ( $\left.a d j^{\prime}\right)$.

The stage $(s t)$ upon which the preparations are placed for examination, the mirror $(m)$ which serves to reflect the light up through the central aperture in the stage and along the tube of the instrument, and the diaphragm (d) below the stage which serves to regulate the amount of light thus thrown up, are all parts the employment of which is readily understood.

It is convenient to begin the study of histology by the examination of the blood,

' The directions for making the principal fluids used in histological work will be found in the Appendix. 
but before doing this the student should endeavour to familiarise himself with the use of the microscope, and at the same time learn to recognise some of the chief objects which are liable to occur accidentally in microscopic specimens. On this account it has been considered desirable to introduce

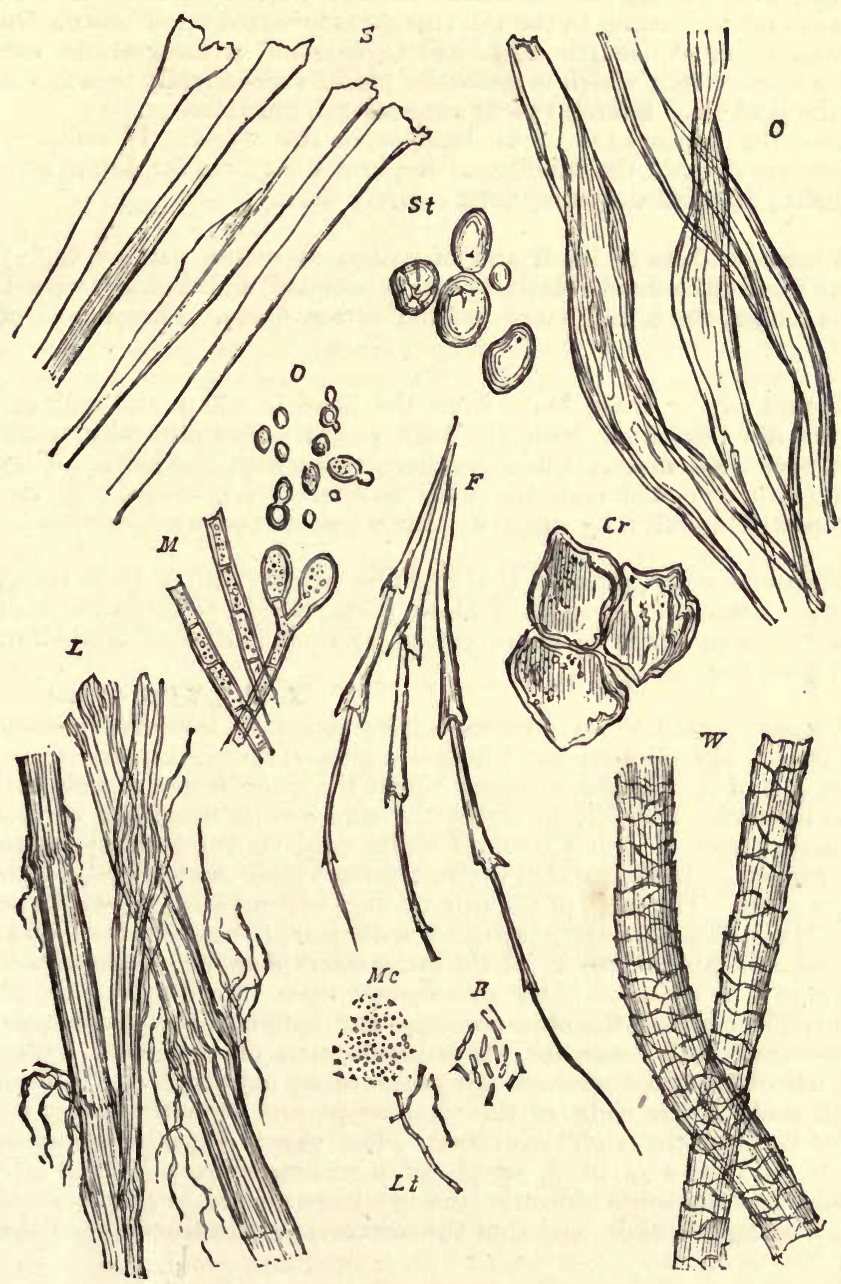

Fig. 3.-Organic matters frequently Present in DUSt. (Heitzmann.)

$S$, fibres of silk ; $C$, of cotton ; $L$, of linen; $W$, of wool ; $F$, feather ; $S t$, starch-granules; $C r$, cork ; $O$, spores of mildew ; $M$, mycelium or threads of mildew ; $M C$, micrococci ; $B$, bacteria; $L t$, leptothrix filaments (500 diameters).

directions for the examination of starch-granules, air-bubbles, linen, cotton, and woollen fibres, and the usual constituents of the dust of a room, into the first practical lesson. 
1. Examination of starch-granules. Gently scrape the cut surface of a potato with the point of a knife; shake the starch-granules so obtained into a drop of water upon a clean slide and apply a cover-glass.

With the low power the starch-granules look like dark specks differing considerably in size; under the high power they are clear, flat, ovoid particles (fig. $3, S t$ ), with a sharp outline when exactly focussed. Notice the change in appearance of the outline as the microscope is focussed up or down. On close examination fine concentric lines are to be seen in the granules arranged around a minute spot which is generally placed eccentrically near the smaller end of the granule. Sketch two or three starch-granules.

Notice the appearance of air-bubbles in the water. If comparatively large they are clear in the middle, with a broad dark border due to refraction of the light; if small they may look entirely dark.

2. Examine fibres of linen and of cotton in water, using a high power. Compare the well-defined, relatively coarse, striated, and slightly twisted linenwith the longer, finer, and more twisted cotton-fibres. Sketch one of each kind.

3. Mount two or three hairs from the head in water and look at them, first with the low, then with the high power. Examine also some fibres from any woollen material and compare them with the hairs. They have the same structure, although the wool is finer and is curled; its structure may be partly obscured by the dye. Draw one or two woollen fibres.

4. Examine some dust of the room in water with a high power. In addition to numerous groups of black particles of carbon (soot) there will probably be seen fibres of linen, cotton, or wool, and shed epithelium-cells derived from the epidermis.

5. Prepare a scale to serve for measuring objects under the microscope. To do this put a stage-micrometer (which is a glass slide ruled in the centre, with the lines $\frac{1}{10}$ and $\frac{1}{10}-$ millimeter apart) under the microscope in such a manner that the lines run from left to right (the microscope must not be inclined). Focus them exactly. Put a piece of white card on the table at the right of the microscope. Look through the instrument with the left eye, keeping the right eye open. The lines of the micrometer will appear projected upon the paper. Mark their apparent distance with pencil upon the card, and afterwards make a scale of lines in ink the same interval apart. A magnified representation is thus obtained of the micrometer scale. Mark upon it the number of the eye-piece and of the objective, and the length of the microscope tube. This scale-card will serve for the measurement of any object without the further use of the micrometer. To measure an object, place the scale-card upon the table to the right of the microscope and view the object with the left eye, keeping the right eye open. The object appears projected upon the scale, and its size in $\frac{1}{10}$ or $\frac{1}{100}$ of a millimeter can be read off. It is important that the same objective and eye-piece should be employed as were used in making the scale, and that the microscope tube should be of the same length. 


\section{LESSON II.}

\section{STUDY OF THE HUMAN BLOOD-CORPUSCLES.}

1. Having cleaned a slide and cover-glass, prick the finger and mount a small drop of blood quickly, so that it has time neither to dry nor to coagulate. Examine it at once with the high power.

Note $(a)$ the coloured corpuscles, mostly in rouleaux and clumps, but some lying apart seen flat or in profile; $(b)$ the colourless corpuscles, easily made out if the cover-glass is touched by a needle, on account of their tendency to stick to the glass, whilst the coloured corpuscles are driven past by the currents set up; (c) in the clear spaces, fibrin filaments and elementary particles or blood-tablets.

Sketch a roll of coloured corpuscles and one or two colourless corpuscles. Count the number of colourless corpuscles in a field of the microscope.

2. To be made like 1 , but the drop of blood is to be mixed upon the slide with an equal amount of 0.6 per cent. salt solution, so that the red corpuscles tend to be less massed together, and their peculiar shape is better displayed.

Sketch a red corpuscle seen on the flat and another in profile (or optical section). Also a crenated corpuscle.

Measure ten red corpuscles, and from the results ascertain the average diameter of a corpuscle.

3. Make a preparation of blood as in $\$ 1$ and put it on one side to coagulate. After fifteen minutes allow a drop of a solution of borax-carmine ${ }^{1}$ to run under the cover-glass. This decolorises the red corpuscles, but stains the nuclei of the white corpuscles and brings the network of fibrin filaments and the elementary particles clearly into view (fig. 7). After a drop of glycerine has been allowed to diffuse into the fluid the cover-glass may be cemented with gold-size and the preparation labelled and kept.

4. Enumeration of the blood-corpuscles. This is readily effected by the hæmacytometer of Gowers. This instrument consists of a glass slide (tig. 4,c), the centre of which is ruled into ${ }_{10}^{1}$ millimeter squares and surrounded by a glass ring $\frac{1}{5} \mathrm{~mm}$. thick. It is provided with measuring pipettes (A and $\left.\mathbf{B}\right)$, a vessel (D) for mixing the blood with a saline solution (sulphate of soda of sp. gr. 1015), glass stirrer (E) and guarded needle (F).

'The mode of proceeding is extremely simple. 995 cubic millimeters of the saline solution are placed in the mixing jar; 5 cubic millimeters of blood are then drawn from a puncture in the finger and blown into the solution. The two fluids are well mixed by the stirrer and a small drop of this dilution is placed in the centre of the cell, the cover-glass gently laid on (so as to touch the drop, which thus forms a layer $\frac{1}{5} \mathrm{~mm}$. thick between the slide and coverglass) and pressed down by two brass springs. In a few minutes the corpuscles have sunk to the bottom of the layer of fluid and rest on the squares. The number in ten squares is then counted, and this, multiplied by 10,000, gives the number in a cubic millimeter of blood.'

1 See Appendix. 


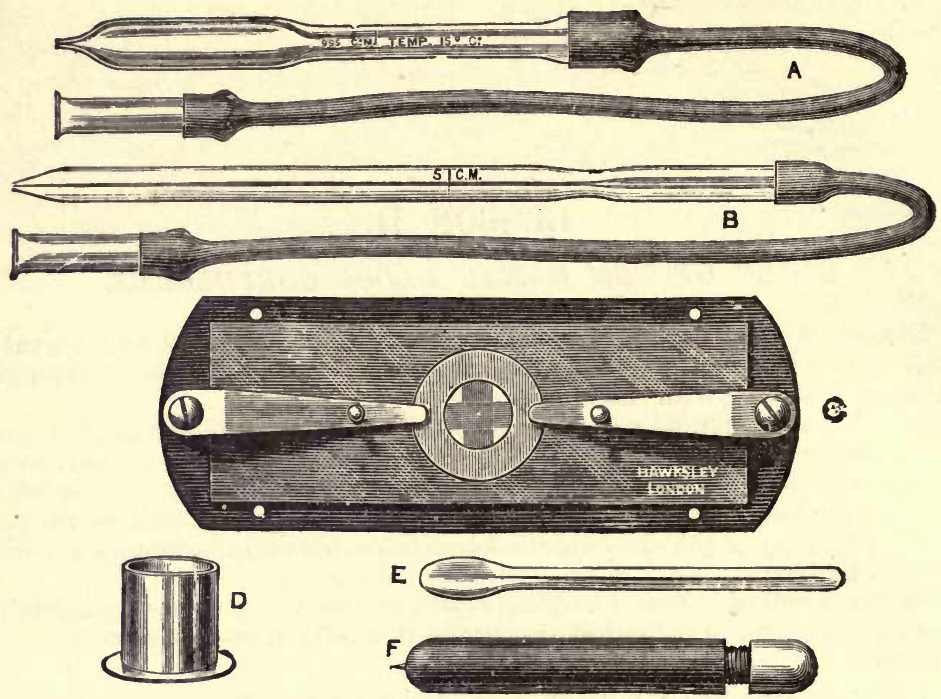

Fig. 4.-Hemacytometer of Gowers.

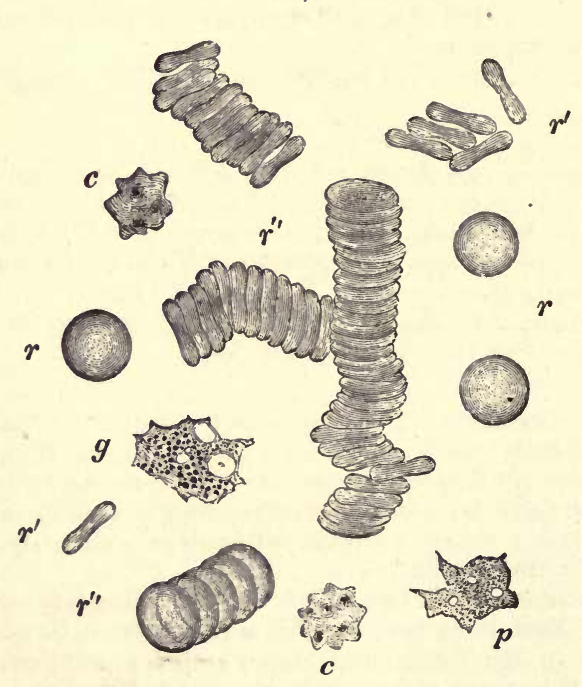

Fig. 5.-Human blood as seen on the warm STAGE. (Magnitied about 1,200 diameters.)

$r, r$, single red corpuscles seen lying flat; $r^{\prime}, r^{\prime}$, red corpuscles on their edge and viewed in profile; $r$, red corpuscles arranged in rouleaux ; $c, c$, crenate red corpuscles; $p$, a finely granular pale corpuscle; $g, a$ coarsely granular pale corpuscle. Both have two or three distinct vacuoles, and were undergoing changes of shape at the moment of observation; in $g$, a nucleus also is visible.

\section{$1(\mathrm{C})$ \\ 8}

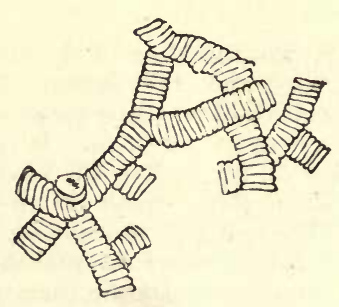

Fig. 6.-Human Red corfusCLES LYING SINGLY AND COLLECTED INTO ROLLS. (As seen under an ordinary high power of the microscope.) 
The coloured blood-corpuscles.-Under the microscope the blood is seen to consist of a clear fluid ( $p$ lasma), in which are suspended the bloodcorpuscles (fig. 5). The latter are of two kinds: the red or coloured $\left(r, r^{\prime}\right)$, which are by far the most numerous, and the white, pale, or colourless $(p, g)$, which from their occurrence in the lymph are also known as lymph-corpuscles. When seen singly the coloured corpuscles are not distinctly red, but appear of a reddish-yellow tinge. In the blood of man and of all other mammals, except the Camelidæ, they are biconcave circular disks. Their central part usually has a slightly shaded aspect, under the ordinary high power (fig. 6,1 ), but this is due to their biconcave shape, not to the presence of a nucleus. They have a strong tendency to become aggregated into rouleaux and clumps when the blood is at rest, but if it is disturbed they readily become separated.

If the density of the plasma is increased in any way, as by evaporation, many of the red corpuscles become shrunken or crenated (c).

The average diameter of the human red corpuscles is 0.077 millimeter (about $\frac{1}{3200}$ inch) ${ }^{1}$

There are from four to five millions of coloured corpuscles in a cubic millimeter of blood.

The colourless corpuscles of human blood are a little larger than the coloured, measuring $0.01 \mathrm{~mm} .\left(\frac{1}{2500}\right.$ inch $)$ in diameter. They are far fewer, numbering not more than ten thousand in a cubic millimeter. Moreover they are specifically lighter, and tend to come to the surface of the preparation. If examined soon after the blood is drawn, they are usually spheroidal in shape, but they soon become irregular (fig. 5, $p, g$ ), and their outline continually alters, owing to the amœba-like changes of form to which they are subject. Some of the colourless corpuscles are very pale and finely granular, others contain coarser and more distinct granules in their protoplasm. The protoplasm may also contain clear spaces or vacuoles, and a reticular structure is described
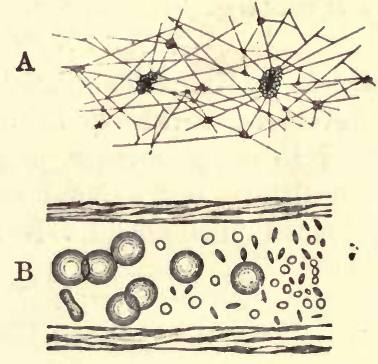

Fig. 7.-Fibrin fildinents aNd bloodTABLETS.

A, network of fibrin, shown after washing away the corpuscles from a preparation of blood that has been allowed to clot; many of the filaments radiate from small clumps of blood-tablets. B (from Osler), blood-corpuscles and elementary particles or blood-tablets, within a small vein. in it by some histologists. Each pale corpuscle has one or more nuclei, which are difficult to see without the aid of reagents.

In the clear fluid in which the corpuscles are suspended, a network of fine straight intercrossing filaments (fibrin) soon makes its appearance (fig. 7). There are also to be seen a certain number of

1 The following list gives the diameter in parts of a millimeter of the red bloodcorpuscles of some of the common domestic animals :-Dog, 0.0073 ; rabbit, 0.0069 ; cat, 0.0065 ; sheep, 0.0050 ; goat, 0.0041 . 
minute round colourless discoid particles, either separate or collected into groups or masses, which may be of considerable size. These are the elementary particles or blood-tablets. Their meaning is not known. Fatty particles may also occur in the plasma.

Development of blood-corpuscles. - In the embryo, the first-formed coloured blood-corpuscles are amœboid nucleated cells, the protoplasm

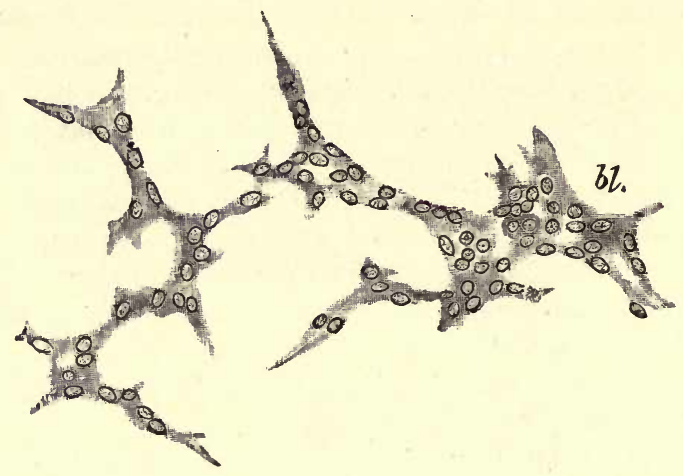

Fig. 8.-DEVElopMeNT OF BLOOD-VESSELS AND BLOOD-CORPUSCLES IN THE VASCUIAAR AREA OF THE GUINEA-PIG.

$b l$, blood-corpuscles becoming free in the interior of a nucleated protoplasmic mass.

of which contains hæmoglobin. These embryonic blood-corpuscles are developed within cells of the mesoderm, which unite with one another so as to form a protoplasmic network (fig. 8). The nuclei then multiply, and around some of them there occurs an aggregation of coloured protoplasm. Next the branched cells become hollowed
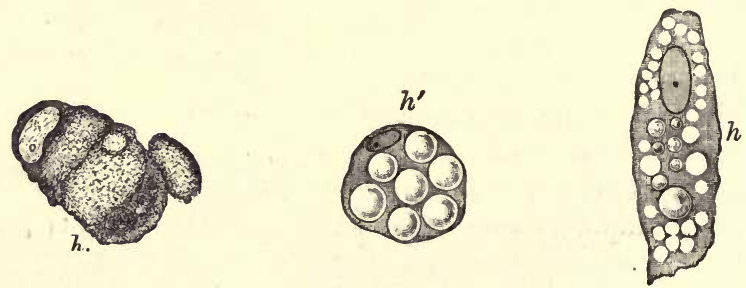

Fig. 9.-BLood-Corpuscles developing within convective-tissue cells.

$h$, a cell containing diffused hæmoglobin; $h^{\prime}$, globu'ar masses of co'oured substance in the protoplasm, within which also are numerous vacuoles; $h^{\prime}$, a cell filled with coloured globules.

out by an accumulation of fluid in their protoplasm so as to form a network of blood-vessels, and then the coloured nucleated portions of protoplasm are set free within them as the embryonic bloodcorpuscles (fig. $8, b l$ ). 
In later embryonic life, and after birth, nucleated coloured corpuscles are no longer present in mammalian blood, but are replaced by the usual discoid corpuscles. These are formed within certain cells of the connective tissue, a portion of the protoplasm of the cell becoming coloured by hæmoglobin, and separated into globular particles (fig. 9, $h, h^{\prime}, h^{\prime \prime}$ ), which are gradually moulded into disk-shaped red corpuscles. In the meantime the cells become hollowed out, and join with similar neighbouring cells to form blood-vessels (fig. 10, $a, b, c$ ). The process is therefore the same as before, except that the cell-nuclei do not participate in it.
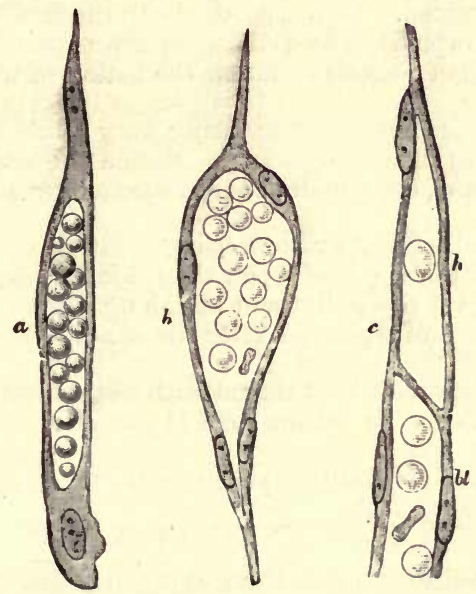

Fig. 10.-Further DEVELOPMENT OF BLOOD-CORPUSCLES in CONNECTIVE-TiSSUE CELLS, AND TRANSFORMATION OF THE LATTER INTO CAPILLARY BLOOD-VESSEIS.

$a$, an elongated cell with a cavity in its protoplasm occupied by fluid and by blood-corpuscles mostly globular; $b, a$ hollow cell the nucleus of which has multiplied. The new nuclei are arranged around the wall of the cavity, the corpuscles in which have now become discoid; $c$ shows the mode of union of a 'hæmapoietic' cell, which in this instance contains only one corpuscle, with the prolongation $(b l)$ of a previously existing vessel. $a$, and $c$, from the new-born rat; $b$, from a fœtal sheep.

Although no nucleated coloured corpuscles are to be seen in the blood in post-embryonic life, they continue to be found in the marrow of the bones (see Lesson XIII.), and it is thought probable that the red disks may be formed in some way from these. Others have supposed that the red disks are derived from the white corpuscles of the blood and lymph, and others again that they are developed from the blood-tablets; but the evidence in favour of these views is insufficient.

The white blood-corpuscles and lymph-corpuscles occur originally as free unaltered embryonic cells, which have found their way into the vessels from the circumjacent tissues. Later they become formed in lymphatic glands and other organs composed of lymphoid tissue, and pass from these directly into the lymphatics and so into the blood. 


\section{LESSON III. \\ ACTION OF REAGENTS UPON THE HUMAN BLOOD. CORPUSCLES.}

1. MAKE a preparation of blood as in Lesson II. 1, and apply a drop of water at one edge of the cover-glass. Examine at a place where the two fluids are becoming mixed. Notice particularly the first effect of water upon both red and white corpuscles, as well as the ultimate action.

Sketch both kinds of corpuscles under the action of water.

2. Repeat on another preparation, using very dilute alkali $(0 \cdot 2$ per cent. potash in salt solution) instead of water. Notice the complete solution first of the white and then of the coloured corpuscles as the alkali reaches them.

3. Repeat on another preparation, using dilute acetic acid ( 1 per cent.). Observe that the effect of the acid upon the coloured corpuscles is similar to that of water, but that it has a different action upon the colourless corpuscles.

Sketch two or three of the latter after the action is completed.

4. Make a preparation of blood mixed with salt solution as in Lesson II. 2, and investigate the action of tannic acid ( 2 per cent. solution) in the same: way.

Sketch two or three coloured corpuscles after the action is complete.

The action of reagents upon the human red blood-corpuscles shows: that, although to all appearance homogeneous, they in reality consist.

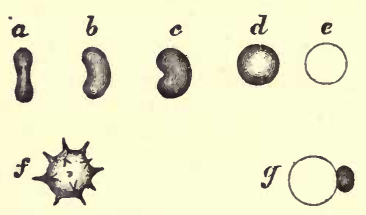

Fig. 11.

$a-e$, successive effects of water upon a red corpusc'e ; $f$, effect of solution of salt; $g$, effect of tannic acid. of an intimate intermixture of the colouring matter or hæmoglobin with certain other substances (globulin, lecithin, cht lesterin), which are left as the colourless stroma, on dissolving out the hæmoglobin, or on causing its discharge by any means from the corpuscle. This separation of the hæmoglobin from the stroma can be effected by water (fig. 11, $a-e)$ and also by dilute acids, by the action of heat $\left(60^{\circ} \mathrm{C}\right.$.), the freezing and thawing of blood, the vapour of chloroform, and the passage of electric shocks through blood. ${ }^{1}$

I In the blood of some animals crystals of hæmoglobin readily form after its separation by any of these means from the red corpuscles. These crystals are rhombic prisms in most animals, but tetrahedra in the guinea-pig, and hexagonal plates in the squirrel. They are most appropriately studied along with the chemical and physical properties of blood, and are therefore omitied here. The same remark applies to the minute dark-brown rhombic crystals (hamin), which are formed when dried blood is heated with glacial acetic acıd, and to the reddish-yellow crystals of hamatoidin, which are found in old blood extravasations. 
The mixing of human blood with the blood or serum of various animals also has a similar action, probably owing to differences of density or alkalinity. Tannic acid produces a peculiar effect (fig. 11, g); the hæmoglobin is discharged from the stroma, but is immediately altered and precipitated, remaining adherent to the stroma in the form of a round or irregular globule of a brownish tinge (hæmatin).

The structure of the colourless corpuscles is also brought out by the action of these reagents. As the water reaches them their amœboid movements cease; they become swollen out into a globular form by imbibition of fluid (fig. 12, 1), and the granules within the proto-

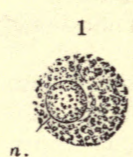

$n$.

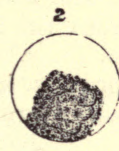

Fig. 12.

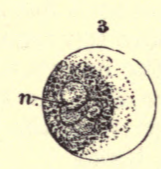

1 , first effect of the action of water upon a white blood-corpuscle ; 2,3 , white corpuscles treated with dilute acetic acid; $n$, nucleus.

plasm can be seen to be in active Brownian motion. Their nuclei also become clear and globular, and are more conspicuous than before. With the further action of the water, the corpuscle bursts and the granules are set free.

Acids have an entirely different action upon the white corpuscles. Their nuclei become somewhat shrunken and very distinct (fig. 12, 2 and 3), and a granular precipitate is formed in the protoplasm around the nucleus. At the same time, a part of the protoplasm generally swells out so as to form a clear bleb-like expansion (an appearance which often accompanies the death of the corpuscle from other (causes). 


\section{LESSON IV.}

\section{STUDY OF THE BLOOD-CORPUSCLES OF AMPHIBIA.}

1. Mount a drop of newt's blood obtained from the cut end of the tail. Examine with the high power. Notice the shape of the coloured corpuscles, both when seen flat and edgeways, and the nucleus within each.

Measure ten corpuscles, and from the results obtain the average dimensions of the newt's blood-corpuscle.

Notice also the colourless corpuscles, smaller than the red, but considerably larger than the pale corpuscles of human blood, although otherwise resembling these.

Sketch two or three red corpuscles and as many white.

Be careful not to mistake the liberated nuclei of crushed red corpuscles for pale corpuscles.

Enormous cells and nuclei belonging to the cutaneous glands as well as the granular secretion of those glands may be present in this preparation.

2. Apply a drop of water to the edge of the cover-glass of the same preparation and notice its action upon the corpuscles.

Sketch two or three corpuscles altered by the action of the water.

3. Mount another drop of blood, and apply dilute acetic acid ( $\mathbf{1}$ per cent.) instead of water at the edge of the cover-glass. Make sketches showing the effect of the acid upon both red and white corpuscles.

4. Examine the corpuscles of newt's blood which has been allowed to flow into boracic acid solution ( 2 per cent.). Notice the effect produced upon the coloured corpuscles. Sketch one or two.

The coloured blood-corpuscles of amphibia (fig. 13), as well as of most vertebrates below mammals, are biconvex elliptical disks, considerably larger than the biconcave circular disks of mammals. ${ }^{1}$ In addition to the coloured body of the corpuscle, which consists, as in mammals, of hæmoglobin and stroma, there is a colourless nucleus, also of an elliptical shape, but easily becoming globular, especially if liberated by any means from the corpuscle. The nucleus resembles that of other cells in structure, being bounded by a membrane, and having a network of filaments traversing its interior (fig. 14). It is not very distinct in the unaltered corpuscle, but is brought clearly into view by the action

1 The following are the dimensions in parts of a millimeter of some of the corpuscles of oviparous vertebrates :-

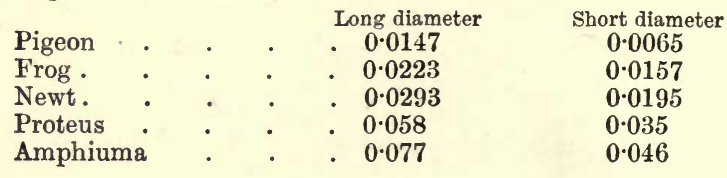


of reagents, especially acetic acid. The action of reagents upon the red corpuscle of amphibia is otherwise similar to that produced upon the mammalian corpuscle, water and acetic acid causing it to swell into a globular form and then to become decolorised; solution of salt causing a shrinking of the corpuscle, and so on. Boracic acid acts like tannic acid in causing the hæmoglobin to be withdrawn from the stroma; but it becomes partially or wholly collected around the nucleus, which may then be extruded from the corpuscle.

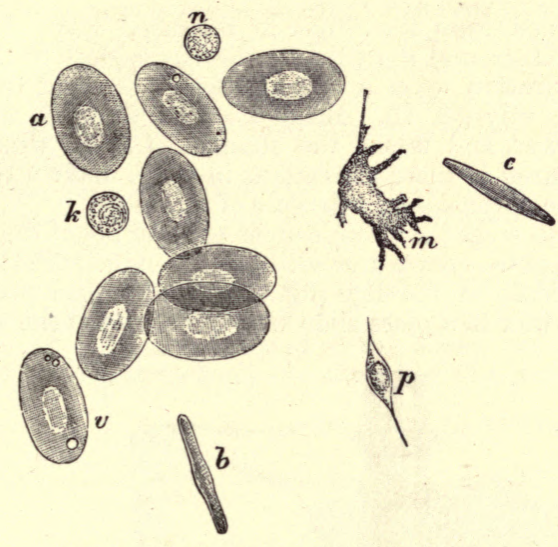

Fig. 13.-Frog's Blood. (Ranvier.)

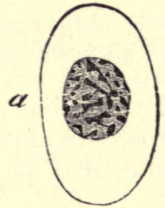

Fig. 14.-COLOURED CORPUSCLE OF SALAMANDER, SHOWING INTRANUCLEAR NETWORK. (Flemming.)

$a$, red corpuscle seen on the flat; $v$, vacuoles in a corpuscle; $b, c$, red corpuscles in profile; $n$, pale corpuscle at rest ; $m$, pale corpuscle, exhibiting amœboid movements ; $p$, coloured fusiform corpusele.

The colourless corpuscles (fig. 13, $k, m, n$ ), although larger, are very similar to those of mammals. Like them, they are of two kinds - either wholly pale or enclosing a number of dark granules. They vary much in size and in the activity of their amœboid movements. They may have one or several nuclei. Reagents have the same effect upon them as on those of mammals. 


\section{LESSON V. \\ THE AMEBOID PHENOMENA OF THE COLOURLESS BLOOD-CORPUSCLES.}

1. Make a preparation of blood from the finger in the usual way. Draw a brush just moistened with oil around the edge of the cover-glass to check evaporation. Place the preparation upon a 'warm stage,' and heat this to about the temperature of the ody $\left(38^{\circ} \mathrm{C}\right)$. Bring a white corpuscle under observation with the high power, and watch the changes of shape which it undergoes. To become convinced of these alterations in form, make a series of outline sketches of the same corpuscle at intervals of a minute.

The simplest form of 'warm stage' is a thin copper plate of about the size of an ordinary slide, perforated in the centre and with a long tongue of the same metal projecting from the middle of one edge (fig. 15). The copper plate is firmly cemented with sealing-wax to a glass slide which rests upon the stage

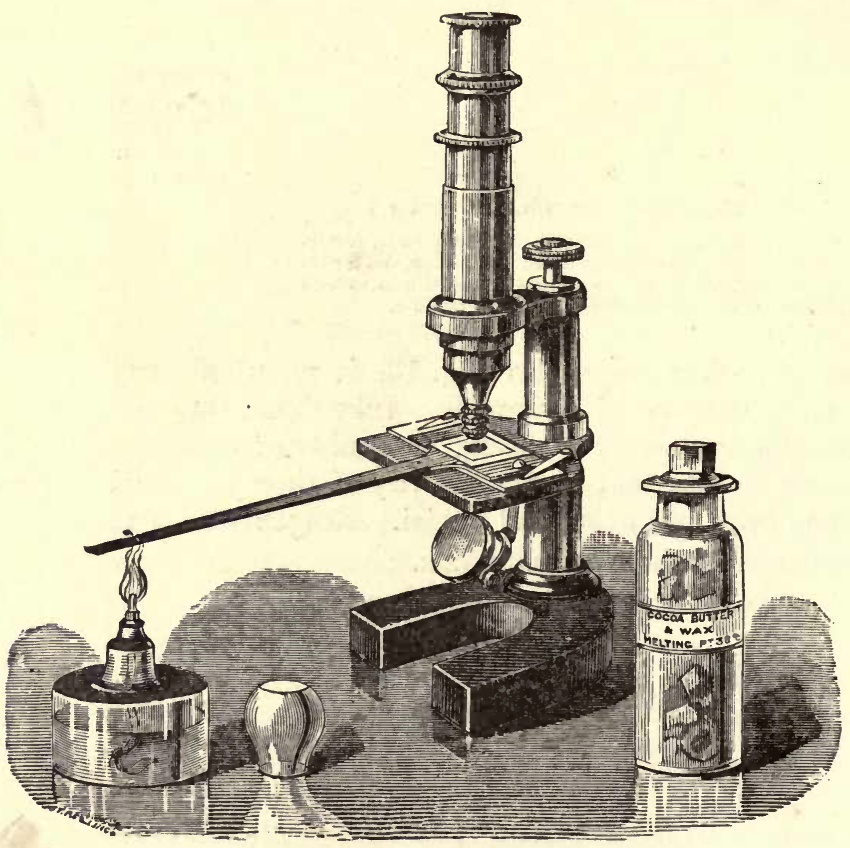

Fig. 15.-Simpie WARMing apparatus, COMPLETE, SHOWN in OPERATION.

of the microscope. The preparation, which should be made upon a rather thin slip of glass, is put on to the warm stage and pressed into contact with it by the brass clips. Heat is applied to the copper tongue by a small spiritlamp flame, and a greater or less amount is conducted to the warm stage and the superjacent preparation according to the point to which the flame is 
applied. To ascertain that the right temperature is got and maintained, put two pieces of paraftin, one melting at $35^{\circ} \mathrm{C}$. $\left(95^{\circ} \mathrm{F}\right.$.) and another at $38^{\circ} \mathrm{C}$. $\left(100^{\circ} \mathrm{F}\right.$.), on the slide, one on either side of the preparation. The temperature must be such that the first piece is melted and remains so whilst the second remains solid. ${ }^{1}$

2. Mount a drop of newt's blood diluted with an equal amount of salt solution, and examine it in the same manner upon the copper stage; the temperature must, however, be kept below $30^{\circ} \mathrm{C}$. Observe the effect of heat in accelerating the amœeboid movements of the pale corpuscles. Sketch one at intervals of a minute $(a)$ in the cold, $(b)$ whilst warmed.

3. Examine some yeast which has been mixed with salt solution. Observe the yeast-cells or torulæ, some of them budding. Sketch two or three.

Now mix a little of the yeast and salt solution with a fresh drop of newt's blood, oiling the edge of the cover-glass as before. Endeavour to observe the inception of torulæ by the white corpuscles. Sketch one or two corpuscles containing torulæ.

Milk-globules or particles of carbon or of vermilion may also be used for this experiment, but the process of inception is most readily observed with the yeast particles.

4. At the commencement of the lesson collect a drop of newt's blood into a fine capillary tube, seal the ends of the tube, and mount it in a drop of oil of cloves. Towards the end of the lesson examine it again to see white corpuscles emigrating from the shrunken clot (see fig. 16).

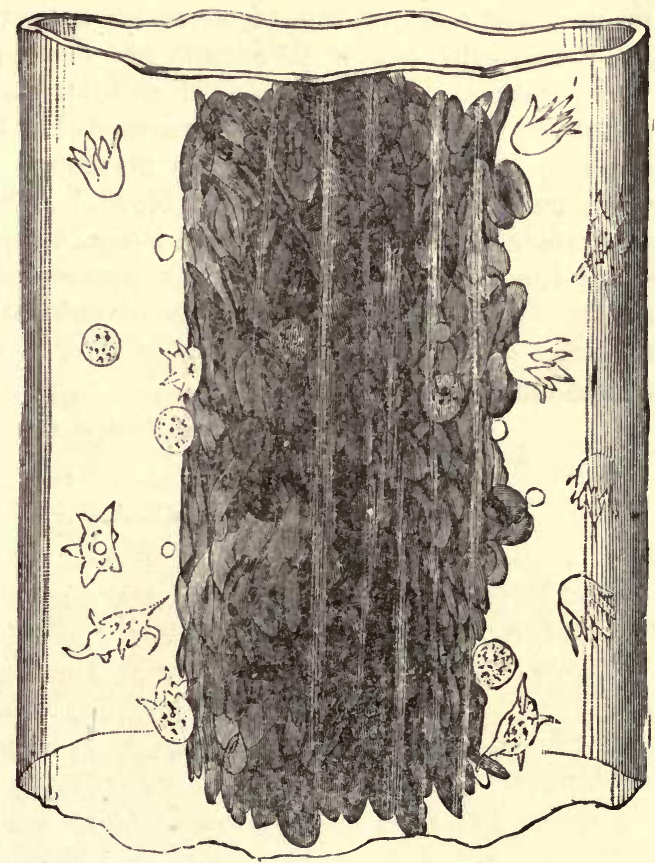

Fig. 16.-White corpuscles of FroG: $\mathrm{S}$ bLOOd Migrated From SHRUNKEN CLOT WITHIN A CAPILLARY TUBE.

1 For exact work, an apparatus somewhat more complex than the above is required. For description of such a one see A Course of Practical Histology, pp. 22, 23. 
The amœboid phenomena which are exhibited by the protoplasm of the colourless blood-corpuscles consist, in the first place, of 'spontaneous changes of form, which when active may also produce changes in place or actual locomotion (migration) of the corpuscle; and, secondly, of a tendency which it presents to enwrap and take into its substance foreign particles with which it may come in contact, and particles thus incepted may then be conveyed by the corpuscle in its locomotory changes from one place to another.

It is probable that particles of organic matter which are taken up by the pale corpuscles may undergo some slow process of intracellular digestion within their protoplasm.

The processes of the granular corpuscles are generally quite clear at first, and the granules afterwards flow into them.

The migration of the colourless corpuscles from the blood-vessels into the surrounding tissue, or from a blood-clot into the surrounding serum (fig. 16), is owing to these amœboid properties.

The conditions which are most favourable to this amœboid activity of the white corpuscles are (1) the natural slightly alkaline medium, such as plasma, serum, or lymph, or, failing these, normal saline solution; (2) the prevention of desiccation. Any increase of density of the medium produces a diminution of amœboid activity, whilst, on the other hand, a slight decrease in its density has the opposite effect; (3) a certain temperature. In warm-blooded animals the phenomena cease below about $10^{\circ} \mathrm{C}$. When gradually warmed they become more and more active up to a certain point, the maximum being a few degrees above the natural temperature of the blood. Above this point they become spheroidal and their protoplasm is coagulated and killed. Acids at once kill the corpuscles and stop the movements. Narcotic gases and vapours, such as carbonic acid gas or chloroform vapour, also arrest the movement, but it recommences after a time if their action is discontinued. 


\title{
LESSON VI.
}

\author{
EPITHELIUM.
}

1. Mount a drop of saliva and examine first with a low, afterwards with the high power. Observe the nucleated epithelium-cells, some single, and others still adhering together by overlapping edges. Measure three or four, and also their nuclei. Sketch one or two on the flat and one edgeways. Notice the salivary corpuscles, which are like white blood-corpuscles swollen out by imbibition of water.

2. Put a small shred of human epidermis into a drop of strong caustic potash solution for one or two minutes. Then break it up in water with needles, cover and examine. Observe the now isolated swollen cells. Measure some.

3. Study the arrangement of the cells in a section through some strarified epithelium, such as that of the mouth, skin, or cornea. The section may be prepared beforehand by the demonstrator; it should be mounted in Canada balsam. ${ }^{1}$ Notice the changes in shape of the cells as they are traced towards the free surface. Measure the thickness of the epithelium. Count the number of layers of cells.

4. Study the minute structure of epithelium-cells and their nuclei, both at rest and dividing, in the tail of the salamander-tadpole. ${ }^{2}$ This preparation may also advantageously be prepared beforehand by the demonstrator.

[The preparation is made as follows: The tail is placed in chromic acid solution ( $0 \cdot 1$ per cent.) for three days, then thoroughly washed for some hours in water to remove the excess of the acid, then placed in dilute hæmatoxylin solution for twenty-four hours, or in 1 p. c. safranin solution for a similar time. After having been again rinsed in water it is rapidly dehydrated in absolute alcohol, then transferred to spirits of turpentine, and finally mounted in Canada balsam.]

Sketch an epithelium-cell with resting nucleus, and others with nuclei in different phases of karyomitosis.

An Epithelium is a tissue composed entirely of cells separated by a very small amount of intercellular substance (cement substance), and generally arranged so as to form a membrane covering either an external or an internal free surface.

The structure of epithelium-cells, and the changes which they undergo in cell-division, are best seen in the epidermis of the tail of the salamander-tadpole, in which the cells and nuclei are much larger than in mammals.

1 The methods of preparing sections are given in the Appendix.

2 If these tadpoles are not obtainable, the structure may be studied in the epidermis of the newt, prepared as recommended in Lesson VII. $\$ 1$ for the study of columnar epithelium, and also in sections stained with hæmatoxylin. 
Structure of the cells. - Each cell consists of protoplasm containing a nucleus. The protoplasm may be either clear or granular, or it may have a reticulated appearance. In some kinds of epithelium it is striated. The nucleus is a round or oval vesicle lying in the protoplasm. Usually there is only one, but there may be two or more in a cell. In the resting condition (i.e. when not undergoing division) the nucleus is composed of a membrane enclosing a clear substance, which is traversed by a network of filaments (fig. 17,a). There may be one

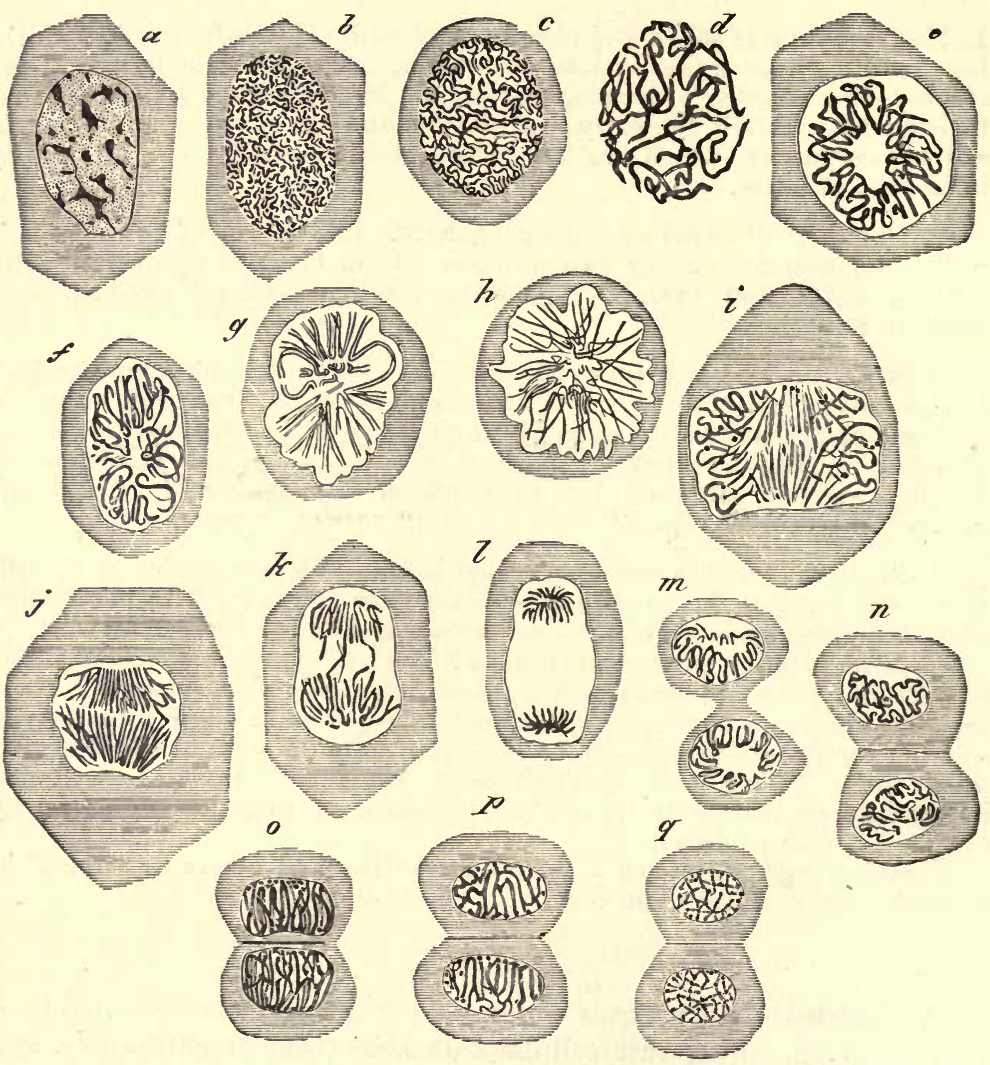

Fig. 17.-Epithelium-Cells of SAlamander LARVA in different Phases OF DIVISION.

or more globular enlargements (nucleoli) on this network. The nucleus is stained more easily by dyes than the protoplasm; this staining affects chiefly the nucleoli, nuclear filaments, and membrane.

Division of the cells. - The division of a cell is preceded by the division of its nucleus. This, in dividing, passes through a series of remarkable changes (fig. 17), which may thus be briefly summarised:-

1. 'The network of filaments of the resting nucleus becomes trans- 
formed into a sort of skein, formed apparently of one long convoluted filament; the nuclear membrane and the nucleoli disappear or are merged into the skein (fig. 17, $b, c, d$ ).

2. The skein becomes arranged in the form of a rosette, the filaments looping in and out, to and from the centre (e).

3. The outer loops of the rosette separate, so that the filament breaks into a number of $\mathrm{V}$-shaped fibres arranged like a star (aster, $f, g, h$.)

4. The V-shaped fibres separate into two groups, the ends of which for a time are interlocked $(i, j, k)$.

5. The two groups pass to the opposite poles of the now elongated nucleus and form a star-shaped figure $(l)$ at each pole (dyaster). Each of the stars represents a daughter nucleus.

$6,7,8$. Each star of the dyaster goes through the same changes as the original nucleus, but in the reverse order-viz. rosette $(m)$, skein $(n)$, and network $(o, p, q)$; passing finally into the condition of a typical resting nucleus. The protoplasm of the cell divides soon after the formation of the dyaster $(m)$. Sometimes fine lines may be seen in the protoplasm, during the process of division, radiating from the poles of the nucleus, and others uniting the two daughter nuclei.

- Classification of epithelia.-Epithelia are classified according to the shape and arrangement of the component cells. Thus we speak of scaly or pavement, cubical, columnar, polyhedral, and spheroidal epithelium. All these are simple epithelia, with the cells only one layer deep. If forming several superposed layers, the epithelium is said to be stratified, and then the shape of the cells differs in the different layers. Where there are only three or four layers in a stratified epithelium, it is termed transitional.

Stratified Epithelium covers the anterior surface of the cornea, lines the mouth, pharynx (lower part), and gullet, and forms the epidermis which covers the skin. In the female it lines the vagina and

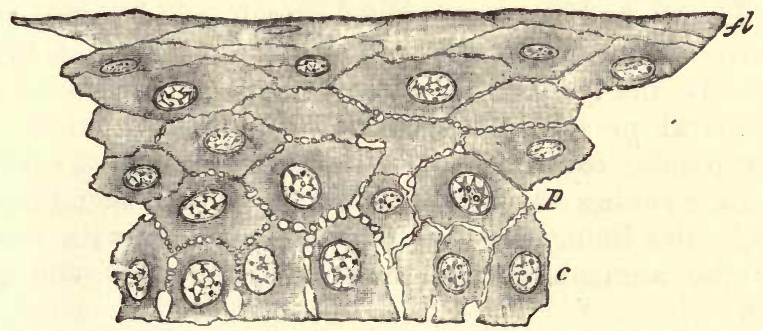

Fig. 18.-Section of the stratified epitheliun Covering the frovt of THE CORNEA OF THE EYE.

$c$, lowermost columnar cells; $p$, polygonal cells above these ; $f$, flattened cells near the : urface.

part of the uterus. The cells nearest the surface are always flattened and scale-like (fig. 18, $f$; fig. 19), whereas the deeper cells are more 
rounded or polyhedral, and those of the deepest layer generally somewhat columnar in shape (fig. 18,c). Moreover, the deeper cells are soft and protoplasmic, and are separated from one another by a system of intercellular channels, which are bridged across by numerous processes passing from cell to cell.

The deeper cells multiply by division, the newly formed cells tending as they enlarge to push those external to them nearer to the surface, from which they are eventually thrown off. As they approach the surface they become hard and horny, and in the case of the epidermis lose entirely their cellular appearance, which can, however, be in a measure restored by the action of potash $(\$ 2)$. The cast-off superficial cells of the stratified epithelium of the mouth, which are seen in abundance in the saliva ( $\S 1$ ), are less altered, and the remains of a nucleus is still visible in them.

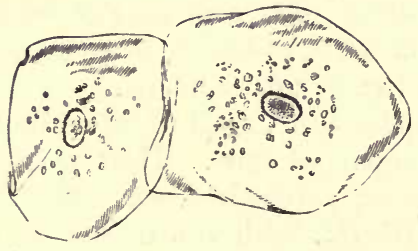

Fig. 19.-EPithelium-SCALES From the inside OF THE MOUTH. (Magnified 260 diameters.)

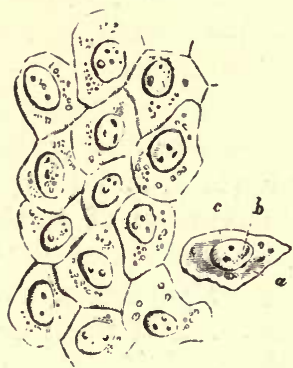

Fig. 20.-Pavement-epithelium from A Serous membrane. (Magnified 410 diameters.)

$a$, cell ; $b$, nucleus ; $c$, nucleoli.

Simple scaly or pavement epithelium is found in the saccules of the lungs, in those of the mammary gland when inactive, in the kidney (in the tubes of Henle), and also lining the cavities of serous membranes (fig. 20), and the heart, blood-vessels, and lymphatics. When occurring on internal surfaces, sutch as those of the serous membranes, blood-vessels, and lymphaties, it is often spoken of as endothelium.

Polyhedral or spheroidal epithelium is characteristic of many secreting glands; columnar and ciliated epithelıum are for the most part found covering the inner surface of mucous membranes, which are membranes lining passages in communication with the exterior, such as the alimentary canal and the respiratory and generative passages.

The detailed study of most of these may be reserved until the organs in which they occur are respectively dealt with.

The hairs and nails and the enamel of the teeth are modified epithelial tissues. 


\section{LESSON VII. \\ COLUMNAR AND CILIATED EPITHELIUM, AND \\ TRANSITIONAL EPITHELIUM.}

1. TAKE a piece of rabbit's intestine which has been two days in chromic acid solution ( 1 part chromic acid to 2,000 normal salt solution). Scrape the inner surface with a scalpel, break up the scrapings in a drop of water on a slide. Add a small piece of hair to avoid crushing, and cover the preparation. Sketch one or two columnar cells and also a row of cells. Measure two or three cells and their nuclei.

To keep this preparation, add a drop of dilute hæmatoxylin (1 drop of the ordinary solution to half a watch-glass-ful of distilled water) at one edge of the cover-glass. When the hæmatoxylin has passed in and has stained the cellnuclei, place a drop of glycerine at the same edge, and allow it slowly to diffuse under the cover-glass. Cement this another day. ${ }^{1}$

2. Break up in glycerine a shred of epithelium from a piece of frog's intestine that has been treated with osmic acid, and has subsequently macerated in water for a few days. The cells easily separate on tapping the cover-glass. They are larger than those of the rabbit and exhibit certain points of structure better. Measure and sketch one or two cells.

The cover-glass may be at once fixed by gold size.

3. Prepare the ciliated epithelium from a trachea that has been in bichromate of potash solution ( $\frac{1}{8}$ per cent.) for two days, in the same way as in \$1. Measure in one or two of the ceils $(a)$ the length of the cell, $(b)$ the length of the cilia, $(c)$ the size of the nucleus. Sketch two or three cells.

This preparation is to be stained and preserved as in $\S 1$.

4. Make a similar teased preparation of the epithelium of the urinary bladder. Observe the large flat superficial cells, and the pear-shaped cells of the second layer. Measure and sketch one or two of each kind.

Stain and preserve as in $\S \S 1$ and 3.

Columnar epithelium.-The cells of a columnar epithelium (fig. 21) are prismatic columns, which are set closely side by side, so that when seen from the surface a mosaic appearance is produced. They often taper somewhat towards their attached end, which is generally truncated, and set upon a basement membrane. Their free surface is covered by a thick striated border (fig. 22 , str), which may sometimes become detached in teased preparations. The protoplasm of the cell is highly vacuolated or reticular, and fine longitudinal striæ may be seen in it, which appear continuous with the striæ of the

' Gentian-violet solution (see Appendix) may be employed instead of hæma toxylin. 
free border. The nucleus $(n)$ is oval and reticular. The lateral borders of the cells are often somewhat irregular or jagged, the result of the pressure of amœboid lymph-cells, which are generally found between the columnar cells, at least in the intestine. After a

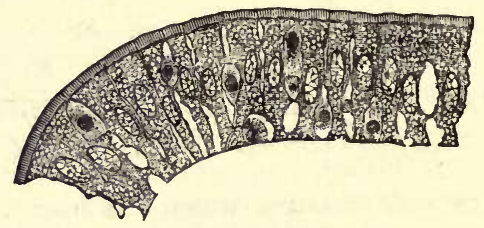

Fig. 21.-A row of columar cells from the intestine of the rabitt.

Smaller cells are seen between the epithelium-cells, probably of the nature of white blood or ly mph corpuscles.

meal containing much fat the cells may be filled with fat-globules, which become stained black in the osmic preparation.

Some of the columnar cells contain mucigen, which may greatly distend the part of the cell nearest the striated border. When the

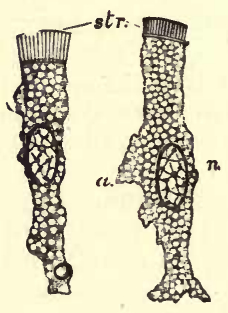

t.

Fig. 22.-Columar epithelium-cells of the rabit's intestine.

The cells have been isolated after maceration in very weak chromic acid. . The cells are much vacuolated, and one of them has a fat-globule near its attached end; the striated border $(s t r)$ is well seen, and the bright disk separating it from the cell-protoplasm; $n$, nucleus with intranuclear network; $a$, a thinned-out wing-like projection of the cell which probably fitted between two adjacent cells.

mucigen is extruded as mucus, this border is thrown off, and the cell takes the form of an open cup or chalice (goblet-cell, fig. 23).

Columnar epithelium-cells are found lining the whole of the interior of the stomach and intestines: they are also present in the ducts of most glands, and sometimes also in their secreting saccules. The epithelium which covers the ovary also has a modified columnar shape, but cells having all the structural peculiarities indicated above are found only in the alimentary canal and in its diverticula.

Ciliated epithelium.-The cells of a ciliated epithelium are also usually columnar in shape (fig. 24), but in place of the striated border the cell is surmounted by a bunch of fine tapering filaments which, 
during life, move spontaneously to and fro, and serve to produce a current of fluid over the surface which they cover.

The cilia are to be regarded as active prolongations of the cellprotoplasm. The border upon which they are set is bright, and appears formed of little juxtaposed knobs, to each of which a cilium is attached. In the large ciliated cells which line the alimentary canal of some molluses (fig. 25) the knob may be observed to be prolonged into the protoplasm of the cell as a fine varicose filament, termed the rootlet of the cilium. These filaments may represent the longitudinal striæ often seen in the protoplasm of the columnar cell, the bunch of cilia being homologous with the striated border. The protcplasm and nucleus have a similar vacuolated or reticular structure in both kinds of cell. Goblet cells may also occur in ciliated epithelia.

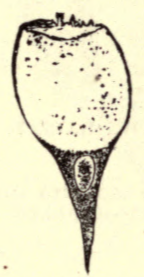

Fig. 23.-Goblet Cell.

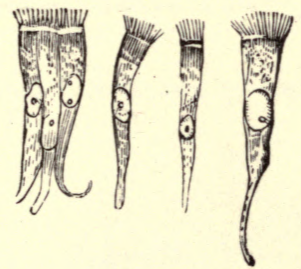

Fig. 24.-Columnar CiliATED EPITHELIUM-CELLS.

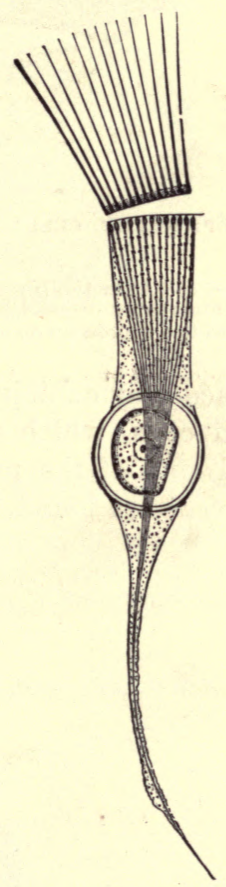

Fig. 25.-Ciliated cell, FROM THE INTESTINE of A MOllusc.

Ciliated epithelium is found throughout the whole extent of the air-passages and their prolongations (but not the part of the nostrils supplied by the olfactory nerves, nor in the lower part of the pharynx); in the Fallopian tubes and the greater part of the uterus; in some of the efferent ducts of the testicle (where the cilia are much longer than 
elsewhere in the body); in the ventricles of the brain, and the central canal of the spinal cord; and in the convoluted tubules of the kidney.

Transitional epithelium is a stratified epithelium consisting of only two or three layers of cells. It occurs in the urinary bladder, the ureter, and the pelvis of the kidney. The superficial cells (fig. $26, a)$ are large and flattened; they often have two nuclei. On their

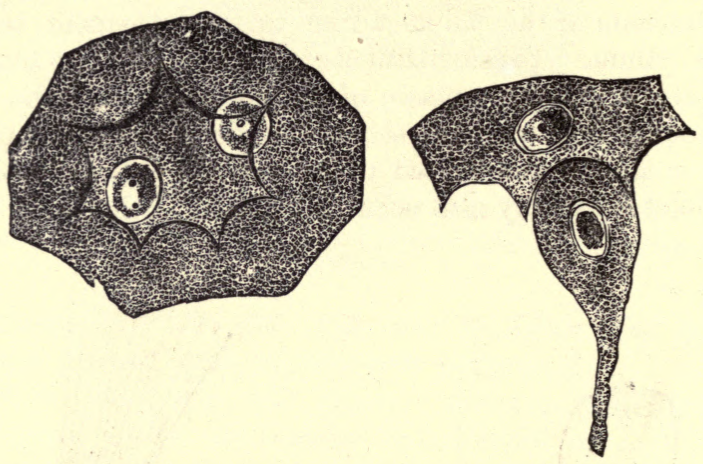

Fig. 26.-Epithelial cells from the bladder of the rabits. (Magnified 500 diameters.)

$a$, large flattened cell from the superficial layer, with two nuclei and with strongly marked ridges and intervening depressions on its under surface; $b$, pear-shaped cell of the second layer adapted to a depression on one of the superficial cells.

under surface they exhibit depressions, into which fit the larger ends of pyriform cells, which form the next layer (fig. 26, $b$ ). Between the tapered ends of the pyriform cells one or two layers of smaller polyhedral cells are found. 


\section{LESSON VIII. \\ STUDY OF CILIA IN ACTION.}

1. Mount in sea-water one or two bars of the gill of the marine mussel (fig. 27). Study the action of the large cilia. Now place the preparation upon the copper warm stage (see Lesson V.) and observe the effect of raising the temperature.

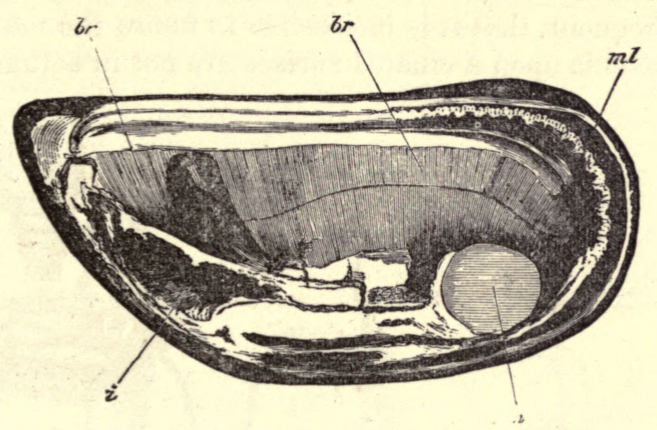

Fig. 27.-VAlve of mussel (mytilus edulis) Showing $b r$, $b r$, The Expanded GILLS OR BRANCHIÆ, WHICH, OWING TO THE LITTLE BARS OF WHICH THEY ARE COMPOSED, FRESENT A STRIATED ASPECT.

$m l$, mantle; $m$, cut adductor musole ; $i$, mass of viscera ; the dark projection just above is the foot.

Keep this preparation until the end of the lesson, by which time many of the cilia will have become languid. When this is the case pass a drop of dilute potash solution (1 part KHO to 1,000 of sea-water) under the cover-glass and observe the effect.

2. Cement with sealing-wax a piece of small glass tubing to a slide so that

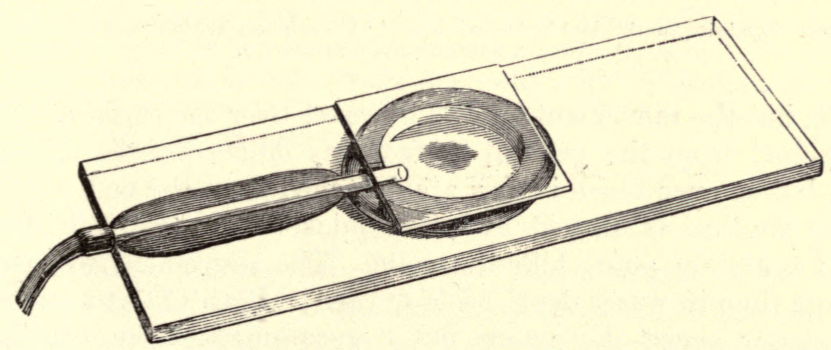

Fig. 28.-MOIST CHAMBER ADAPTED For PASSING A GAS OR VAPOUR to A PREPARATION UNDER THE MICROSCOPE.

one end of the tube comes nearly to the centre of the slide. On this put a ring of putty or modelling wax half an inch in diameter so as to include the 
end of the tube, and make a deep notch in the ring opposite the tube. Place a small drop of water within the ring (fig. 28).

Put a bar from the gill upon a cover-glass in the least possible quantity of sea-water; invert the cover-glass over the putty ring, and press it gently down. The preparation hangs in a moist chamber within which it can be studied through the cover-glass, and into which gases or vapours can be passed and their effects observed.

Pass $\mathrm{CO}_{2}$ through the chamber, and after observing the effect replace it by air (see fig. 29). Repeat with chloroform vapour instead of $\mathrm{CO}_{2}$.

The Movement of Cilia.-When in motion a cilium is bent quickly over in one direction with a lashing whip-like movement, immediately recovering itself. When vigorous the action is so rapid, and the rhythm so frequent, that it is impossible to follow the motion with the eye. All the cilia upon a ciliated surface are not in action at the same

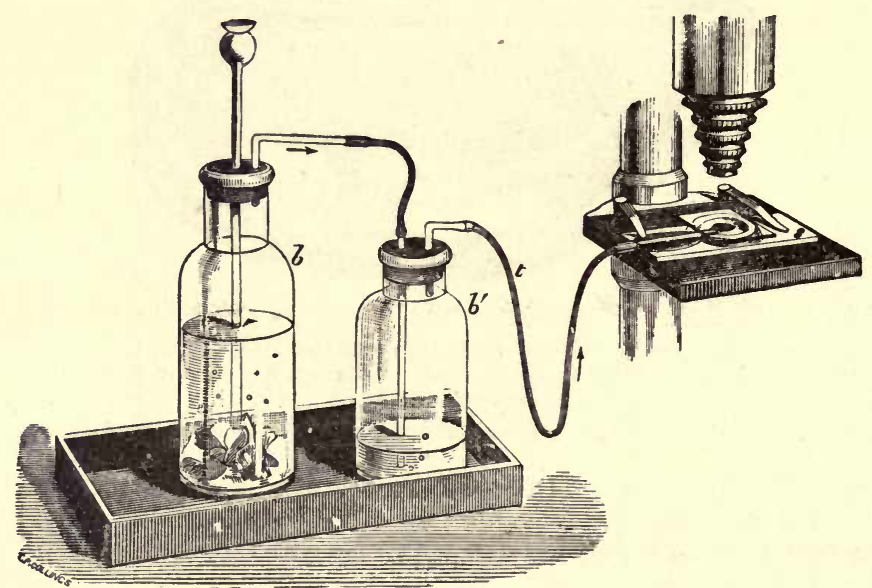

Fig. 29.-Method of subjecting a preparation to a stream of carbonic ANHYDRIDE.

$b$, bottle containing marble and hydroch'oric acid; $b^{\prime}$ wash-bott'e, connected by indiarubber tube, $t$, with the moist chamber, $s$.

instant, but the movement travels in waves over the surface. If a cell is detached from the general surface, its cilia continue to act for a while, but at once cease if they are detached from the cell.

The rhythm is slowed by cold, quickened by warmth, but heat beyond a certain point kills the cells. The movement will continue for some time in water deprived of oxygen. Both $\mathrm{CO}_{2}$ gas and chloroform vapour arrest the action, but it recommences on restoring air. Dilute alkaline solutions quicken the activity of cilia, or may even restore it shortly after it has ceased. 


\title{
LESSON IX.
}

\section{THE CONNECTIVE TISSUES.}

\author{
AREOLAR AND ADIPOSE TISSUE.
}

1. TAKE a little of the subcutaneous tissue or of the intermuscular connective tissue of a rabbit or guinea-pig and spread it out with needles on a dry slide into a large thin film. Keep the centre moist by occasionally breathing on it, but allow the edges to dry to the slide. Before commencing put a drop of salt solution on a cover-glass, and now invert this over the film. Examine with a high power. Sketch one or two bundles of white fibres and also one or two elastic fibres, distinguishable from the former by their sharp outline, isolated course, and by their branching. Sketch also one or more connective-tissue corpuscles, if any such are visible in the clear interspaces. Look also for migratory cells (lymph-corpuscles). Next carefully remove the cover-glass and replace the salt solution by dilute acetic acid. Watch its effect in swelling the white fibres and bringing more clearly into view the elastic fibres and corpuscles. Look for constricted bundles of white fibres.

2. Make another film in the same way, but mount in dilute magenta solution ${ }^{1}$ instead of saline solution. The elastic fibres are deeply stained by the dye; the cells are also well shown. When the staining is completed pass dilute glycerine under the cover-glass and cement this at once with gold size.

3. Prepare another film of the subcutaneous tissue, including a little adipose tissue. Mount in glycerine and water, coloured by magenta, with a piece of hair under the cover-glass to keep this from pressing unduly upon the fat-cells. Cement at once with gold size. Examine first with a low and afterwards with a high power. The nucleus and envelope of the fat-cell are well brought out by the magenta, and if from a young animal, fat-cells will be found in process of formation. Measure and sketch two or three of the cells.

4. Spread out another large film of connective tissue, letting its edges dry to the slide. Place on its centre a large drop of nitrate of silver solution (1 per cent.). After ten minutes wash this away with distilled water, mount in Farrant ${ }^{1}$, and expose to the sunlight until stained brown. Sketch the outlines of two or three of the cell-spaces.

The connective tissues include areolar tissue, adipose tissue, elastic tissue, fibrous tissue, retiform and lymphoid tissue, cartilage and bone. All these tissues agree in certain microscopical and chemical characters. They, for the most part, have a large amount of intercellular substance in which fibres are developed, and these fibres are of two

1 See Appendix. 
kinds-white and yellow or elastic. Moreover, there are many points of similarity between the cells which occur in these several tissues; they are also developed from the same embryonic formation, and they tend to pass imperceptibly the one into the other. Besides this, their use is everywhere similar; they serve to connect and support the other tissues, performing thus a passive mechanical function. They may therefore be grouped together, although differing considerably in external characters. Of these connective tissues, however, there are three which are so intimately allied as to be naturally considered together, being composed of exactly the same elements, although differing in the relative development of those elements; these are the areolar, elastic, and fibrous tissues (adipose tissue may be looked upon as a special modification of areolar tissue). Areolar tissue being the commonest and, in one sense, the most typical, its structure may be considered first.

Areolar tissue.-The areolar tissue presents to the naked eye an appearance of fine transparent threads and laminæ which intercross in every direction with one another, leaving intercommunicating meshes, or areolæ, between them. When examined with the microscope, these threads and fibres are seen to be principally made up of wavy bundles of exquisitely fine transparent fibres (white fibres, fig. 30). The bundles run in different directions, and may branch and intercommunicate with one another; but the individual fibres, although they pass from one bundle to another, never branch or join other fibres. The
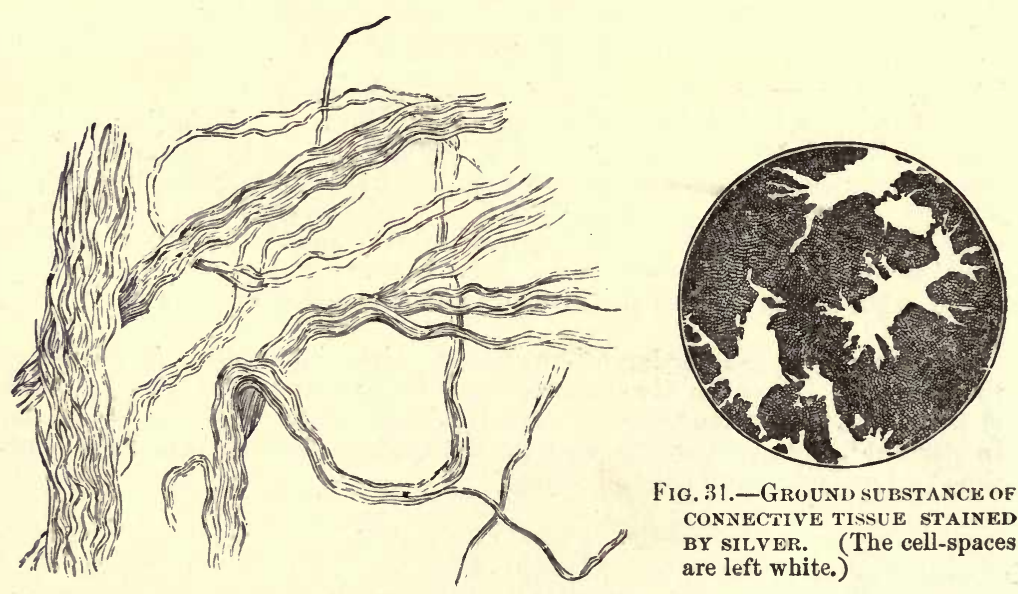

Fig. 31.-Ground SUBSTANCE OF CONNECTIVE TISSUE STAINED BY sILVER. (The cell-spaces are left white.)

Fig. 30.-Bundles of THE WHITE Fibres OF AREOLAR TISSUE PARTLY UNRAVELLED.

fibres are cemented together into the bundles by a clear substance containing mucin, and the same clear material forms also the basis or ground-substance of the tissue, in which the bundles themselves course, and in which also the corpuscles of the tissue lie embedded. This 
ground-substance between the bundles can with difficulty be seen in the fresh tissue on account of its extreme transparency; but it can be brought to view by staining with nitrate of silver, as in $\$ 4$. The whole of the tissue is thereby stained of a brown colour, with the exception of the spaces which are occupied by the corpuscles (cell-spaces, fig. 31).

Besides the white fibres of connective tissue here described, fibres of a different kind (fig. 32) may be made out in the preparations;

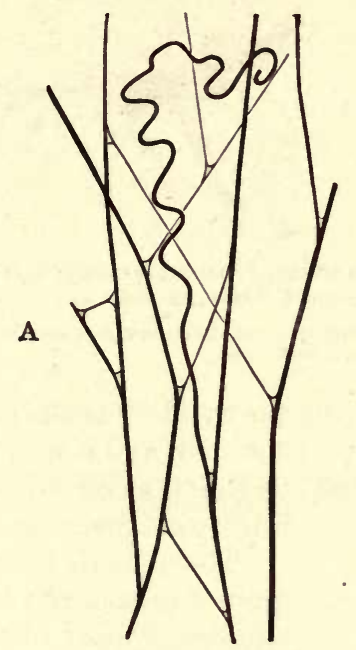

FIG. 32.

A. Elastic fibres of areolar tissue. From the subcutaneous tissue of the rabbit. B (from Toldt). A white bundle swonen by acetic acid. From the subarachnoid tissue at the base of the brain.

these are the elastic fibres. They are especially well seen after treatment with acetic acid, and after staining with magenta ; but they can be detected also in the fresh preparation. They are characterised by their distinct outline, their straight

B

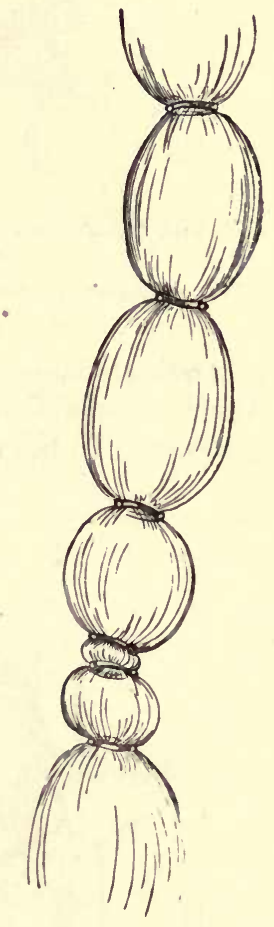
course, the fact that they never run in bundles, but singly, and that they branch and join neighbouring fibres. If broken by the needles in making the preparation, the elastic recoil causes them to curl up, especially near the broken ends. Besides the microscopical differences, the two kinds of fibres differ also in their chemical characters. Thus the white fibres are dissolved by boiling in water, and yield gelatin; whereas the substance of which the elastic fibres are composed (elastin) resists for a long time the action of boiling water. Moreover, the white fibres swell and become indistinct under the action of acetic acid; the elastic fibres are unaltered by this reagent.

The bundles of white fibres which have been swollen out by acid sometimes exhibit curious constrictions (fig. 32). These are due either 
to elastic fibres coiling round the white bundles, or to cell processes encircling them, or to an investment or sheath which remains unbroken at certain parts, and thus prevents the swelling up of the bundle at these places.

The cells of areolar tissue are generally flattened and more or less branched (fig. 33), but may be of an elongated form. They usually con-
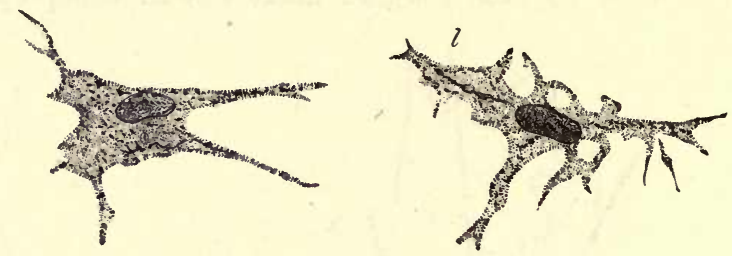

Fig. 33.-Two FLATtened AND BRANCHED CONNECTIVE-TISSUE Corpuscles FROM THE SUBCUTANEOUS AREOLAR TISSUE.

Opposite $l$ a secondary lamella, projecting towards the observer, is seen in optical section as a dark line.

tain a single large oval nucleus having the usual structure of cell-nuclei. Their protoplasm is generally much vacuolated, and it may also contain granules. In the middle coat of the eye the connective-tissue cells are filled with granules of pigment.

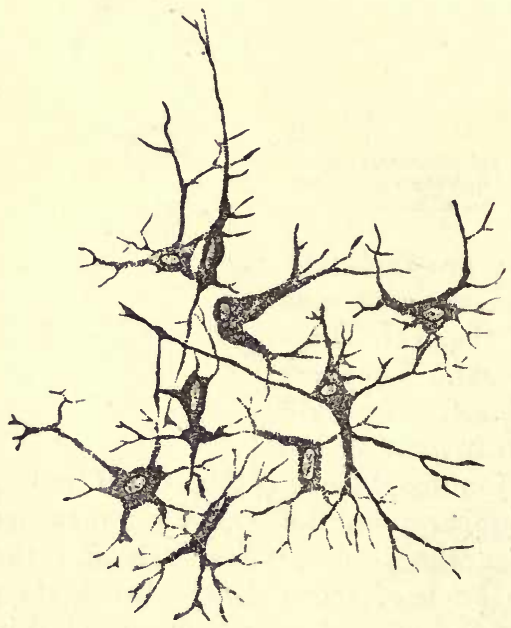

Fig. 34. - Ramified connective-tissue CORPUSCLES, From ARTICULAR SYNOVIAI. MEMBRANE OF OX.

The cells lie in spaces in the ground-substance between the bundles of white fibres. In some parts of the connective tissue the white bundles are developed to such an extent as to pervade almost the whole of the groundsubstance, and then the connective-tissue corpuscles become squeezed into the interstices, flattened lamellar expansions of the cells extending between the bundles, as in tendon (see next resson). The cells are frequently joined either into a network by branching processes (fig. 34), or edge to edge, like the cells of an epithelium (fig. 35); in either case the cell-spaces are also conjoined equally intimately.

Besides the flattened, branched, and elongated connective-tissue corpuscles, others are met with which are like very large lymph-corpuscles in appearance, and are filled with distinct granules, which are stained deeply by aniline dyes. These cells are very common in the neighbourhood of blood-vessels, espe- 


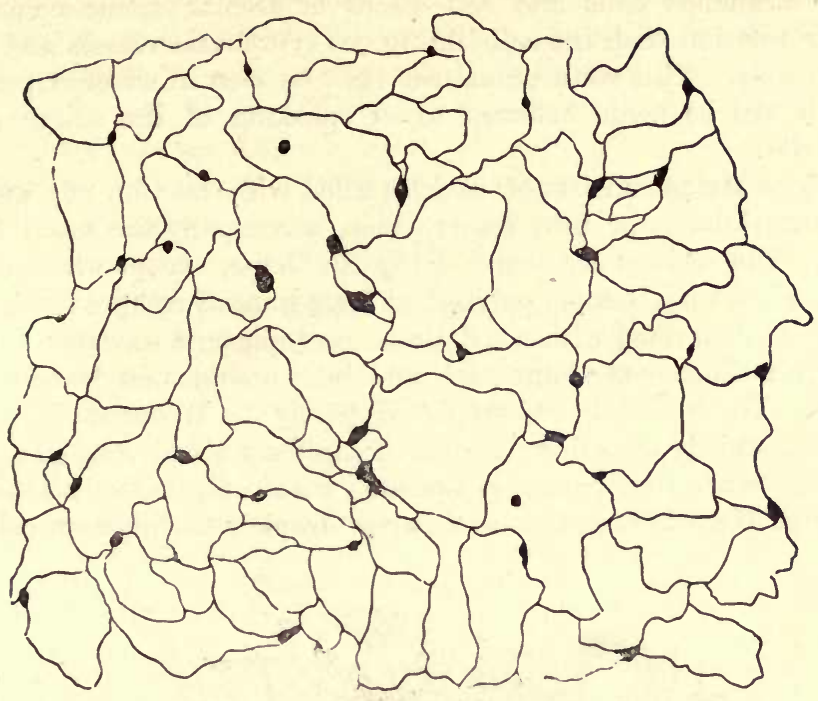

Fig. 35.-Epithelioid cells of convective tissue, from the surface of

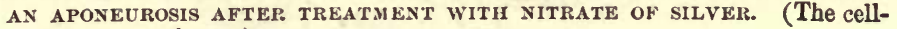
nuclei are not shown.)

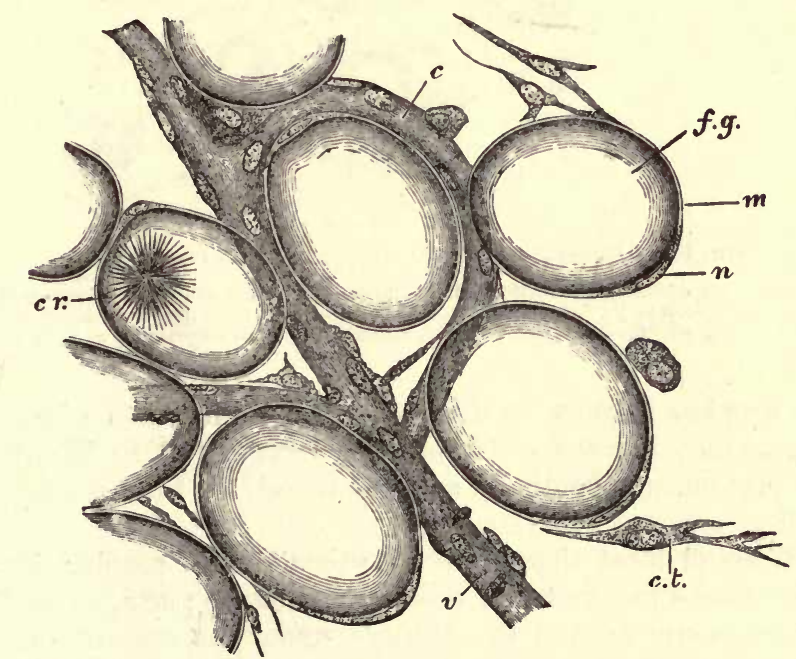

Fig. 36.-A FEW cells from the MARgin of a FAt-Lobule.

$f g$, fat-globule distending a fat-cell ; $n$, nucleus ; $m$, membranous envelope of the fat-cell ; $c r$, bunch of crystals within a fat-cell ; $c$, capillary vessel ; $v$, venule ; $c t$, connectivetissue cell; the fibres of the connective tissue are not represented.

cially where fat is becoming developed (see fig. 37); they exhibit slight indications of amœboid movement, and are known as the plasma-cells. Migratory or lymph-cells may also be seen here and there in the tissue. 
The branched cells and cell-spaces of areolar tissue come into intimate relation with the cells lining the lymphatic vessels and small blood-vessels. This connection can best be seen in silvered preparations; it will be again referred to in speaking of the origin of the lymphatics.

Adipose tissue consists of vesicles filled with fat (fig. 36), and collected into lobules or into tracts which accompany the small bloodvessels. The vesicles are round or oval in shape, except where closely packed, when they become polyhedral from mutual compression. The fat-drop is contained within a delicate protoplasmic envelope (fig. 36, $m$ ) which is thickened at one part, and here includes an oval flattened nucleus. The vesicles are supported partly by filaments of areolar tissue, but chiefly by a fine network of capillary blood-vessels.

The fat when first formed is deposited within plasma-cells of areolar tissue (fig. 37). It is at first in separate droplets within each cell, but

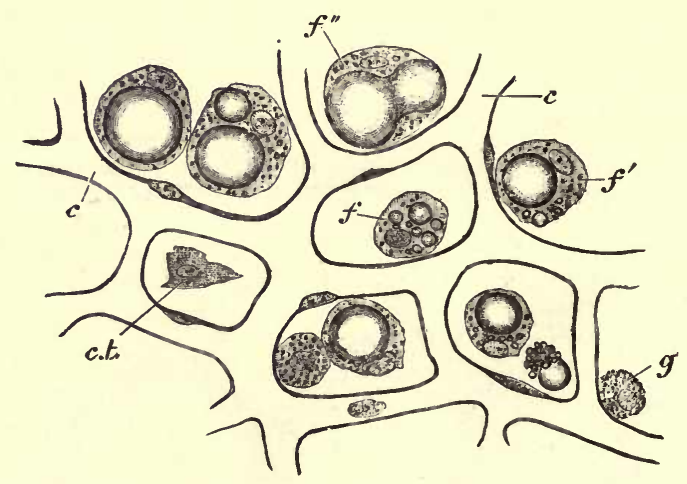

Fig. 37.-Deposition uf FAT in Convective-Tissue cells.

$f$, a cell with a few isolated fat-droplets in its protoplasm ; $f^{\prime}$, a cell with a single large and several minute drops; $f^{\prime \prime}$, fusion of two large drops; $g$, granular or plasma cell, not yet exhibiting any fat-deposition; $c t$, flat connective-tissue corpuscle; $c, c$, network of capillaries.

as these droplets increase in size they run together into a larger drop, which gradually fills the cell more and more, swelling it out so that the cell-protoplasm eventually appears merely as the envelope of the fat-vesicle.

Fat is found most abundantly in subcutaneous areolar tissue, and under the serous membranes; especially in some parts, as at the back of the peritoneum around the kidneys, under the epicardium, and in the mesentery and omentum. The marrow of the long bones is also principally composed of fat. 


\section{LESSON X. \\ THE CONNECTIVE TISSUES (continued).}

\section{Elastic tissue, FIBRous tissue, SPECIAL VARIETIES, DEVElopMent OF CONNECTIVE TISSUE.}

1. TEASE out as finely as possible a small shred of elastic tissue (ligamentum nuchæ of the ox or ligamenta subflava of man) in Farrant's solution ' and cover the preparation. Note the large well-defined fibres constantly branching and uniting with one another. Look for transverse markings on the fibres. Measure three or four. Sketch a small part of the network. Note the existence of bundles of white fibres amongst the elastic fibres.

2. Mount in Farrant a thin transverse section of ligamentum nuchæ which has been hardened in 2 per cent. solution of bichromate of potash. Observe the grouping of the fibres and their angular shape. Sketch one or two groups.

3. Pinch off the end of the tail of a dead mouse or rat, draw out the long silk-like tendons and put them into saline solution. Take two of the longest threads and stretch them along a slide, letting the ends dry firmly to the slide but keeping the middle part moist. Put a piece of hair between them and cover in saline solution. Observe with a high power the fine wavy fibrillation of the tendon. Draw. Now run dilute acetic acid under the cover-glass, watch the tendons where they are becoming swollen by the acetic acid. Notice the oblong nucleated cells coming into view between the tendon bundles. Sketch three or four cells in a row. Lastly, lift the cover-glass, wash away the acid with distilled water, place a drop of hæmatoxylin solution on the tendons, and leave the preparation for fifteen minutes or more; then wash away the logwood and mount the preparation in acidulated glycerine. Cement the cover-glass with gold size.

4. Immerse one or two other pieces of tendon in nitrate of silver solution (1 per cent.) for ten minutes, then wash them in distilled water, stretch them upon a slide, mount in Farrant, and expose to the sunlight.

5. Stain with magenta solution ${ }^{1}$ a thin section of a tendon which has been hardened in alcohol. Mount in dilute glycerine and cement the coverglass at once. Sketch a portion of the section under a low power.

Elastic tissue is a variety of connective tissue in which the elastic fibres preponderate. It is found most characteristically in the ligamentum nuchæ of quadrupeds and the ligamenta subflava of the vertebræ, but the connective tissue of other parts may also have a con-

1 See Appendix. 
siderable development of elastic fibres. It occurs also in an almost pure form in the walls of the air-tubes, and uniting the cartilages of the larynx. It also enters largely into the formation of the walls of the blood-vessels, especially the arteries.

In the ligamentum nuchæ the fibres are very large and angular (fig. 38) ; they often exhibit cross-markings or even transverse clefts. When dragged asunder, they break sharply across; they constantly branch and unite, so as to form a close network. In transverse section they are seen to be separated into small groups (fig. 39) by intervening white bundles of connective tissue.
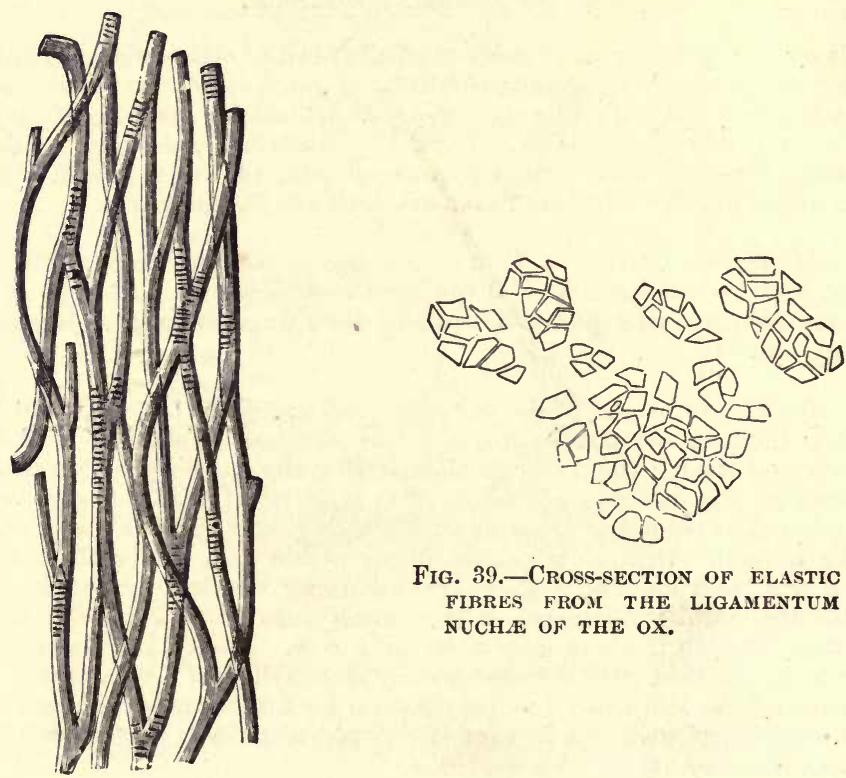

Fig. 39.-Cross-section of elastic FIBRES FROM THE LIGAMENTUM NUCHA OF THE OX.

Fig. 38.-Elastic fibres fron the ligaMENTUM NUCHE OF THE OX, SHOWING TRANSVERSE MARKINGS ON THE FIBRES.

Elastic tissue does not always take the form of fibres, but may occur as membranes (as in the blood-vessels). Sometimes the fibres are very small, but their microscopical and chemical characters are always very well marked (see p. 31).

Fibrous tissue is almost wholly made up of bundles of white fibres running in a determinate direction. These again are collected into larger bundles, which give the fibrous appearance to the tissue. The bundles are constantly uniting with one another in their course, although their component fibres remain perfectly distinct.

The interspaces between the larger bundles are occupied by areolar tissue (fig. 40) in which the blood-vessels and lymphatics of the fibrous 


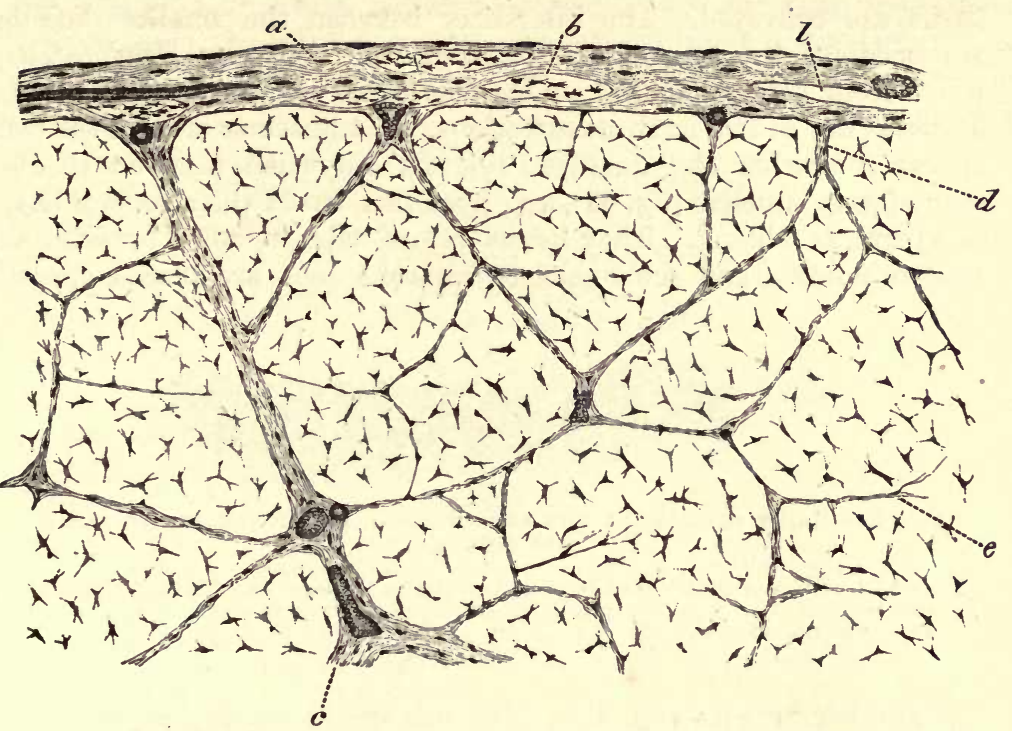

Fig. 40.-Part of a large tendon in transverse section.

$a$, areolar sheath of the tendon, with the fibres for the most part running transversely, but with two or three longitudinal bundles, $b ; l$, lymphatic cleft in the sheath; immediately over it a blood-vessel is seen cut across, and on the other side of the figure a small artery is shown cut longitudinally; $c$. large septum of areolar tissue; $d$, smaller septum; $e$, still smaller septum. The irregularly stellate bodies are the tendon cells in section.

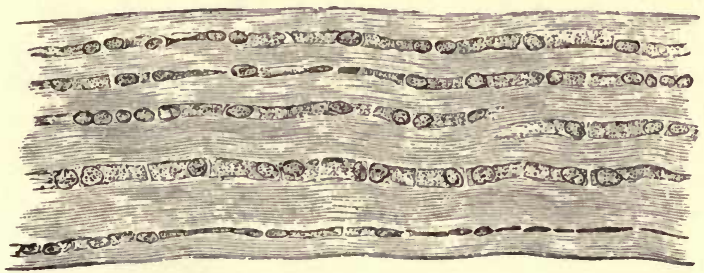

Fig. 41.-Tendon of mouse's tail, stained with log woon; showing chains OF CELLS BETWEEN THE TENDON-BUNDLES. (175 diameters.)

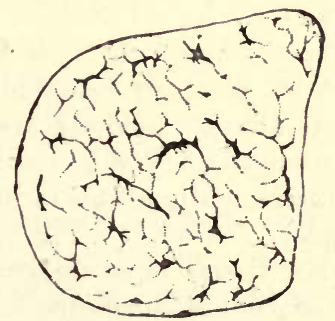

Fig. 42.-Transverse section of tendon of Mouse's tail, stalNed. (175 diameters.)

The flattened processes of the tendon-cells appear in section as lines, frequently coming off ac right angles from the body of the cell. 
tissue are conveyed. The interstices between the smallest bundles are occupied by rows of connective-tissue corpuscles (tendon-cells), which from being squeezed up between three or more bundles become ilattened out in two or three directions. In transverse section the cells appear somewhat stellate (figs. 40,42), but when seen on the flat they appear lamellar (fig. 41), and from this aspect their general shape is square or oblong. They lie, as before said, in rows between the tendon-bundles, and the nuclei of adjacent cells are placed opposite

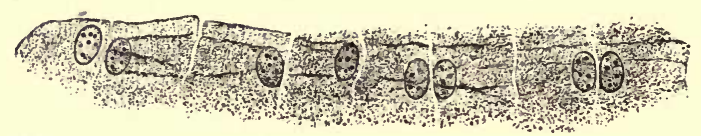

Fig. 43.-Eight cells from the same tendon as Represented in FiG. 41. (425 diameters.)

The nuclei, with their numerous nucleoli, are coloured by the logwood. The dark lines on the surface of the cells are the optical sections of lamellar extensions directed towards or away from the observer.

one another in pairs (fig. 43). The cell-spaces correspond in general figure and arrangement to the cells which occupy them (fig. 44).

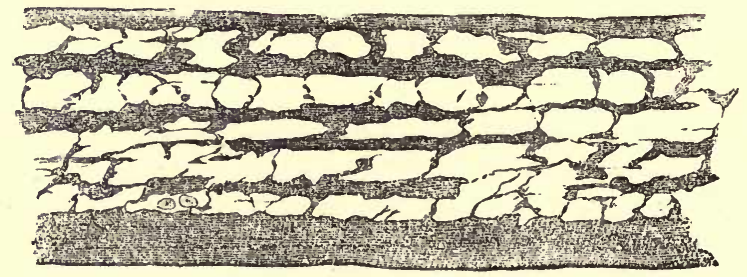

Fig. 44.-Cell-spaces of tendon of MoUsi's tail, Brovght into View by TREATMENT WITH NITRATE OF SILIER. (175 diameters.)

Fibrous tissue forms the tendons and ligaments, and also certain membranes, such as the dura mater, the fibrous pericardium, the fasciæ of the limbs, the fibrous covering of certain organs, \&c. It is found wherever great strength combined with flexibility is concerned. It receives a few blood-vessels, disposed longitudinally for the most part, and contains many lymphatics. Tendons and ligaments also receive nerve-fibres, which, in some cases, end in small localised ramifications like the end-plates of muscle, while others terminate in end-bulbs or in simple Pacinian corpuscles.

Retiform or reticular tissue is a variety of connective tissue in which the intercellular or ground substance has mostly disappeared or is replaced by fluid, very few or no fibres having been developed in it; and these, when present, are enwrapped by the cells. The tissue is composed almost entirely of the cells, which are ramified and united 
with one another into a network or sponge-work by their processes; in some cases, the cell-nuclei have disappeared (as at $b$, fig. 45).

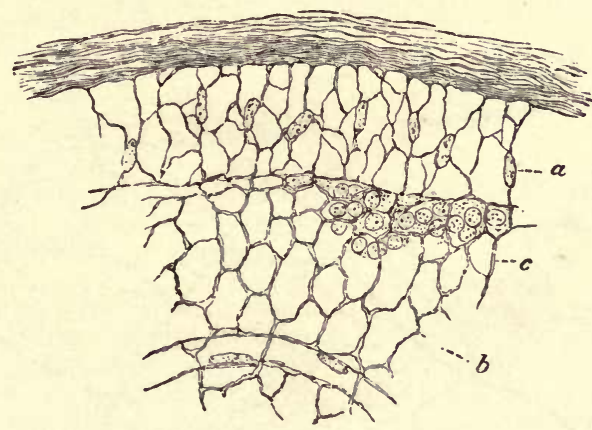

Fig. 45.-Thin section From the cortical PART OF a lymphatic gland, MAGNIFIED.

A network of fine trabeculæ formed by retiform tissue, from the meshes of which the lymph-corpuscles have been washed out, except at $c$, where they are left.

Lymphoid or adenoid tissue is retiform tissue in which the meshes of the network are largely occupied by lymph-corpuscles. This is by far the most common condition of a retiform tissue, and is met with in the lymphatic glands and allied structures (see Lesson XXII.), and also in the tissue of the alimentary mucous membrane, and in some other situations.

Basement membranes (membranæ propriæ) are homogeneous-looking membranes, which are found forming the surface-layers of connective-tissue expansions in many parts, especially where there is a covering of epithelium, as on mucous membranes, in secreting glands, and elsewhere. They are generally formed of flattened connectivetissue cells joined together to form a membrane; but, in some cases, they are evidently formed not of cells, but of condensed groundsubstance, and in others they are of an elastic nature.

Jelly-like connective tissue, although occurring largely in the embryo, is found only in one situation in the adult-viz. forming the vitreous humour of the eye. It seems to be composed entirely of soft ground-substance, with cells scattered here and there through it, and with very few fibres, or none at all. These several varieties of connective tissue will be more fully described in connection with the organs where they occur.

Development of connective tissue. Connective tissue is always developed in the mesoblast or mesoderm of the embryo. In those parts of this layer which are to form connective tissue, the embryonic cells become separated from one another by a muco-albuminous semifluid intercellular substance (ground-substance), but the cells generally remain connected by their processes. The connective-tissue fibres, 
both white and elastic, are deposited in this ground-substance, the elastic substance usually in the form of granules (fig. $47, g$ ), which

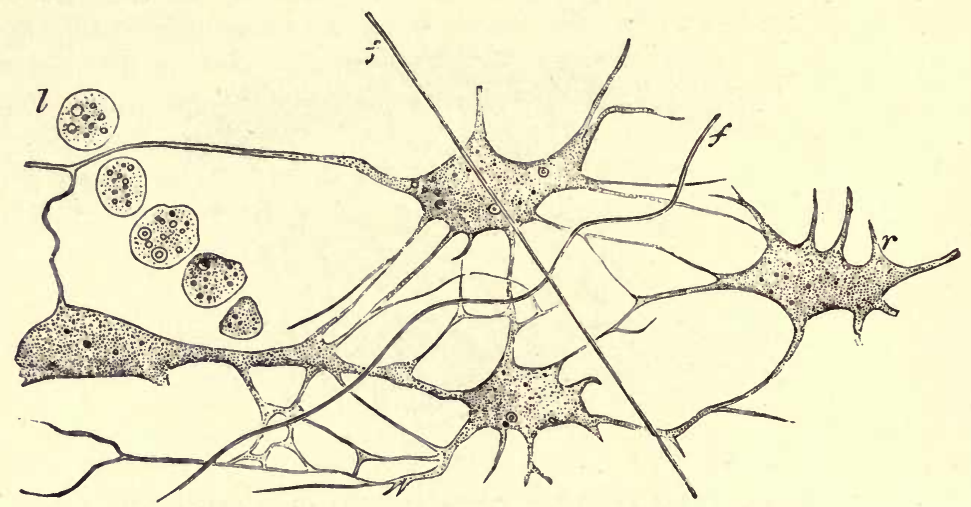

Fig. 46.-JeLLy OF Wharton.

$r$, ramified cells intercommunicating by their branches; $l$, a row of lymph-cells ; $f$, fibres developing in the ground-substance.

subsequently become connected together into elastic fibres or laminæ, as the case may be, the white fibres appearing at first in the form of very fine bundles, which afterwards become gradually larger; so that in fibrous tissue the whole ground-substance is eventually pervaded by

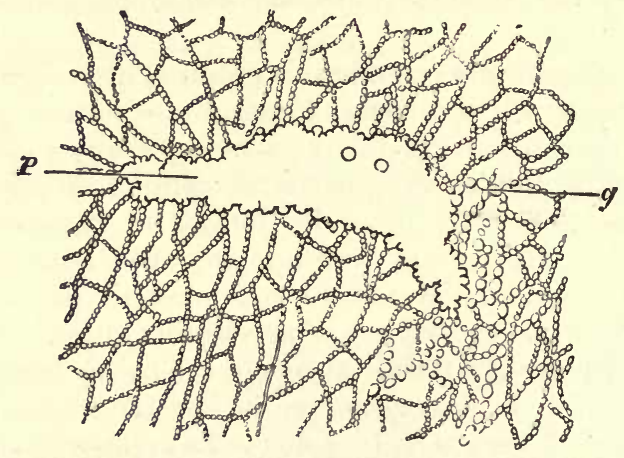

Fig. 47.-Development of elastic tissue by deposition of fine GRANULES.

$g$, fibres being formed of rows of 'elastin' granules; $p$, flat platelike expansion of elastic substance formed by the fusion of 'elastin' granules.

them, and the cells of the tissue become squeezed up into the intervals between them. Before any considerable development of fibres has taken place, the embryonic connective tissue has a jelly-like appearance; in this form it occurs in the umbilical cord, where it is known as the jelly of Wharton (fig. 46). 


\section{LESSON XI. \\ THE CONNECTIVE TISSUES (continued).}

\section{ARTICULAR CARTILAGE.}

1. Cut two or three very thin tangential slices of the fresh cartilage of a joint, mount them in saline solution and examine with a high power. Observe carefully the form and grouping of the cells. Look at the thin edge of the section for spaces from which the cells have dropped out. Measure two or three cells and their nuclei, and sketch one or two groups. Now replace the saline solution by water and set the preparation aside for a little while. On again examining it, many of the cartilage-cells will be found to have shrunk away from their containing capsules.

2. Make other sections of the cartilage (1) from near the middle, (2) from near the edge. Mount in magenta solution, and when stained add dilute glycerine and cement the cover-glass. In (2) look for branched cartilage-cells. Draw one or two.

3. Make vertical sections of articular cartilage from a bone which has been for several days in $\frac{1}{2}$ per cent. chromic acid solution, and mount the sections in Farrant. Sketch the arrangement of the cells in the different layers.

4. Wash a fresh joint with distilled water; drop 1 per cent. nitrate of silver solution over it; after ten minutes wash away the nitrate of silver and expose in water to the light. When browned, cut thin sections from the surface and mount in Farrant. The cells and cell-spaces show white in the brown ground-substance. Draw.

Cartilage or gristle is a translucent bluish-white tissue, firm, and at the same time elastic, and for the most part found in connection with bones of the skeleton, most of which are in the embryo at first represented entirely by cartilage. Two chief varieties of cartilage are distinguished. In the one, which is termed hyaline, the matrix or ground-substance is clear, and free from obvious fibres; in the other, which is termed fibro-cartilage, the matrix is everywhere pervaded by connective-tissue fibres. When these are of the white variety, the tissue is white fibro-cartilage; when they are elastic fibres, it is yellow or elastic fibro-cartilage.

Hyaline cartilage occurs principally in two situations-namely (1) covering the ends of the bones in the joints, where it is known as articular cartilage; and (2) forming the rib-cartilages, where it is known as costal cartilage. It also forms the cartilages of the nose, the 
external auditory meatus, the larynx, and the windpipe; in these places it serves to maintain the shape and patency of the orifices and tubes.

Articular cartilage. The cells of articular cartilage are mostly scattered in groups of two or four throughout the matrix (fig. 48). The latter is free from fibres, except at the extreme edge of the

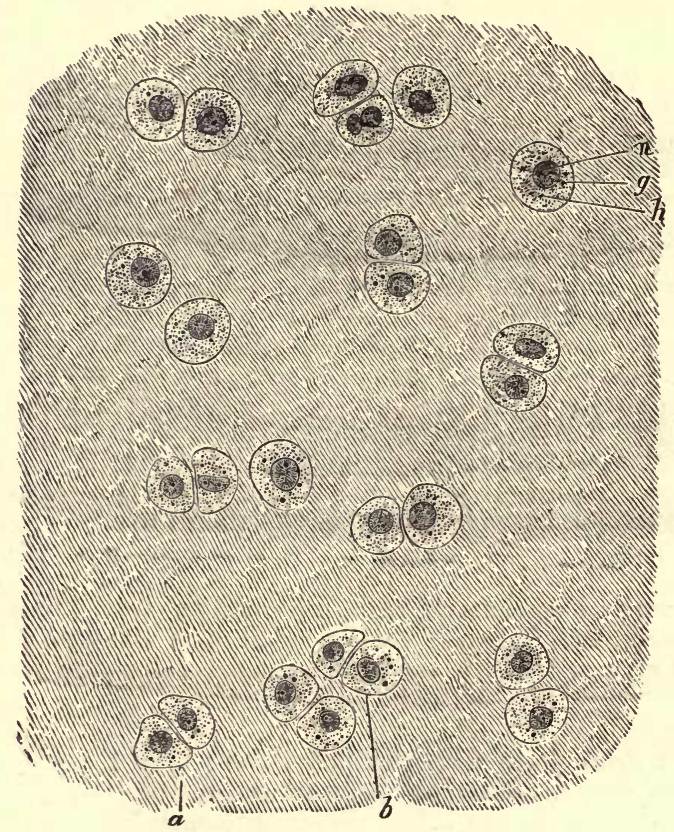

Fig. 48.-Articular cartilage from head of metatarsal bone of man (OSMIC ACID PREPARATION). THE CELL-BODIES ENTIRELY FILL THE SPACES IN THE MATRIX. (340 diameters.)

$a$, group of two cells ; $b$, group of four cells ; $h$, protoplasm of cell, with $g$, fatty granules $n$, nucleus.

cartilage, where the connective-tissue fibres from the synovial membrane extend into it; and here also the cartilage-cells are often branched, and offer transitions to the branched connective-tissue corpuscles of that membrane (transitional cartilage, fig. 49). By long maceration, however, some observers have obtained evidence of a fibrous structure even in the matrix of true hyaline cartilage. The matrix immediately around the cartilagie-cells is often marked off from the rest by a concentric line or lines, this part being known as the capsule of the cell. The cells are bluntly angular in form, the sides opposite to one another in the groups being generally flattened. The protoplasm is very clear, but it may contain droplets of fat; and with a high power fine interlacing filaments and granules have been observed in it (fig. 50). During life the protoplasm entirely fills the 
cavity or cell-space which it occupies in the matrix; but after death, and in consequence of the action of water and other agents, it tends to shrink away from the capsule. The nucleus is round, and shows the usual intranuclear network.

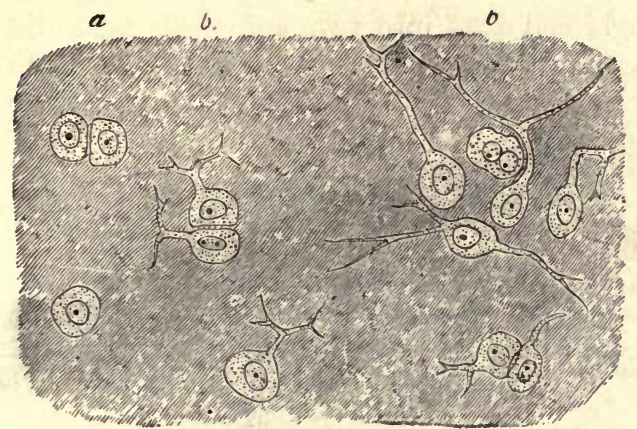

Fig. 49.-Border of ARTICULAR CARTILAgE SHOWING TRANSITION OF CARTILAGE-CELLS IN'TO CONNECTIVETISSUE CORPUSCLES OF SYNOVIAL MEMBRANE. From head of metatarsal bONE, hUMAN. (A bout 340 dianneters.)

$a$, ordinary cartilage-cells; $b, b$, with branching processes.

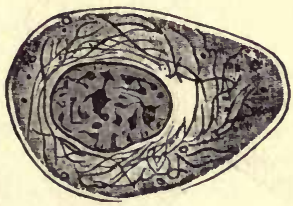

Fig. 50.-A CARTILAGE-CKLL IN THE LIVING STATE, FROM THE SALAMANDER. Highly magnified.

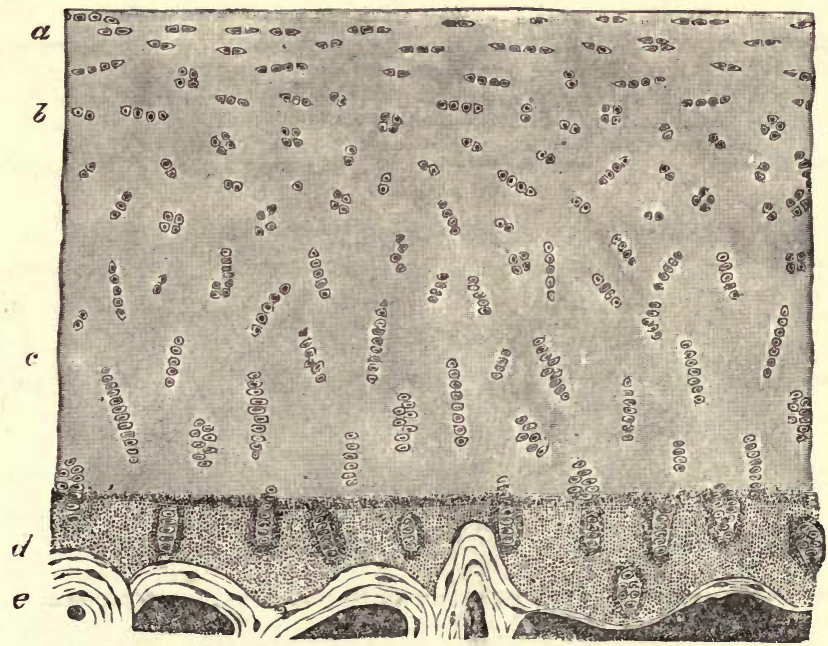

Fig. 51.-Vertical seotion of articular cartilage Covering the Lower END OF THE TiBIA, HUMAN. (Magnified about 30 diameters.)

$a$, cells and cell-groups flattened conformably with the surface; $b$, cell-groups irregularly arranged; $c$, cell-groups disposed perpendicularly to the surface; $d$, layer of calcified cartilage; $e$, bone.

In vertical section (fig. 51) the deeper cell-groups $(c)$ are seen to be arranged vertically to the surface, the more superficial ones $(a)$ parallel to the surface; whilst in an intermediate zone the groups are irregu- 
larly disposed (b). In the deepest part of the cartilage, next the bone, there is often a deposition of calcareous salts in the matrix (calcified cartilage, d).

The disposition of the cells of cartilage in groups of two, four, and so on, is apparently due to the fact that these groups have originated from the division of a single cell first into two, and these again into two, and so on (fig. 52). It would' seem that the matrix is formed of

A.

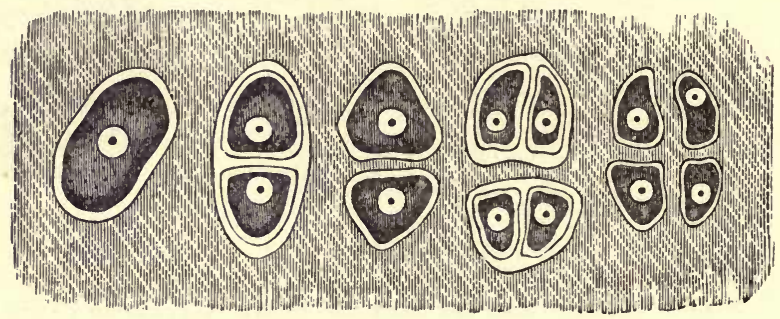

Fig. 52.-Plat of the multiplication of cells of cartilage.

$A$, cell in its capsule; B, divided into two, each with a capsule; $\mathbf{c}$, primary capsule disappeared, secondary capsules coherent with matrix; D, tertiary division; E, secondary capsules disappeared, tertiary coherent with matrix.
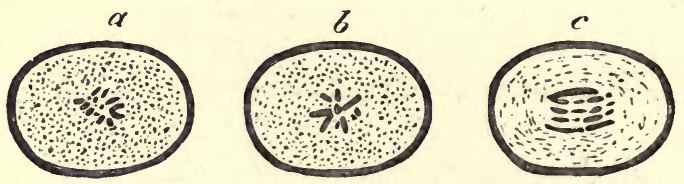

$e$
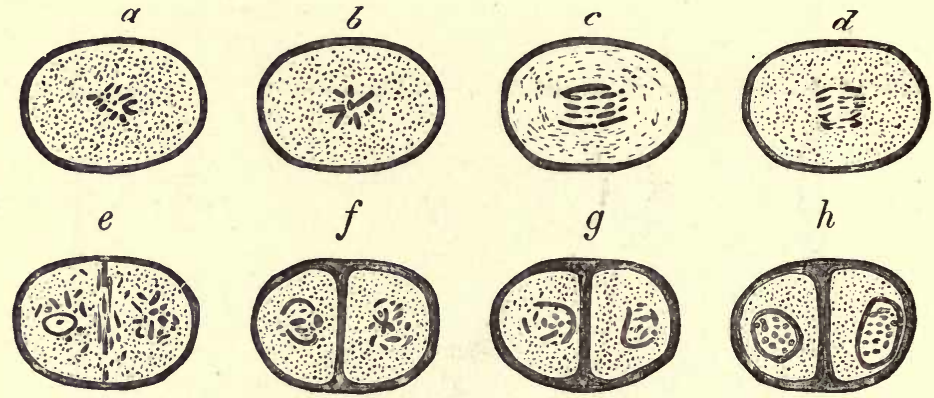

Fig. 53.-Division of a cartilage-cell.

$a-h$, stages of division of a cell, as seen in the living cartilage of the salamander (the connection of the nuclear filaments could not be made out in the fresh condition), $a, b$, stellate phase; $c, d$, commencing separation of the nuclear filaments; the further stages of separation are not represented; $e$, filaments fully separated into two groups, and a sentum beginning to be formed between them; $f$, septum completed, seen to be doub'e and continuous with capsules of daughter cells; $g, h$, further stages in the formation of the daughter nuclei.

successive portions, which are deposited around each cartilage-cell as the so-called 'capsules, each newly formed portion soon blending in its turn with the previously formed matrix, whilst a new capsule is formed within it. The division of the cartilage-cell, like that of other cells, is accompanied by a process of karyomitosis. 


\title{
LESSON XII.
}

\author{
THE CONNECTIVE TISSUES (continued).
}

\section{COSTAL CARTILAGE. FIBRo-CARTILAGE.}

1. MAKE transverse and tangential sections of a rib-cartilage, stain them with magenta, and mount in dilute glycerine, cementing at once. Sketch a part of a transverse section under a low power and a cell-group from one of the tangential sections under a high power. Notice especially the arrangement of the cells, somewhat concentric near the surface but radial near the centre. The costal cartilages are often ossified near the middle.

2. Make sections of the cartilage of the external ear. Mount in dilute glycerine faintly coloured with magenta. If from the ox, notice the very large reticulating elastic fibres in the matrix. Notice also the isolated granules of elastin, and around the cartilage-cells the area of clear groundsubstance. Draw a small portion of the section.

3. Mount a section of the epiglottis in the same way. Notice the closer network of much finer fibres.

4. Cut sections of white fibro-cartilage (intervertebral disk), and stain them with dilute magenta. Mount in dilute glycerine. Observe the wavy fibres in the matrix and the cartilage-cells lying in clear areas often con. centrically striated. Look for branched cartilage-cells. Sketch three or four cells and the adjoining fibrous matrix.

Costal cartilage.-In the costal cartilages the matrix is not always so clear as in the matrix of the joints, for it often happens that fibres

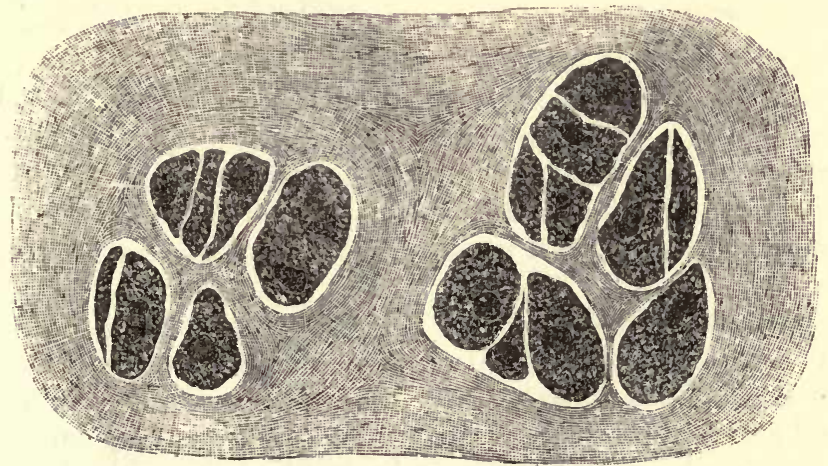

Fig. 54.-SECtion OF RiB-CARTILAGE, SHOWING TWo CELL-Groups in A SOMEWHAT FIBROUS-LOOKING MATRIX.

become developed in it. The cells are generally larger and more angular than those of articular cartilage, and collected into larger groups 
(fig. 54). Near the circumference, and under the perichondrium or fibrous covering of the cartilage, they are flattened and parallel to the surface, but in the deeper parts they have a more irregular or a radiated arrangement. They frequently contain fat. The cartilages of the larynx and windpipe and of the nose resemble on the whole the costal cartilages, but the study of them may be deferred until the organs where they occur are dealt with.

Elastic or yellow fibro-cartilage occurs in only a few situations. These are, the cartilage of the external ear and that of the Eustachian tube, the epiglottis and cartilages of Santorini of the larynx, and in some animals, e.g. the ox, the upper third of the arytenoids. The matrix is everywhere pervaded with well. defined branching fibres, which unite with one another to form a close network (fig. 55). These

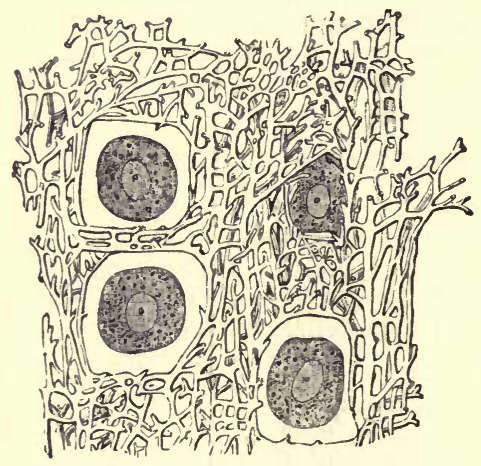

Fig. 55.-Section of the elastic cartiLAGE OF THE EAR. (Highly magnified.)

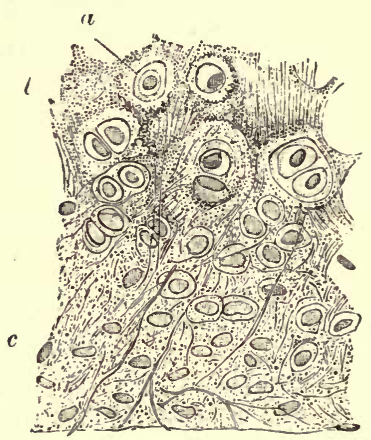

Fig. 56.-SECTION OF PART OF THE CARTILAGE OF THE EPIGLOTTIS.

$a$, cartilage-cell in clear area; $b$, granularlooking matrix near the middle of the cartilage, the granular appearance being due partly to the fine reticulum of elastic fibres, partly to the presence of granules of elastic substance in the matrix; $c$, clearer matrix with longer fibres.

fibres resist the action of acetic acid, and are stained deeply by magenta; they are evidently elastic fibres. In the ox they are very large, but smaller in man, especially in the cartilage of the epiglottis (fig. 56). They appear to be developed by the deposition of granules of elastin in the matrix, which at first lie singly, but afterwards become joined to form the fibres.

White fibro-cartilage is found wherever great strength combined with a certain amount of rigidity is required: thus we frequently find fibro-cartilage joining bones together, as in the case of the intervertebral disks and other symphyses. Fibro-cartilage is frequently employed to line grooves in which tendons run, and may also be found in the tendons themselves. It is also employed to deepen cup-shaped articular surfaces; and in the case of the interarticular cartilages, such as those of the knee and lower jaw, to allow greater freedom of movement whilst diminishing the liability to dislocation. Under the microscope 
white fibro-cartilage looks very like fibrous tissue, but its cells are cartilage-, not tendon-, cells (fig. 57). They are rounded or bluntly angular

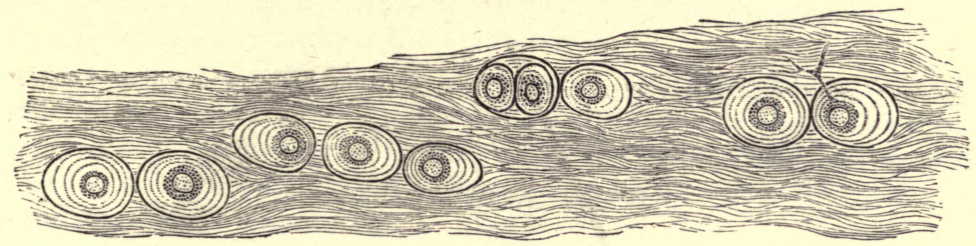

Fig. 57.-White Fibro-cartilage from an intervertebral disk, human. (Highly magnified.)

The concentric lines around the cells indicate the limits of deposit of successive capsules. One of the cells has a forked process which extends beyond the hyaline area surrounding the cell, amongst the fibres of the genera! matrix.

and surrounded by a concentrically striated area of clear cartilagematrix. In some parts of the intervertebral disk many of the cells are branched, and may be looked upon as transitional forms to connectivetissue corpuscles. 


\section{LESSON XIII.}

\section{BONE AND MARROW.}

1. In thin sections of hard bone made by grinding, observe the Haversian canals, lamellæ, lacunæ, canaliculi, \&c. Make a sketch first under a low and afterwards under a high power.

2. With fine forceps strip off a thin shred from a bone which has been decalcified in nitric acid and afterwards kept for some time in dilute alcohol. Mount the shred in water. Observe the fibrous structure of the lamellæ. Look for perforating fibres or the holes from which they have been dragged out. Sketch a small piece of the thin edge of a lamella.

3. Stain with dilute magenta very thin sections of compact bone which has been decalcified in chromic or picric acid, and mount in dilute glycerine, cementing at once. Look for fibres of Sharpey piercing the circumferential lamellæ. The elastic perforating fibres are more darkly stained than the others. Notice the stained nuclei of the bone-corpuscles in the lacunæ. In the thinnest parts of the sections try to make out the blood-vessels and other structures in the Haversian canals.

4. Mount in Canada balsam sections of marrow (from a long bone) stained with hæmatoxylin or borax-carmine.' Observe the fat-cells, the reticular tissue supporting them, the proper marrow-cells in this tissue, \&c.

5. Tease in saline solution some of the red marrow from the rib of a recently killed animal. Observe and sketch the proper marrow-cells and look for myeloplaxes and nucleated coloured blood-corpuscles. If examined carefully, amœboid movements may be detected in the latter and in the marrow-cells.

Bone is a connective tissue in which the ground-substance is impregnated with salts of lime, chiefly phosphate, these salts constituting about two-thirds of the weight of the bone. When bones are macerated this earthy matter prevents the putrefaction of the animal matter. When bones are calcined they lose one-third of their weight, owing to the destruction of the animal matter; when steeped in acid the earthy salts are dissolved and only the animal matter is left. This, like areolar and fibrous tissue, is converted into gelatine by boiling.

Bony tissue is either compact or cancellated. Compact bone is dense like ivory; cancellated is spongy with obvious interstices. The outer layers of all bones are compact, and the inner part is generally cancellated, but the shaft of a long bone is almost entirely made up of

\section{See Appendix.}


compact substance except along the centre, which is hollow and filled with malrow. The interstices of cancellated bone are also occupied by marrow. Externally bones are covered except at the joints by a vascular fibrous membrane, the periosteum.

True bone is always made up of lamella, and these again are composed of fine fibres lying in a calcified ground-substance. Between the lamellæ are branched cells, the bone-corpuscles, which lie in cell. spaces or lacunce. The ramified passages which contain the cell-processes are termed canaliculi.

In cancellated bone the blood-vessels run in the interstices supported by the marrow. In compact bone they are contained in little canalsthe Haversian canals - which everywhere pervade the bone. These canals are about $0.05 \mathrm{~mm}$. ( $\frac{1}{500}$ inch) in diameter, but some are smaller, others larger than this. Their general direction is longitudinal, i.e. parallel to the long axis of the bone, but they are constantly united by transversely and obliquely running passages. In a section across

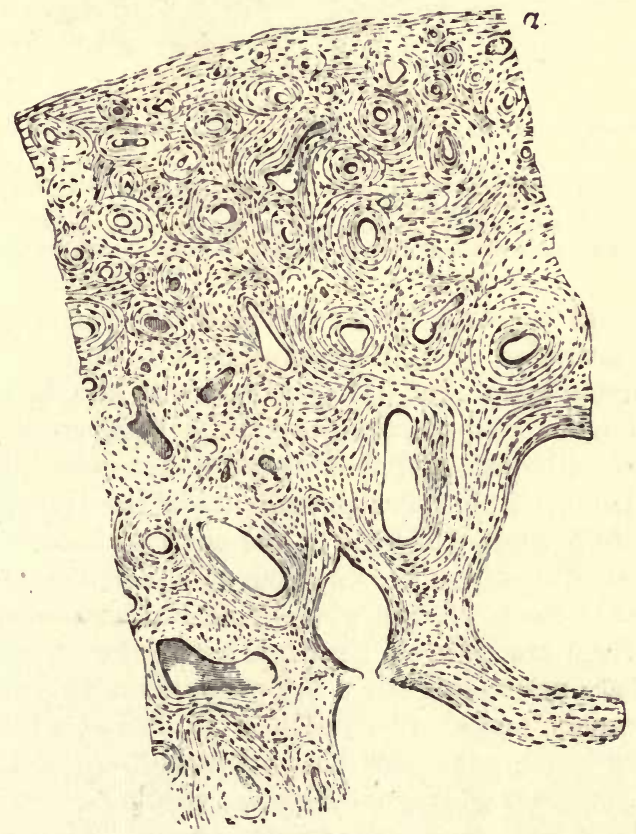

Fig. 58.-Transverse section of a bone (clasa). (Mrgnifie! 20 diameter3.)

The openings of the Haversian cana's are seen encircled by concentric lamellæ. Other lamellæ run parallel with the surface $(a)$.

the shaft of a long bone they are seen as small rounded or irregular holes (fig. 58). When the section has been made by grinding, the holes get filled up with air and débris, and they then look black by 
transmitted light, as do also the lacunæ and canaliculi (fig. 59). Most of the lamellæ in compact bone are disposed concentrically around the Haversian canals ; they are known as the Haversian lamellæ, and with

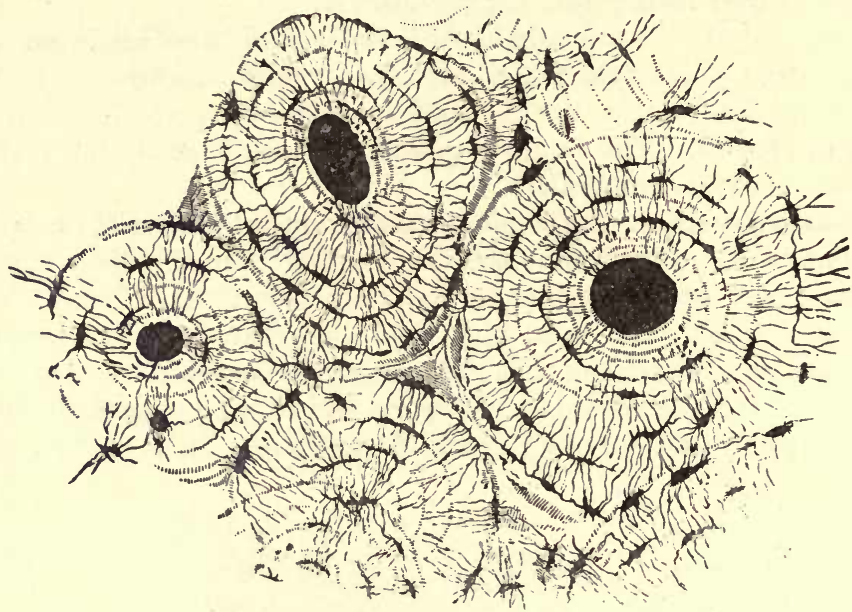

Fig. 5̆9.-Transverse section of compact tissue (of humerus). (Magnified about 150 diameters.)

Three of the Haversian canals are seen, with their concentric rings; also the lacunæ, with the canaliculi extending from them across the direction of the lamellæ, The Haversian apertures had become filled with air and débris in grinding down the section, and therefore appear black in the figure, which represents the object as viewed with transmitted light.

the included canal form what is known as a Haversian system. The lacunæ of a Haversian system communicate with one another and with the Haversian canal, but not as a rule with the lacunæ of other Haversian systems. The angular interstices between the Haversian systems are generally occupied by bony substance, which is fibrous but not distinctly lamellar. Besides the lamellæ of the Haversian systems there is a certain thickness of bone at the surface, immediately underneath the periosteum, which is composed of lamellæ arranged parallel with the surface; these are the circumferential or periosteal lamella (fig. 58,a). They are pierced here and there by canals for bloodvessels, which are proceeding from the periosteum to join the system of Haversian canals, and also by calcified bundles of white fibres and by elastic fibres which may also be prolonged from the periosteum. These are the perforating fibres of Sharpey (fig. 60).

The lamellæ of bone are fibrous in structure. This may be seen in shreds torn off from the superficial layers of a decalcified bone (fig. 61). The fibres often cross one another in adjacent lamellæ, and in the Haversian systems they run in some lamellæ concentrically, in others parallel with the Haversian canal. In shreds of lamellæ which have been peeled off from the surface the perforating fibres may sometimes be seen projecting from the surface of the shred, having been 


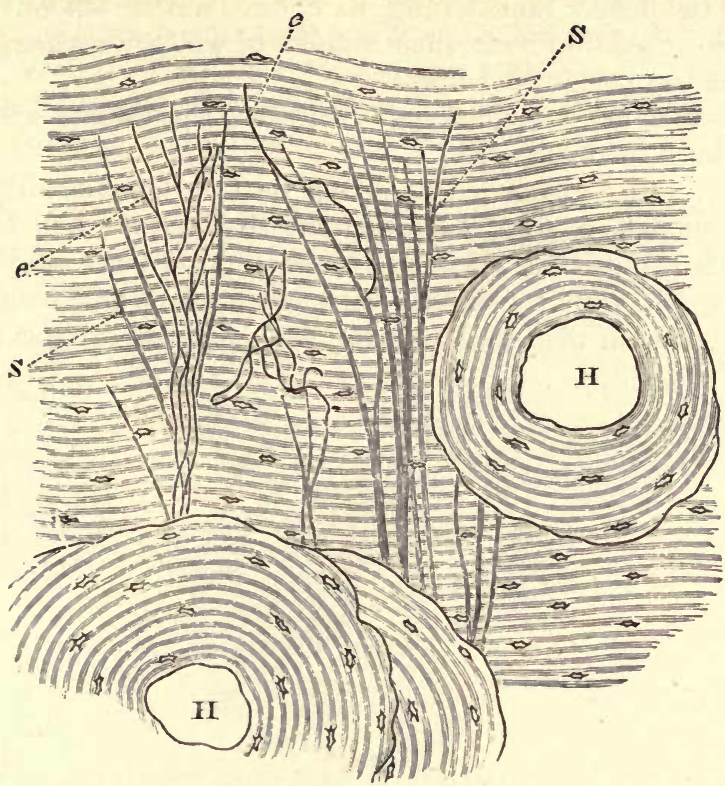

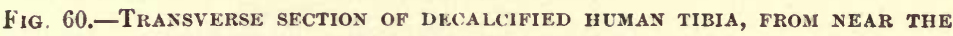
SURFACE OF THE SHAFT.

H, H, Haversian canals, with their systems of concentric lamellæ; in all the rest of the figure the lamellæ are circumferential; s, ordinary perforating fibres of Sharpey; $e, e$, elastic perforating fibres. Drawn under a power of about 150 diameters.

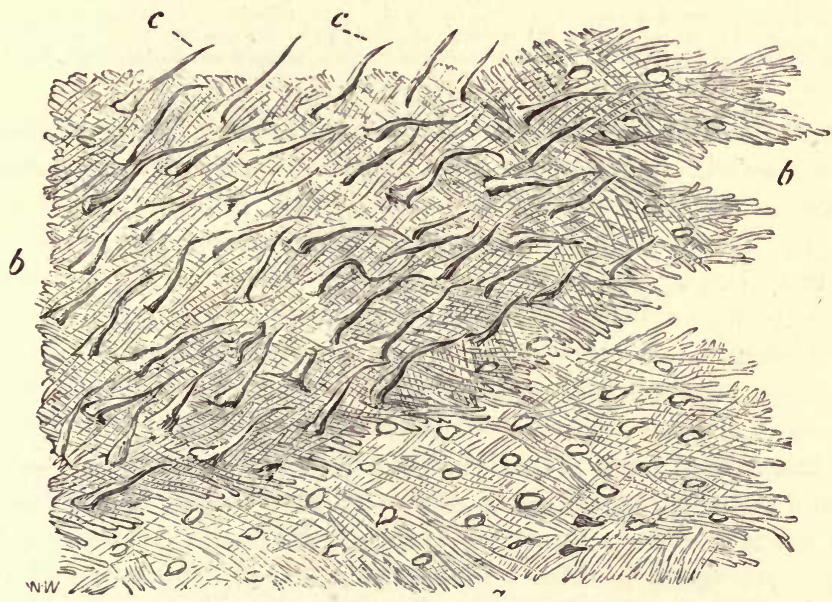

Fig. 61.-LAMELLE TORN OFF FROM A DECALCIFIKD HUMAN PARIETAL IONE AT SOME DEPTH FRON THE SURFACE.

$a$, lamellæ, showing decussating fibres; $b, b$, thicker part, where several lamellæ are superposed; $c$, $c$, perforating fibres: the fibrils which compose them are not shown in the figure. Apertures through which perforating fibres had passed are seen, especially in the lower part, $a, a$, of the figure. Magnitude as seen under a power of 200 , but not drawn to a scale. 
torn out of the deeper lamellæ (fig. $61 c, c$ ). Where tendons or ligaments are inserted into bone, their bundles of white fibres are prolonged into the bone as perforating fibres.

The lacunæ are occupied by nucleated corpuscles, which send branches along the canaliculi.

The Haversian canals contain one or two blood-capillaries and nervous filaments, besides a little connective tissue; and the larger ones may also contain a few marrow-cells. There are also cleft-like lymphatic spaces running parallel with the vessels and connected by means of canaliculi with neighbouring lacunæ.in the osseous substance (fig. 62).

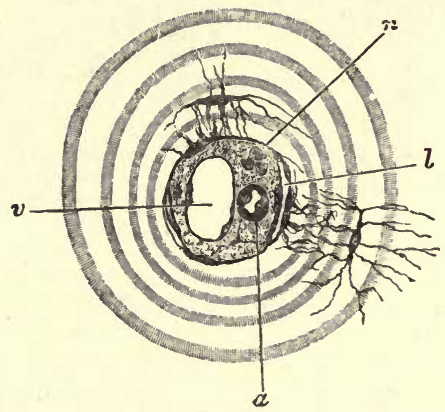

Fig. 62.-Section of a Haversian canal, showing its contents. (Highly magnified.)

$a$, small arterial capillary vessel; $v$, large venous capillary: $n$, pale nerve-fibres cut across ; $l$, cleft-like lymphatic vessel: one of the cells forming its wall communicates by fine branches with the branches of a bone-corpuscle. The substance in which the vessels run is connective tissue with ramified cells; its finely granular appearance is probably due to the cross-section of fine fibrils. The canal is surrounded by several concentric lamellæ.

The periosteum, which is best studied in sections from a bone which has been decalcified in chromic or picric acid, is a fibrous membrane composed of two layers, the inner of which contains many elastic fibres. In the outer layer numerous blood-vessels ramify and send from it branches to the Haversian canals of the bone. The periosteum ministers to the nutrition of the bone, partly on account of the bloodvessels it contains, partly, especially in young animals, on account of the existence between it and the bone of a layer of osteoblasts or boneforming cells, a remainder of those which originally produced the bone.

The marrow of bone is of a yellow colour in the shafts of the long bones, and is there largely composed of adipose tissue, but in the cancellated tissue it is red, the colour being partly due to the large amount of blood in its vessels. This red marrow is chiefly composed of round nucleated cells-the marrow-cells (fig. $63, e-i$ )-which resemble large lymph-corpuscles, and, like these, are amœboid. There are also to be seen mingled with them a number of corpuscles somewhat smaller in size, but nucleated and amœboid, and of a reddish tint (fig. $63, j-t)$; these are believed to be cells in process of development into coloured 
blood-corpuscles (hamatoblasts). Lastly the marrow contains a certain number of very large cells with multiple nuclei, the myeloplaxes (fig. $63, a, b, c, d)$. These are especially numerous wherever bone is becoming absorbed. The marrow is very vascular, the capillaries and veins being large and thin-walled; indeed, according to some authorities,

$\alpha$
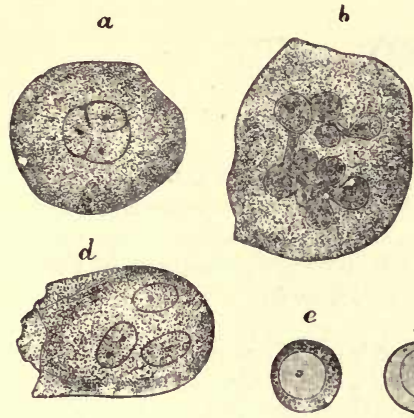
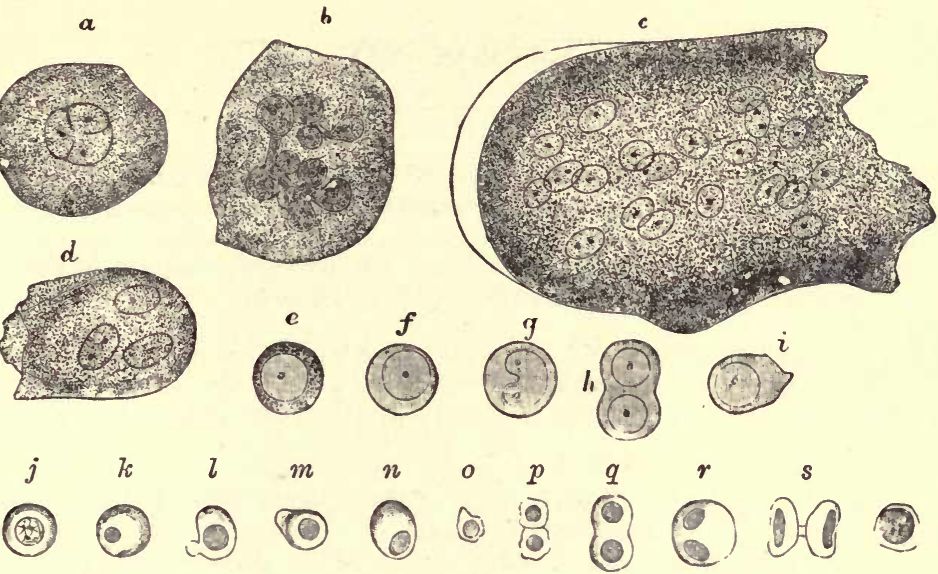

Fig. 63.-Cells of the red marrow of the guinea-pig. (Highly magnified.)

$a$, a large cell, the nucleus of which appears to be partly divided into three by constrictions; $b$, a cell the enlarged nucleus of which shows an appearance of being constricted into a number of smaller nuclei ; $c$, a so-called giant-cell or myeloplaxe with many nuclei; $d$, a smaller myeloplaxe with three nuclei; $e-i$, proper cells of the marrow ; $j-t$, various forms of coloured nucleated cells, some in process of division.

the walls of the capillaries are imperfect, so that there is an open communication between them and the interstices of the tissue, and in this way it is supposed that the coloured blood-disks, which are believed to be produced from the coloured nucleated cells of the marrow, may get into the circulation. 


\title{
LESSON XIV.
}

\author{
DEVELOPMENT OF BONE.
}

1. Mount in Canada balsam a section of the lower jaw of a fotus which has been stained in bulk with magenta or hæmatoxylin and embedded in paraffin.' Find the part where the lower jaw-bone is becoming ossified, and carefully study the appearances which it presents. The bone is prolonged in the form of osteogenic fibres which are covered with osteoblasts.

2. Intramembranous ossification may also be studied in the parietal bone of a fotus which has been preserved in Müller's fluid. A piece of the growing edge is scraped free from its investing membranes and mounted in glycerine or Farrant.

3. Mount in Canada balsam sections of a fotal limb which has been stained with magenta. The bones will be found in different stages of ossification, those of the digits being least developed. Make sketches illustrating the three chief stages of endochondral ossification. Notice the peculiar terminal ossification of the third phalanx.

4. Make with a sharp scalpel a longitudinal section at the line of ossification in a more advanced bone which has not been decalcified. These sections will show the mode of progress of the calcification. The sections can be mounted in Farrant's solution.

True bone is essentially formed in all cases by an ossification of connective tissue. Sometimes the bone is preceded by cartilage, which first of all becomes calcified, and this is then invaded, and for the most part removed, by an embryonic tissue which re-deposits bony matter in the interior of the cartilage, whilst at the same time.layers of bone are being formed outside underneath the periosteum. This is intracartilaginous or endochondral ossification. Sometimes the bone is not preceded by cartilage, and then the only process which occurs is one corresponding to the subperiosteal ossification of the former variety; the ossification is then known as intramembranous.

Ossification in Cartilage.-This may be described as occurring in three stages. In the first stage the cells in the middle of the cartilage become enlarged and arranged in rows radiating from the centre (fig. 64), and fine granules of calcareous matter are deposited in the matrix. Simultaneously with this the osteoblasts underneath the

1 For the methods of staining and embedding 1 and 3, see Appendix, ' Embedding in Paraffin.' 
periosteum deposit a layer or layers of fibrous lamellæ upon the surface of the cartilage, and these lamellæ also become calcified (fig. $64, \mathrm{im}$ ). As they are formed some of the osteoblasts $(0)$ are included between them and become bone-corpuscles.

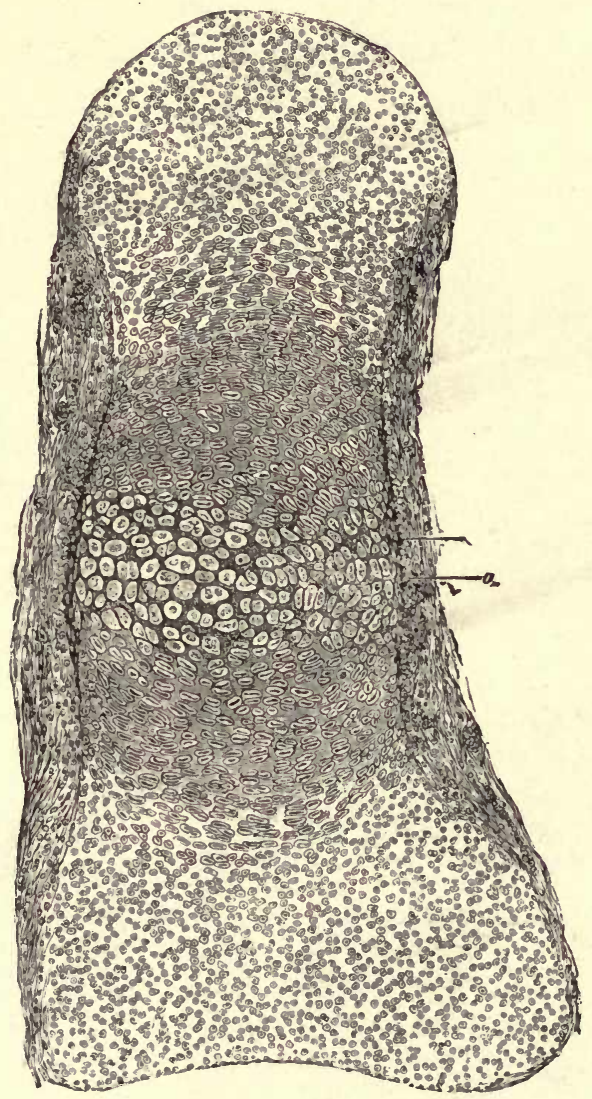

Fig. 64.-Section of phalangeal bone of human foetus, at the time of Commencing ossification. (Magnified about 75 diameters.)

The cartilage-cells in the centre are enlarged and separated from one another by dark-looking calcified matrix; im, layer of bone deposited underneath the periosteum ; $o$, layer of osteoblasts by which this layer has been formed. Some of the osteoblasts are already embedded in the new bone as lacunæ. The cartilage-cells are becoming enlarged and flattened and arranged in rows above and below the calcified centre. At the ends of the cartilage the cells are small and the groups are irregularly arranged; the fibrous periosteum is not sharply marked off from the cartilage.

In the second stage some of the subperiosteal tissue eats its way through the newly formed layer of bone and into the centre of the calcified cartilage (fig. 65, ir). This is freely absorbed before it, so that large spaces are produced which are filled with osteoblasts and contain 
numerous blood-vessels which have grown in at the same time. The spaces are termed medullary spaces, and this second stage may be termed the stage of irruption.

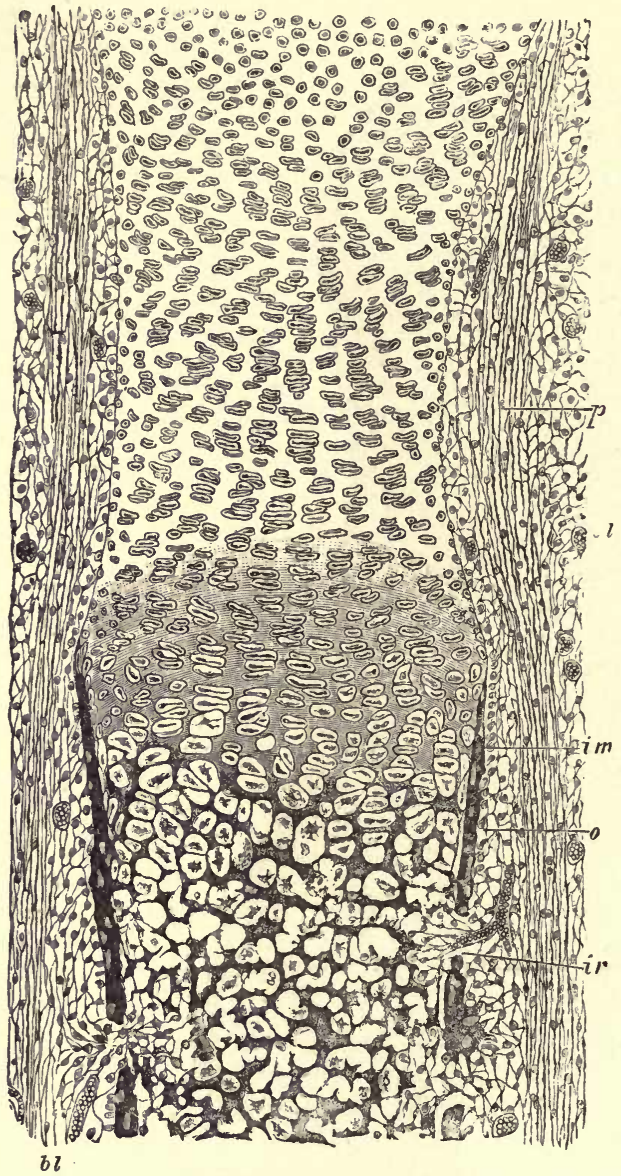

Fig. 65.-Shction of PART of ONE OF THE LIMB-BONES OF A FOETAL CAT, AT A MORE ADVANCED STAGE OF OSSIFICATION THAN IS REPIRESENTED IN FIG. 64, AND SONEWHAT MORE HIGHIY MAGNIFIED.

The calcification of the cartilagematrix has advanced from the centre, and is extending between the groups of cartilage-cells which are arranged in characteristic rows. The subperiosteil bony deposit $(\mathrm{im})$ has extended pari passu with the calcification of the cartilage-matrix. The cartilage-cells in the primary areolæ are mostly shrunken and stellate; in some cases they have dropped out of the space. At ir and in two other places an irruption of the subperiosteal tissue, composed of ramified cells with osteoblasts and growing bloodvessels, has penetrated the subperiosteal bony crust, and has begun to excavate the secondary areolæ or medullary spaces; $p$, fibrous layer of the periosteum; $o$, layer of osteoblasts, some of them are embedded in the osseous layer as bone-corpuseles in la. cunæ; $b l$, blood-vessels occupied by blood-corpuscles. Beyond the line of ossific advance the periosteum may be noticed to be distinctly incurved. This incurvation is gradually moved on, the cartilage expanding behind it until the head of the bone is reached, when it forms the periosteal notch or groove represented in fig. 66, p. 57 .

In the third stage of endochondral ossification there is a gradual advance of the ossification towards the extremities of the cartilage, and at the same time a gradual deposition of fresh bony lamellæ and spicules on the walls of the medullary spaces, and on the surface of the new bone under the periosteum. The advance into the cartilage always takes place by a repetition of the same changes, the cartilagecells first enlarging and becoming arranged in rows, the matrix between the rows becoming calcified, and then the calcified cartilage becoming excavated from behind by the osteoblastic tissue so as to form new medullary spaces (fig. 67). The walls of these are at first formed 
only by remains of the calcified cartilage-matrix (fig. $67, c$ ), but they soon become thickened by lamellæ of fibrous bone $(b)$ which are

Fig. 66.-Longituininal secTION THROUGH THE UPPER HALF OF THE DECALCIFIED HUMERUS OF A FEETAL SHEEP, AS SEEN UNDER A MAGNIFYING POWER OF ABOUT 30 DIAMETERS.

$i c$, the part of the shaft which was primarily ossified in cartilage; what remains of the primary bone is represented as dark, enveloped by the clear secondary deposit. The areolæ of the bone are occupied by embryonic marrow with osteoblasts, and bloodvessels variously cut, represented as dark lines. One long straight vessel $(b v)$ passes in advance of the line of ossification far into the cartilaginous head, most of the others loop round close to the cartilage. At one or two places in the older parts of the bone elongated groups of cartilagecells $(c)$ may still be seen, which have escaped absorption. im, the part of the boue that has been ossified in membrane, that is to say, in the osteoblastic tissue under tlie periosteum. It is well marked off from the central portion, and is bounded, veripherally, by a jagged edge, the projections of which are inclistinctly seen to be prolonged by bunches of osteogenic fibres. A row of osteoblasts covers the superficial layer of the bone. The subperiosteal layer is prolonged above into the thickening $(y)$, which encroaches upon the cartilage of the head of the bone, and in which are seen, amongst numerous osteoblasts and $a$ few b ood-vessels, the straight longitudinal osteogenic fibres (of), and some other fibres ( $p f)$ crossing them, and perliaps representing fibres of Sharpey. The calcareous salts having been removed by an acid, the granu ar ossific deposit passing up between the rows of cartilage-cells is not seen in this specimen; it would have extended as far as a line joining the marks $x \times$. Observe the general tendency of the osseous trabeculæ and the vascular channels between them to radiate from the original centre of ossification. This is found to prevail more or less in all bones when they are first formed, although the direction of the trabeculæ may afterwards become modified in relation with varying physiological conditions, and especially as the result of pressure in different directions.

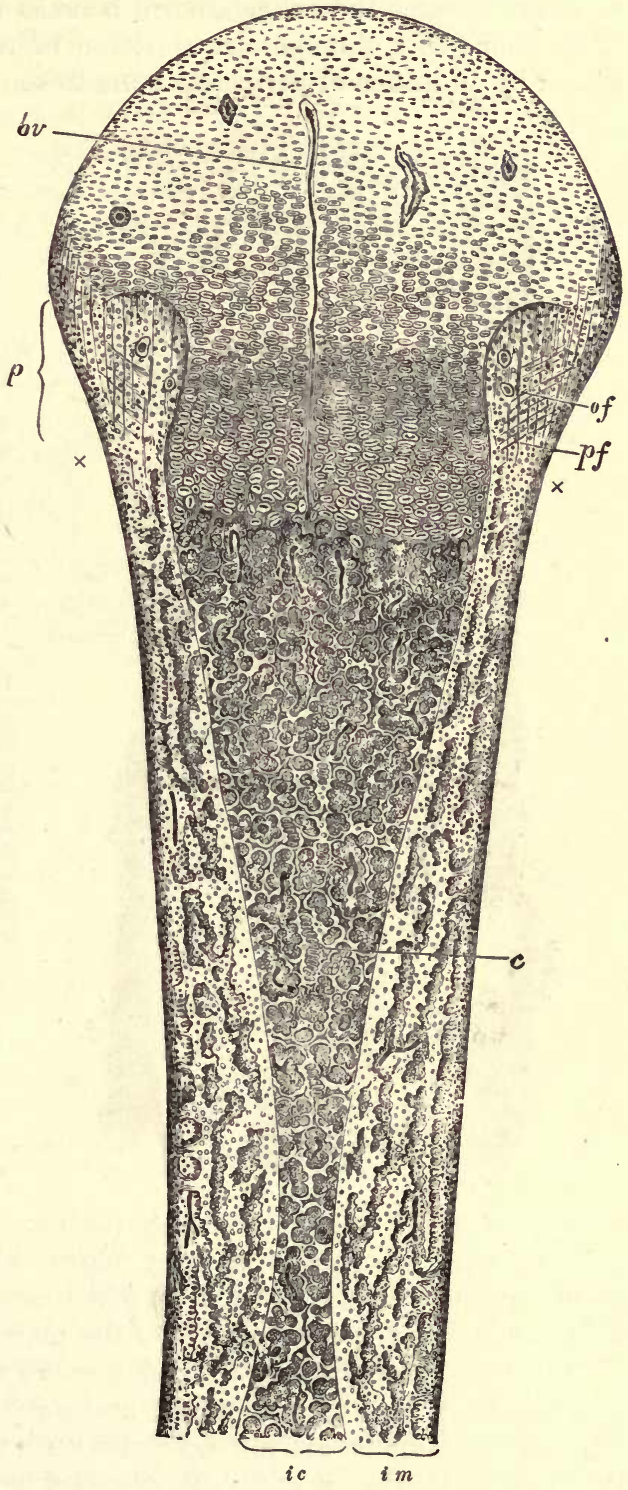

deposited by the osteoblasts, and between which bone-corpuscles become included, as in the case of the subperiosteal bone. The latter 
advances pari passu with the endochondral calcification, but beyond this the uncalcified cartilage grows both in length and breadth, so that the ossification is always advancing into larger and larger portions of cartilage ; hence the endochondral bone as it forms assumes the shape of an hour-glass, the cylindrical shape of the whole bone being maintained by additions of periosteal kone to the outside (see fig. 66). The

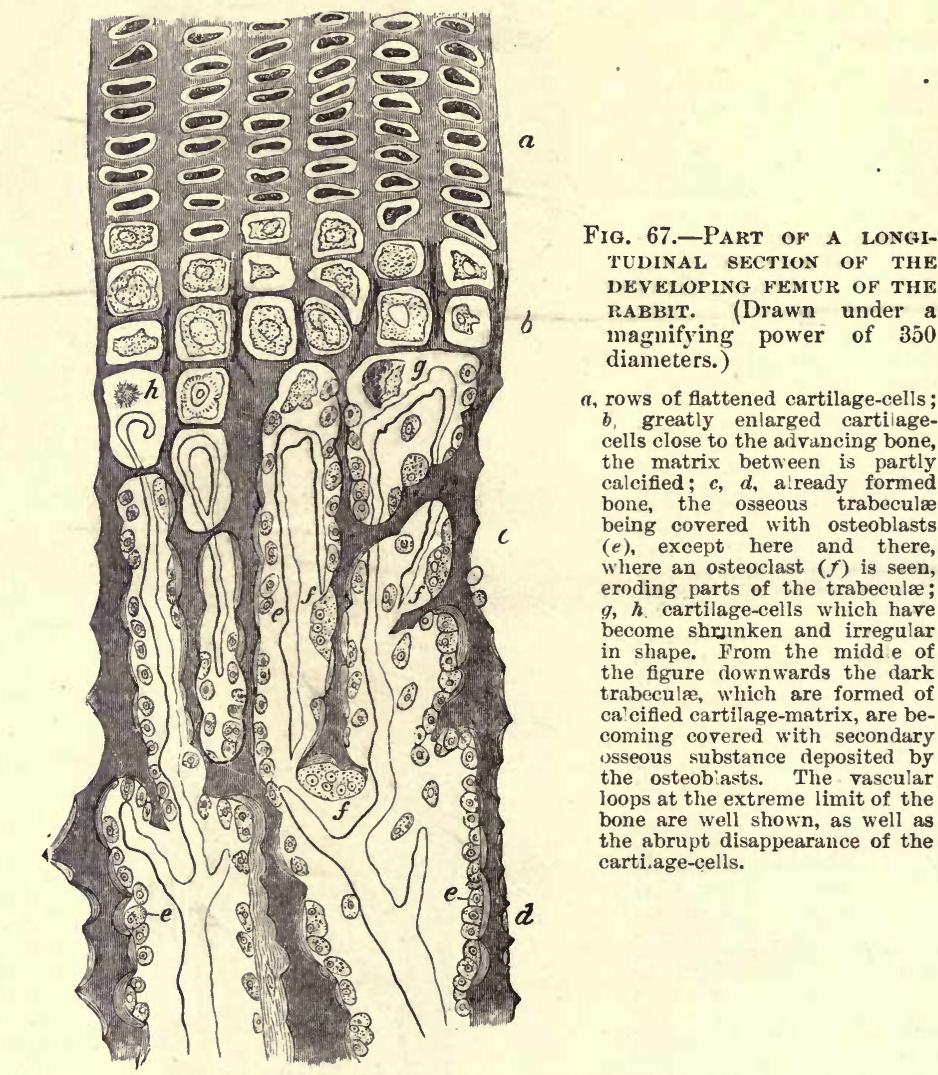

absorption of the calcified cartilage-matrix appears to be effected, as is the case with absorption of bony matter wherever it occurs, by large multinucleated cells (fig. $67, f, f$ ) which are termed osteoclasts. They are the same as the myeloplaxes of the marrow.

The bone which is first formed is more reticular and less regularly lamellar than that of the adult, and contains no Haversian systems. The regular lamellæ are not deposited until some little time after birth, and their deposition is generally preceded by a considerable amount of absorption. It is about this time also that the medullary canal of the long bones is formed by the absorption of the bony tissue which originally occupies the centre of the shaft. 
After a time the cartilage in one or both ends of the long bones begins to ossify independently, and the epiphyses are formed. These are not joined to the shaft until the growth of the bone is completed. Growth takes place in length by an expansion of the cartilage (intermediate cartilage) which intervenes between the shaft and the epiphyses, and by the gradual extension of the ossification into it; in width entirely by the deposition of fresh bony layers under the periosteum. In the terminal phalanges of the digits the ossification starts, not from the middle of the cartilage, but from its distal extremity.

For the regeneration of portions of bone which have been removed by disease or operation it is essential that the periosteum be left.

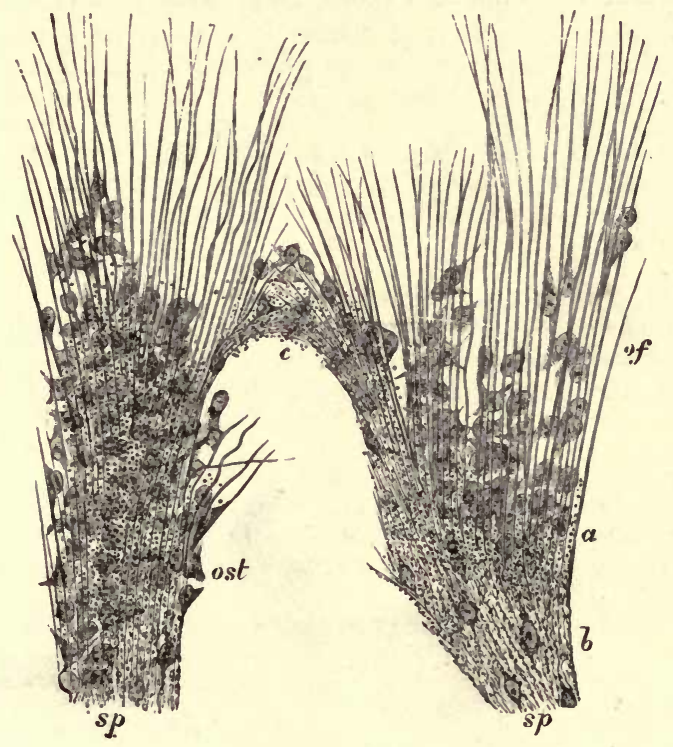

Fig. 68.-Part of the growing enge of the developing parietal bone OF A FETAL CAT, $1 \frac{1}{2}$ INCH LONG.

$s p$, bony spicules, with some of the osteoblasts embedded in them, producing the lacunæ; of, osteogenic fibres prolonging the spicules, with osteoblasts (ost) between them and applied to them.

Intramembranous ossification. In this variety of ossification, the bone is not preceded by cartilage at all, and therefore no endochon. dral bone is formed, but the calcification occurs in a sort of embryonic fibrous tissue which contains numerous osteoblasts and blood-vessels (fig. 68). The fibres of this tissue (osteogenic fibres), which, like those of fibrous tissue, are collected into small bundles, become enclosed in a calcareous matrix; and as the fibres grow, the calcification extends further and further, so that bony spicules are formed, which, as they become thickened, run together to form reticular layers, leaving spaces filled with osteoblasts around the blood-vessels. The osteogenic 
fibres are covered with osteoblasts, and as the bone forms, some of these become left as bone-corpuscles within lacunæ. Thus in every particular the development of these bones resembles that of the subperiosteal layer of endochondral bone, which is also to be considered as an instance of intramembranous ossification, although taking place on the surface of cartilage. Moreover, it is the same subperiosteal tissue which deposits the true or secondary bone upon those parts of the calcified cartilage-matrix which have escaped absorption; and this must also, therefore, be reckoned as developed according to the same type. In fact, even in intracartilaginous ossification, very little of the calcified cartilage-matrix eventually remains; this being almost wholly replaced by true or fibrous bone which has been formed by osteoblasts. 


\title{
LESSONS XV. AND XVI.
}

\author{
STRUCTURE OF MUSCLE.
}

\section{LESSON XV.}

1. TAKE a shred of muscle from a recently killed mammal, and on a dry slide carefully separate long pieces of muscular fibre (single fibres if possible) and stretch them out, keeping them moist during the process by breathing on the slide. Put a drop of serum on the cover-glass before placing this over the preparation. Study first with a low, then with a high power. Sketch all the appearances to be seen in a small piece of a fibre, focussing carefully the most superficial layers. Notice the oval nuclei immediately under the sarcolemma. Then allow a little dilute acetic acid to run under the cover-glass and watch its effect.

2. Prepare some fibres of frog's muscle in the same way, but mount in salt solution instead of serum. Notice the muscular substance shrinking away here and there from the sarcolemma, which then becomes distinctly visible. Sketch a piece of sarcolemma bridging across an interval thus produced.

3. Mount in Farrant transverse sections of fresh muscle which have been cut whilst frozen, and at once placed in 1 per cent. nitrate of silver solution. Take care to prevent the cover-glass from pressing on the sections. Examine first with a low and then with a high power. Sketch the appearances which are seen. fibres.

In each of the above preparations measure the diameter of some of the

\section{LESSON XVI.}

1. CuT off the head of a water-beetle and bisect the trunk with scissors so as to expose the interior. Notice two kinds of muscular tissue, the one belonging to the legs greyish in colour, the other attached to the wings yellowish. Preparations of both kinds of muscle are to be made in the same way as living mammalian muscle (see previous Lesson), but they are to be mounted either without the addition of any fluid or in a drop of the insect's blood. In both preparations the dark-looking air-tubes or tracheæ form prominent objects ramifying amongst the fibres. Observe the structure of the two kinds of muscle, noting especially the fine fibrils of the wing muscles and the musclerods in the large fibres of the leg muscles. If the preparation is made quickly, waves of contraction will probably be observed passing along the last-named fibres.

The remainder of the water-beetle may be put into spirit (pinned upon a cork so that the muscles are well stretched) and preparations made on a future day after the manner described in the next preparation.

2. Place in logwood a small shred of mammalian muscular tissue which has been stretched upon a cork and hardened in alcohol. When it is deeply 
stained, wash it in water and with needles break the fibres up in Farrant's solution into as fine fibrils as possible. Cover and examine with a high power.

3. Tear off a small shred of the muscular coat of a piece of intestine which has been from 24 to 48 hours in ( $\frac{1}{8}$ per cent.) bichromate of potash solution. Hold the shred with forceps in a drop of water and fray it out with a needle. In this process many cells will be set free and can be seen with a low power. The preparation may then be covered and examined with a high power. Sketch one of the cells. Then allow dilute logwood to pass under the coverglass and lastly a drop of glycerine. Sketch another cell after staining. Measure two or three cells and their nuclei.

Voluntary muscle is composed of long cylindrical fibres, measuring on an average about ${ }_{50}^{\frac{1}{0}} \pi$ inch in diameter in mammalian muscles, but having a length of an inch or more. Each fibre has an elastic sheath, the sarcolemma, which encloses the contractile substance. The sarcolemma is seldom distinct, unless the contained substance becomes broken (fig. 69).

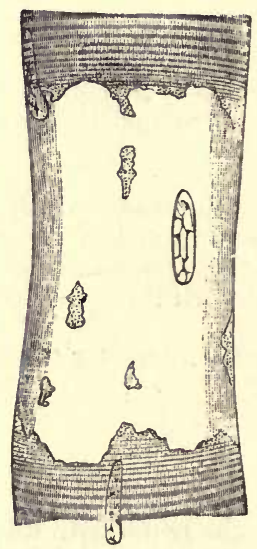

FIG. 69.-SARCOLEUMA OF MAMMALIAN MUSCLE, HIGHLY MAGNIFIED.

The fibre is represented at a place where the muscular substance has become ruptured and has shrunk away, leaving the sarcolemma (with a nucleus adhering to it) clear. The fibre had been treated with serum acidulated with acetic acid.

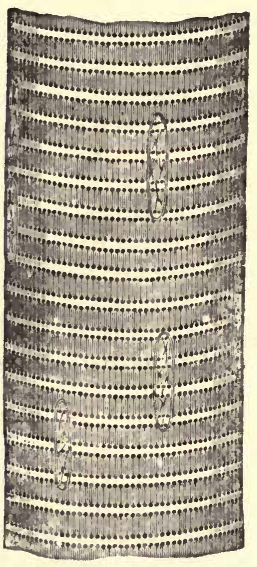

Fig. 70. - Muscular FIBRE OF A MAMMAL EXAMINED FRESH IN SERUM, HIGHLY MAGNIFIED, THE SURFACE OF THE FIBRE BEING ACCURATELY FOCUSSED.

The nuclei are seen on the flat at the surface of the fibre, and in profile at the edges.

The contractile substance of the fibre is characterised by the alternate dark and light stripes which run across the length of the fibre; hence the name, cross-striated or striped muscle. On focussing, it 
can be seen that the stripes pass through the whole thickness of the fibre; they may therefore be looked upon as representing alternate disks of dark and light substance. If the surface be very carefully focussed, double rows of granules are seen lying in or at the boundariès of the light streaks, and very fine longitudinal lines may, with a good microscope, be detected running through the dark streak, and uniting the minute granules (fig. 70). These fine lines, with their enlarged extremities the granules, are known as muscle-rods; they are more conspicuous in the muscles of insects.

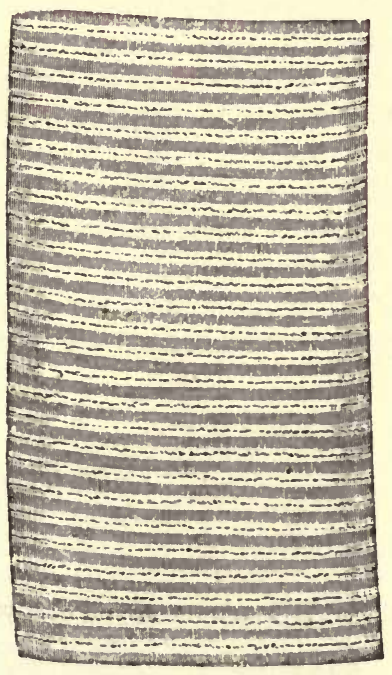

Fig. 71.-Portion of A MediumSIZEI) HUMAN MUSCULAI? FIBRE, SHOWING THE INTERMEDIATE LINE MENTIONED IN THE TEXT.

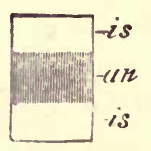

Fig. 72.-DiagramaTIC REPRESENTATION OF A MUSCLE-CASE.

s:n, musc'e-prism, consisting of a bund'e of musclerods; is, tluid substance.

If instead of focussing the surface of the fibre it be observed in its depth, a different appearance is frequently visible, namely a fine dotted line bisecting each clear stripe (fig. 71) ; but this appearance is probably due to an optical effect caused by the light being transmitted between disks of different refrangibility.

The fine line bisecting the clear stripe is, however, taken by many histologists to represent a definite structure, and is often known as Krause's membrane, having been described by Krause as connected with the sarcolemma and dividing the muscle-fibre into so many flat compartruents. Krause further described these compartments as divided longitudinally by fine membranes, so that the muscle-substance may, according to him, be regarded as composed of little cylindrical 'cases ' (fig. 72) each containing in the centre a portion of the dark disk, and, above and below this, portions of the light disks (which are fluid according to this author). ${ }^{1}$

1 For other views regarding the structure of striated muscular fibre, the student is referred to Quain's Anatomy, 9th edition, vol. ii. pp. 127 to 129. 
Besides the sarcolemma and striated substance, a muscular fibre also exhibits a number of oval nuclei which have the usual reticular structure of cell-nuclei. Sometimes there is a little granular substance (protoplasm) at each pole of the nucleus. In mammalian muscle the nuclei lie immediately under the sarcolemma (figs. 69, 70), except in certain fibres, e.g. those which compose the red muscles of some animals, such as the hare and rabbit, and which occur scattered amongst the ordinary fibres in mammalia generally. In these the nuclei are distributed through the thickmess of the fibre, and this is also the case in all the muscular fibres of the frog.

The transverse section of a muscle shows the fibres to be nearly cylindrical in figure. Between the fibres there is a certain amount of areolar tissue, which serves to support the blood-vessels and also unites them into fasciculi; the fasciculi are again united together by a larger amount of this intramuscular connective tissue.

On examining the transverse section of a fibre with a high power, it is seen to be subdivided everywhere into small angular fields, the areas of Cohnheim. These probably represent sections of the longitudinal fibrils into which a muscular fibre splits after death, or after being hardened in certain reagents, e.g. alcohol, chromic acid, or osmic acid.

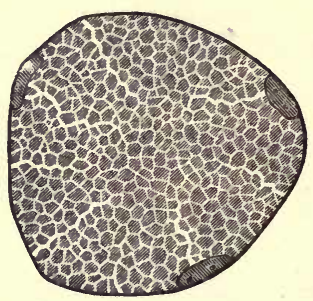

Fig. 73.-Section of a muscular fibre SHOWING AREAS OF CoHNhEIM.

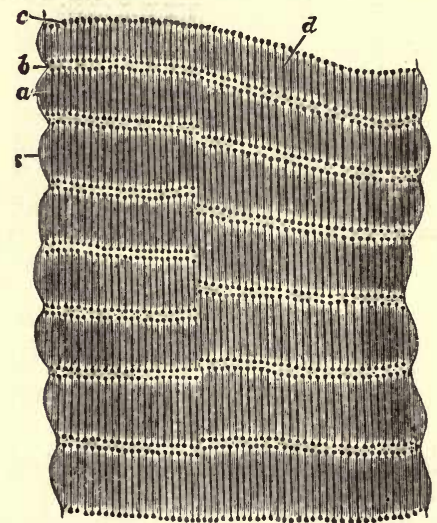

Fig. 74.-LiviNg MUSCLE OF WATERBEETLE (DYTISCUS MARgINALIS). (Highly magnified.)

$s$, sarcolemma ; $a$, dim stripe; $b$, bright stripe; $c$, row of dots in bright stripe which seem to be the enlarged ends of rod-shaped particles, $d$.

In the muscles of insects the stripes are relatively broad, and their structure can be more readily seen than in mammals. In the living fibres from the muscles of the legs, the appearance of fine longitudinal lines traversing the dark stripes, and terminating within the light stripes in rows of dots, is very obvious. When the fibres contract, the light stripes are seen, as the fibre shortens and thickens, to become 
dark, an apparent reversal being thereby produced in the striæ. This reversal is due to the enlargement of the rows of dark dots, and the formation by their juxtaposition and blending of dark disks, whilst the muscular substance between these disks has by contrast a bright appearance.

The wing-muscles of insects are easily broken up into very fine fibres or fibrils, which also show alternate dark and light striæ. The number and relative thickness of these differ, however, considerably, according to the amount of stretching of the fibres (fig. 75). Musclerods are not seen in these fibres.

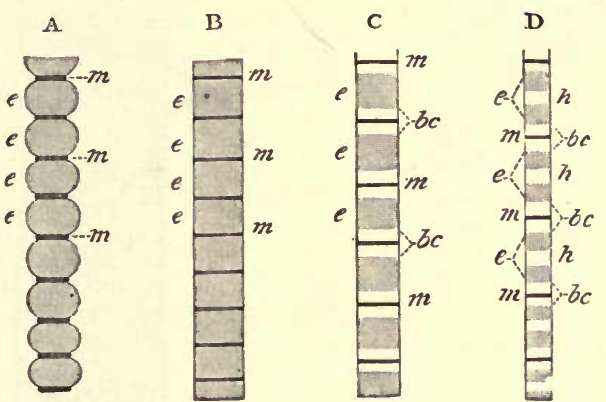

Fig. 75.-FirRes of the WiNg-muscles of an insect.

The fibres are in different conditions of extension, from A least extended, to D most extended. $e, e$, chief substance of the fibre; $m, m$, intermediate lines or disks; the light bands, $b c$, on either side of these only come to view when the fibre is sufficiently stretched (C); with further extension (D), the middle of the dark band appears lighter, $h$.

In muscular tissue which has been hardened in alcohol and certain other reagents, the structural appearances are a good deal altered from those of the living muscle, although the cross-striæ are still very obvious. There is also a considerable tendency for the fibres to split up longitudinally into fibrils, and by some authorities the finest of such fibrils are regarded as the ultimate elements of the fibre. Certain other reagents, such as dilute hydrochloric acid, cause a transverse splitting of the fibres into disks, and these effects of reagents led Bowman to form the opinion that the muscular substance may be in reality composed of minute prismatic particles set side by side in rows or planes to form the disks, and adhering end to end longitudinally to form the fibrils. To these constituent particles of the muscular substance he gave the name of ' sarcous elements.'

When living muscular fibres are examined by polarised light, the whole of the muscular substance except the muscle-rods is seen to be doubly refracting, looking bright in the dark field produced by crossing the axes of the Nichol's prisms. Contracted muscle and dead muscle show, however, alternate bands of dark and light under those circumstances.

Ending of muscle in tendon.-A small tendon-bundle passes to 
each muscular fibre and becomes firmly united with the sarcolemma, which extends over the end of the fibre (fig. 76). Further, the areolar tissue between the tendon-bundles is continuous with that which lies between the muscular fibres, so that the connection of a muscle to its tendon is very firm.

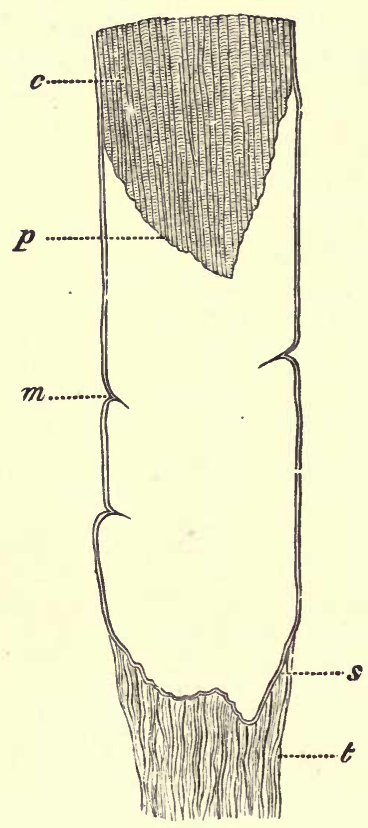

Fig. 76.-Termination of a musCULAR FIBRE IN TENDON.

$m$, sarcolemma ; $s$, the same membrane passing over the end of the fibre; $p$, extremity of muscular substance, $c$, retracted from the lower end of the sarco!emma-tube; $t$, tendon-bundle passing to be fixed to the sarcolemma.

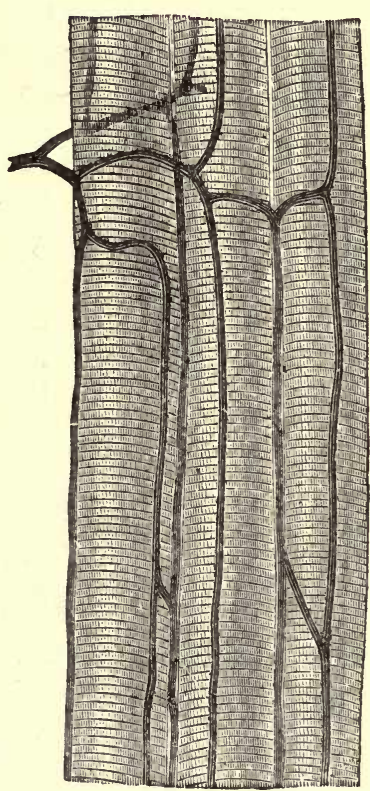

Fig. 77.-Capit.lary vessels of MUSCLE.

Blood-vessels of muscle.-The capillaries of the muscular tissue are very numerous. They run, for the most part, longitudinally, with transverse branches, so as to form long oblong meshes (fig. 77). In the red muscles of the rabbit and hare, the transverse capillaries have small dilatations upon them. No blood-vessels ever penetrate the sarcolemma.

Lymphatic vessels, although present in the connective-tissue sheath (perimysium) of a muscle, do not penetrate between its component fibres.

The nerves of voluntary muscles pierce the sarcolenma and terminate in a ramified expansion known as an end-plate (see Lesson XIX.). 
Voluntary muscular fibres are developed from embryonic cells of the mesoderm, which become elongated, and the nuclei of which become multiplied, so as to produce long multi-nucleated fusiform or cylindrical fibres. These become cross-striated at first along one side, the change gradually extending around the fibre and also towards the centre; but the middle of the fibre, to which the nuclei are at first confined, remains for some time unaltered (fig. 78). Eventually the change in structure exteids to this also, and the nuclei pass gradually to occupy their ordinary position under the sarcolemma, which by this time has become formed.

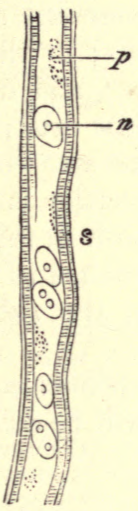

Fig. 78.-Developing MUSCULAR FIBRE, FROM FETUS OF 2 MONTHS.

$p$, central protoplasm with several nuclei, $n$, scattered in it ; $s$, commencing sarcolemma, with striated muscular substance developing immediately beneath it.

Fig. 79.-MUSCULAR Fibre-CELlS From the MUSCU. LAR COAT OF THE SMALL INTESTINE, HIGHLY MAGNIFIED.

A. A complete cell, showing the nucleus with intra-nuclear network, and the longitudinal fibrillation of the cell-substance, with finely vacuolated protoplasm between the fibrils. B. A cell broken in the process of isolation; the delicate enveloping membrane projects at the broken end a little beyond the substance of the cell.

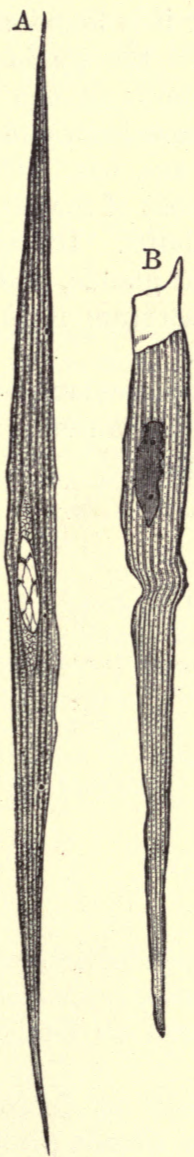

Involuntary or plain muscular tissue is composed of long, somewhat flattened, fusiform cells (fig. 79), which vary much in length, but are usually not more than $\frac{1}{600}$ inch long. Each cell has an oval or 
rod-shaped nucleus, which shows the usual intra-nuclear network and commonly one or two nucleoli. The cell-substance is longitudinally striated, but does not exhibit cross-striæ like those of voluntary muscle. There appears to be a delicate sheath to each cell. There is a little intercellular cementing substance uniting the cells together, and which can be stained by nitrate of silver. The fibres are collected into fasciculi.

Plain muscular tissue is found chiefly in the walls of hollow viscera ; thus it forms the muscular coat of the whole of the alimentary canal below the œsophagus, and occurs abundantly in the muscular coat of that tube also, although it is here intermixed with cross-striated muscle; it is found also in the mucous membrane of the alimentary canal; in the trachea and its ramifications; in the urinary bladder and ureters; in the uterus, Fallopian tubes, and ovary; in the prostate, the spleen, and muscle of Müller in the orbit, and in the ciliary muscle, and iris. The walls of gland-ducts also contain it, and the middle coat of the arteries, veins, and lymphatics is largely composed of this tissue. It occurs also in the skin, both in the secreting part of the sweat-glands, and in small bundles attached to the hair-follicles; in the scrotum it is found abundantly in the subcutaneous tissue (dartos).

The muscular tissue of the heart constitutes a special variety of involuntary muscular tissue (cardiac), and will be described along with that organ. 


\title{
LESSON XVII.
}

\author{
STRUCTURE OF NERVE-FIBRES.
}

1. Tease a piece of fresh nerve in saline solution, injuring the fibres as little and obtaining them as long and straight as possible. Study the medullated fibres, carefully noticing all the structures that are visible-viz., nodes of Ranvier, nuclei of primitive sheath, double contour of medullary sheath, medullary segments, \&c. Measure the diameter of half a dozen fibres. Draw a short length of a fibre very exactly.

2. Prepare a piece of the sympathetic nerve in the same way. Measure and sketch as before.

3. Separate (in dilute glycerine or Farrant) into its fibres a small piece of nerve that has been twenty-four hours in $\frac{1}{2}$ per cent. osmic acid. The nerve should have been moderately stretched on a piece of cork by means of pins before being placed in the acid. Keep the fibres as straight as possible and only touch them near their ends with the needles. Sketch two portions of a fibre under a high power, one showing a node of Ranvier and the other a nucleus of the primitive sheath. Look for fibres of Remak. Measure the length of the nerve-segments between the nodes of Ranvier.

4. Mount in Canada balsam sections of a nerve which has been hardened in picric acid and stained with picro-carmine. The nerve should have been stretched out before being placed in the hardening solution. Examine the sections first with a low and afterwards with a high power. Notice the lamellar structure of the perineurium, the varying size of the nerve-fibres, the axis cylinder in the centre of each fibre, \&c. Measure the diameter of five or six fibres, and sketch a small portion of one of the sections.

Nerve-fibres are of two kinds, medullated and non-medullated. The cerebro-spinal nerves and the white matter of the nerve-centres are composed of medullated fibres; the sympathetic and its branches is chiefly made up of non-medullated.

The medullated or white fibres are characterised, as their name implies, by the presence of the so-called medullary sheath or white substance. This is a layer of soft substance, chiefly of a fatty nature, which encircles the essential part of a nerve-fibre, viz. the axis-cylinder. Outside the medullary sheath is a delicate but tough homogeneous membrane, the primitive sheath or nucleated sheath of Schwann, but 
this is not present in all medullated fibres, being absent in tnose which are within the nerve-centres.

The medullary sheath is composed of a highly refracting fatty

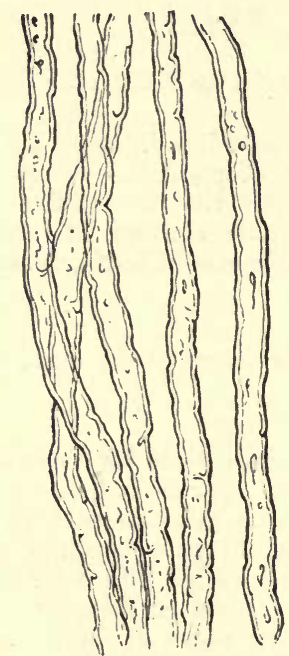

Fig. 80.-White or medullated NERVE - FIBRES, SHOWING THE SINUOUS OUTLINE AND DOUBLE CONTOURS.

Fig. 81.-Portions of TWo NerVe-Fibres STAINED WITH OSMIC ACID (From A YOUNG RABBIT). (425 diameters.)

R, R. Nodes of Ranvier, with axis-cylinder passing through. $a$, primitive sheath of the nerve. $c$, opposite the midd'e of the segment, indicates the nucleus and protoplasm lying between the primitive sheath and the medullary sheath. In $\mathbf{A}$ the nodes are wider, and the intersegmental substance more apparent than in $B$.

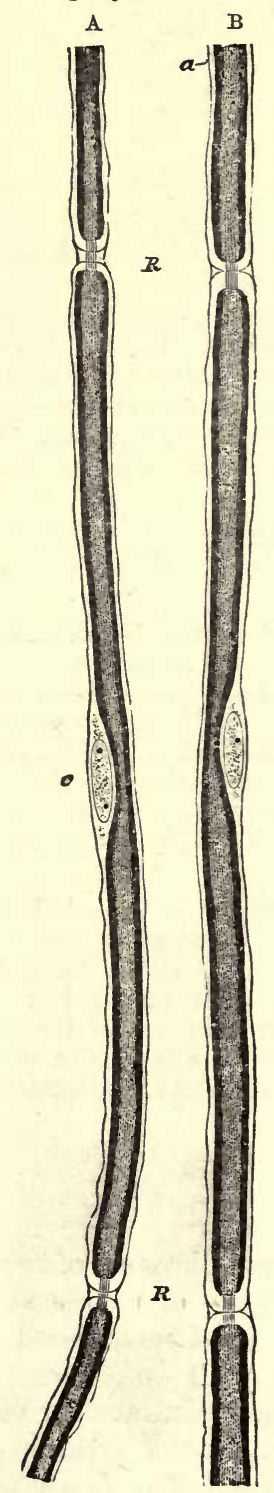

material, which gives a characteristic dark contour and tubular appearance to the nerve-fibres. It affords a continuous investment to the axis-cylinder, except that it is interrupted at regular intervals in the 


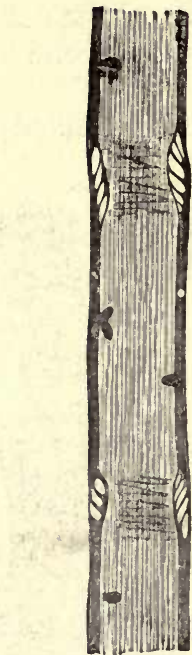

Fig. 82.-A small PART OF A MedulLATED FIBRE, HIGHLY MAGNIFIED.

The fibre looks in optical section like a tubehence the term tubu'ar, formerly applied to these fibres. Two partial breaches of continuity are seen in the medu'lary sheath, which at these places exhibits a tendency to split into laminæ. The primitive sheath is here and there apparent outside the medullary sheath, and the delicate striæ which are visible in the middle of the fibre probably indicate the fibrillated axis-cylinder.

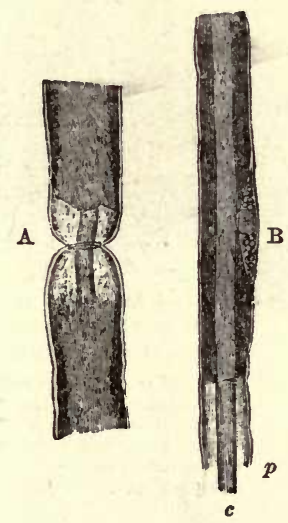

Fig. 84.-Two PoRtions OF MEDUllated NERVE-FIBRE: AFTER TREATMENT WITH OSMIC ACID, SHOWING THE AxISCYLINDER, AND THE MEDULLARY AND PRIMTITE SHEATHS.

A. Node of Ranvier. B. Middle of an internode with nuc eus. $c$, axis-cylinder, projecting; $p$, primitive sheath, within which the medullary sheath, which is stained dark by the osmic acid, is somewhat retracted.

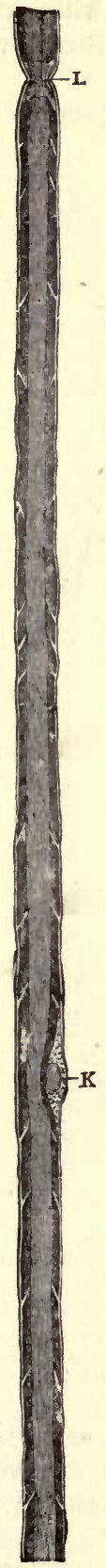

course of the peripheral nerve-fibres, the axis-cylinder at these places being encompassed only by the primitive sheath. Hence the primitive sheath appears at these spots to produce a constriction in the nerve-fibre, and the interruptions of the medullary sheath are accordingly known as the constrictions or nodes of Ranvier (fig. 81, $\mathrm{R}, \mathrm{R}$; fig. $83, \mathrm{~L})$, the term nodes being applied from the resemblance which they bear to the nodes of a bamboo. The length of nerve between two successive nodes may be termed an internode; in the middle of each internode is one of the nuclei of Schwann's sheath. Besides these interruptions the medullary sheath shows a variable number of oblique clefts (fig. 83) which subdivide it into irregular portions, which have been termed medullary segments, but there is reason to believe that the clefts are artificially produced. Osmic acid stains the Fig. 83. - Nerve- medullary sheath
Fibre WITH OSMIC ACID. black. 
The axis-cylinder, which runs along the middle of the nerve-fibre, is a soft transparent thread which is continuous from end to end of the nerve.

On account of the peculiar refractive power of the medullary sheath

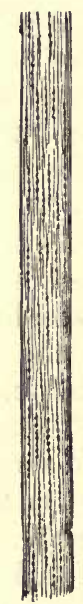

Fig. 85. - AxisCYLINDER, HIGHLY MAGNIFIED, SHOWING THE FIBRILS COMPOSING IT.

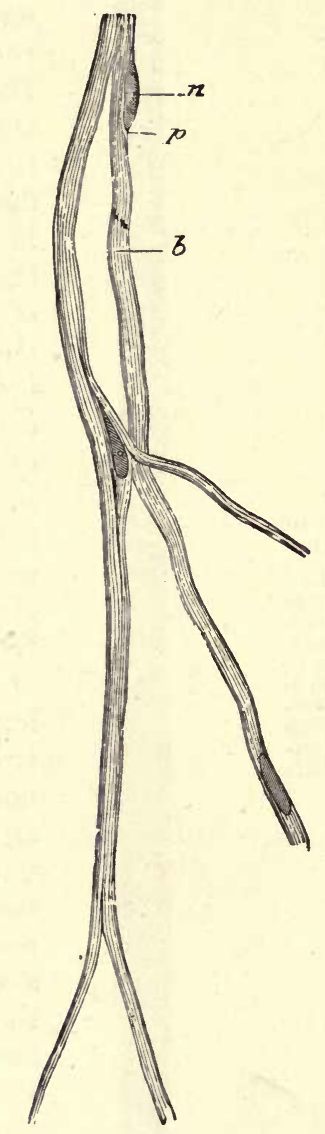

Fig. 86. - Portion of THE NETWORK OF FIBRES OF REMAK FROM THE PNEUMOGASTRIC OF THE DOG.

$n$, nucleus; $p$, protoplasm surrounding it; $b$, striation caused by fibrils.

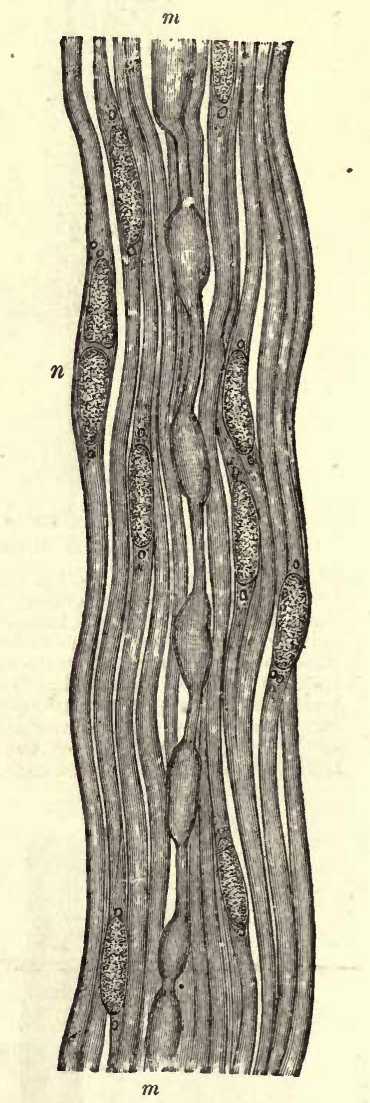

Fig. 87.-A SMALL BUNDLE OF NERVE-FIBRES FROM THE SYMPATHETIC NERVE.

The bund'e is composed of pale nerve-fibres, with the exception of the fibre $m, m$, which is en. closed here and there by a thin medullary sheath; $n, n$, nuclei of pale fibres.

it is difficult to see the axis-cylinder in the fresh nerve except at the nodes, where it may be observed stretching across the interruptions in the medullary sheath, and it may also sometimes be seen projecting from a broken end of a nerve-fibre. It is longitudinally striated, being really made up of exceedingly fine fibrils (ultimate fibrils, fig. 85), which are darkly stained by chloride of gold. Staining with nitrate 
of silver produces a curious transversely striated appearance in the axiscylinder, but it is not known if this indicates a pre-existent structure.

Intermingled with the medullated fibres there may always, even in the cerebro-spinal nerves, be found a certain number of pale fibres devoid of the dark double contour which is characteristic of the presence of a medullary sheath. There are the non-medullated fibres, also called, after their discoverer, fibres of Remak (fig. 86). They frequently branch, which the medullated fibres never do except near their termination, and they are beset with numerous nuclei which perhaps belong to a delicate sheath. The sympathetic nerve is in many animals chiefly made up of fibres of this nature, but in some animals, on the other hand, most of the fibres of the sympathetic possess some small amount of medullary sheath (fig. 87).

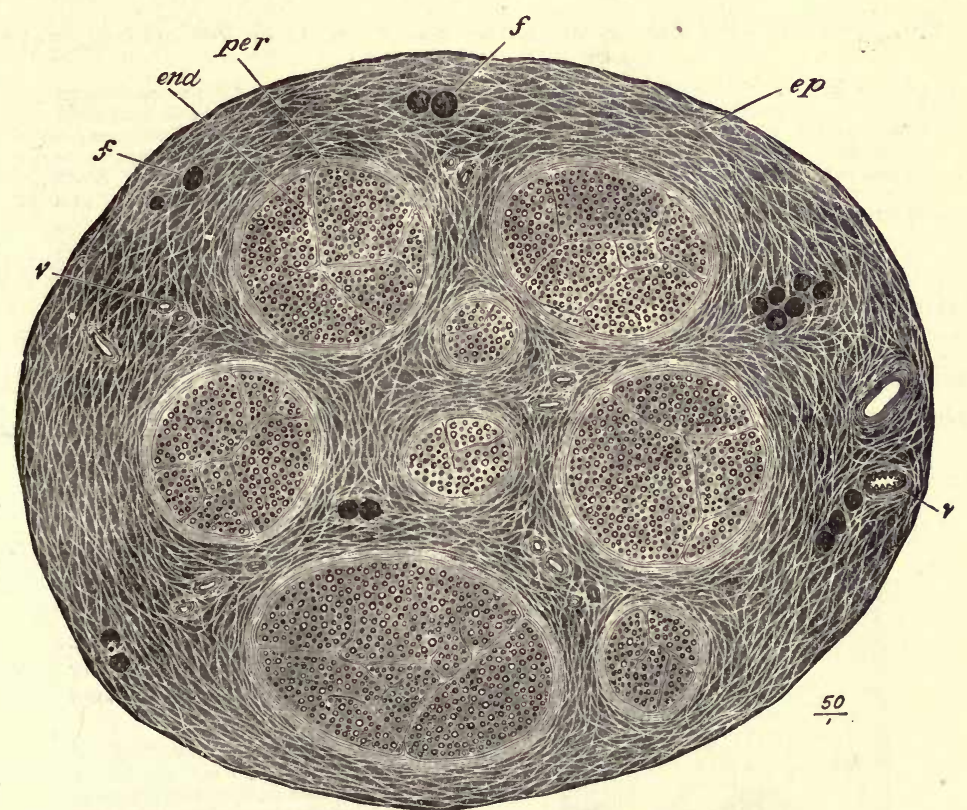

Fig. 88.-Section of the saphenous nerve (human), Made after being STAINED IN OSMIC ACID AND SUBSEQUENTLY HARDENED IN AI.COHOL. (Drawn as seen under a very low magnifying power.)

$e p$, epineurium, or general sheath of the nerve, consisting of connective-tissue bundles of variable size separated by cleft-like areolæ, which appear as a network of clear lines with here and there fat-cells and blood-vessels : $f, f$, funiculi enc'osed in their lamellated connective-tissue sheaths (perineurium, $p$ ); end, interior of funiculus, showing the cut ends of the medullated nerve-fibres, which are embedded in the connective tissue within the funiculus (endoneurium). The fat-cells and the nerve-fibres are darkly stained by the osmic acid, but the connective tissue of the nerve is only slightly stained.

Structure of the nerve-trunks. - In their course through the body the nerve-fibres are gathered up into bundles or funiculi, and the funiculi may again be united together to form the nerves which we meet with in dissection. The connective tissue which unites the 
funiculi and invests the whole nerve, connecting it to neighbouring parts and conveying to it blood-vessels, lymphatics, and even nervefibres destined for its coats, is termed the epineurium (fig. 88, ep). That which ensheaths the funiculi is known as the perineurium (fig. 88 , per). It has a distinctly lamellar structure (fig. 89, $p$ ), the lamellæ

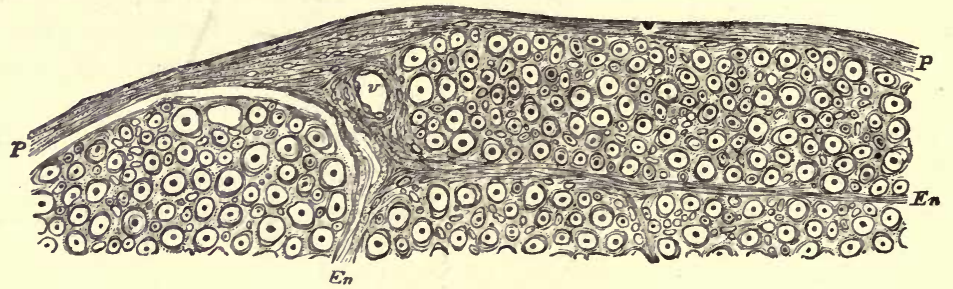

Fig. 89.-Part of a section of one of the funiclli of the sciatic Nerve OF MAN. (Magnified.)

$P$, perineurium, consisting of a number of c'ose'y arranged lamellæ. En, processes from the perineurium, passing into the interior of the funiculus, and becoming continuous with the endoneurium, or delicate connective tissue between the nerve-fibres. The connectivetissue fibri s of the endoneurium are seen cut across as fine points, often appearing to ensheath the nerve-fibres with a circle of minute dots (fibril-sheath of Key and Retzius). Numerous uuclei of connective-tissue cells are embedded in the endoneurium; $r$, section of a blood-ressel.

being composed of connective tissue and covered on both surfaces by flattened epithelioid cells (fig. 90). Between the lamellæ are clefts for the conveyance of lymph to the lymphatics of the epineurium. The delicate connective tissue which lies between the nerve-fibres of the funiculus is the endoneurium (fig. 88, end; fig. 89, En). It assists in

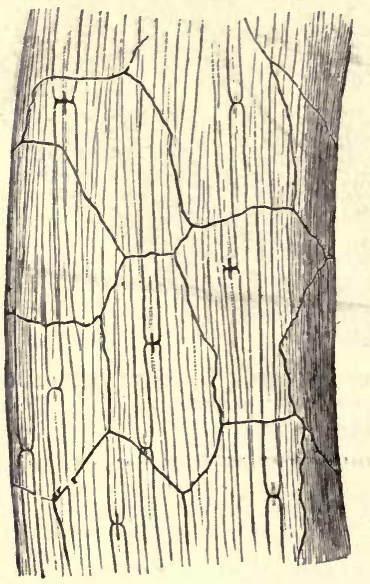

Fig. 90. - Nerve-funiculus STAINED WITH NITRATE OF SILVFR, SHOWING THE OUTLINES OF EPITHELIOID CELLS OF THE PERINEURIUM.

The dark crosses on the nerve-fibres at the nodes of Ranvier are due to the staining of the axis-cylinder and of $\mathrm{a}$ band of intercellular substance which encircles the axis-cylinder at the node (constricting band of Ranvier).

supporting the longitudinally arranged meshwork of blood-capillaries, and its interstices communicate with the lymphatic clefts of the perineurium.

The nerve-trunks themselves receive nerve-fibres (nervi nervorum) which ramify chiefly in the epineurium and terminate in this in endbulbs. 


\section{LESSON XVIII.}

\section{STRUCTURE OF NERVE-CELLS.}

1. TEAsE in Farrant's solution or in dilute glycerine a small piece of a spinal ganglion that has been preserved with osmic acid. Notice the spheroidal ganglion-cells; their large nuclei and distinct nncleoli. Look for cells which still retain the axis-cylinder process and for $\mathrm{T}$-shaped junctions of nervefibres with this.

2. Prepare a piece of sympathetic ganglion in the same way. Cells may be found with three or more axis-cylinder processes. If from a rabbit observe that the cells are bi-nucleated.

3. Mount stained sections of ganglia in Canada balsam. These will serve to show the arrangement of the cells and fibres in a ganglion and the nucleated sheaths around the nerve-cells.

4. Tease out a portion of the grey matter from a piece of spinal cord that has been a day or two in dilute chromic acid ( $\frac{1}{20}$ per cent.). Before covering, look for the nerve-cells with a low power, and if possible get out one or two clear of the surrounding substance. Mount in water with a thick hair under the cover-glass. Notice the large branching cells some with a mass of pigment near the nucleus. Observe the fibrillation of the cell-processes. Notice also the reticular character of the tissue in which the cells are embedded. Many axis-cylinders ' will be seen in this preparation deprived wholly or partially of their medullary sheath, and their fibrillar structure can then also be well seen. Carefully sketch these appearances. To keep this preparation run very dilute logwood solution or osmic acid under the cover-glass, and when the cells are stained allow a drop of glycerine to pass in by diffusion.

Measure two or three cells in each of the above preparations.

Nerve-cells only occur in the grey matter of the nerve-centres, and in little groups on the course of certain of the peripheral nerves, these groups often causing nodular enlargements of the nerves, which are known as ganglia. The most important ganglia are those which are found upon the posterior roots of the spinal nerves, upon the reots of some of the cranial nerves, and upon the trunk and principal branches of the sympathetic nerve. Minute ganglia are also found very numerously in connection with the nerves which are supplied to involuntary muscular tissue, as in the heart, alimentary canal, bladder, uterus, \&c.

Nerve-cells vary much in size and shape; they are mostly large, some being amongst the largest cells met with in the body, but others are quite small. The nucleus is generally large, clear, and 
spherical, with a single large and distinct nucleolus. The shape depends a good deal on the number of processes, and the manner in which they come off from the cell. If there is but one process, the cell is generally spherical. This is the case with the cells of the

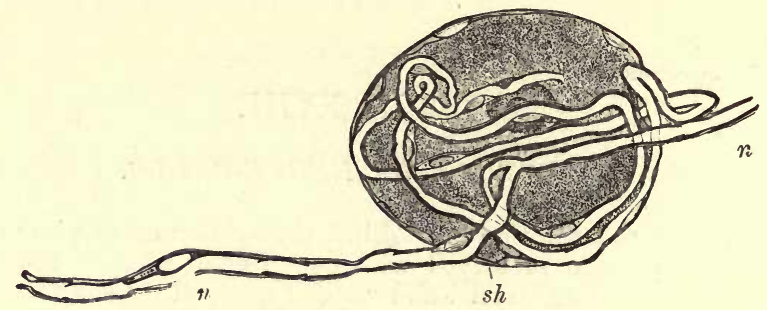

Fig. 91.-Cell from a spinat ganglion.

$s h$, nucleated sheath of the cell ; $n, u^{\prime}$, the nerve-fibre which the single process of the cell, after a number of coils, joins.

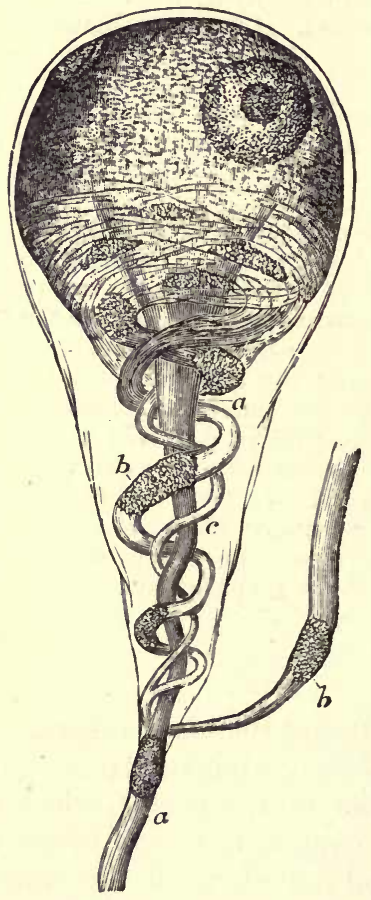

Fig 92.-GaNglion-Celi, of a FROG, HIGHLY MAGNIFIED.

$a$, $a$, straight fibre; $b, b$. coiled fibre; $c$, smaller one joining it.

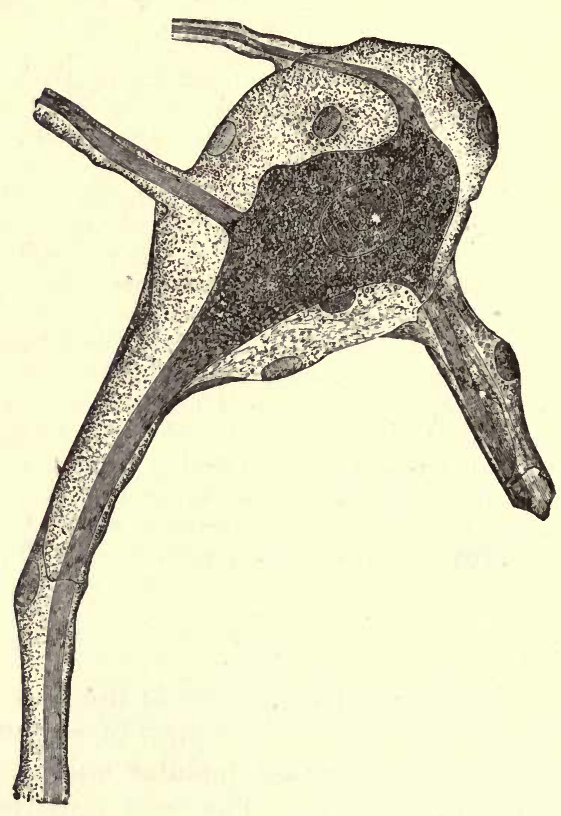

Fig. 93. - A GANglion-CElL within ITS SIEATH; FROM THE HUMAN SYMPATHETIC. (Highly magnified.)

spinal ganglia (fig. 91); in these the single process, after a short course, joins one of the nerve-fibres which is traversing the ganglion. When there are two processes, they often go off in opposite directions 
from the cell, which is thus rendered somewhat spindle-shaped, but occasionally they emerge at the same part, and the cell, being tapered in their direction, becomes pyriform (fig. 92). In these cases one fibre often coils spirally round the other (fig. $92, b$ ) before they separate to proceed in opposite directions as the axis-cylinders of nerve-fibres. When there are three or more processes, the cell becomes irregularly angular or stellate. Sometimes, as in the sympathetic ganglia (fig. 93), all the processes appear to become nerve-fibres, but in other instances, as in the large cells of the grey matter of the spinal cord, only one

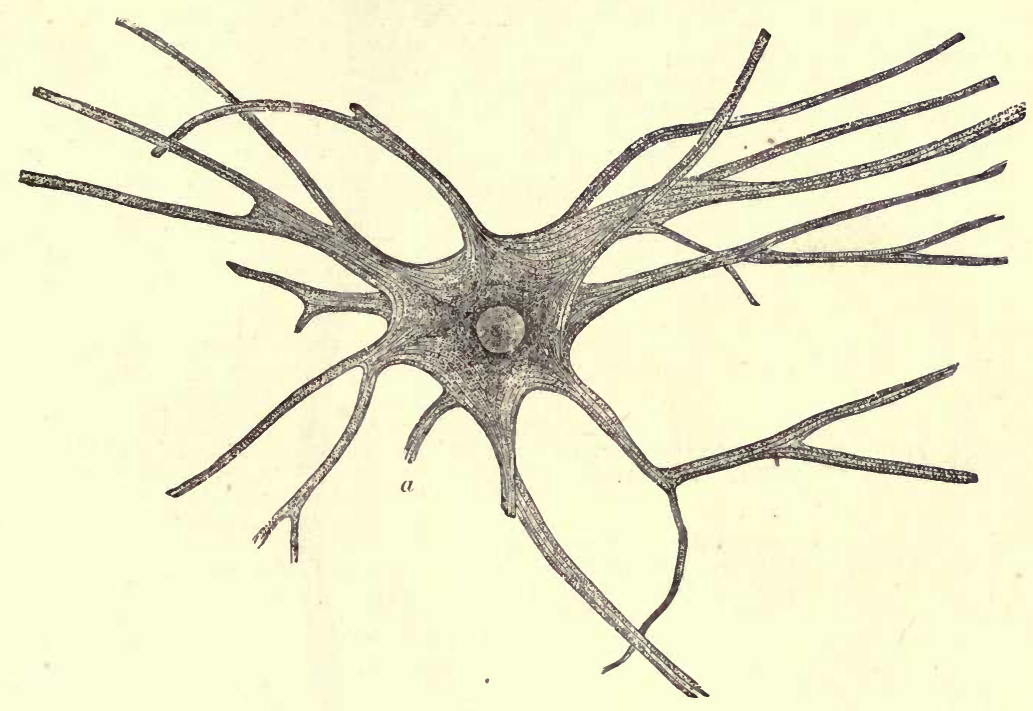

Fig. 94.-Nerve-Cell from spinal cord of ox, isolated After maceration IN VERY DILUTE CHROMIC ACID. (Magnitied 175 diameters.)

The cell has a well-defined, clear, round nucleus, and a bright nucleolus. The cell processes are seen to be finely fibrillated, the fibrils passing from one process into another through

the body of the cell. a, axis-cylinder process broken a short distance from the cell.

process becomes the axis-cylinder of a nerve-fibre (process of Deiters), the others dividing and subdividing in a ramified manner until their further course can no longer be traced. Their ultimate branches appear to lose themselves in a network which pervades the whole of the grey matter.

According to the number of their processes, nerve-cells are termed uni-, bi-, or multi-polar.

Many nerve-cells, and notably those of the spinal cord, have a finely fibrillar structure. The fibrils can be traced into the branches of the cells and into the axis-cylinders of nerve-fibres which are connected with the cells (fig. 95). Otherwise the cells have a finely granular appearance; often with a clump of black, brown, or yellow pigmentgranules placed at one side of the nucleus. 
In the ganglia the nerve-cells have a nucleated sheath (figs. 9193) which is continuous with the primitive sheath of the nerve-fibres with which they are connected. In the spinal ganglia, and in many o: the ganglia at the roots of the cranial nerves, the cells are unipolar
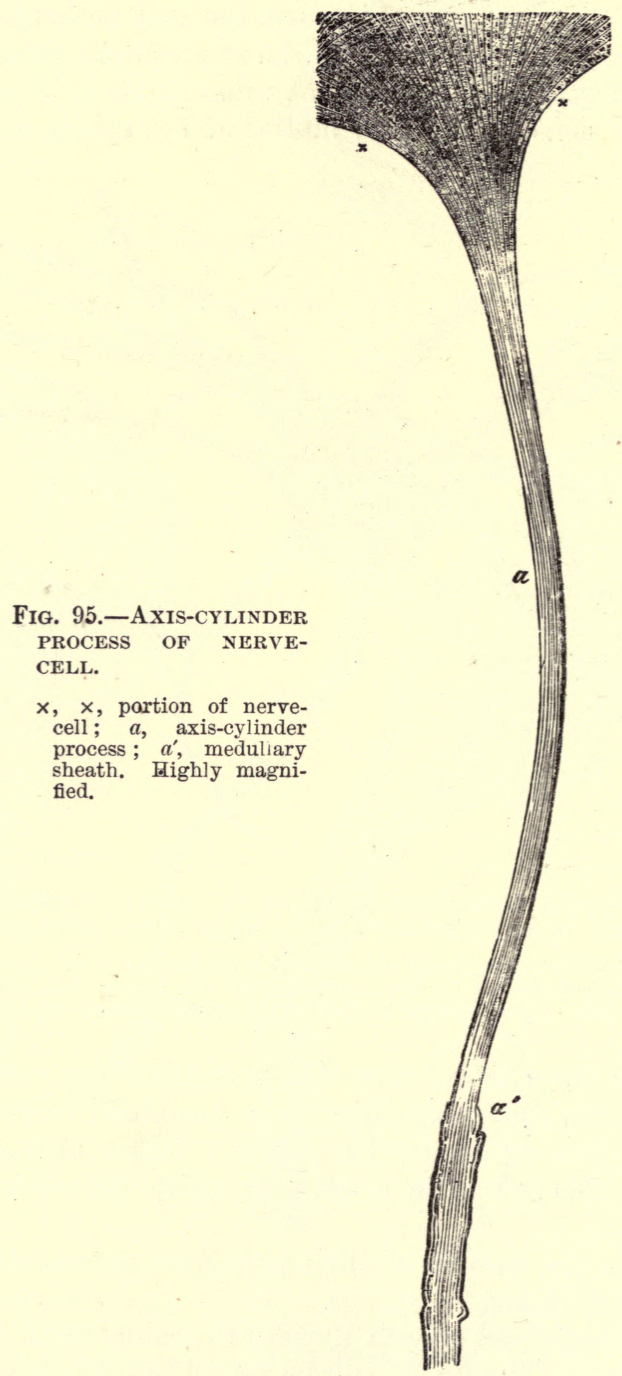

and the cell-process joins a traversing nerve-fibre by a T-shaped junction (fig. 91). In the sympathetic ganglia they are multipolar. The cells are disposed in aggregations of different size, separated by the bundles of nerve-fibres which are traversing the ganglion (fig. 96). 
The ganglion if large is enclosed by an investing capsule of connective tissue which is continuous with the epi-and peri-neurium of the entering and issuing nerve-trunks.

The structure of the nerve-centres and the arrangement of the sells and fibres in them are given in Lessons XXXVI. to XXXIX.

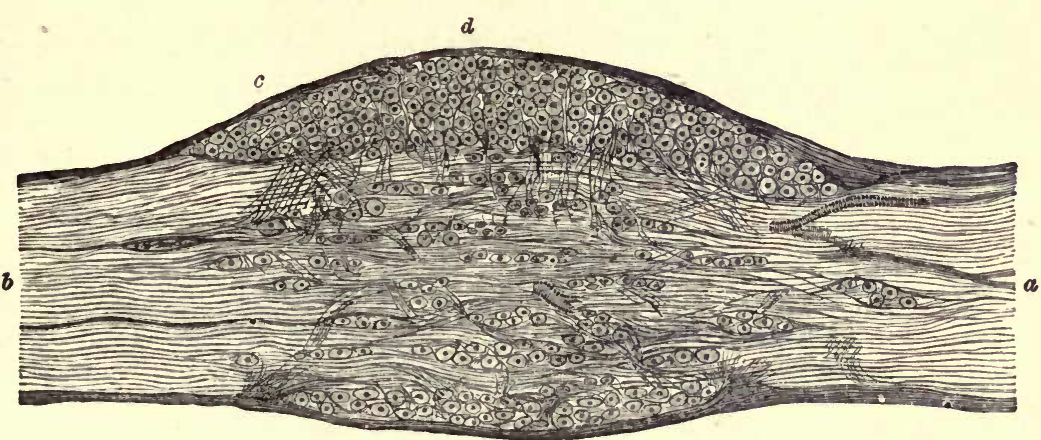

Fig. 96.-Longituninat. Section throvgh THE MidDle of a ganglion on THE PUSTERIOR ROOT OF ONE OF THE SACRAL NERVES OF THE DOG, AS SEEN UNDER A LOW MAGNIFYING POWEL.

$a$, nerve-root entering the ganglion; $b$, fibres leaving the ganglion to join the mixed spinal nerve; $c$, connective tissue coat of the ganglion; $d$, principal group of nerve-cells, with fibres passing down from amongst the cells, probably to unite with the longitudinally coursing nerve-fibres by $T$-shaped junctions.

Development.-The cells and fibres of the nervous tissue are developed from cells derived from the ectoderm or epiblast of the embryo. The nerve-fibres are at first developed as pale fibres like the fibres of Remak; it is uncertain whether they are formed by the coalescence of a number of cells, or whether they grow out as the processes of nerve-cells. The medullary sheath is subsequently added.

When a nerve is cut, the fibres beyond the section as far as their terminations undergo a process of degeneration, the medullary sheath being broken up and the axis-cylinder interrupted and eventually absorbed. New nerve-fibres are at length produced by a growth of the axis-cylinders in the proximal end of the nerve. 


\section{LESSON XIX.}

\section{MODES OF TERMINATION OF NERVE-FIBRES.}

1. Shell out a Pacinian corpuscle from a piece of cat's mesentery which has been kept for two or three days in $\frac{1}{20}$ per cent. chromic acid, and clear it as much as possible of adhering fat, but be careful not to prick or otherwise injure the corpuscle itself. Mount in water with a thick hair to prevent crushing with the cover-glass. Sketch the corpuscle under a low power, and afterwards draw under a high power the part of the core where the nerve enters and the part where it terminates. Notice the fibrous structure of the lamellar tunics of the corpuscle and the oval nuclei belonging to flattened epithelioid cells which cover the tunics. The distinct lines which when seen in the fresh corpuscle are generally taken for the tunics, are really the optical sections of these flattened cells.

2. Mount in Farrant one or more sections of a rabbit's cornea which has been stained with chloride of gold. Notice the arrangement in plexuses of the darkly stained nerve-fibres and fibrils, (1) in the connective-tissue substance, (2) under the epithelium and (3) between the epithelial cells. Make one or two sketches showing the arrangement of the fibrils.

3. Spread out a small piece of muscle which has been stained with chloride of gold by Löwit's method; and examine it with a low power to find the nerve-fibres crossing the muscular fibres and distributed to them.

Try and separate those parts of the muscular fibres to which nerves appear to go, and mount them in glycerine. Search thoroughly for the close terminal ramifications (end-plates) of the axis-cylinders immediately within the sarcolemma.

It is rather difficult to dissociate the fibres, and much patience is sometimes required in searching for the nerve-terminations, but when they are found the trouble is amply repaid. ${ }^{1}$

Modes of ending of sensory nerve-fibres.-Nerve-fibres which are distributed to sensory parts end either in special organs or in terminal ramifications or plexuses. There are three chief kinds of special organs, termed respectively Pacinian corpuscles, tactile corpuscles, and end-bulbs. In the tactile corpuscles and end-bulbs the connectivetissue sheath of a medullated fibre expands to form a somewhat solid bulbous enlargement, which is either cylindrical or spheroidal in the end-bulbs and ellipsoidal in the tactile corpuscles. In both kinds of endorgan there is a capsule of connective tissue within which is generally a sort of core containing numerous nucleated cells. As the nerve-fibre enters the corpuscle (which in the tactile corpuscle only happens after it has reached the distal part of the corpuscle having wound spirally

1 For methods of staining with chloride of gold see Appendix. 
once or twice round it) it loses its sheaths and is prolonged as an axiscylinder only, which terminates after either a straight or a convoluted course within the organ (see figs. 98 to 101). Tactile corpuscles occur

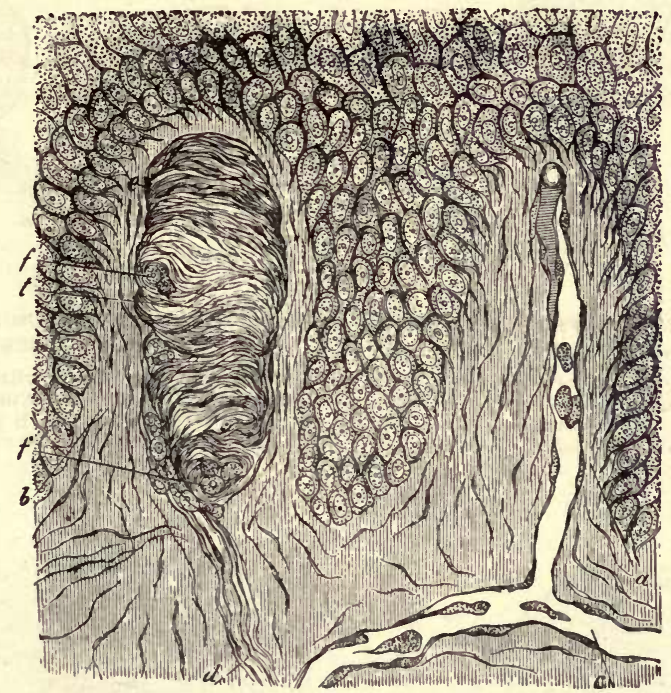

Fig. 97.-Section of SKIN SHOWING TWO PAPILle AND DEEPER LAYERS of EPIDERMIS.

$a$, vascular papilla with capillary loop passing from subjacent vessel, $c ; b$, nerve-papilla with tactile corpuscle, $t$. The latter exhibits transverse fibrous markings; $d$, nerve passing up to it $; f, f$, sections of spirally winding nerve-fibres.

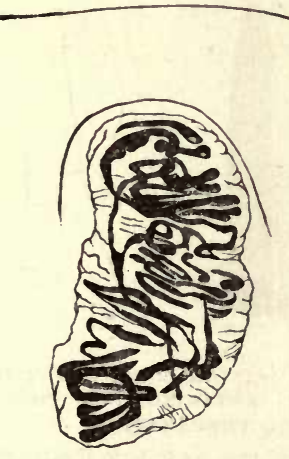

Fig.98.-TACTILE CORPUSCLE WITHIN A PAPILLA OF THE SKIN OF THE HANI, STAINED WITH CHLORIDE OF GOLD.

The convo'utions of the nervefibres within the corpuscle are seen. Ep. epidermis.

\section{Ep}
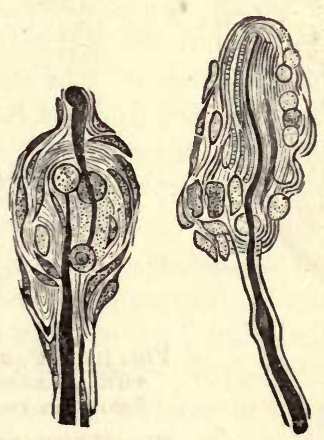

Fig. 99.-Simple tactile END-ORGANS FROM THE CLITORIS OF THE RABBIT.

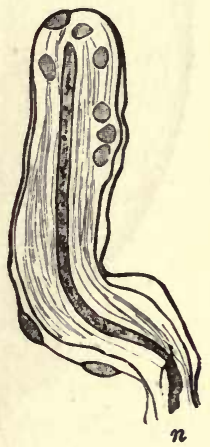

Fig. 100.-CyLiNDRICAL KND-BULB FROM THE CONJUNCTIVA OF THE CALF. 


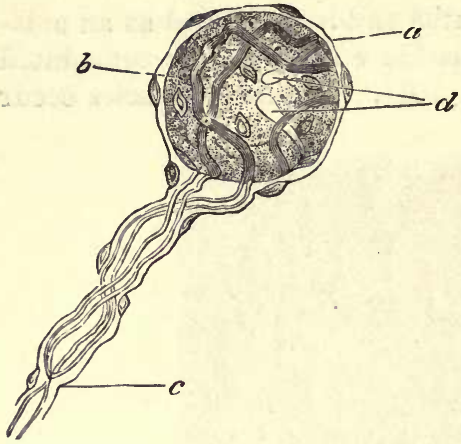

Fig. 101.-END-BULb From the humaN CONJUNCTIVA.

$a$, nucleated capsule; $b$, core, the outlines of its cells are not seen; $c$, entering fibre, branching, and its two divisions passing to terminate in the core at $d$.

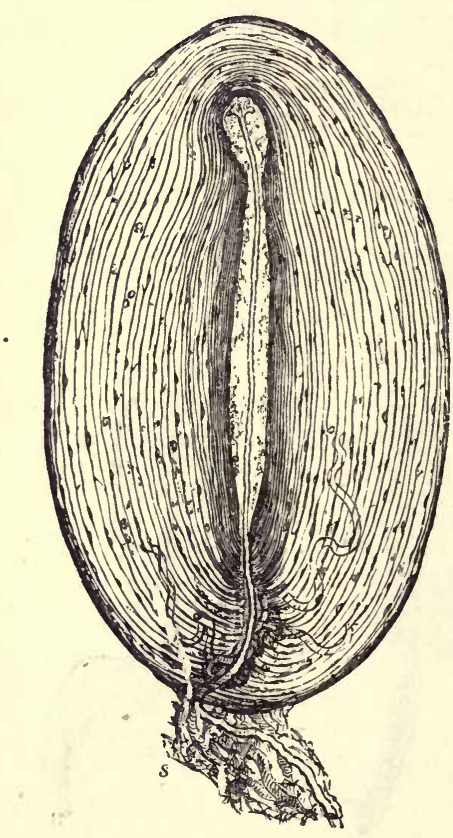

Fig. 103.-MAgnified view of A PACINIAN BODY FROM THE CAT's MESEN'TERY.

s, stalk with nerve-fibre passing to the corpuscle. One or two canillaries are also seen issuing from it between the tunics.
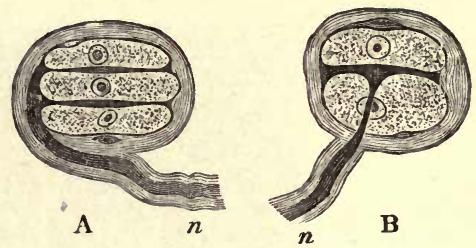

Fig. 102.-Tactile Corpuscles from the DUCK'S TONGUE.

A, composed of three cells, with two interposed disks, into which the axis-cylinder of the nerve, $n$, is observed to pass; in B there is but one tactile disk enclosed between two tactile cells.

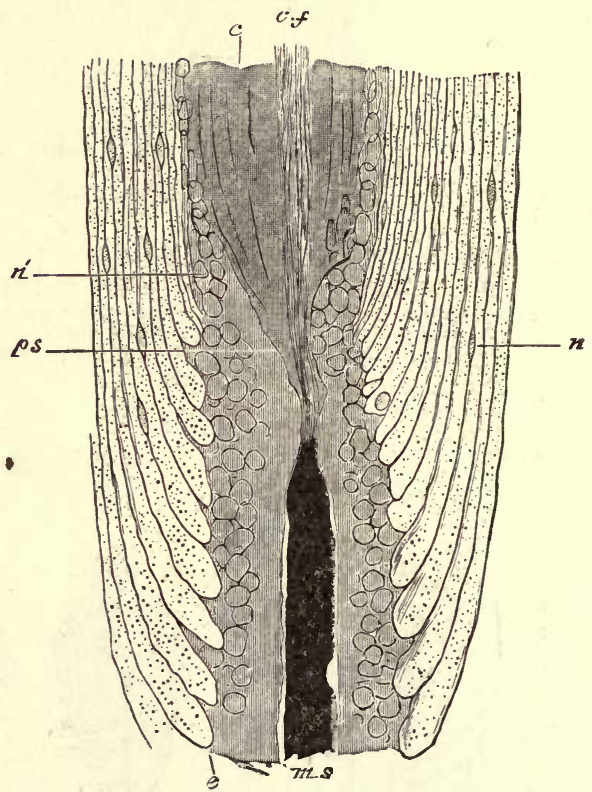

Fig. 104.-Part of Pacinian body, showing TIE NERVE-FIBRES ENTERING TIIE CORE. From aN OSMIC ACID PREPARATION.

$m s$, entering nerve-fibre, the medullary sheath of which is stained darkly, and ende abruptly at the core; $p s$, pro'ongation of primitive sheath, passing towards the nuter part of the core: $c_{\text {. }} f$. axis-cylinder passing through the core of the central fibre $e$, some of the inner tunics of the corpuscle, enlarged where they abut against the canal through which the nerve-fibre nasses-the dots within them are sections of the fibres of which they are composed; $n$, nnc'ei of the tunics; $n^{\prime}$, nuc'ei of the entoneurium, continued by others in the outer part of the core. 
in some of the papillæ of the skin of the hand and foot, in sections of which they may be afterwards studied (see Lesson XXIII.) End-bulbs are found in the conjunctiva of the eye, where in most animals they have a cylindrical or oblong shape (fig. 100), but in man are spheroidal (fig. 101). They have also been found in papillæ of the lips and tongue, and in the epineurium of the nerve-trunks, and somewhat similar sensory end-organs also occur in the integument of the external genital organs of both sexes (fig. 99). In the skin covering the bill, and in the tongue of certain birds (e.g. duck), a simple form of endorgan occurs, consisting of two or more cells arranged in rows within a capsule, with the axis-cylinder terminating in flattened expansions between the cells (corpuscles of Grandry, fig. 102).

The Pacinian corpuscles are larger, and have a more complex structure, than the tactile corpuscles and end-bulbs (fig. 103). They are composed of a number of concentric coats arranged like the layers of an onion, and enclosing the prolonged end of a nerve-fibre. A single medullated nerve-fibre goes to each Pacinian corpuscle encircled by a prolongation of perineurium, and within this by endoneurium; when it reaches the corpuscle, of which it appears to form the stalk, the lamellæ of the perineurium expand to form some of the tunics of the corpuscle. The nerve passes on, piercing the other tunics, and still provided with medullary sheath, and surrounded by endoneurium, to reach the centre of the corpuscle. Here the endoneurium is prokonged to form a sort of soft cylindrical core, along the middle of which the nerve-fibre, now deprived of its medullary and primitive sheaths, passes in a straight course as a simple axis-cylinder (fig. 104, c.f.) to terminate at the farther end of the core in a bulbous enlargement. Occasionally the fibre is branched.

The tunics of the corpuscle are composed of connective tissue, the fibres of which for the most part ran cireularly. They are covered on both surfaces with a layer of flattened epithelioid cells, and here and there cleft-like lymph-spaces can be seen between them like those between the layers of the perineurium (see p. 74).

When sensory nerve-fibres terminate in plexuses, they generally branch once or twice on nearing their termination. The sheaths of the fibres then successively become lost, first the connective tissue or perineural sheath, then the medullary sheath, and lastly the primitive sheath, the axis-cylinder being alone continued as a bundle of primitive fibrils (fig. $105, n$ ). This branches and joins with the ramifications of the axis-cylinders of neighbouring nerve-fibres to form a primary plexus. From the primary plexus smaller branches $(a)$ come off, and these form a secondary plexus $(e)$ nearer the surface, generally immediately under the epithelium if the ending is in a membrane covered by that tissue. Finally, from the secondary plexus nerve-fibrils proceed and form a terminal plexus or ramification amongst the epitheliumcells (fig. 106, $p$ ), the actual ending of the fibrils being generally in little knob-like enlargements $(b)$. Such a mode of ending in terminal 


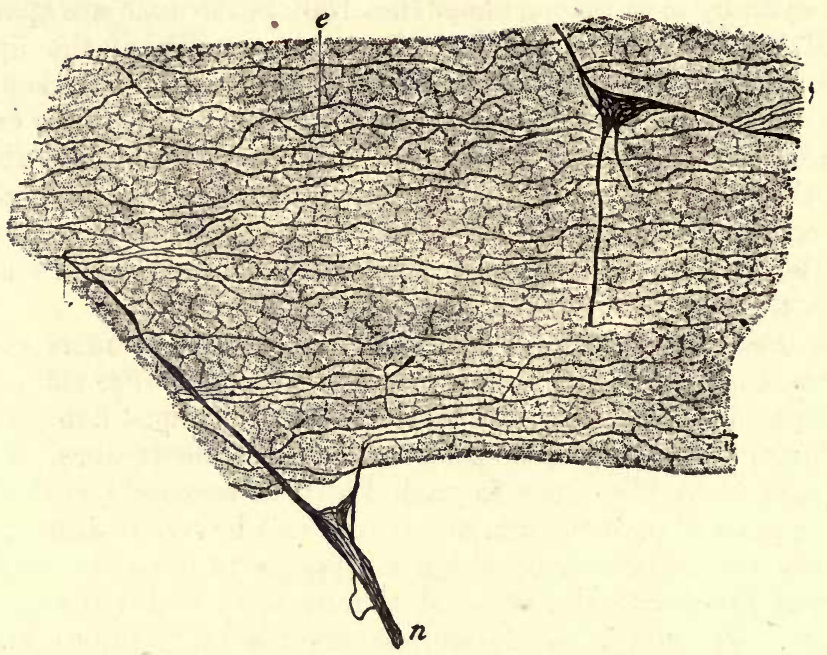

Fig. 105.-Sub-epithelial Plexus of the cornea treated with Chloride of GOLD. (Ranvier.)

$n$, branch of primary plexus; $a$, small branch passing to join the sub-epithelial plexus, $e$.

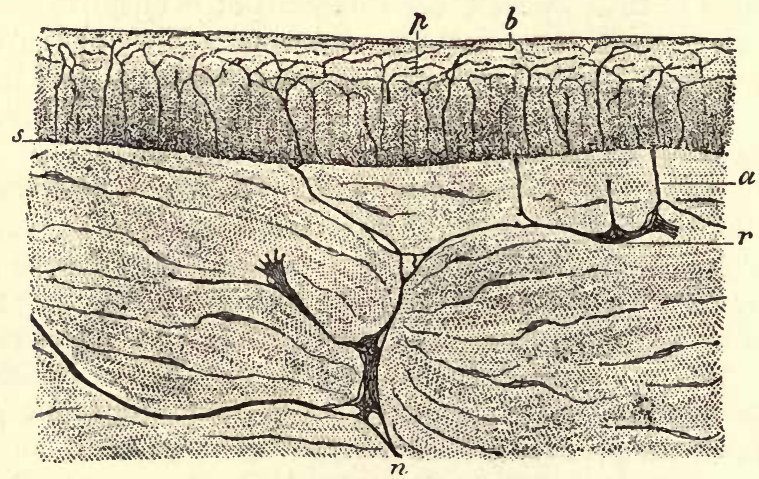

Fig. 106 - Vertical section of cornea stained with Chloride of gold. (Ranvier.)

$n, r$, primary plexus in connective tissue of cornea ; $a$, branch passing to sub-epithelial plexus, 8 ; $p$, intra-epithelial plexus; $b$, terminations of fibrils. 
plexuses is most characteristically seen in the cornea of the eye. The nerve-tibrils may be brought distinctly into view by staining with chloride of gold, and then the fibrillar structure of the ramifications of the axis-cylinders also becomes very apparent.
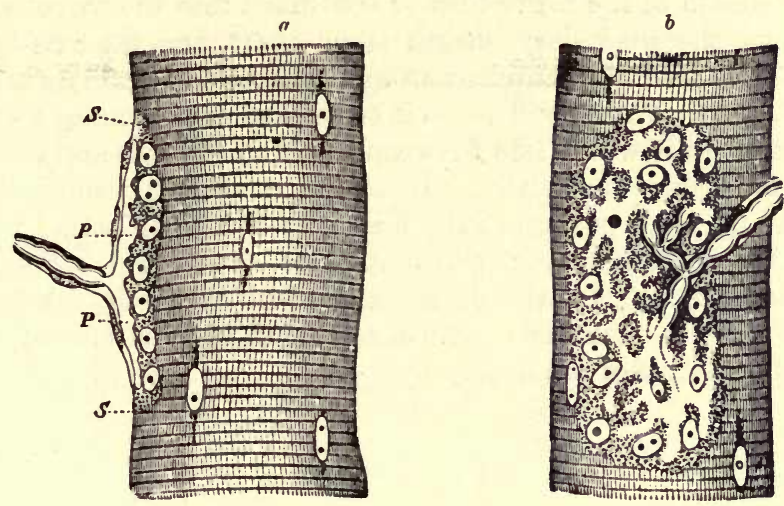

Fig. 107.-Nerve-ending in muscular fibre of a lizakd (Lacerta viridis)

$a$, end-plate seen edgeways; $b$, from the surface; $s, s$, sarcolemma; $p, p$, expansion of axiscylinder. In $b$ the expansion of the axis-cylinder appears as a clear network branching from the divisions of the medullated fibres.
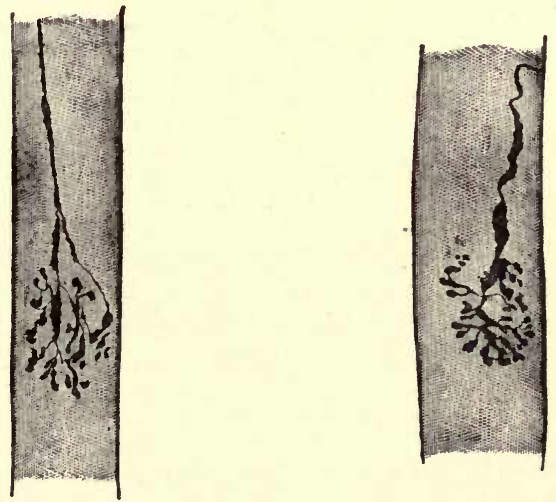

Fig. 108-Terminal RAMIFICATIONS OF THE AXIS-CYLINDER IN END-PLATES OF MUSCLE, STAINED WITH CHLORIDE OF GOLD. (Ranvier.)

Ending of motor nerves.-Lastly the nerves to muscles also terminate either in special organs or in plexuses. The latter is the case with the nerves going to involuntary muscle, and here the primary plexuses are generally furnished with ganglion-cells in abundance. From these other nerve-fibres pass which form secondary plexuses and terminal ramifications amongst the contractile fibre-cells. These nerves will be more fully studied in connection with the intestine (see Lesson XXIX.) 
In voluntary muscle the nerves, which are always medullated, terminate in special organs, the so-called end-plates. A medullated fibre will branch two or three times before terminating, and then each branch passes straight to a muscular fibre. Having reached this, the primitive sheath of the nerve-fibre is continued into the sarcolemma of the muscle, the medullary sheath stops short, and the axis-cylinder ends in a close terminal ramification with varicosities upon its branches (figs. 107, 108). This ramification is embedded in a granular nucleated protoplasmic mass which lies between the sarcolemma and the crossstriated muscular substance. In some cases the ramification is restricted to a small portion of the muscular fibre, and forms with the granular bed a slight prominence (eminence of Doyère). This is the case in mammals. In the lizard the ramification is rather more extended than in mammals, whilst in the frog it is spread over a considerable length of the fibre. 


\section{LESSON XX.}

\section{STRUCTURE OF THE LARGER BLOOD-VESSELS.}

1. Sections of a medium-sized peripheral artery and vein. In this preparation the limits of the vascular coats can be well seen and also the differences which they present in the arteries and veins respectively. The sections may either be stained with hæmatoxylin and mounted in Canada balsam, or they may be stained in dilute magenta and mounted in glycerine and water.

2. Mount in Canada balsam a thin slice cut from the inner surface of an artery which, after having been cut open longitudinally and washed with distilled water, has been treated with nitrate of silver solution and exposed to the light in spirit. This preparation will show the outlines of the epithelioid cells which line the vessel.

3. A piece of an artery which has been macerated for two or three days in 30 per cent. alcohol ( 1 part rectified spirit to two parts water) is to be teased so as to isolate some of the muscular cells of the middle coat and portions of the elastic layers (networks and fenestrated membranes) of the inner and middle coats. The tissue may be stained cautiously with dilute logwood solution, and glycerine afterwards added. The muscular cells are recognisable by their irregular outline and long rod-shaped nucleus. Sketch one or two and also a piece of fenestrated membrane.

4. Transverse section of aorta. Notice the differences in structure between this and the section of the smaller artery.

5. Transverse section of vena cava inferior. Notice the comparatively thin layer of circular muscle, and outside this the thick layer of longitudinal muscular bundles.

Make sketches from 1, 4, and 5, under a low power, from 2 and 3 under a high power.

An artery is usually described as being composed of three coats, an inner or elastic, a middle or muscular, and an external or areolar (fig. 109, $b, c, d$ ). It would, however, be more correct to describe the wall of an artery as being composed of muscular and elastic tissue lined internally by a pavement-epithelium and strengthened externally by a layer of connective tissue. For the present, however, we may adhere to the generally received mode of description. The inner coat of an artery is composed of two principal layers. The inner one is a thin layer of pavement-epithelium (often spoken of as the endothelium), the cells of which are somewhat elongated in the direction of the axis of the vessel (fig. 110), and form a smooth lining to the tube. After death they become easily detached. Next to this comes an elastic 
layer in the form either of elastic networks or of a fenestrated memorane. In some arteries there is a layer of fine connective tissue intervening between the epithelium and the fenestrated membrane (sub. epithelial layer).

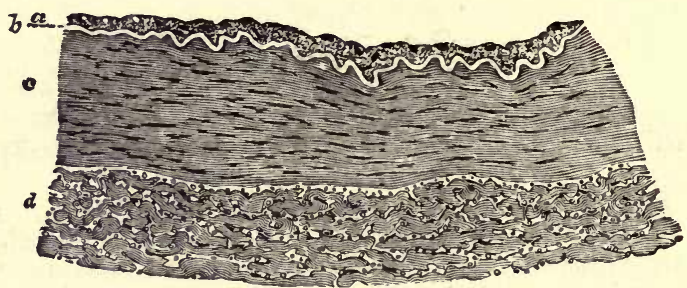

Fig. 109. - Transverse section of Part of the wall of the posterior tibiat ARTERY. (75 diameters.)

$a$, epithelial and sub epithelial layers of inner coat; $b$, elastic layer (fenestrated membrane) of inner coat, appearing as a bright line in section; $c$, muscular layer (middle coat); $d$, outer coat, consisting of connective-tissue bundles. In the interstices of the bundles are some connective-tissue nuclei, and, especially near the muscular coat, a number of elastic fibres cut across.

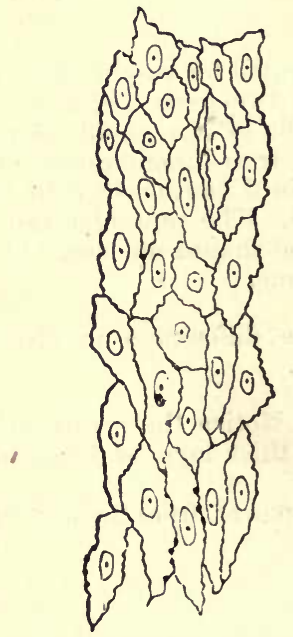

Fig. 110.-Epithelial Layer Lining THE POSTERIOR TIBIAL ARTERY. (250 diameters.)

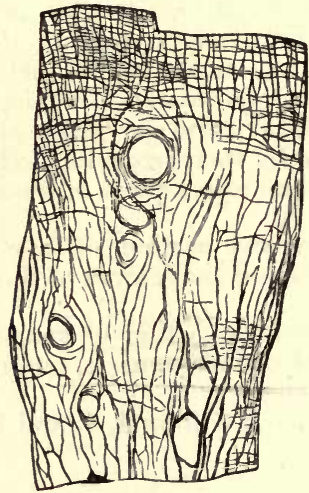

Fig. 111.-Portion of FENESTRaTED MEMBRANE FROM AN ARTERY. (Toldt.) $a, b, c$, perforations.

The middle coat consists mainly of circularly disposed plain muscular fibres, but it is also pervaded in most arteries by a network of elastic fibres which are connected with the fenestrated membrane of the inner coat and are sometimes almost as much developed as the muscular tissue itself. This is especially the case with the larger arteries such as the carotid and its immediate branches, but in the smaller arteries of the limbs the middle coat is almost purely composed of muscular tissue. 
The outer coat is formed of connective tissue with a good many elastic fibres, especially next the middle coat. The strength of an artery depends largely upon this coat; it is far less easily cut or torn than the other coats, and it serves to resist undue expansion of the

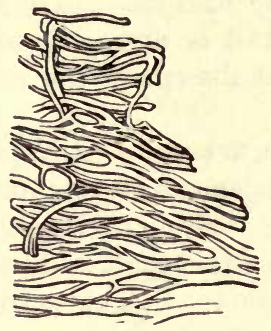

Fig. 112.-Elastic NETWORK OF ARTERY. (Toldt.)
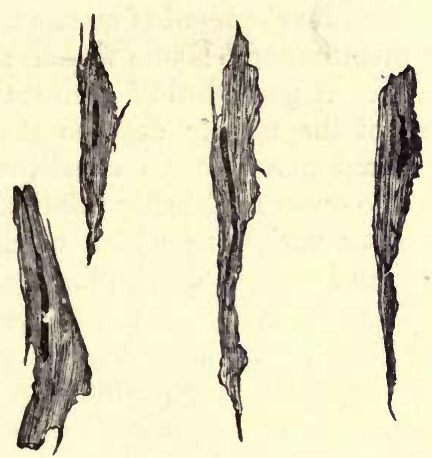

Fig. 113.-MUSCULAR FIBRE-CELLS FROM SUPERIOI THYROID ARTERY. (340 diameters.)

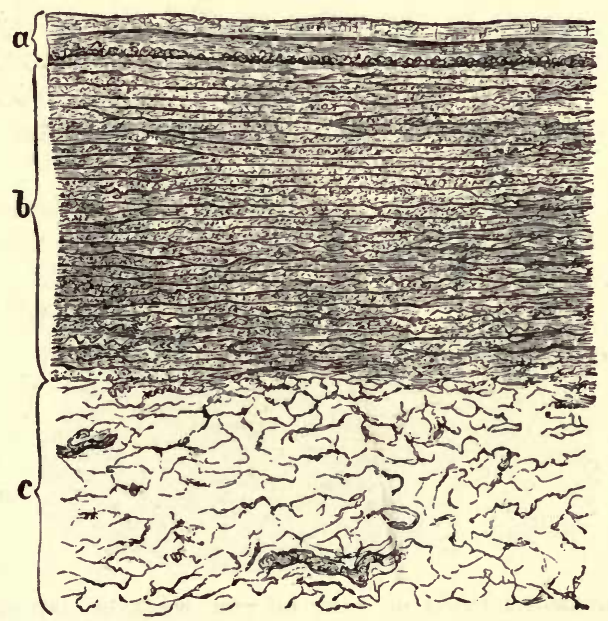

Fig. 114.-Section of thoracic AORTA As SEen under a low power. (Toldt.)

$a$, the inner coat consisting of three layers, viz.: 1. Epithelium seen as a fine line. 2. Subepithelial. 3. Elastic lavers. In the rart of the inner coat, at its junction with the middle, a layer of longitudinal muscular fibres is represented as cut across. $b$, middle coat with its elastic membranes; $c$, outer coat with two vasa vasorum.

vessel. Its outer limit is not sharply marked, for it tends to blend with the surrounding connective tissue (hence it has been termed tunica adventitia).

Variations in structure.-The aorta (fig. 114) differs in some 
respects in structure from an ordinary artery. Its inner coat contains a considerable thickness of sub-epithelial connective tissue, but its elastic layers are chiefly composed of fine fibres, and are not especially marked off from those of the middle coat, so that the inner and middle coats appear almost blended with one another. On the other hand, there is a very great development of elastic tissue in the middle coat, this tissue forming membranous layers which alternate with layers of the muscular tissue. A good deal of connective tissue also takes part in the formation of the middle coat, so that the wall is unusually strong. The inner and middle coats constitute almost the entire thickness of the wall, the outer coat being relatively thin.

The other variations which occur in the arterial system chiefly have reference to the development and arrangement of the muscular tissue. Thus in many of the larger arteries there are longitudinal muscular fibres at the inner boundary of the middle coat, and in some arteries amongst the circular fibres of the middle coat. This is the case in the aorta. In some parts of the umbilical arteries there is a complete layer of longitudinal fibres internal to the circular fibres and. another external to them, whilst the amount of elastic tissue is very small. Longitudinal fibres are also present in some other arteries (iliac, superior mesenteric, splenic, renal, \&c.), external to the circular fibres, and therefore in the outer coat of the artery. The larger arteries themselves receive blood-vessels, vasa vasorum, which ramify chiefly in the external coat. Nerves, derived for the most part from the sympathetic system, are distributed to the muscular tissue of the middle coat.

The veins (fig. 115) on the whole resemble the arteries in structure, but they present certain differencess. In the internal coat the same

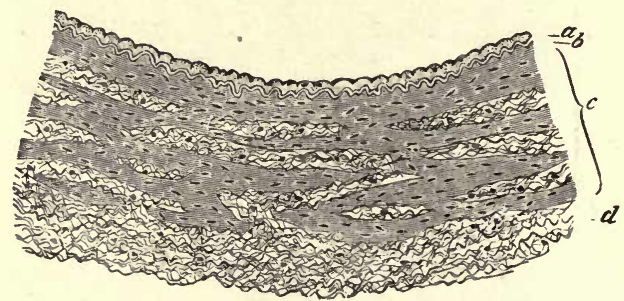

Fig. 115.-Transverse section of part of the wall of one of the posterior TIBIAL VEINS (MAN).

$a$, epithelial and sub-el)ithelial layers of inner coat; $b$, elastic layers of inner coat ; $c$, middle coat consisting of irregular layers of muscular tissue, alternating with connective tissue and passing somewhat gradually into the outer connective tissue and elastic coat, $d$.

layers may be present, but the elastic tissue is less developed and seldom takes the form of a complete membrane. The epithelium-cells are less elongated than those of the arteries. The middle coat (c) contains less elastic tissue and also less muscular tissue, being partly occupied by bundles of white connective-tissue fibres. These are derived 
from the external coat, which is relatively better developed in the veins than in the arteries, so that, although thinner, their walls are often stronger.

Many of the veins are provided with valves, which are semilunar folds of the internal coat strengthened by a little fibrous tissue: a few muscular fibres may be found in the valve near its attachment. The layer of the inner coat is rather thicker, and the epithelium-cells are more elongated on the side which is subject to friction from the current of blood than on that which is turned towards the wall of the vessel.

Variations in different veins. - The veins vary in structure more than do the arteries. In many veins longitudinal muscular fibres are found in the inner part of the middle coat, as in the iliac, femoral, umbilical, \&c. ; in others they occur external to the circularly disposed fibres, and are described as belonging to the outer coat. This is the case in the inferior vena cava and also in the hepatic veins and in the portal vein and its tributaries. In the superior and in the upper part of the inferior vena cava the circular fibres of the middle coat are almost entirely absent. The veins of the following parts have no muscular tissue, viz. pia mater, brain and spinal cord, retina, bones, and the venous sinuses of the dura mater and placenta.

It is only the larger veins and especially those of the limbs that possess valves. They are wanting in most of the veins of the viscera, in those within the cranium and vertebral canal, in the veins of the bones, and in the umbilical vein. 


\section{LESSON XXI.}

SMALLER BLOOD-VESSELS. LYMPHATIC SYSTEM.

1. TAKE a piece of pia mater which has been stained with logwood, and separate from it some of the small blood-vessels of which it is chiefly composed. Mount the shreds in Farrant. The structure of the small arteries can be studied in this preparation, the nuclei of the epithelium and of the muscular coat being brought distinctly into view by the logwood. The veins, however, possess no muscular tissue. Capillary vessels which have been dragged out from the brain in removing the pia mater may also be seen in this preparation. Sketch two small arteries of different sizes, giving also their measurements.

2. Mount in Canada balsam a piece of the omentum of the rabbit stained with silver nitrate. The membrane should be stretched over a cork or a plate of glass, rimsed with distilled water, treated for five minutes with 1 per cent. nitrate of silver solution, again washed and exposed to the light in spirit. When stained brown the spirit is replaced by oil of cloves. Pieces may now be cut off from the membrane and mounted, as directed, in Canada balsam; they should include one or more blood-vessels.

This preparation is intended to show the epithelium of the smaller bloodvessels and accompanying lymphatics and also the epithelium of the serous membrane. Sketch a small piece showing the epithelium of the vessels.

3. Mount in Canada balsam a piece of the central tendon of the rabbit's diaphragm which has been similarly prepared (except that the pleural surface has first been brushed to remove the superficial epithelium so as to enable the nitrate of silver more readily to penetrate to the network of lymphatic vessels underlying that surface). Observe the lymphatic plexus under a low power; sketch a portion of the network. If the peritoneal surface is focussed, the epithelium which covers that surface will be seen, and opposite the clefts between the radially disposed tendon-bundles stomata may be looked for in this epithelium.

4. Carefully study the circulation of the blood either in the web of the frog's foot or in the mesentery or tongue of the frog or toad, or in the tail of the tadpole.

The coats of the smaller arteries and veins are much simpler in structure than those of the larger vessels, but they contain at first all the same elements. Thus there is a lining epithelium and an elastic layer forming an inner coat, a middle coat of circularly disposed plain muscular tissue, and a thin outer coat. The same differences also are found between the arteries and veins, the walls of the veins being thinner and containing far less muscular tissue (fig. 116), and the lining epithelium-cells, mueh elongated in both vessels, are far longer 
and narrower in the small arteries than in the corresponding veins (fig. 117).

In the smallest vessels it will be found that the elastic layer has disappeared in the veins, and the muscular tissue is considerably reduced in thickness in both kinds of vessels. Indeed, it is soon represented by but a single layer of contractile cells, and even these no longer form a complete layer. By this time also, the outer coat and the elastic layer of the inner coat have entirely disappeared both from arteries and veins. The vessels are reduced, therefore, to the condition of a tube formed of pavement epithelium cells, with a partial covering of circularly disposed muscular cells.

Even in the smallest vessels, which are not capillaries, the differ-
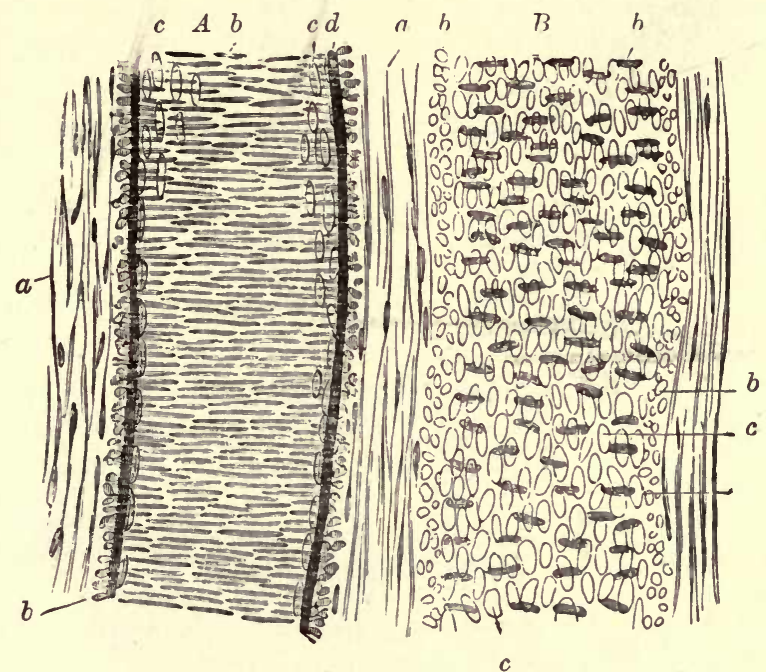

Fig. 116.-A small artery, $A$, with a corresponding vein, $B$, treated with ACETIC ACID. (Magnified 350 diameters.)

$a$, external coat with elongated nuclei; $b$, nuclei of the transverse muscular tissue of the middle coat (when seen endwise, as at the sides of the vessel, their outline is circular);

$c$, nuclei of the epithelium-cells; $d$, elastic layers of the inner coat.

ences between arteries and veins are still manifested. These differences may be enumerated as follows:-The veins are larger than the corresponding arteries; they branch at less acute angles; their muscular cells are fewer, and their epithelium-cells less elongated; the elastic layer of the inner coat is always less marked, and sooner disappears.

Capillary vessels. - When traced to their smallest branches, the arteries and veins eventually are seen to be continued into a network of the smallest blood-vessels or capillaries. The walls of these are composed only of flattened epithelium-cells (fig. 118) continuous with those that line the arteries and veins; these cells can be exhibited by staining a tissue with nitrate of silver. The capillaries vary somewhat in size and in the closeness of their meshęs; their arrangement in 
different parts, which is mainly determined by the disposition of the tissue-elements, may best be studied when the structure of the several organs is considered.

In the transparent parts of animals, the blood may be seen flowing through the capillary network from the arteries into the veins. The

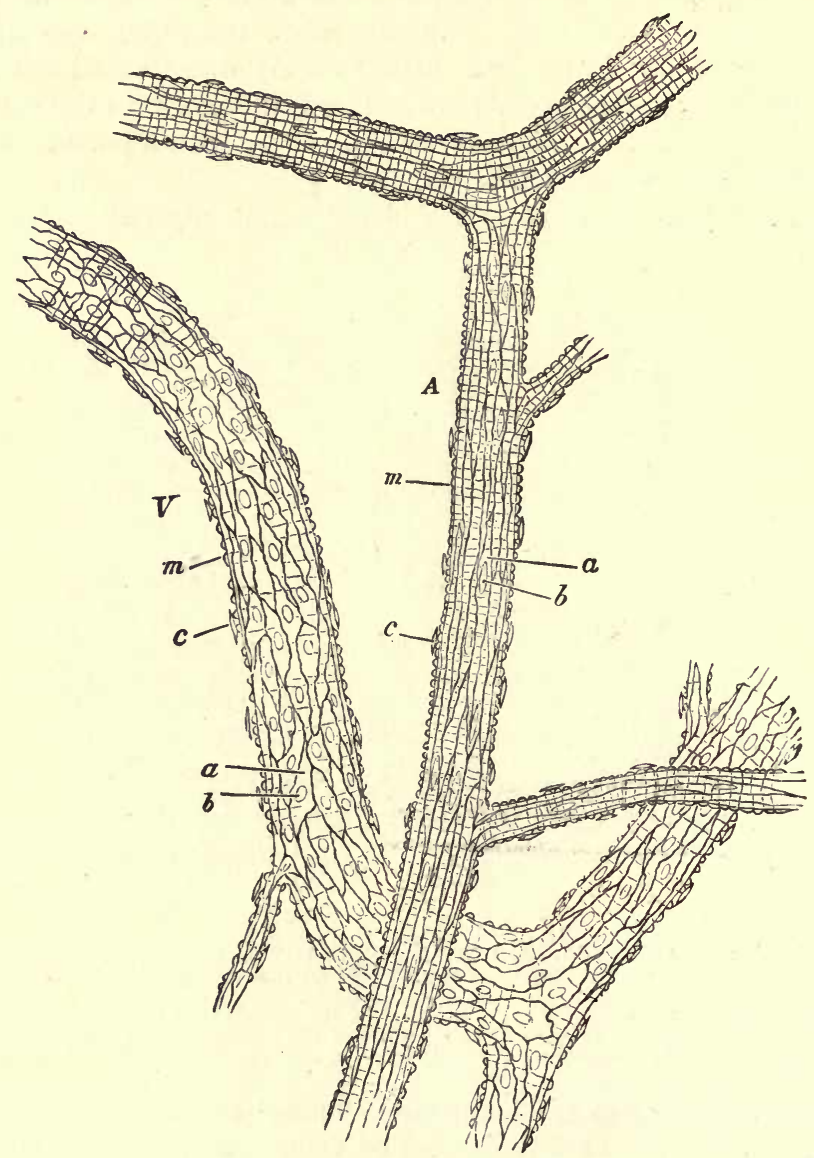

Fig. 117.-A small artery, $A$, AND vein, $V$, From the sUbCutanfous connective TissUe OF THE RAT, TREATED WITH Nitrate OF SILVER. (17j diameters.)

$a$, $a$, epitheloid cells with $b, b$, their nuclei ; $m, m$, transverse markings due to staining of substance between the muscular fibre-cells; $c$, $c$, nuclei of connective-tissue corpuscles attached to exterior of vessel.

current is very rapid in the small arteries, somewhat less so in the veins, and comparatively slow in the capillaries. The current is fastest in the centre of the vessel, slowest near the wall (inert layer), and with care it may be observed-especially where there is any commencing inflammation of the part, as in the mesentery in consequence of 
exposure-that the white blood corpuscles, which always tend to pass into the inert layer, and to adhere occasionally to the inner sur-

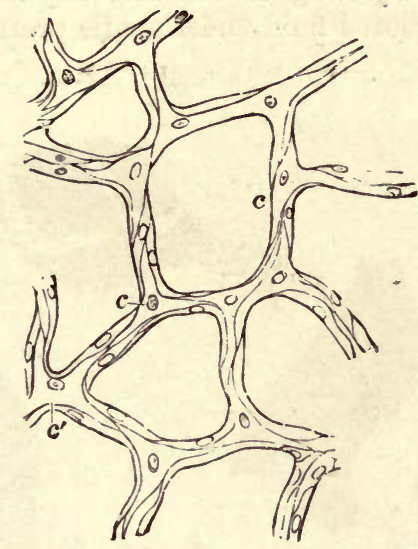

Fig. 118.-Capillary Vesshlo from THE BLADDER OF THE (AT, MAGNIHIED.

The outlines of the cells are stained by nitrate of silver.

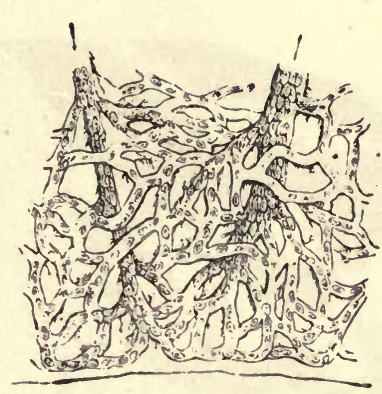

Fig. 119.-Capillary BloodVESSELS IN THE WEB OF A FROG'S FOOT, AS SEEN WITH THE MICROSCOFE.

The arrows indicate the course of the blood.

face of the blood-vessels, here and there pass through the coats of the small vessels, and appear as migratory cells in the surrounding connective tissue.

\section{LYMPHATIC SYSTEM.}

To the lymphatic system belong not only the lymphatic vessels and lymphatic glands, but also the cavities of the serous membranes, which are moistened with lymph and are in open communication with the lymphatic vessels in their parietes.

The larger lymphatic vessels somewhat resemble the veins in structure, except that their coats are much thinner and their valves much more numerous. In lymphatics of somewhat smaller size, the wall of the vessel is formed, first, by a lining of pavement-epithelium cells (endothelium of some authors), which are elongated in the direction of the axis of the vessel ; and, secondly, by a layer of circularly and obliquely disposed muscular fibres. In the smallest vessels (lymphatic capillaries), which, however, are generally considerably larger than the blood-capillaries, there is nothing but the epithelium remaining, and the cells of this are frequently not more elongated in one direction than in another, but have a characteristic wavy outline (fig. 121).

Lymphatics begin in two ways-either in the form of plexuses, as in membranes (fig. 120), or as lacunar interstices, as is the case in some of the viscera. 
In order to show the lymphatic vessels, it is generally necessary to stain a tissue with nitrate of silver; but they may easily be injected by sticking the nozzle of an injecting canula into any tissue which contains them, and forcing coloured fluid under gentle pressure into the interstices of the tissue.

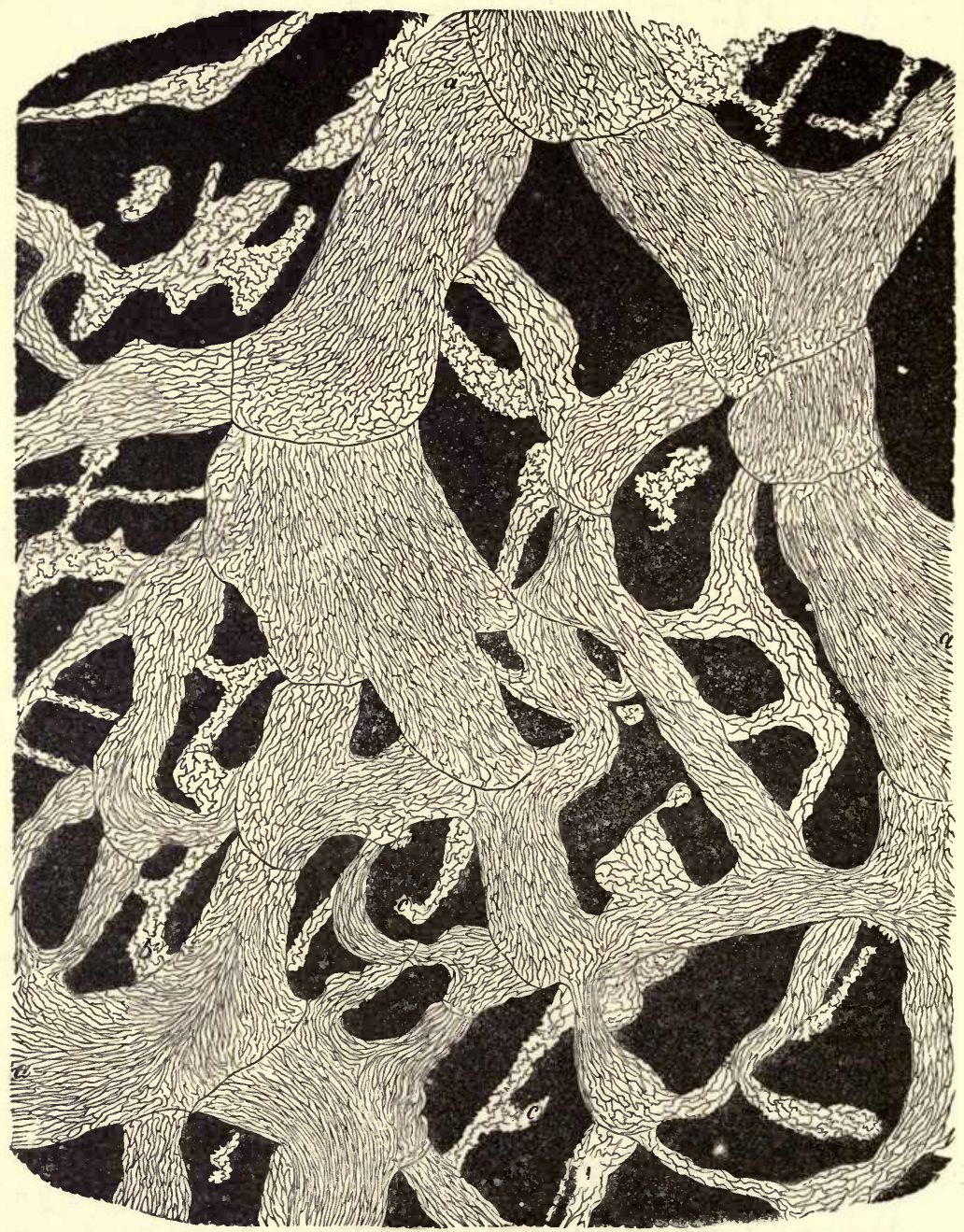

Fig. 120.-Lymphatic plexus of cextral texion of maphragm of Rabit, PLEURAL SIDE.

$a$, larger vessels with lanceolate cells and numerous valves; $b, c$, lymphatic capillaries with wavy-bordered cells.

In silvered preparations it may be observed that the lymphatics always appear in the form of clear channels in the stained ground-sub- 


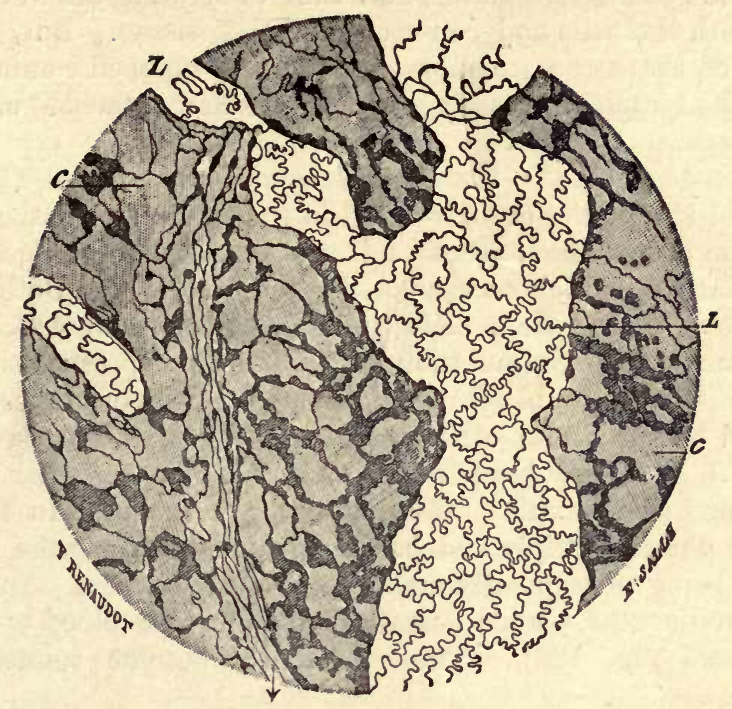

Fig. 121.-A small part of the lymphatic plexus on tile pleural surface OF THE DIAPHRAGM. (Magnified 110 diameters.) (Ranvier.)

$L, 1$ ymphatic vessel with characteristic epithelium; $c$, cell-spaces of the connective tissue, here and there abutting against the lymphatic.

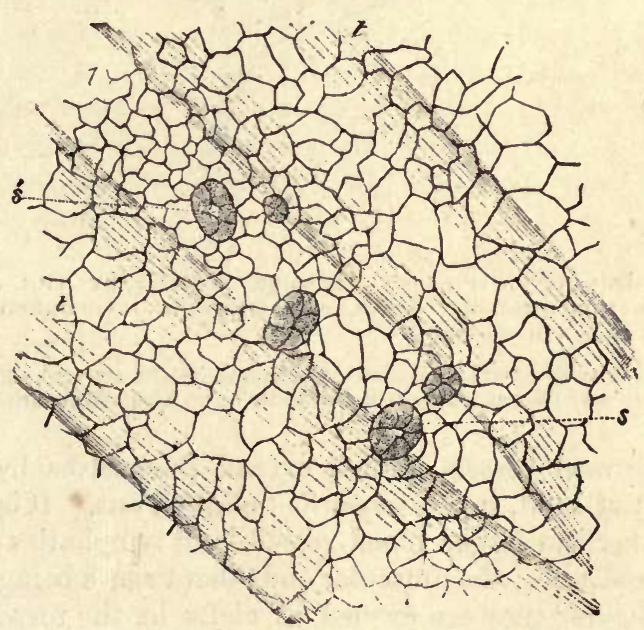

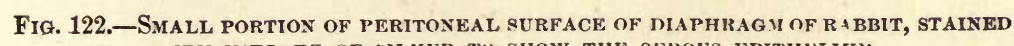
WITH NITRATE OF SILVER TU SHOW THE SEROUS EPITHELIUM.

$l$, lymph-channel below the surface, lying between tendon bundles, $t, t$, and over which the surface-cells are seen to be relatively smaller, and to exhibit five stomata, $s, s$, leading into the lymphatic. The epithelium of the lymphatic channel is not represented. 
stance of the connective tissue, and that their walls are in close connection with the cells and cell-spaces of that tissue. But, except in the case of the serous membranes, there is no open communication between the lymphatic vessels and the interstices (areolæ) of the connective tissue.

Development of the blood-vessels and lymphatics.-The bloodvessels and lymphatics are developed in the connective tissue or in the mesoblastic tissue which precedes it, the first vessels being formed in the vascular area which surrounds the early embryo. Both linds of vessels are developed from cells (vaso-formative cells) which become hollowed out by an accumulation of fluid in their protoplasm, and in the case of developing blood-vessels coloured blood-corpuscles may also be formed within these cells (see Development of Blood-corpuscles, Lesson II.) The cells branch and unite with one another to form a network, and their cavities extend into the branches. In the meantime their nuclei multiply and become distributed along the branches, cell-areas being subsequently marked out around them. In this way intercommunicating vessels - capillaries containing blood or lymphare produced (fig. 123). These presently become connected with

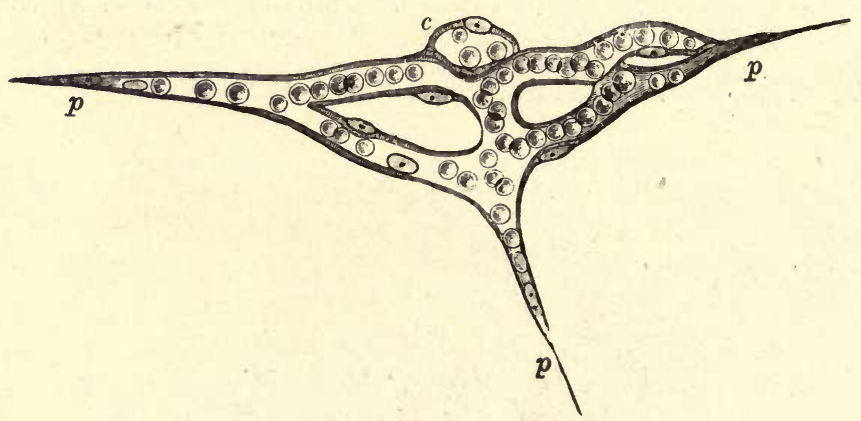

Fig. 123.-Isolated CAPILlaRY NETWORK FORMED BY THE JUNCTION OE SEVERAL HOLLOWED-OUT CELLS, AND CONTAINING COLOURED BLOOD-CORpUscles in a Clear fletid.

$c$, a hollow cell the cavity of which does not yet communicate with the network; $p, p$, pointed cell-processes, extending in different directions for union with neighbouring capillaries.

previously formed vessels, which extend themselves by sending out sprouts, at first solid, and afterwards hollowed out. It is not precisely known whether the larger blood-vessels and lymphatics are developed at first as capillaries, the muscular and other tissues being subsequently added, or whether they are formed as clefts in the mesoblastic tissue which become bounded by flattened cells.

The se ous membranes, which may conveniently be studied in connection with the lymphatic system, are delicate membranes of connective tissue which surround and line the internal cavities of the body, and are reflected over many of the thoracic and abdominal viscera; in 
passing to which they form folds, within which blood-vessels, lymphatics, and nerves pass to the viscera.

The inner surface is lined by a continuous layer of pavementepithelium (fig. 122), which is very distinct in nitrate of silver preparations. In some places there are apertures in the epithelium which lead direct into subjacent lymphatic vessels. These apertures are called stomata, and are surrounded by small protoplasmic cells (fig. 122, s, s). They are most numerous upon the peritoneal surface of the diaphragm, but are present in all serous membranes, and they serve to prevent any undue accumulation of lymph within the serous cavity during health. The pavement-epithelium rests upon a homogeneous basement-membrane, which is especially well marked in the serous membranes of man. The rest of the thickness of the membrane is composed of connective tissue, with a network of fine elastic fibres near the inner surface.

The cavities of the serous membranes are originally formed in the embryo as a cleft in the mesoblast (pleuro-peritoneal split) which becomes lined with epithelium, and its wall eventually becomes differentiated into the serous membrane.

The synovial membranes, which are often compared with the serous membranes, and are indeed, like the latter, connective-tissue membranes which bound closed cavities moistened with fluid, are not so intimately connected with the lymphatic system, nor is the fluid (synovia) which moistens them of the nature of lymph. Moreover, it is only here and there that there is a lining of epithelıum-like cells, in place of the continuous lining of epithelium which we find in the serous membranes. Curious villus-like projections occur in many parts ; they are covered by small rounded cells, and probably serve to extend the surface for the secretion of synovia. The blood-vessels of synovial membranes are numerous, and approach close to the inner surface of the membrane. 


\section{LESSON XXII. \\ LYMPHATIC GLANDS, TONSIL, THYMUS.}

1. Sections of a lymphatic gland which has been stained in bulk with magenta and embedded in paraffin. ${ }^{1}$ Notice $(1)$ the fibrous and muscular capsule, with trabeculæ extending inwards from it through the cortex and anastomosing with one another in the medulla, (2) the dense lymphoid tissue (adenoid tissue of authors) forming large masses in the cortex (cortical nodules) and rounded cords in the medulla (medullary cords). Notice also the clearer channel or lymph-sinus which everywhere intervenes between the fibrous tissue and the lymphoid tissue. Observe the fine fibres and branched cells which bridge across this channel.

Make a general sketch under a low power of a portion of the cortex together with the adjoining part of the medulla, and under a high power drawings of small portions of cortex and medulla.

2. In sections of tonsil prepared similarly to those of the lymphatic gland, notice the large amount of lymphoid tissue only imperfectly collected into nodules. Observe also that the stratified epithelium, which covers the mucous membrane here as elsewhere in the mouth, is infiltrated with lymph-corpuscles. Here and there pit-like recesses may be met with glands opening into the pits.

3. A similar preparation of the thymus gland of an infant. Notice that the masses of lymphoid tissue which form the lobules of the gland are separated by septa of connective tissue, and that they show a distinction into two parts, cortical and medullary. Observe the differences of structure of these two parts, and especially notice the concentric corpuscles in the medullary part.

Make a sketch of one of the lobules under a low power and of a small part of the medulla under a high power, including one or two concentric corpuscles. Measure the latter.

Structure of a lymphatic gland.-A lymphatic gland is composed of a fibrous and muscular framework, which encloses and supports the proper glandular substance, but is everywhere separated from it by a narrow channel, bridged across by cells and fibres, which is known as the lymph-channel. The framework consists of an envelope or capsule (fig. 124,c), and of trabeculce (tr), which pass at intervals inwards from the capsule, and after traversing the cortex of the gland divide and reunite with one another so as to form a network of fibrous bands. At one part of the gland there is usually a depression (hilus), and at the bottom of this the medulla comes to the surface and its fibrous bands are directly continuous with the capsule.

1 See Appendix. 


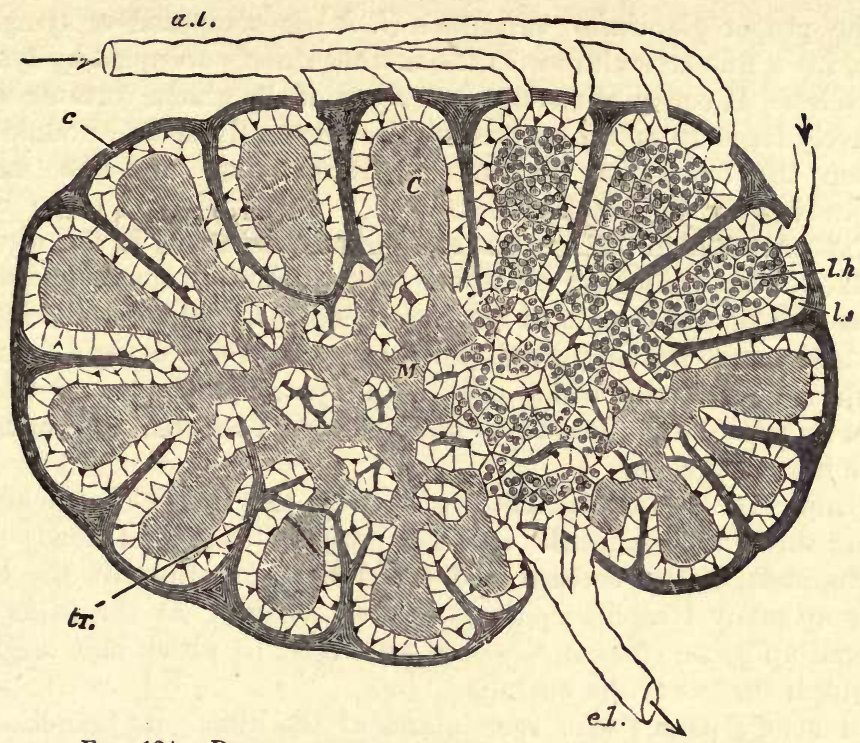

Fig. 124.-Diagrammatic Section of LYMPhatic GLAND.

a. $l$. afferent, $e . l$. efferent lymphatics; $C$, cortical substance; $M$, reticulating cords of medullary substance; $l$.s. lymph-sinus; $c$, fibrous coat sending trabeculæ, $t r$, into the substance of the gland.

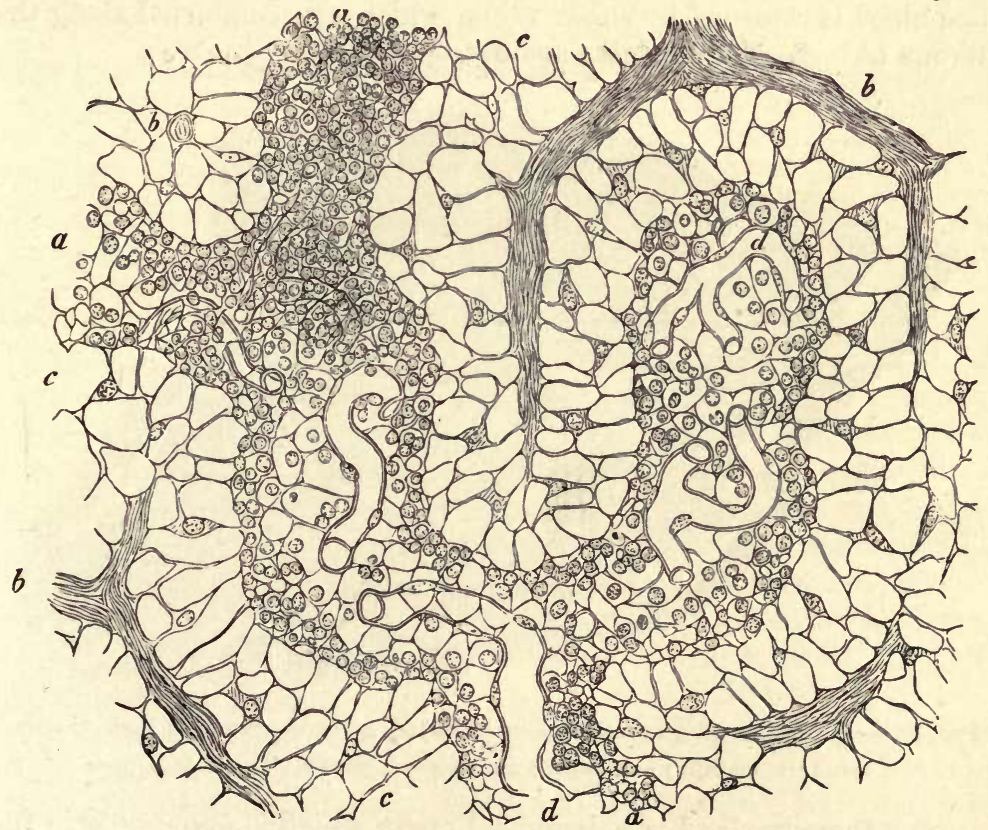

Fig. 125.-Section of the medullary substance of a lymphatic gland (ox). (300 diameters.)

$a, a, a$, lymphoid cords ; $c$, lymph-sinus; $b, b$, trabeculæ ; $d, d$, capillary blood-vessels. 
The proper glandular substance $(l . h$.$) is composed of lymphoid$ tissue, i.e. a fine reticulum with the meshes thickly occupied by lymphcorpuscles. It occupies all the interstices of the gland, forming comparatively large rounded masses in the cortex (lymphoid nodules, $C$ ) between the trabeculæ, and smaller retiulating cord-like masses (lymphoid cords, $M$ ) in the medulla.

The cells which bridge across the lymph-channel in the medulla (fig. 125, c) are branching nucleated cells which often contain pigment, so that this part of the gland Las a dark colour. The lymph-channel is bridged across not only by these, but also by fibres derived from the capsule and trabeculæ, which pass to the lymphoid tissue and become lost in its reticulum. But these fibres are often covered and concealed by the branched cells.

Lymphatic vessels (fig. 124, a. l.) enter the lymph-channels after passing through the capsule, and the lymph is conveyed slowly along the channels of the cortical and medullary part towards the hilus, taking up many lymph-corpuscles in its passage. At the hilus it is gathered up by an efferent vessel or vessels $(e . l$.) which take origin in the lympln-sinuses of the medulla.

An artery passes into each gland at the hilus; its branches are conveyed at first along the fibrous cords, but soon pass into the lymphoid tissue, where they break up into capillaries (fig. 125, d). The blood is returned by small veins, which are conducted along the fibrous trabeculæ to the hilus again.

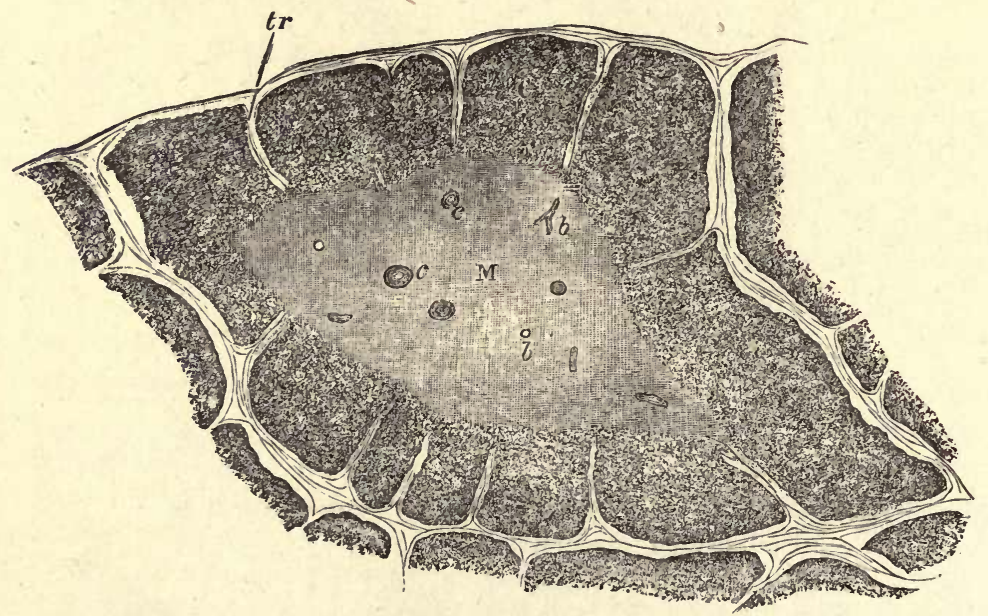

Fig. 126.-A lobule of the thymus of a Child as Sefin UNier a low power.

C, cortex; $\mathrm{M}$, medulla ; $c$, concentric corpuscles; $b$, blood-vessels; $t r$, trabeculæ.

The thymus gland is a lymphoid organ which is found only in the embryo and during infancy. It is composed of a number of larger and smaller lobules (fig. 126), which are separated from one anotuer by 
septa of connective tissue, along which the blood-vessels and lymphatics pass to and from the lobules. Each lobule shows plainly, when examined with the low power, a distinction into an outer cortical and an inner medullary portion. The cortical part of the lobule is imperfectly divided into nodules by trabeculæ of connective tissue, and is very similar in structure to the lymphoid tissue of the lymplatic glands and tonsils, but the medulla is more open in its texture, and the reticulum is composed of larger, more transparent, flattened cells, and contains fewer lymph-corpuscles. Moreover, there are found in

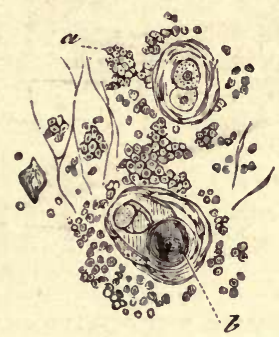

Fig. 127.-Elements of the thymus. (300 diameters.) (Cadiat.) $a$, lymph-corpuscles; $b$, concentric corpuscle.

the medulla peculiar concentrically striated bodies (the concentric corpuscles, fig. 127), which are usually composed of a number of flattened cells arranged concentrically around one or more central cells. Sometimes these corpuscles are compound, two or three being grouped together and similarly enclosed by flattened cells. The lymphoid tissue is abundantly supplied with capillary blood-vessels, and large lymphatic vessels issue from the organ, but in what way the latter are connected with the lobules has not been ascertained.

Lymphoid tissue occurs in many other parts of the body in addition to the lymphatic glands, tonsils, and thymus gland, although it may not, as in these structures, constitute the bulk of the organ. Thus it is found in many mucous membranes, such as those of the intestine and of the respiratory tract, both in a diffuse form and also collected into nodular masses which are like the cortical nodules of a lympratic gland, and may, like those, be partially surrounded by a lymph-sinus. In the spleen also a large amount of lymphoid tissue is found sheathing the smaller arteries, and also expanded into nodular masses (Malpighian corpuscles of the spleen). In these organs it will, however, be studied in subsequent Lessons.

Lymphoid tissue also occurs in considerable amount in the serous membranes, especially in young animals ; in the adult it is often transformed into adipose tissue. The tissue is generally developed in connection with lymphatic vessels, an accumulation of retiform tissue and lymph-cells taking place either external to and around the lymphatic 
(perilymphatic nodule); or the lymphatic is dilated into a sinus and

A

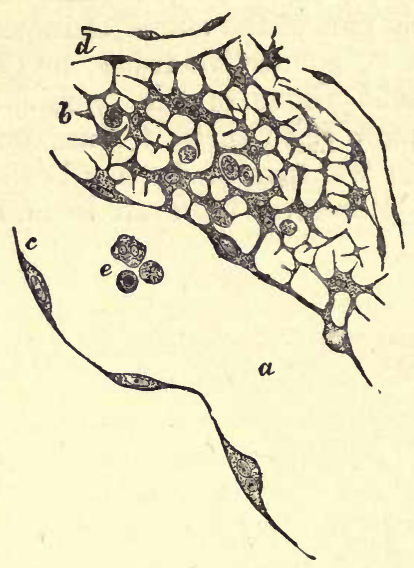

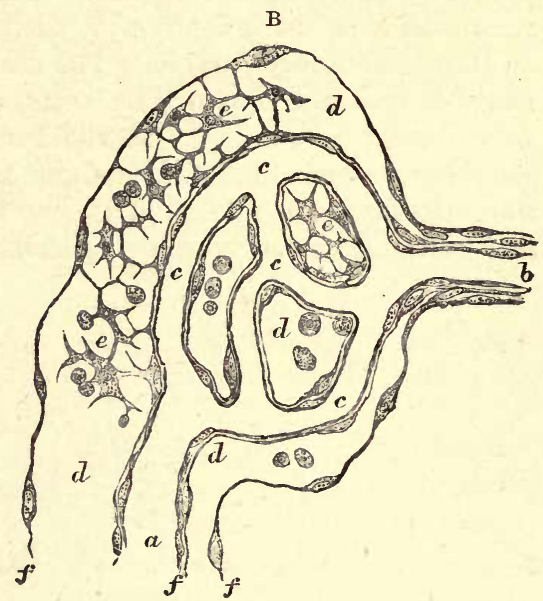

Fig. 128.-Developing Lymphatic nodvles, From the omentum of A guineaPIG.

A, perilymphatic nodule ; $a$, lymphatic vessel ; $c$, part of its epithelial wall, seen in optical section ; $e$, lymph-corpuscles within the vessel ; $b$, lymphoid tissue of the nodule ; $d$, bloodcapillaries; $\mathrm{B}$, endolymphatic nodule; $a$, vein ; $b$, artery ; $c$, capillaries ; $d$, a lymphatic vessel, in which this whole system of blood-vessels is enclosed; $e$, lymphoid tissue within the lymphatic vessel; $f$, wall of the lymphatic in optical section.

the formation of lymphoid tissue occurs within it (endolymphatic nodule) (see fig. 128). 


\title{
LESSON XXIII.
}

\author{
THE SKIN.
}

1. Sections of skin from the palmar surface of the fingers. The sections are to be made vertical to the surface, and should extend down as far as the subcutaneous tissue. They may be stained with logwood or picro-carmine and mounted in Canada balsam. In these sections notice the layers of the epidermis and their different behaviour to the staining fluid. Notice also the papillæ projecting from the corium into the epidermis, and look for tactile corpuscles within them. In very thin parts of the sections the fine intercellular channels in the deeper parts of the epithelium (see Lesson VI. p. 22) may be seen with a high power. The convoluted tubes of the sweat-glands will be seen here and there in the deeper parts of the corium, and in thick sections the corkscrew-like channels by which the sweat is conducted through the epidermis may also be observed. Make a sketch showing the general structure under a low power, and other sketches to exhibit the most important details under a high power. Measure the thickness of the epidermis and the length of the papillæ.

2. Sections of the skin of the scalp, vertical to the surface and parallel to the slope of the hair-follicles, and others parallel to the surface, and therefore across the hair-follicles. Stain and mount in the same way as in the last preparation. Examine also the structure of the hairs.

In these preparations the details of structure of the hairs and hair-follicles together with the sebaceous glands and the little muscles of the hair-follicles are to be made out.

3. Vertical sections across the nail and nail-bed, cut with a strong scalpel or razor. The sections are stained with hæmatoxylin or picro-carmine. Notice the ridges (not papillæ) of the corium projecting into the epidermis. Observe also the distinction of the epidermis into Malpighian layer and nail proper.

4. Mount in Canada balsam a section from a portion of skin of which the blood-vessels have been injected, and notice the distribution of the capillaries to the sweat-glands, to the hair-follicles, and to the papillary surface of the corium.

The skin is composed of two parts, epidermis and cutis vera.

The epidermis, or scarf skin, is a stratified epithelium (fig. 129). It is composed of a number of layers of cells, the deeper of which are soft and protoplasmic, and form the rete mucosum of Malpighi, whilst the superficial layers are hard and horny ; this horny portion sometimes constituting the greater part of the thickness of the epidermis. The deepest cells of the rete mucosum, which are set on the surface of the cutis vera, are columnar (fig. 129, c) in shape. In the coloured 
races of mankind these cells contain pigment-granules. In the layers immediately above them the cells are polyhedral (fig. 129, $p$ ). Between all these cells of the rete mucosum there are fine intercellular clefts which separate the cells from one another, but are bridged across by fine fibres, which pass from cell to cell. The intercellular channels serve for the passage of lymph, and within them occasional lymphcorpuscles may be found, often having a stellate figure from compression. The most superficial layer of the rete mucosum is formed of somewhat flattened granular cells (stratum granulosum, s.gr). Im-

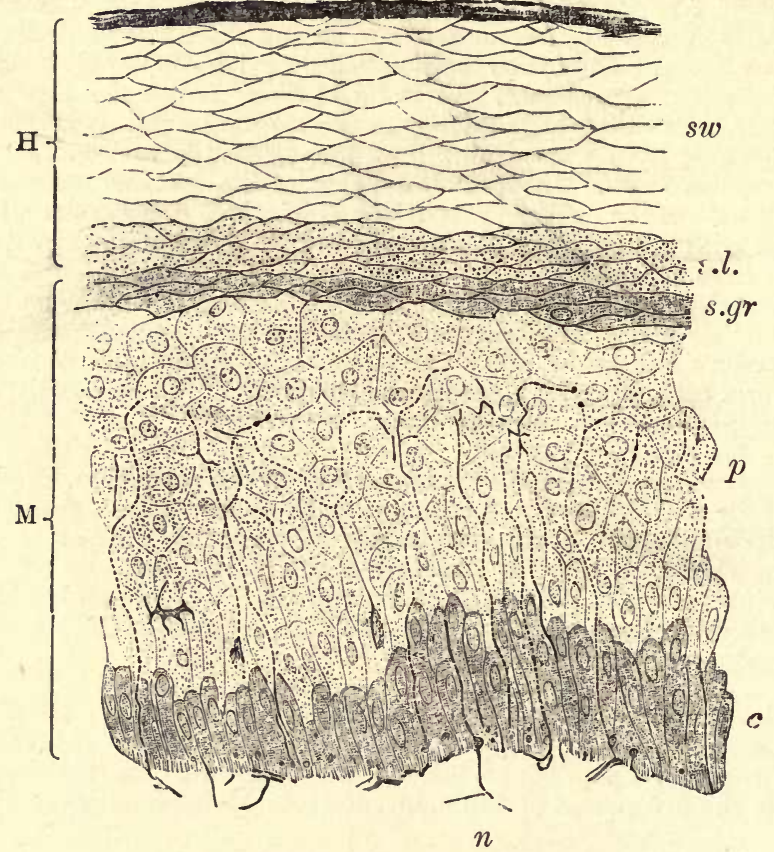

Fig, 129.-SECTION OF EPIDERMis.

H, horny laver, consisting of $s$, superficial borny scales; sw, swollen-out horny cells; s.l. stratum lucidum; $\mathbf{M}$, rete muc sum or Malpighian layer, consisting of $p$, prickle-cells, several rows (lee); $c$, elongated cells forming a single stratum near the corium ; and s.gr. stratum granulosum of Langerhans, just below the stratum lucidum; $n$, part of a plexus of nerve-fibres in the superficial layer of the cutis vera. From this plexus fine varicose nerve-fibrils may be traced passing up between the epithelium-cells of the Malpighian layer.

mediately above this layer, the horny part of the epidermis commences, as a layer of clear compressed cells several deep (stratum lucidum, s.l.). Above this comes the main part of the horny layer. It is composed of a number of layers of somewhat swollen cells $(s w$.$) , the nuclei of$ which are no longer visible. These cells become flatter as they approach the surface, where they eventually become detached in the form of thin horny scales $(s)$.

The growth of the epidermis takes place by a multiplication of the 
cells of the deeper layers. The newly formed cells, as they grow, push towards the surface those which were previously formed, and in their progress the latter undergo a chemical transformation, which converts their protoplasm into horny material. This change seems to occur at the stratum granulosum (see fig. 130); the granules which occupy the cells of that layer being composed of a substance termed eleidin, which is transformed into keratin.

No blood-vessels pass into the epidermis, but it receives nerves which ramify between the cells of the rete mucosum in the form of fine varicose fibrils (fig. 129).

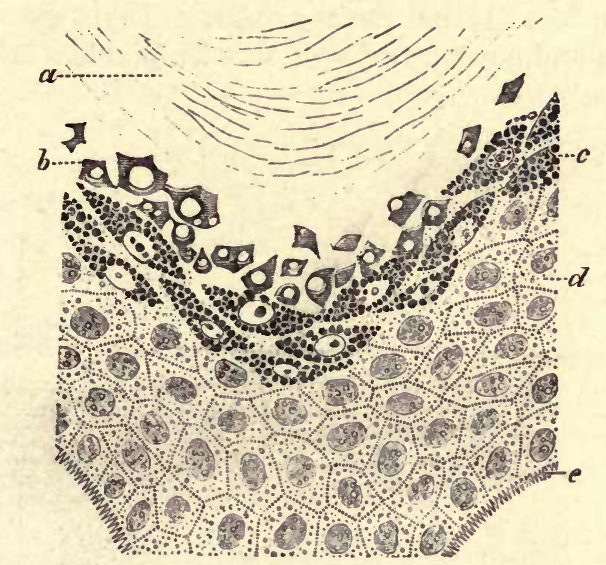

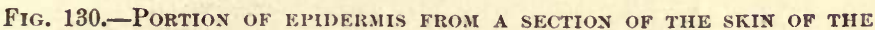
FiNGer, COloured With PICrocarminate of ammonia. (Ranvier.)

, stratum corneum; $b$, stratum lucidum with diffused flakes of eleidin; $c$, stratum granulosum, the cells filled with drops of eleidin; $d$, prickle-cells; $e$, dentate projections by which the deepest cells of the epidermis are fixed to the cutis vera.

The cutis vera or corium is composed of dense connective tissue, which becomes more open and reticular in its texture in its deeper part, where it merges into the subcutaneous tissue. The superficial or vascular layer of the corium bears minute papilla, which project up into the epidermis, which is moulded over them. These papillæ for the most part contain looped capillary vessels (fig. 137), but some, especially those of the palmar surface of the hand and fingers, and the corresponding part of the foot, contain tactile corpuscles, to which medullated nerve-fibres pass (fig. 97, $b$ ).

In some parts of the body (scrotum, penis, nipple, and areola), involuntary muscular tissue occurs in the deeper portions of the cutis vera, and in addition, wherever hairs occur, small bundles of this tissue are attached to the hair-follicles.

The blood-vessels of the skin are distributed almost entirely to the surface, where they form a close capillary network, sending up loops 
into the papillæ.' Special branches are also distributed to the various appendages of the skin, viz. the sweat-glands and hair-follicles, with their sebaceous glands and little muscles, as well as to the little masses of adipose tissue which may be found in the deeper parts of the cutis.

The lymphatics originate near the surface in a network of vessels, which is placed a little deeper than the blood-capillary network. They receive branches from the papillæ, and pass into larger vessels, which are valved, and which run in the deeper or reticular part of the corium. From these the lymph is carried away by still larger vessels, which course in the subcutaneous tissue.

The appendages of the skin are the nails, the hairs, with their sebaceous glands and the sweat-glands. They are all developed as thickenings and downgrowths of the Malpighian laver of the epidermis.

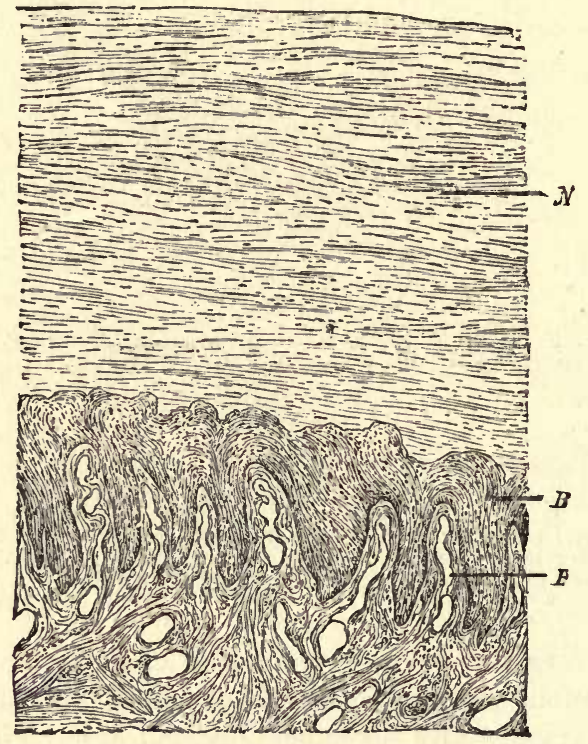

-Fig. 131.-Section ACross THE NAIL AND NAIL-BED. (100 diameters.) (Heitzmann.)

$P$, ridges with blood-vessels ; $B$, rete mucosum; $N$, nail.

The nails are thickenings of the stratum lucidum of the epidermis, which are developed over a specially modified portion of the corium, which is known as the bed of the nail, the depression at the posterior part of the nail-bed from which the root of the nail grows being known as the nail-groove. The distal part of the nail forms the free border, and is the thickest part of the body of the nail. The horny substance of the nail (fig. 131, $N$ ) is composed of clear horny cells, each containing the remains of a nucleus; it rests immediately upon a Malpighian layer $(B)$ similar to that which is found in the epidermis 
generally. The corium of the nail-bed is beset with longitudinal ridges instead of the papillæ which are present over the rest of the skin; these, like the rest of the superficial part of the corium, are extremely vascular. The nails are developed in the fœtus at about the third month, the groove being formed at this time in the corium, and the nail-rudiment appearing in it as a thickening of the stratum lucidum, which extends forward over the bed. It becomes free in the sixth month, its free end being at first thin, but as it grows forward over the bed it appears to receive additions on its under surface, so that after a time the distal part becomes the thicker. The superficial layers of the cuticlo which originally covered the developing nail become detached, and, after birth, only remain as the narrow border of cuticle which overlies the lunula.

The hairs are growths of the epidermis, which are developed in little pits-the hair-follicles - which extend downwards into the deeper part of the corium, or even into the subcutaneous tissue. The hair grows from the bottom of the follicle, the part which thus lies within the follicle being known as the root.

The substance of a hair is mainly composed of a pigmented, horny, fibrous material (fig. 132, $f$ ), which can be separated by the action of sulphuric acid into long tapering cells, the nuclei of which are still visible. This fibrous substance of the hair is covered by a layer of delicate imbricated scales termed the hair-cuticle $(c)$. In many hairs, but not in all, the centre is occupied by a dark-looking axial substance (medulla, $m$ ), formed of angular cells which contain granules of eleidin, particles of dark pigment, and frequently minute air-bubbles. The latter may also occur in interstices in the fibrous substance. When they are present, the hair looks white by reflected light. The root has the same structure as the body of the hair, except at its extremity, which is enlarged into a knob (fig. 133, b); this is composed mainly of soft, growing cells, and fits over a vascular papilla $(p)$, which projects up into the bottom of the follicle. The follicle, like the skin itself, of which it is a recess, is composed of two parts : one epithelial, and the other connective tissue. The epithelial or epidermic part of the follicle closely invests the hair-root, and is often in great part dragged out with it; hence it is known as the root-sheath. It consists of an outer layer of soft columnar and polyhedral cells, like the Malpighian layer of the epidermis - the outer root-sheath (figs. 133, $f$; $134, e)$; and of an inner, thinner, horny stratum next the hair-the inner root-sheath (figs. 133, $g ; 134, f$ ). The inner root-sheath itself consists of three layers, the outermost being composed of oblong cells without nuclei (Henle's layer), the next of flattened polyhedral nucleated cells (Huxley's layer), and the third-the cuticle of the rootsheath-being a thin layer of downwardly imbricated scales, which fit over the upwardly imbricated scales of the hair itself.

The connective tissue or dermic part of the hair-follicle (fig. 134, $a, c, d)$ is composed internally of a vascular layer, separated from the 


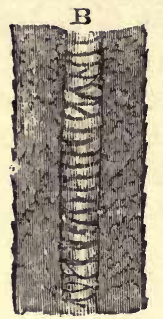

A.

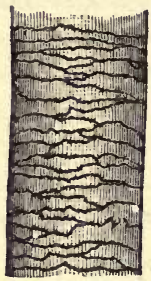

Fit. 132.-Piece of human HAIR. (Magnified.)

$A$, seen from the surface; $B$, in optical section. $c$, cuticle; $f$, fibrous substance; $m$, medulla, the air having been expelled by Canada balsam.

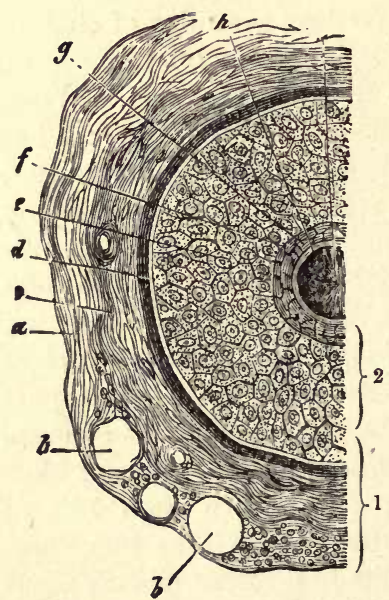

F1G. 134.-SECTION OF HAIRFOLLICLE.

1, dermic coat of follicle; 2, epidermic coat or root-sheath; $a$, outer layer of dermic coat, with blood-vessels, $b, b$, cut across; $c$, middle layer $; \boldsymbol{d}$, inner or hyaline layer ; $e$, outer root-sheath; $f, g$, inner root-sheath; $h$, cuticle of root-sheath ; $l$, hair.

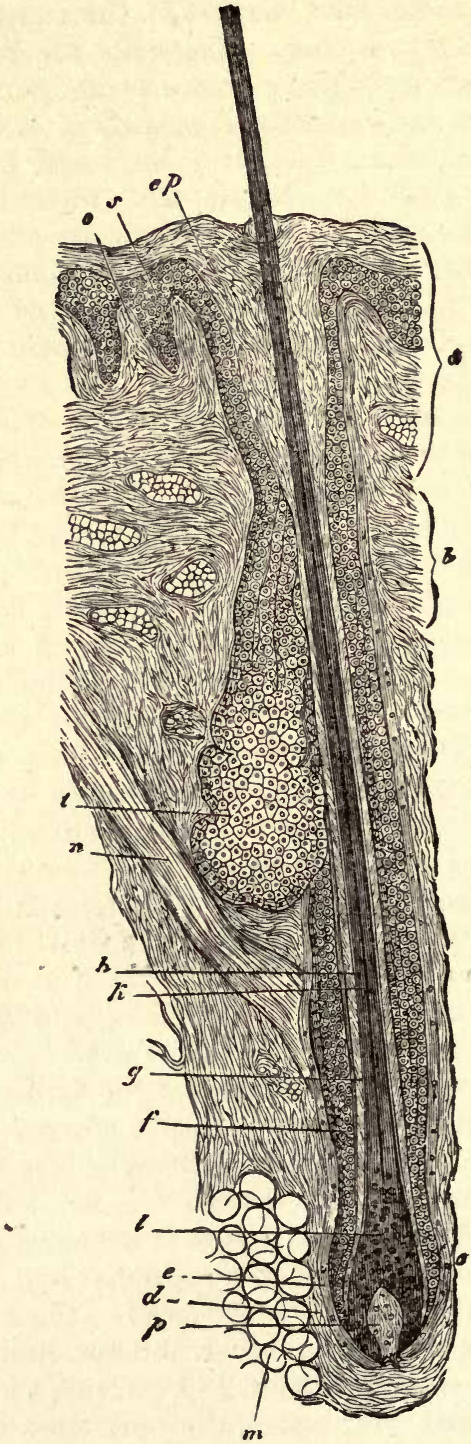

Fig. 133.-HAIR-FoLLICLE IN LONGITUDINAL SECTION.

$a$, mouth of follicle; $b$, neck ; $c$, bulb ; $d, e$, dermic coat ; $f$, outer root-sheath; $g$, inner root-sheath : $h$, hair; $k$, its medulla; $l$, hair-knob; $m$, adipose tissue ; $n$, hair-muscle; $o$, papilla of skin; $p$, papilla of hair ; s, rete mucosum, continuous with outer root-sheath; $e p$, horny layer; $t$, sebaceous gland. 
root-sheath by a basement-membrane termed the hyaline layer of the follicle. This inner vascular layer corresponds to the superficial layer of the cutis vera. Its fibres and cells have a regular circular arrangement around the follicle, the cells being flattened against the hyaline layer. Externally the dermic coat of the follicle has a more open texture, corresponding to the reticular part of the cutis, and containing the larger branches of the arteries and veins. In the large tactile hairs of animals, the veins near the bottom of the follicle are dilated into sinuses, so as to produce a kind of erectile structure.

The hair grows from the bottom of the follicle by multiplication of the soft cells which cover the papilla, these cells becoming elongated to form the fibres of the fibrous substance, and otherwise modified to produce the medulla and cuticle.

When a hair is eradicated, a new hair is produced from these cells. It is not uncommon to find hair-follicles in which the whole of the lower part has degenerated in such a way that the vascular papilla, and the soft, growing cells which cover it, may have entirely disappeared. The hair then ceases to grow, and eventually becomes lost, but its place may be again supplied by a new hair, which becomes formed in a downgrowth from either the bottom or the side of the hair-follicle, a new papilla first becoming formed at the extremity of the downgrowth (fig. 135). If not previously detached, the old hair may be pushed from out the follicle by the one which replaces it.

The hairs are originally developed in the embryo in the form of small solid downgrowths from the Malpighian layer of the epidermis (fig. 136, A). The hair-rudiment, as it is called, is at first composed entirely of soft, growing cells ; but presently those in the centre become differentiated, so as to produce a minute hair invested by inner rootsheath, and its base resting upon a papilla which has grown up into the extremity of the hair-rudiment from the corium (fig. 136, B). As the minute hair grows, it pushes its way through the superficial layers of the epidermis, which it finally perforates (C). The hair-rudiments commence at the third or fourth month of fotal life; their growth is completed about the fifth' or sixth month, and they form a complete hairy covering

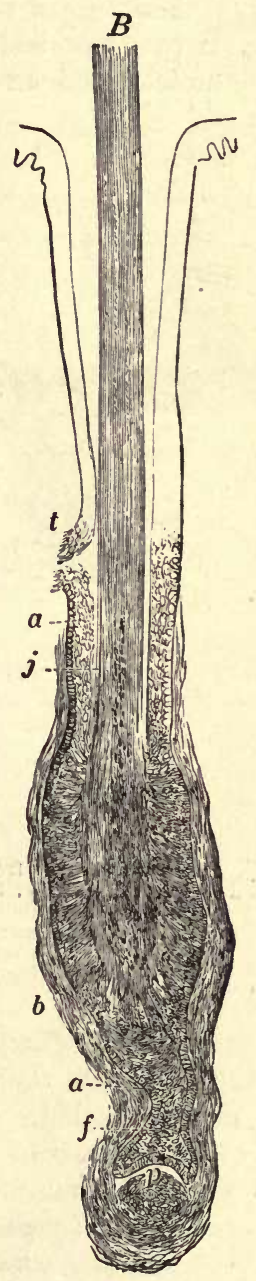

Fig. 135.-CoMMENCING REPLACEMENT OF OLD BY NEW HAIR. (Toldt.)

$a$, outer root-sheath; $b$, dermic coat of follicle; $f$, downgrowth of epithelium to form new hair-follicle ; $p$. papil'a of new hair commencing ; $j$, root of old hair ; $t$, duct of sebaceous gland. 
termed the lanugo. This is entirely shed within a few months of birth. the new hairs being formed in downgrowths from the old hair-follicles in the manner already mentioned.

Hairs grow at the rate of half an inch per month. They are found all over the body except on the palms of the hands and the soles of the feet, and on the distal phalanges of the fingers and toes. They

$\mathbf{A}$

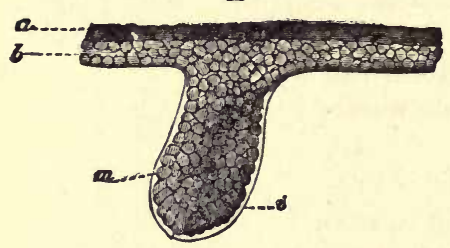

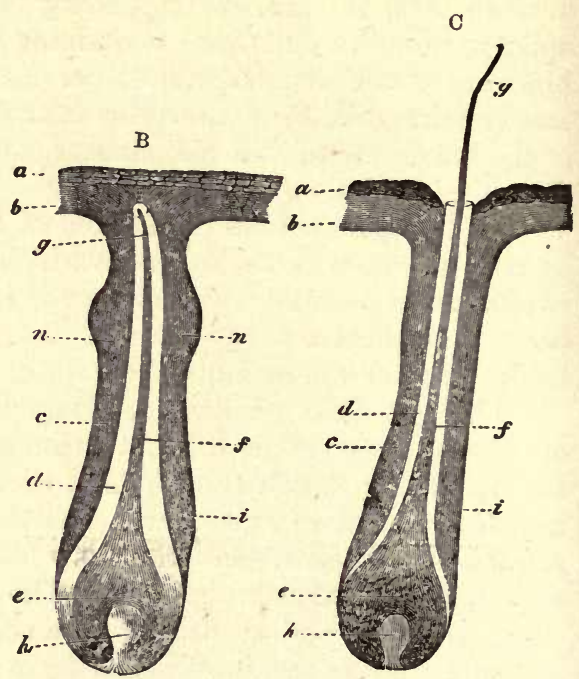

FIG. 136.

A. Hair-rudiment from an embryo of six weeks $a$, horny, and $b$. mucous or Malpighian layer of cuticle; $i$, basement-membrane; $m$, cells. some of which are assuming an oblong figure, which chiefly form the future hair. B. Hair-rudiment, with the young hair formed but not yet risen through the cuticle. $a$, horny, $b$, Malpighian layer of epidermis; $c$, outer, $d$, inner, root-sheath ; $e$, hair-knob : $f$, stem, and $g$, point of the hair; $h$, hair-papilla ; $n, n$, commencing sebaceous follicles. C. Hair-follicle with the hair just protruded.

usually slant, and in the negro the hair-follicles are even considerably curved. On the scalp they are set in groups, as is well seen in a horizontal section.

The hairs of animals are often curiously marked by the arrangement of their medulla, the markings being often characteristic of the particular species.

Muscles of the hairs. - A bundle of plain muscular tissue is attached to each hair-follicle ; passing from the superficial part of the corium, on the side to which the hair slopes, obliquely downwards, to be attached near the bottom of the follicle (arrector pili, fig. 133, n). When the muscle contracts, the hair becomes erected, and the follicle is dragged upwards so as to cause a prominence on the general surface of the skin; whilst the part of the corium from which the little muscle arises is correspondingly depressed; the roughened condition known as ' goose skin' being in this way produced.

The sebaceous glands (fig. 133, $t$ ) are small saccular glands, the 
ducts from which open into the mouths of the hair-follicles. Both the duct and the saccules are lined by epithelium, which becomes charged with fatty matter. This sebaceous matter is discharged into the cavity of the saccule, probably owing to the disintegration of the cells within which it is formed. There may be two or more sebaceous glands attached to each follicle.

The sebaceous glands are developed as outgrowths from the outer root-sheath.

The sweat-glands are abundant over the whole skin, but they are most numerous on the palm of the hand and on the sole of the foot. They are composed of coiled tubes, which lie in the deeper part of the integument and send their ducts up through the cutis to open on the surface by corkscrew-like channels which pierce the epidermis (fig. 137).

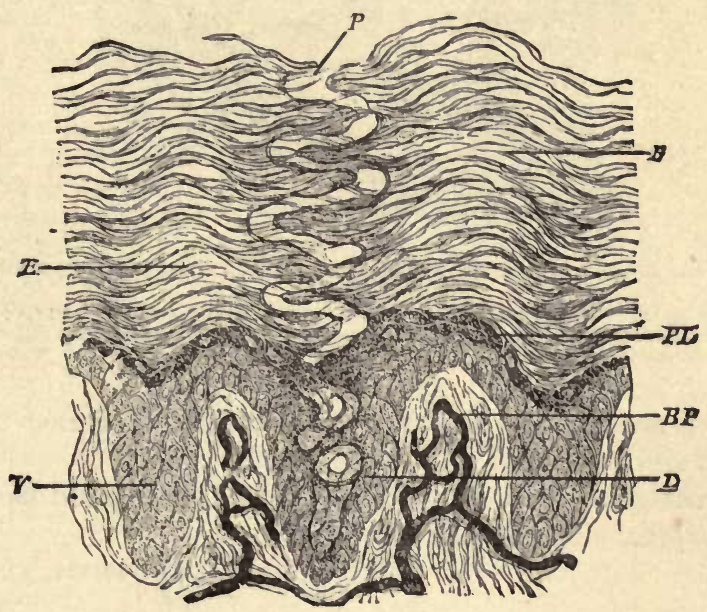

Fig. 137.-DUCt of a SWEAT-GiaNi PASSING THLUUGi the EPIDERMis. (Magnitied 200 diameters.) (Heitzmann.)

$B P$, papillæ with blood-vessels injected; $V$, rete mucosum betweeu the papillæ; $E$, stratum corneum ; $P L$, stratum granulosum; $D$, duct, opeuing on the surface at $P$.

The glandular or secreting tube is a convoluted tube composed of a basement-membrane lined by a single layer of cubical or columnar epithelium-cells, and with a layer of longitudinally disposed plain muscular fibres betwesn the epithelium and basement-membrane. It is considerably larger than the efferent tube or duct, which begins within the gland and usually makes several convolutions before leaving this to traverse the cutis vera. The efferent tube has an epithelium consisting of two or three layers of cells, within which is a well. marked cuticular lining, but there is no muscular layer. The passage through the epidermis has no proper wall, but is merely a channel excavated between the epithelium-cells.

The ceruminous glands of the ear are modified sweat-glands. 
The sweat-glands are developed, like the hairs, from downgrowths of the Malpighian layer of the epidermis into the corium, the rudi-

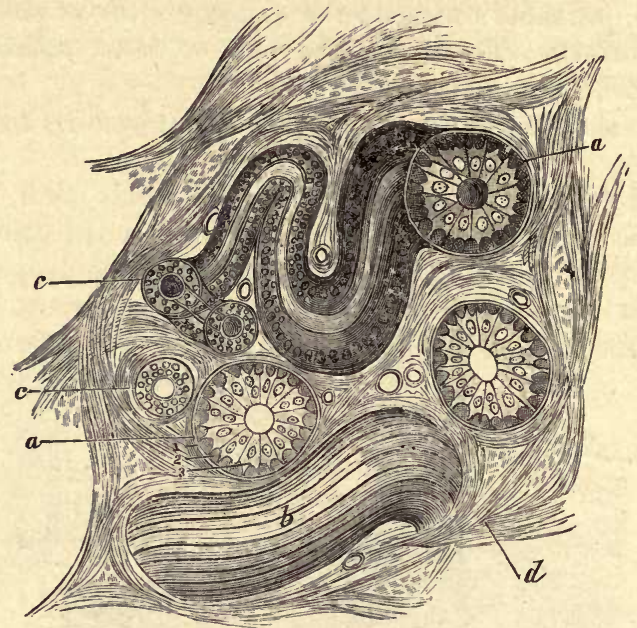

Fig. 138.-Section of a sweat-gland in the Skin of man.

$a, a$, secreting tubes in section ; $b$, a tube seen from above ; $c$, $c$, efferent tubes ; $d$, intertubular connective tissue with blood-vessels. 1, basement mémbrane; 2, muscular fibres cut across ; 3 , secreting epithelium of a tubule.

ments which are thus formed becoming eventually coiled up at their extremities and converted into hollow tubes.

The sweat-glands receive nerve-fibres, and each gland has a special cluster of capillary blood-vessels. 


\section{LESSON XXIV. STRUCTURE OF THE HEART.}

1. In a section through the wall of the auricle which has been stained with magenta and mounted in glycerine, notice the relative thickness of the epicardium, myocardium, and endocardium. Observe the blood-vessels and nerve-fibres under the epicardium, often embedded in fat; here and there a ganglion may be seen under this membrane. Notice also the elastic networks under both the pericardium and endocardium. Make a general sketch from this section.

2. Section through the wall of the ventricle, stained with logwood and mounted in Canada balsam. The muscular fibres are variously cut. In those cut longitudinally, notice the branching of the fibres and their union into a network. Notice also that although the fibres are cross-striated this is less distinct than in voluntary muscle, and the nuclei lie in the centre of each fibre. Transverse markings may also be seen passing across the fibres between the nuclei and indicating a division into cells. The endocardium is very thin, especially over the columnæ carneæ.

3. The lymphatics of the heart are easily injected with Berlin blue by sticking the nozzle of the injecting syringe into the muscular substance, in the interstices of which the lymphatics arise. These ammencing lymphatics lead to efferent vessels which pass to the base of the heart under the epicardium.

4. Section through one of the valves of the heart, stained and mounted as preparation 2.

5. The epithelium which covers the epicardium, and that which lines the endocardium, may be studied in preparations of the fresh organ which have been treated with nitrate of silver and subsequently exposed to the light and hardened in alcohol.

The muscular substance of the heart (myocardium) is composed of transversely striated muscular fibres (fig. 139), which differ from those of voluntary muscle in the following particulars: their striations are less distinct; they have no sarcolemma; they branch and unite with neighbouring fibres, and their nuclei lie in the centre of the fibres. Moreover, the fibres are composed of a series of short cylindrical cells (fig. 140) joined together end to end, each corresponding to one of the nuclei. The lines of junction of these cells may sometimes be seen in longitudinal sections stained with hæmatoxylin or magenta; but they come much more distinctly into view in sections of the fresh tissue stained with nitrate of silver. 
In the interstices of the muscular tissue there is a little areolar tissue in which run the very numerous blood-capillaries and the lacunar lymphatics.

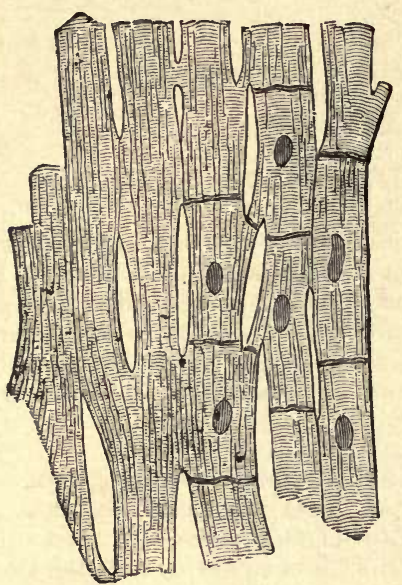

FIG. 139.-MUSCULAR FIBRES FROM THE HEART, MAGNIFIED, SHOWING THEIR CROSS-STRIA, DIVISIONS, AND JUNCTIONS.

The nuclei and cell-junctions are only represented on the right-hand side of the figure.

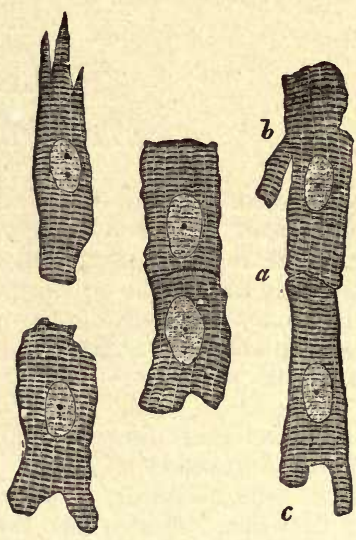

Fig. 140.-Six muscular fibrf-Cells FROM THE HEART. (Magnitied 425 diameters.)

$a$, line of junction between two cells; $b, c$, branching of cells.

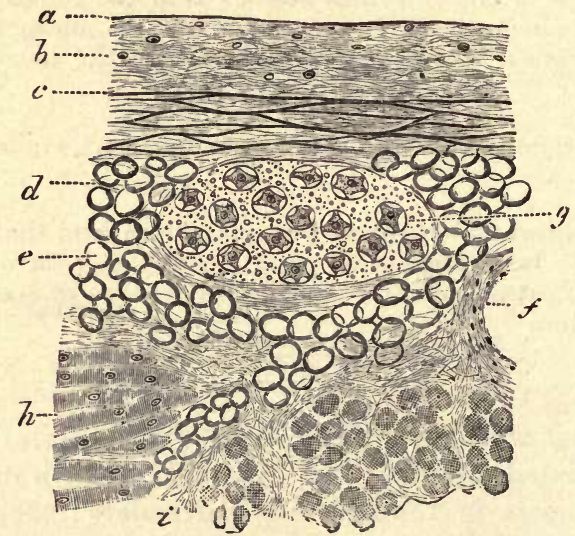

Fig. 141.-SECtion of THE EPICARDiUM OF THE RIGHT AURICLE.

$a$, serous epithelium in section; $b$, connective-tissue layer; $c$, elastic network ; $d$, subserous areolar tissue ; $e$, fat; $f$. section of a blood-vessel ; $g$, a small ganglion; $h$, muscular fibres of the myocardium; $i$, intermuscular areolar tissue.

The myocardium is covered externally by a layer of serous membrane-the epicardium (cardiac pericardium, fig. 141)-composed, like other serous membranes, of connective tissue and elastic fibres, the 
latter being most numerous in its deeper parts. Underneath the epicardium run the blood-vessels, nerves, and lymphatic vessels of the heart, embedded in areolar and adipose tissue; this tissue being continuous with that which lies between the muscular bundles.

The endocardium (fig. 142) has a structure not very unlike the pericardium. It is lined by a pavement-epithelium, like the epithelium of a serous membrane, and consists of connective tissue with elastic fibres in its deeper part, between which there may, in some parts, be

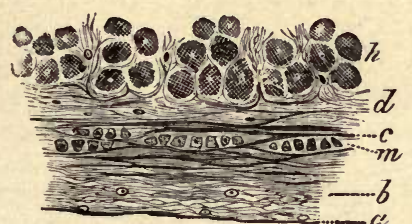

Fig. 142.- Section of THE ENDOCarDIUM OF THE RIGHT AURICLK.

$a$, lining epithelium; $b$, connective tissue with fine elastic fibres; $c$, layer with coarser elastic fibres ; $\boldsymbol{d}$, sub-endocardial connective tissue continuous with the intermuscular tissue of the myocardium; $h$, muscular fibres of the myocardium; $m$, plain muscular tissne in the endocardium.

Fig. 143.-Section throvgh one of THE FLAPS OF THE AORTIC VALVE, AND PART OF THE CORRESPONDING sinus of VALSALVA, WITH THE ADJOINING PART OF THE VENTRICULAR WALL.

$\boldsymbol{\alpha}$, endocardium, prolonged over the valve; $b$, sub-endocardial tissne; $c$, fibrous tissue of the valve, thickened at $c^{\prime}$ near the free edge ; $d$, section of the lunula; $e$, section of the fibrous ring ; $f$, muscular fibres of the ventricle attached to it; $g$, loose areolar tissue at the base of the ventricle; s. $V$. sinus Valsalvæ; 1, 2, 3, inner, middle, and outer coats of the aorta.

found a few plain muscular fibres. Fat is sometimes met with under the endocardium.

In some animals, e.g. the sheep, and sometimes also in man, large beaded fibres are found under the endocardium. These are formed of large clear cells joined end to end, and generally containing in their centre two nuclei, whilst the peripheral part of the cell is formed of crossstriated muscular tissue; they are known as the fibres of Purkinje.

The valves of the heart are formed of folds of the endocardium strengthened by fibrous tissue (fig. 143). This tissue forms a thicken- 
ing near the free edge of the valve $\left(c^{\prime}\right)$. At the base of the auriculoventricular valves a little of the muscular tissue of the auricle may be found passing a short distance into the valve.

The nerves of the heart are seen in sections underneath the epicardium of both auricles and.ventricles; in the former situation, they are connected at intervals with small ganglia (fig. 141, g). Their branches pass to the muscular substance, but their mode of termination has not been ascertained. 


\section{LESSON XXV. \\ THE TRACHEA AND LUNGS.}

1. In sections of trachea, stained with logwood or borax-carmine, and mounted in Canada balsam, notice the ciliated epithelium, the basement-membrane (of some thickness in the human trachea), the lymphoid tissue of the mucous membrane, the elastic tissue external to this, and lastly the fibrous membrane containing the cartilages. In the mucous membrane and submucous areolar tissue look for sections of mucous glands, ducts of which may be seen opening on the surface. At the back of the trachea notice the plain muscular fibres transversely arranged; there may be larger mucous glands external to these.

2. In sections of lung similarly prepared, notice the sections of the alveoli collected into groups (infundibula). Find sections of bronchial tubes, some cut longitudinally and passing at their extremities into the infundibula, others cut across; the latter show the structure of the tubes best. In each tube notice the ciliated epithelium internally. Next to this the mucous membrane containing numerous elastic fibres and often thrown into folds; then the layer of circular muscular fibres, and outside this, loose fibrous tissue in which in larger bronchial tubes the pieces of cartilage may be seen embedded. Small mucous glands may also be observed in the fibrous tissue sending their ducts through the other layers to open on the inner surface. Notice always accompanying a section of a bronchial tube the section of a branch of the pulmonary artery.

In the sections of the alveoli observe the capillary vessels passing from one side to the other of the intervening septa; and in places where the thin wall of an alveolus is to be seen in the section, try and make out the network of blood-capillaries upon it. Notice within the alveoli nucleated corpuscles which very frequently contain dark particles in their protoplasm. They appear to be amœboid cells which have migrated from the blood-vessels and have taken in inhaled particles of carbon. They seem to pass back into the lung tissue, for similar cells may be seen in this. Make a sketch of part of the wall of a bronchial tube and of one or two of the alveoli.

3. Mount in Canada balsam a section of lung in which the pulmonary vessels have been injected. Study the general arrangement of the vessels with a low power, and the network of capillaries of the alveoli with a high power. Observe that the veins run apart from the arteries. Sketch the capillary network of one or two adjoining alveoli.

The trachea or windpipe is a fibrous and muscular tube, the wall of which is rendered somewhat rigid by $\mathbf{C}$-shaped hoops of cartilage which are embedded in the fibrous tissue. The muscular tissue, which is of the plain variety, forms a flat band, the fibres of which run transversely at the back of the tube. The trachea is lined by a mucous 
membrane (fig. $144, a-c$ ), which has a ciliated epithelium upon its inner surface. The epithelium-cells have been already described (Lesson VII.); they rest upon a thick basement-membrane. The mucous membrane proper consists of areolar and lymphoid tissue, and contains numerous blood-vessels and lymphatics. In its deepest part is a well-marked layer of longitudinal elastic fibres $(d)$. Many small glands for the secretion of mucus are found in the wall of the trachea. They may lie either within the mucous membrane or in the submucous

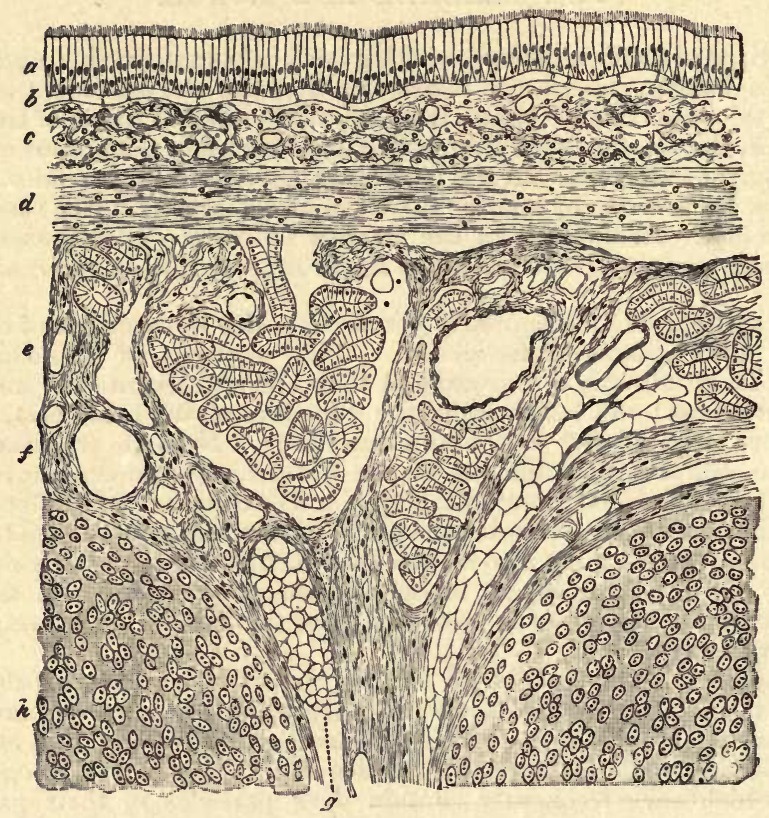

Fig. 144.-Jongitudinal section of tile human trachea, incluing portions of two Cartilaginous Rings. (Moderately magnified.)

$a$, ciliated epithelium ; $b$, basement-membrane ; $c$, superficial part of the mucous membrane, containing the sections of numerous capillary blood-vessels and much lymphoid tissue ; $d$, deeper part of the mucous membrane, consisting mainly of elastic fibres; $e$, submucous areolar tissue, containing the larger blood-vessels, small mucous glands (their ducts and alveoli are seen in section ), fat, \&c. ; $f$, fibrous tissue investing and uniting the cartilages; $g$, a small mass of adipose tissue in the fibrous layer; $h$, cartilage.

areolar tissue (e), or, lastly, at the back of the trachea, outside the transverse muscular fibres.

The two divisions of the trachea, the bronchi, are precisely similar in structure.

The larynx is also very like the trachea so far as the structure of the mucous membrane is concerned, but over the true vocal cords and upon the epiglottis, as well as here and there in the part above the glottis, stratified epithelium is found, and taste-buds (see Lesson XXVI.) may occur in this epithelium, except over the vocal cords. 
The lymphoid tissue is especially abundant in the mucous membrane of the ventricle of Morgagni, and a large number of mucous glands open into this cavity and into that of the sacculus.

The true vocal cords are composed of fine elastic fibres.

The cartilages of the trachea and larynx are hyaline, except the epiglottis and the cartilages of Santorini and of Wrisberg, which are composed of elastic fibro-cartilage.

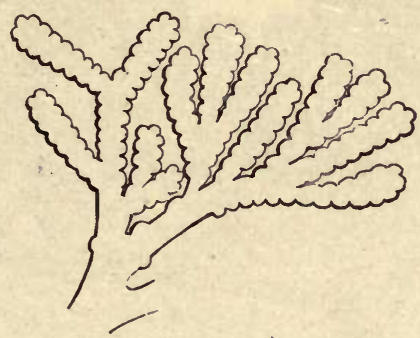

Fig. 145.-Diagramatic Representation of the ending of a BRONCHIAL TUBE IN SACCULATED INFUNDIBULA.

The lungs are formed by the ramifications of the bronchial tubes and their terminal expansions, which form groups of sacculated dilatations (infundibula), beset everywhere with small hemispherical bulgings, known as the air-cells or pulmonary alveoli.

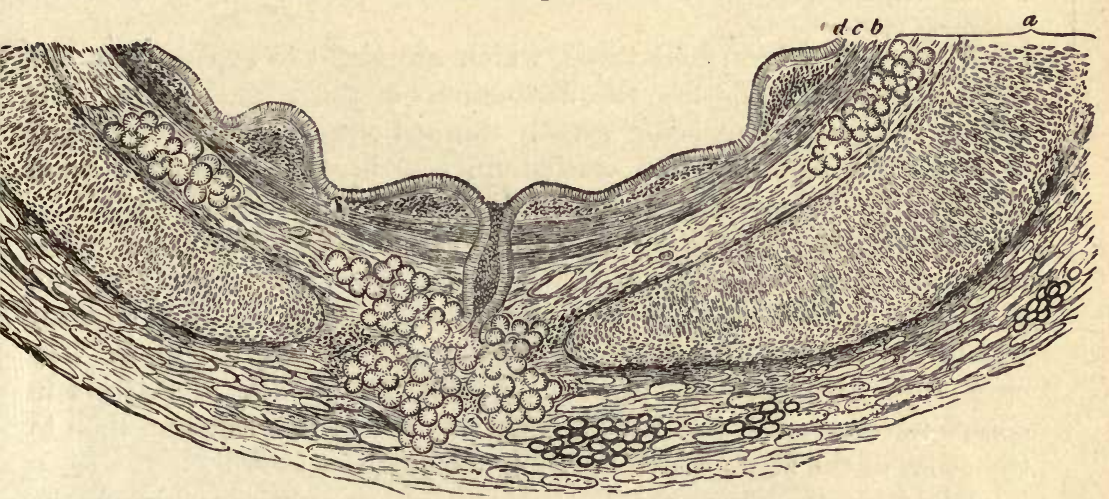

Fig. 146.-Portion of a transverse section of a bronchial tube, human 6 MM. IN DIAMETER. (Magnified 30 diameters.)

$a$, cartilage and fibrous layer with mucous glands, and, in the outer part, a little fat ; in the middle, the duct of a gland opens on the inner surface of the tube; $b$, annular layer of involuntary muscular fibres; $c$, elastic layer, the elastic fibres in bundles which are seen cut across ; $d$, columnar ciliated epithelium.

The bronchial tubes (figs. 146, 147) are lined in their whole extent by ciliated epithelium which rests on a basement-membrane. External to this is the corium of the mucous membrane, containing a large number of longitudinal elastic fibres and some lymphoid tissue. 
Outside this again is a complete layer of plain muscular fibres encircling the tube. Next comes a loose fibrous layer in which, in the larger tubes (fig. 146), small plates of cartilage are embedded. Mucous glands are also present in this tissue.

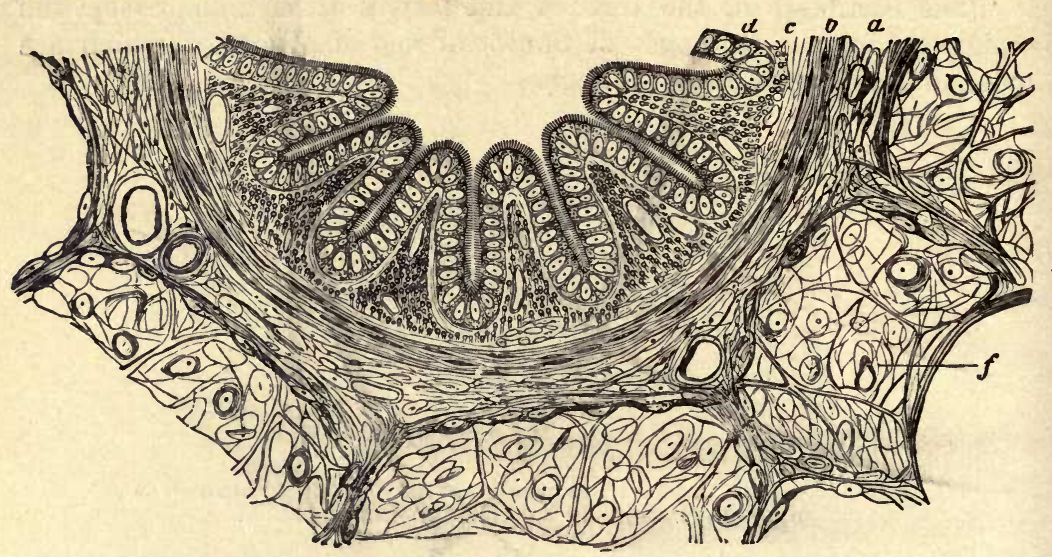

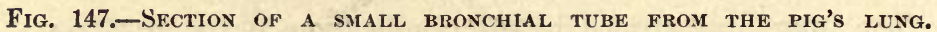
(This section is much more magnified than that represented in the previous figure.)

$a$, fibrous layer; $b$, muscular layer; $c$, mucous membrane in longitudinal folds, with numerous longitudinally running elastic fibres cut across; $d$, ciliated epithelium; $f$, surrounding alveoli.

The smallest bronchial tubes, which are about to expand into the infundibula, gradually lose the distinctness of the several layers, their wall at the same time being greatly thinned out and becoming bulged to form the alveoli. The epithelium also becomes changed; from columnar and ciliated it becomes cubical and non-ciliated.

In the alveoli themselves, besides small groups of cubical cells, there are large irregular flattened cells (fig. 148) which form an extremely delicate layer, separating the blood-capillaries from the air within the alveoli. The capillary network of the alveoli is very close (fig. 149), and the capillary vessels of adjoining alveoli are in complete continuity, the vessels passing first to one side and then to the other of the septa which separate the adjacent alveoli.

Blood-vessels.-Branches of the pulmonary artery accompany the bronchial tubes to be distributed to the capillary networks upon the alveoli, from which the blood is returned by the pulmonary veins which, pursuing a separate course through the tissue of the lung, join in their course with others to form larger vessels which pass to the hilus. Branches from the bronchial arteries are distributed to the walls of the bronchial tubes, and to the connective tissue of the lung. This tissue intervenes everywhere in small quantity between the infundibula (interstitial tissue), and forms a distinct layer, containing much elastic tissue, covering the surface of the lung underneath the 


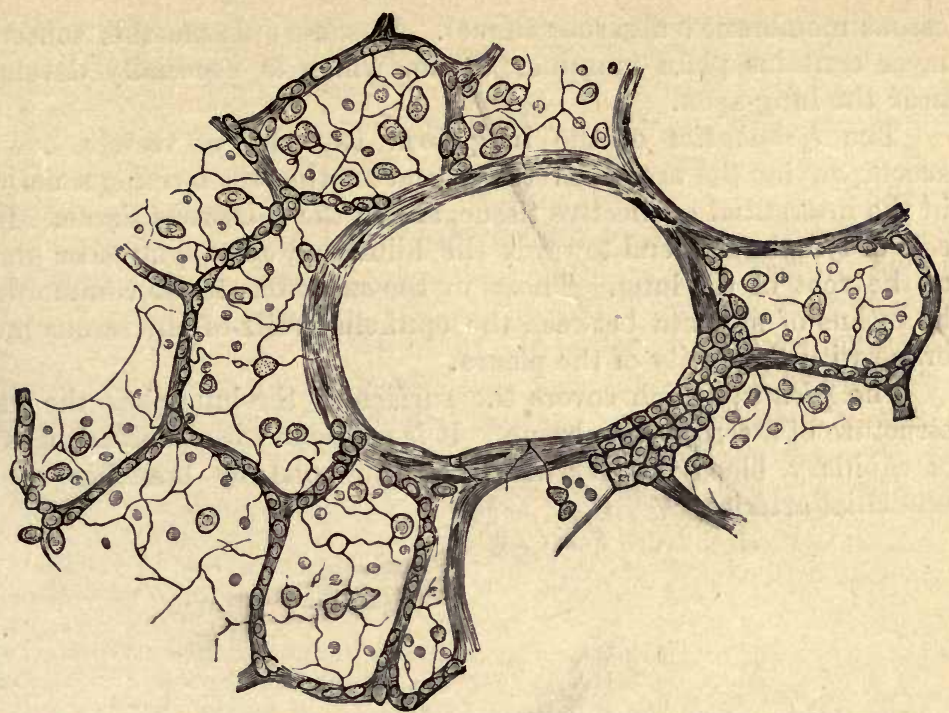

Fig. 148.-Section of PART of cat's LUNG, StAINEd with Nitrate of Silver. (Highly magnified.)

The small granular and the large flattened cells of the alveoli are shown. In the middle is a section of a lobular bronchial tube, with a patch of the granular parement-epithelium cells on one side.

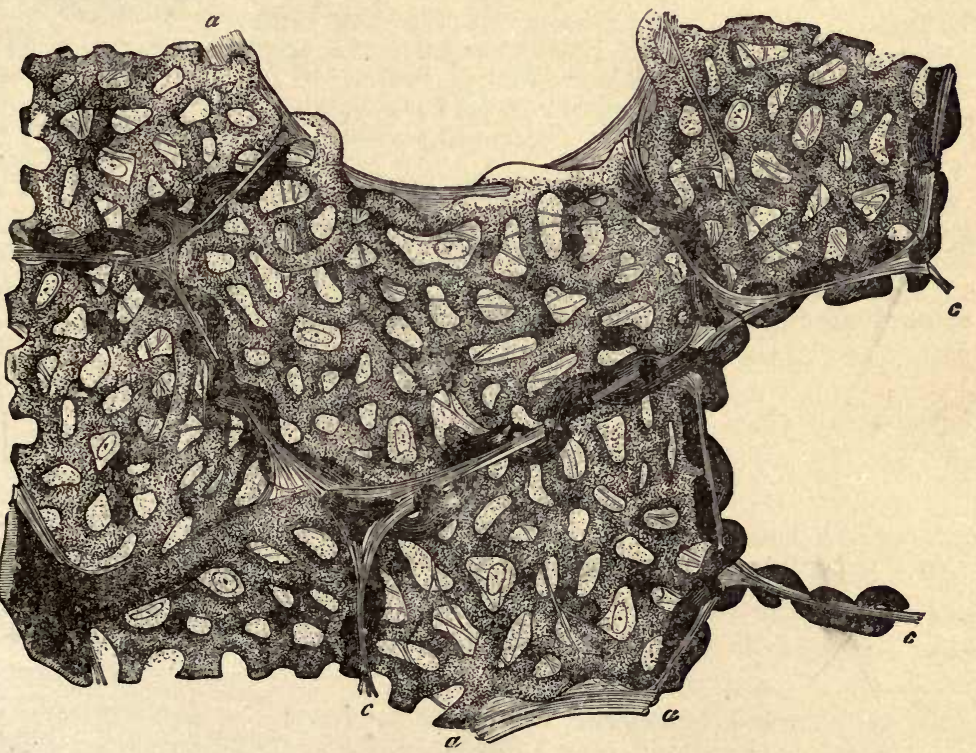

Fig. 149.-Section of injecten tryg, incluning several contiguous alveoli. (Highly magnified.)

$a, a$, free edges of alveoli; $c, c$, partitions between neighbouring alveoli, seen in section: $b$, small arterial branch giving off capillaries to the alveoli. The looning of the vessels to either side of the partitions is well exhibited. Between the capillaries is seen the homogeneous alveolar wall with nuclei of connective-tissue corpuscles, and elastic fibres. 
serous membrane (subserous tissue). In some animals this subserous layer contains plain muscular tissue, which is especially developed near the lung-apex.

The lymphatics of the lung form two sets of vessels, one set accompanying the bronchial tubes, and another set forming a network in the interstitial connective tissue, and in the subserous tissue. Both sets of lymphatics tend towards the hilus and enter lymphatic glands at the root of the lung. Those in the subserous tissue communicate by means of stomata between the epithelial cells of the serous membrane with the cavity of the pleura.

The pleura, which covers the surface of the lung, has the usual structure of a serous membrane. It is provided with a special network of capillary blood-vessels, which are supplied by branches of the bronchial arteries. 


\section{LESSON XXVI.}

\section{STRUCTURE OF THE TEETH, THE TONGUE, AND MUCOUS MEMBRANE OF THE MOUTH.}

1. Study first with the low power and afterwards with the high power a longitudinal section of a human tooth which has been prepared by grinding. It is better to purchase this specimen, for the process of preparation is difficult and tedious without the aid of special apparatus. Examine carefully the enamel, the dentine, and the cement. The dark appearance of the dentinal tubules is due to their containing air in the dried specimen. Measure the diameter of the enamel prisms and of some of the dentinal tubules. Make sketches from each of the tissues.

2. Mount in Canada balsam a section of a tooth in situ, which has been decalcified in chromic or picric acid and stained with logwood or boraxcarmine. In this section the mode of implantation of a tooth, as well as the structure of the pulp, can be made out. Make a general sketch under a low power, and under a high power draw a small piece of the pulp showing the processes of the odontoblasts extending into the dentinal tubules.

3. The development of the teeth and the formation of their tissues are studied in sections made across the snout and lower jaw of fotal animals. The preparation should be stained in bulk with alcoholic magenta, boraxcarmine, or hæmatoxylin, and embedded in paraffin, and the sections mounted by the shellac-creosote process (see Appendix).

4. Section across the whole tongue of a small mammal; stain with log. wood, and mount in Canada balsam. In these sections the arrangement of the muscular fibres and the structure of the papillæ of the mucous mem. brane may be studied; and if the organ have been previously injected, the arrangement of the blood-vessels in the muscular tissue and in the mucous membrane will also be well seen.

\section{T'HE TEETH.}

A tooth consists of three calcified tissues : the enamel, which is of epithelial origin, the dentine, and the cement, or crusta petrosa. The dentine forms the main substance of a tooth, the enamel covers the crown, and the cement is a layer of bone which invests the root (fig. 150).

The enamel is formed of elongated hexagonal prisms (fig. 151), which are set vertically, or with a slight curvature upon the surface of the dentine. They are marked at tolerably regular intervals with slight transverse shadings producing an indistinct cross-striated appearance. Sometimes coloured lines run through the enamel across the direction of its fibres. 
The dentine is composed of a hard dense substance like bone, but containing no Haversian canals or lacunæ. It is pierced everywhere, however, by fine canaliculi (dentinal tubules, figs. 152, 153), which radiate outwards from a central cavity which, during life, contains the pulp. The tubules branch at acute angles as they pass outwards; their branches become gradually finer towards the periphery of the

Fig. 150-Vertical SECTION OF A TOOTH in situ. (15 diameters.)

$c$, is placed in the pulpcavity, opposite the cervix or neck of the tooth; the part above is the crown, that below is the root (fang). 1 , enamel with radial and concentric markings; 2 , dentine with tubules and incremental lines ; 3 , cement or crusta petrosa, with bone corpuscles; 4 , dental periosteum; 5 , boue of lower jaw.

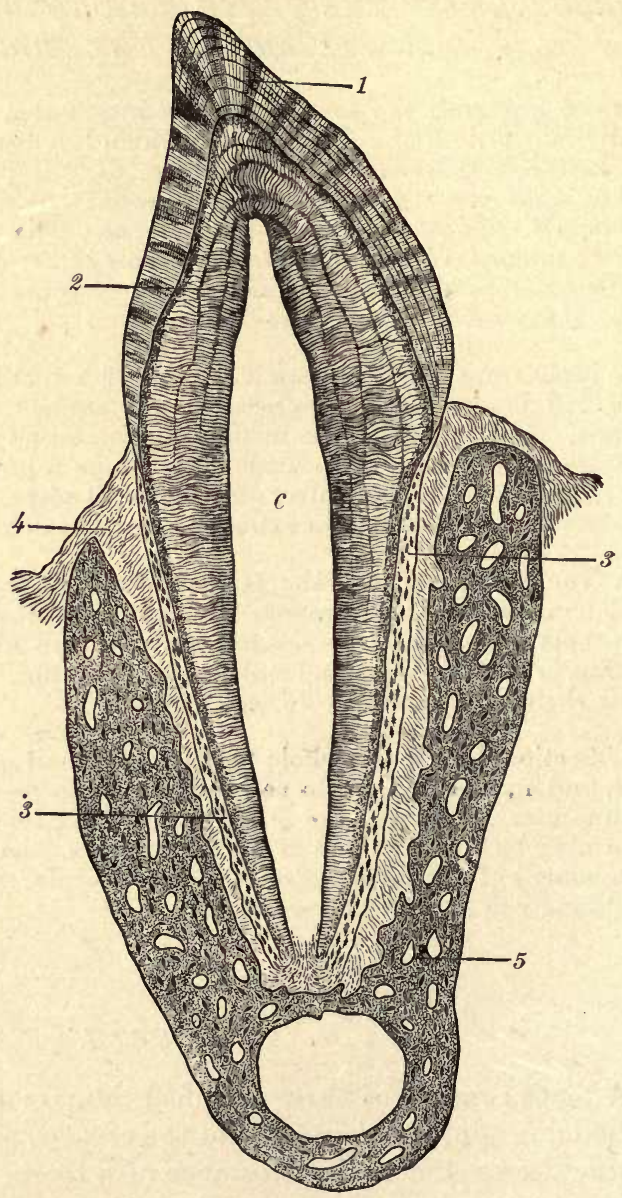

dentine. The tubules have a proper wall of their own, which can be isolated by steeping a section of tooth in strong hydrochloric acid. In the living tooth they are occupied by protoplasmic fibres, which are prolonged from the superficial cells of the pulp.

The intertubular substance is for the most part homogeneous, but here and there indications can be seen of its deposition in the form of globules. This is especially the case near the surface of the dentine, 


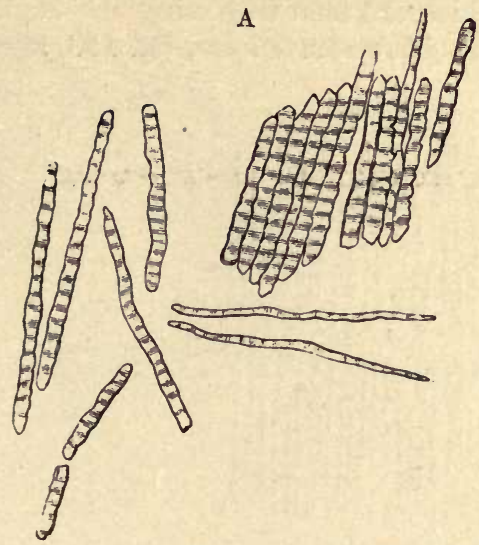

B

Fig. 151.-ENAMEL PRISMS. ( 350 diameters.)

A, fragments and single fibres of the enamel, isolated by the action of hydrochloric acid. B, surface of a small fragment of enamel, showing the hexagonal ends of the fibres.

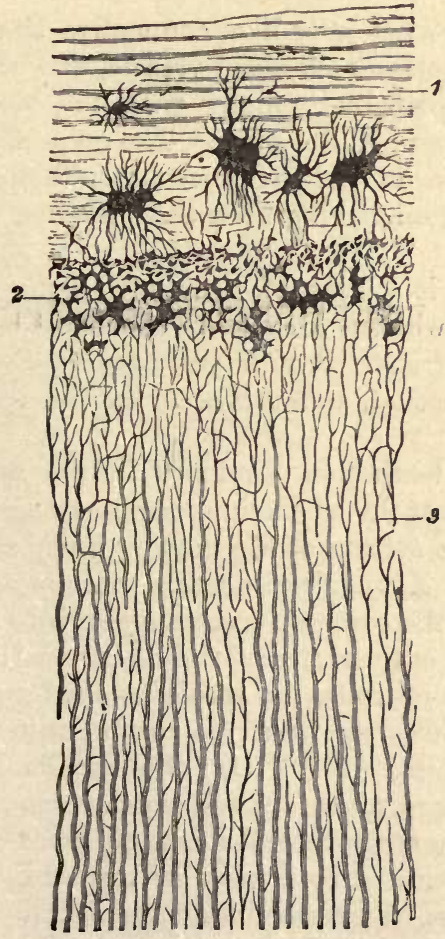

Fig. 152.- Section of FANG, PA. RALLEL TO THE DFNTINAL TU. BULEs. (Magnified 300 diameters.)

1, cement, with large bone-lacunæ and indications of lamellæ; 2, granular layer of Purkinje (interglobular spaces); 3 , dentinal tubules.

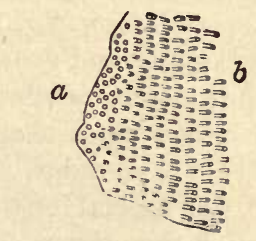

Fig. 153.-SEctioxs of DENTINAL TUBULES.

$a$, cut across: $b$, cut ob'iquely.

(About 300 diameters.)

where the globular deposit and the interglobular spaces may produce a granular appearance (granular layer, fig. 152, 2), and also in the 
course of certain lines or clefts which are seen traversing the dentine across the direction of the tubules (incremental lines, fig. 150, shown magnified in fig. 154).

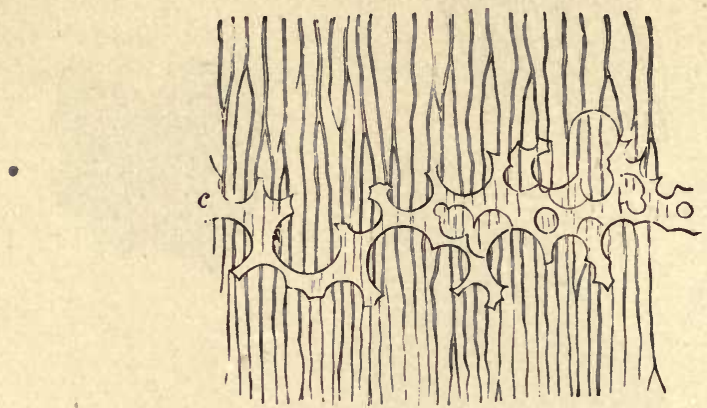

Fig. 154.-A sMali, portion of the Dentine With iNTERglobular sPaCes. ( 350 diameters.)

c, portion of incremental line formed by the interglobular spaces, which are here filled up by a transparent material.

The pulp consists of a soft, somewhat jelly-like, connective tissue, containing many branched cells, a network of blood-vessels, and some nerve fibres which pass into the pulp-cavity along with the bloodvessels by a minute canal at the apex of the fang. The superficial cells of the pulp form an almost continuous layer, like an epithelium. They are known as odontoblasts, from having been concerned in the formation of the dentine.

The crusta petrosa (fig. 152, 1) is a layer of lamellated bone including lacunæ and canaliculi, but without Haversian canals, at least normally in the human teeth. It is covered with periosteum (dental periosteum), which also lines the socket, and serves to fix the tooth securely.

Formation of the teeth.-The teeth are developed in the same manner as the hairs. A thickening of the epithelium occurs along the line of the gums, and grows into the corium of the mucous membrane (common enamel-germ, fig. 155, A). At regular intervals there is yet a further thickening and growth from the common enamel-germ into the tissue of the mucous membrane, each of these special rudiments swelling out below into a flask-shaped mass of cells, the special enamel-germ, fig. 155, B). A vascular papilla grows up from the corium into the bottom of the special enamel-germ (fig. $155, \mathrm{C}, \mathrm{D}$ ) ; this papilla has the shape of the crown of the future tooth. Each special enamelgerm, with its included papilla, presently becomes cut off from the epithelium of the mouth, and surrounded by a vascular membranethe dental sac. The papilla becomes transformed into the dentine of the future tooth, and the enamel is deposited upon its surface by the epithelial cells of the enamel-germ. The root of the tooth, with its covering of cement, is formed at a later period, when the tooth is 
A

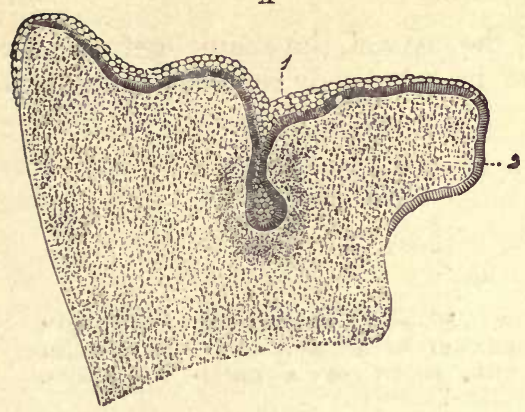

B

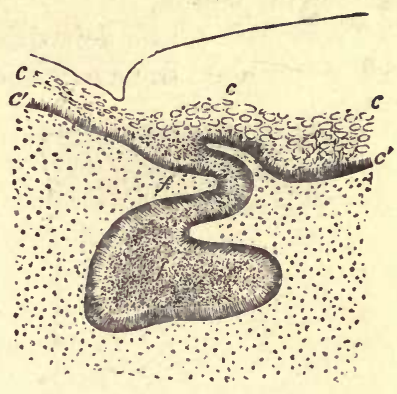

$\mathrm{D}$
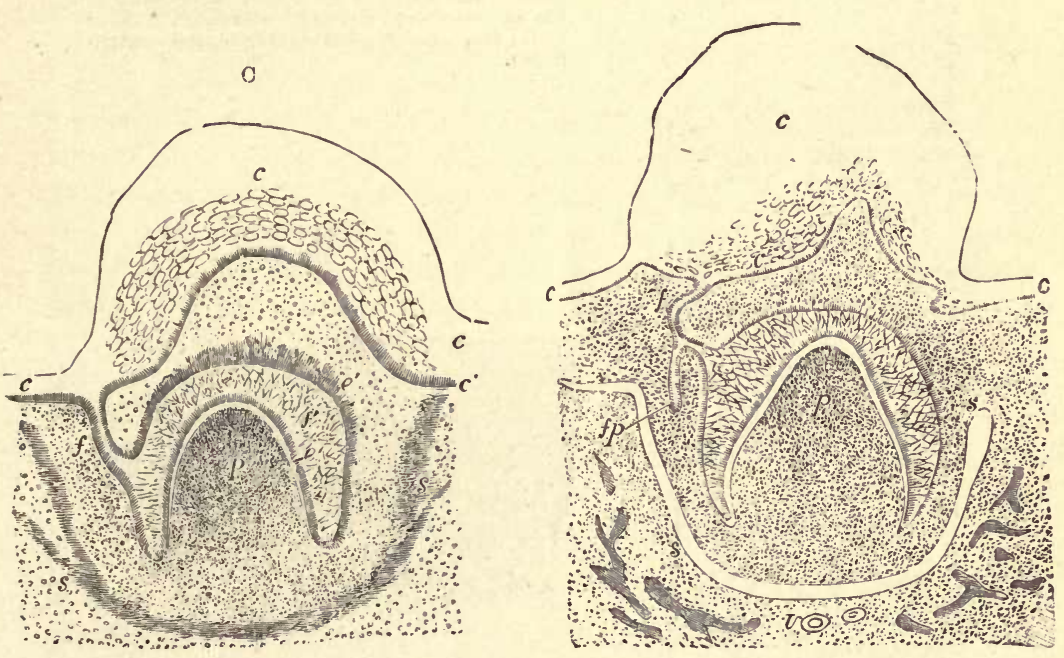

Fig. 155.

A. Section across the upper Jaw of a feetal sheep. (3 centimeters long.)

1, common enamel-germ dipping down into the mucous membrane where it is half surrounded by a semilunar-shaped more dense-looking tissue, the germ of the dentine and dental sac; 2 , palatine process of the maxilla.

B. Section smilar to that shown in the previous figure, but passing THROUGH ONE OF THE SPECIAL ENAMEL-GERMS HERE BECOMING FLASK-SHAPED.

$c, c^{\prime}$, epithelium of mouth; $f$, neck; $f^{\prime}$, body of special enamel-germ.

C and D. Sections at later stages than A and B, the papilla having BeCOME FORMED AND INDENTED THE ENAMEL-GERM, WHICH HAS AT TIE SAME TIME GROWN PARTLY ROUND IT.

$c$, epithelium of gum, sketched in outline ; $f$, neck of enamel-germ; $f^{\prime}$, enamel-organ ; $e$, its deeper columnar cells; $e^{\prime}$. projections into the corium ; $p$, papilla; $s$, dental sac forming. In $\mathrm{D}$, the enamel-germ $(f p)$ of the corresponding permanent tooth has become formed. 
beginning to grow up through the gum, by a gradual elongation of the base of the papilla.

Previously to the deposition of the enamel, the enamel-germ undergoes a peculiar transformation of its previously rounded epithelium-

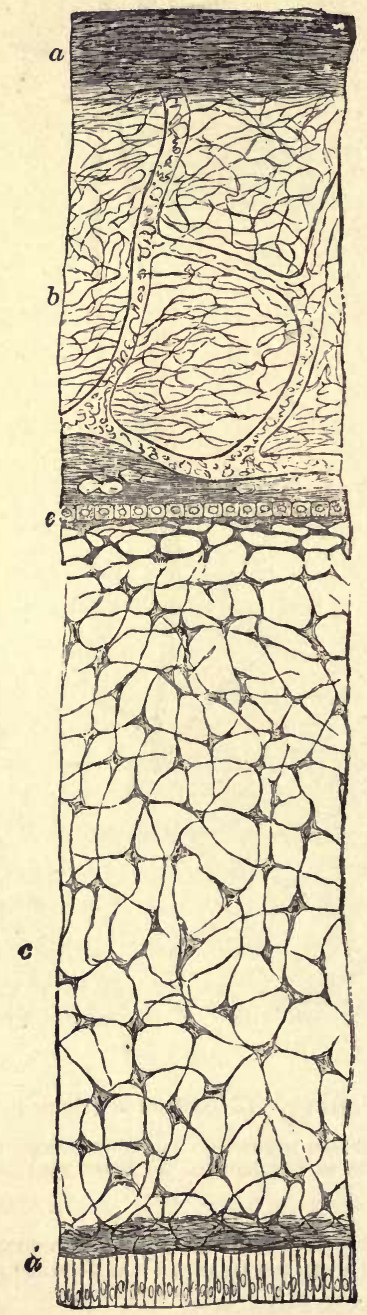

Fig. 156. - A Section throdgh The ENAMELORGAN AND DENTAL SAC FROM THE TOOTH OF A CHILD AT BIRTH. (250 diameters.)

$a$, outer dense layer of the dental sac; $b$, inner looser texture of the same with capillary bloodvessels and a somewhat denser layer towards the enamel organ; $c$, spongy substance; $d$, inner cells ; and $e$, outer cellular layer of the enamelorgan.

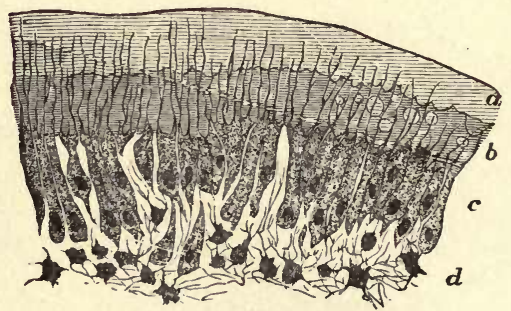

FIG. 157.-PART OF SECTION OF DEVELOPING TOOTH OF YOUNG RAT, SHOWING THE MODE OF DEPOSITION OF THE DENTINF. (Highly magnified.)

$a$, outer layer of fully calcified dentine; $b$, uncalcified matrix, with a few nodules of calcareous matter; $c$, odontoblasts with processes extending into the dentine; $d$, pulp. The section is stained with carmine, which colours the uncalcified matrix, but not the calcified part.

cells into three layers of modified cells. One of these is a layer of columnar cells (fig. 156, $d$ ), which immediately covers the surface of the dentine. These columnar cells form the enamel-prisms either by a deposition of calcareous salts external to them, or by a direct calcification of their protoplasm. The cells next to the dental sac form a single layer of cubical epithelium (e), all the other cells of the 
enamel-germ become transformed into branching corpuscles (c) communicating by their processes, and thus forming a continuous network. The enamel-germ, after it is thus modified, is known as the enamel-organ.

The dentine of the tooth is formed by calcification of the surface of the papilla. At this surface there is a well-marked layer of odontoblasts (fig. 157), and these produce a layer of dentinal matrix which forms a sort of cap to the papilla, and which soon becomes calcified by the deposition of globules of calcareous matter. Processes of the odontoblasts remain in the dentine as it is forming, and thus the dentinal tubules are produced. Subsequently other layers of dentine are formed within the first by a repetition of the same process, and in this way the papilla gradually becomes calcified. A part, however, remains unaltered in the centre of the tooth, and with its covering of odontoblasts forms the pulp.

The ten milk-teeth are formed in each jaw in this manner. These, however, become lost within a few years after birth, and are replaced by permanent teeth in much the same way that a new succession of hairs occurs. A small outgrowth takes place at an early period from the enamel-germ of each of the milk-teeth (fig. 155, D, f p), and this eventually becomes the germ of the corresponding permanent tooth. It gradually enlarges, acquires a papilla, forms an enamelorgan, in short, passes through the same phases of development as its parent germ, and when the milk-tooth drops out of the jaw in consequence of the absorption of its roots (by osteoclasts) the permanent tooth grows up into its place.

But there are six permanent teeth in each jaw which do not succeed milk-teeth ; these are the permanent molars. They are developed from an extension backwards of the original epithelial thickening (common enamel-germ) and the downgrowth from this into the corium of three successive special enamel-germs at comparatively long intervals of time. Within these the tissues of the permanent molars become formed in a manner exactly similar to that in which the milk-teeth are developed.

\section{THE TONGUE.}

The tongue is mainly composed of striated muscular fibres, running, some longitudinally, and others transversely. It is covered by a mucous membrane, the epithelium of which, like that of the rest of the mouth, is thick and stratified, and conceals microscopic papillæ (fig. 158) like those of the skin. Besides these, the upper surface of the organ is covered with larger papillæ, which give it a rough appearance. These, which are termed the lingual papilla, are of three kinds: (1) About twelve or thirteen comparatively large circular projections, each of which is surrounded by a narrow groove (fossa), external to which the mucous 


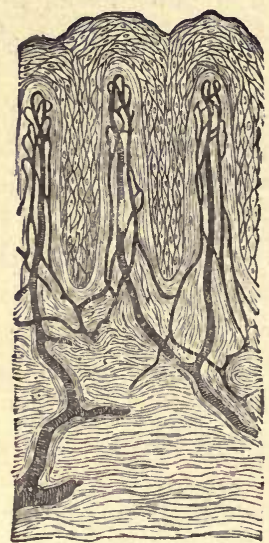

Fig. 158.-SeCtion of MUCOUS MEMIRANE OF MOUTH, SHOWING THREE MICROSCOPIC PAPILLE AND STRATIFIED ERTHELIUM. THE BLOODVESSELS HAVE BEEN INJECTED. (Toldt.)

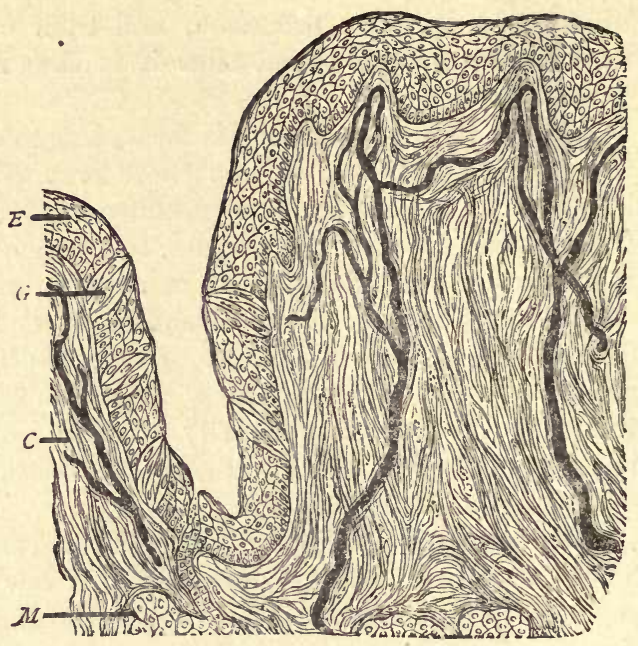

Fig. 159.--Section of circumyallate papilla, HUMAN. THE FIGURE INCLUDFS ONE SIDE OF THE PAPILLA AND THE ADJOINING PAITT OF THE VALLUn. (Magnified 150 diameters.) (Heitzmann.) $E$, epithelium ; $G$, taste-bud ; $C$, corium with injected bloodvessels; $M$, gland with duct.

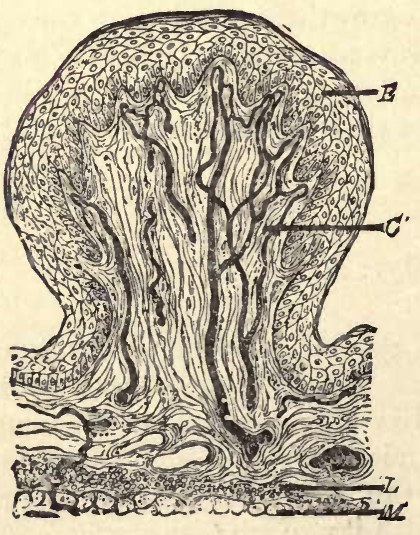

Fig. 160.-Section of FUNGIFORM papilla, human. (Heitzmann.)

$E$, epithelium; $C$, corium; $L$, lymphoid tissue; $M$, muscular fibres of tongue.

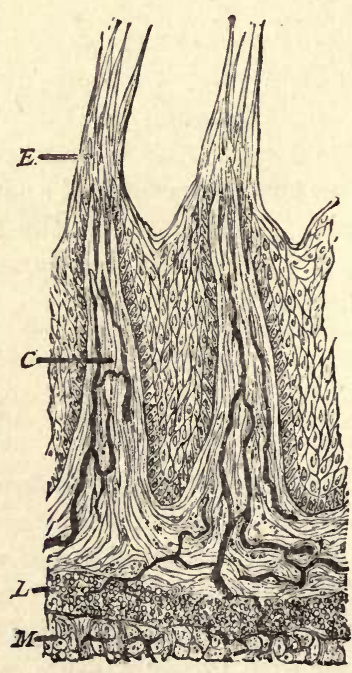

Fig. 161.-Section of two FILIFORM PAPILLE, HUMAN. (Heitzmann.) (Letters as in previous figure.) 
membrane is raised above the general level (vallum) (fig. 159). These papillæ form a V-shaped line towards the back of the tongue; they receive filaments of the glosso-pharyngeal nerve, and have taste-buds in the epithelium which covers their sides, and in that of the side of the vallum. They are known as the circumvallate papilla. (2) All the rest of the papillary surface of the tongue is covered by conical papilla, so named from the conical pointed cap of epithelium which is borne by each ; sometimes this cap is fringed with fine epithelial filaments, when they are termed filiform (fig. 161). (3) Scattered here and there amongst the conical papillæ are other larger papillæ, the fungiform (fig. 160). These are very vascular, ano lie partly embedded in little depressions of the mucous membrane.

Small tubular glands may be seen between the superficial muscular fibres sending their ducts to the surface. Most of them secrete mucus, but those which open into the trenches of the circumvallate papillæ, and a few others elsewhere, yield a serous secretion.

The mucous membrane at the back of the tongue contains a large amount of lymphoid tissue.

The taste-buds. - The minute gustatory organs which are known as taste-buds may be seen in sections which pass through the papillæ vallatæ or the papillæ fungiformes; they are also present here and there in the epithelium of the general mucous membrane of the tongue, especially at the back and sides, and occur also upon the under surface of the soft palate, and on the epiglottis. But they are most easily studied in the papillæ foliatæ of the rabbit, two small oval areas lying

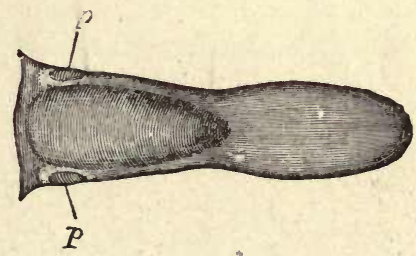

Fig. 162.-Tongue of RABbit, Showing the situation of the PAPILle FOLIATE, $p$.

on either side of the back of the tongue and marked transversely with a number of small ridges or laminæ with intervening furrows (see figs. 162 and 163). Sections across the riagges show numerous taste-buds embedded in the thick epithelium which clothes their sides.

The taste-buds are ovoid clusters of epithelium-cells which lie in cavities in the stratified epithelium (fig. 164). The base of the tastebud rests upon the corium of the mucous membrane, and receives a branch of the glosso-pharyngeal nerve; the apex is narrow and com- 
municates with the cavity of the mouth by a small pore in the superficial epithelium (gustatory pore, fig. $164, p$ ).

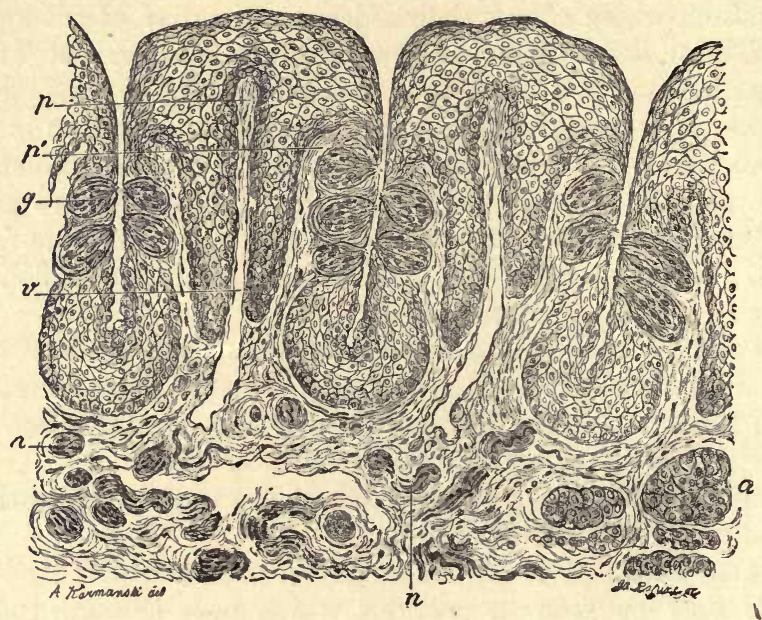

Fig. 163.-Vertical section of papilla foliata of the rabitt, Passing ACROSS THE FOLIA. (Ranvier.)

$p$, central lamina of the corinm; $v$, section across a vein, which traverses the whole length of the folia; $p^{\prime}$, lateral lamina in which the nerve-fibres run; $g$, taste-bud; $n$, sections of nerve-bundles; $a$, serous gland.

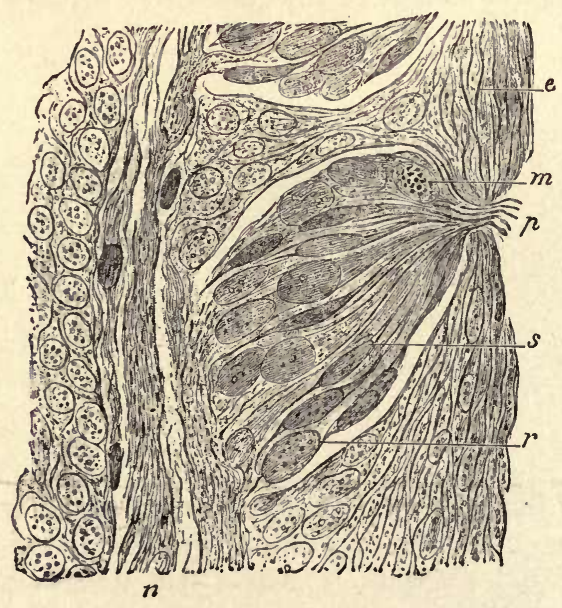

Fig. 164.-Section throvgh the middle of a taste-bud, (Ranvier.)

$p$, gustatory pore ; $s$, gustatory cell ; $r$, sustentacular cell ; $m$, lymph-cell, containing fatty granules; $e$, superficial cells of the stratified epithelium; $n$, nerve-fibres.

The cells which compose the taste-bud are of two kinds, viz.: 1. The gustatory cells (fig. 165, a), which are delicate fusiform or 
bipolar cells composed of the cell-body or nucleated enlargement, and of two processes, one distal, the other proximal. The distal process is nearly straight, and passes towards the apex of the taste-bud, where it terminates in a small, highly refracting cilium-like appendage, which projects into the pore.above mentioned. The proximal process is more delicate than the other, and is often branched and varicose; it is
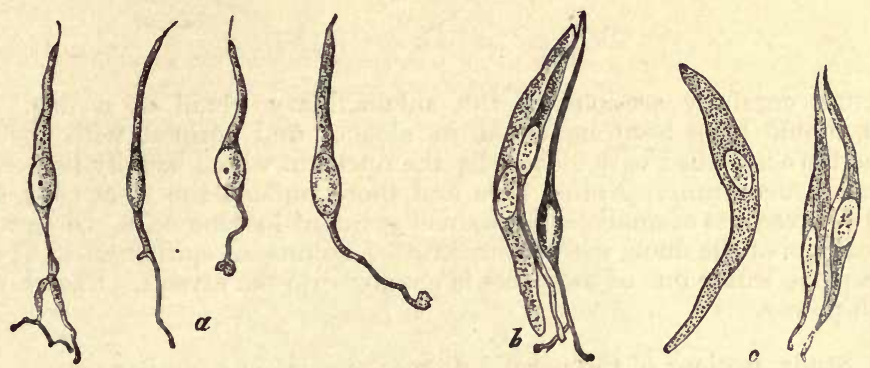

Fig. 165-VArious Cells From taste-bUd of RABbit. (600 diameters.)

$a$, four gustatory cells from central part ; $b$, two sustentacular cells, and one gustatory cell, in connection ; $c$, three sustentacular cells.

believed to be directly connected with an entering nerve-fibre. 2 . The sustentacular cells (fig. 165, c). These are elongated cells, mostly flattened, and pointed at their ends; they lie between the gustatory cells, which they thus appear to support, and in addition they form a sort of envelope or covering to the taste-bud. Between the cells of the tastebud lymph-corpuscles are often seen, having probably wandered here from the subjacent mucous membrane. 


\section{LESSON XXVII.}

\section{THE SALIVARY GLANDS.}

1. STUdy carefully sections of the submaxillary gland of a dog. The gland should have been hardened in alcohol and stained with logwood. Notice the acini filled with clear cells, the nuclei of which usually lie near the basement-membrane. Notice here and there, outside the clear cells, demilunes or crescents of small darkly stained granular-looking cells. Observe also the sections of the ducts with their striated columnar epithelium. Try and find a place where one of the ducts is passing into the alveoli. Sketch under a high power.

2. Study sections of the parotid gland prepared in a similar way.

3. Examine small pieces of both submaxillary and parotid gland fresh in saline solution. In the submaxillary gland notice that the alveolar cells are swollen out with clear mucigen, but that those of the parotid are filled with granules (zymogen). ${ }^{1}$ Make a sketch from each preparation under a high power.

4. Prepare a transverse section of the œesophagus. Notice the thick muscular coat partly containing cross-striated fibres and the mucous membrane with its papillæ and stratıfied epithelium. Look for mucous glands in the areolar coat. Sketch under a low power.

The salivary glands may be looked upon as typical of secreting glands in general. They are composed of a number of lobules bound together loosely by connective tissue. Each small lobule is formed of a group of saccular or somewhat tubular alveoli or acini (fig. 166) from which a duct passes, and this, after uniting with other ducts to form larger and larger tubes, eventually leaves the gland to open upon the surface of the mucous membrane of the mouth.

The alveoli are enclosed by a basement-membrane, which is reticular (fig. 167). This basement-membrane is continued along the ducts. Within it is the epithelium, which in the alveoli is composed of polyhedral cells (fig. 168, a), but in the ducts is regularly columnar, except in that part of the duct which immediately opens into the

1 To study the changes which the alveolar cells undergo during secretion, pilocarpine is injected subcutaneously into an animal in sufficient amount to produce copious salivation; after which the animal is killed and its salivary glands are examined as in preparation 3 . The granules are not seen in preparations that have been in alcohol, but osmic acid preserves them; they are best seen, however, in the fresh tissue. 


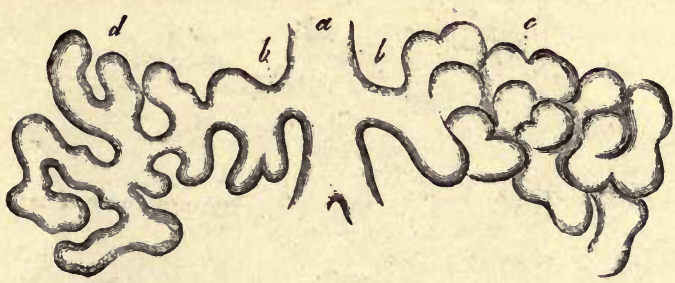

Fig. 166.-Diagram of the construction of a lobule of a tubulo-Racemose (ACINO-TUBULAR) MUCOUS GLAND.

$a$, duct; $b$, a branch of the duct; $c$, alveoli as they lie together in the gland; $d$, the same separated, showing their connection as an irregular tube.

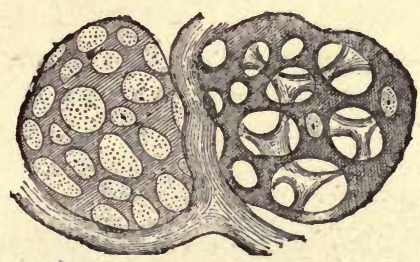

Fig. 16i.-Membrand propria of two alveoli isolated.

The preparation is taken from the orbital gland of the dog, which is similar in structure to a mucous salivary gland.

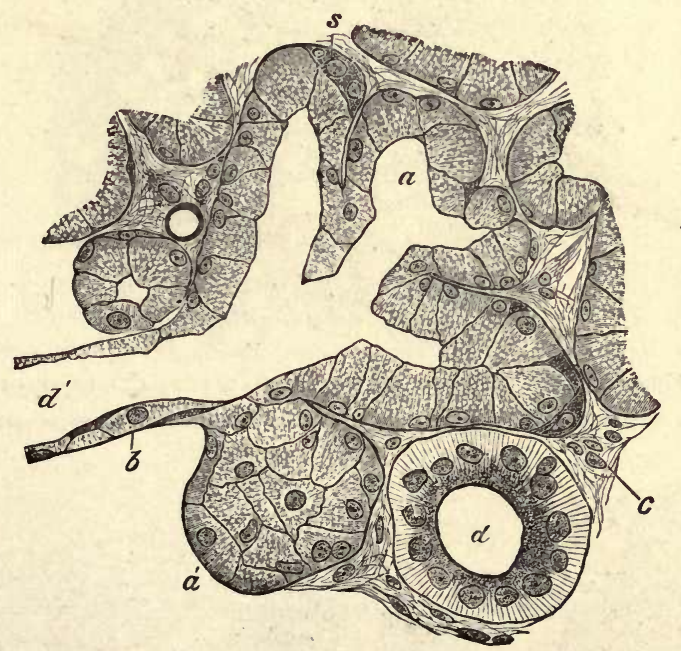

Fig. 168.-Section of the submaxillary gland of the dog, showing the CoMmencement of A DUCT in the Alveoli. (Magnified 425 diameters.)

$\boldsymbol{a}$, one of the alveoli, several of which are in the section shown grouped around the commencement of the duct, $d^{\prime} ; a^{\prime}$, an alveolus, not opened by the section; $b$, basementmembrane in section; $c$, interstitial connective tissue of the gland; $d$, section of a duct which has passed away from the alveoli, and is now lined with characteristically striated columnar cells ; s, semilunar group of darkly stained cells at the periphery of an alveolus. 


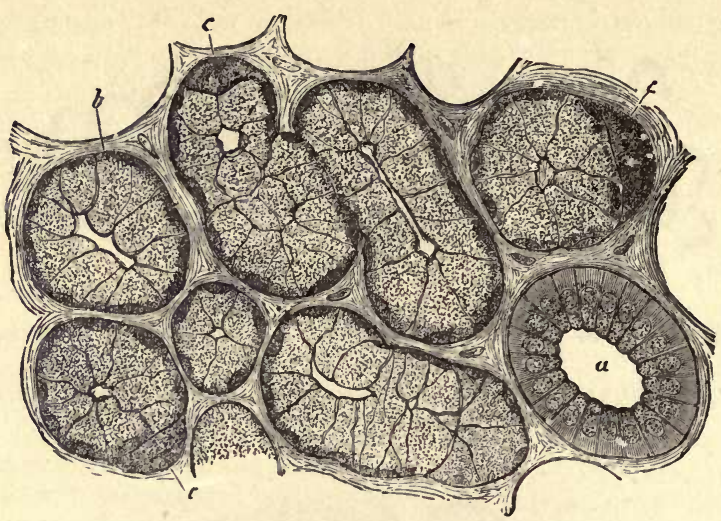

Fig. 169.-Section of dog's surmaxillary, stained. $a$, duct ; $b$, alveolus ; $c$, crescent

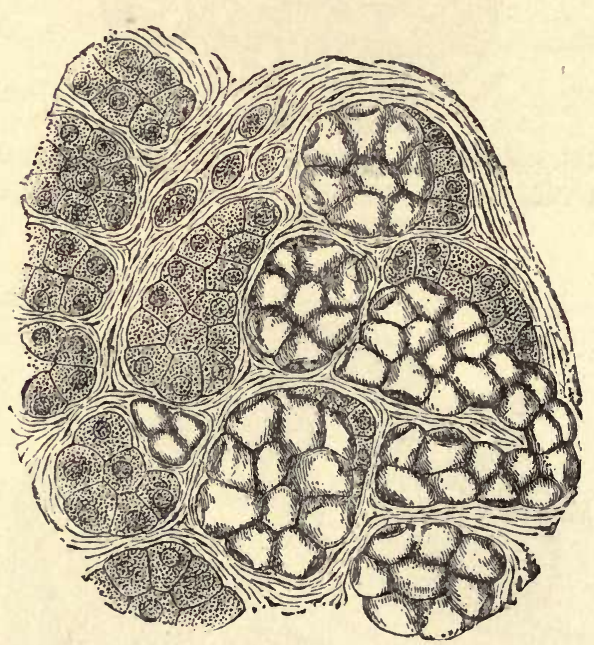

Fig. 170.-Section of PaRT OF the human submaxiliary GLAND.

To the right of the figure is a group of mucous alveoli, to the left a group of serous alveoli.
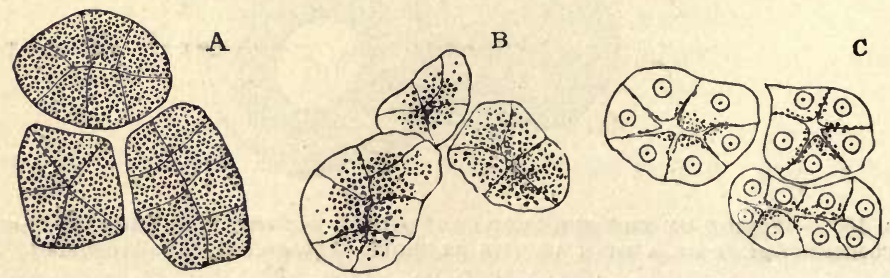

Fig. 171,-Alveoli of a serous gland. A, at rest. B, After a short peirod OF ACTIVITY. C, AFTER A PROLONGED PERIOD OF ACTIVITY.

In $\mathrm{A}$ and $\mathrm{B}$ the nuclei are obscured by the granules of zymogen. 
alveoli; in this it is flattened $\left(d^{\prime}\right)$. 'The columnar epithelium of the ducts is peculiar, in that the cells show a distinction into two unequal zones, an outer, larger, striated zone, and an inner, smaller, granular one (fig. $168, d$ ).

The cells of the alveoli differ according to the substance they secrete. In alveoli which secrete mucus, such as all the alveoli of the dog's submaxillary, and some of the alveoli of the same gland in man (fig. 170), the cells are clear and swollen out with mucigen, which is discharged into the ducts when the gland is stimulated to activity. But in each alveolus there are some smaller cells which do not contain mucigen, and these generally form crescentic groups which lie next to the basement-membrane (fig. 169, $c$ ). These are the so-called crescents of Gianuzzi ; their constituent cells are also known as marginal cells. In alveoli, on the other hand, which do not secrete mucus, but watery or serous saliva, such as the parotid in all animals, and some of the alveoli of the human submaxillary, the cells are filled with granules when the gland is at rest, although the outer part of each cell may become clear after a long period of secretion (fig. 171).

The largest ducts have a wall of connective tissue outside the basement-membrane, and also a few plain muscular cells. The blood-vessels of the salivary gland form a capillary network around each alveolus. The lymphatics commence in the form of lacunar vessels encircling the alveoli. The nerve-fibres, which are derived both from the cerebrospinal nerves and from the sympathetic, have not been satisfactorily traced to their termination, but they probably become connected with the alveolar cells.

\section{THE PHARYNX AND FiSOPHAGUS.}

The pharynx is composed of a fibrous membrane, which is encircled by striated muscles, the constrictors, and lined by mucous membrane. The mucous membrane is lined in the upper part of the pharynx and on the upper surface of the soft palate with ciliated epithelium, which is continuous with that of the nostrils, and through the Eustachian tube with that of the tympanum. Below the level of the soft palate the epithelium is stratified like that of the mouth and gullet, into which it passes. In certain parts the mucous membrane contains a large amount of lymphoid tissue, especially at the back, where it forms a projection which is sometimes termed the pharyngeal tonsil, and there are numerous mucous glands opening on its surface.

The œsophagus or gullet, which passes from the pharynx to the stomach, consists, like the pharynx, of a fibrous covering, a muscular coat, a lining mucous membrane, and intervening connective tissue (areolar coat) (fig. 172). The muscular coat is much more regularly arranged than that of the pharynx, and is composed of striated muscle in about its upper third only, the rest being of the plain variety. There 
are two layers of the muscular coat, an outer layer, in which the fibres run longitudinally, and an inner, in which they course circularly. The mucous membrane is lined by a stratified epithelium, into which microscopic papillæ from the corium project. The corium is formed of areolar tıssue, and its limits are marked externally by a narrow layer of longi-

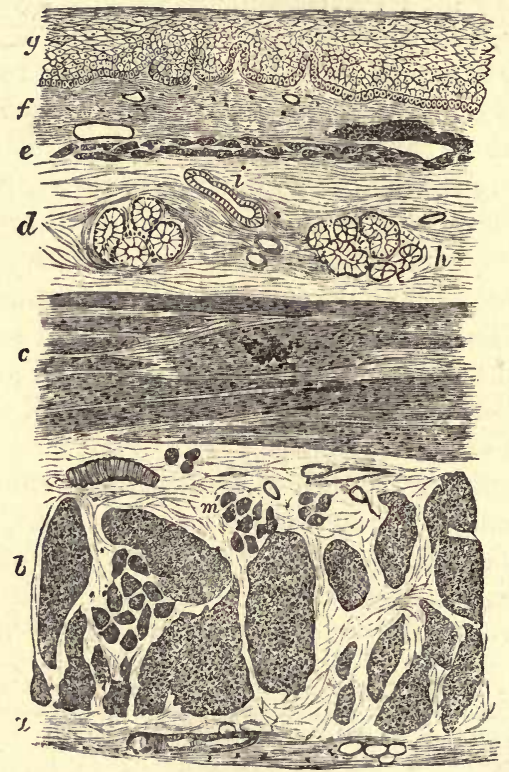

Fig. 172.-Section of the human cesophagus.

The section is transverse, and from near the middle of the gullet. $a$, fibrous covering; $b$, divided fibres of the longitudinal muscular coat ; $c$, transverse muscular fibres; $d$, submucous or areolar layer; $e$, muscularis mucosæ ; $f$, papillæ of mucous membraue ; $g$, laminated epithelial lining; $h$, mucous gland; $i$, gland duct.

tudinally disposed plain muscular fibres, the muscularis mucosa. This is separated from the proper muscular coat by the areolar coat, which contains the larger branches of the blood-vessels and lymphatics, and also most of the mucous glands of the membrane. 


\title{
LESSON XXVIII.
}

\author{
THE STRUCTURE OF THE STOMACH.
}

1. Sections of the cardiac region of the dog's stomach, cut perpendicularly to the surface of the mucous membrane. 'The tissue is to be stained with logwood or borax-carmine, and the sections are to be mounted in Canada balsam.

In these sections the general arrangement of the coats of the stomach is to be studied and sketches are to be made under a low power illustrating this arrangement, and others under a high power showing the structure of the glands of the mucous membrane.

Measure the whole thickness of the mucous membrane, the thickness of the muscular coat, the size of the columnar epithelium-cells of the surface, and that of the cells in the deeper parts of the glands.

2. Sections of the mucous membrane of the same region, cut parallel to the surface.

These sections will show better than the others the arrangement of the cells in the glands.

3. Vertical sections of the mucous membrane from the pyloric region of the dog's stomach. Make a sketch under a low power of one of the glands in its whole length, filling up some of the details with the high power.

4. Study the arrangement of the blood-vessels of the stomach in vertical sections of the wall of an organ the vessels of which have been injected.

The wall of the stomach consists of four coats, which, enumerated from without in, are as follows, viz.: serous, muscular, areolar or submucous, and mucous membrane.

The serous coat is a layer which is derived from the peritoneum. It is deficient only along the lines of the lesser and greater curvatures.

The muscular coat consists of three layers of plain muscular fibres. Of these the bundles of the outer layer run longitudinally, those of the middle layer circularly, and those of the inner layer obliquely. The longitudinal and circular bundles become thicker and stronger towards the pylorus, at which they pass into the corresponding layers of the small intestine; at the pylorus itself the circular layer is greatly thickened to form the sphincter muscle. The oblique fibres are only present in the left or cardiac part of the stomach.

The areolar or submucous coat is a layer of areolar tissue, which serves to unite the mucous membrane loosely to the muscular coat; in it ramify the larger branches of the blood-vessels and lymphatics. 


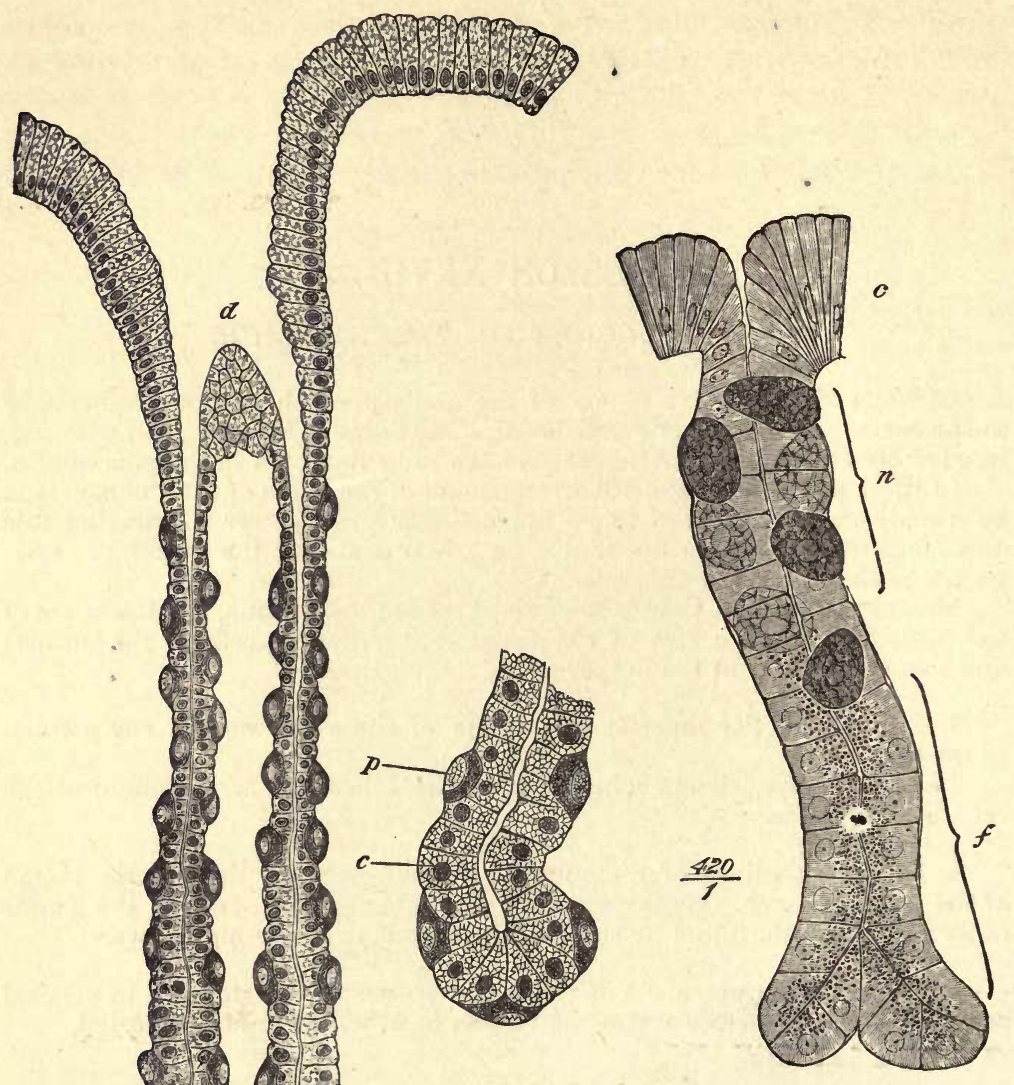

Fig. 174.-A CARDIAC GLAND OF SIMPLE FORM, FROM THE BAT'S STOMACH. (Osmic acid preparation.)

$c$, columnar epithelium of the surface; $n$, lleck of the gland with central and parietal cells ; $f$, base or fundus, occupied only by principal or central cells, which exhibit the granules accumulated towards the lumen of the gland.

Fig. 173,-A cakdiac gland from the dog's stomach. (Highly magnified.) $d$, duct or mouth of the gland; $b$, base or fundus of one of its tubules. On the right the base of a tubule more highly magnified; $c$, central cell; $p$, parietal cell. 
The mucous membrane is a soft, thick layer, generally somewhat corrugated in the empty condition of the organ. Its thickness is mainly due to the fact that it is made up of long tubular glands, which open upon the inner surface. Between the glands the mucous membrane is formed of areolar with much lymphoid tissue. Externally it is bounded by the muscularis mucosa, which consists of an external longitudinal and an inner circular layer of plain muscular fibres. The glands are formed of a basement-membrane lined wilh epithelium. Each gland consists of three or four secreting tubules, which oper towards the surface into a larger common tube, the duct of the gland. The duct is in all cases lined by columnar epithelium of the same character as that which covers the inner surface of the mucous membrane, but the epithelium of the secreting tubules is somewhat different from this, and, moreover, differs somewhat in the glands of the cardiac and pyloric regions of the organ.

In the cardiac glands (fig. 173) the secreting tubules are long, and the duct short. The epithelium of the tubules is composed of two kinds of cells. Those of the one kind, which form a continuous lining to the tubule, are somewhat polyhedral in shape, and in stained sections look clearer and smaller than the others, but in the fresh glands, and in osmic preparations, they appear filled with granules (fig. 174). ${ }^{1}$ These cells are believed to secrete the pepsin of the gastric juice, and are termed the chief cells of the cardiac glands, or, from their relative position in the tubule immediately surrounding the lumen, the central cells. Scattered along the tubule, and lying between the chief cells and the basement membrane, are a number of other spheroidal or ovoidal cells, which become stained by logwood and other reagents more darkly than the central cells. These are the superadded or parietal cells (oxyntic ${ }^{2}$ cells of Langley).

In the pyloric glands (fig. 175) the ducts are much longer than in the cardiac glands, and the secreting tubules possess cells of only one kind. These correspond to the chief cells of the cardiac glands. They are of a columnar or cubical shape, and in the fresh condition of a granular appearance, and quite unlike the columnar epithelium-cells of the surface, which are long tapering cells, the outer part of which is filled with mucus. At the pylorus itself the pyloric glands become considerably lengthened, and are continued into the submucous tissue, the muscularis mucosæ being here absent ; they thus present transitions to the glands of Brunner, which lie in the submucous tissue of the duodenum, and send their ducts through the mucous membrane to the inner surface.

The blood-vessels of the stomach (fig. 176) are very numerous, and

1 The granules are most numerous at the inner part of the cell, a small outer zone being left clear. After prolonged activity this outer zone increases in size while the granules diminish in number as in the analogous cases of the pancreas and parotid glands.

${ }^{2}$ So called because they produce the acid of the gastric secretion. 
pass to the organ along its curvatures. The arteries pass through the muscular coat, giving off branches to the capillary networks of the muscular tissue, and ramify in the areolar coat. From this, small arteries pierce the muscularis mucosæ, and break up into capillaries near the bases of the glands. The capillary network extends between the glands to the surface, close to which it terminates in a plexus of

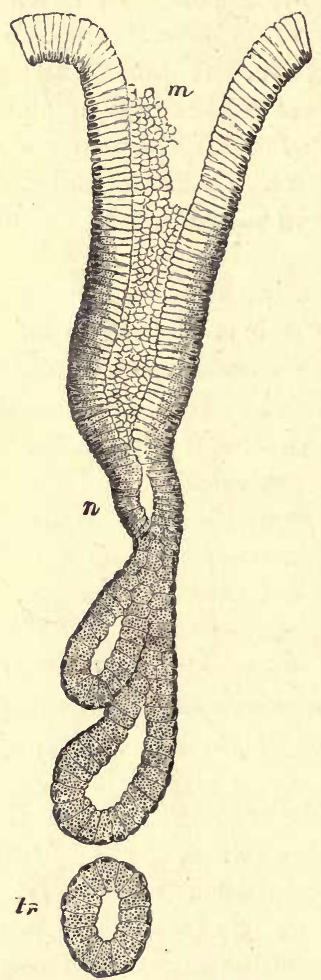

Fig. 175.-A PYLoric gLAND, FROM A SECTION OF THE DOG'S STOMACH.

$m$, mouth; $n$, neck; $t r$, a deep portion of a tubule cut transversely.

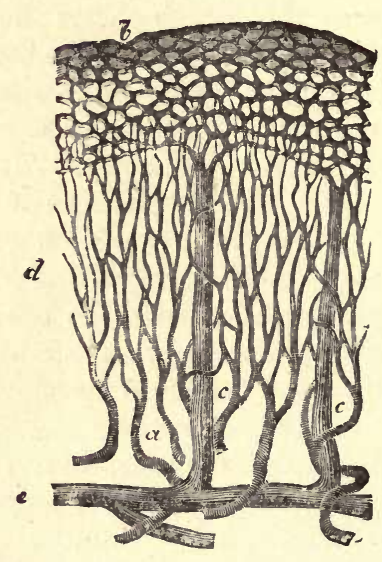

Fig. 176.-PLAN OF THE BLOODVESSELS OF THE STOMACH.

$a$, small arteries passing to break up into the fine capillary network, $d$, between the glands; $b$, coarser capillary network around the mouths of the glands; $c, c$, veins passing vertically downwards from the superficial network; $e$, larger vessels in the submucosa.

relatively large venous capillaries which encircle the mouths of the glands. From this plexus straight venous radicles pass through the mucous membrane, pierce the muscularis mucosæ, and join a plexus of veins in the submucous tissue. From these veins blood is carried away from the stomach by efferent veins, which accompany the entering arteries.

The lymphatics (fig. 177) arise in the mucous membrane by a 
plexus of large vessels dilated at intervals, and looking in sections like clefts in the interglandular tissue. From this plexus the lymph is carried into larger valved vessels in the submucous coat, and from these, efferent vessels pass through the muscular coat to reach the

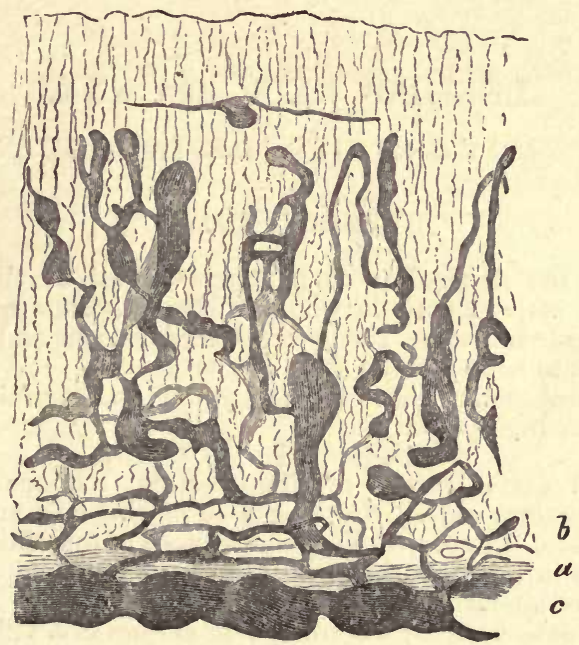

Fig. 177.-Lymphatics of the human Gastric mucous membrane, injected.

The tubules are only faintly indicated; $a$, muscularis mucosæ; $b$, plexus of fine vessels at base of glands ; $c$, plexus of larger valved lymphatics in submucosa.

serous membrane, underneath which they pass away from the organ. The muscular coat has its own network of lymphatic vessels. These lie between the two principal layers, and their lymph is poured into the efferent lymphatics of the organ.

The nerves have the same arrangement and mode of distribution as those of the small intestine (see next Lesson). 


\section{LESSONS XXIX. AND XXX.}

\section{STRUCTURE OF THE INTESTINE.}

\section{LESSON XXIX.}

1. Sections of the duodenum or jejunum vertical to the surface. The tissue is to be stained with logwood or borax-carmine and the sections mounted in Canada balsam. The general arrangement and structure of the intestinal wall is to be studied in these sections.

Make a general sketch under the low power and carefully sketch part of a villus under the high power.

2. From the same portion of the intestine, sections parallel to the surface, and therefore across the long axis of the villi and glands of the mucous membrane. In order to keep the sections of the villi together so that they are not lost in the mounting, it will be necessary to employ the creosote. shellac method of mounting the sections (see Appendix).

In this preparation sketch the transverse section of a villus.

3. Transverse vertical sections of the ileum passing through a Peyer's patch. Observe the nodules of lymphoid tissue which constitute the patch and which extend into the submucous tissue. Notice also the sinus-like lymphatic or lacteal vessel which encircles the base of each nodule. Make a general sketch under a low power.

4. To study the process of fat-absorption, kill a rat three or four hours after feeding it with fat meat. Put a very small piece of the mucous membrane of the intestine into osmic acid $(0.5$ per cent.) and another piece into chromic acid $(0 \cdot 2$ per cent.) containing a few drops of osmic acid solution. After forty-eight hours teased preparations may be made from the osmic acid preparation, in the same manner as directed in Lesson VII. $\$ 2$; the rest may be then placed in alcohol. The prece in chromic and osmic acid may also after two or three days be placed in alcohol. When hardened and dehydrated in this, the pieces of tissue are embedded in paraffin, and sections are made and mounted by the shellac-creosote process.

\section{LESSON XXX.}

1. Sections of small intestine the blood-vessels of which have been injected. Notice the arrangement of the vessels in the several layers. Sketch carefully the vascular network of a villus.

2. From a piece of intestine which has been stained with chloride of gold tear off broad strips of the longitudinal muscular coat, and mount them in Farrant's solution. It will generally be found that portions of the nervous plexus of Auerbach remain adherent to the strips, and it can in this way easily be studied.

From the remainder of the piece of intestine tear off with forceps the 
fibres of the circular muscular layer on the one side, and the mucous mem. brane on the other side so as to leave only the submucous tissue and the muscularis mucosæ. This tissue is also to be mounted flat in Farrant's solution: it contains the plexus of Meissner.

Sketch a small portion of each plexus under a high power.

3. Sections of the large intestine, perpendicular to the surface. These will show the general structure and arrangement of the coats. Sketch under a low power.

4. Sections of the mucous membrane of the large intestine parallel to the surface, and therefore across the glands. Sketch some of the glands and the interglandular tissue under a high power.

5. The arrangement of the blood-vessels of the large intestine may be studied in sections of the injected organ.

\section{THE SMALL INTESTINE.}

The wall of the small intestine consists, like the stomach, of four coats.

The serous coat is complete except over part of the duodenum.

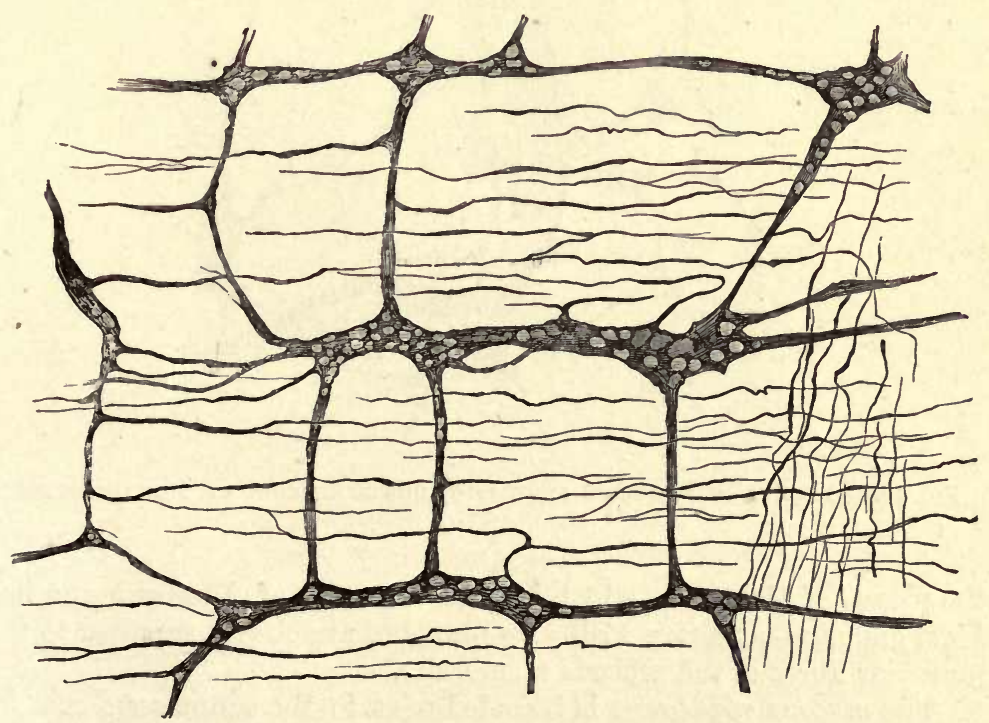

Fig. 178.-Plexus of Auerbach, Between the two layers of the muscular COAT OF THE INTESTINE. (Cadiat.)

The muscular coat is composed of two layers of muscular tissue, an outer longitudinal and an inner circular. Between them lies a network of lymphatic vessels and also the close gangliated plexus of non- 
medullated nerve-fibres known as the plexus myentericus of Auerbach. The ganglia of this plexus may usually be seen in vertical sections of the intestinal wall, but the plexus, like the one in the submucous coat immediately to be described, can only be properly displayed in preparations made with chloride of gold (fig. 178).

The submucous coat is like that of the stomach; in it the bloodvessels and lymphatics ramify before entering or after leaving the mucous membrane, and it contains a gangliated plexus of nerve-fibres-

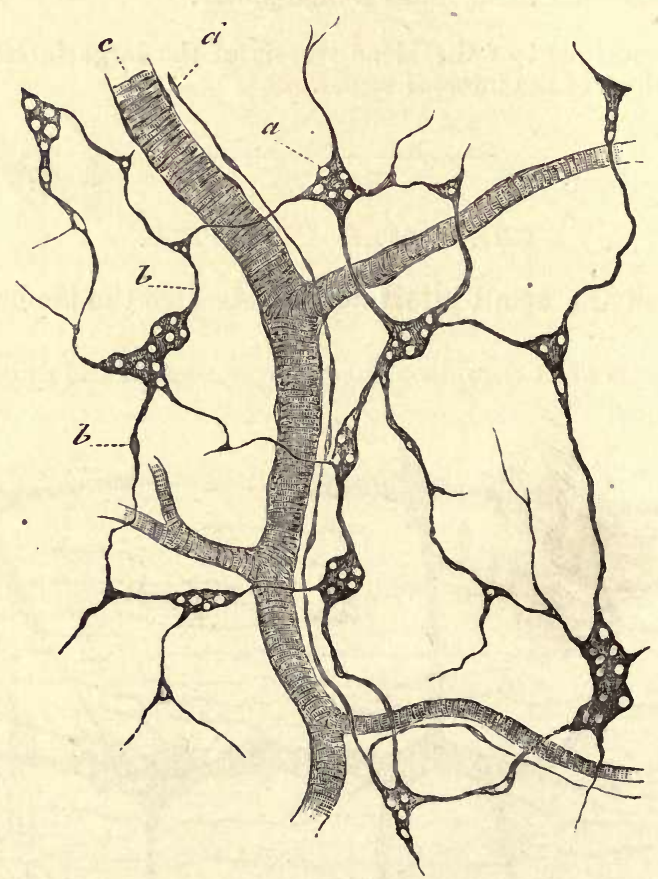

Fig. 179.-Plexus of Meissner from the submucous coat of the intestine. (Cadiat.)

the plexus of Meissner-which is finer than that of Auerbach and has fewer ganglion-cells (fig. 179). Its branches are chiefly supplied to the muscular fibres of the mucous membrane.

The mucous membrane is bounded next to the submucous coat by a double layer of plain muscular fibres (muscularis mucosa). Bundles from this pass inwards through the membrane towards its inner surface and penetrate also into the villi. The mucous membrane proper is pervaded with simple tubular glands - the crypts of Lieberkühnwhich are lined throughout by a columnar epithelium like that which covers the surface and the villi. The mucous membrane between these 
glands is mainly composed of lymphoid tissue, which is aggregated at intervals into more solid nodules (fig. 181) constituting when they occur singly the so-called solitary glands of the intestine, and when aggregated

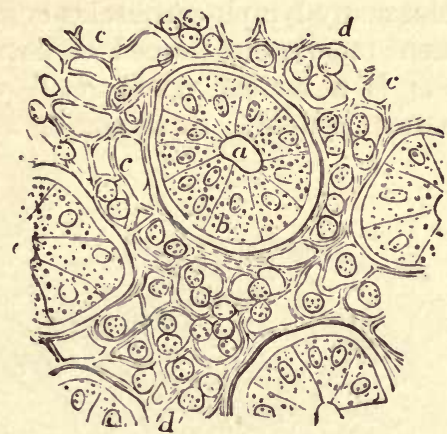

Fig. 180.-Cross-section of A SMall Fragment of the Mucous Membrane OF THE INTESTINE, INCLUDING ONE ENTIRE CRYPT OF LIEBERKÜHN AND Parts of three others. (Magnified 400 diameters.)

$a$, cavity of the tubular glands or crypts ; $b$, one of the lining epithelial cells ; $c$, the interglandular tissue; $d$, lymph-cells.

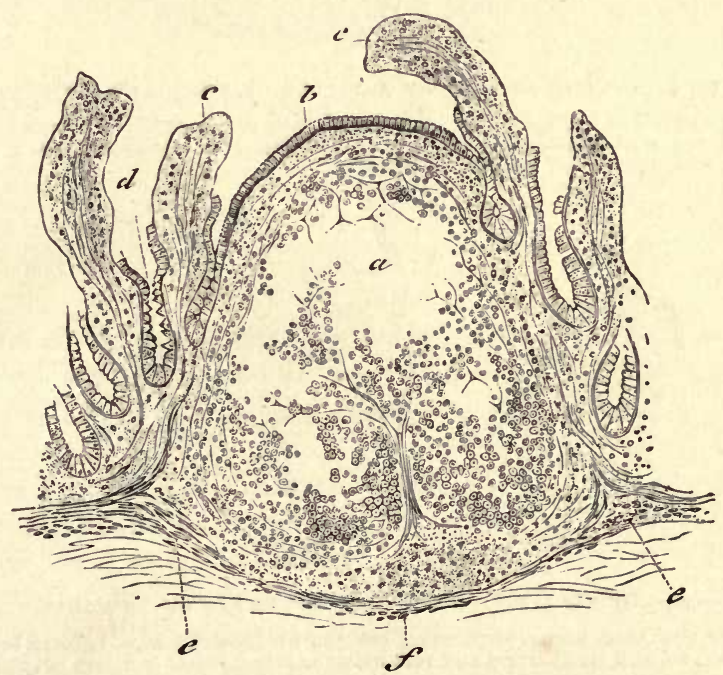

Fig. 181.-Section of the ileum throvgh a lymphoid nodule. (Cadiat.)

$a$, middle of the nodule with the lymphoid tissue partly fallen away from the section; $b$, epithelium of the intestine; $c$, villi : their epithelium is partly broken away ; $d$, crypts of Lieberkühn.

together form the agminated glands or patches of Peyer. The latter occur chiefly in the ileum.

The villi with which the whole of the inner surface of the small 
intestine is closely beset are clavate or finger-shaped projections of the mucous membrane, and are composed, like that, of lymphoid tissue and covered with columnar epithelium (fig. 182). The characters of this have been already described (Lesson VII.). Between and at the base of the epithelium-cells many lymph-corpuscles occur. The epithelium rests upon a basement-membrane formed of flattened cells. In the middle of the villus is a lacteal vessel $(c . l)$ which is somewhat enlarged near its commencement. Surrounding this vessel are small

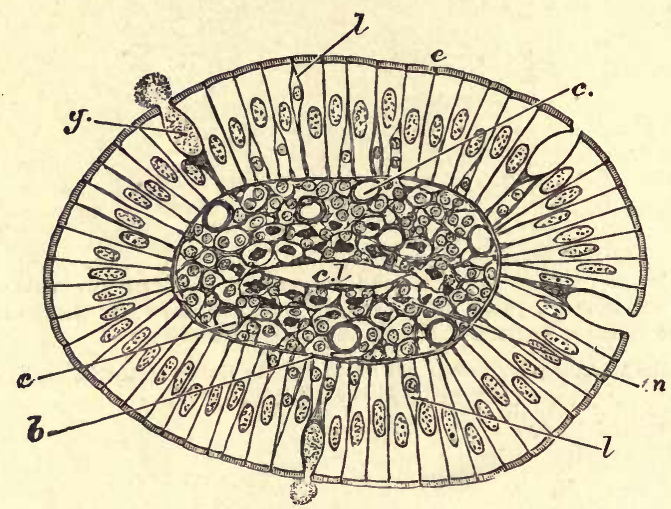

Fig. 182.-Cross-section of a villus of the cat's intestine.

$e$, columnar epithelium; $g$, goblet cell, its mucus is seen partly exuded; $l$, lymph-corpuscles between the epithelium-cells; $b$, basement-membrane; $c$, blood-capillaries; $m$, section of plain muscular fibres; $c$. $l$, central lacteal.

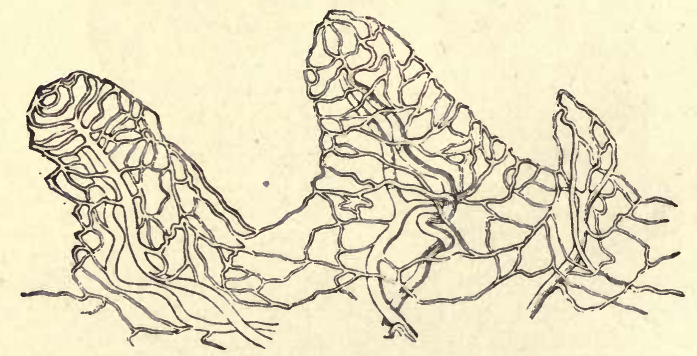

Fig. 183.-MAgNified view of THE BLOOD-vessels of THE INTESTINAL villi.

The drawing was taken from a preparation injected by Lieberkiuhn, and shows, belonging to each villus, a small artery and vein with the intermediate capillary network.

bundles of plain muscular tissue prolonged from the muscularis mucosæ. The network of blood-capillaries (fig. 183) lies for the most part near the surface within the basement-membrane; it is supplied with blood by a small artery which joins the capillary network at the base of the villus; the corresponding vein generally arises nearer the extremity.

The lymphatics (lacteals) of the mucous membrane (fig. 184), after 
receiving the central lacteals of the villi, pour their contents into a plexus of large valved lymphatics which lie in the submucous tissue and form sinuses around the bases of the lymphoid nodules. From the submucous tissue efferent vessels pass through the muscular coat, receiving the lymph from an intramuscular plexus of lymphatics, and are conveyed away between the layers of the mesentery.

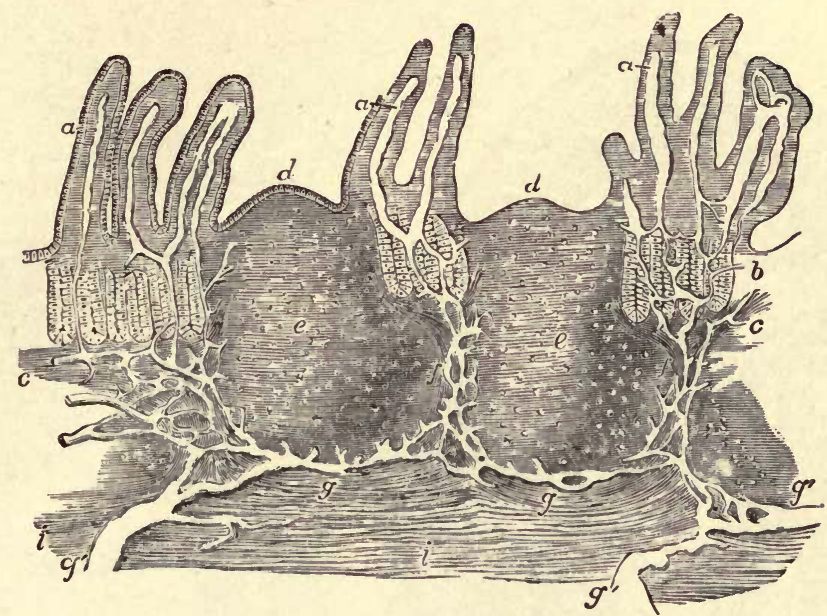

Fig. 184.-Vertical section of a portion of a patci of Peyer's glands, with THE LACTEAL VESSELS INJECTED. (32 diameters.) (Frey.)

The specimen is from the lower part of the ileum: $a$, villi, with their lacteals left white; $b$, some of the tubular glands : $c$, the muscular layer of the mucous membrane; $d$, cupola or projecting part of the nodule; $e$, central part; $f$, the reticulated lacteal vessels occupying the lymphoid tissue between the nodules, joined above by the lacteals from the villi and mucous surface, and passing below into $g$, the sinus-like lacteals under the nodules, which again pass into the large efferent lacteals, $g^{\prime} ; i$, part of the muscular coat.

Absorption of fat.-The lymph-corpuscles of the villi are the chief agents in effecting the passage of fat-particles into the lacteals. In order to study this process of transference, it is convenient to stain the fat-particles with osmic acid, which colours them black. It can then be observed that in animals which have been fed with fat these particles are present $(1)$ in the columnar epithelium-cells ; $(2)$ in the lymph-cells; and (3) in the central lacteal of the villus. The lymphcells are present not only in the reticular tissue of the villus, but also in considerable number between the epithelium-cells; and they can also be seen in thin sections from osmic preparations within the commencing lacteal; but in the last situation they are in every stage of disintegration.

Since the lymph-cells are amœboid, it is probable from these facts that the mechanism of fat-absorption consists of the following processes-viz. (1) absorption of fat into the columnar epithelium-cells of the surface; (2) inception of fat by the lymph-corpuscles in the epithelium, partly from the epithelium-cells, and partly, perhaps, directly 
from the intestinal contents; (3) migration of the lymph-corpuscles carrying the incepted fat-particles by their amœboid movements through the tissue of the villus and into the central lacteal ; (4) disintegration and solution of the immigrated lymph-corpuscles, and setting free both of their fatty contents and also of the proteid matters of which they are themselves composed.

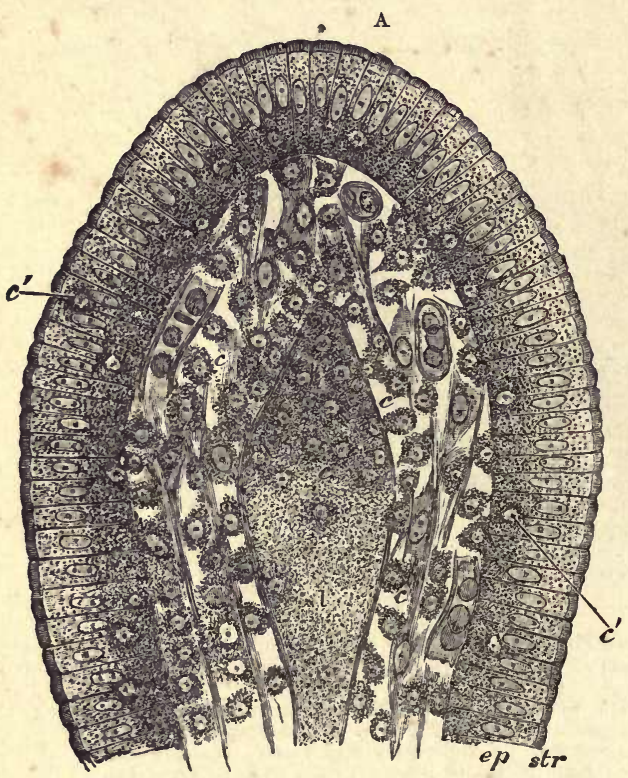

B

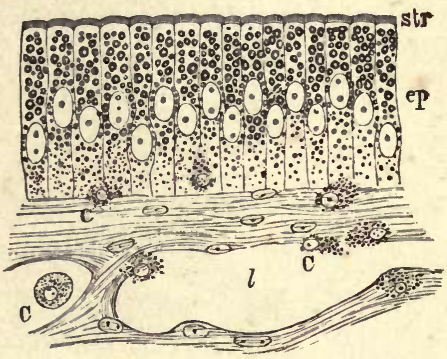

Fig. 185, A.-Section of the villus of a rat killed dURing fat-ABsorption.

$e p$, epithelium ; str, striated border; $c$, lymph-cells; $c^{\prime}$, lymph-cells in the epithelium; $l$, central lacteal containing disintegrating lymph-corpuscles.

Fig. 185, B.-Mucous Membrane of Frog's intestine during FAT-ABSorption. $e p$, epithelium ; str, striated border; $c$, lymph-corpuscles ; $l$, lacteal.

This migration of the lymph-corpuscles into the lacteals of the villi is not a special feature of fat-absorption alone, but occurs even when absorption of other matters is proceeding; so that the transference of fat-particles is merely a part of a more general phenomenon accompanying absorption.

\section{THE LARGE INTESTINE.}

The large intestine has the usual four coats, except near its termination, where the serous coat is absent. The muscular coat is peculiar in the fact that along the cæcum and colon the longitudinal muscular fibres are gathered up into three thickened bands which produce puckerings in the wall of the gut. 
The mucous membrane of the large-intestine is beset with simple tubular glands somewhat resembling the crypts of Lieberkühn of the small intestine, and lined by columnar epithelium similar to that of the inner surface of the gut, but containing many more mucussecreting or goblet cells (fig. 186). The extremity of each gland is usually slightly dilated. The interglandular tissue is like that of the
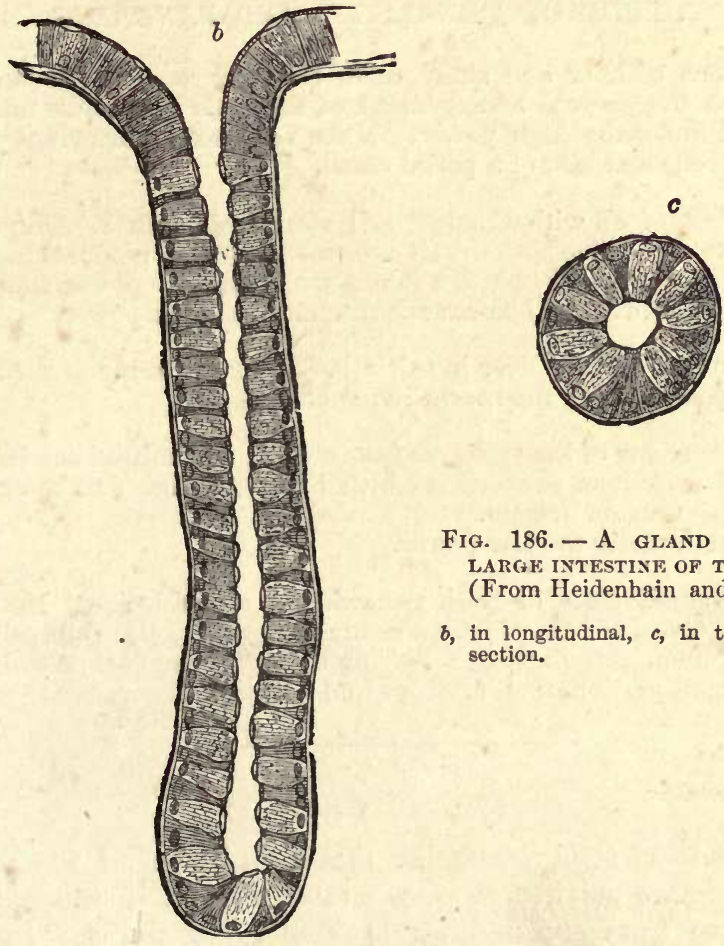

Fig. 186. - A GLAND OF THE LARGE INTESTINE OF TIIE DOG. (From Heidenhain and Klose.)

$b$, in longitudinal, $c$, in transverse section.

stomach, as is also the arrangement of the blood-vessels and lymphatics in it. The nerves of the large intestine also resemble those of the small intestine and stomach in their arrangement.

At the lower end of the rectum the circular muscular fibres of the gut become thickened a little above the anus so as to form the internal sphincter muscle. In this region also there are a number of compound racemose mucous glands opening on to the surface of the mucous membrane. 


\section{LESSON XXXI.}

\section{STRUCTURE OF THE LIVER AND PANCREAS.}

1. MAKE sections of liver and study them carefully with a low and high power. Sketch the general arrangement of the cells in a lobule under the low power and under the high power. Make very careful drawings of some of the hepatic cells and also of a portal canal.

2. Study, first of all with the low and afterwards with a high power, a section of the liver in which both the blood-vessels and the bile-ducts have been injected. Make a general sketch of a lobule under the low power and draw a small part of the network of bile-canaliculi under the high power.

3. Tease a piece of fresh liver in salt solution for the study of the appearance of the hepatic cells in the recent living condition.

4. Prepare sections of the pancreas from a gland which has been hardened in alcohol. The sections are stained with borax-carmine and mounted in the usual way in Canada balsam.

Make a sketch under the low power.

5. Tease a small piece of fresh pancreas in salt solution. Notice the granules in the alveolar cells, chiefly accumulated in the half of the cell which is nearest the lumen of the alveolus, leaving the outer zone of the cell clear.

Sketch a small portion of an alveolus under a high power.

\section{THE LIVER.}

The liver is a solid glandular mass, made up of the hepatic lobules. These are polyhedral masses (about $1 \mathrm{~mm}$. in diameter) of cells, separated from one another by connective tissue. In some animals, as in the pig, this separation is complete, and each lobule is isolated, but in man it is incomplete. There is also a layer of connective tissue underneath the serous covering of the liver, and forming the so-called capsule of the organ.

The blood-vessels of the liver (portal vein and hepatic artery) enter it on its under surface, where also the bile-duct passes away from the gland. The branches of these three vessels accompany one another in their course through the organ, and are enclosed by loose connective tissue (capsule of Glisson), in which are lymphatic vessels, the whole being termed a portal canal (fig. 187). The smallest branches of the vessels penetrate to the intervals between the hepatic lobules, and are known as the interlobular branches. The blood leaves the liver at the back of the organ by the hepatic veins: the branches of these run through the gland unaccompanied by other vessels (except 
lymphatics) and can also be traced to the lobules, from each of which they receive a minute branch (intralobular vein) which passes from the centre of the lobule, and opens directly into the (sublobular) branch of the hepatic vein.

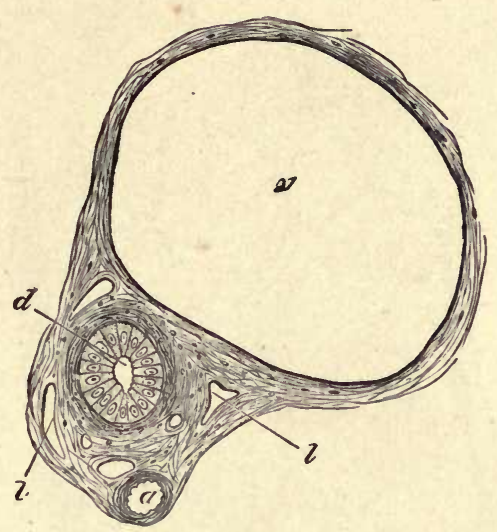

Fig. 187.-Section of a portal canal.

$a$, branch of hepatic artery ; $v$, branch of portal vein; $d$, bile-duct; $l, l$, lymphatics in the areolar tissue of Glisson's capsule which encloses the vessels.

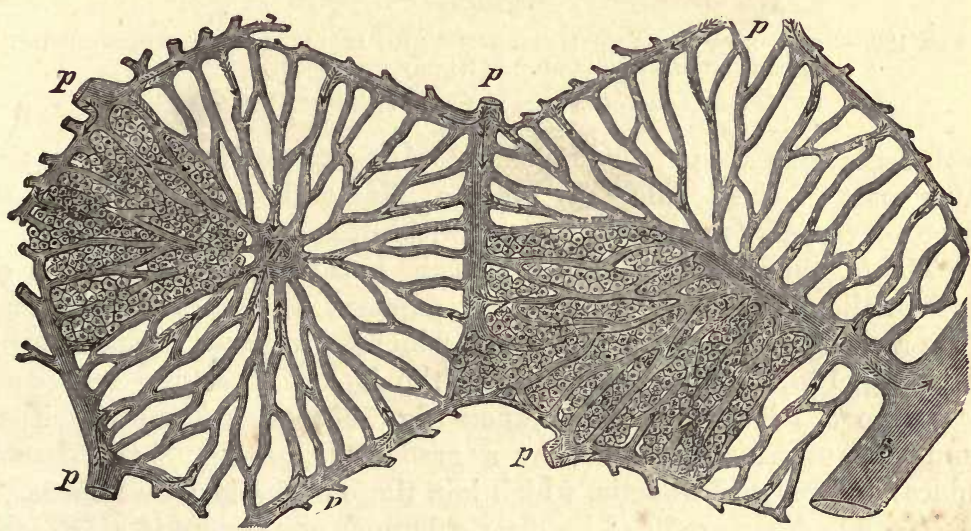

Fig, 188.-Diagramatic representation of two hepatic lobules.

The left-hand lobule is represented with the intralobular vein cut across ; in the right-hand one the section takes the course of the intralobular vein. $p$, interlobular branches of the portal vein; $h$ intralobular branches of the hepatic veins; $s$, sublobular vein; $c$. capillaries of the lobules. The arrows indicate the direction of the course of the blood. The liver-cells are only represented in one part of each lobule.

Each lobule is a mass of hepatic cells pierced everywhere with a network of blood-capillaries (fig. 188), which arise at the periphery of the lobule, there receiving blood from the interlobular branches of the portal vein $(p)$, and converge to the centre of the lobule, where they unite to form the intralobular branch of the hepatic vein. The inter- 
lobular branches of the hepatic arteries join this capillary network a short distance from the periphery of the lobule.

The hepatic cells (fig. 189), which everywhere lie between and surround the capillaries, are polyhedral, somewhat granular-looking cells,

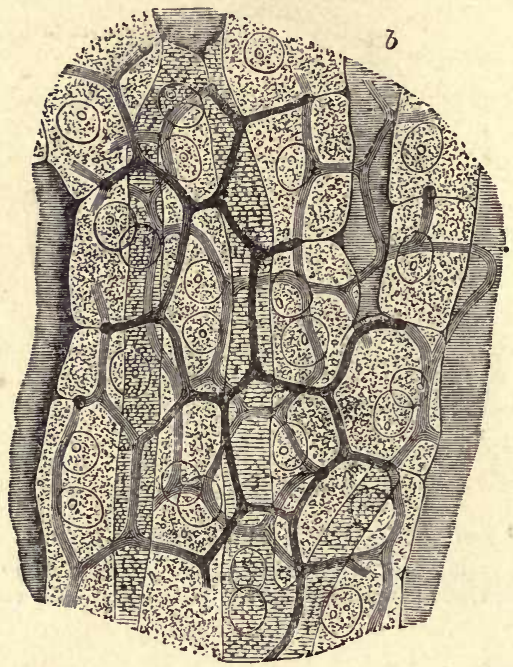

Fig. 189.-SECTION OF RABBIT'S LIVER WITH THE INTERCELLULAR NETWORK OF BILE-CANALICULI INJECTED. (Highly magnified.) (Hering.)

Two or three layers of cells are represented ; $b, b$, blood-capillaries.

each containing a spherical nucleus. After a meal, the cells in the outer part of the lobule may become filled with fat, and masses of glycogen can also frequently be seen within the cells.

The bile-ducts commence between the hepatic cells in the form of fine canaliculi, which lie between the adjacent sides of two cells, and form a close network, the meshes of which correspond in size to the cells (fig. 189). At the periphery of the lobule these fine canaliculi pass into the interlobular bile-ducts (fig. 190), the columnar epithelium-cells of which become, by a gradual transition, changed into cubical and polyhedral cells, which join those of the hepatic lobules.

The bile-ducts are lined by clear columnar epithelium (fig. 187, d). Outside this is a basement-membrane, and in the larger ducts some fibrous and plain muscular tissue. Many of the larger ducts are beset with sinall cæcal diverticula.

The gall-bladder is in its general structure similar to the larger bileducts. It is lined by columnar epithelium, and its wall is formed of fibrous and muscular tissue.

The lymphatics of the liver are said to commence as perivascular lymphatic spaces enclosing the capillaries of the lobules. Efferent lymphatics pass away from the organ in the connective tissue which invests the portal and hepatic veins. 


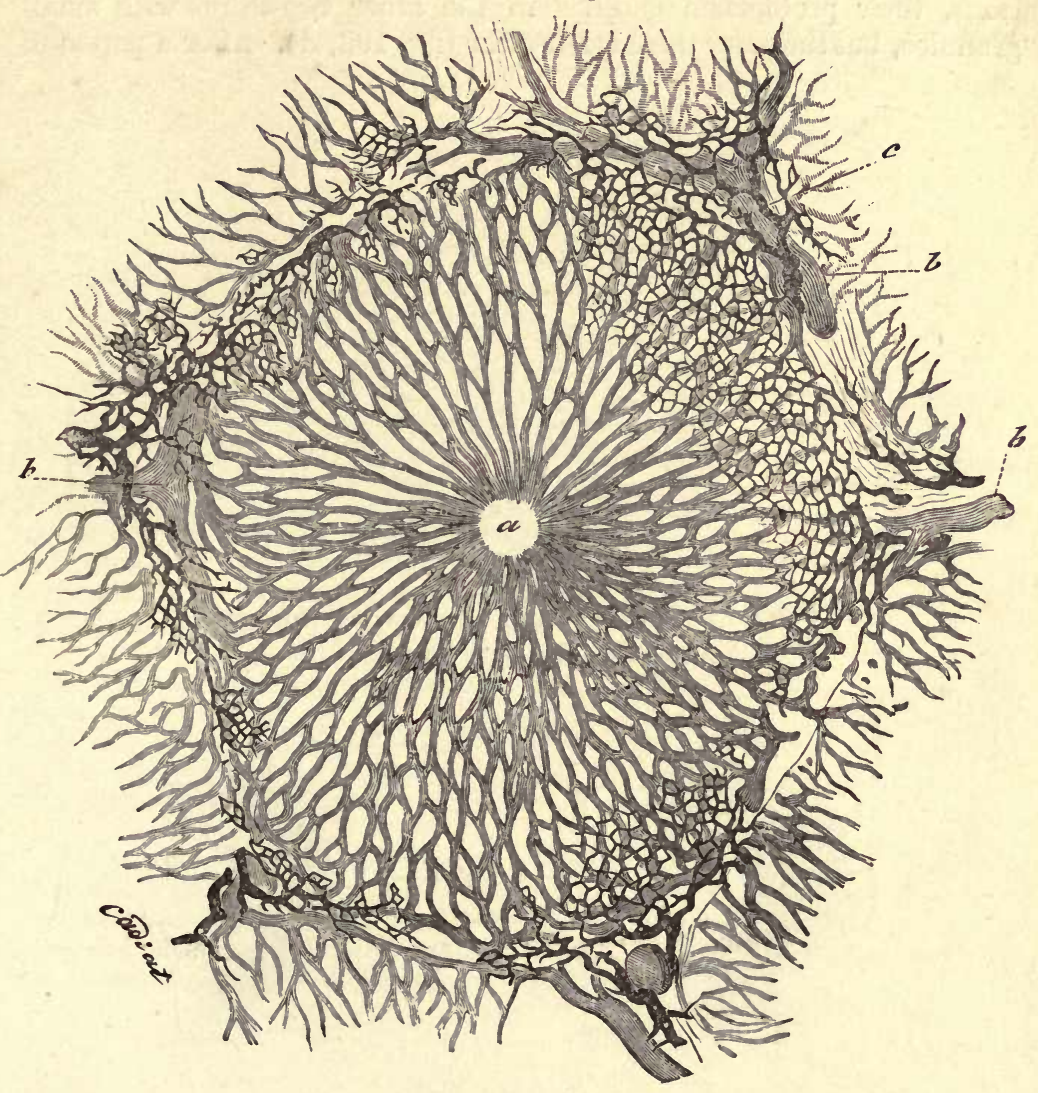

Fig. 190.-Lobule of RABbit's Liver, vessels ANd bILE-DUCtS INJECted. (Cadiat.)

$a$, central vein ; $b, b$, peripheral or interlobular veins; $c$, interlobular bile-duct.

\section{THE PANCREAS.}

The pancreas is a tubulo-racemose gland, resembling the salivary glands, so far as its general structure is concerned, but differing from them in the fact that the alveoli, in place of being saccular, are longer and more tubular in character (fig. 191). Moreover, the connective tissue of the gland is somewhat looser, and there occur in it at intervals small groups of epithelium-like cells, which are supplied with a close network of convoluted capillary vessels; their function is unknown, but their presence is very characteristic of the pancreas.

The cells which line the alveoli are columnar or polyhedral in shape. When examined in the fresh condition, or in osmic prepara- 
tions, their protoplasm is filled in the inner two-thirds with small granules, but the outer third is left clear (fig. 192, $A$ ). After a period of

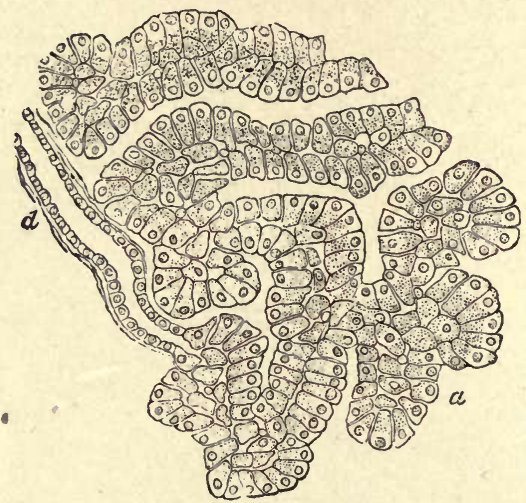

Fig. 191.-Section of the PANCreas of THE Dog.

$d$, termination of a duct in the tubular alveoli, alv.

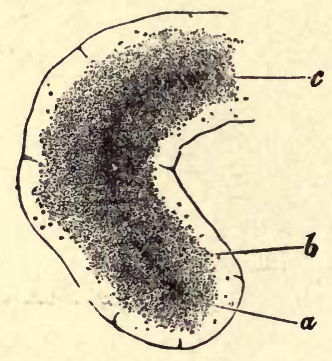

4 .

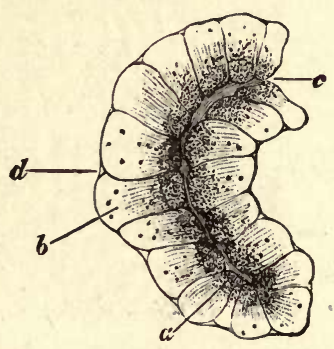

$B$.

Fig. 192.-Part of an alveolus of the rabbit's pancreas, $A$, at rest; $B$, AFter ACtive Secretion. (Foster, after Kühne and Lea.)

$a$, the inner granular zone, which in $A$ is larger and more closely studded with fine granules than in $B$, in which the granules are fewer and coarser; $b$, the outer transparent zone, small in $A$, larger in $B$, and in the latter marked with faint striæ; $c$, the lumen, very obvious in $B$, but indistinct in $A ; d$, an indentation at the junction of two cells, only seen in $B$.

activity the clear part of the cell becomes larger, and the granular part smaller $(B)$. In stained sections the outer part is coloured more deeply than the inner.

In the centre of each acinus there may generally be seen some spindle-shaped cells, the nature of which (whether epithelial or connective tissue) has not been determined (centro-acinar cells of Langerhans). 


\section{LESSON XXXII.}

\section{STRUCTURE OF THE SPLEEN, SUPRARENAL CAPSULE, AND} THYROID BODY.

1. Sections of the spleen stained with logwood. Notice the trabeculæ extending into the substance of the organ from the capsule. Notice also that the glandular substance is of two kinds, (1) lymphoid tissue accumulated round the small arteries and here and there massed to form lymphoid nodules- the Malpighian corpuscles of the spleen-and, (2) a tissue consisting of a reticulum of branched and flattened cells containing blood in its interstices and pervaded by capillaries and venous radicles.

Sketch part of a section under a low power and a small portion of the reticulum under a high power.

2. Sections across a suprarenal capsule. Examine first with a low power, noticing the general arrangement and extent of the cortical and medullary parts of the organ, making a general sketch which shall include both. Afterwards sketch carefully under the high power a group of cells from each part of the organ.

3. Sections of the thyroid body stained with logwood. Notice the vesicles lined with cubical epithelium and filled with a 'colloid' substance which becomes stained by the logwood. Notice also in some parts of the sections a peculiar highly vascular retiform tissue. Sketch a part of this tissue and also one or two vesicles. Measure several vesicles.

\section{THE SPLEEN.}

The spleen is the largest of the so-called ductless glands. It appears to be connected in some way with the elaboration of the blood, white blood-corpuscles being certainly formed and the coloured bloodcorpuscles being probably submitted to destruction within it.

Like the lymphatic glands, the spleen is invested with a fibrous and muscular capsule (fig. 193, A), and this again has a covering derived from the serous membrane. The capsule sends fibrous bands or trabeculæ $(b)$ into the organ, and these join with a network of similar trabeculæ which pass into the gland at the hilus along with the bloodvessels. In the interstices of the fibrous framework thus constituted lies a soft pulpy substance containing a large amount of blood, and therefore of a deep red colour, dotted within which are here and there to be seen small whitish specks, the Malpighian corpuscles of the spleen $(c, c)$. These are composed of lymphoid tissue which is gathered up into masses which surround the smaller arteries, whilst the red pulp which everywhere surrounds them and which forms the bulk of the 
organ is composed of a cl.jse network or spongework of flattenea and branched cells like connective-tissue corpuscles. Coursing through the pulp and communicating with its interstices are capillary blood-vessels

A.

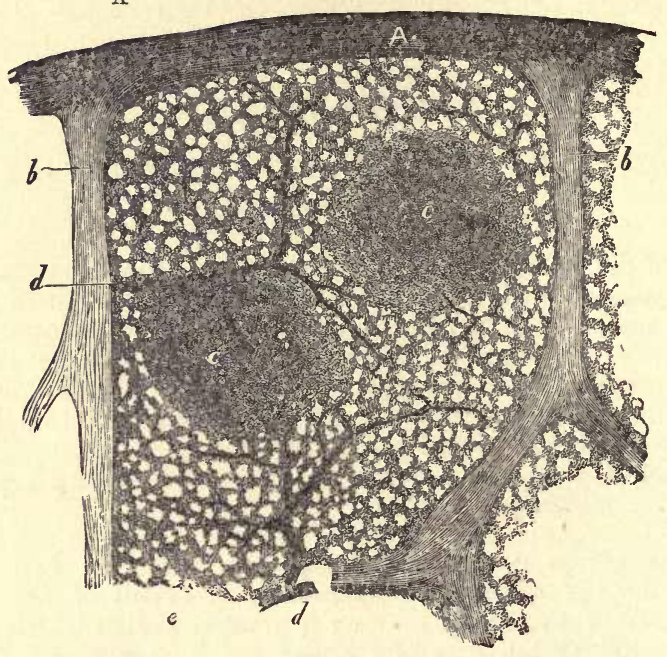

Fig. 193.-Vertical section of a small superficial portion of the human SPLEEN, AS SEEN WITH A LOW POWER.

A, peritoneal and fibrous covering ; $b$, trabeculæ; $c, c$, Malpighian corpuscles, in one of which an artery is'seen cut transversely, in the other longitudinally; $d$, injected arterial twigs; $e$, spleen-pulp.

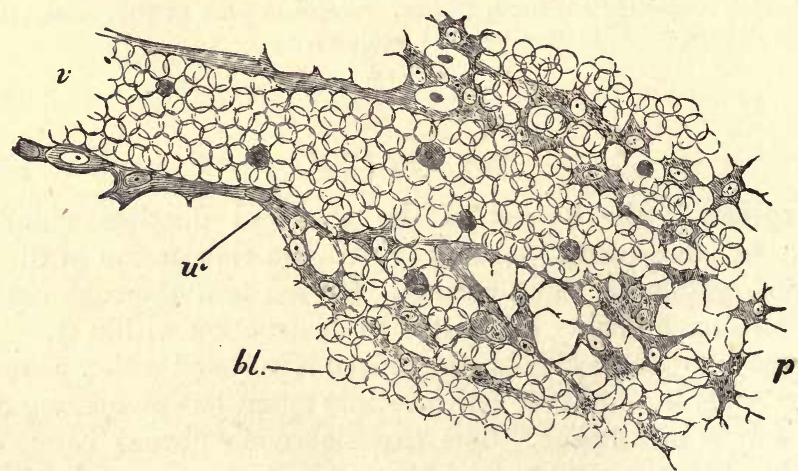

Fig. 194.-Thin section of SPLeen-PUlp, Highly MAGNified, showing the MODE OF ORIGIN OF A SMALL VEIN IN THE INTERSTICES OF THE PULP.

$v$, the vein, filled with blood-corpuscles, which are in continuity with others, $b l$, filling up the interstices of the retiform tissue of the pulp; $w$, wall of the vein. The shaded bodies amongst the red blood-corpuscles are pale corpuscles.

which are connected with the terminations of the arteries; whilst in other parts venous channels arise from the pulp, and bring the blood which has passed into its interstices from the arterial capillaries 
towards the larger veins of the organ, which run in the trabeculæ, and are by them conducted to the hilus. The arteries, which are also at first conducted from the hilus along the trabeculæ into the interior of the organ, presently leave the trabeculæ, and their external coat becomes converted into a thick sheath of lymphoid tissue which invests them in the remainder of their course, and in places becomes swollen into the Malpighian corpuscles already mentioned. These small arteries distribute a few capillaries to the Malpighian corpuscles, and then break up into pencils of small vessels which open into the pulp in the manner already mentioned.

The cellular elements of the spleen-pulp are of three kinds, viz. large, amœboid, connective-tissue cells, also called splenic cells, lymphcorpuscles, and the branched, flattened cells which form the spongework. The first-named are frequently found to contain coloured blood-corpuscles in their interior in various stages of transformation into pigment.

The lymphatics of the spleen run partly in the trabeculæ and capsule, and partly in the lymphoid tissue ensheathing the arteries. They join to form larger vessels which emerge together at the hilus.

\section{THE SUPRARENAL CAPSULES.}

The suprarenal capsules belong to the class of bodies known as ductless glands, but they are entirely different in structure from the spleen and lymphatic glands. A section through the fresh organ (fig. 195)

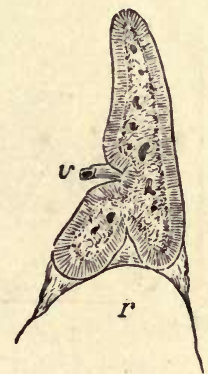

Fig. 195.-A vertical section of the suprareNal body OF A FETUS, Twice THE NATURAL SIZE, SHOWING THE DISTINCTION BETWEEN THE MEDULLARY AND CORTICAL SUBSTANCE.

$v$, issuing vein ; $r$, summit of kidney.

shows a cortical zone which is striated vertically to the surface, and of a yellowish colour, and a medulla which is soft and highly vascular, and of a brownish-red colour. The whole organ is invested by a fibrous capsule which sends fibrous septa inwards to the cortical substance (fig. 196), subdividing this for the most part into columnar groups of cells (zona fasciculata, $c$ ). Immediately underneath the capsule, however, 
the groups are more rounded (zona glomerulosa, $b$ ), whilst next to the medulla they have a closely reticular arrangement (zona reticularis, $d$ ), and a similar disposition both of the cells and the connective tissue is noticeable throughout the medulla.

The cells which form the rounded groups and columns of the cortical substance are polyhedral in form (fig. 197); each contains a clear round nucleus, and there are often yellowish oil-globules in their protoplasm.

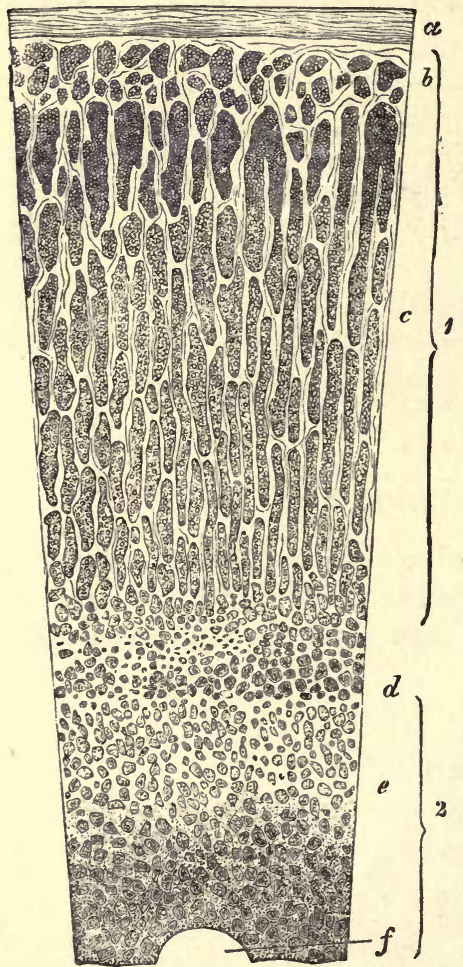

Fig. 196.-V'GrTical Section OF SUPRA RENAL BOIN. (Magnified.)

1, cortical substance; 2 , medullary substance ; $a$, capsule; $b$, zona glomerulosa ; $c$, zona fasciculata; $d$, zona reticularis ; $e$, groups of medullary cells ; $f$, section of a large vein.

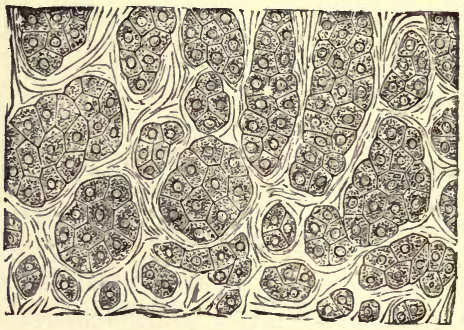

Fig. 197.-Chlls and Cell-groups FRON THE OUTERNOST LAYER OF THE CORTICAL SUBSTANCE OF THE SUPRARENAL BODY.

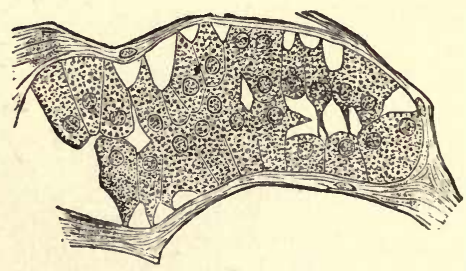

Fig. 198.-A SMALL PORTION OF THE MEDULlary PART OF THE SUPRARENAL CAPSULE OF THE OX.

No blood-vessels penetrate between these cells, both the blood-vessels and lymphatics of the cortex running in the fibrous septa between the columns; the lymphatics have been stated to communicate with fine spaces which run between the cells of the columns.

The cells of the medulla (fig. 198) are more irregular in shape, and are often branched. Their protoplasm is either clear, or it may in some animals contain a brownish pigment, but in man the dark red colour of 
the medulla is largely due to the blood contained in the large venous spaces by which it is pervaded, and which receive the blood after it has traversed the capillaries of the cortex. Investing the larger veins are bundles of plain muscular fibres ; and numerous nerves, after traversing the cortical substance, are distributed throughout the medulla, where they form a close plexus provided with ganglion-cells.

\section{THE THYROID BODY.}

The thyroid body consists of a framework of connective tissue enclosing numerous spherical or oval vesicles (fig. 199) which are lined with cubical epithelium. The cavities of the vesicles are filled with a peculiar viscid liquid which is coagulated by alcohol and which then becomes stained by hæmatoxylin. A similar material has been found

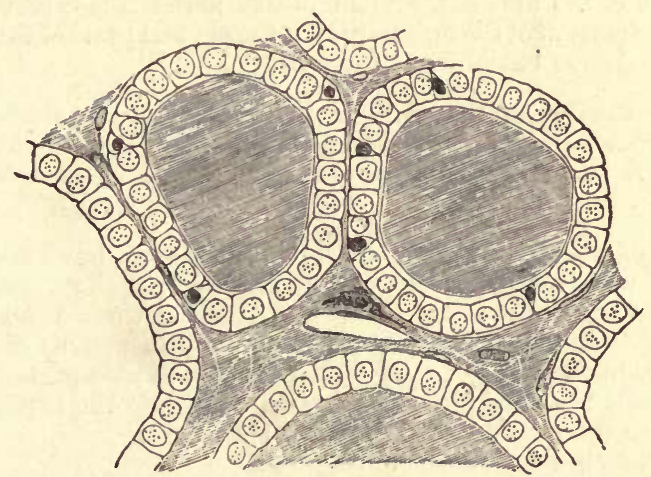

Fig. 199.-SECTION OF THE THYROID GLAND OF A CHILd.

Two complete vesicles and portions of others are represented. The vesicles are filled with colloid, which also occupies the interstitial spaces. In the middle of one of the spaces a blood-vessel is seen cut obliquely, and close to it is a plasma-cell. Between the cubical epithelium-cells, smaller cells like lymph-corpuscles are here and there seen.

in the lymphatics of the gland, and may sometimes be detected also in the ir.terstices of the connective tissue.

The blood-vessels of the thyroid are exceedingly numerous, and the capillaries form close plexuses round the vesicles. Some of the bloodvessels are distributed to a peculiar highly vascular retiform tissue which occurs in patches here and there in the organ.

Disease of the thyroid or its extirpation is accompanied by remarkable changes in the chemical composition of the blood and many of the tissues, resulting chiefly in the accumulation within them of a large amount of mucin; a condition of general myxœdema, and eventually of cretinism, being produced. 


\section{LESSON XXXIII.}

\section{STRUCTURE OF THE KIDNEY.}

1. Secrions passing through the whole kidney of a small mammal, such as a mouse or rat. These sections will show the general arrangement of the organ and the disposition of the tubules and of the Malpighian corpuscles.

A general sketch should be made of one of these sections under a low power.

2. Thin sections of the kidney of a larger mammal, such as the dog, may next be studied. In some the direction of the section should be parallel with the tubules of the modulla, and in others across the direction of those tubules. The characters of the epithelium of the several parts of the uriniferous tubules are to be made out in these sections.

3. Separate portions of the uriniferous tubules may be studied in teased preparations from a kidney which has been subjected to some process which renders it possible to unravel the uriniferous tubules for a certain distance. ${ }^{1}$

4. Sections of a kidney in which the blood-vessels have been injected. Examine these with a low power of the microscope. Try and follow the course of the arteries-those to the cortex sending their branches to the glomeruli, those to the medulla rapidly dividing into pencils of fine vessels which run between the straight uriniferous tubules of that part. Notice also the efferent vessels from the glomeruli breaking up into the capillaries which are distributed to the tubules of the cortical substance.

Make sketches showing these points.

The kidney is a compound tubular gland. To the naked eye it appears formed of two portions-a cortical and a medullary-the latter being subdivided into a number of pyramidal portions (pyramids of Malpighi), the base of each being surrounded by cortical substance, while the apex projects in the form of a papilla into the dilated commencement of the ureter (pelvis of the kidney). ${ }^{2}$ Both cortex and medulla are composed entirely of tubules-the uriniferous tubules-which have a straight direction in the medulla and a contorted arrangement in the cortex; but groups of straight tubules also pass from the medulla through the thickness of the cortex (medullary rays).

The uriniferous tubules begin in the cortical part of the organ in dilatations, each enclosing a tuft or glomerulus of convoluted capillary

1 For a method which may be employed for this purpose, see Course of Practical Histology, p. 209.

2 In many animals the whole kidney is formed of only a single pyramid, but in man there are about twelve. 
blood-vessels, the dilated commencement of the tubule being known as the capsule (fig. 200, 1). The tubule leaves the capsule by a narrow neck (2); it is at first convoluted (first convoluted tubule, 3 ), but

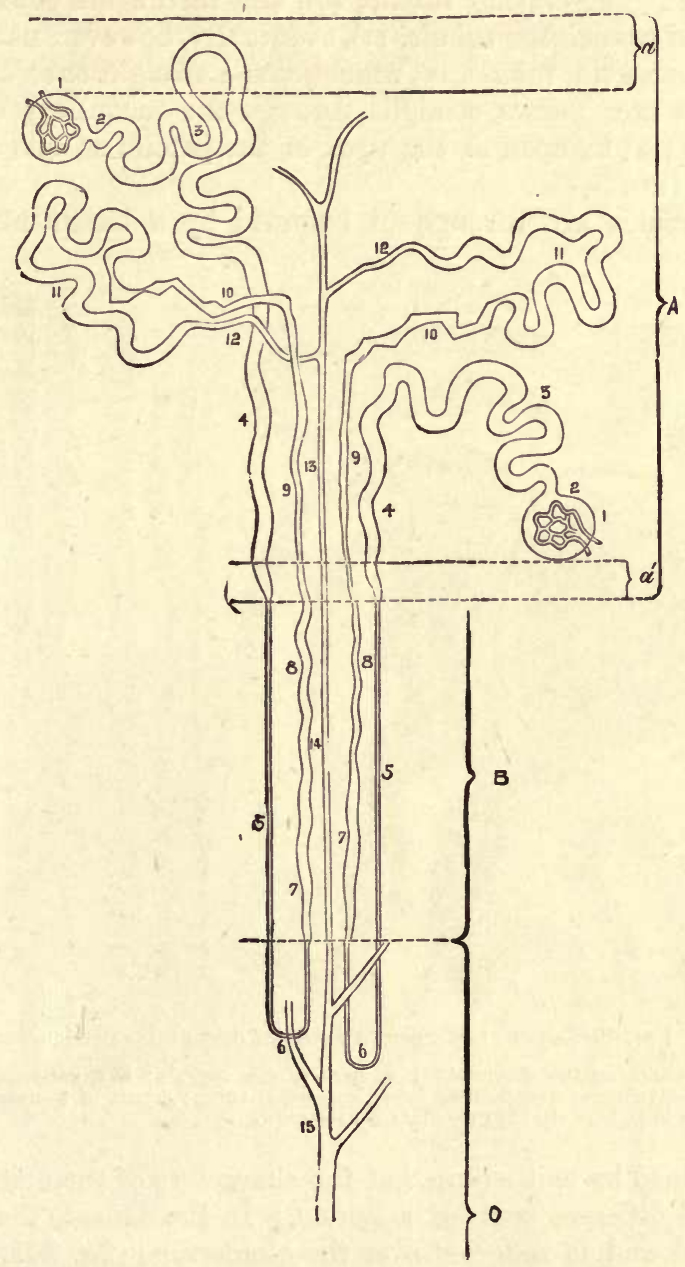

Fig. 200.-Diagram of the course of two Uriniferous tubules.

A, cortex; B, boundary zone; c, papillary zone of the thedulla ; $a, a^{\prime}$, superficial and deep layers of cortex, free from glomeruli. For the explanation of the numerals, see the text.

soon becomes nearly straight or slightly spiral only (spiral tubule, 4), and then, rapidly narrowing, passes down into the medulla towards the dilated commencement of the ureter as the descending tubule of Henle (5). It does not at once, however, open into the pelvis of the kidney, 
but before reaching the end of the papilla it turns round in the form of a loop (loop of Henle, 6) and passes upwards again towards the cortex, parallel to its former course and somewhat larger than before (ascending tubule of Henle, 7, 8, 9). Arrived at the cortex, it at first becomes irregularly zigzag (zigzag tubule, 10), and then again convoluted as at first (second convoluted tubule, 11), eventually, however, narrowing into a vessel (junctional tubule, 12) which joins a straight or collecting tubule (13). This now passes straight through the medullary substance of the kidney (14) to open at the apex of the papilla as one of the ducts of Bellini (15).

The tubules are throughout bounded by a basement-membrane,
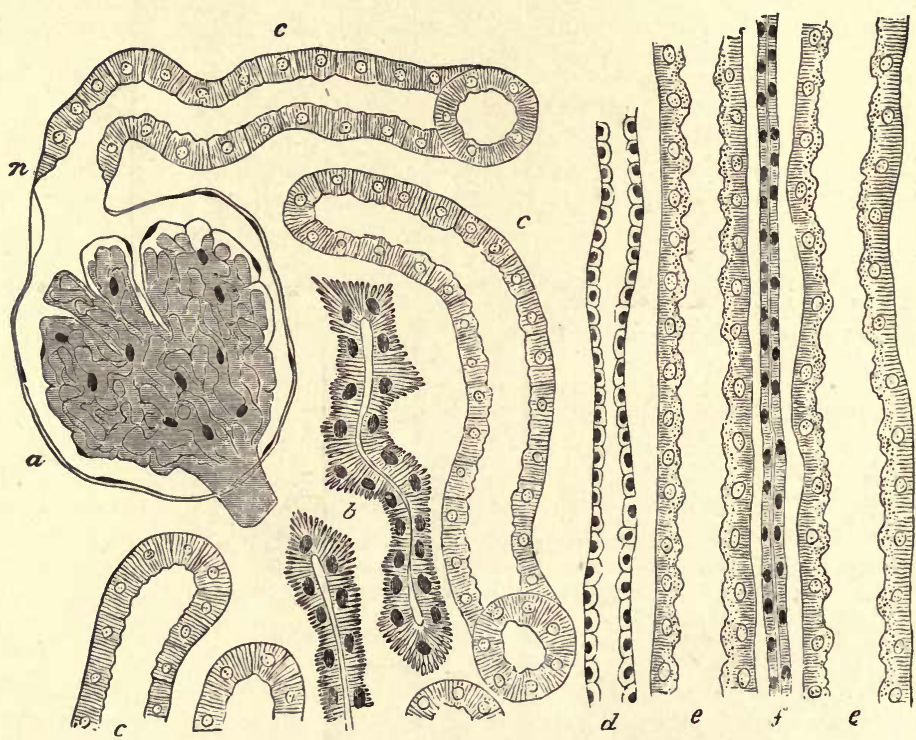

Fig. 201.-TUBULES From A SECTION OF THE DOG'S KIDNEY.

$\boldsymbol{a}$, Capsule, enclosing the glomerulus; $n$, neck of the capsule; $c, c$, convoluted tubules ; $b$, irregular tubules; $d$, collecting tube; $e, e$, spiral tubes; $f$, part of the ascending limb of Henle's loop, here (in the medullary ray) narrow.

which is lined by epithelium, but the characters of the epithelium-cells vary in the different parts of a tubule. In the capsule the epithelium is flattened and is reflected over the glomerulus (fig. 201, ai. In the first convoluted and spiral tubules it is thick, and the cells show a marked fibrillar structure (figs. 202, 203). Moreover, they interlock laterally and are difficult of isolation ; in many animals they have been shown to be ciliated. In the narrow descending limb of the looped tubule (fig. 204,c), and in the loop itself, the cells are clear and flattened and leave a considerable lumen; in the ascending limb they again acquire the striated structure and nearly fill the lumen. The fibrillations of the cells are still more marked in the zigzag tubules (fig. 201, $b$ (, and a similar structure is present also in the second 

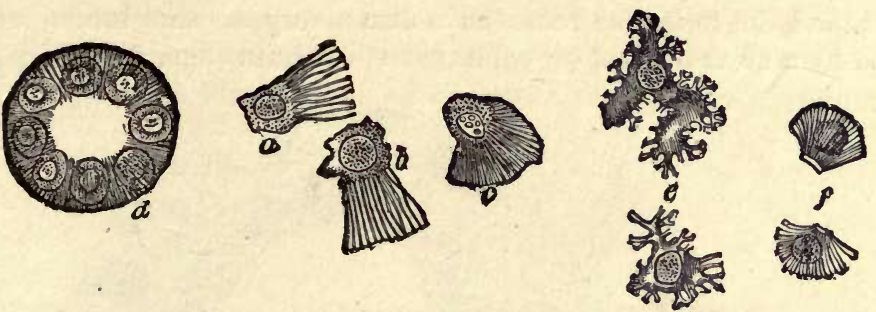

Fig. 202.-Structure of the epithelium of THE Convoluted tubules.

$d$, section of a convoluted tubule from the rat, showing the unaltered protoplasm occupying a circular area around the nucleus of each cell; $a, b, c$, isolater cells from the convoluted tubules of the rat; $e$, isolated cells from the dogs kidney, viewed from the imner surface, and showing the irregular contour of the protoplasm; $f$, isolated cells from the newt, showing the rods and the bomogeneous cuticular layer.

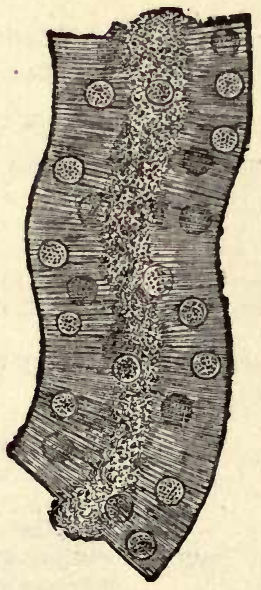

Fig. 203.-PART of a conVOLUTED TUBULE FROM THE DOG'S KIDNEY.

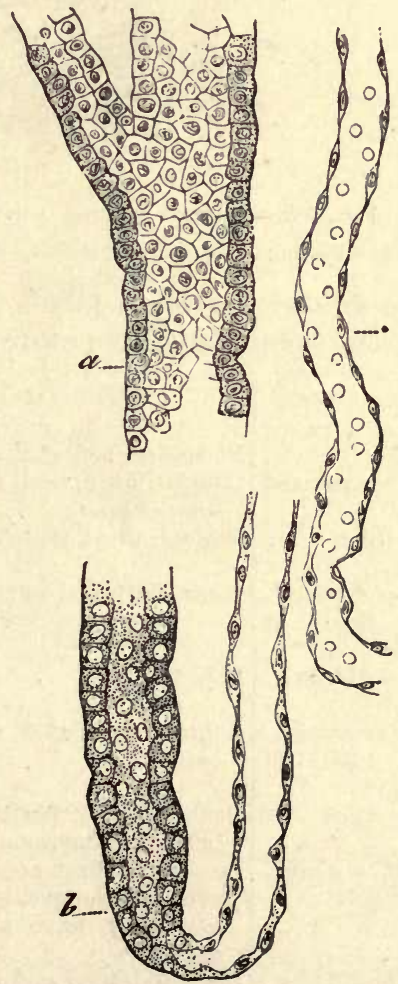

Fig. 204.-Portions of tubules, isolated. (Cadiat.)

$a$, large collecting tubu'e; $b$, loop of Henle; $c$, descending tubule of Henle. 
convoluted tubules, into which these pass. On the other hand, the junctional tubule has a large lumen and is lined by clear flattened cells, and the collecting tubes have also a very distinct lumen and are lined by a clear cubical or columnar epithelium (figs. 201, $d ; 204, a$ ).

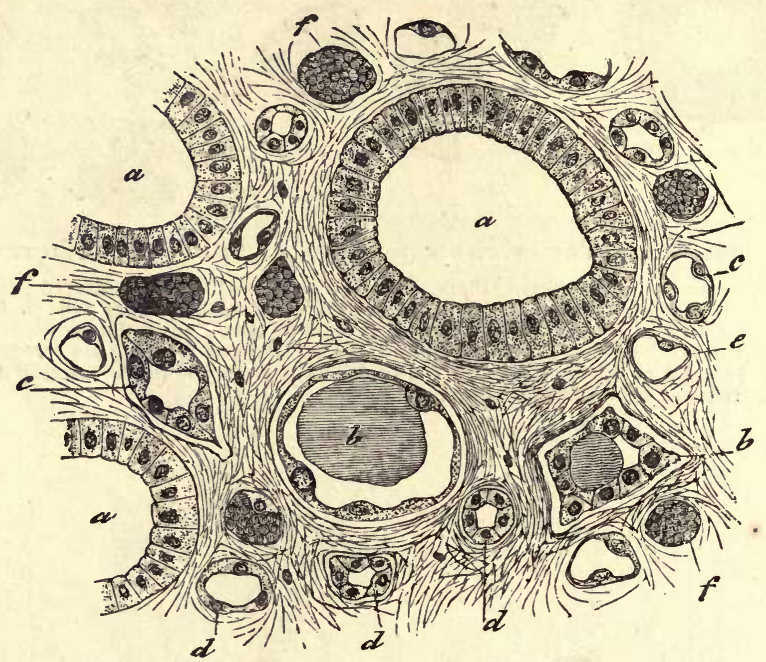

Fig. 205.-Section across a papilla of the kinney. (Cadiat.)

$a$, large collecting tubes (ducts of Bellini); $b, c, d$, tubules of Henle; $e, f$, blood-capillaries.

The following gives a tabular view of the parts which compose a uriniferous tubule, and the nature of the epithelium in each part:

\begin{tabular}{|c|c|c|}
\hline Portion of tubule & Nature of epithelium & Position of tubule \\
\hline Capsule & Flattened, reflected over glomerulus . & Labyrinth of cortex \\
\hline $\begin{array}{l}\text { First convoluted } \\
\text { tube }\end{array}$ & $\begin{array}{l}\text { Cubical, fibrillated, ciliated, the cells } \\
\text { interlocking }\end{array}$ & Labyrinth of cortex \\
\hline Spiral tube & Cubical, fibrillated (like the last) . & $\begin{array}{l}\text { Medullary ray of cor } \\
\text { tex }\end{array}$ \\
\hline $\begin{array}{l}\text { Small or descend- } \\
\text { ing tube of } \\
\text { Henle }\end{array}$ & Clear, flattened cells & $\begin{array}{l}\text { Boundary zone and } \\
\text { partlypapillary zone } \\
\text { of medulla }\end{array}$ \\
\hline Loop of Henle. & Like the last & $\begin{array}{l}\text { Papillary zone of } \\
\text { medulla }\end{array}$ \\
\hline $\begin{array}{l}\text { Larger or ascend- } \\
\text { ing tube of } \\
\text { Henle }\end{array}$ & $\begin{array}{l}\text { Cubical, fibrillated, sometimes imbri- } \\
\text { cated }\end{array}$ & $\begin{array}{l}\text { Medulla, and medul } \\
\text { lary ray of cortex }\end{array}$ \\
\hline Zigzag tube & $\begin{array}{l}\text { Cells strongly fibrillated; varying in } \\
\text { height; lumen small }\end{array}$ & Labyrinth of cortex \\
\hline $\begin{array}{l}\text { Second convo- } \\
\text { luted tube }\end{array}$ & $\begin{array}{l}\text { Similar to first convoluted tube, but } \\
\text { cells are longer, with larger nuclei, } \\
\text { and they have a more refractive } \\
\text { aspect }\end{array}$ & Labyrinth of cortex \\
\hline Junctional tube . & Clear flattened and cubical cells & $\begin{array}{l}\text { Labyrinth passing to } \\
\text { medullary ray }\end{array}$ \\
\hline $\begin{array}{l}\text { Straight or col- } \\
\text { lecting tube }\end{array}$ & Clear, cubical and columnar cells & $\begin{array}{l}\text { Medullary ray and } \\
\text { medulla }\end{array}$ \\
\hline Duct of Bellini . & Clear, columnar cells. & $\begin{array}{l}\text { Opens at apex of } \\
\text { papilla }\end{array}$ \\
\hline
\end{tabular}


Blood-vessels. - The artery of the kidney divides into branches on entering the organ, and these branches pass towards the cortex, forming incomplete arches between the cortex and the medulla (fig. $206, a)$. The branches of the renal vein form similar but more complete arches $(g)$. From the arterial arches vessels pass through the

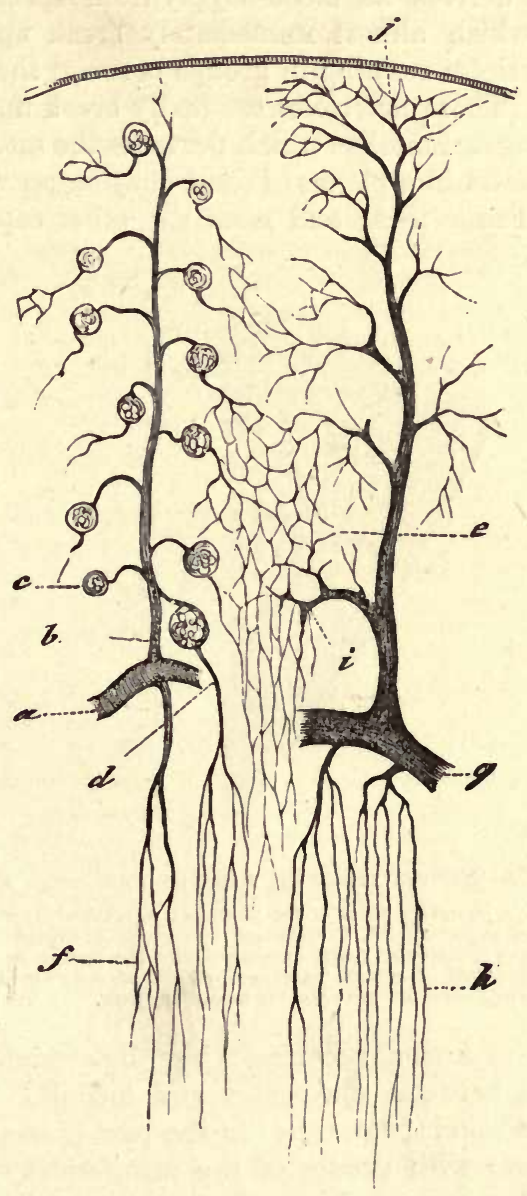

Fig. 206.-VASCULAR SUPPLY OF KIDNEY. (Cadiat.)

$a$, part of arterial arch; $b$, interlobular artery ; $c$, glomerulus ; $d$, efferent vessel passing to medulla as false art. rect.; $e$, ca!nillaries of cortex $;$, capillaries of medulla; $g$, venous arch; $h$, straight veins of medulla; $j$, vena stellula; $i$, interlobular vein.

cortex (interlobular arteries, $b$ ), and give off at intervals small arterioles (efferent vessels of the glomeruli), each of which enters the dilated commencement of a uriniferous tubule, within which it forms a glomerulus. From the glomerulus a somewhat smaller efferent vessel passes out, and this at once again breaks up into capillaries, which 
are distributed amongst the tubules of the cortex $(e)$; their blood is collected by veins which accompany the arteries and join the venous arches between the cortex and the medulla, receiving in their course certain other veins which arise by radicles which have a somewhat stellate arrangement near the capsule (vence stellula, $j$ ).

The medulla derives its blood-supply from special offsets of the arterial arches, which almost immediately break up into pencils of fine straight arterioles running in groups between the straight tubules of the medulla. These arterioles gradually break up into a capillary network with elongated meshes which pervades the medulla (fig. 206, $f$ ), and which terminates in a plexus of somewhat larger venous capillaries in the papillæ. From these and from the other capillaries the veins

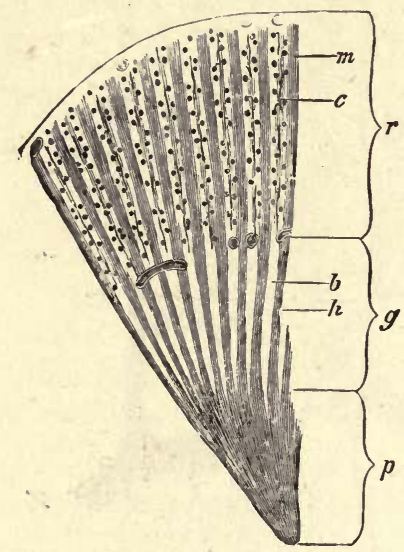

Fig. 207.-Section throvgh PART OF the nog's KIDNEy.

$\boldsymbol{p}$, papillary, and $g$, boundary zones of the medulla; $c$, cortical laver; $h$. bundles of tubules in the boundary layer, separated by spaces, $b$, containing bunches of vessels (not here represented), and pro'onged into the cortex as the medullary rays, $m ; c$, intervals of cortex, composer chiefly of convoluted tubules, with irregular rows of glomeruli, between the medullary rays.

collect the blood, and pass, accompanying the straight arterioles, into the venous arches between the cortex and medulla. The groups of small arteries and veins (vasa recta) in the part of the medulla nearest the cortex alternate with groups of the uriniferous tubules, and this arrangement confers a striated aspect upon this portion of the medulla (boundary zone, see fig. 207).

The efferent vessels of those glomeruli which are situated nearest to the medulla may also break up into pencils of fine vessels (false arteria recta) and join the capillary network of the medulla (fig. 198, $d$ ).

Between the uriniferous tubules, and supporting the blood-vessels, is a certain amount of connective tissue (fig. 205), within which are cleft-like spaces from which the lymphatics of the organ originate. 


\title{
LESSON XXXIV.
}

\author{
STRUCTURE OF THE URETER, BLADDER, AND MALE \\ GENERATIVE ORGANS.
}

1. Section across the ureter.

2. Section of the urinary bladder vertical to the surface.

In the sections of the ureter and of the urinary bladder, notice the transitional epithelium resting on a mucous membrane, which is composed chiefly of areolar tissue without glands, and the muscular coat outside this. In the ureter there is some fibrous tissue outside the muscular coat, and at the upper part of the bladder there is a layer of serous membrane covering the muscular tissue. Sketch a section of the ureter under a low power, and the epithelium of the bladder under the high power.

3. Section across the penis. The blood-vessels of the organ should have been injected so as the better to exhibit the arrangement of the renous spaces which constitute the erectile tissue. Notice the large venous sinuses of the corpora cavernosa and the smaller spaces of the corpus spongiosum, in the middle of which is seen the tube. of the urethra.

4. Section across the testis and epididymis. The sections are best made from a rat's testis which has been hardened in alcohol and pieces of which have been stained in bulk in dilute logwood. In these sections notice the strong capsule surrounding the gland, the substance of which consists of tubules which are variously cut, and the epithelium in which is in different conditions of development in the different tubules. Observe the strands of polyhedral interstitial cells lying in the loose tissue between the tubules and the lymphatic clefts in that tissue. Notice also in sections through the epididymis the ciliated epithelium of that tube.

Sketch carefully under a high power the contents of some of the semini. ferous tubules so as to illustrate the mode of formation of the spermatozoa.

5. Examination of spermatozoa. The spermatozoa are to be obtained fresh from the testis or seminal vesicles of a recently killed animal and examined in saline solution. Their movements may be studied on the warm stage; to display their structure a very high power of the microscope is necessary. Measure and sketch three or four spermatozoa.

The ureter is a muscular tube lined by mucous membrane. The muscular coat consists of three layers of plain muscular tissue, an outer and inner longitudinal and a middle circular. Outside the muscular coat is a layer of fibrous tissue in which the blood-vessels and nerves ramify before entering the muscular layer.

The mucous membrane is composed of areolar tissue and is lined by transitional epithelium. 
The urinary bladder has a muscular wall lined by a strong mucous membrane and covered in part by a serous coat.

The muscular coat consists of three layers, but the innermost is incomplete. The principal fibres run longitudinally and circularly, and the circular fibres are collected into a layer of some thickness which immediately surrounds the commencement of the urethra, forming the sphincter vesica. The mucous membrane is lined by a transitional stratified epithelium like that of the ureter. The shape and structure of the cells have already been studied.

The penis is mainly composed of cavernous tissue which is collected into two principal tracts-the corpora cavernosa, one on each side, and the corpus spongiosum in the middle line inferiorly. All these are bounded by a strong capsule of fibrous and muscular tissue, containing also many elastic fibres and sending in strong septa or trabeculæ,

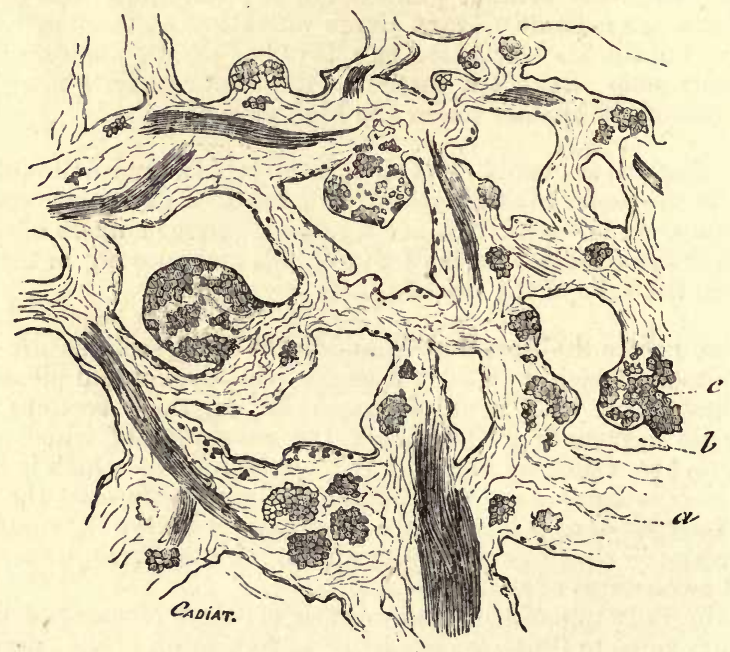

Fig. 208.-Section of ERectile tissue. (Cadiat.)

$a$, trabeculæ of connective tissue, with elastic fibres, and bundles of plain muscular tissue $(c) ; b$, venous spaces.

which form the boundaries of the cavernous spaces of the erectile tissue (fig. 208). The arteries of the tissue run in these trabeculæ, and their capillaries open into the cavernous spaces. On the other hand, the spaces are connected with efferent veins. The arteries of the cavernous tissue may often in injected specimens be observed to form looped or twisted projections into the cavernous spaces (helicine arteries of Müller).

Urethra.-The cross-section of the urethra appears in the middle of the corpus spongiosum in the form of a transverse slit. It is lined by columnar epithelium, except near its orifice, where the epithelium is stratified. The epithelium rests upon a vascular mucous membrane, 
and this again is supported by a coating of submucous tissue, containing two layers of plain muscular fibres-an inner longitudinal and an outer circular. Outside this again is a close plexus of small veins which are connected with, and may be said to form part of, the corpus spongiosum.

The mucous membrane of the urethra is beset with small mucous glands, simple and compound (glands of Littré). There are also a number of oblique recesses termed lacunæ. Besides these small glands and glandular recesses, two compound racemose glands open into the bulbous portion of the urethra (Cowper's glands). Their acini are lined by clear columnar cells which secrete mucus.

The prostate, which surrounds the commencement of the urethra, is a muscular and glandular mass, the glands of which are composed of tubular alveoli, lined by columnar epithelium, with smaller cells lying between them and the basement-membrane. Their ducts open upon the floor of the urethra.

The integument of the penis contains numerous special nerve endorgans of the nature of end-bulbs, and Pacinian bodies have also been

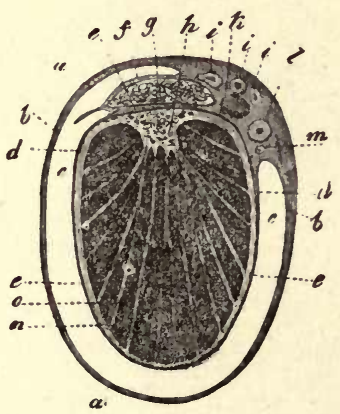

Fig. 209.-Transverse SECTION THROCGH THE RIGHT TESTICLE AND THE TUNICA VAGINALIS.

$a$, connective tissue enveloping the parietal layer of the tunica vaginalis; $b$, this layer itself ; $c$, carity of the tunica vaginalis; $d$, reflected or visceral layer adhering to $e$, the tunica albuginea ; $f$, covering of epididymis $(g) ; h$, mediastinum testis; $i$, branches of the spermatic artery; $k$, spermatic vein ; $l$, vas deferens ; $m$, small artery of the vas deferens; $n, 0$, septa or processes from the mediastinum to the surface.

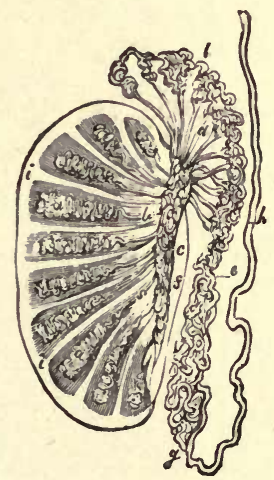

Fig. 210.-Plan of a VerTICAL SECTION OF THE TESTICLE, SHOWING THE ARRANGEMENT OF THE DUCTS.

The true length and diameter of the ducts have been disregarded. $a, a$, tubuli seminiferi coiled up in the separate lobes; $b$, vasa recta: $c$, rete vasculosum; $d$, vasa efferentia ending in the coni vasculosi ; $l, e, g$, convoluted canal of the epididymis; $h$, vas deferens; $f$, section of the back part of the tunica albuginea; $i, i$, fibrous processes running between the lobes ; $f$. to $s$, mediastinum.

found upon the nerves. Lymphatic vessels are numerous in the integument of the organ and also in the submucous tissue of the urethra. 
The testicle is enclosed by a strong fibrous capsule, the tunica albuginea (fig. 209,e). This is covered externally with a layer of serous epithelium reflected from the tunica vaginalis. From its inner surface there proceed fibrous processes or trabecula, which imperfectly subdivide the organ into lobules, and posteriorly the capsule is prolonged into the interior of the gland in the form of a mass of fibrous tissue, which is known as the mediastinum (fig. 209, h). Attached to the posterior margin of the body of the gland is a mass (epididymis) which when investigated is found to consist of a single convoluted tube, receiv-

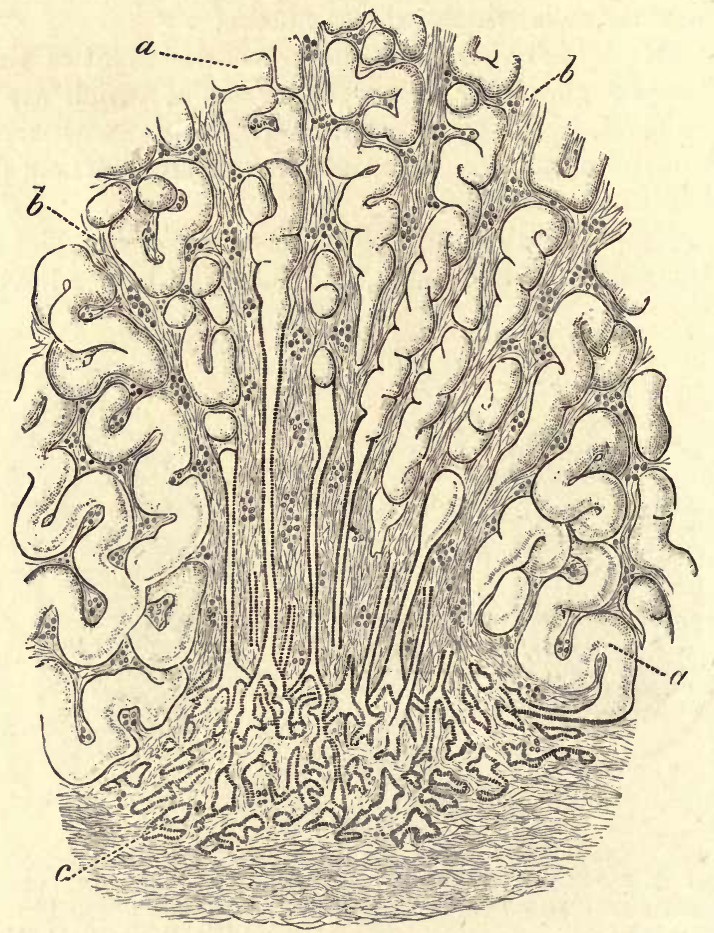

Fig. 211.-Passage of Convoluted seminiferous tubules into Straight tUbUles AND OF THESE INTO THE RETE TESTIS.

$a$, seminiferous tubules; $b$, fibrous stroma continued from the mediastinum testis; $c$, rete testis.

ing at its upper end the efferent ducts of the testis and prolonged at its lower end into a thick-walled muscular tube, the vas deferens, which conducts the secretion to the urethra.

The glandular substance of the testicle is wholly made up of convoluted tubules, which when unravelled are of very considerable length. Each commences near the tunica albuginea, and after many windings terminates, usually after joining one or two others, in a straight tubule (fig. 211), which passes into the mediastinum, and there forms, by 
uniting with the other straight tubules, a network of intercommunicating vessels. which is known as the rete testis. From the rete a certain number of efferent tubules arise, and after a few convolutions pass into the tube of the epididymis.

Structure of the tubules.-The seminiferous tubules are formed of a basement-membrane, and contain several layers of epithelium-cells. Of these layers, the one next the basement-membrane is a uniform layer of clear cubical cells (lining epithelium), the nuclei of which, for the most part, exhibit the irregular network which is characteristic of

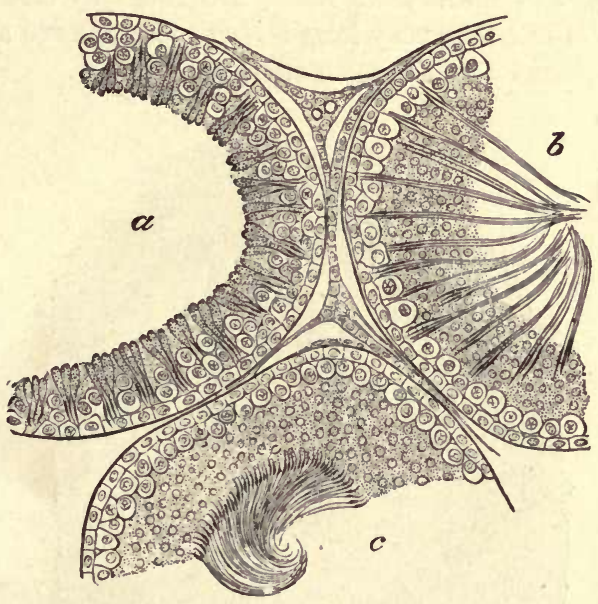

Fig. 212.-Section of parts of three seminiferous tubules of the rat.

$a$, with the spermatozoa least advanced in development; $b$, more advanced; $c$, containing fully developed spermatozoa. Between the tubules are seen strands of interstitial cells with blood-vessels and lymph-spaces.

the resting condition. Here and there these epithelium-cells appear enlarged and to project between the more internal layers.

Next to this layer of cubical epithelium there is seen a layer of larger cells (spermatogenic cells), the nuclei of which have the skeinlike aspect which is typical of commencing division; these cells may be two, three, or more deep (as in the tubule $a$, fig. 212). Next to them, and most internal, are to be seen in some tubules ( $b$ and $c$ ) a large number of small protoplasmic corpuscles with simple circular nuclei (spermatoblasts). In other tubules these corpuscles are elongated, and the nucleus is at one end, and in others again these elongated cells are converted into evident spermatozoa, which lie in groups with their heads projecting between the deeper cells and connected with one of the enlarged cells of the lining epithelium, and their tails emerging into the lumen of the tubule (fig. 212, $b$ ). As they become matured they gradually pass altogether towards the lumen, where they eventually become free $(c)$. During the time that this crop of spermatozoa has been forming, another set of spermatoblasts has been produced by the 
division of the spermatogenic cells, and on the discharge of the spermatozoa the process is repeated as before.

The straight tubules which lead from the convoluted seminiferous tubes into the rete testis (fig. 211) are lined only by a single layer of clear flattened or cubical epithelium. The tubules of the rete also have a simple epithelial lining, but the basement-membrane is here absent, the epithelium being supported directly by the connective tissue of the mediastinum.

The efferent tubules which pass from the rete to the epididymis, and the tube of the epididymis itself, are lined by columnar ciliated epithelium, the cilia being very long; these tubes have a considerable amount of plain muscular tissue in their wall.

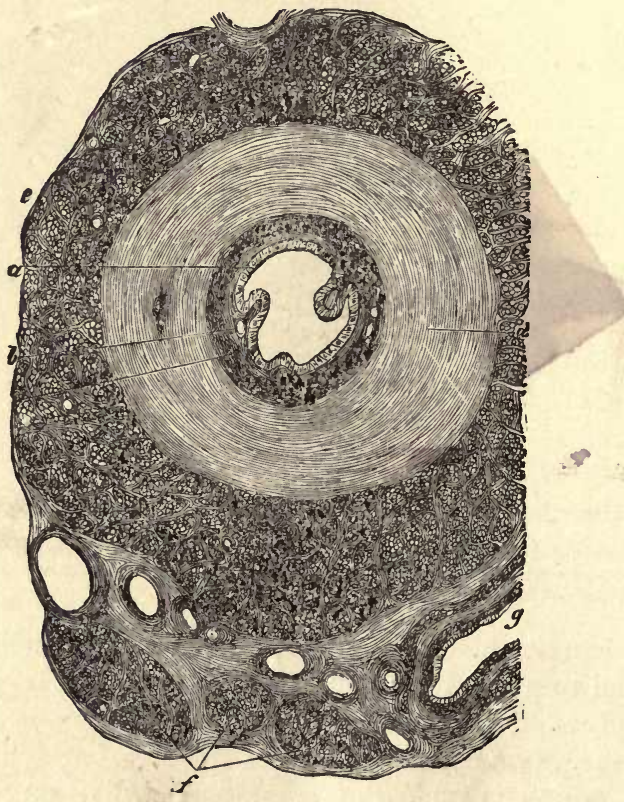

Fig. 213.-Section across the commencement of THE VAS DEFERENS.

$a$, epithelium ; $b$, mucous membrane ; $c, d, e$, inner, middle, and outer layers of the muscular coat; $f$, bundles of the internal cremaster muscles; $g$, section of a blood-vessel.

The vas deferens (fig. 213) is a thick tube, the wall of which is formed of an outer thick layer of longitudinal bundles of plain muscular tissue; within this an equally thick layer of circular bundles of the same tissue, and within this again a thin layer of longitudinal muscle. The tube is lined by a mucous membrane, the inner surface of which is covered by columnar non-ciliated epithelium.

The connective tissue between the tubules of the testis is of very 
loose texture, and contains numerous lymphatic clefts, which form an intercommunicating system of commencing lymphatic vessels. Lying in this intertubular tissue are strands of polyhedral epithelium-like cells (interstitial cells) of a yellowish colour; they are much more abundant in some species of animals (cat, boar) than in others. They accompany the blood-vessels before these break up to form the capillary networks which cover the walls of the seminiferous tubules.

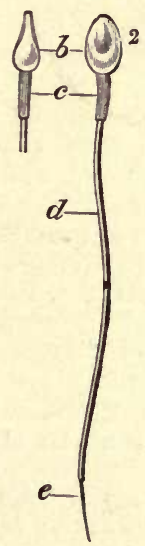

Fig. 214.-HUMAN SPERMATOZOA.

1 , in profle; 2 , viewed on the flat : $b$, head; $c$, middle-piece; $d$, tail ; e, end-piece of the tail.

The spermatozoa.-Each spermatozoon consists of three parts, a head, a middle part or body, and a long tapering and vibratile tail. In man (fig. 214) the head is of a flattened oval shape, somewhat more. flattened and pointed anteriorly; and the middle-piece is short and cylindrical, and appears to have a spiral fibre passing round it ; but in different animals the shape of the head and the extent of the middlepiece vary greatly. In the rat the head is long, and is recurved anteriorly ; it is set obliquely on the middle-piece, which is also of considerable extent, and has a closely wound spiral filament encircling it in its whole length. The tail is the longest part of the spermatozoon, and during life is in continual vibratile motion, the action resembling that of the cilia of a ciliated epithelium-cell.

The spermatozoa are developed from the small cells or spermatoblasts which form the innermost strata of the seminal epithelium, and are themselves produced by the division of the large cells of the second layer. The nucleus of the spermatoblast forms the head of the spermatozoon, while the protoplasm forms first the middle-piece and subsequently the tail ; but a portion of the protoplasm of each spermatoblast containing a number of small darkly staining particles (seminal granules) appears always to become detached and disintegrated before the spermatozoon is fully mature. 


\section{LESSON XXXV.}

\section{GENERATIVE ORGANS OF THE FEMALE, AND MAMMARY GLANDS.}

1. Secrions of the ovary of the rabbit. Study the sections with the low power, observing the small and large Graafian vesicles, each enclosing an ovum, scattered through the stroma. Measure some Graafian vesicles of different sizes; make a general sketch of a section under the low power. Then sketch carefully two or more of the Graafian vesicles with their contents.

2. Sections across the Fallopian tube. Sketch a section under the low power.

3. Section across the body of the uterus. Observe with the naked eye the thickness of the muscular and mucous coats respectively. Notice the ciliated columnar epithelium lining the organ and extending into the glands of the mucous membrane. Draw a part of the section under the low power.

4. Sections of the mammary gland from an animal killed during lactation. Notice the fat-globules in the alveoli and also in the alveolar cells. Draw an alveolus under the high power.

The ovary is a small solid organ, composed of a stroma of fibrous tissue, with many spindle-shaped cells, and also containing, especially near its attachment to the broad ligament, a large number of plain

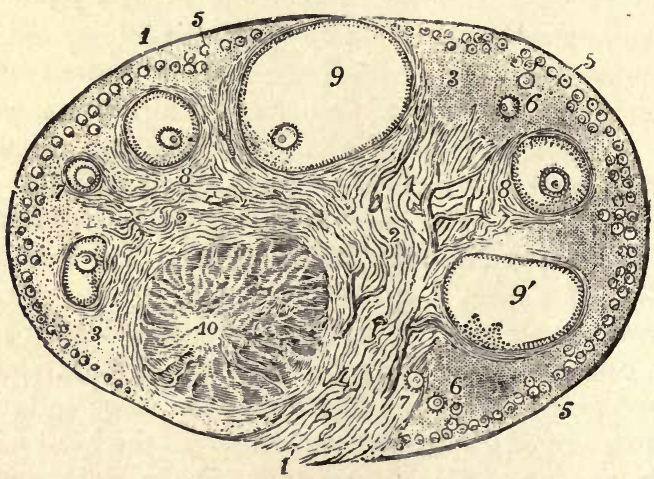

Fig. 215.-Section of the ovary of the Cat. $\frac{6}{1}$

1, outer covering and free border of the ovary; 1 ', attached border ; 2 , the central ovarian stroma, presenting a fibrous and vascular structure; 3 , peripheral stroma; 4, bloodressels; 5 , Graafian follicles in their earliest stages lying near the surface; $6,7,8$, more advanced follicles which are cmbedded more deeply in the stroma; 9 , an almost mature follicle containing the ovum in its deepest part; 9 , a follicle from which the ovum has accidentally escaped; 10, corpus luteum. 
muscular fibres. It is covered by a layer of small columnar epitheliumcells (germinal epithelium, fig. $216, a$ ), between which are here and there to be seen a few larger spheroidal cells, with large round nuclei (primitive ova, fig. 218, $c$ ). In the young subject the epithelium may occasionally dip down into the subjacent stroma.

The stroma is beset with vesicles of different sizes, the smallest

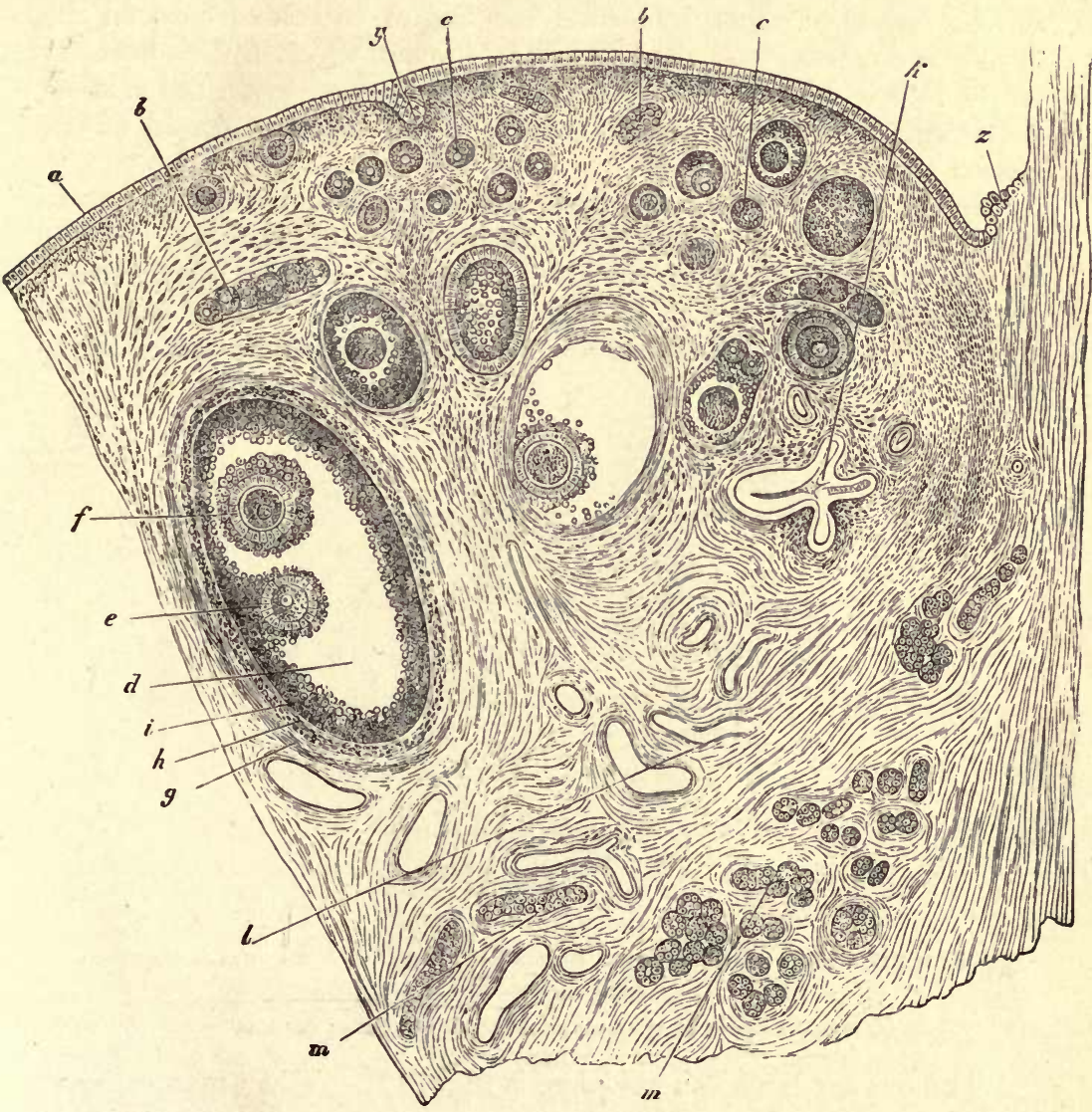

Fig. 216.-Section of the ovary of AN ADULt BItch.

$a$, germ-epithelium ; $b$, egg-tubes ; $c, c$, sinall follicles ; $d$, more advanced follicle ; $e$, discus proligerus and ovum : $f$, second ovum in the same follicle (this occurs but rarely); $g$, outer tunic of the follicle; $h$, inner tunic; $i$, membrana granulosa; $k$, collapsed retrograded follicle ; $l$, blood-vessels; $m, m$. longitudinal and transverse sections of tubes of the parovarium ; $y$, involuted portion of the germ-epithelium of the surface ; $z$, place of the transition from peritoneal to germinal or ovarian epithelium.

being near the surface of the organ, the larger ones placed more deeply in the stroma, although, as they increase in size, they may again tend to approach the surface.

These vesicles are the Graafian follicles. Each Graafian follicle 
has a proper wall of basement-membrane, strengthened by a layer derived from the stroma, and contains an ovum, surrounded by $\mathbf{a}$ number of epithelium-cells. In the smallest follicles the ovum is small, and the epithelium of the follicle is formed of a single layer of cells, which are flattened against the ovum. In somewhat larger follicles the epithelium-cells are in two layers, and these are columnar in shape. In still larger ones, each of these two layers is formed of several strata of cells, and fluid has begun to collect between the layers at one part. Of the two layers, the one which lines the cavity of the follicle is termed the membrana granulosa, while the mass of cells which more immediately surrounds the ovum is known as the discus proligerus.

In the largest follicles the fluid has much increased in amount, so that the follicle has become gradually larger and more tense. Finally it reaches the surface of the ovary, and projects from that surface, where it eventually bursts, and the liquor folliculi, with its contained ovum, is set free. This event occurs at about the time of menstruation. ${ }^{1}$

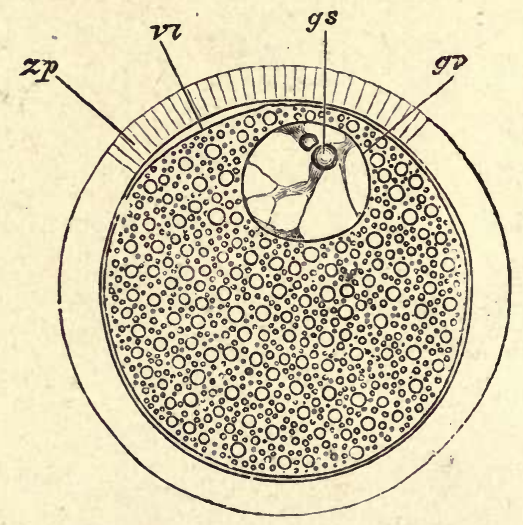

Fig. 217.-Semi-diagrammatic Representation of a mammalian ovum. (Highly magnified.)

$z p$, zona pellucida ; $v i$, vitellus; $g v$, germinal vesicle; $g s$, germinal spot.

The ova are large spheroidal cells (fig. 217). When mature, as in the largest Graafian follicles, each ovum is surrounded by a thick transparent striated membrane (zona pellucida). Within this is the protoplasm of the cell (vitellus), filled with fatty and albuminous granules. Lying in the vitellus, generally eccentrically, is the large clear round nucleus (germinal vesicle), which contains an intranuclear network, and usually one well-marked nucleolus (germinal spot). Both the ova and the epithelium of the Graafian follicles are developed

' Some of the Graafian follicles do not burst, but, after attaining a certain stage of maturity, undergo a process of retrograde metamorphosis and eventually disappear. 
originally from the germinal epithelium. In the embryo, this forms a thick layer, covering the fibrous and vascular stroma. After a time solid cords of epithelium-cells, which in some animals are partly tubular (ovarian tubes of Pflüger), grow down into the stroma, whilst this at the same time grows into the epithelium. The cords presently become broken up by the ingrowths of stroma into small isolated nests of epithelium-cells, each of which may represent a Graafian follicle. To form the ova, some of the germinal epithelium-cells become enlarged, and usually there is one such enlarged cell in each of the isolated nests. The remaining cells form the epithelium of the follicle (see fig. 218).

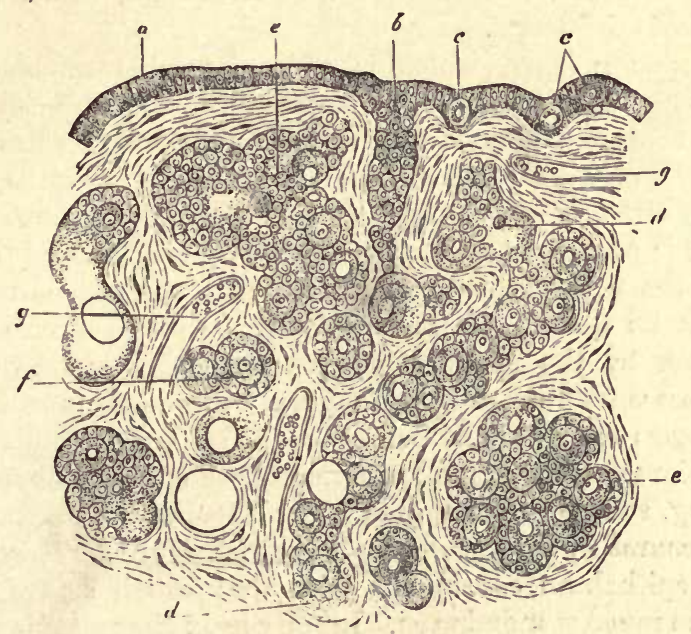

Fig. 218.-Section of the ovary of a NEwly born child. (Highly magnified.)

$a$, ovarian or germinal epithelium; $b$, formation of an ovarian tube ; $c, c$, primordial ova lying in the germ-epithelium; $\boldsymbol{d}, \boldsymbol{d}$, longer tube becoming constricted so as to form nests of cells; $e, e$, larger nests; $f$, distinctly formed follicle with ovum and epithelium; $g, g$, blood-ressels.

The stroma of the ovary contains, besides the spindle-shaped connective-tissue cells and plain muscular fibres already mentioned, a number of epithelium-like interstitial cells, like those found in the intertubular tissue of the testis. They are most abundant near the hilus. Corpora lutea may also be seen in the stroma. These are large yellow nodules, which are developed out of the Graafian follicles after the ovum has become extruded. They consist of columns of large yellowish cells, with intervening vascular fibrous tissue, which converge to a central strand of connective tissue occupying the axis of the nodule. The columns of yellow cells are not unlike those of the cortex of the suprarenal capsule. The cells are derived from the membrana granulosa of the follicle, which becomes thickened and folded; between the folds connective tissue and blood-vessels grow in towards the centre, and in this way the corpus luteum becomes 
developed. After persisting for a time it gradually shrinks and disappears, but it becomes larger and remains longer persistent in the event of pregnancy supervening.

The Fallopian tubes are chiefly composed of plain muscular tissue disposed circularly. They are lined by a mucous membrane which is covered with ciliated epithelium. Externally they are covered by a serous coat, within which is a thin layer of longitudinal muscular fibres overlying the circular fibres.

The uterus is usually described as composed of two parts, the body and cervix. The wall of the uterus is formed of the following layers:

1. A serous layer, derived from the peritoneum, which covers the greater part of the fundus.

2. A muscular layer, which is of considerable thickness and is composed of plain muscular fibres disposed in two imperfectly separated strata. Of these the outer is much the thinner, and its fibres are disposed partly longitudinally, partly circularly. The inner muscular layer, on the other hand, is very thick ; its fibres run in different directions, and it is prolonged internally into the deeper part of the mucous membrane, the extremities of the uterine glands extending between and amongst its fibres. It is imperfectly separated from the thinner external layer by the ramifications of the larger blood-vessels, and probably represents a much-hypertrophied muscularis mucosæ.

3. A mucous membrane, which is very thick and is composed of soft connective tissue containing a large number of spindle-shaped cells. It contains long, simple, tubular glands (fig. 219), which take a curved or convoluted course in passing through the membrane. They are lined by ciliated epithelium continuous with that which covers the inner surface of the mucous membrane. In the cervix the mucous membrane is marked by longitudinal and oblique ridges, and the glands are shorter than those of the body of the uterus. Near the os uteri the epithelium becomes stratified and overlies vascular papillæ of the corium. The mucous membrane is exceedingly vascular, and it also contains a large number of lymphatic vessels.

At each menstrual period the greater part of the mucous membrane of the body undergoes a process of disintegration accompanied by an escape of blood from the capillaries of the membrane. This is succeeded by a rapid renewal of the membrane. Should gestation supervene, the process of renewal results in the formation of a greatly thickened mucous membrane, with long convoluted glands, which is then known as the decidua.

The mammary gla-ds are compound racemose glands which open by numerous ducts upon the apex of the nipple. The ducts are dilated into small reservoirs just before reaching the nipple. If traced backwards, they are found as in other compound racemose glands to commence in groups of saccular alveoli. The walls of the ducts and alveoli are formed of a basement-membrane lined by a simple layer of flattened epithelium (fig. 220, A). But during lactation. when the gland is in 
Fig. 219.-SeCtion of THE MUCOUS MEMBRANE OF THE UTERUS FROM NEAR THE FUNDUS.

$a$, epithelium of inner surface: $b, b$, uterine glands; $c$.interglandular connective tissue; $d$, muscular tissue.

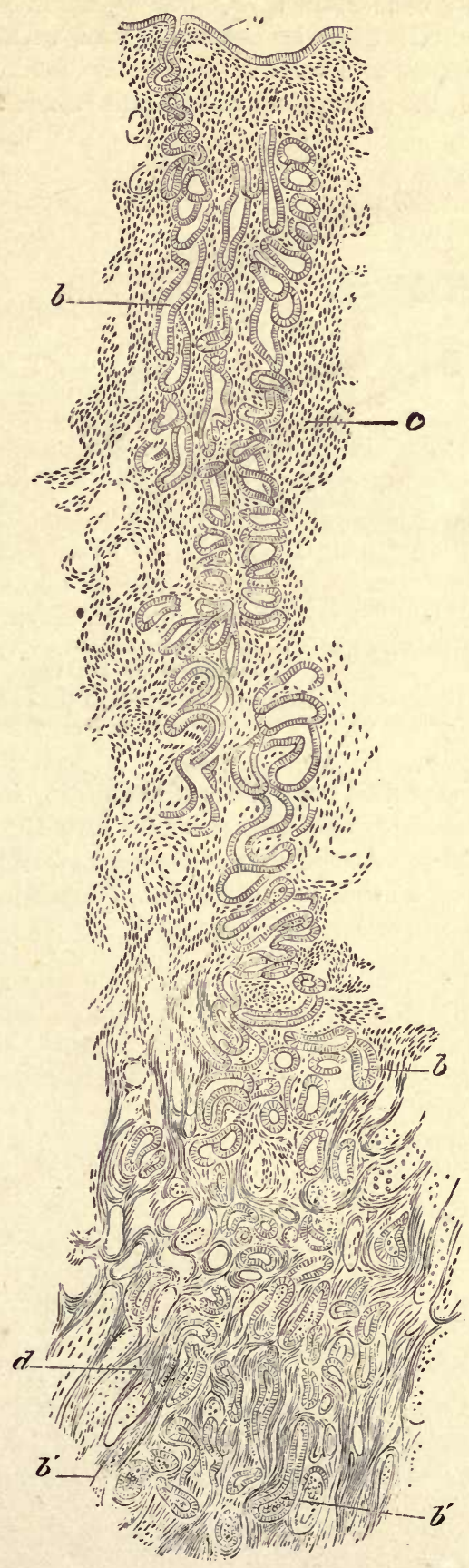


activity, the cells of the alveoli become much enlarged and of a columnar shape, and fatty globules become formed within them $(B)$. These fatty globules appear to become set free by the breaking down of the inner part of the cell, the protoplasm of the cells becoming partially dissolved and
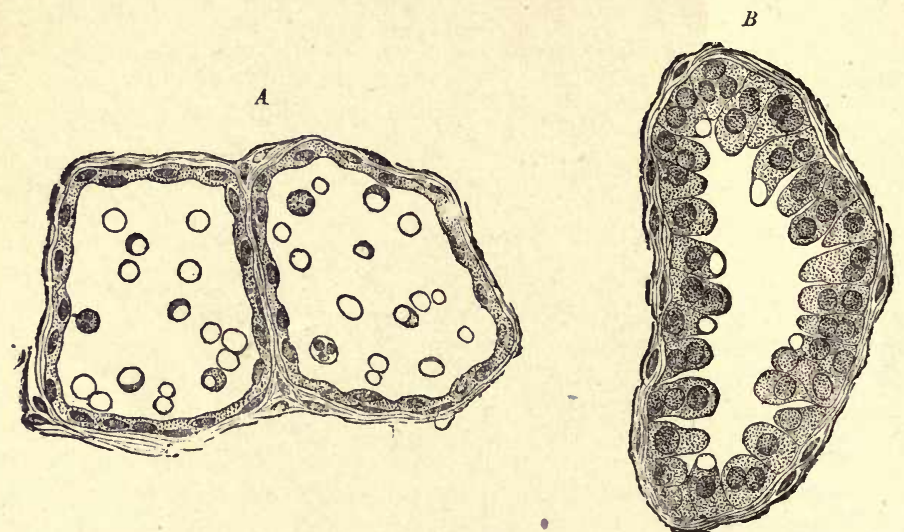

Fig. 220.-ALveoli of the MAMmary gLAND OF THE BITCH UNDER DifFerent CONDITIONS OF ACTIVITY.

$A$, section through the middle of two alveoli at the commencement of lactation, the epithelium-cells being seen in profile; $B$, an alveolus in full secretory activity.

forming the proteid substances of the milk. According to some authors, lymph-corpuscles may also carry fat into the alveoli and there become disintegrated. At the commencement of lactation this disintegration is imperfect, so that numerous cells containing fat-particles appear in the secretion (colostrum corpuscles). 


\section{LESSON XXXVI.}

STRUCTURE OF THE SPINAL CORD.

1. Sections of the spinal cord from the cervical, dorsal, and lumbar regions.

[The spinal cord is hardened by being immersed for about a month in bichromate of ammonia solution ( 2 per cent.); the sections are made with a freezing microtome and are stained with aniline blue black. $\left.{ }^{1}\right]$

Notice the relative extent of the grey as compared with the white matter in the different regions of the cord. In the white matter the stained dots are sections of the axis-cylinders of the longitudinally disposed medullated nerve-fibres; the tissue between the nerve-fibres is neuroglia. In the grey matter the branched nerve-cells are conspicuous, lying in a reticular substance which is formed of neuroglia together with an interlacement of nervefibres and cell-processes.

Sketch a section from each region under a low power. Sketch also a small portion of the white substance, two or three nerve-cells, and the central canal with its lining epithelium and surrounding neuroglia under the high power.

Measure the diameter of some of the nerve-fibres in the anterior columns, in the lateral columns, and in the posterior columns.

The spinal cord is composed of grey matter in the centre and of white matter externally. It is closely invested by a layer of connective tissue containing numerous blood-vessels (pia mater), and less closely by two other membranes. One of these is an areolar membrane, resembling a serous membrane in general structure, but non-vascular and more delicate in texture (arachnoid). The other, which lines the vertebral canal, is a strong fibrous membrane known as the dura mater. At the middle of the anterior and posterior surfaces the pia mater dips into the substance of the cord in the anterior and posterior median fissures, so as to divide it almost completely into two lateral halves. These are, however, united by an isthmus or bridge, which is composed anteriorly of transversely crossing white fibres (white commissure), posteriorly of grey matter (grey commissure), in the middle of which is a minute canal lined by ciliated epithelium (central canal, fig. 221, e).

Each lateral half of the spinal cord contains a crescent of grey matter, which is joined to the corresponding crescent of the opposite side by the grey commissure. Of the two cornua of the crescent the 
posterior is the narrower and comes near the surface of the cord; opposite to it the bundles of the posterior nerve-roots enter the cord. The bundles of the anterior nerve-ronts enter the anterior cornu.

The white matter of each half of the cord is subdivided by the passage of the nerve-roots into the cornua into three principal columns -anterior, lateral, and posterior. It is composed of longitudinally coursing medullated nerve-fibres, which in stained sections appear as clear circular areas with a stained dot, the axis-cylinder, near the middle (fig. 222).

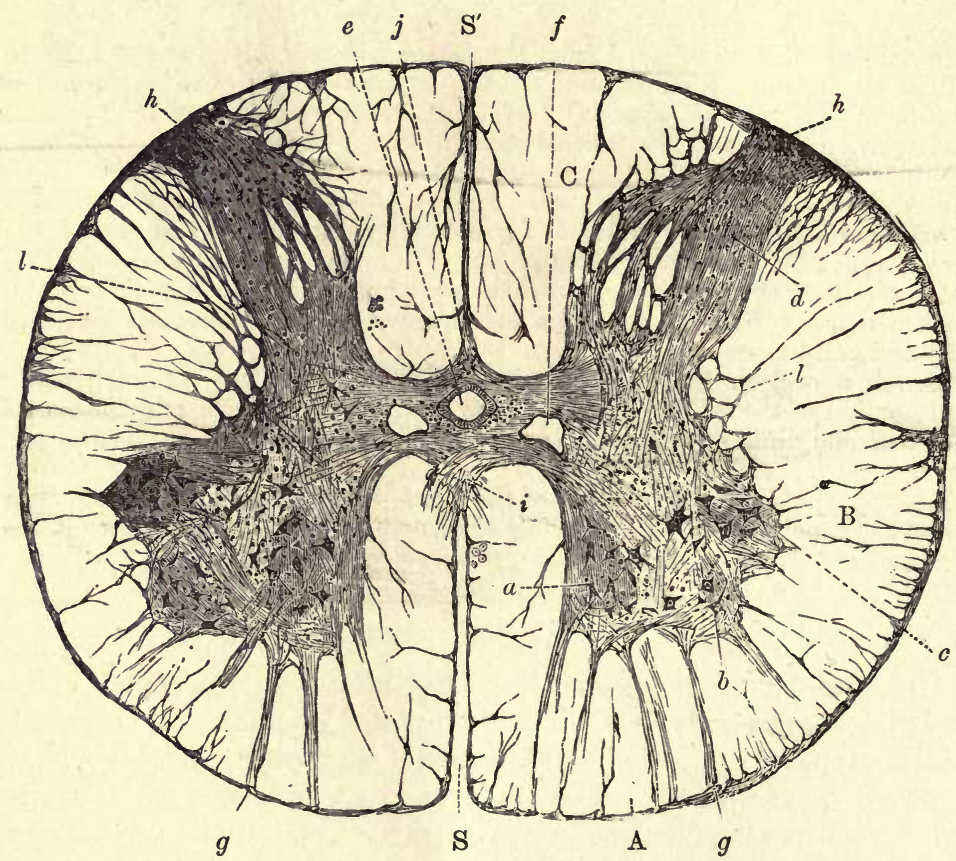

Fig. 221.-Section of the spinal cord in the lower dorsal region. (Cadiat.)

A, B, C, anterior, Jateral, and posterior columns; s, s', anterior and posterior median fissures. $a, b, c$, cells of anterior cornu ; $d$, posterior cornu and substantia gelatinosa of Rolando ; $e$, central canal; $f$, veins; $g$, anterior root-bundles; $h$, posterior root-bundles ; $i$, white commissure ; $j$, grey conımissure ; $l$, reticular formation.

The medullated fibres are supported by a peculiar reticular tissue (neurogiia) which contains a number of nuclei embediled in it and appears to be composed of branched fibrillated cells (neuroglia-cells). The neuroglia is accumulated in greater amount at the surface of the cord underneath the pia mater (fig. 222), and it extends into the grey matter, of which it may be said to form the basis, and in which it is especially accumulated at the apex of the posterior cornu and around the central canal (substantia gelatinosa of Rolando). 
The grey matter, besides neuroglia, consists of an interlacement of nerve-fibres and of the branching processes of the nerve-cells which are embedded in it.

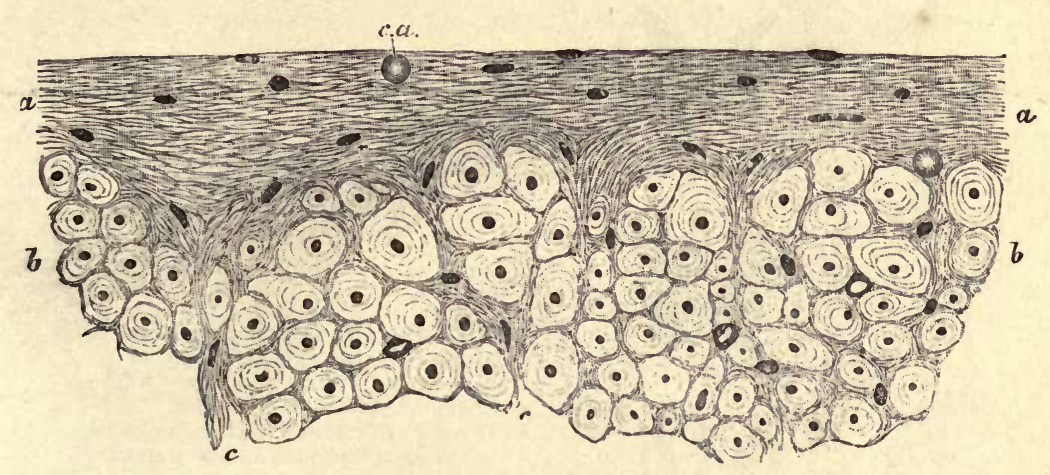

Fig. 222.-A small portion of a transverse section of the human spinal Cord iN THE REgION OF THE LATERAL COLUMN, TO SHOW THE SUPERFICIAL NElROGLIA.

$a, a$, superficial neuroglia ; $b, b$, transverse section of part of the lateral column of the cord, in
which the dark points are the axis-cylinders, and the clear areas the medullary substance
of the nerve-fibres. The superficial neuroglia is seen to exhibit the appearance of a fine net-
work in which numerous nuclei and one or two corpora amylacea are embedded, and to
extend inwards among the nerve-fibres.

Disposition of the nerve-fibres of the white columns in tracts.The nerve-fibres vary in size in different parts of the white columns. They are smallest in the posterior columns, especially in that part of the posterior column which is next the posterior median fissure (pasterior median column), and largest in the posterior part of the lateral column, but also of considerable size in the anterior column. This difference corresponds in some measure with the functions of the nerve-fibres in those parts respectively. Thus the posterior part of the lateral column which is characterised by the large size of its nervefibres constitutes the tract along which voluntary motor impressions pass down the spinal cord from the opposite side of the brain, after having crossed at the pyramids of the medulla oblongata (crossed pyramidal tract, fig. 223, c.p.t.). The large fibres which lie in the anterior columns next to the anterior median fissure, and the upper part of the cord, belong to a portion of the same tract which has not undergone decussation (direct pyramidal tract, d.p.t.). The small fibres of the posterior median column belong to a tract which is only distinct above the middle of the dorsal region of the cord, and is known as the tract of Goll (fig. 223, g). The rest of the posterior column is termed the cuneate fasciculus, and is chiefly composed of the fibres of the posterior nerve-roots which run for a short distance in it before entering the grey matter. In the lateral column there is another tract which is distinct in the cervical and dorsal regions. This lies external 
to the crossed pyramidal tract, and consists of large fibres which pass up into the cerebellum (direct cerebellar tract, c.t.).

$v$.

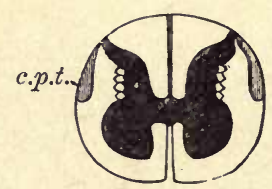

IV.

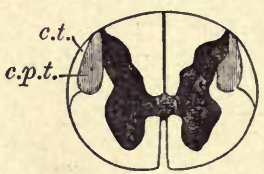

III.

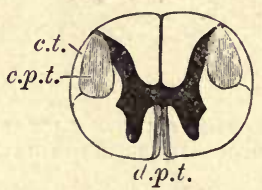

I1.

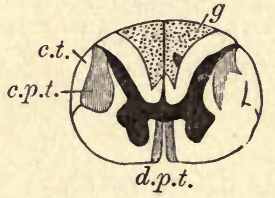

I.

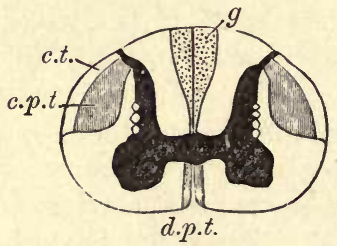

Fig. 223. - Diagramiatic SecTIONS OF THE SPINAL CORD AT DIFFERENT PARTS, TO SHOW THE CHIEF LOCALISED TRACTS OF FIBRES IN THE WHITE SUBSTANCE. (One and a half times the natural size.)

1. at the level of the sixth cervical nerve; $I I$. of the third dorsal ; $I I I$. of the sixth dorsal; $I V$. of the twelfth dorsal ; $V$. at the level of the fourth lumbar. d.p.t. direct or anterior pyramidal tracts; c.p.t. crossed or lateral pyramidal tracts ; c.t. direct cerebellar tract; $g$, tract of Goll.

The course of the nerve-tracts in the spinal cord, and in other parts of the central nervous system, can best be made out by the study of sections of the fœtal cord, for it is found that the development of the medullary substance occurs sooner in some tracts than in others, so that it is easy to make out the distinction between them. Another method consists in investigating the course which is pursued by degenerations of the nerve-fibres in consequence of lesions produced accidentally or purposely.

Disposition of the nerve-cells in the grey matter.-The nerve-cells which are scattered through the grey matter are in part disposed irito definite groups. Thus there is a group of large multipolar nerve-cells in the anterior cornu; their axis-cylinder processes pass out into the anterior nerve-roots (column of the anterior cornu, fig. $221, a, b, c)$. In the middle dorsal region there is a well-marked group of large rounded 
nerve-cells at the base of the posterior cornu (Clarke's column). Another group is seen on the outer side of the grey matter lying in a projection which is sometimes known as the lateral cornu (intermediolateral tract). This also is only distinct in the dorsal region. The cells of the posterior cornu are not collected into a special group.

Course of the nerve-roots in the spinal cord.-The anterior roots enter the anterior cornu in a number of bundles (fig. 224, $a, a$ ). Some of their fibres $(1,1)$ are directly connected with the nerve-cells there, others pass through the grey matter into the posterior cornu, others into

Fig. 224.-DI.IGRAM TO ILLUSTRATE THE PROBABLE COURSE TAKEN BY THE FIBRES OF THE NERVE-ROOTS ON ENTERING THE SPIVAT. CORD.

$a, a$, two bundles of the anterior root of a nerve; 1,1 , some of their fibres passing into the lateral cells of the anterior cornu ; 1', 1', otbers passing into the mesial cells of the same cornu; 2, 2, fibres passing to the lateral column of the same side without joining nerve-cells ; 3 , 3, fibres passing towards the posterior cornu: 4, 4, fibres passing across the anterior commissure, to enter nerve-cells in the anterior coruu of the other side; $p$, bundle of the posterior root; $p, l$, fibres of its lateral or external division coming through and around the gelatinous substance of Rolando; some of these, 5, are represented as beconing longitudinal in the latter; others, 6 , 6 , as passing towards the anterior cornu, either directly

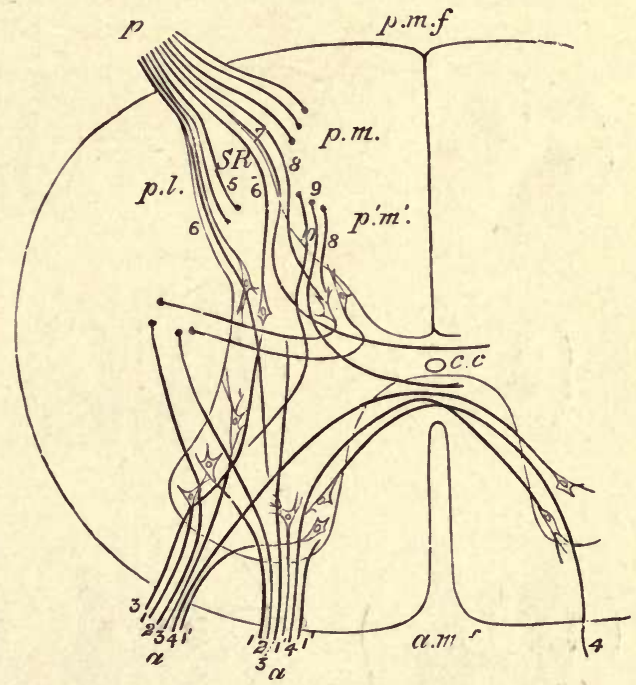

or after joining cells in the posterior cornu, and others, 7 , as curving inwards towards the grey commissure; $p, m$. fibres of the mesial or inner division, entering the posterior column and then becoming longitudinal ; $\mu^{\prime}, m^{\prime}$, fibres from a posterior root which had joined the cord lower down and entered the posterior column, now passing into the grey matter at the root of the posterior cornu. Of these, 8 is represented as entering Clarke's column, 9 , as curving around this and coursing to the anterior commissure, and 10 as passing towards the anterior cornu. The axis-cylinder processes of the cells of Clarke's column are shown arching round, and taking the direction of tho lateral column. $u_{.} m . f$. , anterior median fissure; $p . m . f$., posterior median fissure ; c.c., central canal; S.R., substautia gelatinosa of Rolaudo.

the lateral white column of the same side of the cord $(2,3)$, and others again pass across in the isthmus to the anterior cornu of the opposite side (4).

The fibres of the posterior roots (fig. $224, p$ ) chiefly pass into the posterior white columns, from which after a short course they enter the grey matter and are partly connected with its cells, and partly pass to the other side of the cord. Some of the fibres of the posterior roots enter the posterior cornu directly, and are believed to be connected with the scattered cells of that cornu.

The central canal of the spinal cord is lined by columnar eiliated epithelium-cells, which are surrounded by a.quantity of neuroglia. 
The cells are best seen in the spinal cord of animals and in the child; in the human adult they have frequently become proliferated, and their cilia are no longer visible.

Characters of the spinal cord in the several regions (fig. 225). In the cervical region the white matter, especially that of the lateral
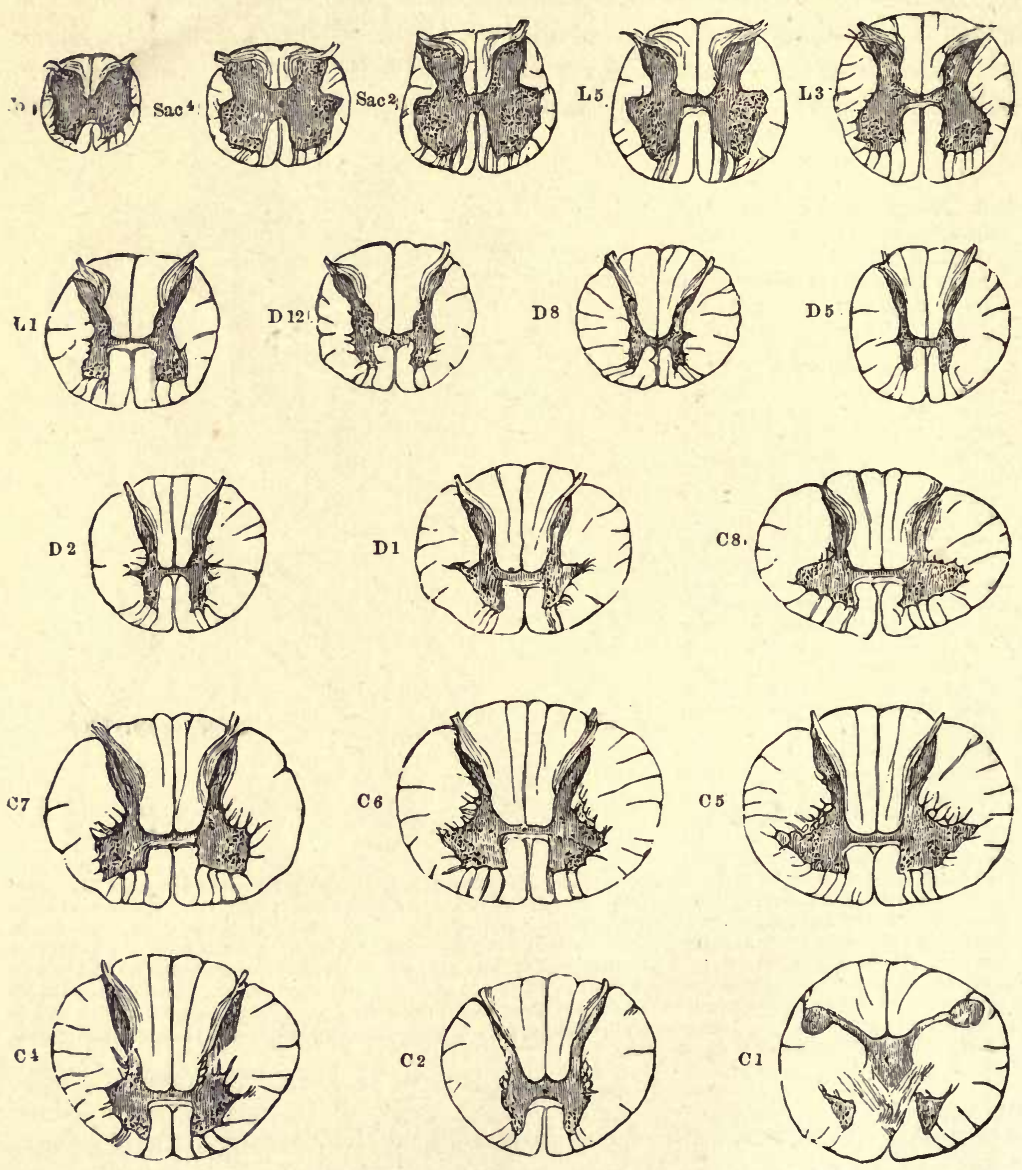

Fig. 225.-Trassyerse sections of the spinal Cord at different levels. (Twice the natural size.)

The letters and numbers indicate the position of each section; Ca. at level of coccygeal nerve ; Sac. 4 of 4 th sacral ; L3 of 3rd lumbar, and so on. The grey substance is shaded dark, and the nerve-cells within it are indicated by dots.

columns, occurs in largest proportion. The grey matter, especially in the cervical enlargement, is in considerable amount (C 5), and it encroaches in the form of a network upon the adjacent part of the lateral white column. The anterior cornua are thick and the posterior slender. The posterior median column is distinctly marked off. 
In the dorsal region the grey matter is small in amount, and both cornua are slender (D 5). The whole cord is smaller in diameter than either in the cervical or lumbar region. The column of nerve-cells known as Clarke's column, and the intermedio-lateral tract, are well marked.

In the lumbar region the crescents of grey matter are very thick, and the white substance, especially the lateral columns, relatively small in amount (L 5). The isthmus lies nearly in the centre of the cord, whereas in the cervical and dorsal regions it is nearer the anterior surface. The posterior median columns are not seen.

In the part of the spinal cord from which the sacral and coccygeal nerve-roots take origin the grey matter largely preponderates, the crescents forming thick irregular masses, and the grey isthmus being also of considerable thickness. 


\section{LESSON XXXVII.}

\section{THE MEDULLA OBLONGATA, PONS, AND MESENCEPHALON.}

1. Sections of the medulla oblongata made, $(a)$ at the level of the decussation of the pyramids, $(b)$ opposite the middle of the olivary body, and $(c)$ just above the olivary body.

2. Section through the middle of the pons Varolii.

3. Sections across the region of the corpora quadrigemina, one at the level of the inferior, the other at the level of the superior, pair.

In all the above sections sketch under a low power the general arrangement of the grey and white matter, inserting the positions of the chief groups of nerve-cells.

[The tissue is hardened and the sections are prepared, stained, and mounted in the same way as the spinal corà.]

The structure of the medulla oblongata can best be made out by the study of a series of sections taken from below upwards, and by tracing in these the changes which occur in the constituent parts of the spinal cord, taking note at the same time of any parts which may be superadded.

A section through the region of the decussation of the pyramicls (fig. 226) has much the same form as a section through the upper part

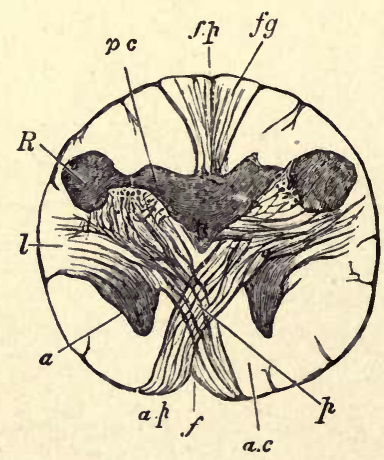

Fig. 226.-Section of the MeDULLA OBLONGATA AT THE MIDDLE OF THE DECUSSATION OF THE PYRAMIDS.

anterior, $f . p$. posterior fissure; a.p. pyramid ; $a$, remains of part of anterior cornu, separated bv the crossing bundles from the rest of the grey matter; $l$, continuation of lateral column of cord ; $R$, continuation of substantia gelatinosa of Rolando; p.c. continuation of posterior cornu of grey matter; f.g. funiculus rracilis.

of the spinal cord, and most of the structures of the cord can be easily recognised. A considerable alteration of the grey matter is, however, produced by the passage of the large bundles of the crossed pyramidal 
tract $(p)$ from the lateral column of the spinal cord on each side through the root of the anterior cornu and across the anterior median fissure to the opposite anterior column of the medulla, where, together with the fibres of the direct pyramidal tract, they constitute the prominent mass of white fibres which is seen on the front of the medulla on each side of the middle line, and is known as the pyramid. By this passage of fibres through the grey matter the tip of the anterior cornu $(a)$ is cut off from the rest and becomes pushed as it were to the side; in sections a little higher up it appears as an isolated mass of grey matter which is known as the lateral nucleus (fig. 22, n.l.)

A change also occurs in the posterior cornu in consequence of the increased development of the posterior median and cuneate fasciculi. This causes the posterior cornu (fig. 226, $p c$ ) to be pushed towards the side, the $\mathrm{V}$ which they form with one another being thus opened out; at the same time the tip of the cornu swells out and causes a prominence upon the surface of the medulla, which is known as the tubercle of Rolando $(R)$. Grey matter also soon becomes formed within the upward prolongations of the posterior median column and of the cuneate fasciculus

Fig. 227.-Section of THE MEDULIA OBLONGATA IN THE REGION OF THE SUPERIOR I'YRAMIDAL DECUSSATION.

a.m. $f$. anterior median fissure; f. $a$. superficial arciform fibres emerging from the fissure; $p y$, pyramid; not nucleus of the arciform fibres; $f^{\prime} \cdot a^{\prime}$. deep arciform fbres beconing superficial; 0 , lower end of olivary nucleus ; $0^{1}$, accessory olivary nucleus; n.l. nucleus lateralis; f.r. formatio reticularis: $t^{\prime} \|^{2}$. arciform fibres proceeding from formatio reticularis; $g$, substantia gelatino $\triangleleft$ of Rolanto; $a . V$. ascending root of fifth nerve; n.c. nucleus cuneatus; n.c. external cuneate nucleus; f.c. funiculus cmeatus; n.g. nucleus gracilis; $f . g$. funiculus gracilis; $p . m, f$. posterior median fissure; c.c. central canal surrounied by grev matter. in which are, n.XI. nucleus of the spinal accessory, and n.XII. nucleus of the hypoglossal; s.d. superior pyramidal decussation.

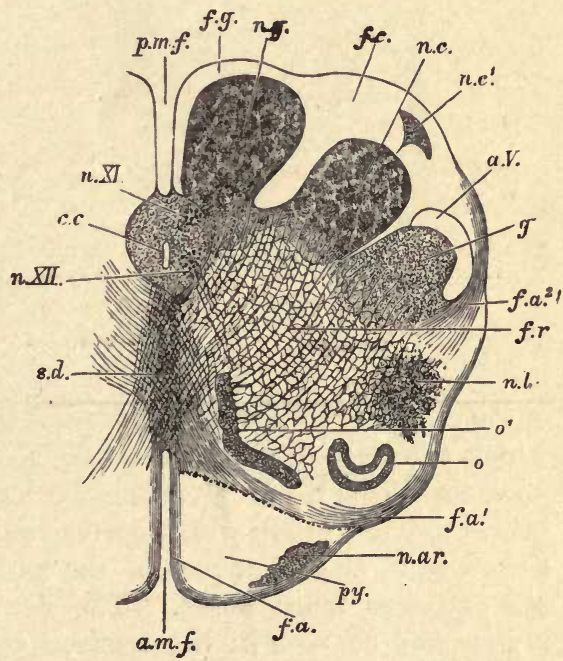

(fig. 227, n.g., n.c.), but most of the grey matter of the cornu becomes broken up, by the passage of bundles of nerve-fibres through it, into a reticular formation $(f . r)$, the production of which is already foreshadowed in the upper part of the spinal cord. The central canal of the spinal cord is still seen in the lower part of the medulla oblongata $(c . c)$, but it comes nearer to the posterior median fissure. The grey matter which surrounds it contains two well-marked groups of nerve-cells; the anterior of these is the mucleus of the hypoglossal or twelfth nerve (n.xIr.), the posterior that of the spinal accessory or eleventh $(n . X I$.$) . Instead of the$ 
comparatively narrow isthmus which joins the two halves of the spinal cord, a broad raphe now makes its appearance; this is formed of obliquely and antero-posteriorly coursing fibres, together with some grey matter containing nerve-cells.

In a section at about the middle of an olivary body (fig. 228), it will be seen that a marked change has been produced in the form of the medulla and the arrangement of its grey matter, by the opening out of the central canal into the fourth ventricle. This causes the grey matter which below surrounded the central canal to be now spread

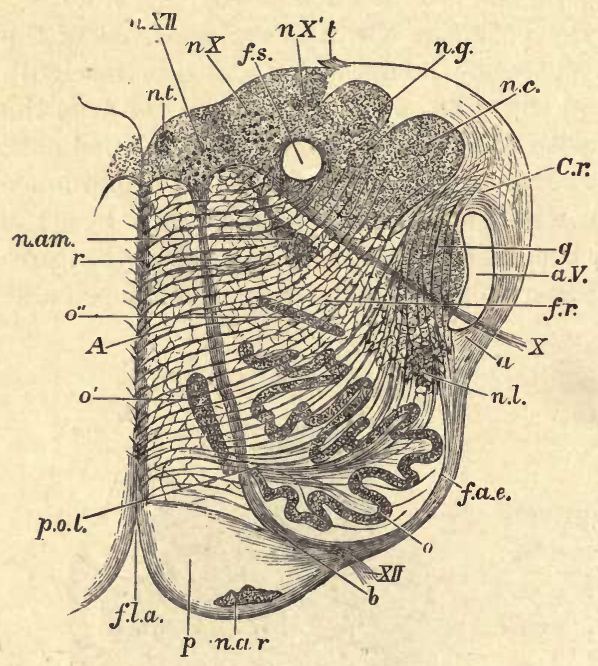

Fig. 228.-SECTION OF THE MEDULLA OBLONGATA AT ABOUT TIIE MIUILE OF THE OLIVARY BODY. $\frac{4}{3}$

f.l.a. anterior median fissure; n.ar. nucleus arciformis; $p$, pyramid; $X I I$. bundle of hypoglossal nerve emerging from the surface; at $b$ it is seen coursing betwcen the pyramid and the olivary nucleus, $o$; f.a.e. external arciform fibres; $n . l$. nucleus lateralis; $a$, arciform fibres passing towards restiform body partly through the substantia gelatinosa, $g$, partly superficial to the ascending root of the fifth nerve, a.V.; $\boldsymbol{X}$, bundle of vagus root, emerging ; f.r. formatio reticularis; $c . r$. corpus restiforme, begiming to be formed, chiefly by areiform fibres, superficial and deep; n.c. nucleus cuneatus; n.g. nucleus gracilis; $t$, attachment of the ligula; $f$.s. funiculus solitarius; n.X. n.X.'. two parts of the vagus nucleus; n.XII. liypoglossal nucleus; n.t. nucleus of the funiculus teres; n.am. nucleus ambiguus: $r$, raphe; $A$, contiuuation of anterior column of cord ; $o^{\prime}, o^{\prime \prime}$, accessory olivary nuclei; olivary nucleus; p.o.l. pedunculus olivæ.

out at the floor of that ventricle, and the collections of nerve-cells from which the hypoglossal and spinal accessory nerves respectively arose now, therefore, lie in a corresponding situation. At this level, however, the outer group which corresponds with the nucleus of the spinal accessory in the lower part of the medulla has become the nucleus of the vagus or tenth nerve. The nerve-bundles of the roots of these nerves can be seen in the sections coursing through the thickness of the medulla and emerging, those of the hypoglossal (xIr.) just outside the pyramids, those of the spinal accessory and vagus $(x$.$) at the side$ of the medulla. The two sets of emerging fibres thus appear to subdivide each lateral half of the medulla into three areas-a posterior, a middle, and an anterior. Of these the posterior is chiefly occupied by the grey matter of the floor of the fourth ventricle, and, with fibres which are passing obliquely upwards and outwards towards the cerebellum, forming its inferior crus (restiform body, c.r.); and in addition there is the continuation upwards of the portions of grey matter forming the nuclei of the funiculus gracilis (n.g.), of the funiculus cuneatus 
(n.c.), and of the tubercle of Rolando $(g)$. The anterior or mesial area is occupied in front by the pyramid $(p)$, and behind this by a reticular formation (reticularis alba, A) composed of longitudinally coursing bundles of fibres interlaced with fibres that are passing obliquely from the opposite side, through the raphe, towards the restiform body (fig. $229, r$.a.). The middle area, which lies between the issuing bundles of the two sets of nerve-roots, consists in its deeper part of a similar

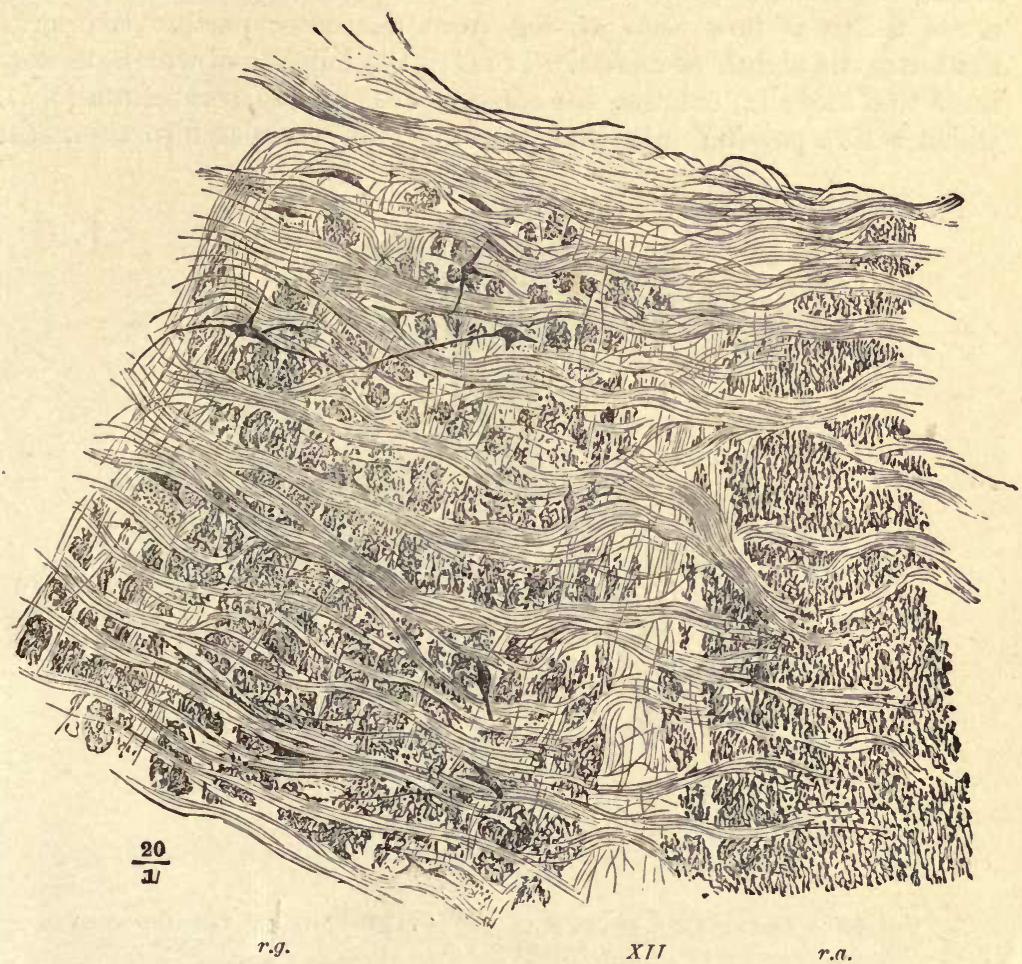

Fig. 229.-PARt of the Reticular formation of the Medulla oblongata.

r.a. reticularis alba ; r.g. reticularis grisea: between them a root-bundle of the hypoglossus $(X I I$.$) . The longitudinal fibres of the reticnlar formation are cut across ; the transversely$ coursing fibres are internal arcuate fibres, passing on the right of the figure towards the raphe.

reticular formation (fig. 228, $f r$ ), but with more grey matter and nerve-cells (reticularis grisea, fig. 229, r.g.) Superficially there is developed within it a peculiar wavy lamina of grey matter containing a large numher of small nerve-cells; this is the nucleus of the olivary body (fig. 228, o).

The floor of the fourth ventricle is covered by a layer of ciliated epithelium-cells, continuous below with those lining the central canal, and above, through the Sylvian aqueduct, with the epithelium of the 
third and lateral ventricles. The fourth ventricle is roofed over by a thin layer of pia mater, with projecting choroid plexuses, the under surface of which is covered by a thin epithelial layer continuous at the side with the ciliated epithelium of the floor.

A section taken just above the level of the olivary prominence will still show very much the same form and structural arrangements as that just described. The nucleus of the hypoglossal (fig. 230, n. XII.) is still visible in the grey matter of the floor of the ventricle, but the nerve which is now seen arising from the outer part of that grey matter is the eighth or auditory (VIII.), the bundles of which, as they leave the medulla, enbrace the inferior crus of the cerebellum (c.r.), which is now passing into that organ. The reticular formation still

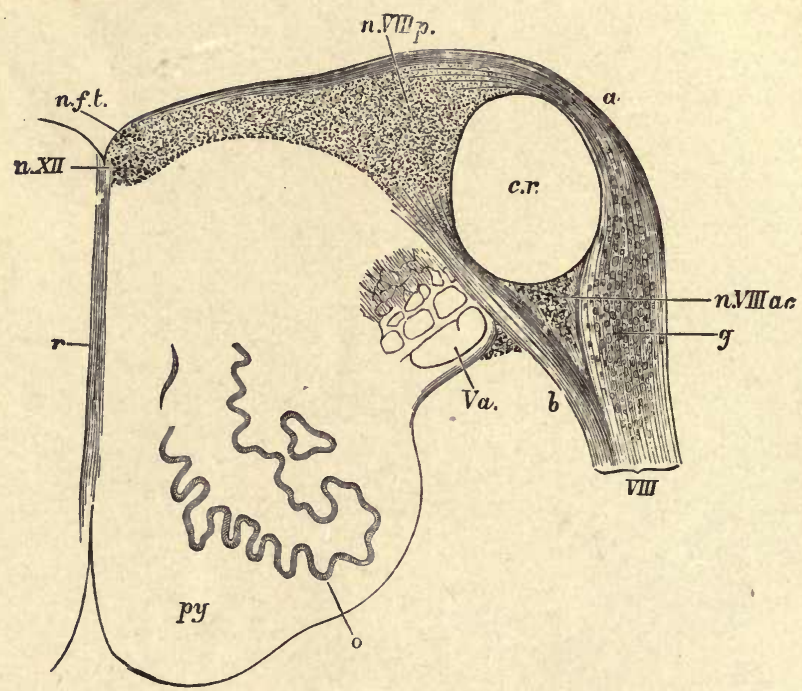

Fig. 230.-Transverse section of the UPPER PART of the MEdulla OBLONGATA. $\frac{4}{1}$

$p y$, pyramid; $o$, o'ivary nucleus; $V . a$. ascending root of the fifth nerve; VIII. inferior (posterior) root of the auditory nerve, formed of two parts, $a$, (striæ acusticæ), and $b$, which enclose the restiform body, c.r.: n.VIII.p. principal nucleus of the auditory nerve; $n$.VIII.ac. accessory nucleus; $g$, ganglion-cells in the root; n.f.t. nucleus of the funiculus teres; $n . X I I$. nucleus of the hypoglossal; $r$, raphe.

occupies the greater part of each lateral half of the medulla between the grey matter at the floor of the fourth ventricle and the pyramids $(p y)$, and a small portion of the olivary nucleus $(o)$ may still be seen, as may also the upward continuation of the grey matter of the tubercle of Rolando; this is intimately connected with some well-marked bundles of nerve-fibres, which are passing up to the pons to join eventually the root of the fifth nerve $(V a)$.

A section through the middle of the pons Varolii (fig. 231) shows very much the same arrangement of grey and white matter as that 
which is met with at the upper part of the medulla, but the general appearance of the section is much modified by the presence of a large number of transversely coursing bundles of nerve-fibres which are passing from one hemisphere of the cerebellum to the other. Intermingled with these bundles is a considerable amount of grey matter. The continuation upwards of the pyramids of the medulla $(p y)$ is embedded between these transverse bundles and separated by them from the reticular formation. The olivary nucleus is no

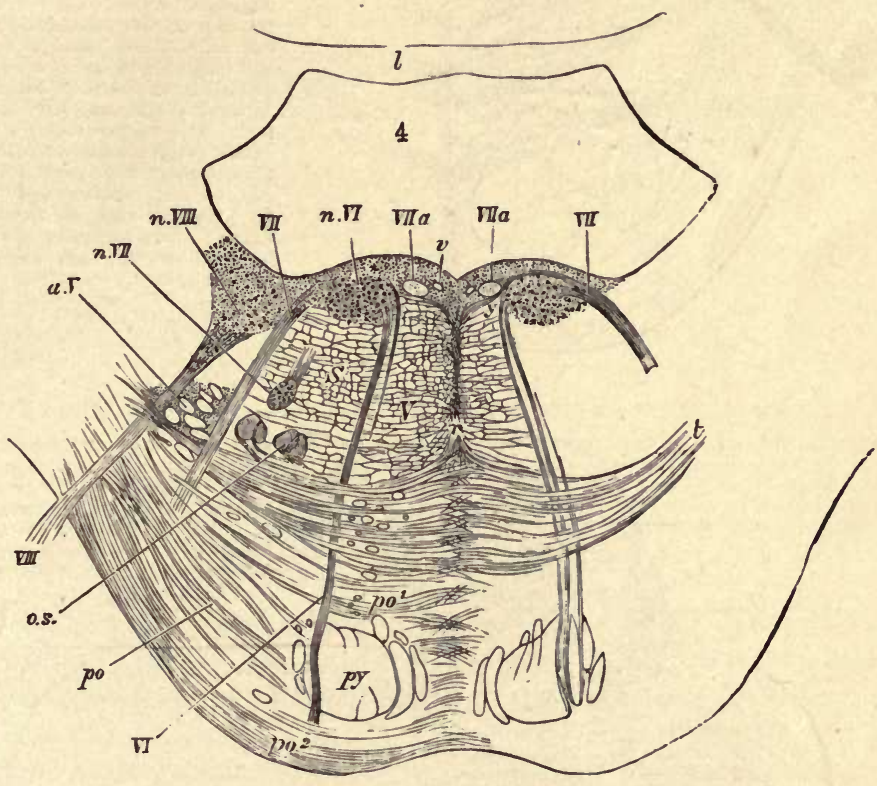

Fig. 231.-SEction ACross the pons at ABout THE MIDDLE OF THE FOURTH VENTRICLE. $\frac{3}{1}$

$p y$, pyramid-bundles continued up from the medulla; $p o$, transverse fibres of the pons passing from the middle crus of the cerebellum, before $(p o)$ and behind $\left(p o^{\prime}\right)$ the chief pyramid bundles; $t$, deeper fibres of the same set, constituting the trapezium; the grey matter between the transverse fibres is not represented either in this or in the two following figures; $r$, raphe; o.s. superior olivary nucleus; $a . V$. bundles of the ascending root of the fifth nerve, enclosed by a prolongation of the grey substance of Rolando; $V I$. the sixth nerve; $n . V I$. its nucleus ; VII. the facial nerve; VIIa. intermediate portion of the same nerve; $n . V I I$. its nucleus; VIII. anterior (superior) root of the auditory nerve; $n . V I I I$. part of its outer or anterior (superior) nucleus ; $v$, section of a vein.

longer seen, but there are one or two small collections of grey matter which lie in the antero-lateral part of the reticular formation and are known as the superior olivary nucleus (o.s.). The nerves which take origin from the grey matter of this region are part of the eighth, the seventh, the sixth, and somewhat higher up the fifth cranial nerves (see figs. 231, 232). Of these the eighth and fifth take origin from groups of nerve-cells which occupy the grey matter opposite the external border of the floor of the ventricle; the sixth from a group which is placed also 


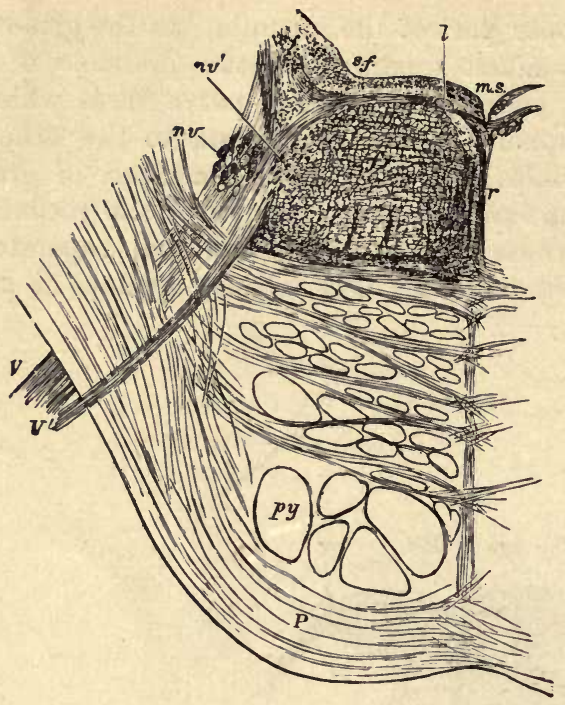

Fig. 232.-Oblique SEction OF THE PONS ALONG THE LINE OF EXIT TRAVERSED BY THE FIETH NERVE. $\frac{3}{1}$

The section passes through the lower part of the motor nucleus $\left(n^{\prime} v\right)$ from which a bnndle of fibres of the motor root is seen passing, $V^{\prime}$; a part of the upper sensory nucleus $(n v)$ is also shown in the section in the form of a number of small isolated portions of grey matter. Amongst these are a few bundles of the ascending root cut across, but most of these have already become diverted outwards to join and assist in forming the issuing part of the main or seusory root, $V ; l$, small longitudinal bundle of fibres near the median sulcus (m.s.), passing ontwards to join the root of the fifth nerve; $f$ formatio reticularis : $r$, raphe; $s, f$. substantia ferruginea.

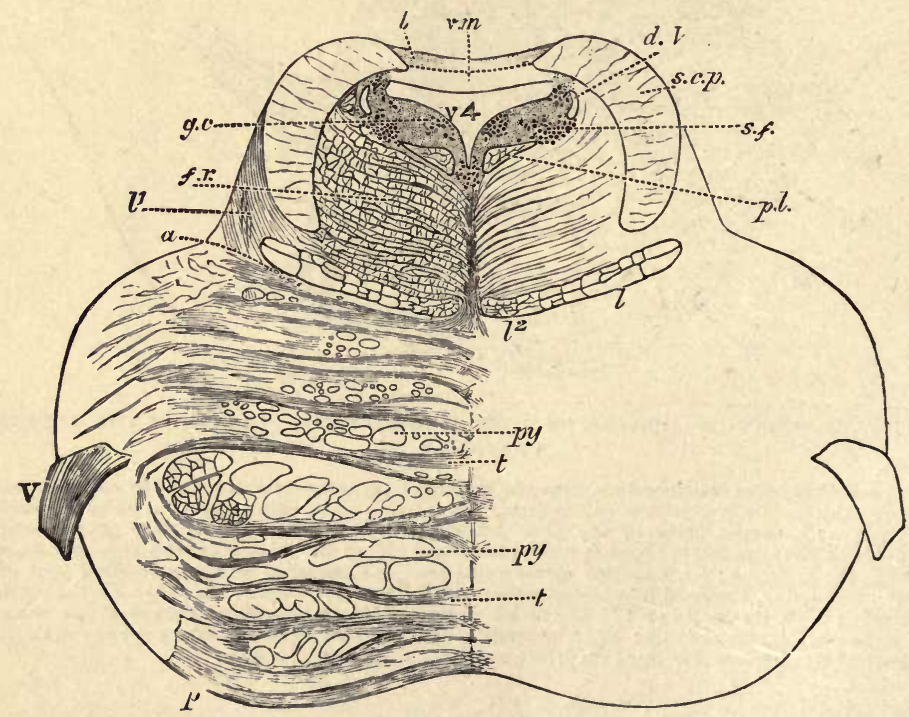

Fig. 233.-Transverse section throtgh the UpPer PART of the PoNs. more than twice the natural size.) 1

$p$, transverse fibres of the pons; $p y, j y$, bundles of the nyramils; $n$, boundary line between the tegmental part of the pons and its ventral part; $l^{\prime}$. oblique fibres of the tillet, passing towards $l, l^{2}$, longitulinal fibres of the fillet; $f . r$. formatio reticnlaris; $p . l$. posterior longitudinal bundle ; s.c.p. superior cerebellar peduncle; $v . m$. superior inedullary velum ; $b$, grey matter of the lingula; $v, 4$, fourth ventricle; in the grey matter which bounds it laterally are seen, $\boldsymbol{d . V}$, the descending root of the fifth nerve, with its nucleus, s.f. substantia ferruginea, g.c. group of cells continuous with the nucleus of the aqueduct.

1 The details of this and of several of the precerling figures are filled in under a somewhat higher magnifying power than that used for tracing the outlines. 
in the grey matter of the floor of the ventricle but nearer the middle line, and the seventh from a nucleus which lies in the formatio reticularis, and from which the fibres of the nerve pass backwards to the floor of the ventricle, then longitudinally upwards for a short distance, finally bending forwards and downwards and emerging between the transverse fibres at the sides of the pons.

At the upper part of the pons (fig. 233) the fourth ventricle narrows considerably towards the Sylvian aqueduct, and behind and on either

A

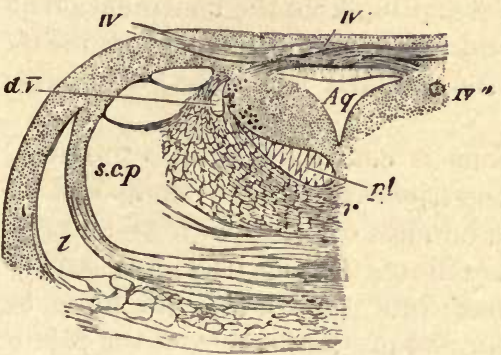

B

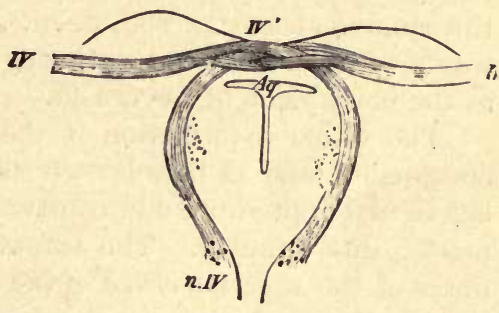

Fig. 234.-SEctions throcgh THE ORIGIN OF THE FOURTH NERVE. $\frac{3}{1}$

A, transverse section at the place of emergence of the nerve-fibres. B, oblique section carried along the course of the bundles from the nucleus of origin to the place of emergence. $A q$, Sylvian aqueduct, with its surrounding grey matter: $I V$. the nerve-bundles emerging : $I V^{\prime}$. decussation of the nerves of the two sides; $I V^{\prime \prime}$. a round bundle passing downwards by the side of the aqueduct to emerge a little lower down; n.IV. nucleus of the fourth nerve. $l$, fillet; s.c.p., superior cerebellar peduncle; $d . V$. descending root of the fifth nerve; $p l$, posterior longitudinal bundle; $r$, raphe.

side of it two considerable masses of longitudinal white fibres make their appearance. These are the superior crura of the cerebellum (s.c.p.), and they tend as they pass upwards gradually to approach the middle line (fig. $234 \mathrm{~A}$ ), across which in the region of the posterior pair of the corpora quadrigemina they pass, decussating with one another, to the formatio reticularis of the opposite side (fig. 235, A).
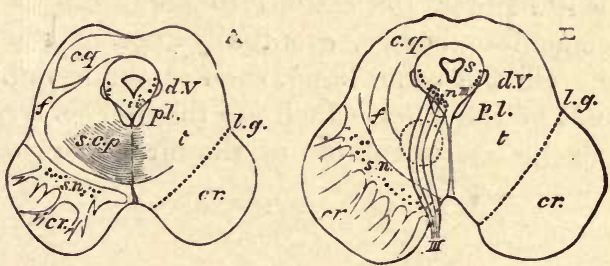

Fig. 235.-Outline of two sections across the nesencephalon. (Natural size.)

A, through the middle of the inferior corpora quadrigemina. $\mathrm{B}$, through the middle of the superior corpora quadrigemina. $c r$, crusta ; s.n. snbstantia nigra; $t$, tegmentum; $s$, Sylvian aqueduct, with its surrounding grey matter; c.q. grey matter of the corpora quadrigemina; l.g. lateral groove; $p . l$. posterior longitudinal bundle; $d . v$. descending root of the fifth nerve; s.c.p. superior cerebellar periuncle $f$, fllet; $n . I I I$. its nucleus; $I I I$. third nerve. The dotted circle in $B$ indicates the situation of the tegmental nucleus.

In sections across the mesencephalon (fig. 235), the upward continuity of the parts which have thus been described in the lower parts of the nerve-centres, can still in great measure be traced. 
The Sylvian aqueduct $(s)$, with its lining of ciliated epithelium, represents the central canal of the cord and the fourth ventricle of the medulla. In the grey matter which surrounds it (central grey matter) there is seen in all sections of the region a group of large nerve-cells lying anteriorly on each side of the middle line, close to the reticular formation. From this group the root-bundles of the fourth nerve arise at the lower part of the mesencephalon and pass obliquely backwards and downwards around the central grey matter, decussating with those of the opposite side to emerge just above the pons Varolii (fig. 234). Higher up the bundles of the third nerve spring from the continuation of the same nucleus (fig. 235, n. IIr.), and these pass forwards and downwards with a curved course through the reticular formation, to emerge at the mesial side of the crusta.

The reticular formation of the pons is continued up into the mesencephalon, and is here known as the tegmentum. It is composed as before of longitudinal and transverse bundles of fibres with much grey matter intermingled. The transverse fibres include the decussating fibres of the superior crura of the cerebellum ;s.c.p.), and the fibres of the fillet $(f)$, which are passing in an oblique manner from the raphe to the side of the mesencephalon, to reach eventually the grey matter of the prominences of the corpora quadrigemina. The pyramid bundles of the pons are continued upwards on each side into the crusta (cr.). This forms a mass of longitudinally coursing bundles of fibres lying on the ventral aspect of each half of the mesencephalon, and diverging above into the internal capsule of the cerebral hemisphere. The crusta is separated from the tegmentum by a layer of grey matter containing a number of very deeply pigmented nerve-cells which give it the name of substantia nigra (s.n.) The crusta and tegmentum, together with the intervening substantia nigra, constitute the crus cerebri.

The prominences of the corpora quadrigemina are formed mainly of grey matter containing numerous small nerve-cells. From each a bundle of white fibres (brachium) passes upwards and forwards towards the geniculate bodies, eventually joining the optic tract of the same side. On the other hand, each of the prominences receives from below fibres of the fillet, which are themselves traceable into the posterior part of the lateral columin of the medulla oblongata. 


\section{LESSON XXXVIII.}

\section{STRUCTURE OF THE CEREBELLUM AND CEREBRUM.}

1. Sectrons of the cerebellum across the direction of the laminæ and vertical to the surface.

2. Section across the whole of one hemisphere of the cerebrum of a monkey or man, passing through the middle of the third ventricle.

3. Vertical sections of the cerebral cortex, one from the ascending frontal gyrus, another from the occipital lobe, and a third across the hippocampal gyrus and hippocampus.

4. Transverse sections of the olfactory tract and bulb.

In all these preparations make sketches under a low power of the general arrangement of the grey and white matter, and also of the nerve-cells in the grey matter. Sketch some of the details under a high power.

The cerebellum is composed of a white centre, and of a grey cortex, both extending into all the folds or laminæ, so that when the laminæ are cut across, an appearance is presented of a white arborescence

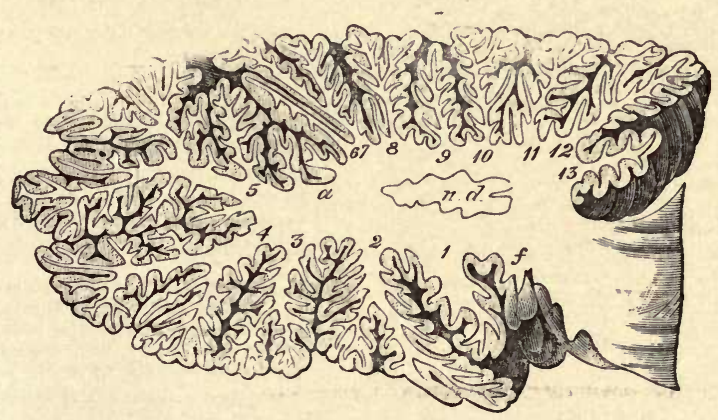

Fig. 236.-Section throvgh oNe of the hemispheres of the CEREbellum, TO SHOW THF MEDULLARY CENTRE AND ITS PROLONGATIONS INTO THE LAMELLE.

covered superficially by grey matter. The white matter is in largest amount in the middle of each cerebellar hemisphere (fig. 236). There is here present also a peculiar wavy lamina of grey matter, similar to that in the olivary body, and known as the mucleus dentatus (n.d.). 
Other isolated grey nuclei lie in the white matter of the middle lobe.

The grey matter of the cerebellum consists of two layers (fig. 237). The inner one (that next the white centre) is composed of a large. number of very small nerve-cells (granule layer, $d$ ). The outer layer

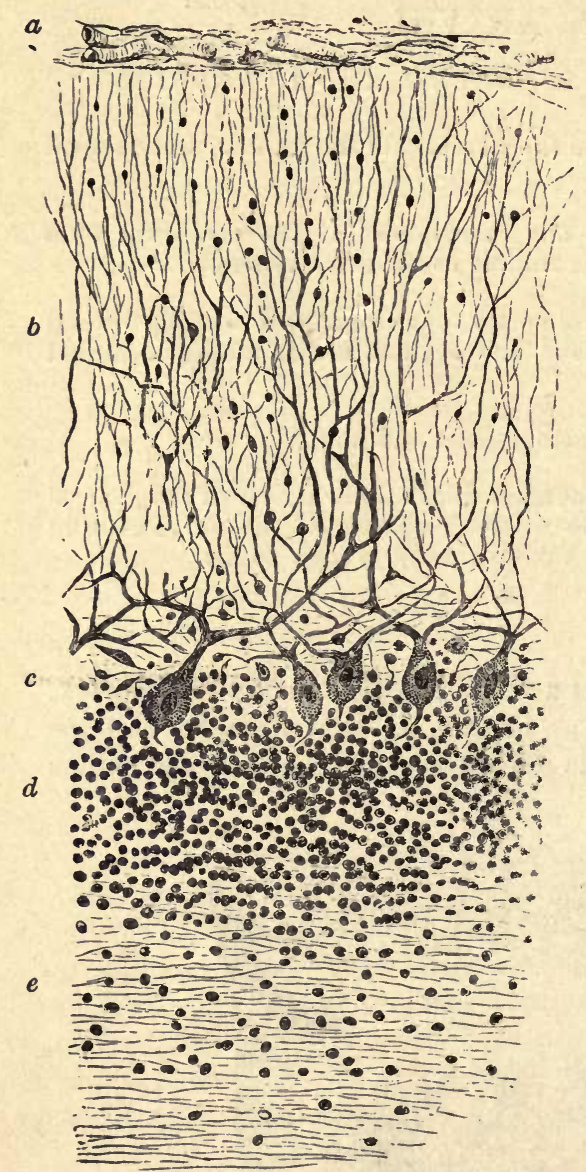

Fig. 237.-Section of CORTEX OF CEREBELLUM.

$a$, pia mater ; $b$, external layer ; $c$, layer of corpuscles of Purkinje; $d$, inner or granule layer; $e$, medullary centre.

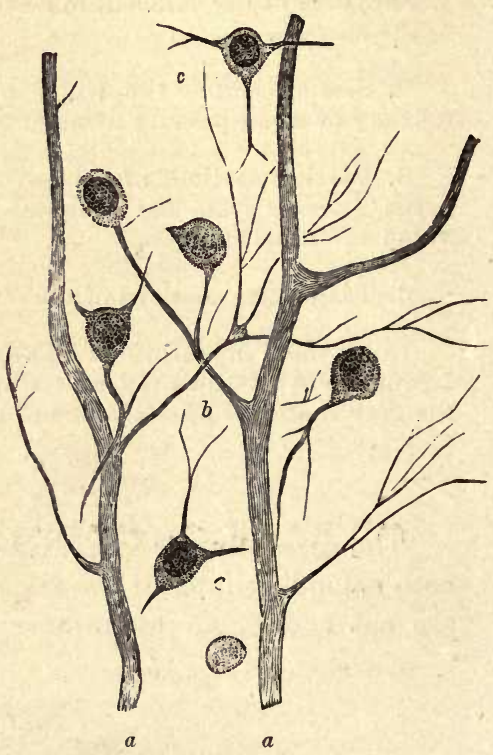

Fig. 238.-Processes of the corpusCles OF PURKINJE, CONNECTED BY THEIR FINER BRANCHES WITH CORPUSCLES OF THE OUTER LAYER OF THE CEREBELLAR CORTEX. (Highly magnified.)

$a, a$, processes of the corpuscles of Purkinje; $b$, a branch from one of them, which is connected with two of the corpuseles of the outer layer ; $c$, $c$, scattered branched corpuscles of this layer.

(b) is thicker, and is formed of neuroglia, with rounded and angular small nerve-cells and neuroglia-cells scattered through it. Into its outer part processes of the pia mater conveying blood-vessels pass vertically, and there are also in this part a number of long tapering cells, somewhat like the Müllerian fibres of the retina. Lying 
between the two layers of the grey matter is an incomplete stratum of large flask-shaped cells (cells of Purkinje, c). Each of these gives off from its base a fine process, which becomes the axis-cylinder of one of the medullated fibres of the white centre, while from the opposite pole of the cell large ramified processes spread out into the superficial layer of the grey matter, and some of these processes become connected with its small nerve. cells (fig. 238).

Structure of the cerebrum. The grey matter of the cerebral cortex is described as being composed of a number of layers, but they are not sharply marked off from one another. The following are. usually distinguished (fig. 239).

1. Most extemally a thin stratum containing a few scattered cells, probably neuroglia-cells. In the most superficial part of this layer immediately under the pia mater, is a very thin stratum of medullated nerve-fibres.

2. A layer of closely set small pyramidal nerve-cells several deep. This layer is also thin.

3. A thick layer containing larger and longer pyramidal cells less closely set. These cells are largest in the deepest part of the layer; and in the region of the motor centres of the brain some of them are of very large size, and are collected into small groups.

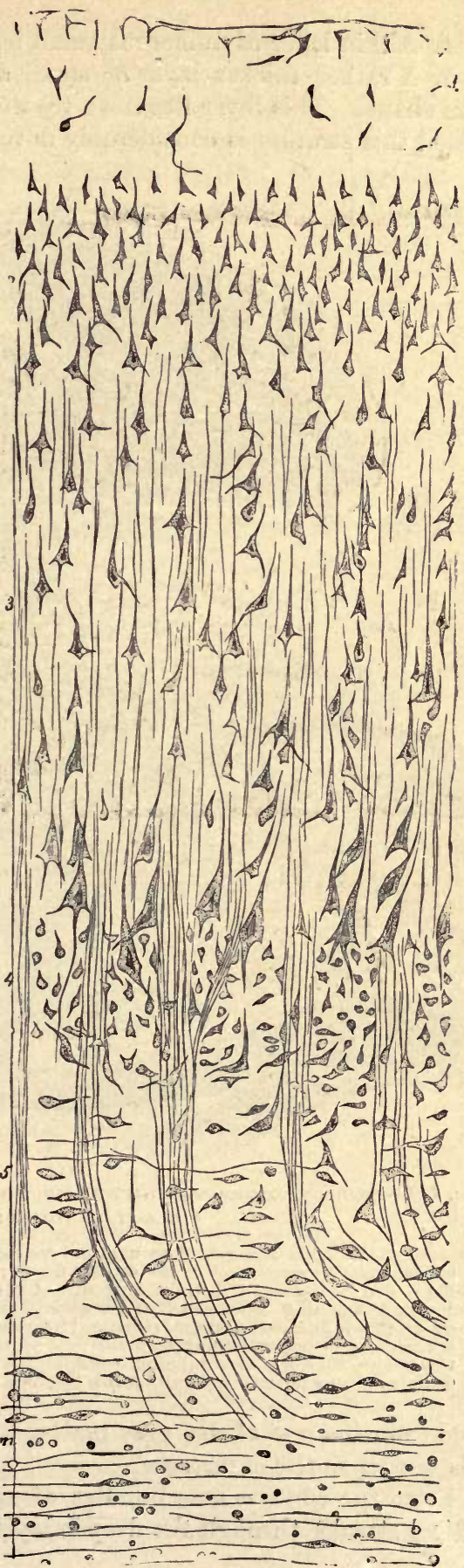


4. A thin layer of numerous small irregular angular cells.

5. A rather thicker layer of small scattered cells, many of a fusiform shape. This layer lies next to the white centre. In the island of Reil this stratum is considerably developed, and is somewhat sepa-

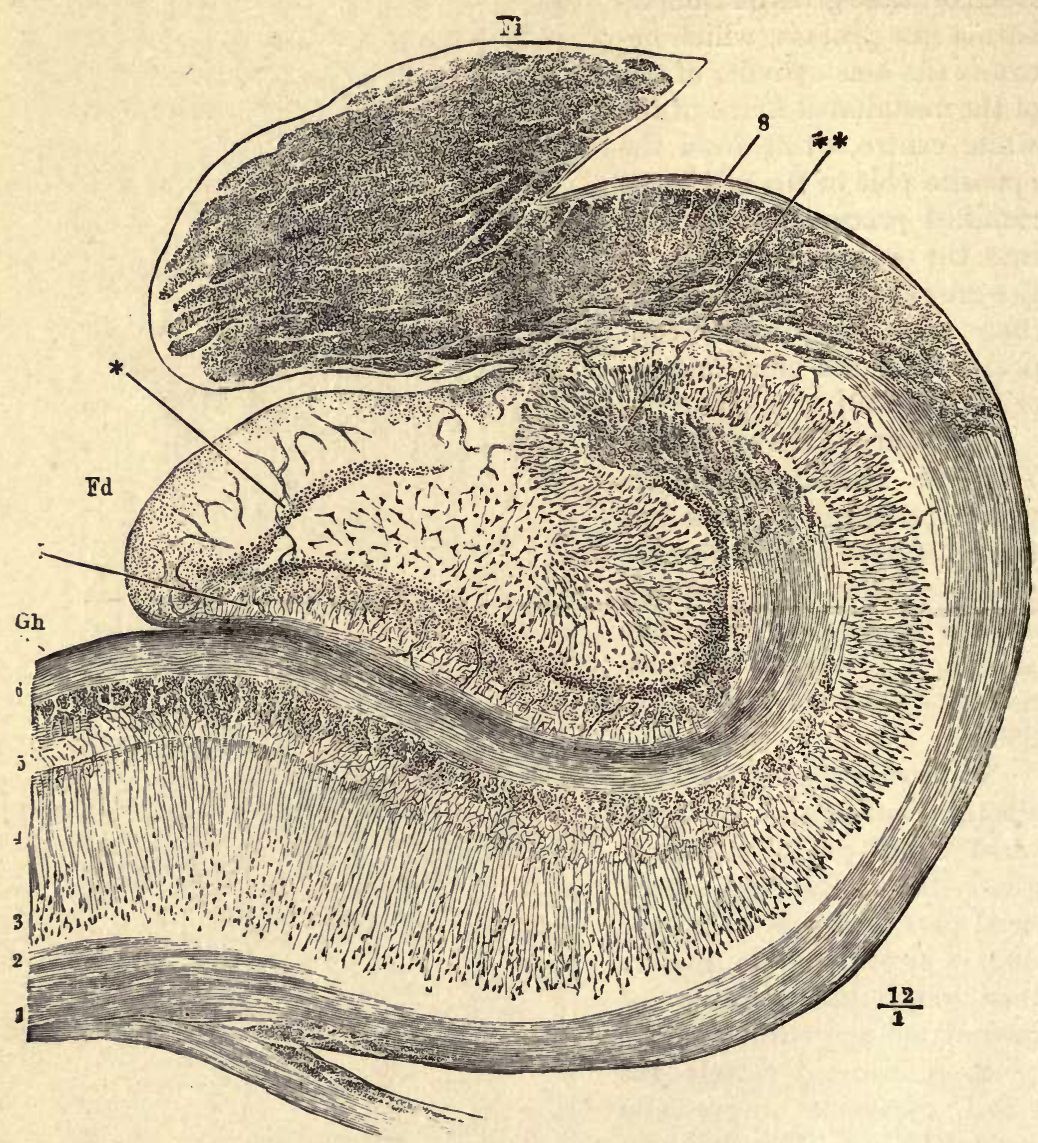

Fig. 240.-Section aCross the hippocampus major, Dentate fissure, Dentate FASCIA AND FIMBRIA.

$G h$, part of the gyrus hippocampi or uncinate convolution; $F d$, fascia dentata, or dentate convo'ution; between them is the dentate fissure; Fi, fimbria, composed of longitudinal fibres here cut across; 1,2 , medullary centre of the hippocampal girus prolonged around the hippocampus, $H$, as the so-called alveus, into the fimbria; 3 , layer of large pyramidal cells ; 4, their processes (stratum radiatum) ; 5 , reticular neurogia (stratum lacinio 6 , superficial medullary lamina, invo uted around the dentate fissure ; $\$$, termination of this lamina, the fibres here running longitudinally; 7 , superficial neuroglia of the fascia dentata ; *, ring of small cells within this (stratum granulosum).

rated from the rest of the grey matter by a layer of white substance. It is known as the claustrum.

From the white centre bundles of medullated nerve-fibres pass in vertical streaks through the deeper layers of the grey matter, to lose 
themselves amongst the pyramidal cells of the more superficial layers, with processes from which they are probably continuous.

In the hippocampal region a remarkable difference of structure manifests itself (fig. 240). The superficial layer of neuroglia and the white stratum, which overlies it as a thin band in other parts of the cortex, are in this region both very strongly marked $(5,6)$, the neuroglia layer having a very distinctly reticular aspect, and being in part beset with small cells. All the rest of the thickness of the grey matter appears to be mainly composed of, or at least to contain, long conical cells $(3,4)$, the distal processes or apices of which are prolonged into fibres which lose themselves in the superficial layer of neuroglia. The pyramidal cells rest upon the white centre, here known as the alveus ( 1 ), which is the part of the hippocampus seen within the ventricle, and which is prolonged externally into the fimbria $(\mathrm{Fi})$, where its fibres become longitudinal in direction.

In the dentate gyrus (fascia dentata, fig. $240, \mathrm{Fd}$ ) the pyramidal cells are arranged in an irregularly radiating manner, occupying the centre of the convolution, and surrounded by a ring of closely packed small cells (*), external to these being the relatively thick layer of superficial neuroglia $(\overline{)})$.

The olfactory tract is an outgrowth of the brain which was originally hollow, and remains so in many animals; but in man the cavity has become obliterated, and the centre is occupied by neuroglia, containing, however, no nerve-cells (fig. 241). Outside the central

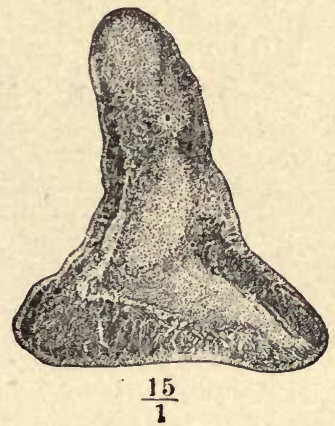

Fig. 241.-Section ACross the OLFACtory tract.

neuroglia lies the white or medullary substance, consisting of bundles of longitudinal white fibres. Most externally is a thin superficial layer of neuroglia.

The olfactory bulb (fig. 242) has a more complicated structure. Dorsally there is a flattened ring of longitudinal white bundles enclosing neuroglia $(1,2,3)$, as in the olfactory tract, but below this ring a number of layers are superadded as follows:

1. A gramule layer (fig. 242,4 ), characterised by the presence of a 
large number of small nerve-cells with reticulating bundles of medullated nerve fibres running between them.

2. An intermediate nerve-cell layer (6) consisting of neuroglia, in which small pyramidal nerve-cells are embedded. This layer is also traversed by medullated nerve-fibres, and is partly separated from the granule layer by irregular clefts (5).

3. The layer of olfactory glomeruli (7) consists of rounded nests of small ganglion-cells, which appear to give origin to the olfactory nervefibres. These pass between the glomeruli to enter the close plexus of non-medullated nerve-fibres which lies directly over the cribriform plate.

4. This is the layer of olfactory nerve-fibres (8), and from it branches (*) pass directly downwards to the nasal fossæ.

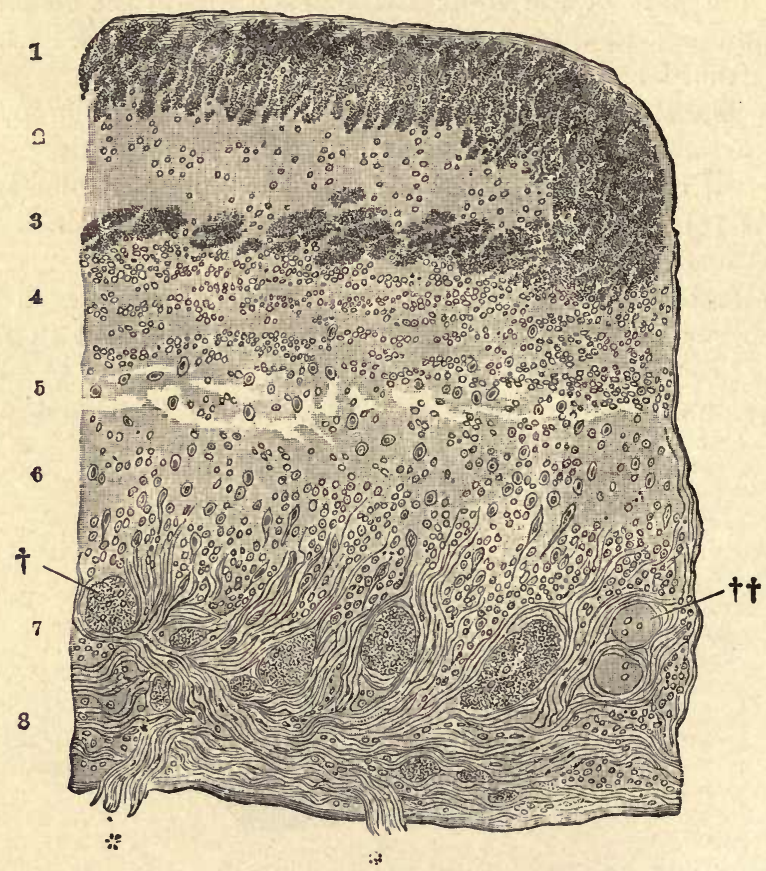

Fig. 242.- Section ACross a part of the Olfactory bULb.

1, 3, bundles of very fine transversely out nerve-fibres, forming the flattened medullary ring, enclosing the central neuroglia, $2 ; 4$, grannle-layer; 5 , loose tissue with irregular spaces (? lymphatic); 6 , intermediate layer; 7 , layer of olfactory glomeruli, $t, \dagger+8$, layer of olfactory nerve-fibres.

Basal ganglia.--Besides the grey matter of the cerebral cortex the cerebral hemispheres conceal in their deeper parts certain other masses of grey substance (fig. 243). The principal of these are the corpus striatum (nucleus caudatus, $c$, and mucleus lenticularis, str) and optic thalamus $(t h)$. Between them run the bundles of white fibres which are 
passing upwards from the crus cerebri, forming a white lamina termed the internal capsule. Above the level of these nuclei the internal capsule expands into the medullary centre of the hemisphere (see also figs. 244 and 245 , with their description).

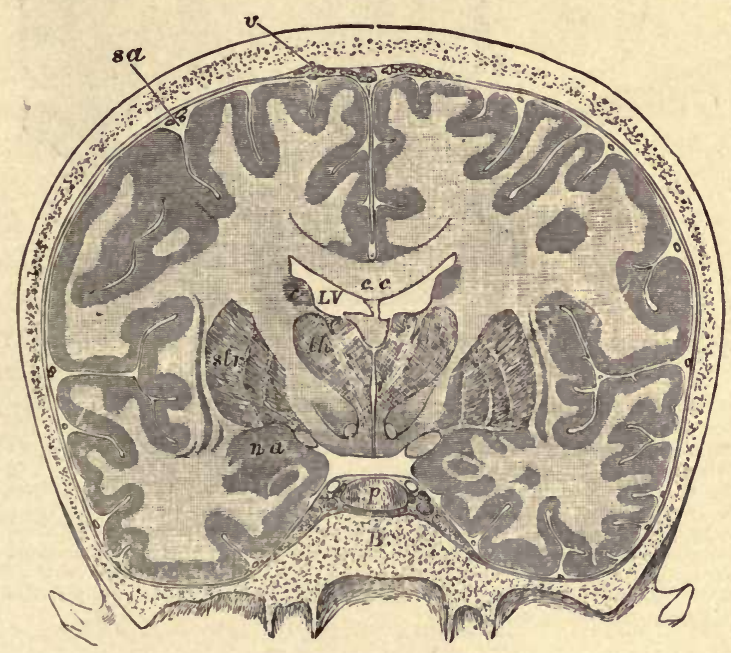

Fig. 243.-Frontal section throUgi trie Brain AND SKULl MADE Whilst FROZEN. $\frac{1}{2}$

\begin{abstract}
c.c. corpus callosum; below its middle part the septum lucidum, and below that again the fornix; $L V$, lateral veutrisle; $t h$, thalanus ; between the two thalami the third ventricle is seen; below the thalamus is the subthalamic prolongation of the tegmental region, and below this the prolongation of the crusta; st $r$, lenticular nucleus of the corpus striatum; $c$, caulate nucleus of the same; between $c, t h$, and $s / r$, the internal capsule is seen ; outside $s t r$ is the thin grey band of the claustrum, and outside this again the island of Reil at the bottom of the Sylvian fissure; n.u. nucleus amygdalæ; immediately within this is the optic tract seen in section; $p$, pituitary body ; $B$, body of the sphenoid bone ; $s a$, subaracbnoid space; $v$, villi of the arachnoid.
\end{abstract}

The nucleus candatus of the corpus striatum is composed of a reddish-grey neuroglia containing both moderately large and small multipolar nerve-cells. It receives fibres from the part of the internal capsule which separates it from the nucleus lenticularis, and next the lateral ventricle it is covered by a thin layer of neuroglia, and over this by the epithelium of the cavity.

The nucleus lenticularis, which corresponds in position internally with the island of Reil externally, is divided by two white laminæ into three zones. It is separated from the nucleus caudatus and optic thalamus by the internal capsule (figs. $244,245, i c$ ), which consists of the bundles of medullary fibres which are passing between the white centre of the hemisphere and the crus cerebri ; it receives on its inner side many white fibres from the capsule, and these impart to it a radially striated aspect. Many of the nerve-cells of the nucleus lenticularis contain yellow pigment. 


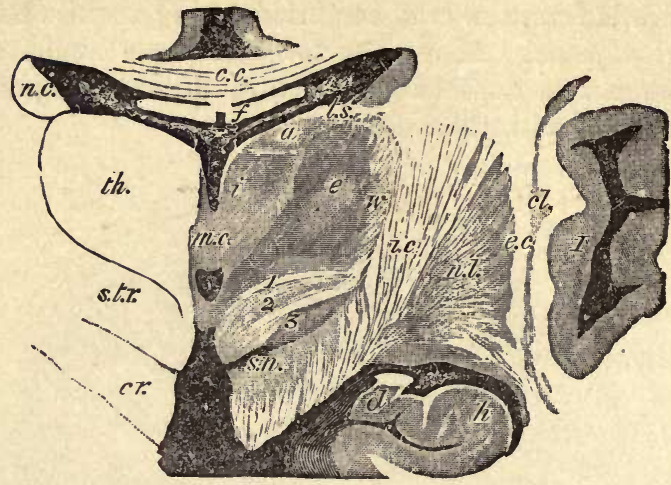

Fig. 244.-Section across the optic thalamus and corpus striatum in the REGION OF THE MIDDLE COMMISSURE. (Natural size.)

th, thalamus; $a, e, i$, its anterior, external, and internal nuclei respectively ; $v$, its external white layer ; m. $c$. midd e commissure; $v$. 3 . third ventricle; a small part is also seen below the middle commissure ; $c$. $c$. corpus callosum ; $f$, foruix, separated from the third ventricle and thalamus by the velum interpositum. In the middle of this are seen the two veins of Galen and the choroid plexuses of the third ventricle; and at its edges the choroid plexuses of the lateral ventricles, v.l.; t.s. tænia semicircularis; $c r$, forward prolougation of the crusta passing laterally into the internal capsule, $i . c . ; s . t$. $r$. subthalamic prolongation of the tegmentum, consisting of (1) the dorsal layer, (2) the zona incertan and (3) the corpus subthalamicum ; s. $n$. substantia nigra; $n$. $c$. nucleus caudatus of the corpus striatum; n.l. uncleus lenticularis ; e. $c$. external capsule ; cl. claustrum; $I$, island of Reil ; $h$, hippocampus ; $d$, fascia dentata.

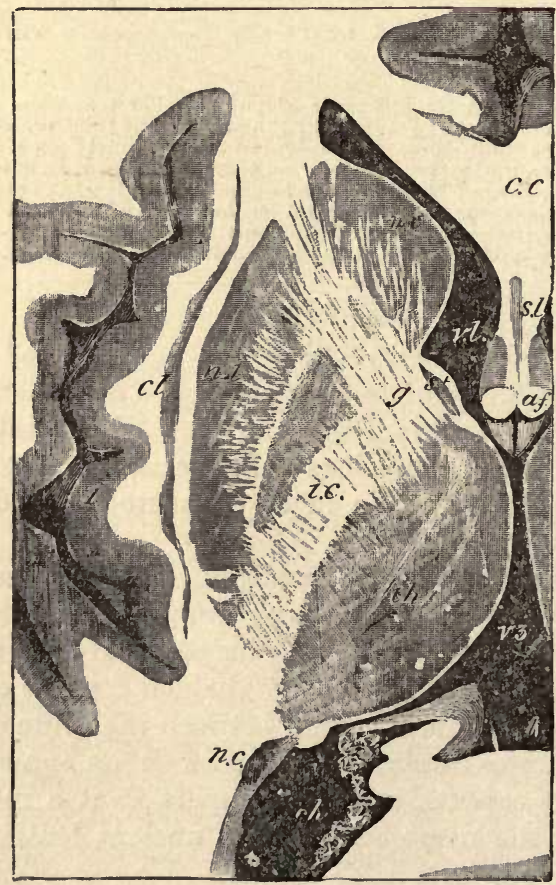

Fig. 245.- HorizontaL SECTION THROUGH THE MIDULE OF THE RIGHT CEREBRAL HEMISPHERE. (Natural size.)

v.l. lateral ventricle, anterior cornu; c. c. corpus callosum ; s.l. septum lucidum; a. $f$. anterior pillars of the fornix; $v .3$, third ventricle; th, thalamus opticus; s.t. stria terminalis; n. $c$. nucleus caudatus, and n. $l$. nucleus lenticu'aris of the corpus striatum; $i$. $c$. internal capsule; $g$, its angle or genu; $n$. $c$. tail of the nuc eus caudatus appearing in the descending cornu of the lateral ventricle; $c l$, claustrum; $I$, island of Reil. 
The optic thalamus, which lies at the side of the third ventricle and forms part of the floor of the lateral ventricle, is covered externally by a layer of white fibres, most marked next to the internal capsule, fibres from which pass into the thalamus and serve to connect it with the hemisphere.

The grey matter of the thalamus (fig. 244) is partially subdivided by an oblique white lamina into a smaller, inner $(i)$, and a larger, outer, nucleus $(e)$; these contain a number of small scattered nervecells. Anteriorly another portion of grey matter $(a)$ is divided off in a similar way; this contains comparatively large nerve-cells.

Attached to the optic thalamus below and externally are the two geniculate bodies which are connected with the optic tract. The outer geniculate body has a lamellated structure consisting of alternating layers of grey and white matter.

The tegmentum of the crus cerebri is prolonged below the thalamus opticus into a mass of grey substance, with longitudinally and obliquely crossing white bundles, which is known under the name of subthalamic region. In it at least three parts differing from one another in structure may be distinguished (see fig. $244,1,2,3$ ).

The pineal gland, which is developed in the roof of the third ventricle, is composed of a number of tubes and saccules lined and sometimes almost filled with epithelium, and containing deposits of earthy salts (brain sand). These may, however, occur in other parts

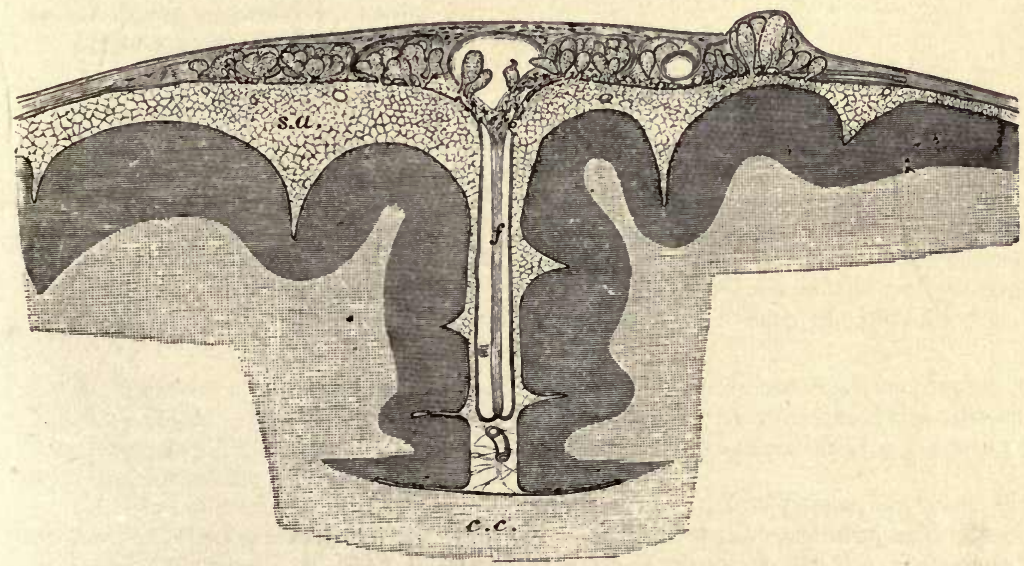

Fig. 246.-SECTION OF THE UPPER PART OF THE BRAIN AND MENINGES TO SHOW THE Relations of the arachNoidal villi. (Magnitied.)

c.c. corpus callosum; $f$, falx cerebri ; s.a. subarachnoid space, pervaded by a network of fine trabecu'æ; from it the fungiform vi'li are seen projecting into the dura mater. Some are projecting into the superior longitudinal sinus, s.

of the brain as well. The follicles are separated from one another by vascular connective tissue derived from the pia mater.

The pituitary body is a small reddish mass which lies in the sella 
turcica, and is connected with the third ventricle by the infundibulum. It consists of two lobes, a larger anterior, and a smaller posterior. The anterior lobe is originally developed as a hollow protrusion of the buccal epithelium. It consists of a number of tubules, which are lined by epithelium, and united by connective tissue. In some of the tubes the epithelium is ciliated, and sometimes a colloid substance like that occurring in the vesicles of the thyroid has been found in them.

The posterior lobe of the pituitary body, although developed from the floor of the third ventricle, contains scarcely any perceptible nervous elements in the adult. It consists chiefly of vascular connective tissue.

The membranes of the brain are similar in general structure to those of the spinal cord, p. 185. The dura mater is, however, more closely adherent to the under surface of the bony cavity than is the case in the vertebral canal. The arachnoid is in many places close to the dura mater, and separated by a wide subarachnoid space, which is bridged across by finely reticulating bands of areolar tissue (subarachnoid trabeculæ, fig. 246, s.a.) from the pia mater. In the vicinity of the longitudinal sinus, small rounded elevations (arachnoidal villi, Pacchionian glands) project into the dura mater, and even become embedded in the skull itself. 


\section{LESSON XXXIX. AND XL. \\ STRUCTURE OF THE EYELIDS AND OF THE PARTS OF \\ THE EYEBALL.}

\section{LESSON XXXIX.}

1. Sectrons of the eyelid vertical to its surfaces and transverse to its long axis. The lid should be hardened in alcohol, and the sections may be stained with hæmatoxylin and mounted in the usual manner.

Notice the long sacculated Meibomian glands lying in dense connective tissue close to the conjunctival surface, and their ducts opening at the margin of the lid. External to these the small fibres of the orbicularis pal. pebrarum cut across; a few of the fibres of the muscle lie on the conjunctival side of the duct. A short distance from the Meibomian gland may be observed another tolerably large sebaceous gland; outside this again are the eyelashes. In the skin covering the outer surface of the eyelid a few small hairs may be seen. At the attached part of the eyelid are some bundles of involuntary muscular fibres cut longitudinally in the section, and in the upper eyelid the fibrous attachment of the elevator muscle may be observed attached to the dense connective tissue.

Make a genera] sketch under a low power.

2. Sections through the posterior part of an eyeball that has been hardened in Müller's fluid. The sections are stained and mounted in the usual way. These sections will show the relative thickness of the several coats and the layers of which each coat is formed. Sections which pass through the point of entrance of the optic nerve will also exhibit the manner in which the nerve-fibres pierce the several coats to reach the inner surface of the retina. The modifications which are found in the neighbourhood of the yellow spot may also be made out if the sections have been taken from the human eye.

3. Sections of the anterior half of an eyeball which has been hardened in Muiller's fluid. These sections should pass through the middle of the cornea The lens may be left in situ, but this renders the preparation of the sections and the mounting of them much more difficult.

In these sections make a general sketch under a low power, showing the relations of the several parts one with another; and study carefully, and sketch in detail, the layers of the cornea, the place of junction of the cornea and sclerotic, the ciliary muscle, the muscular tissue of the iris, the mode of sus. pension of the lens, and the pars ciliaris retinæ.

4. Mount in glycerine thin tangential sections of a cornea stained with chloride of gold by Cohnheim's method. Sketch three or four of the con. nective-tissue cells (corneal corpuscles). The arrangement and distribution of the nerve-fibres and their termination amongst the epithelium-cells as shown in chloride of gold preparations have been already studied (Lesson XIX.) 
5. Mount in Canada balsam sections of a cornea which has been stained with nitrate of silver. Notice the branched cell-spaces corresponding with the connective-tissue cells of the last preparation.

[This preparation is best made by rubbing the surface of the cornea with lunar caustic after scraping off the epithelium. After ten or fifteen minutes (by which time the nitrate of silver will have penetrated the thickness of the cornea) the eye is washed with distilled water, placed in spirit, and exposed to the light. When brown and sufficiently hardened, tangential sections may be made with a razor.]

\section{LESSON XL.}

1. Remove the sclerotic from the anterior part of an eye which has been preserved in Müller's fluid, and tear off thin shreds from the surface of the choroid, including amongst them portions of the ciliary muscle. Stain the shreds with logwood and mount them in Farrant's solution. Sketch the branched pigment-cells, the elastic network, the mode of attachment of the fibres of the ciliary muscle, \&c.

2. Injected preparation of choroid and iris. Mount in Canada balsam portions of the choroid coat and iris from an eye, the blood-vessels of which have been filled with coloured injection. Make sketches showing the arrangement of the capillaries and veins.

3. Teased preparation of retina. Break up with needles in a drop of glycerine a minute fragment of retina which has been placed in 1 per cent. osmic acid solution for a few hours, and has subsequently been kept in dilute glycerine. Complete the separation of the retinal elements by tapping the cover-glass. Draw carefully under a high power some of the isolated elements-e.g. the rods and cones with their attached fibres and nuclei, the inner granules, the ganglion-cells, the fibres of Müller, hexagonal pigmentcells, \&c. In some of the fragments the arrangement of the elements in the retinal layers may be made out even better than in actual sections.

Measure the length and diameter of some of the cones, the length of the cone-fibres, and the diameter of some of the outer and inner nuclei.

4. Teased preparation of lens. Separate in water the fibres of a crystalline lens which has been macerated for some days in weak bichromate of potash solution. Sketch some of the fibres, together and separate.

The eyelids (fig. 247) are covered externally by the skin, and internally or posteriorly by a mucous membrane, the conjunctiva, which is reflected from them over the globe of the eye. They are composed in the main of connective tissue, which is dense and fibrous under the conjunctiva, where it forms what is known as the tarsus.

Embedded in the tarsus is a row of long sebaceous glands (the Meibomian glands, $f$ ), the ducts of which open at the edge of the eyelid. The rest of the thickness of the eyelid is composed of a somewhat loose connective tissue, and contains the bundles of the orbicularis muscle $(b)$. In the upper eyelid the levator palpebrce is inserted into the tarsus by a fibrous expansion, and some bundles of involuntary muscle are also present near the attachment of the eyelid. The skin 
has the usual strueture; it contains small sweat-glands and the follicles of small hairs, and, in addition, at the edge of the eyelid, the large hair-follicles from which the eyelashes grow. The epithelium of the conjunctiva palpebræ is columnar, passing at the edge of the lid into the stratified epitheliurn of the skin; it also becomes stratified in

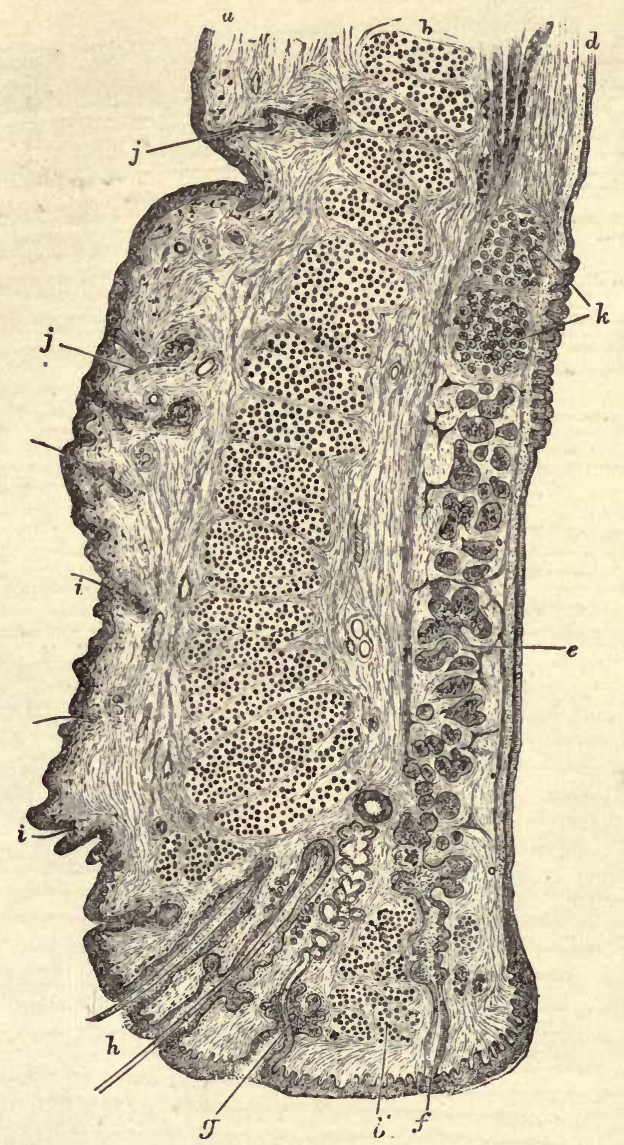

Fig. 247.-Vertical Section throvgh the UPPER Eyelid, (Magnified.)

$a$, skin ; $b$, orbicn'aris ; $b^{\prime}$, ciliary bundle ; $c$, involun+ary muscle of eyelid: $a$, conjuuctiva ; $e$, tarsus; $f$, Meibomian gland; $q$, sebaceous gland near eyelashes, with modified sweatgland opening with it; $h$, eyelashes; $i$, small hairs in outer skin; $j$, sweat-glands; $k$, posterior tarsal glands.

the part which is reflected over the globe of the eye. The nerves of the conjunctiva terminate for the most part in end-bulbs, which in man are spheroidal, and formed chiefly of a small mass of polyhedral cells, but in the calf and most animals they are elliptical, and consist of a core and lamellated sheath (see Lesson XIX.) 
The lachrymal gland may be briefly mentioned in connection with the eyelid. It is a compound racemose gland, yielding a watery secretion, and resembling in structure the serous salivary glands, such as the parotid.

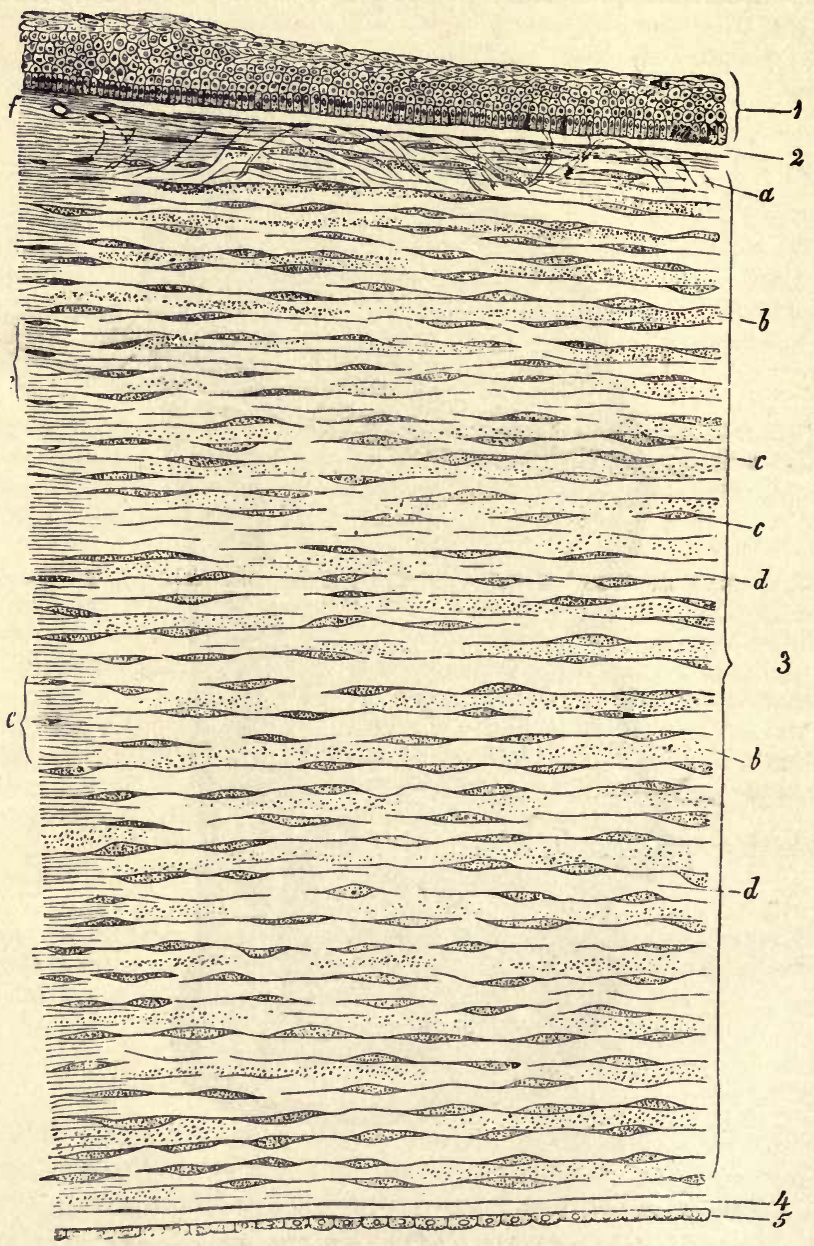

Fig. 248.-Vertical Section of human Cornea from Near tile margin. (Magnified.)

1, epithelium; 2, anterior homogeneous lamina ; 3. snbstantia propria corneæ; 4, posterior homogeneous (elastic) lamina; 5 , epithelium of the anterior cliamber; $a$, oblique fibres in the anterior layer of the substantia propria; $b$, lamellæ, the fibres of which are cut across, producing a dotted appearance ; $c$, corneal corpuscles appearing fusiform in section ; $d$, bundles of fibres cut longitudinally; $e$, transition to the sclerotic, with more distinct fibrillation, and surmounted by a thicker epithelium; $f$, small blood-vessels cut across near the margin of the cornea.

The sclerotic coat is composed of dense fibrous tissue, the bundles of which are intimately interlaced. It is thickest at the back of the 
eyeball. It is covered externally with a lymphatic epithelium, while internally it is lined by a layer of connective tissue containing pigment-cells, which give it a brown appearance (lamina fusca). At the entrance of the optic nerve the sclerotic is prolonged into the sheath of that nerve, the bundles of which, piercing the coat, give a sieve-like aspect to the part (lamina cribrosa, fig. $257, \mathrm{~L}$ ).

The cornea (fig. 248) consists of the following layers enumerated from before back:

1. A stratified epithelium continuous with the epithelium of the conjunctiva ( 1 ).

2. A thin lamina of homogeneous connective tissue (membrane of Bowman), upon which the deepest cells of the epithelium rest (2).

3. A thick layer of fibrous connective tissue which forms the proper substance of the cornea (3). This is continuous laterally with the tissue of the sclerotic. It is composed of bundles of white fibres arranged in regular laminæ, the direction of the fibres crossing one another at right angles in the alternate laminæ. Between the laminæ lie flattened connective-tissue corpuscles, which are branched and united by their processes into a continuous network; there is of course a corresponding network of cell-spaces (fig. 249, A, B). In vertical

A

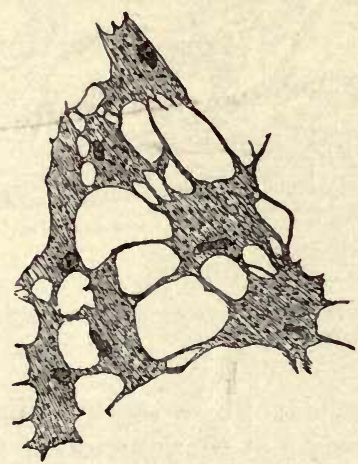

I

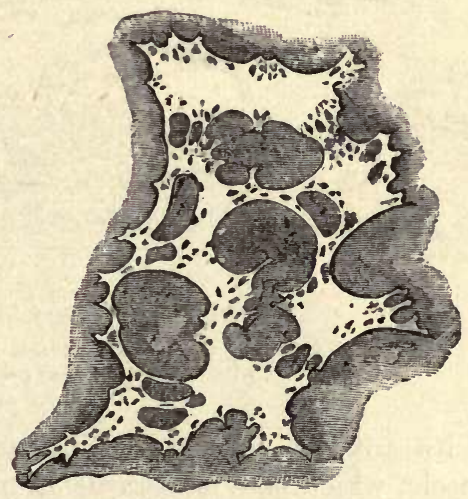

Fig. 249.-A. Corpuscles of the Rat's Cornea. (From a preparation treated with chloride of gold.) B. CELL-spaces of THE RAT's CORNEA. (From a preparation stained with nitrate of silver.)

sections the cells appear narrow and spindle-shaped (fig. 248, c). In the superficial laminæ there are a few bundles of fibres which run obliquely towards the surface $(a)$.

4. A homogeneous elastic layer (membrane of Descemet) (fig. 248, 4). This completely covers the back of the cornea, but at the angle which the cornea forms with the iris it breaks up into separate fibres, which are continued into the iris as the ligamentum pectinatum, or pillars of the iris. 
5. A layer of pavement-epithelium covering the posterior surface of the elastic lamina, and lining the front of the anterior chamber of the eye (fig. 348,5 ). At the sides it is continued over the ligamentum pectinatum into a similar epithelium, covering the anterior surface of the iris (fig. $253, N$ ).

The nerves of the cornea pass in from the periphery, losing their medullary sheath as they enter the corneal substance. They form a primary plexus in the substantia propria, a secondary or sub-epithelial plexus immediately under the epithelium which covers the anterior surface, and a terminal plexus of fine fibrils which pass from the sub-epithelial plexus in pencil-like tufts and become lost between the epithelium-cells (see figs. 105, 106, Lesson XIX.) There are no blood-vessels or lymphatics in the cornea, although they come close up to its margin.

The choroid or vascular coat of the eye is of a black colour in many animals, but in the human eye is dark brown. It is composed of connective tissue, the cells of which are large and filled with pigment (fig. 251), and it contains in its inner part a close network of blood-vessels, and

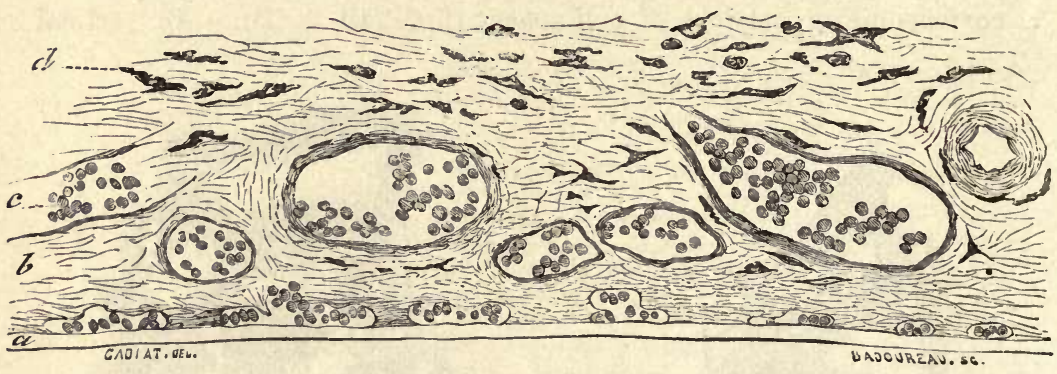

Fig. 250.-SeCtion of CHOROID. (Caciat.)

$a$, membrane of Bruch: the chorio-capillaris is just above it; $b$, vascular layer; $c$, vessels with blood-corpuscles; $d$, lamina suprachoroidea.

in its anterior part the involuntary muscular fibres of the ciliary muscle, which pass backwards from their origin at the junction of the cornea and sclerotic, to be inserted into the choroid. The choroid is separable into the following layers, enumerated from without in (fig. 250) :

1. The lamina suprachoroidea (fig. $250, d$ ). This is a thin membrane composed of homogeneous connective tissue pervaded by a network of fine elastic fibres, and containing many large branched pigment-cells and lymph-corpuscles (fig. 251). It is covered superficially by a delicate lymphatic epithelium, and is separated from the lamina fusca by a cleft-like lymphatic space which is bridged across here and there by the passage of vessels and nerves, and by bands of connective tissue.

2. The vascular layer of the choroid (fig. $250, b$ ), which resembles 


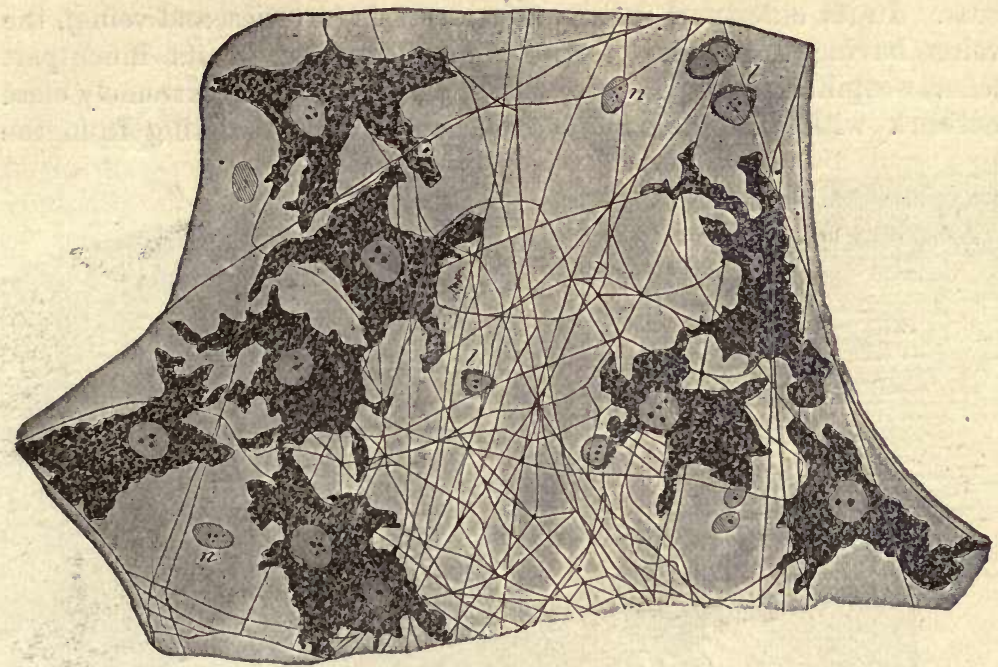

Fig. 251.-A siall portion of the lamina suprachoroidea. (Highly magnified.) $p$, pigment-cells; $f$, elastic fibres; $n$, nuclei of epithelioid cells (the outlines of the cells are not indlcated); $l$, lymph-cells.

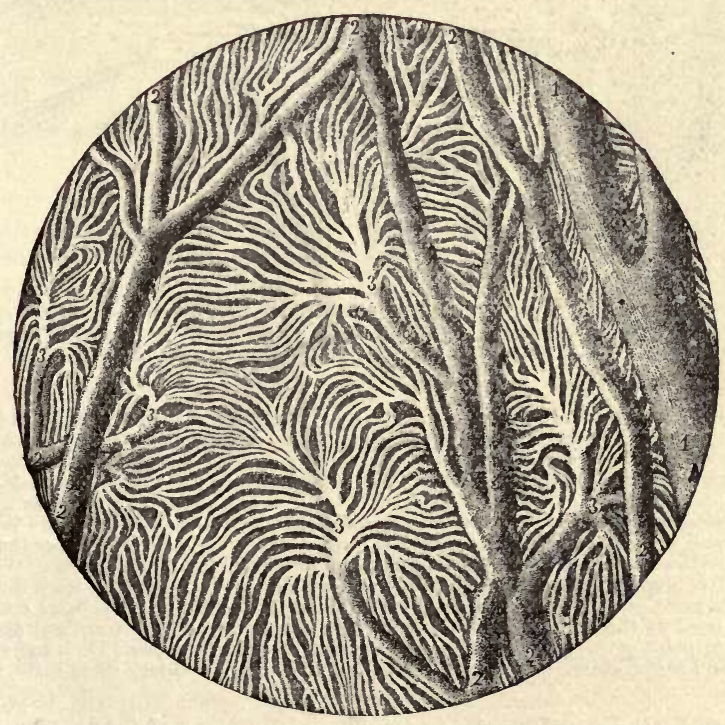

Fig. 252.-INJECTED BLOOD-VESSELS OF THE CHOROIN COAT.

1 , one of the larger veins; 2, small anastomosing vessels; 3 , branches dividing into the smallest vessels. 
the suprachoroidea in structure, but contains the blood-vessels of the coat. In its outer part are the larger vessels (arteries and veins), the veins having a peculiar vorticose arrangement; in its inner part (chorio-capillaris) are the capillaries, which form an extremely close network with elongated meshes, the capillaries radiating from the

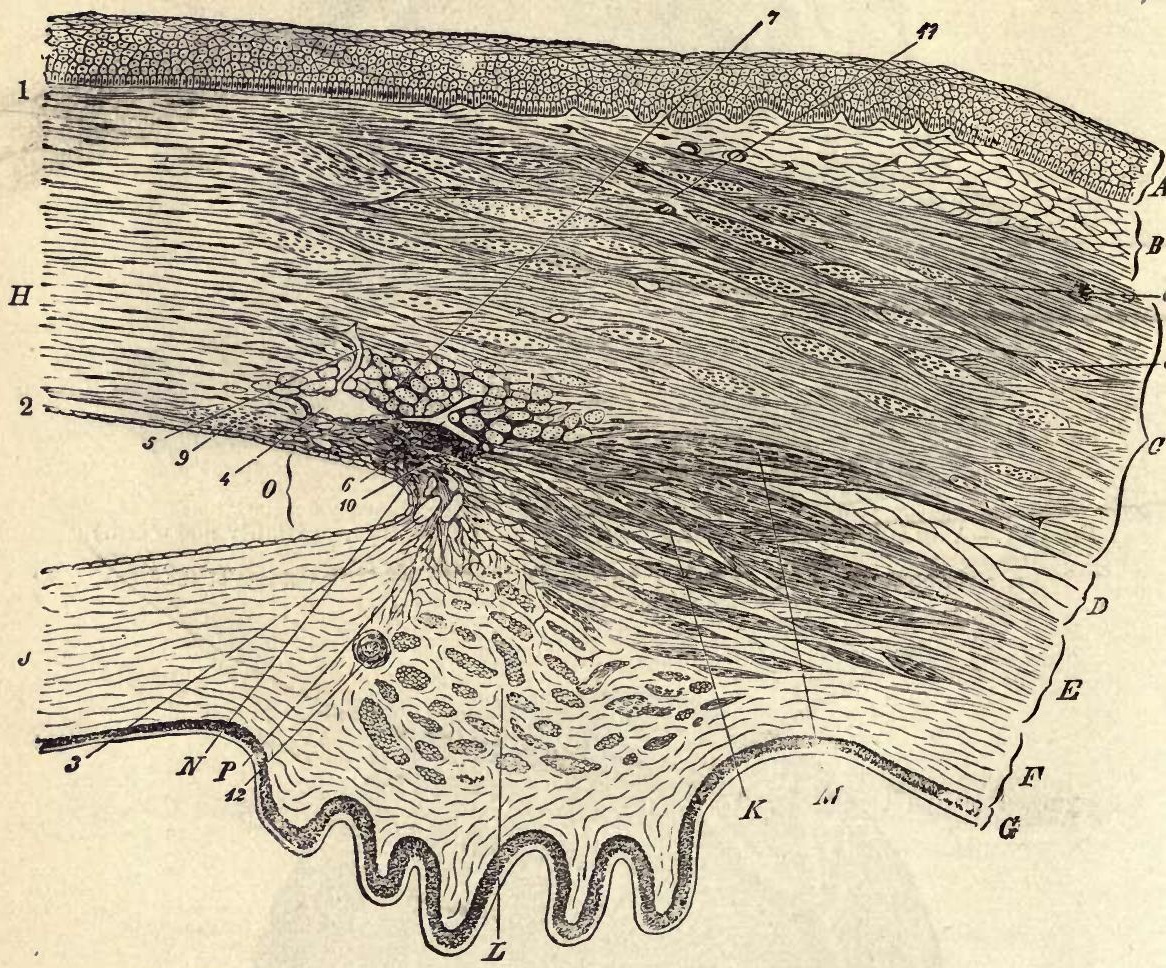

Fig. 253.-Sectiox (Fron the Fye of a MAN, AgED 30), SHOWing the RELATIONS OF THE CORNEA, SCLEROTIC, AND IRIS, TOGETHER WITH THE CILIARY MUSCLE, AND THE CAVERNOUS SPACES NEAR THE ANGLE OF THE ANTERIOR CHAMBER. (Magnified.)

A, epithelium ; B, conjunctival mucous membrane ; , sclerotie ; D, membrana suprachoroidea; $\mathrm{F}$, opposite the ciliary muscle ; F, choroid, with ciliary processes; $\mathrm{G}$, tapetum nigrum and pars ciliaris retinæ; $\mathrm{H}$, cornea (substantia propria); J, iris; $\mathrm{K}$, radiating and meridional, and $\mathrm{L}$, circular or annular bundles of the ciliary muscle; $\mathrm{M}$. bundles passing to the selerotic; $\mathrm{N}$, ligamentum pectinatum iridis at the angle. o, of the anterior chamber; p, line of attachment of the iris. 1, nnterior homogeneous lamina of the cornea ; 2, posterior homogeneous lamina, covered with epithelial cells which are continued over the front of the iris ; 3 , cavernous spaces at the angle of the anterior chamber (spaces of Fontana); 4 , canal of Schlemm, with epithelial lining, and with a vessel, 5 , lcading from it; 6 , other ressels; 7 , bundles of fibres of the selerotic having a cireular direction, eut across: 8. larger ones in the substance of the sclerotic; 9, fine bundles cut across, at limit of cornea ; 10, point of origin of meridional bundles of ciliary muscle; 11 , blood-vessels in sclerotic and conjunctiva, cut across; 12 , section of one of the ciliary arteries.

extremities of the small arteries and veins in a highly characteristic manner (fig. 252). In the ciliary processes the vessels have for the most part a longitudinal direction, but there are numerous convoluted transversely disposed capillaries uniting the longitudinal vessels (fig. 255). 
3. Lining the inner surface of the choroid is a very thin transparent membrane known as the membrane of Bruik (fig. 250, a).

The ciliary muscle of Bowman consists of involuntary muscular bundles which arise at the corneo-sclerotic junction, and pass meridionally backwards to be inserted into the choroid (fig. 253, k). Many of the deeper-seated bundles take an oblique direction, and these pass gradually into others which run circularly around the circumference of the iris, and on a level with the ciliary processes. This set of circularly arranged bundles constitutes the circular ciliary muscle of H. Muller (L); it is most marked in hypermetropic eyes.

The iris is that part of the vascular coat of the eye which extends in front of the lens. It is continuous with the choroid and has a

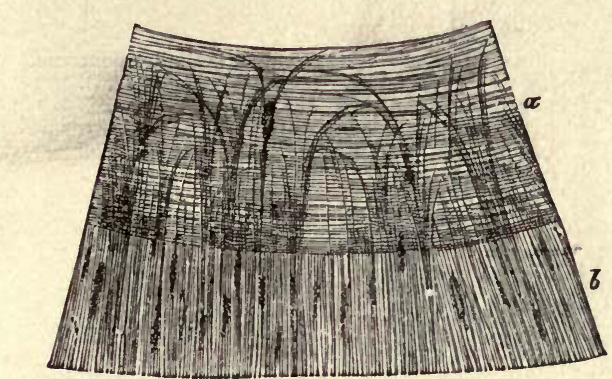

FIG. 254.-SEgMENT OF THE IRIS, SEEN FRON THE POSTERIOR STRFACE AFTER REMOVAL OF THE LVEAL PIGMENT.

$a$, sphincter muscle; $b$, dilatator muscle of the pupil.

Fig. 255.-VESSELS OF THE CHOROID, CILIARY PROCESSES, AND IRIS $\mathrm{CF}$ A CHILD. (10 diameters.)

$a$, capillary network of the prsterior part of the choroid, ending at $b$, the ora serrata ; $c$, arteries of the corona ci'iaris, supplying the ciliary processes, $d$, and passing into the iris, $e ; f$, the capillary uetwork close to the pupillary margin of the iris.

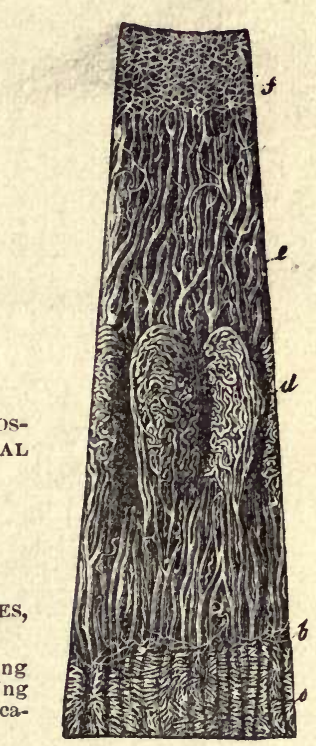

similar structure, but its pigment-cells often contain coloured pigment. Besides the homogeneous connective tissue, with numerous elastic fibres and blood-vessels of which it is chiefly composed, it contains two sets of plain muscular fibres. The one set forms the sphincter muscle (fig. $245, a$ ), which encircles the pupil, the other set consists of a flattened layer of radiating fibres which extend from the attachment of the iris nearly to the pupil, lying close to the posterior surface and constituting the dilaiator muscle $(b)$.

The back of the iris is covered by a thick layer of pigmented epithelium (uvea) continuous with the epithelium of the pars ciliaris retinæ.

The blood-vessels of the iris converge towards the pupil (fig. 255, e). 
Near the pupil the small arteries form a small anastomotic circle, from which capillaries arise and pass still nearer the pupil, around which they form a close capillary network.

A large number of nerve-fibres are distributed to the choroid and iris, probably going chiefly to the muscular tissue (ciliary muscle and sphincter and dilatator iridis).

The retina consists of the eight layers shown in the accompanying figure (fig. 256), numbered as they occur from within out.

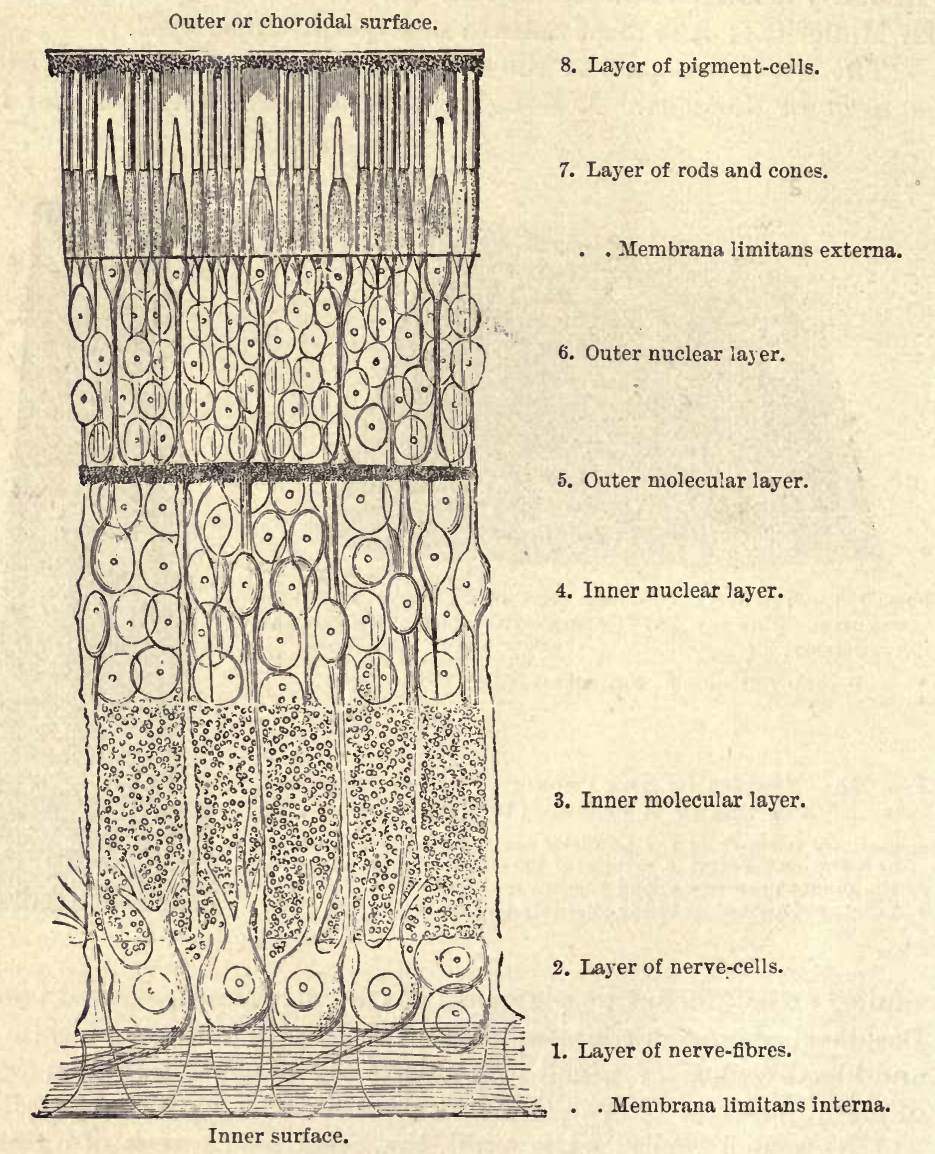

Fig. 256.-Diagramiatic section of the humax retina.

The inner surface of the retina, which is smooth, rests upon the hyloid membrane of the vitreous humour. It is formed of the united bases of the fibres of Müller, which will be afterwards described.

The layer of nerve-fibres is formed by the expansion of the 
optic nerve after it has passed through the coats of the eye (fig. 257). At its entrance it forms a slight eminence (colliculus nervi optici). The nerve-fibres lose their medullary sheath on reaching the retina. The layer becomes gradually thinner in the anterior part of the retina.

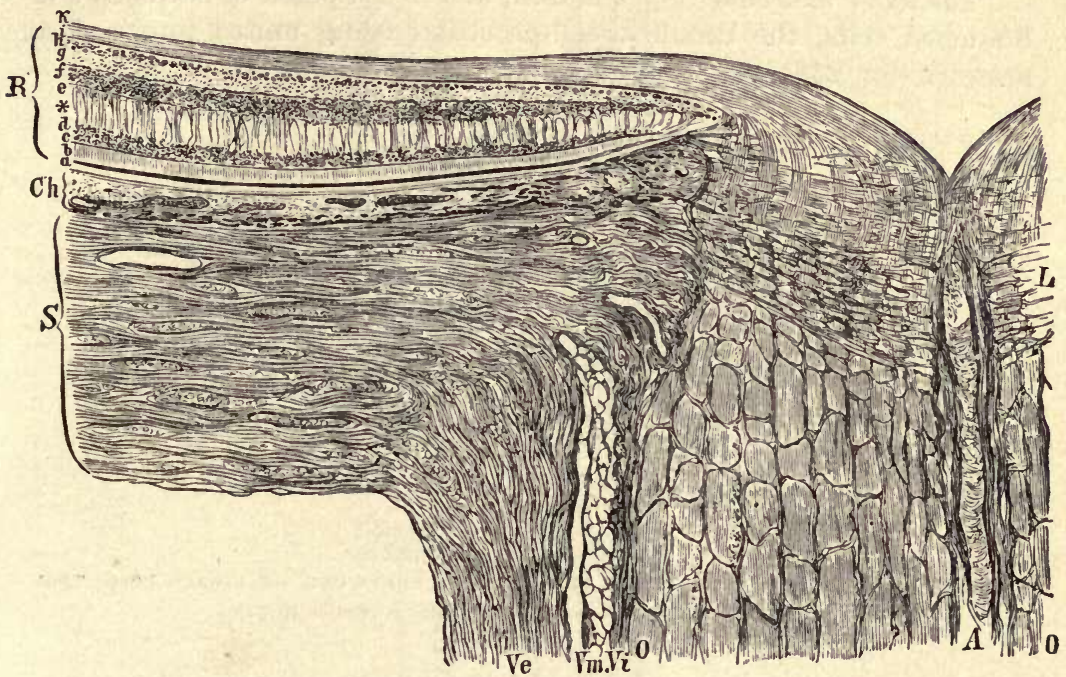

Fig. 257.-Section through the coAts of the eyeball at the point of ENTRANCE OF THE OPTIC NERVE. (Toldt.)

$V e$, dural sheath : $V m$, arachnoidal sheath, and $V i$, pia-matral sheath of the optic nerve, with lymphatic spaces between them; $O, O$, funiculi of the nerve ; $L$, lamiha cribrosa ; $A$, central artery : $S$, sclerotic ; $C h$, choroid; $R$, retina. The small letters refer to the various parts of the retina, $b$ being the layer of rods and cones, and $i$ that of nerve-fibres.

The layer of nerve-cells, or ganglionic layer, is composed of large nerve-cells somewhat like the cells of Purkinje of the cerebellum, and having on the one side a fine axis-cylinder process prolonged into a nerve-fibre, and on the other a thick branching process, the ramifications of which become lost in the next layer.

The inner molecular layer is comparatively thick, and has an appearance very like the neuroglia of the grey matter of the nervecentres. A few nuclei are scattered through it, and it is traversed by the processes of the nerve-cells and of the inner granules, as well as by the fibres of Müller.

The inner nuclear layer is mainly composed of bipolar cells containing large nuclei (inner granules). The processes of these cells extend on the one hand inwards through the inner molecular layer, probably to join with nerve-fibres or with the processes of the ganglion-cells, whilst the other process is directed outwards, and is connected with the extremity of a rod or cone fibre. Besides these bipolar cells, there are other inner granules which are different in character, being devoid of processes and resting on, or even embedded in, the 
inner molecular layer, with the formation of which they are probably connected. Others, which are larger and more rounded, are applied to the outer molecular layer. The fibres of Müller have nucleated enlargements in the inner nuclear layer.

The outer molecular layer is thin, and is composed of flattened and branched cells, the ramified cell-processes being united into a close network (fig. 258).

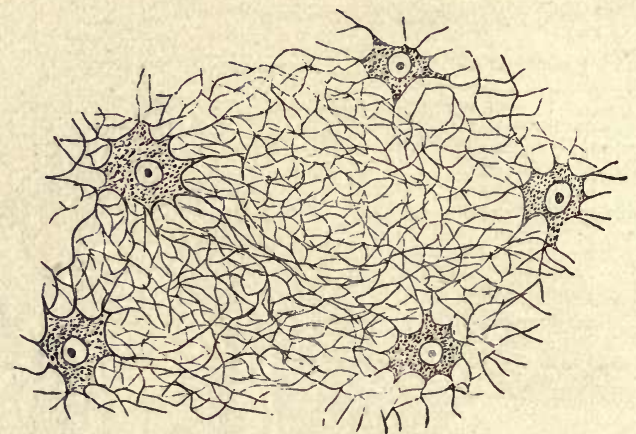

Fig. 258.-BRaNChed CELLS With THE UNITING FELTWORK OF FIBRES FRoM the OUTER MOLECULAR LAYER OF THE HORSE'S RETINA.

As far as the outer molecular layer the retina may be said to consist of nervous elements, but beyond this layer it is formed of modified epithelium-cells.

The outer nuclear layer and the layer of rods and cones are composed of elements which are continuous through the two layers, and they should properly, therefore, be described as one. It may be termed the neural or sensory epithelium of the retina (fig. 260, 6 and 7). The elements of which the neural epithelium consists are elongated, nucleated cells of two kinds. The most numerous, which we may term the rod-elements, consist of peculiar rod-like structures (rods proper) set closely side by side, and each of which is prolonged internally into a fine varicose fibre (rod-fibre) which swells out at one part of its course into a nucleated enlargement. The rod proper consists of two segments, an outer cylindrical and transversely striated segment, which during life has a purplish-red colour, and an inner slightly bulged segment, which in part of its length is longitudinally striated. The nucleus of the rod-element often has, in the fresh condition, a transversely shaded aspect (fig. 259). The cone-elements are formed of a conical tapering external part, the cone proper, which is directly prolonged into a nucleated enlargement, from the farther side of which the conefibre, considerably thicker than the rod-fibres, passes inwards, to terminate by an expanded base at the outer molecular layer. The cone proper, like the rod, is formed of two segments, the outer of which, much the smaller, is transversely striated, the inner, bulged segment 


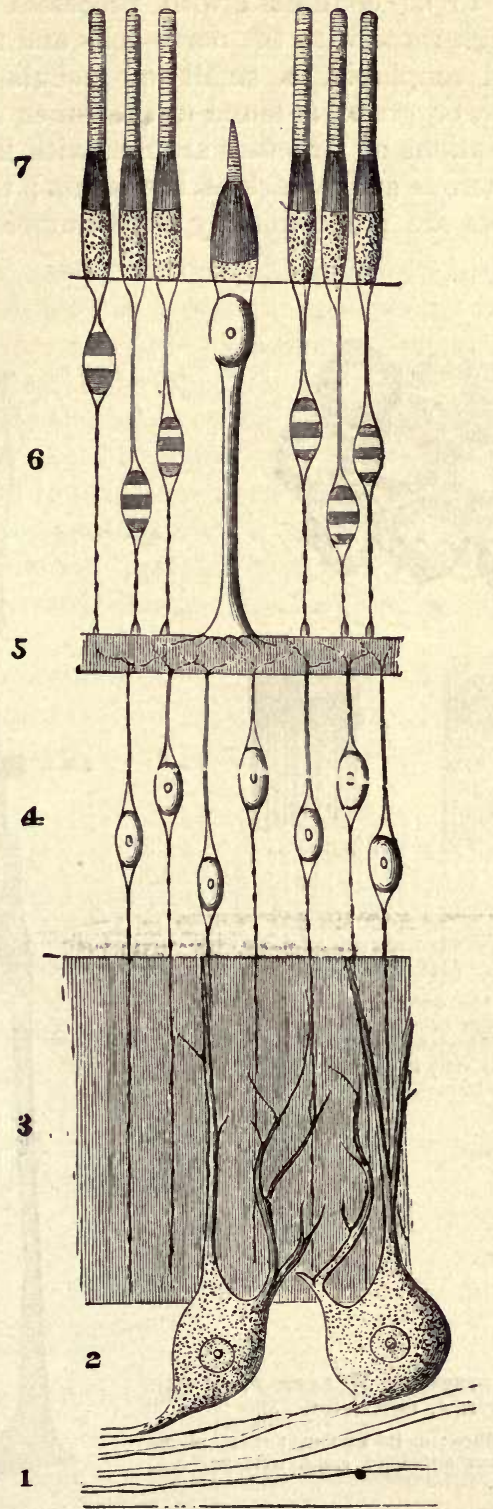

Fig. 259,-Diagrammatic Representation of the Nervous aNd ePITHELIAL ELEMENTS OF THE RETINA.

1 to 5 , nervous elements. 6,7 , epithelial elements. The designation of the numbers is the same as in fig. 346. The extent of the molecular layers is indicated merely by linear shading. 
being longitudinally striated. The inner ends of the rod-and conefibres are believed to be connected with processes from the inner granules, and through these with the nerve-cells and nerve-fibres. In birds, reptiles, and amphibia, a small oil-globule, often brightly coloured red, yellow, or green, is found in the inner segment of each cone, and other variations of structure are met with in animals. The cones are most numerous at the back of the retina; they are fewer in number, and the rods are proportionally more numerous, towards the anterior part.
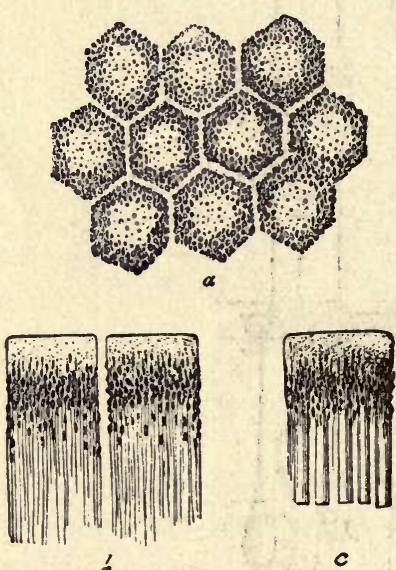

c

Fig. 260.-Pigmented epitheidum of the HUMAN RETINA. (Highly magnified.)

$a$, cells seen from the outer surface with clear lines of intercellular substance between; $b$, two cells seen in profile with fine offsets extending inwards; $c$, a cell still in connection with the outer ends of the rods.

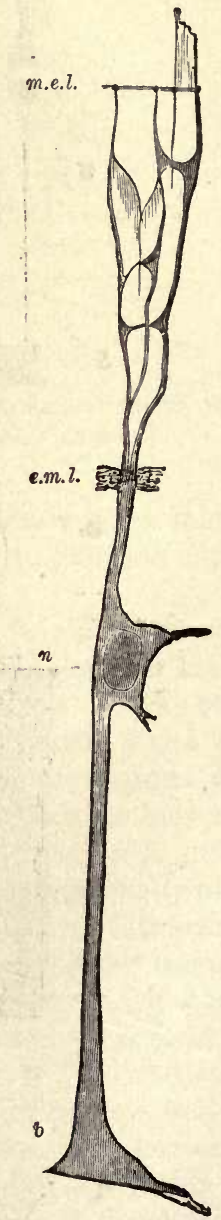

The pigmentary layer is the most external part of the retina. It is formed of hexagonal epithelium-cells (fig. 260), which are smooth externally where they rest against the choroid, but are prolonged internally into fine filaments which extend between the rods. The pigment-granules, 
many of which are in the form of minute crystals, lie in the inner part of the cell, and after prolonged exposure to the light they are found extending along the cell-processes between the rods $(b, c)$, their function being probably connected with the restoration of the purple colouring matter which has been bleached by the light.

Fibres of Mïller. - The fibres of Müller (fig. 261) are long stiff fibres which pass through several of the retinal layers. Commencing at the inner surface of the retina by expanded bases which unite with one another to form the so-called internal limiting membrane (fig. 356), the fibres pass through all the layers in succession, until they reach the outer nuclear layer. Here they branch and expand into a sort of reticular tissue which serves to support the fibres and nuclei of the rod-and cone-elements. At the bases of the rods and cones, this sustentacular tissue ceases, being here bounded by a distinct margin which has been called the external limiting membrane $(m . c . l$.), but delicate sheaths have been described passing from it round the bases of the rods and cones. Each Müllerian fibre, as it passes through the inner nuclear layer, has a nucleated enlargement $(n)$, indicating the original cell nature of the fibre.

There are two parts of the retina which call for special description.

The macula lutea (yellow spot, fig. 263), with its central fovea, lies in the visual axis, and is the part of the retina which is most immedi-

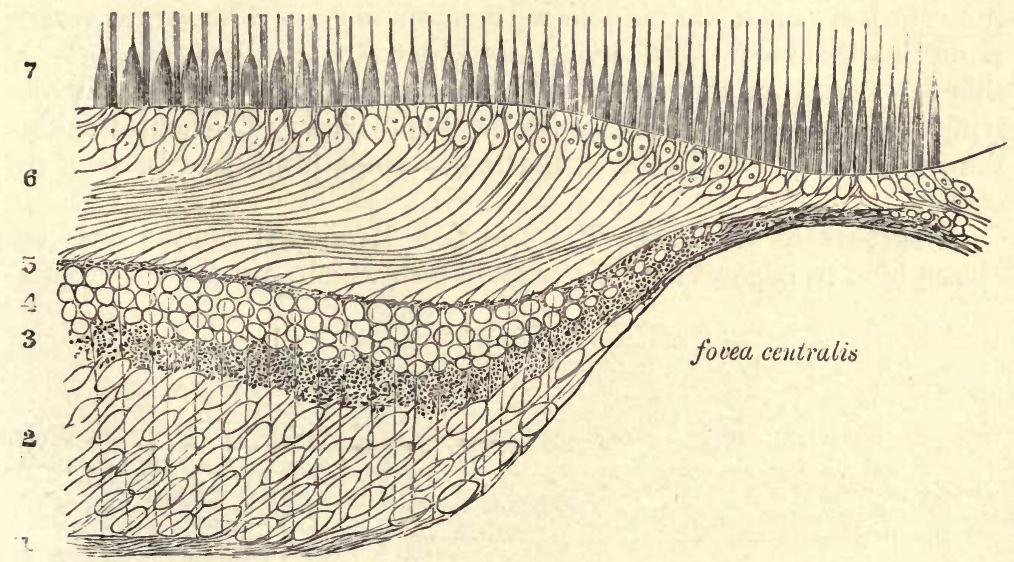

Fig. 262.-VErtical Section throvgh the macela lutea AND fovea Cextralis ; DIAGRAMMATIC.

1, nerve-layer; 2, gang:ionic layer ; 3. inner molecular ; 4, inner nuclear ; and 5, onter molecular layers: 6 , onter nuclear layer, the inner part with only cone-fibres forming the so-called external fibrous layer; 7 , cones and rods.

ately concerned in direct vision. It is characterised first by its greater thickness (except at the fovea), secondly by the large number of ganglion-cells, which are all distinctly bipolar (2), and thirdly by the large number of cones it contains as compared with the rods. In the 
central fovea itself there are no rods, and the cones are very long and slender; moreover, all the other layers become gradually thinned down almost to complete disappearance, so that the middle of the central fovea is the thinnest part of the retina. Since there are few rods, the outer nuclear layer (6) loses in great measure its appearance of being composed of closely packed nuclei, and the cone-fibres are very distinct. The direction of all the fibres is very oblique in this part of the retina.

The pars ciliaris retince which commences at the ora serrata, where the retina proper abruptly ends, is composed of two epithelial layers (fig. 263), and has no nervous structures. Of the two layers, the

F1G. 263.-A SMALL PORTION OF THE CILIARY PART OF THE RETINA. (350 diameters.)

1, pigment-cells; 2, columnar cells.

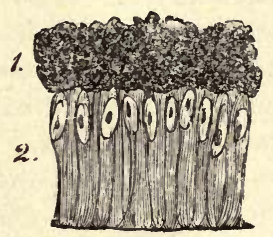

external is a thick stratum of pigmented epithelium formed of rounded cells and continuous with the pigmentary layer of the retina on the one hand, and with the uvea of the iris on the other; the inner is a layer of columnar cells each containing an oval nucleus.

The retina contains but few blood-vessels. The artery enters and the vein leaves it in the middle of the optic nerve. The larger vessels ramify in the nerve-fibre layer, and there are capillary networks in this layer and in the inner nuclear layer. There are peri-vascular lymphatic spaces around the veins and capillaries. The neural epithelium receives no blood-vessels, but is nourished from the vessels of the choroid.

Structure of the lens. The lens is a laminated fibrous body enclosed by a transparent elastic capsule into which, around the circum-

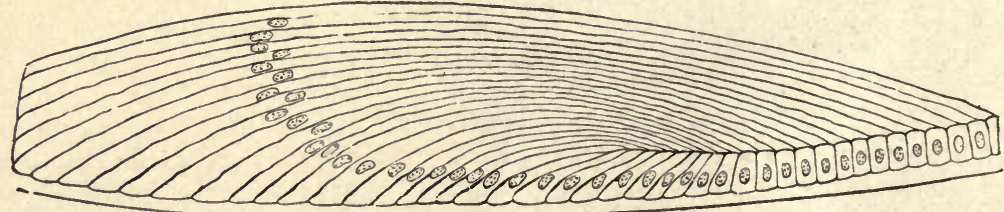

Fig. 264.-Section throvgh the margin of the Rabbit's LeNs, Showing the TRANSITION OF THE EPITHELIUM INTO THE LKNS-FIBRES.

ference, the fibres of the suspensory ligament are inserted. Immediately within the capsule, in front and at the sides, there is a layer of cubical epithelium termed the epithelium of the capsule, but at the margin of the lens the cells become longer and pass by a gradual transition into the lens-fibres (fig. 264). The fibres which compose the lens are long and riband-shaped, with finely serrated edges (fig. 265, A); 
in transverse section they appear prismatic (B). Many of the superficial fibres are nucleated $(c)$, the lens-fibres having originally been developed by the elongation of epithelium-cells.

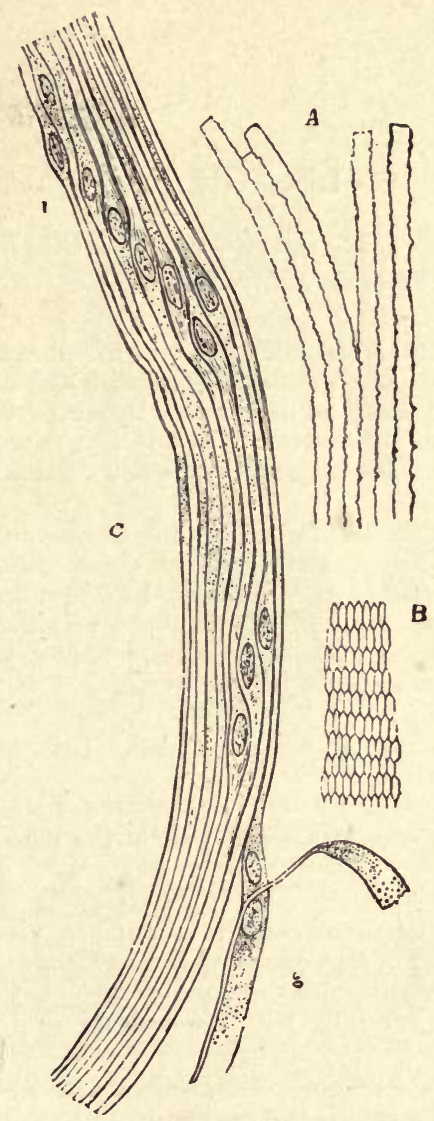

Fig. 265.-Fibres of the CRYstalline lexs. (350 diameters.)

A, longitudinal view of the fibres of the lens from the ox, showing the serrated edges. B, transverse section of the fibres of the lens from the human eye. C, longitudinal view of a few of the fibres from the equatorial region of the human lens. Most of the fibres in $\mathrm{C}$ are seen edgeways, and, towards 1 , present the swellings and nuclei of the 'nuclear zone ;' at 2 , the flattened sides of two fibres are seen.

The vitreous humour is composed of soft gelatinous tissue, apparently structureless when examined in the fresh condition, but containing a few scattered amœboid cells, the processes of which are often long and varicose, and the cell-bodies distended by large vacuoles. The hyaloid membrane, which invests the vitreous humour, is homogeneous and structureless except in the region of the ciliary processes, where it is fibrous in structure, forming the zonule of Zinn and spreading out into the suspensory ligament of the lens. 


\title{
LESSON XLI.
}

\author{
STRUCTURE OF THE OLFACTORY MUCOUS MEMBRANE \\ AND OF THE EXTERNAL AND MIDDLE EAR.
}

1. Vertical sections of the olfactory mucous membrane. The sections may be carried either across the middle turbinate bone, after decalcification in $0 \cdot 2$ per cent. chromic acid, or across the upper part of the nasal septum. Make a sketch under the low power. Notice the difference in the character of the epithelium in the olfactory and respiratory parts of the membrane.

2. Teased preparation of the epithelium of the olfactory mucous membrane. A piece of the membrane is placed quite fresh in osmic acid (1 per cent.) for a few hours, and is then macerated for two days or more in water. The epithelium is broken up in dilute glycerine; the cells easily separate from one another on tapping the cover-glass. Notice the two kinds of cells. Sketch some of the cells under a high power.

3. Sections of the external ear (these have been already studied for the cartilage, Lesson XII.)

4. Sections across the cartilaginous part of the Eustachian tube. Sketch under the low power.

5. Preparation of the membrana tympani. A piece of the membrane, stained with hæmatoxylin. and mounted flat in Canada balsam.

Determine the composition of the membrane-i.e. the several layers composing it-by focussing carefully with the high power.

\section{STRUCTURE OF THE OLFACTORY MUCOUS MEMBRANE.}

The olfactory region of the nasal fossæ includes the upper and middle turbinate processes and the upper third of the septum. It is covered by a soft vascular mucous membrane of a yellow colour in man.

'The epithelium of the olfactory mucous membrane (figs. 266,267 ) is very thick and is composed of long tapering cells, set closely side by side and bounded superficially by a cuticular lamina, through which the free ends of the cells project. The cells are of two kinds : 1. Long narrow spindle-shaped or bipolar cells consisting of a larger part or body $(b)$, containing the nucleus, and of two processes or poles, one $(c)$ straight and cylindrical and extending to the free surface, the other $(d)$ very delicate and varicose, looking not unlike a nerve-fibril and extending 
down to the corium. The position of the nuclear enlargement varies, and with it the relative length of the two processes. 'The distal or free process terminates in a small clear projection, which passes beyond the cuticular membrane; in amphibia, reptiles, and birds, and perhaps in some

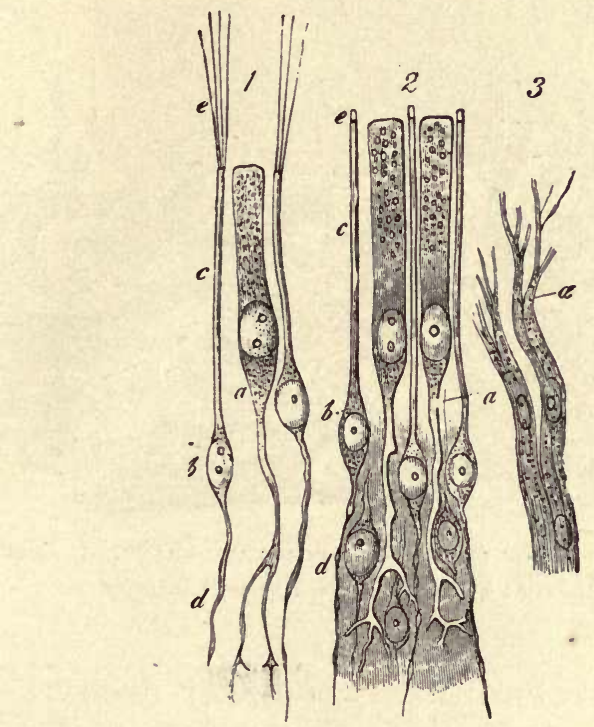

Fig. 266.-Cells and terninal Nerve-fibres of the olfactory Region. (Highly magnified.)

1, from the frog; 2 , from man; , epithelial cell, extending deeply into a ramified process; $b$, olfactory cells; $c$, their peripheral rods; $e$, their extremities, seen in 1 to be prolonged into fine liairs; $d$, their central filaments.

mammals, it bears fine stiff hairlike filaments $(e)$. The proximal or varicose process becomes lost amongst the plexus of olfactory nerve-fibrils at the base of the epithelium, and is believed to be connected with a fibril. These cells have accordingly been termed olfactory cells. 2. Long columnar epithelium cells $(a)$, with comparatively broad cylindrical nucleated cell-bodies placed next the free surface, and long, forked, and branching tail-like processes extending down to the corium. These are usually regarded not as sensory epithelium-cells, but merely as serving to support the proper olfactory cells; but, according to Exner, they are also connected with the olfactory fibres, and there is no sharp distinction between them and the bipolar cells. 3. Tapering cells are present, at least in some animals, in the deeper part of the epithelium. They rest by their bases upon the corium, and project between the other cells, which they assist to support.

The corium of the olfactory mucous membrane is also very thick (fig. 267). It contains numerous blood-vessels, bundles of the olfactory nerve-fibres (which are non-medullated), and a large number of serous 
glands known as Bowman's glands (b). which open upon the surface by fine ducts passing between the epithelium-cells.

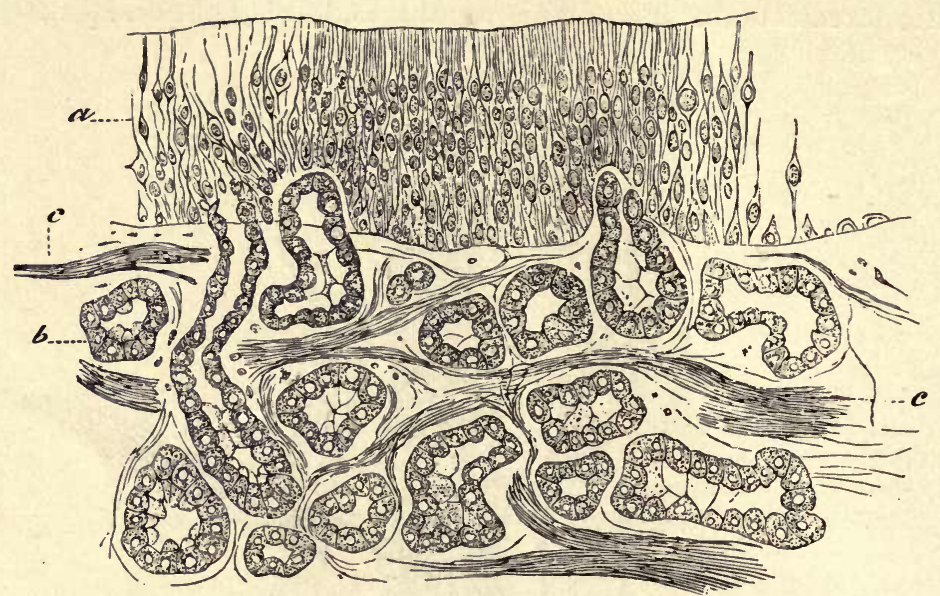

Fig. 267.-Section of olfactory micous menirane. (Cadiat.) $a$, epithelium; $b$, glands of Bowman; $c$, uerve-bundles.

STRUCTURE OF THE AUDITORY ORGAN.

The external ear proper (pinna) is composed of elastic fibro-cartilage, invested by a thin, closely adherent skin. The skin is covered by small hairs, and connected with these are the usual sebaceous follicles. In some parts-e.g. the lobule-there is a considerable amount of adipose tissue; and voluntary muscular fibres are in places attached to the cartilage and may be seen in sections of the ear.

The external auditory meatus is a canal formed partly of cartilage continuous with that of the pinna, partly of bone. It is lined by a prolongation of the skin and is closed by the membrana tympani, over which the skin is prolonged as a very thin layer. Near the orifice the skin has hairs and sebaceous glands, and the meatus is also provided throughout the cartilaginous part with small convoluted tubular glands of a brownish-yellow colour, which yield a waxy secretion (ceruminous glands). They appear to represent modified sweat-glands.

The tympanum is lined by a mucous membrane which is continuous through the Eustachian tube with the mucous membrane of the pharynx; it is also prolonged into the mastoid cells. The epithelium is columnar and ciliated in some parts, but in others-e.g. roof, promontory, ossicles, and membrana tympani-it is a pavement-epithelium.

The membrana tympani is a thin membrane formed of fibrous bundles which radiate from the umbo. Within the radial fibres are a few annular bundles. Covering the fibrous membrane externally is a thin 
layer continuous with the skin of the meatus ; covering it internally is another thin layer, derived from the mucous membrane of the tympanic cavity. Blood-vessels and lymphatics are distributed to the membrane chiefly in the cutaneous and mucous layers.

The Eustachian tnbe is the canal leading from the tympanum to the pharynx. It is formed of bone near the tympanum, but below,

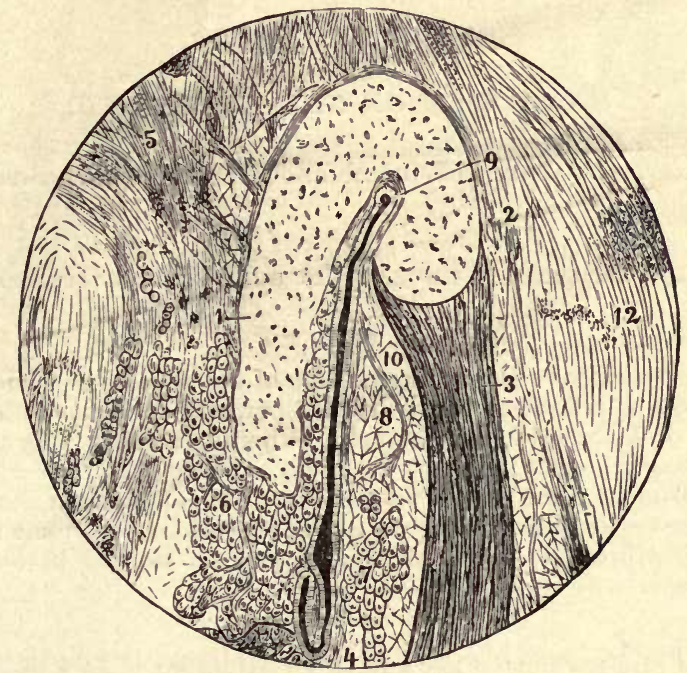

Fig. 268.-Section across thf. Cartilaginous PART of the Eustachian tube.

1,2 , bent cartilaginous plate; 3 , musc. dilatator tubæ; to the left of 4 , part of the attachment of the levator palati muscle: 5, tissue uniting the tube to the base of the skull; 6 and 7, mucous glanis; 8,10 , fat; 9 to 11 , lumen of the tube ; 12 , connective tissue ou the lateral aspect of the tube.

near the pharynx, it is bounded partly by a bent piece of cartilage (fig. 268, 1, 2), partly by fibrous tissue. The latter contains numerous mucous glands $(6,7)$, which open into the tube, and on the outer side a band of muscular tissue (3) which joins the tensor palati. The epithelium is ciliated. 


\section{LESSON XLII. \\ STRUCTURE OF THE LABYRINTH.}

1. Sections across one of the membranous semicircular canals of a fish (skate).

2. Longitudinal sections through the ampulla of a semicircular canal (skate).

3. Vertical sections through the middle of the cochlea of a mammal.

The cochlea is put quite fresh into $0 \cdot 2$ per cent. chromic acid containing a few drops of 1 per cent. osmic acid. When decalcified, it is well washed, and then placed in spirit for a day or more.

In preparing sections of the above three preparations it is advisable, in order that the epithelium should be kept in position, to mount them by the creosote-shellac process. They may previously be stained in bulk either by alcoholic magenta or borax-carmine.

4. Teased preparations of the auditory epithelium of an ampulla or of the macula of the utricle, from the fish.

5. Teased preparations of the epithelium of the organ of Corti from the guinea-pig.

Both 4 and 5 are made from osmic preparations.

Make sketches from all these preparations under the high power.

The labyrinth, which is the essential part of the auditory organ, consists of a complex membranous tube lined by epithelium and filled with endolymph, contained within a bony tube-the osseous labyrinth -of corresponding complexity of shape (figs. 269, 270). The membranous labyrinth does not wholly fill the bony cavity; the rest of the space is occupied by perilymph. The membranous labyrinth (fig. 269) is composed of the utricle $(u)$, and the three semicircular canals, each with an enlargement or ampulla which opens into it, the saccule ( $s$ ) and the canal of the cochlea (c. c.)

The branches of the auditory nerve pass to certain parts only of the membranous labyrinth, viz.: the macula of the utricle and saccule; the cristæ of the ampullæ, and along the whole length of the canal of the cochlea (the shaded parts in fig. 269). 
At these places the lining epithelium is specially modified to form a sensory or nerve-epithelium; elsewhere it is a simple pavementepithelium.

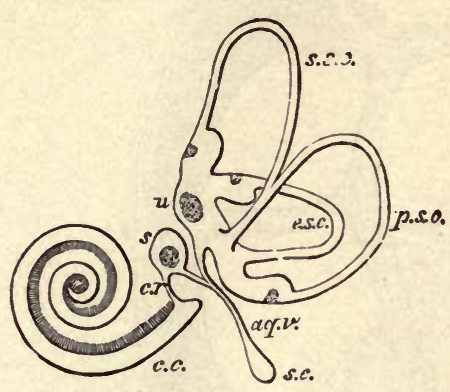

Fig. 269.-PLAN of THE RIGHT MEMBRANOUS LABYRINTH VIEWED FRON THE MESIAL ASPECT. 23

$u$ utricle, with its macula and the three semicircular canals with their ampullæ; s, saccule; $a q . v$. aquæuluctus vestibuli ; s.e. saccus endolymphaticus; $c . r$. canalis reuniens; c.c. canal of the cochlea.

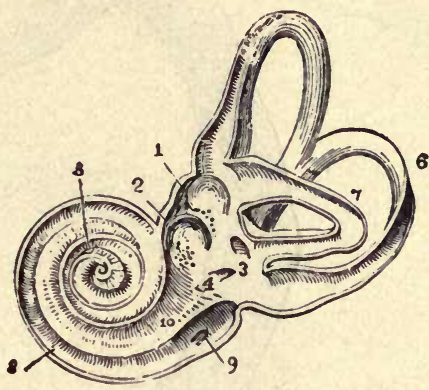

FIG. 270.-VIEI OF THE INTERIOR OF THE LEFT OSSEOUS LABYRINTH. $\frac{2}{1}$

The bony wall of the labyrinth is remored superiorly and externally. 1, fovea hemielliptica; 2 , forea hemispliærica; 3 , common opening of the superior and posterior semicircular canals; 4 , opening of the aquerluct of the vestibule; 5 , the superior, 6 , the posterior, and 7 , the extermal semicircular canals; 8 , spiral tube of the cochlea; 9 , scala tympani; 10 , scala vestibuli.

The membranous semicircular canals and the utricle and saccule are composed of fibrous tissue, which is adherent along one side to the endosteum of the bony canal; from the opposite side bands of fibrous tissue pass across the perilymph. Within the fibrous membrane is a thick clear tunica propria, which, in the semicircular canals, forms papillary elevations in the interior of the tube (figs. 271, 272).

The places of entrance of the nerve-fibres into the ampullæ are marked by a transverse, inwardly projecting ridge (crista), in the saccule and utricle by a thickening of the tunica propria (macula). The epithelium at these places is formed of columnar cells (fig. 273), which are surmounted by long, stiff, tapering hairs (auditory hairs, fig. $273, h$ ), and to these hair-cells the axis-cylinders of the nerve-fibres pass directly (fig. 274); they are therefore-like the rod-and coneelements of the retina, the bipolar cells of the olfactory membrane, and the gustatory cells of the taste-buds-sensory or neural epithelium-cells. Between them are a number of thin and somewhat rigid nucleated cells (fibre-cells of Retzius, fig. 274, f), which rest upon the basementmembrane, and are connected at their free extremity with a cuticular membrane, through which the auditory hairs project.

The auditory liairs do not project free into the endolymph, but into a soft mucus-like substance, of a dome-like form, in the ampullæ (fig. 273), and which in the saccule and utricle has a mass of calcareous particles (otoliths) embedded in it. 


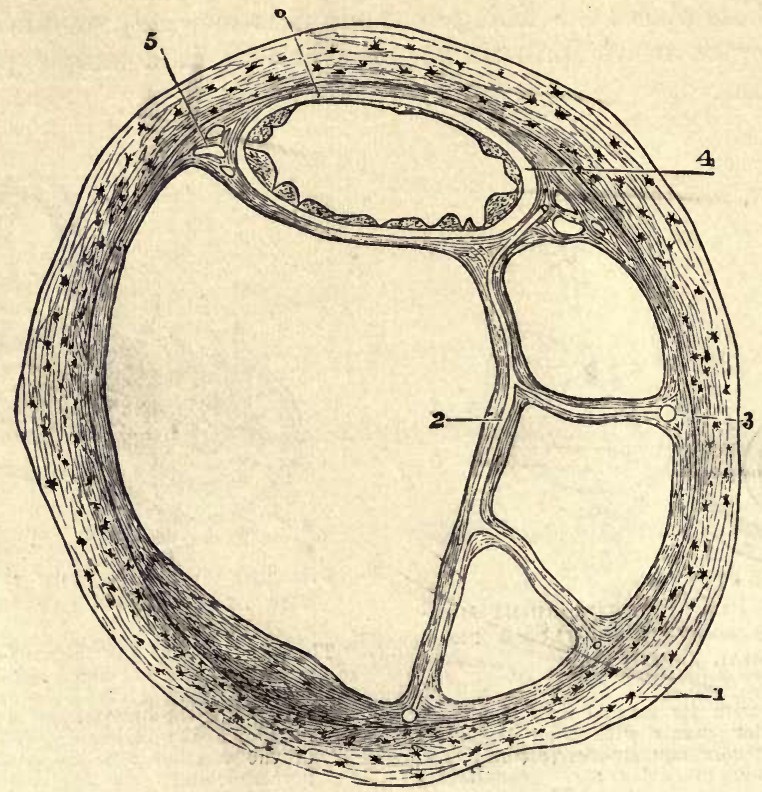

Jig. 271.-Section of one of the human semicircular canals. (Magnified.)

1, osseous wall ; 2 , fibrous bands with included b'ood-vesse's, united at 3 with the periosteum; 4 , membranous canal with its three layers: 5 , short fibrous bands (with intervening spaces) uniting the membranous canal firmly to the veriosteum ; 6 , union of its outermost layer with the periosteum.

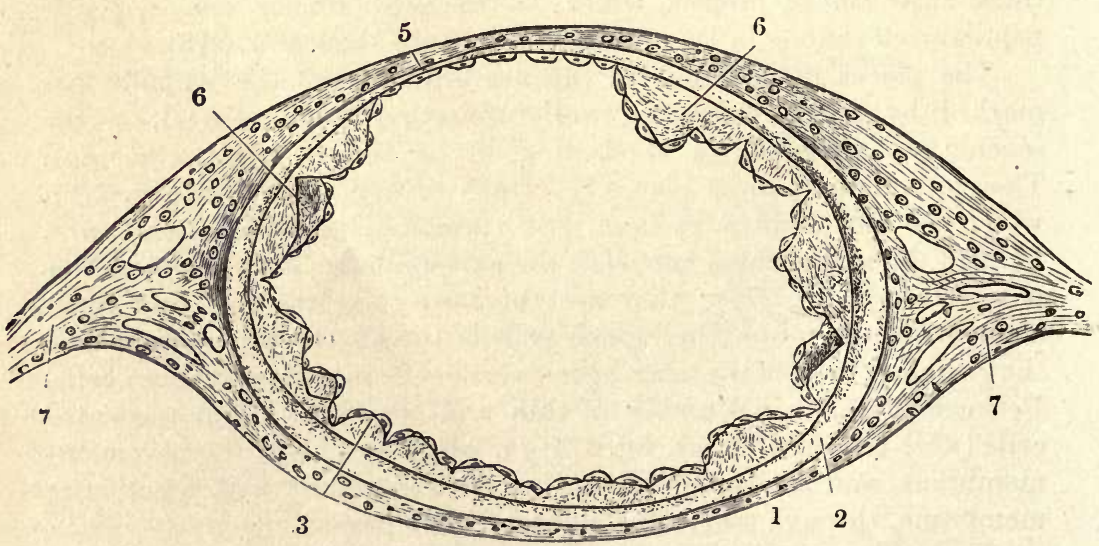

Fig. 272.-Section of membranous semicircular canal. (Much magnified.)

1. outer fibrous layer; 2 , tunica propria ; 3, 6, papilliform projections with epithelial covering; 5 , fixed side of the canal, with very thin tunica propria without papillæ; 7 , fibrous bands passing to periosteum. 


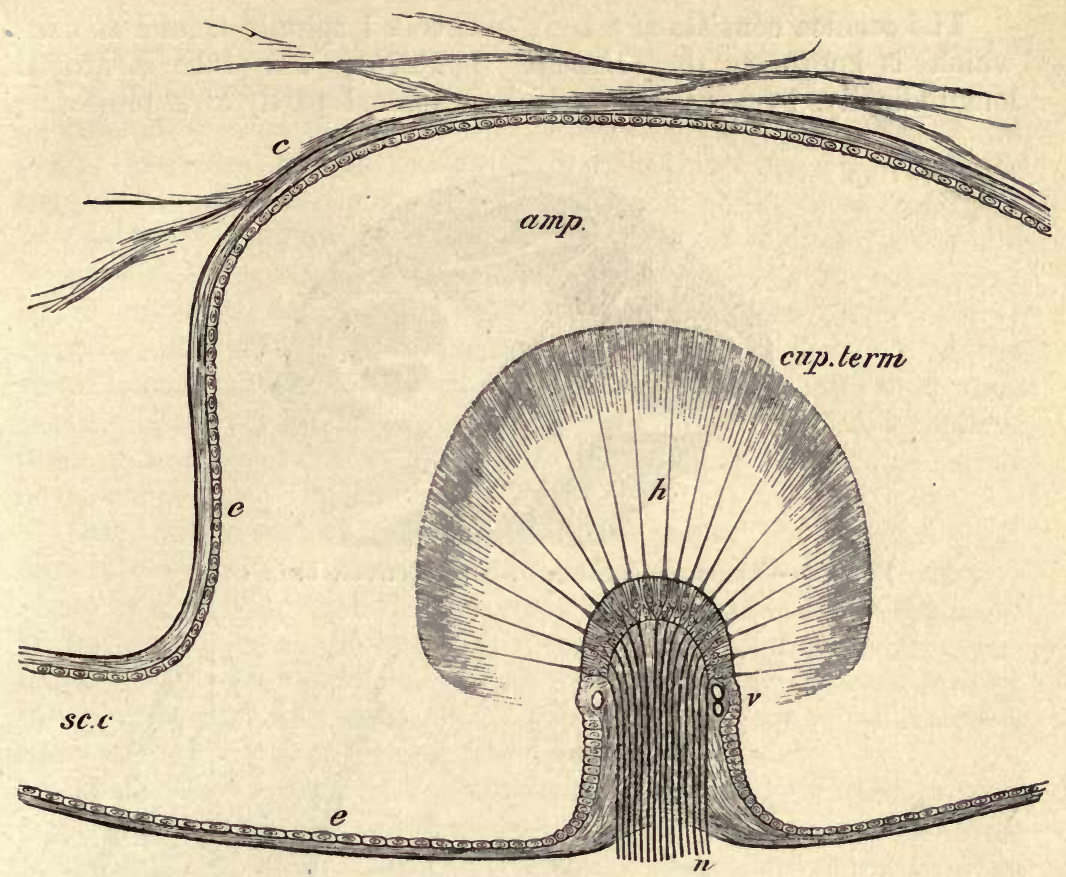

Fig. 273.-Longitudixal section of Ax AMptlla throvgh the crista acustica.

ump, cavity of the ampulla; sc.c, semicircular canal opening out of it; $c$, connective tissue attached to the wall of the nembranous ampulla and traversing the perilymph; $e, e$, flattened epithelium of ampulla ; $h$, auditory hairs projecting from the columnar cells of theauditory epithelium into the cuupla, cup. lerm.; $r$, blood-vessels; $n$, nerve-fibres entering the base of the crista and passing into the columnar cells.

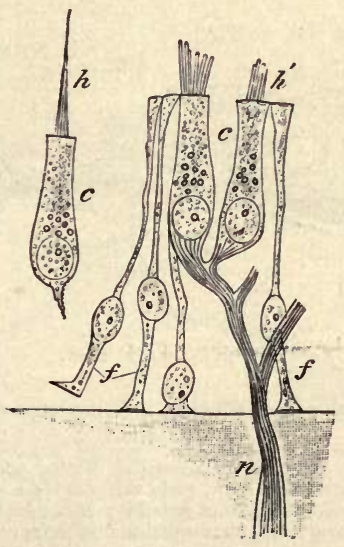

Fig. 274.-A Aditory epithelivm From the maCela ACUSTICA OF the SACCULE OF AN ALLigator. (Hi

$c, c$, columnar hair-cells ; $f, f$, fibre-cells ; $n$, nerve-fibre, losing its medullary sheath and passing to terminate in the columnar auditory cells ; $h$, auditory hair $h^{\prime}$, base of auditory hairs, split up into fibrils. 
The cochlea consists of a bony tube coiled spirally around an axis, which is known as the columella (fig. 275). The tube is divided longitudinally by a partition which is formed partly by a projecting

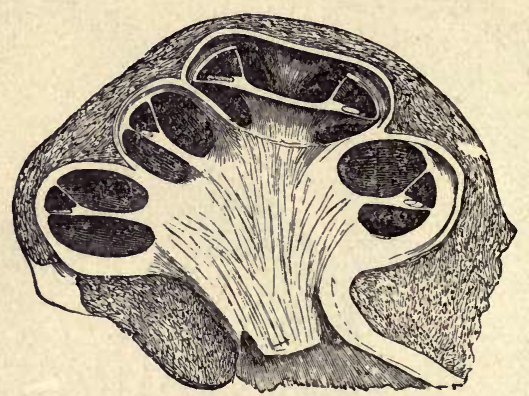

Fig. 275. - VERTiCAL SECTION OF THE COCHLEA OF A CALF.

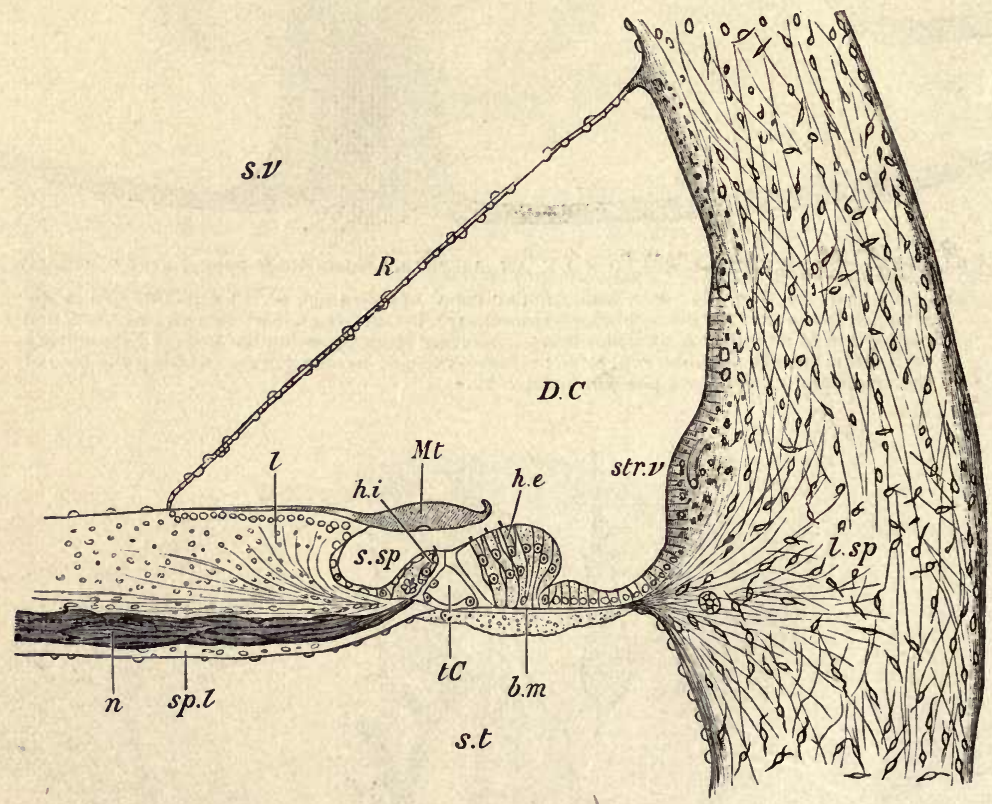

Fig. 276.-VERTical Section of the First tURN of the himan cochlea. (Retzius.)

s.v. scala vestibuli ; s.t. scala tympani; D.C. eanal of the cochlea; sp.l. spiral lamina; $n$, nerve-fibres; l.sp. spiral ligament; str.v. stria vascularis; s. sp. spiral groove; $R$, section of Reissner's membrane; $l$, limbus laminæ spiralis; $M . l$. membrana tectoria; $l . C$. tunnel of Corti ; b.m. basilar membrane; h.i., h.e., Internal and external hair-cells.

lamina of bone (spiral lamina), partly by a flat membrane (basilar membrane), into two parts or scala; the upper (supposing the cochlea resting base downwards) being termed the scala vestibuli (fig. $276, s . v$.) 
the lower the scala tympani (s.t.); the latter is closed at its larger end by the membrane of the fenestra rotunda. The scalæ are lined by endosteum, and are filled with perilymph, continuous with that of the rest of the osseous labyrinth at the commencement of the scala vestibuli; they communicate at the apex by a small opening, the helicotrema.

The scala vestibuli does not occupy the whole of that part of the bony tube of the cochlea which is above the partition. Its outer third is cut off by a delicate connective-tissue membrane (membrane of Reissner, fig. 276, R), which springs from near the end of the spiral lamina, and passes upwards and outwards to the outer wall, thus separating a canal $(D C)$ triangular in section, which is lined by epithelium, and represents the membranous labyrinth of the cochlea (canal of the cochlea).

Canal of the cochlea. The floor of the canal of the cochlea is formed (1) of the extremity of the spiral lamina, which is thickened above by a peculiar kind of connective tissue, forming an overhanging projection known as the limbus (fig. $276, l) ;(2)$ of the basilar membrane $(b . m$.$) , which stretches across from the end of the bony lamina$ to the outer wall, and is attached to this by a projection of reticular connective tissue termed the spiral ligament (l.sp.)

The basilar membrane is composed of stiff, straight fibres, which extend from within out, and themselves rest on a homogeneous stratum. It is covered below by a layer of connective tissue continuous with the endosteum of the scala tympani ; above the modified epithelium which forms the organ of Corti rests upon it. It becomes gradually broader in the upper turns of the cochlea (rather more than twice as broad in the uppermost as in the lowermost turn), and its constituent fibres become therefore gradually longer.

The organ of Corti consists of the following structures:

1. The rods of Corti, two series (inner and outer) of stiff, striated fibres of a peculiar shape, the inner rods somewhat like a human ulna, the outer like a swan's head and neck (fig. 277). They rest by one extremity (the foot) on the basilar membrane a short distance apart, and

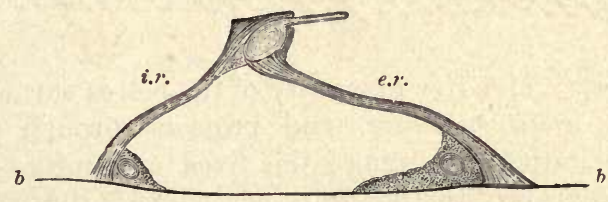

Fig. 277.-A pair of rods of Corti, fron the rabits's cochlea, in Side v1Ew. (Highly magnitied.)

$b, b$, basilar membrane; i.r. inner rod; e.r. onter rod. The nucleated protoplasmic masses at the feet are also shown.

are inclined towards one another, their larger ends (heads) being jointed together; the series of rods thus enclose a sort of tunnel, the floor of which is formed by a part of the basilar membrane. Close to their 


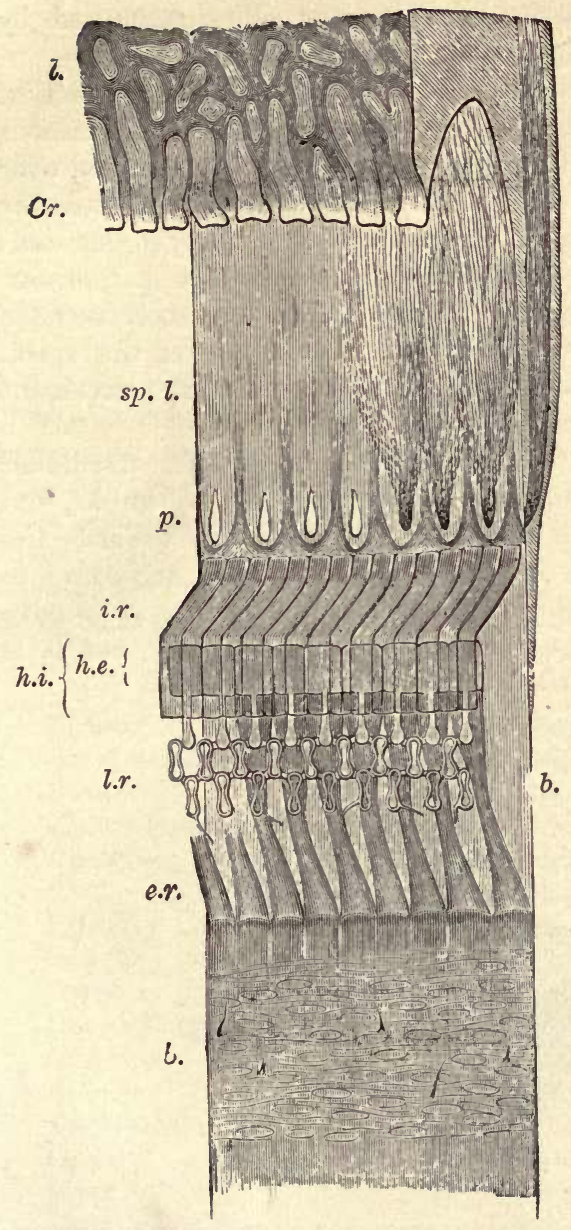

FIG. 279. - SEMI-DIAGRAMMATIC VIEW OF PART OF THE BASILAR MEMBRANE and TUNNel OF Corti of THE RABBIT, FIROM ABOVE AND THE SIDE. (Much magnified.)

l. limbus ; $\mathrm{Cr}$. extremity or erest of limbus with tooth-like projections ; b.b. basilar menbraue; sp.l. spiral lamina with, $p$, perforations for transmiscion of nerve-fibies. In part of the suiral lamina here represented the nerve-fibres are left, and are supuosed to be seen through the upper layer of that lamina. con verging to three of the perforations; to the right, in the section of the lamina, they are shown occupy ing a cutual, or eleft, in the osseous substance; $i . r$. fifteen of the inner roils of Corti ; h.i. their flatteneit heads seen from above; e.r. nine onter rods of Corti; h.e. their hends, with the phalangeal processes extenling out wuril from them and forming. with the two rows of phalanges, the lamina reticularis, i.r. On the left of the figure the connective-tissue fibres and nuclei of the undermost layer of the basilar membrane are scen through the upper layers. Portions of the basilar processes of the outer hair-cells remain attached bere and there to the membrane at this part.

FIG. 280.-AN OUTER HAIR-CELL IN CONNECTION WITH ITS BASILAR Process. From the guinea-pig. (Highly magnitied.)

$h$, one or two hairlets which have remained attached to the cell ; $b$, bulged lower end of cell ; $p$, basilar process, protoplasmic above, but becoming cuticular below and slightly expanded at the extremity, $f$, which is broken away from the basilar membrane. 
Corti. They form a single series of columnar cells surmounted by auditory hairs, lying in close apposition to the inner rods.

The rest of the epithelium-cells have no important characteristics. They are long and columnar next the outer hair-cells, but soon diminish in size, becoming cubical, and in this form they are continued over the outer wall of the cochlear canal. Here they cover a very vascular membrane (stria vascularis, fig. 276, str), which is frequently pigmented; its capillary blood-vessels may even penetrate between the epitheliumcells. Internal to the inner hair-cells the epithelium also soon becomes cubical; it is prolonged in this form over the limbus of the spiral lamina. The epithelium of Reissner's membrane is of the pavement variety.

The membrana tectorix (fig. 276, M.t.) is a soft, fibrillated structure, which is attached along the upper surface of the limbus, and lies like a pad over the organ of Corti. It thins out towards the distal margin, here becoming somewhat reticular, and, according to Retzius, attached to the lamina reticularis. In sections it usually appears raised a short distance above the auditory hairs, but it is possible that it may rest upon them during life.

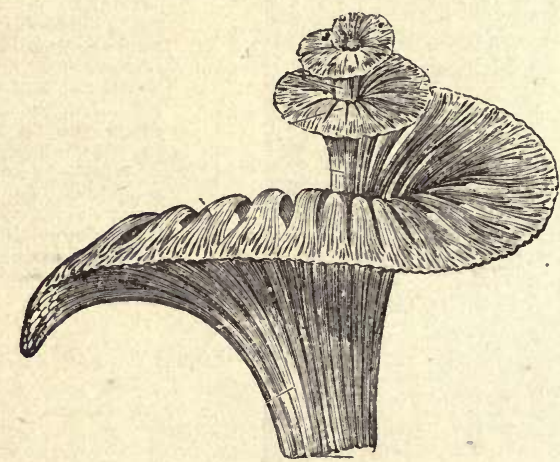

Fig. 281.-General view of the mode of Distribution of the cochlear nerve, ALL THE OTHER PARTS HAVING BEEN REMOVED.

The fikres of the cochlear branch of the auditory nerve enter the base of the columella, and run in canals through its substance, being gradually deflected outwards as they pass upwards into the spiral lamina, at the base of which they swell out into a ganglionic cord (spiral ganglion).

After traversing the spiral lamina they emerge in bundles, and the fibres then, having lost their medullary sheath, pass into the epithelium of the inner hair-cell region. Here some of them are connected directly with the inner hair-cells, whilst others pass in the form of delicate fibrils across the tumnel of Corti, to become connected with the outer hair-cells (fig. 278). 


\section{APPENDIX.}

General Methods of Preserving and Hardening Tissues and Organs. ${ }^{1}-$ The fluids which are most commonly used are alcohol, chromic acid solution ( 1 in 500), picric acid solution (saturated), bichromate of potash solution (2 per cent.), Müller's fluid (bichromate of potash $2 \frac{1}{2}$ parts ; sulphate of soda 1 part; water 100 parts), and bichromate of ammonia ( 2 per cent.) The following are the methods of hardening the several tissues and organs which are found to give the best general results :-

\section{Tissue or Organ Hardening Fluid}

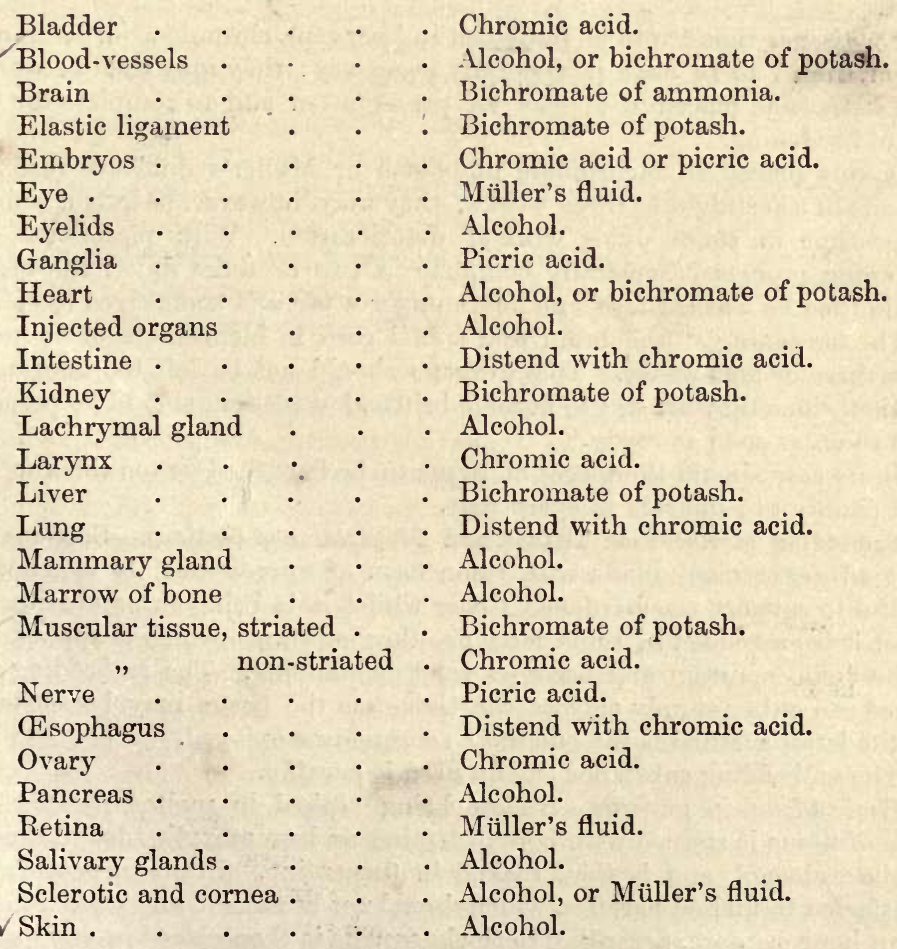

1 Methods of preparation required for special purposes are given in the Lessons. 


\section{Tissue or Organ}

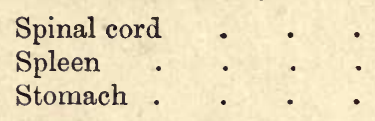

Suprarenal capsule .

Tendon and ligament

Testis

Thymus gland .

Thyroid gland .

Tongue

Tonsils

Trachea

Ureter

Uterus

\section{Hardening Fluid}

- Bichromate of ammonia.

- Bichromate of potash.

- Distend with chromic acid or with alcohol.

- Alcohol.

- Alcohol.

- Alcohol.

- Alcohol.

- Alcohol.

- Bichromate of potash.

- Alcohol.

- Chromic acid.

- Chromic acid.

- Chromic acid.

Tissues to be hardened in alcohol may either be placed at oace in strong spirit (90 per cent. alcohol), or the hardening may be effected gradually, the tissue being placed first in weak spirit (50 per cent.) for twenty-four hours, then in somewhat stronger, and finally in strong spirit or absolute alcohol. They are ready for cutting after having been twenty-four hours in strong spirit.

For tissues that are to be hardened in $\frac{1}{5}$ per cent. chromic acid, an immersion of from 7 to 14 days is generally necessary; they may then be washed with water, and placed in alcohol for preservation and to complete the process of hardening.

Organs placed in bichromate of potash or Müller's fluid are ready for sections in a fortnight or three weeks; they may, however, be left for a much longer time in those fluids without deterioration. With picric acid the hardening process is generally complete in two or three days; the organs should then be washed for some hours under a tap and transferred to spirit.

The hardening of the brain and spinal cord in bichromate of ammonia takes three or four weeks. These organs should not be left too long in the solution, since they are apt to become brittle, but sections should be prepared from them as soon as ready.

In no case should the pieces of tissue to be hardened be too thick for the fluid readily to penetrate to every part.

Embedding of Hardened Tissues, and Preparation of Sections.- Sections are most advantageously made with some form of microtome. It is generally needful to support the hardened tissue whilst it is being cut, and with this object it is embedded in some fatty or other substance which is applied to it in the fluid condition and becomes solid on standing. The embedding substance can either simply enclose the tissue, or the tissue may be soaked in it: the latter method is the one most commonly employed.

The embedding substance chiefly used is paraffin.

Embedding in paraffin.-Before being soaked in melted paraffin, the piece of tissue is stained with borax-carmine or hæmatoxylin, dehydrated by absolute alcohol, and is then soaked in turpentine. From turpentine it is transferred to melted paraffin, which should not be too hot, and soaked in this for an hour or more, according to thickness. It is then placed in any desired position in a paper tray or on the microtome and surrounded by melted 
paraftin. When cold, thin sections can be cut, the paraffin dissolvea out by turpentine, and the sections mounted.

Preparation of frozen sections.-The bichromate solutions are the best fluids to use for preserving tissues which are to be frozen in place of being embedded. The tissue in such cases should not be put into alcohol, but merely requires to be dipped in strong gum before being placed upon the freezIng microtome. Portions of the central nervous system need to be soaked in gum to which a little syrup has been added.

Staining and mounting of sections.-The fluids most commonly employed for the staining of sections are:-1. A dilute watery solution of hæmatoxyln and alum; 2. A solution of carmine; 3. A solution of picro-carminate of ammonia. The time of immersion in the staining fluid varies according to the strength of the fluid and the mode by which the tissue has been hardened. The necessity of staining sections may be avoided if the piece of tissue is stained in bulk before embedding. For this purpose a carmine solution is mostly used, on account of its penetrative power, that known as borax-carmine being the best. The tissue must be left in it for twenty-four hours or more, and then placed in acidulated alcohol. An alcoholic solution of magenta can be used for staining in bulk; from this the tissue goes into a small quantity of oil of cloves or into turpentine, and, after being soaked with this, into the melted paraffin.

If the tissues have not been stained in bulk, the following is the order of transference of the sections (they are supposed, if cut from paraffin, to have been freed from this by immersion in turpentine) :-

1. From turpentine to absolute alcohol (5 minutes).

2. From alcohol to distilled water ( $\frac{1}{2}$ minute).

3. From distilled water to hæmatoxylin or carmine (5 minutes or more).

4. From hæmatoxylin to distilled water ( $\frac{1}{2}$ minute).

5. From distilled water to alcohol ( 2 or 3 minutes).

6 . From alcohol to oil of cloves (1 minute).

7. From oil of cloves to Canada balsam.

If the tissues have already been stained in bulk, the sections are simply mounted in Canada balsam after the paraffin used for embedding has been dissolved away from them in turpentine.

Creosote-shellac method of mounting.-Friable sections, such as sections of small embryos, and ribands of sections such as are cut with many microtomes, are mounted in the following way:-The slide is smeared with a solution of shellac in creosote. the sections are placed in this and warmed so as to melt their paraffin. They are thus fixed by the shellac, and the slide can be immersed in turpentine to remove the paraffin, and the sections then covered in Canada balsam. For this method the tissue should always have been previously stained in bulk.

Solutions employed for Staining:-1. Solution of hamatoxylin in water.Rub together in a mortar 10 grammes of powdered alum and 5 grammes of extract of hæmatoxylin with 25 cubic centimeters of 70 per cent. alcohol, gradually adding 100 cubic centimeters of distilled water. Decant into a bottle and add a drop or two of ammonia. Let the mixture stand a few days, occasionally shaking it. For staining, add two or three drops to a watch-glass full of distilled water, and filter if necessary.

2. Grenacher's hamatoxylin. -To 150 cubic centimeters of a saturated 
solution of alum in water, add 4 cubic centimeters of saturated solution of hæmatoxylin in alcohol. Let the mixture stand 8 days, then decant, and add 25 cubic centimeters of glycerine, and 25 cubic centimeters of methylic alcohol.

3. Kleinenberg's hamatoxylin.-This serves better for staining in bulk. Saturate 70 per cent. alcohol first with calcium chloride and then with alum, and after filtration add six to eight volumes of 70 per cent. alcohol.

Take a freshly prepared saturated solution of hæmatoxylin in absolute alcohol, and add it drop by drop to the above mixture until it is of a distinct purplish colour.

This solution improves on keeping. It may if necessary be diluted with more of the mixture.

When hæmatoxylin solutions become red instead of blue, a trace of ammonia will restore the requisite colour.

4. Carminate of ammonia.-Prepared by dissolving carmme in ammonia and allowing the excess of ammonia to escape by slow evaporation. The salt should be allowed to dry and be dissolved in water as required.

5. Picro-carminate of ammonia (picro-carmine).-To a saturated solution of picric acid add a strong ammoniacal solution of carmine, until a precipitate begins to form. Evaporate on the water-bath to $\frac{1}{5}$ th ; filter from the sediment and evaporate the filtrate to dryness. Make a 5 per cent. solution of the residue, diluting further as required.

6. Borax-carmine.-a. Dissolve 4 grammes borax and 3 grammes carmine in 100 cubic centimeters of warm water. Add 100 cubic centimeters of 70 per cent. alcohol, filter and let stand. This solution improves on keeping. It is useful for staining in bulk.

3. Boil 0.5 gramme carmine and 1 gramme borax in 100 cubic centimeters water. Filter and add acetic acid drop by drop until the original violet colour becomes crimson; then filter once more. This solution is used for staining sections.

After staining with borax-carmine, the tissue should in all cases be placed in 70 per cent. alcohol containing 5 drops of hydrochloric acid to 100 cubic centimeters.

7. Magenta. - This may be kept in solution in alcohol $(0.5$ to 1 per cent.) For fresh tissues and for sections to be mounted in glycerine, an excellent staining fluid is obtained by adding one or two drops to a watch-glass of water. For sections to be mounted in Canada balsam a solution in oil of cloves is used. This is best made by adding a drop of the alcoholic solution to a little oil of cloves in a watch-glass: the sections after being stained are washed in spirit of turpentine.

8. Gentian viclet.-Mix 20 cubic centimeters water with 10 cubic centimeters alcohol and 10 cubic centimeters glycerine, and add to the mixture 10 drops of a 1 per cent. solution of gentian violet in alcohol and 10 drops of a 25 per cent. solution of formic acid in water.

This solution gives excellent results with fresh tissues, especially with epithelium.

9. Safranin.-A saturated alcoholic solution is used for staining cellnuclei. The tissue elements having been fixed by dilute chromic acid or by alcohol, small shreds or thin sections are placed for 12 to 24 hours in a little of the solution, mixed with half its bulk of water. The shreds are rinsed in absolute alcohol (which must contain no trace of free acid) until the colour is 
washed out from everything except the nuclei; they are then at once trans. ferred to turpentine, and from this are mounted in Canada balsam.

10. Aniline blue-black.-Dissolve 1 gramme of aniline blue-black in a mixture of 30 parts of water with 20 of alcohol. This serves for staining the central nervous system either in bulk or in sections.

11. Staining with chloride of gold.-a. Cohnheim's method.-Place the fresh tissue for from 30 to 60 minutes in $\frac{1}{2}$ per cent. solution of chloride of gold; then wash and transfer to a large quantity of water just acidulated with acetic acid. Keep for 2 or 3 days in the light in a warm place.

B. Löwit's method.-Place small pieces of the fresh tissue in a mixture of 1 part of formic acid to 2 to 4 parts of water for $\frac{1}{2}$ to 1 minute; then in 1 per cent. chloride of gold solution for 10 to 15 minutes; then back again into the formic acid mixture for 24 hours and then into pure formic acid for 24 hours more. After removal from the gold, and whilst in the acid, the tissue must be kept in the dark.

$\gamma$. Ranvier's method.-Immerse in lemon-juice for 5 to 10 minutes, then wash with water and place in 1 per cent. gold chloride solution for 20 minutes. Then treat either as in Cohnheim's or in Löwit's method.

12. Staining with nitrate of silver.-Wash the fresh tissue with distilled water; immerse in $\frac{1}{2}$ to 1 per cent. nitrate of silver solution for 5 to 10 minutes; rinse with distilled water and expose to bright sunlight either in water, alcohol, or glycerine.

Mounting Solutions:-1. Saline solution.-A 0.6 per cent. solution of common salt is used in place of serum for mounting fresh tissues for imme. diate examination.

2. A mixture of glycerine and vater in equal parts.

3. Farrant's solution.-Take a mixture of equal parts of glycerine and saturated watery solution of arsenious acid, and stir gum arabic with it until a thick syrupy fluid is formed. Filter.

4. Canada balsam, from which the volatile oils have been driven off by heat, dissolved in benzole. 




\section{LEA BROTHERS \& CO.'S}

(Late HENRY C. LEA'S SON \& CO.)

\section{CLASSIFIED CATALOGUE}

OF

\section{MEDICALAND SURGICAL} PUBLICATIONS.

In asking the attention of the profession to the works advertised in the following pages, the publishers would state that no pains are spared to secure a ccntinuance of the confidence earned for the publications of the house by their careful selection and accuracy and finish of execution.

The large number of inquiries received from the profession for a finer class of bindings than is usually placed on medical books has induced us to put certain of our standard publications in half Russia; and, that the growing taste may be encouraged, the prices have been fixed at so small an advance over the cost of sheep as to place it within the means of all to possess a library that shall have attractions as well for the eye as for the mind of the reading practitioner.

The printed prices are those at which books can generally be supplied by booksellers throughout the United States, who can readily procure for their customers any works not kept in stock. Where access to bookstores is not convenient books will be sent by mail postpaid on receipt of the price, and as the limit of mailable weight has been removed, no difficulty will be experienced in obtaining through the post-office any work in this catalogue. No risks, however, are assumed either on the money or on the books, and no publications but our own are supplied, so that gentlemen will in most cases find it more convenient to deal with the nearest bookseller.

\section{LEA BROTHERS \& CO.}

Nos. 706 and 708 Sansom St., Philadelphia, September, 1887.

\section{PROSPECTUS FOR 1887.}

\section{The American Journal of the Medical Sciences.}

Quarterly, 300-350 pages, with illustrations. Price, $\$ 5.00$ per annum.

WITH the year 1886 The American Journal of The Medical Sciences became $W$ in Great Britain the recognized organ of the profession-a position similar to that occupied by it in America for sixty-six years. On its announcement, this project for an international journal was welcomed abroad with acclamation, and one hundred and thirty-five of the foremost English practitioners authorized the use of their names as contributors in order to aid in extending over their country the benefits which American medicine has enjoyed from the existence of THE JoURNaL during two generations This friendly challenge was accepted by an almost equal-number of Americans, to whose proved ability this country can well afford to entrust her reputation.

In thus becoming the medium of communication between the two nations distinguished above all others by the practical character of their labors, The JoURNAL undoubtedly forms the most efficient factor in medical progress which the world has yet seen. Already this generous spirit of rivalry has proved that the ample space devoted to Original Articles will continue to be filled with a series of contributions unapproachable in value. 


\section{THE AMERICAN JOURNAL of the MEDICAL SCIENCES.}

(Continued from first page.)

But it is not only in the Original Department that the JouRnaL of the future will seek to eclipse all its efforts in the past. The mass of contributions to medical literature and science increases with such rapidity, that if the reader is to keep abreast with them the matter must be carefully sifted, and arranged so as to enable him to grasp it understandingly with the least possible expenditure of time. In the Bibliographical Department, therefore, separate reviews are devoted only to works of exceptional importance. As a rule, new books are considered in groups of cognate subjects, the reviewer setting forth tersely the merits of the individual volumes with a condensed statement of the views of the authors. In this manner the reader is kept advised of the products of the press in the most convenient manner.

A similar plan is adopted in the Quarterly Summary of Progress. The various branches of medical science have been assigned to the following gentlemen, who will furnish well-digested résumés of progress, paying special attention to clinical application: Anatomy, George D. Thane, M. R. C. S.; Physiology, Gerald F. Yeo, M. D.; Materia Medica, Therapeutics and Pharmacology, Roberts Bartholow, M. D., LL. D.; Medicine, William Osler, M.D.; Surgery, in America, R. J. Hall, M. D.; in Europe, Frederick Treves, F. R. C. S.; Ophthalmology, L. Webster Fox, M. D. ; Otology, Charles H. Burnett, M. D.; Laryngology, J. Solis Cohen, M. D.; Dermatology, Louis A. Duhring, M. D., and H. W. Stelwagon, M. D.; Midwifery and Gynecology, D. Berry Hart, M. D.; Jurisprudence, Matthew Hay, M. D.; Public Health, Shirley F. Murphy, M. R. C. S.

The publishers feel an honest pride in thus being the means of bringing together the professions of the two great English-speaking peoples, and in laying before them a periodical which must be universally recognized as marking a new era in medical progress. Believing that it will be regarded as indispensable by all intelligent physicians on both sides of the Atlantic, they feel themselves warranted, by the expectation of a large increase in circulation, in maintaining the present very moderate subscription price, notwithstanding the greatly augmented expenditure entailed by the change.

The JouRnaL will continue to be published quarterly, as heretofore, on the first of January, April, July and October.

Price, FIVE DOLLARS Per Annum, in Advance.

\section{THE MEDICAL NEWS.}

\section{A National Weekly Periodical, containing 28 to 32 Quarto Pages} in Each Issue.

THE continually increasing appreciation of THE MEDICAL NEWs by the profession 1 throughout the country, is a most gratifying recognition of the policy pursued by the managers of this journal in their unceasing efforts to enhance its value to the practitioner.

Possessing a most efficient organization THE NEwS unites the best features of the medical magazine and newspaper. Its large and able Editorial Staff discusses in each issue the important topics of the day in a thoughtful and scholarly manner, while its corps of qualified reporters and correspondents, covering every medical centre, insures that its readers shall be promptly and thoroughly posted upon all matters of interest in the world of medicine. On account of the position conceded to The News, it has become the medium chosen by the leading minds of the profession for the publication of their most important contributions to medical science. The valuable instruction afforded in Clinical Lectures, and the rich experience gained in the leading Hospitals of the world are constantly laid before the readers of The News, while prompt and authentic reports of Society Proceedings are received from special reporters in various sections of the country by mail and Telegraph. In the pages devoted to the progress of Medical Science are found early notes of all important advances, gleaned from the principal journals of both hemispheres. Ample space is devoted to Reviews, News Items, Correspondence and Notes and Queries In short, every branch of medicine is adequately represented in The News, and the details of plan and typography have been carefully studied in order to economize the time and secure the comfort of the reader in every possible way.

$$
\text { Price, FIVE DOLLARS Per Annum, in Advance. }
$$

\section{COMMUTATION RATE.}

To subscribers paying in advance for $1887:-$

AMERICAN JouRnal OF THE MEDiCAL ScIEnchs (quarterly) $\}$ To one address for $\$ 9.00$ The Medical News (weekly)

SPECIAL OFFERS.

Advance-paying subscribers to either or both of the above-named periodicals may take advantage of any one of the following offers:

(1). The Medical News Visiting List for 1887, dated, either for 30 patients per week ( 1 vol.), or for 60 patients ( 2 vols.), or for 90 patients $(3$ vols.), will be sent 
post-paid on receipt of 75 cents per volume (regular price \$1.25). (2). THE YEAR-BooK oF TREATMENT for 1886 will be sent on receipt of 75 cents (regular price \$1.25). (3). An advance remittance of $\$ 10$ will procure ThE NEws and THE JouRNAL for one year, together with any one volume of The Medical News Visiting List and The YearBook of Treatment for 1886, as offered above. Subsequent volumes of The Medical News Visitivg LisT may be procured by advance-paying subscribers for 75 cents each (regular price \$1.25). Thumb-letter Index for quick use, 25 cents additional.

Subscribers can obtain, at the close of each volume, cloth covers for THE JouRNaL (one annually), and for THE NEWs (one annually), free by mail, by remitting Ten Cents for the JournaL cover, and Fifteen Cents for the NEws cover.

The safest mode of remittance is by bank check or postal money order, drawn to the order of the undersigned; where these are not accessible, remittances for subscriptions may be sent at the risk of the publishers by forwarding in registered letters. Address,

LEA BROTHERS \& CO., 706 and 708 Sansom Street, PhInadelphia.

\section{THE MEDICAL NEWS VISITING LIST FOR $188 \%$.}

Containing Calendar for two years. Obstetric diagrams. Scheme of Dentition. Tables of weights and measures and comparative scales. Instructions for examining the urine. List of disinfectants. Table of eruptive fevers. Lists of new remedies and remedies not generally used, Incompatibles, Poisons and Antidotes. Artificial respiration. Table of doses, prepared to accord with the last revision of the U. S. Pharmacopoia, an extended table of Diseases and their remedies, and directions for Ligation of Arteries. Blanks for all records of practice and Erasable tablet. Handsomely bound in limp Morocco, with tuck, pencil, rubber and catheter scale.

In response to numerous requests, The Medical News Visiting List for 1887 is issued in three sizes, viz. Dated, for 30 patients per week, 1 vol.; dated, for 60 patients, 2 vols.; dated, for 90 patients, 3 vols.; Price per volume, $\$ 1.25$. Also, furnished with Ready Reference Thumb-letter Index for quick use, 25 cents additional. For special offers, including VISITING LIST, see above.

\section{THE MEDICAL NEWS PHYSTCIANS' LEDGER.}

Containing 400 pages of fine linen "ledger" paper, ruled so that all the accounts of a large practice may be conveniently kept in it, either by single or double entry, for a long period. Strongly bound in leather, with cloth sides, and with a patent flexible back, which permits it to lie perfectly flat when opened at any place. Price, $\$ 5.00$. Also, a small special lot of same Ledger, with 300 pages. Price, $\$ 4.00$.

\section{HARTSHORNE, HENRY, A. M., M. D., LL. D.,}

Latsly Professor of Hygiene in the University of Pennsylvania.

A Conspectus of the Medical Sciences; Containing Handbooks on Anatomy, Physiology, Chemistry, Materia Medica, Practice of Medicine, Surgery and Obstetrics. Second edition, thoroughly revised and greatly improved. In one large royal $12 \mathrm{mo}$. volume of 1028 pages, with 477 illustrations. Cloth, $\$ 4.25$; leather, $\$ 5.00$.

The object of this manual is to afford a conven- $/$ industry and energy of its able editor-Boston lent work of reference to students during the brief Medical and Surgical Journal, Sept. 3, 1874.

moments at their command while in attendance We can say with the strictest truth that it is the upon medical lectures. It is a favorable slgn that best work of the kind with which we are acit has been found necessary, in a short space of quainted. It embodies in a condensed form all time, to issue a new and carefully revised edition. recent contributions to practical medicine, and is The illustrations are very numerous and unusu- therefore useful to every busy practitioner throughally clear, and each part seems to have received out our country, besides being admirably adapted its due share of attention. We can conceive such to the use of students of medicine. The book is a work to be useful, not only to students, but to faithfully and ably executed.-Charleston Medical practitioners as well. It reflects credit upon the Journal, April, 1875.

\section{NEILL, JOHN, M. D., and SMTTH, H. G., M.. D.,}

Late Surgeon to the Penna. Hospital.

Prof. of the Institutes of Med. in the Univ. of Penna.

An Analytical Compendium of the Various Branches of Medical Science, for the use and examination of Students. A new edition, revised and improved. In one large royal $12 \mathrm{mo}$. volume of 974 pages, with 374 woodcuts. Cloth, $\$ 4$; leather, $\$ 4.75$.

\section{LUDLOW, J. L., M. D.,}

Consulting Physician to the Philadelphia Hospital, etc.

A Manual of Fxaminations upon Anatomy, Physiology, Surgery, Practice of Medicine, Obstetrics, Materia Medica, Chemistry, Pharmacy and Therapeutics. To whicn is added a Medical Formulary. 3d edition, thoroughly revised, and greatly enlarged. In one $12 \mathrm{mo}$. volume of 816 pages, with 370 illustrations. Cloth, $\$ 3.25$; leather, $\$ 3.75$.

The arrangement of this volume in the form of question and answer renders it especially suitable for the office examination of students, and for those preparing for graduation. 


\section{DUNGLISON, ROBLEY, M. D.,}

Late Professor of Institutes of Medicine in the Jefferson Medical College of Philadelphia.

MEDICAL LEXICON; A Dictionary of Medical Science: Containing a concise Explanation of the various Subjects and Terms of Anatomy, Physiology, Pathology, Hygiene, Therapeutics, Pharmacology, Pharmacy, Surgery, Obstetrics, Medical Jurisprudence and Dentistry, Notices of Climate and of Mineral Waters, Formulæ for Officinal, Empirical and Dietetic Preparations, with the Accentuation and Etymology of the Terms, and the French and other Synonymes, so as to constitute a French as well as an English Medical Lexicon. Edited by KICHARD J. DunGLISON, M.D. In one very large and handsome royal octavo volume of 1139 pages. Cloth, $\$ 6.50$; leather, raised bands, $\$ 7.50$; very handsome half Russia, raised bands, $\$ 8$.

The object of the author, from the outset, has not been to make the work a mere lexicon or dictionary of terms, but to afford under each word a condensed view of its various medical relations, and thus to render the work an epitome of the existing condition of medical science. Starting with this view, the immense demand which has existed for the work has enabled him, in repeated revisions, to augment its completeness and usefulness, until at length it has attained the position of a recognized and standard authority wherever the language is spoken. Special pains have been taken in the preparation of the present edition to maintain this enviable reputation. The additions to the vocabulary are more numerous than in any previous revision, and particular attention has been bestowed on the accentuation, which will be found marked on every word. The typographical arrangement has been greatly improved, rendering reference much more easy, and every care has been taken with the mechanical execution. The volume now contains the matter of at least four ordinary octavos.

About tne first book purchased by the medical student is the Medical Dictionary. The lexicon explanatory of technical terms is simply a sine qua nor. In a science so extensive and with such collaterals as medicine, it is as much a necessity also to the practising physician. To meet the wants of students and most physicians the dictionary must be condensed while comprehensive, and practical while perspicacious. It was because Dunglison's met these indications that it became at once the dictionary of general use wherever medicine was studied in the English language. In no former revision have the alterations and additions been so great. The chief terms have been set in black letter, while the derivatives follow in small caps; an arrangement which greatly facilitates reference. - Cincinnati Lancet and Clinic, Jan. 10, 1874.

A book of which every American ought to be proud. When the learned author of the work

passed away, probably all of us feared lest the book should not maintain its place in the advancing science whose terms it defines. Fortunately, Dr. Richard J. Dunglison, having assisted his father in the revision of several editions of the work, and having been, therefore, trained in the methods and imbued with the spirit of the book, has been able to edit it as a work of the kind should be edited-to carry it on steadily, without jar or interruption, along the grooves of thought it has travelled during its lifetime. To show the magnitude of the task which Dr. Dunglison has assumed and carried through, it is only necessary to state that more than six thousand new subjects have been added in the present edition. - Philadelphia Medical Times, Jan. 3, 1874.

It has the rare merit thatit certainly has no rival in the English language for accuracy and extent of in the English language for accuracy
references.-London Medical Gazette.

\section{HOBLYN, RICHARD D., M. D.}

A Dictionary of the Terms Used in Medicine and the Collateral Sciences. Revised, with numerous additions, by IsAAC HAYs, M. D., late editor of The American Journal of the Medical Sciences. In one large royal $12 \mathrm{mo}$. volume of 520 double-columned pages. Cloth, $\$ 1.50$; leather, $\$ 2.00$.

It is the best book of definitions we have, and ought always to be upon the student's table.-Southern Medieal and Surgical Journal.

\section{STUDENTS' SERIES OF MANUALS.}

A Series of Fifteen Manuals, for the use of Students and Practitioners of Medicine and Surgery, written by eminent Teachers or Examiners, and issued in pocket-size 12mo. volumes of 300-540 pages, richly illustrated and at a low price. The following volumes are now ready: TREves' Manual of Surgery, by various writers, in three volumes, each, \$2; BeLL's Comparative Physiology and Anatomy, \$2; GoulD's Surgical Diagnosis, \$2; RoBertson's Physiological Physics, \$2; Bruce's Materia Medica and Therapeutics, \$1.50; Power's Human Physiology, \$1.50; CLARKe and Lockwoon's Dissectors' Manual, \$1.50; RALFE's Clinical Chemistry, \$1.50; Treves' Surgical Applied Anatomy, \$2; PEPPER's Surgical Pathology, \$2; and KLEIN's Elements of Histology, \$1.50. The following are in press: Bellamr's Operative Surgery, PEPPER's Forensic Medicine, and CuRNow's Medical Applied Anatomy. For separate notices see index on last page.

\section{SERIES OF CLINICAL MANUALS.}

In arranging for this Series it has been the design of the publishers to provide the profession with a collection of authoritative monographs on important clinical subjects in a cheap and portable form. The volumes will contain about 550 pages and will be freely illustrated by chromo-lithographs and woodcuts. The following volumes are now ready: Hutchinson on Syphilis, $\$ 2.25$; Marsh on the Joints, $\$ 2$; OwEN on Surgical Diseases of Children, \$2; Morris on Surgical Diseases of the Kidney, \$2.25; PICK on Fractures and Dislocations, \$2; Buturs on the Tongue, $\$ 3.50 ;$ Treves on Intestinal Obstruction, \$2; and SAvAGE on Insanity and Allied Neuroses, \$2. The following are in active preparation: CARTer \& Frost's Ophthalmic Surgery, Bryant on the Breast, Broadbent on the Pulse, LUCAs on Diseases of the Urethra, and BaLL on the Rectum and Anus. For separate notices see index on last page. 
GRAY, HENRY, F.R.S.,

Lecturer on Anatomy at St. George's Hospital, London.

Anatomy, Descriptive and Surgical. The Drawings by H. V. CARTER, M. D., and Dr. WestmacotT. The dissections jointly by the AUthor and Dr. CARTER. With an Introduction on General Anatomy and Development by T. Holmrs, M. A., Surgeon to St. George's Hospital. Edited by T. Prckering PICK, F. R. C. S., Surgeon to and Lecturer on Anatomy at St. George's Hospital, London, Examiner in Anatomy, Royal College of Surgeons of England. A new American from the eleventh enlarged and improved London edition, thoroughly revised and re-edited by William W. KEen, M. D., Professor of Anatomy in the Pennsylvania Academy of the Fine Arts, etc. To which is added the second American from the latest English edition of LANDMARKs, MEDICAL AND SURGICAL, by LUTHer Holden, F. R. C. S. In one imperial octavo volume of about 1100 pages, with about 650 large and elaborate engravings on wood. Price of edition in black (see below): Cloth, $\$ 6$; leather, $\$ 7$; half Russia, $\$ 7.50$. The price of edition in colors will be announced shortly.

This work covers a more extended range of subjects than is customary in the ordinary text-books, giving not only the details necessary for the student, but also the application to those details to the practice of medicine and surgery. It thus forms both a guide for the learner and an admirable work of reference for the active practitioner. The engravings form a special feature in the work, many of them being the size of nature, nearly all original, and having the names of the various parts printed on the body of the cut, in place of figures of reference with descriptions at the foot. In this edition a new departure will be taken by the issue of the work with the arteries, veins and nerves distinguished by different colors. The engravings thus form a complete and splendid series, which will greatly assist the student in forming a clear idea of Anatomy, and will also serve to refresh the memory of those who may find in the exigencies of practice the necessity of recalling the details of the dissecting-room. Combining, as it does, a complete Atlas of Anatomy with a thorough treatise on systematic, descriptive and applied Anatomy, the work will be found of great service to all physicians who receive students in their offices, relieving both preceptor and pupil of much labor in laying the groundwork of a thorough medical education.

For the convenience of those who cannot afford the slight increase in cost necessitated by the use of colors, the volume will be published also in black alone, and maintained in this style at the price of former editions, notwithstanding the largely increased size of the work.

Landmarks, Medical and Surgical, by the distinguished Anatomist, Mr. Luther Holden, has been appended to the present edition as it was to the previous one. This work gives in a clear, condensed and systematic way all the information by which the practitioner can determine from the external surface of the body the position of internal parts. Thus complete, the work, it is believed, will furnish all the assistance that can be rendered by type and illustration in anatomical study.

\section{Also For SAle SEPARATE-}

\section{HOLDEN, LUTHER, F. R. C.S.,}

Surgeon to St. Bartholomew's and the Foundling Hospitals, London.

Landmarks, Medical and Surgical. Second American from the latest revised English edition, with additions by W. W. KeEn, M. D., Professor of Artistic Anatomy in the Pennsylvania Academy of the Fine Arts, formerly Lecturer on Anatomy in the Philadelphia School of Anatomy. In one handsome 12mo. volume of 148 pages. Cloth, \$1.00.

This little book is all that can be desired within / cians and surgeons is much to be encouraged. It its scope, and its contents will be found simply in- inevitably leads to a progressive education of both valuable to the young surgeon or physician, since the eye and the touch, by which the recognition of they bring before him such data as he requires at disease or the localization of injuries is vastly asevery examination of a patient. It is written in sisted. One thoroughly familiar with the facts here language so clear and concise that one ought taught is capable of a degree of accuracy and a almost to learn it by heart. It teaches diagnosis by confidence of certainty which is otherwise unatexternal examination, ocular and palpable, of the tainable. We cordially recommend the Landmarks body, with such anatomical and physiological facts to the attention of every physician who has not as directly bear on the subject. It is eminently yet provided himself with a copy of this useful, the student's and young practitioner's book. - Phy. practical guide to the correct placing of all the sician and Surgeon, Nov. 1881.

The study of these Landmarks by both physi-

\section{WILSON, ERASMUS, F. R. S.}

A System of Human Anatomy, General and Special. Edited by W. H. Gobrecht, M. D., Professor of General and Surgical Anatomy in the Medical College of Ohio. In one large and handsome octavo volume of 616 pages, with 397 illustrations. Cloth, $\$ 4.00$; leather, $\$ 5.00$.

\section{CLELAND, JOHN, M. D., F. R. S.,}

Professor of Anatomy and Physiology in Queen's College, Galway.

A Directory for the Dissection of the Human Body. In one 12mo. volume of 178 pages. Cloth, $\$ 1.25$. 


\section{ALLEN, HARIRISON, M. D.,}

Professor of Physiology in the University of Pennsylvania.

A System of Human Anatomy, Including Its Medical and Surgical Relations. For the use of Practitioners and Students of Medicine. With an Introductory Section on Histology. By E. O. SHAKESPEA RE, M. D., Ophthalmologist to the Philadelphia Hospital. Comprising 813 double-columned quarto pages, with 380 illustrations on 109 full page lithographic plates, many of which are in colors, and 241 engravings in the text. In six Sections, each in a portfolio. Section I. Histology. Section II. Bones and Jornts. Section III. Muscles and Fascla. Section IV. Arterims, Venns and Lymphatics. Section V. Nervous System. Section VI. Organs of Sense, of Digestion and Gentto-Urinary Organs, Embryology, Development, Triratology, Superfictal Anatomy, Post-Mortem Examinations, AND GeNeral aNd Clinical Indexes. Price per Section, $\$ 3.50$; also bound in one volume, cloth, $\$ 23.00$; very handsome half Russia, raised bands and open back, $\$ 25.00$. For sale by subscription only. Apply to the Publishers.

Extract from Introduction.

It is the design of this book to present the facts of human anatomy in the manner best suited to the requirements of the student and the practitioner of medicine. The author believes that such a book is needed, inasmuch as no treatise, as far as he knows, contains, in addition to the text descriptive of the subject, a systematic presentation of such anatomical facts as can be applied to practice.

A book which will be at once accurate in statement and concise in terms; which will be an acceptable expression of the present state of the science of anatomy; which will exclude nothing that can be made applicable to the medical art, and which will thus embrace all of surgical importance, while omitting nothing of value to clinical medicine,-would appear to have an excuse for existence in a country where most surgeons are general practitioners, and where there are few general practitioners who have no interest in surgery.

It is to be considered a study of applied anatomy $\mid$ care, and are simply superb. There is as much in its widest sense-a systematic presentation of of practical application of anatomical points to such anatomical facts as can be applied to the the every-day wants of the medical clinician as practice of medicine as well as of surgery. Our to those of the operating surgeon. In fact, few author is concise, accurate and practical in his general practitioners will read the work without a statements, and succeeds admirably in infusing feeling of surprised gratification that so many an interest into the study of what is generally con- points, concerning which they may never have sidered a dry subject. The department of Histol- thought before are so well presented for their conogy is treated in a masteriy manner, and the sideration. It is a work which is destined to be ground is travelled over by one thoroughly famil. the best of its kind in any language.-Medical iar with it. The illustrations are made with great Record, Nov. 25, 1882.

\section{CLARKE,W. B., F.R.C.S. \& LOCKWOOD,C.B., F.R.C.S.}

Demonstrators of Anatomy at St. Bartholomew's Hospital Medical School, London.

The Dissector's Manuial. In one pocket-size $12 \mathrm{mo}$. volume of 396 pages, with 49 illustrations. Limp cloth, red edges, $\$ 1.50$. See Students' Series of Manuals, page 4.

Messrs.Clarke and Lockwood have written abook | intimate associatlon with students could have that can hardly be rivalled as a practical aid to the given. With such a guide as this, accompanied dissector. Their purpose, which is "how to de- by so attractive a commentary as Treves' Surgical scribe the best way to display the anatomical Applied Anatomy (same series), no student could structure," has been fully attained. They excel in fail to be deeply and absorbingly interested in the a lucidity of demonstration and graphic terseness study of anatorny.-Newo Orleans Medical anã Surof expression, which only a long training and gical Journal, April, 1884.

\section{TREVES, FREDERICK, F. R. C.S., .}

Senior Demonstrator of Anatomy and Assistant Surgeon at the London Hospital.

Surgical Applied Anatomy. In one pocket-size 12mo. volume of 540 pages, with 61 illustrations. Limp cloth, red edges, $\$ 2.00$. See Students Series of Manuals, page 4.

He has produced a work which will command a quickened by daily use as a teacher and practilarger circle of readers than the class for which it tioner, has enabled our author to prepare a work was written. This union of a thorough, practical which it would be a most difficult task to excel.acquaintance with these fundamental branches, The American Practitioner, Feb. 1884.

\section{CURNOW, JOHN, M. D., F. R. C. P.,}

Professor of Anatomy at King's College, Physician at King's College Hospital.

Medical Applied Anatomy. In one pocket-size 12mo. volume. Preparing. See Students' Series of Manuals, page 4.

\section{BELLAMY, EDWARD, F. R. C. S.,}

Senior Assistant-Surgeon to the Charing-Cros8 Hospital, London.

The Student's Guide to Surgical Anatomy: Being a Description of the most Important Surgical Regions of the Human Body, and intended as an Introduction to operative Surgery. In one $12 \mathrm{mo}$. volume of 300 pages, with 50 illustrations. Cloth, $\$ 2.25$. 


\section{DRAPER, JOHIN C., M. D., LL. D.,}

Professor of Chemistry in the University of the City of New York.

Medical Physics. A Text-book for Students and Practitioners of Medicine. In one octavo volume of 734 pages, with 376 woodcuts, mostly original. Cloth, $\$ 4$.

\section{FROM THE PREFACE.}

The fact that a knowledge of Physics is indispensable to a thorough understanding of Medicine has not been as fully realized in this country as in Europe, where the admirable works of Desplats and Gariel, of Robertson and of numerous German writers constitute a branch of educational literature to which we can show no parallel. A full appreciation of this the author trusts will be sufficient justification for placing in book form the substance of his lectures on this department of science, delivered during many years at the University of the City of New York.

Broadly speaking, this work aims to impart a knowledge of the relations existing between Physics and Medicine in their latest state of development, and to embody in the pursuit of this object whatever experience the author has gained during a long period of teaching this special branch of applied science.

This elegant and useful work bears ample testi- explained, acoustics, optics, heat, electricity and mony to the learning and good jndgment of the magnetism, closing with a section on electroauthor. He has fitted his work admirably to the blology. The applications of all these to physiology exigencies of the situation by presenting the and medicine are kept constantly in view. The reader with brief, clear and simple statements of text is amply illustrated and the many difficult such propositions as he is by necessity required to points of the subject are brought forward with remaster. The subject matter is well arranged, markable clearness and ability.-M M edical and Surgliberally lllustrated and carefully indexed. That ical Reporter, July 18, 1885.

It will take rank at once among the text-books is That this work will greatly facilltate the study certain, and it is to be hoped that it will find a of medical physics is apparent upon even a mere place upon the shelf of the practical physician, cursory examination. It is marked by that scienwhere, as a book of reference, it will be found tific accuracy which always characterizes Dr. useful and agreeabl3.-Louisville Medical News, Draper's writings. Its pecullar value lies in the September 26, 1885.

Certainly we have no text-book as full as the excellent one he has prepared. It begins with a statement of the properties of matter and energy. After these the special departments of physics are Draper's writings. Its pecullar value lies in the
fact that it is written from the standpoint of the medical man. Hence much is omitted that appears in a mere treatise on physical science, while much is inserted of peculiar value to the physician.-Medical Record, August 22, 1885.

\section{ROBERTSON, J. MCGREGOR, M. A., M. B.,}

Muirhead Demonstrator of Physiology, University of Glasgovo.

Physiological Physics. In one $12 \mathrm{mo}$. volume of 537 pages, with 219 illustrations. Limp cloth, $\$ 2.00$. See Students' Series of Manuals, page 4.

The title of this work sufficiently explains the / ments. It will be found of great value to the nature of its contents. It is designed as a man- practitioner. It is a carefully prepared book of ual for the student of medicine, an auxlliary to reference, concise and accurate, and as such we his text-book in physiology, and it would be particu- heartily recommend it.-Journal of the Amerncan larly useful as a guide to his laboratory experi- Medical Association, Dec. 6, 1884.

\section{DALTON, JOHN C., M. D.,}

Professor Emeritus of Physiology in the College of Physicians and Surgeons, New York.

Doctrines of the Circulation of the Blood. A History of Physiological Opinion and Discovery in regard to the Circulation of the Blood. In one handsome 12mo. volume of 293 pages. Cloth, \$2.

Dr. Dalton's work is the fruit of the deep research | revolutionized the theories of teachers, than the of a cultured mind, and to the busy practitioner it discovery of the circulation of the blood. This cannot fail to be a source of instruction. It will explains the extraordinary interest it has to all inspire him with a feeling of gratitute and admir- medical historians. The volume before us is one ation for those plodding workers of olden times, of three or four which have been written within a who laid the foundation of the magnificent temple few years by American physicians. It is in several of medical science as it now stands. - New Orleans respects the most complete. The volume, though Medical and Surgical Journal, Aug. 1885.

In the progress of physiological study no fact tributions from an American pen to medical history was of greater moment, none more completely that has appeared.-Med. \& Surg. Rep., Dec. 6, 1884.

\section{BELL, F. JEFEREY, M. A.,}

Professor of Comparative Anatomy at King's College, London.

Comparative Physiology and Anatomy. In one 12mo. volume of 561 pages, with 229 illustrations. Limp cloth, $\$ 2.00$. See Students' Series of Manuals, page 4.

The manual is preëminently a student's book-1 it the best work in existence in the English clear and simple in language and arrangement. language to place in the hands of the medical It is well and abundantly illustrated, and is read- student.-Bristol Medico-Chirurgical Journal, Mar., able and interesting. On the whole we consider 1886.

\section{ELLIS, GEORGE VINER,}

Emeritus Professor of Anatomy in University College, London.

Demonstrations of Anatomy. Being a Guide to the Knowledge of the Human Body by Dissection. From the eighth and revised London edition. In one very handsome octavo volume of 716 pages, with 249 illustrations. Cloth, $\$ 4.25$; leather, $\$ 5.25$.

\section{ROBERTS, JOHN B., A. M., M. D.,}

Prof. of Applied Anat. and Oper. Surg. in Phila. Polyclinic and Coll. for Graduates in Medicine.

The Compend of Anatomy. For use in the dissecting-room and in preparing for examinations. In one 16 mo. volume of 196 pages. Limp cloth, 75 cents. 


\section{CHAPMAN, HENRY C., M. D.,}

Professor of Institutes of Medicine and Medical Jurisprudence in the Jefferson Medical College of Philadelphia.

A Treatise on Human Physiology. In one handsome octavo volume of about 1000 pages, profusely illustrated. Shortly.

\section{DALTON, JOHN C., M. D.,}

Professor of Physiology in the College of Physicians and Surgeons, New York, etc.

A Treatise on Human Physiology. Designed for the use of Students and Practitioners of Medicine. Seventh edition, thoroughly revised and rewritten. In one very handsome octavo volume of 722 pages, with 252 beautiful engravings on wood. Cloth, $\$ 5.00$; leather, $\$ 6.00$; very handsome half Russia, raised bands, $\$ 6.50$.

This edition of Dr. Dalton's well-known work | which it could be revlewed in the ordinary sense. bears evinence of having been thoroughly and The work is eminently one for the medical pracc:areruily revised. From the first appearance of titioner, since it treats most fully of those branches the book it has been a favorite, owing as well to of physiology which have a direct bearing on the the author's renown as an oral teacher as to the diagnosis and treatment of disease. The work is charm of simplicity with which, as a writer, he one whlch we can highly recorumend to all our always succeeds in investing even intricate sub- readers.-Dublin Journal of Medical Science, Feb.'83. jects It must be gratifying to him to observe the Certainly no physiological work as ever issued frequency with which his work, written for students from the press that presented its subject-matter in and practitioners, is quoted by other writers on a clearer and more attractive light. Almost every physiology. This fact attests its value, and, in page bears evidence of the exhaustive revision great measure, its originality. It now needs no that has taken place. The material is placed in a such seal of approbation, however, for the thou- more compact form, yet its delightful charm is resands who have studied it in its various editions tained, and no subject is thrown into obscurity. have never been in any doubt as to its sterling Altogether this edition is far in advance of any worth. $-N$. Y. Medical Journal and Obstetrical Re- previous one, and will tend to keep the profession view, Oct. 1882 .

Professor Dalton's well-known and deservedly- $\begin{aligned} & \text { posted as to the most recent additions to our } \\ & \text { plichical knowledge.-Michigan Medical News, }\end{aligned}$ appreciated work has long passed the stage at / April, 1882.

\section{FOSTER, MCTCHALL, M. D., F. R. S.,}

Prelector in Physiology and Fellow of Trinity College, Cambridge, England.

Text-Book of Physiology. Third American from the fourth English edition, with notes and additions by E. T. Reichert, M. D., Professor of Physiology in the University of Pennsylvania. In one handsome royal 12mo. volume of 908 pages, with 271 illustrations. Cloth, $\$ 3.25$; leather, $\$ 3.75$.

Dr. Foster's work upon physiology is so well- to know and what may be passed over by them as known as a text-book in this country, that it needs not important. From the beginning to the end, but little to be said in regard to it. There is physiology is taught in a systematic manner. To scarcely a medical college in the United States this third American edition numerous additions, where it is not in the hands of the students. The corrections and alterations have been made, so author, more than any other writer with whom that in its present form the usefulness of the book we are acquainted, seems to understand what will be found to be much increased.-Cincinnati portions of the science are essential for students Medical News, July 1885.

\section{POWER, HENRY, M. B., F. R. C. S.,}

Examiner in Physiology, Royal College of Surgeons of England.

Human Physiology. In one handsome pocket-size $12 \mathrm{mo}$. volume of 396 pages. with 47 illustrations. Cloth, $\$ 1.50$. See Students' Series of Manuals, page 4.

The prominent character of this work is that of to every one of our readers.-The American Jourjudicious condensation, in which an able and suc- nal of the Medical Sciences, October, 1884.

cessful effort appears to have been made by its This little work is deserving of the highest accomplished author to teach the greatest number praise, and we can hardly conceive how the main of facts in the fewest possible words. The result facts of this science could have been more clearly is a specimen of concentrated intellectual pabu- or concisely stated. The price of the work is such lum seldom surpassed, which ought to be care- as to place it within the reach of all, while the exfully ingested and digested by every practitioner cellence of its text will certainly secure for it most who desires to keep himself well informed upon favorable commendation-Cincinnati Lancet and this most progressive of the medical sciences. Clinic, Feb. 16, 1884.

The volume is one which we cordially recommend

\section{SIMON, W., Ph. D., M. I.,}

Professor of Chemestry and Toxicology in the College of Physicians and Surgeons, Baltimore, and Professor of Chemistry in the Maryland College of Pharmacy.

Manual of Chemistry. A Guide to Lectures and Laboratory work for Beginners in Chemistry. A Text-book, specially adapted for Students of Pharmacy and Medicine. In one 8 vo. vol. of $410 \mathrm{pp}$., with 16 woodcuts and 7 plates, mostly of actual deposits, with colors illustrating 56 of the most important chemical reactions. Cloth, $\$ 3.00$; also without plates, cloth, $\$ 2.50$.

This book supplies a want long felt by students $\mid$ plates, beautifully executed, illustrating precipiof medicine and pharmacy, and is a concise but tates of various reactions, form a novel and valuthorough treatise on the subject. The long expe- able feature of the book, and cannot fail to be aprience of the author as a teacher in schools of preciated by both student and teacher as a help medicine and pharmacy is conspicuous in the over the hard places of the science._Maryland perfect adaptation of the work to the special needs Medical Journal, Nov. 22, 1884.

of the student of these branches. The colored

Wöhler's Outlines of Organic Chemistry. Edited by Fitrig. Translated by Ira Remsen, M. D., Ph. D. In one $12 \mathrm{mo}$. volume of 550 pages. Cloth, $\$ 3$.

LEHMANN'S MANUAL OF CHEMICAL PHYS- CARPENTER'S PRIZE ESSAY ON THE USEAND IOLOGY. In one octavo volume of 327 pages, with 41 illustrations. Cloth, \$2.25.

CARPENTER'S HUMAN PHYSIOLOGY. Edited by Henry Powrr. In one octavo volume. 


\section{FRANKLAND, E., D. C.L., F.R.S., \&JAPP, F.R., F.I. C., Professor of Chemistry in the Normal School of Science, London. \\ Assist. Prof. of Chemistry in the Normal School of Science, London.}

Inorganic Chemistry. In one handsome octavo volume of 677 pages with 51 woodcuts and 2 plates. Cloth, $\$ 3.75$; leather, $\$ 4.75$.

This work should supersede other works of its | This excellent treatise will not fail to take its class in the medical colleges. It is certainly better place as one of the very best on the subject of edapted than any work upon chemistry, with which we.are acquainted, to impart that clear and full knowledge of the science which students of medicine should have. Physicians who feel that their chemical knowledge is behind the times, would do well to devote some of their leisure time to the study of this work. The descriptions and demonstrations are made so plain that there is no difficulty in understanding them.-Cincinnati Medical which it treats. We have been much pleased
which with the comprehensive and lucid manner in which the difficulties of chemical notation and nomenclature have been cleared up by the writers. It shows on every page that the problem of rendering the obscurities of this science easy of comprehension has long and successfully engaged the attention of the authors.-Medical and Surgical Reporter, October 31, 1885. News, January, 1886.

\section{FOWNES, GEORGE, Ph. J.}

A Manual of Elementary Chemistry; Theoretical and Practical. Embodying WATrs' Physical Inorganic Chemistry. New American edition. In one large royal $12 \mathrm{mo}$. volume of 1061 pages, with 168 illustrations on wood and a colored plate. Cloth, $\$ 2.75$; leather, $\$ 3.25$.

Fownes' Chemistry has been a standard text- / chemistry extant.-Cincinnati Medical Newos, Ocbook upon chemistry for many years. Its merits tober, 1885 .

are very fully known by chemists and physicians Of all the works on chemistry intended for the everywhere in this country and in England. As use of medical students, Fownes' Chemistry is the science has advanced by the making of new perhaps the most widely used. Its popularity is discoveries, the work has been revised so as to based upon its excellence. This last edition conkeep it abreast of the times. It has steatily tains all of the material found in the previous, maintained its position as a text.bonk with medi- and it is also enriched by the addition of Watts cal students. In this work are treated fully: Heat, Physical and Inorganic Chemistry. All of the matLight and Electricity, including Magnetism. The ter is brought to the present standpoint of chemiinfluence exerted by these forces in chemical cal knowledge. We may safely predict for this action upon health and disease, etc., is of the most work a continuance of the fame and favor it enjoys important kind, and should be familiar to every among medical students.- New Orleans Medical medical practitioner. We can commend the and Surgical Journal, March, 1886.

work as one of the very best text-books upon

\section{ATTFLELD, JOHN, Ph. 1).,}

Professor of Practical Chemistry to the Pharmaceutical Society of Great Britain, etc.

Chemistry, General, Medical and Pharmaceutical; Including the Chemistry of the U. S. Pharmacopoia. A Manual of the General Principles of the Science, and their Application to Medicine and Pharmacy. A new American, from the tenth English edition, specially revised by the Author. In one handsome royal $12 \mathrm{mo}$. volume of 728 pages, with 87 illustrations. Cloth, $\$ 2.50$; leather, $\$ 3.00$.

A text-book which passes through ten editions to put himself in the student's place and to apprein sixteen years must have good qualities. This ciate his state of mind.-American Chemical Jourremark is certainly applicable to Attfield's Chem- nal, April, 1884.

istry, a book which is so well known that it is It is a book on which too much praise cannot be hardly necessary to do more than note the appear- bestowed. As a text-book for medical schools it ance of this new and improved edition. It seems, however, desirable to point out that feature of the science, and having been prepared with a special book which, in all probability, has made it so view towards medicine and pharmacy, it is alike popular. There can be little doubt that it is its indispensable to all persons engaged in those dethoroughly practical character, the expression partments of science. It includes the whole being used in its best sense. The author under- chemistry of the last Pharmacopoeia.-Pacific Medi-

\section{BLOXAM, CHARLES L.,}

Professor of Chemistry in King's College, London.

Chemistry, Inorganic and Organic. New American from the fifth London edition, thoroughly revised and much improved. In one very handsome octavo volume of 727 pages, with 292 illustrations. Cloth, $\$ 3.75$; leather, $\$ 4.75$.

Comment from us on this standard work is al- the best manuals of general chemistry tn the Engmost superfluous. It differs widely in scope and aim from that of Attfield, and in its way is equally beyond criticism. It adopts the most direct methods in stating the principles, hypotheses and facts of the science. Its language is so terse and lucid, and its arrangement of matter so logical in $8 \theta-$ quence that the student never has occasion to complain that chemistry is a hard study. Much attention is paid to experimental illustrations of ehemical principles and phenomena, and the mode of conducting these experiments. The book maintains the position it has always held as one of

lish language.-Detroit Lancet, Feb. 1884

Professor Bloxam's book is a very satisfactory one. We know of no treatise on chemistry which contains so much practical information in the same number of pages. The book can be readily adapted not only to the needs of those who desire a tolerably complete course of chemistry, but also to the needs of those who desire only a general knowledge of the subject. We take pleasure in recommending this work both as a satisfactory text.book, and $\mathbf{s}$ a useful book of reference,-Boston Medical and Surgical Journal, June 19, 1884.

\section{REMSEN, IRA, M. D., Ph. D.,}

Professor of Chemistry in the Johns Hopkins University, Baltimore.

Principles of Theoretical Chemistry, with special reference to the Constitution of Chemical Compounds. New (third) and revised edition. In one handsome royal $12 \mathrm{mo}$. volume of about 250 pages. In press. 
CHARLES, T. CRANSTOUN, M. D., F. C. S., M. S.,

Formerly Asst. Prof. and Denionst. of Chemistry and Chemical Physics, Queen's College, Belfast.

The Elements of Physiological and Pathological Chemistry.

Handbook for Medical Students and Practitioners. Containing a general account of Nutrition, Foods and Digestion, and the Chemistry of the Tissues, Organs, Secretions and Excretions of the Body in Health and in Disease. Together with the methods for preparing or separating their chief constituents, as also for their examination in detail, and an outline syllabus of a practical course of instruction for students. In one handsome octavo volume of 463 pages, with 38 woodcuts and 1 colored plate. Cloth, $\$ 3.50$.

This is, we believe, the first complete work of has treated it in a competent and instructive manthe kind in the English language, and may well ner. We cannot recommend a better book than serve to show what progress is being made in medi- the present. In fact, it fills a gap in medical textcine. The student will find condensed in one vol- books, and that is a thing which can rarely be said ume such a store of knowledge as would formerly nowadays. Dr. Charles has devoted much space have cost him much reading to gather. The book to the elucidation of urinary mysteries. He does will fully repay anyone to read, and every library this with much detail, and yet in a practical and should have it for reference.-New Orleans Medical intelligible manner. In fact, the author has filled and Surgical Journal, April, 1885.

Dr. Charles is fully impressed with the import-

ance and practical reach of his subject, and he ord, December 20, 1884.

\section{HOFIMANN, F., A.M., Ph.D., \& POWER F.B., Ph.D., \\ Public Analyst to the State of New York. \\ Prof. of Anal. Chem. in the Phil. Coll. of Pharmacy.}

A Manual of Chemical Analysis, as applied to the Examination of Medicinal Chemicals and their Preparations. Being a Guide for the Determination of their Identity and Quality, and for the Detection of Impurities and Adulterations. For the use of Pharmacists, Physicians, Druggists and Manufacturing Chemists, and Pharmaceutical and Medical Students. Third edition, entirely rewritten and much enlarged. In one very handsome octavo volume of 621 pages, with 179 illustrations. Cloth, $\$ 4.25$.

We congratulate the author on the appearance | tion of them singularly explicit. Moreover, it is of the third edition of this work, published for the exceptionally free from typographical errors. We first time in this country also. It is admirable and have no hesitation in recommending it to those the information it undertakes to supply is both who are engaged either in the manufacture or the extensive and trustworthy. The selection of pro- testing of medicinal chemicals.-London Pharmacesses for determining the purity of the substan- ceutical Journal and Transactions, 1883.

ces of which it treats is excellent and the descrip-

\section{CLOWES, FRANK, D. Sc., London,}

Senior Science-Master at the High School, Newcastle-under-Lyme, etc.

An Elementary Treatise on Practical Chemistry and Qualitative Inorganic Analysis. Specially adapted for use in the Laboratories of Schools and Colleges and by Beginners. Third American from the fourth and revised English edition. In one very handsome royal $12 \mathrm{mo}$. volume of 387 pages, with 55 illustrations. Cloth, $\$ 2.50$.

We may simply repeat the favorable opinion which we expressed after the examination of the previous edition of this work. It is practical in its aims, and accurate and concise in its statements. - American Journal of Pharmacy, August, 1885.

The style is clear, the language terse and vigorous. Beginning with a list of apparatus necessary

for chemical work, he gradually unfolds the subject from its simpler to its more complex divisions. It is the most readable book of the kind we have yet seen, and is without doubt a systematic, intelligible and fully equipped laboratory guide and text-book. - Medical Record, July 18, 1885.

\section{RALFE, CHARLES H., M.D., F.R. C.P.,}

Assistant Physician at the London Hospital.

Clinical Chemistry. In one pocket-size 12mo. volume of 314 pages, with 16 illustrations. Limp cloth, red edges, \$1.50. See Students' Series of Manuals, page 4.

This is one of the most instructive little works $\mid$ cine. Dr. Ralfe is thoroughly acquainted with the that we have met with in a long time. The author latest contributions to his science, and it is quite is a physician and physiologist, as well as a chem- refreshing to find the subject dealt with so clearly ist, consequently the book is unqualifiedly prac- and simply, yet in such evident harmony with the tical, telling the physician just what he ought to modern scientific methods and spirit.-Medical know, of the applications of chemistry in medi- Record, February 2, 1884.

\section{CLASSEN, ALEXANDER,}

Professor in the Royal Polytechnic School, Aix-la-Chapelle.

Elementary Quantitative Analysis. Translated, with notes and additions, by Edgar F. Smith. Ph. D., Assistant Professor of Chemistry in the Towne Scientific School, University of Penna. In one $12 \mathrm{mo}$. volume of 324 pages, with 36 illust. Cloth, \$2.00.

It is probably the best manual of an elementary | and then advancing to the analysis of minerals and nature extant, insomuch as its methods are the such products as are met with in applied chemisbest. It teaches by examples, commencing with try. It is an indispensable book for students in single determinations, followed by separations, 1 chemistry.-Boston Journal of Chemistry, Oct. 1878.

\section{GREENE, WILLIAM H., M. D.,}

Demonstrator of Chemistry in the Medical Department of the University of Pennsylvania.

A Manual of Medical Chemistry. For the use of Students. Based upon Bowman's Medical Chemistry. In one $12 \mathrm{mo}$. volume of 310 pages, with 74 illus. Cloth, $\$ 1.75$.

It is a concise manual of three hundred pages, the recognition of compounds due to pathological giving an excellent summary of the best methods of analyzing the liquids and sollds of the body, both for the estimation of their normal constituents and conditions. The detection of poisons is treated with sufficient fulness for the purpose of thestudent or practitioner.-Boston Jl. of Chem. June,'80. 
BRUNTON, T. LAUDER, M.D., D.Sc., F.R.S., F.R.C.P., Lecturer on Materia Medica and Therapeutics at St. Bartholomew's Hospital, London, etc.

A Text-book of Pharmacology, Therapeutics and Materia Medica; Including the Pharmacy, the Physiological Action and the Therapeutical Uses of Drugs. In one handsome octavo volume of 1033 pages, with 188 illustrations. Cloth, $\$ 5.50$; leather, $\$ 6.50$.

It is a scientific treatise worthy to be ranked with | upon the subject of Pharmacology the comprethe highest productions in physiology, either in hensive work of Brunton is clearly the mest our own or any other language. Everything is important, and is beyond question the foremost practical, the dry, hard facts of physiology being English handbook of Materia Medica and Therapressed into service and applied to the treatment peutics since the appearance of Pereira's Elements of the commonest complaints. The information of Materia Medica in 1842. It is original both in is so systematically arranged that it is available the arrangement of the subjects and in the mode for immediate use. The index is so carefully of treatment, and develops in a comprehensive compiled that a reference to any special point is manner the foundation principles of the science at once obtainable. Dr. Brunton is never satisfied of Pharmacology without leaving the needs of the with vague generalities, but gives clear and pre- practitioner out of sight for an instant. In fact, cise directions for prescribing the various drugs the author has written a book which deserves to and preparations. We congratulate students on be known far beyond the boundaries of England, being at last placed in possession of a sclentific and can serve as a model for the pharmacologlcal treatise of enormous practical importance.- The works of the continent on account of its happy London Lancet, June $27,1885$.

Of all the numerous publications of this year Jahresbericht, Berlin, 1886.

\section{BARTHOLOW, ROBERTS, A. M., M. D., LL. D.,}

Professor of Materia Medica and General Therapeutics in the Jefferson Medical College of Philadelphia.

New Remedies of Indigenous Source: Their Physiological Actions and Therapeutical Uses. In one octavo volume of about 300 pages. Preparing.

\section{PARRISH, EDWARD,}

Late Professor of the Theory and Practice of Pharmacy in the Philadelphia College of Pharmacy.

A Treatise on Pharmacy: designed as a Text-book for the Student, and as a Guide for the Physician and Pharmaceutist. With many Formulæ and Prescriptions. Fifth edition, thoroughly revised, by Thomas S. WIEGAND, Ph. G. In one handsome octavo volume of 1093 pages, with 256 illustrations. Cloth, $\$ 5$; leather, $\$ 6$.

No thorough-going pharmacist will fail to possess | Each page bears evidence of the care bestowed himself of so useful a guide to practice, and no upon it, and conveys valuable information from physician who properly estimates the value of an the rich store of the editor's experience. In fact, accurate knowledge of the remedial agents em- all that relates to practical pharmacy - apparatus ployed by him in daily practice, so far as their processes and dispensing - has been arranged and miscibility, compatibllity and most effective meth- described with clearness in Its various aspects, so ods of combination are concerned, can afford to as to afford ald and advice alike to the student and leave this work out of the list of their works of to the practical pharmacist. The work is judireference. The country practitioner, who must clously illustrated with good woodeuts-American always be in a measure his own pharmacist, will Journal of Pharmacy, January, 1884.

find it indispensable. - Louisville Medical News, There is nothing to equal Parrish's Pharmacy March 29, 1884.

This well-known work presents itself now based upon the recently revised new Pharmacopoia.

in this or any other language.-London Pharmam ceutical Journal.

\section{$\operatorname{HERMANN,Dr}$. L.,}

Professor of Physiology in the University of Zurich.

Experimental Pharmacology. A Handbook of Methods for Determining the Physiological Actions of Drugs. Translated, with the Author's permission, and with extensive additions, by RoBert Meade Smith, M. D., Demonstrator of Physiology in the University of Pennsylvania. In one handsome 12mo. volume of 199 pages, with 32 illustrations. Cloth, $\$ 1.50$.

\section{MAISCH, JOHN M., Phar. D.,}

Professor of Materia Medica and Botany in the Philadelphia College of Pharmacy.

A Manual of Organic Materia Medica; Being a Guide to Materia Medica of the Vegetable and Animal Kingdoms. For the use of Students, Druggists, Pharmacists and Physicians. Third edition In one handsome royal $12 \mathrm{mo}$. volume of about 550 pages, with about 250 illustrations. Shortly.

\section{BRUCE, J. MITCHELL, M. D., E. R. C.P.,}

Physician and Lecturer on Materia Medica and Therapeutics at Charing Cross Hospital, London.

Materia Medica and Therapeutics. An Introduction to Rational Treatment. Fourth edition. In one pocket-size $12 \mathrm{mo}$. volume of 591 pages. Limp cloth, \$1.50. See Students' Series of Manuals, page 4.

\section{GRIFHITH, ROBERT EGLESFIELD, M. D.}

A Universal Formulary, containing the Methods of Preparing and Administering Officinal and other Medicines. The whole adapted to Physicians and Pharmaceutists. Third edition, thoroughly revised, with numerous additions, by JоHN M. MAISCH, Phar. D., Professor of Materia Medica and Botany in the Philadelphia College of Pharmacy. In one octavo volume of 775 pages, with 38 illustrations. Cloth, $\$ 4.50$; leather, $\$ 5.50$. 
STILLÉ, A., M.D., LL. D., \& Professor Emeritus of the Theory and Practice of Medicine and of Clinical Medicine in the University of Pennsylvania.

NEW (FOURTH) EDITION.

\section{The National Dispensatory.}

CONTAINING THE NATURAL HISTORY, CHEMISTRY, PHARMACY, ACTIONS AND USES OF MEDICINES, INCLUDING THOSE RECOGNIZED IN THE PHARMACOPCIAS OF THE UNITED STATES, GREAT BRITAIN AND GERMANY, WITH NUMEROUS

\section{REFERENCES TO THE FRENCH CODEX.}

Fourth edition, revised to October, 1886, and covering the new British Pharmacopceia.

In one magnificent imperial octavo volume of 1794 pages, with 311 elaborate engravings. Price in cloth, $\$ 7.25$ : leather, raised bands, $\$ 8.00$; very handsome half Russia, raised bands and open back, $\$ 9.00$.

* * This work will be furnished with Patent Ready Reference Thumb-letter Index for $\$ 1.00$ in addition to the price in any style of binding.

In this new edition of THE Nationil Dispensatory, all important changes in the recent British Pharmacopœia have been incorporated throughout the volume, while in the Addenda will be found, grouped in a convenient section of 24 pages, all therapeutical novelties which have been established in professional favor since the publication of the third edition two years ago. Detailed information is thus given of the following among the many drugs treated: Antipyrin, Cocaine Hydrochlorate, Cascara Sagrada, Fabiana, Franciscea, various new Glycerins, Gymnocladus, Hydroquinon, Hypnone, Iodol, Jacaranda, Lanolin, Menthol, Phormium, Sulphophenol, Thallin and Urethan. In this edition, as always before, The National Dispensatory may be said to be the representative of the most recent state of American, English, German and French Pharmacology, Therapeutics and Materia Medica.

The National Dispensatory is so well and favorably known on both sides of the Atlantic that scarcely anything else is left to the reviewer than to call attention to the fact that another edition of this valuable work has made its appearance. This last edition surpasses even its predecessors in thoroughness and accuracy. The fact that in 1884, when tho third edition was published, no revision of the British Pharmacopoia had been made for seventeen years, has necessitated a thorough going over of the whole work in order that references might correspond to the last revision of the work of our British cousins. In addition to these changes is a fairly full résumê, in the form of addenda, of the more important drugs which have come into general use in the last two or three years.-The American Journal of the Medical Sciences, April, 1887.

This, the most comprehensive of the several commentaries on the Pharmacopceias of the United States, Great Britain and Germany which has yet appeared, has by this last revision been brought

fully up to the existing knowledge upon the subject treated. Its references to the British Pharmacopois have been amended for the late and much-changed new edition of that work; an "addenda" of twenty-five pages has been appended, treating of the latest and most important additions to the materia medica. This work should be in the hands of every physician and pharmsc1st.-Boston Medical and Surgical Journal, Feb. 10, 1887.

We think it a matter for congratulation that the profession of medicine and that of pharmacy have shown such appreciation of this great work as to call for four editions within the comparatively brief period of eight years. The matters with which it deals are of so practical a nature that neither the physician nor the pharmacist can do without the latest text-books on them, especially those that are so accurate and comprehensive as this one. The book is in every way creditable both to the authors and to the publishers.-New York Medical Journal, May 21, 1887.

\section{FARQUHARSON, ROBERT, M. D.,}

Lecturer on Materia Medica at St. Mary's Hospital Medical School.

A Guide to Therapeutics and Materia Medica. Third American edition, specially revised by the Author. Enlarged and adapted to the U. S. Pharmacopœia by Frank Woodbury, M.D. In one handsome $12 \mathrm{mo}$. volume of 524 pages. Cloth, $\$ 2.25$.

Dr. Farquharson's Therapeutics is constructed / umned pages-one side containing the recognized upon a plan which brings before the reader all the physiological action of the medicine, and the other essential points with reference to the properties of the disease in which observers (who are nearly aldrugs. It impresses these upon him in such a way ways mentioned) have obtained from it good reas to enable him to take a clear view of the actions sults-make a very good arrangement. The early of medicines and the disordered conditions in chapter containing rules for prescribing is excelwhich they must prove useful. The double-col- lent.-Canada Med. and Surg. Journal, Dec. 1882.

\section{EDES, ROBERT T., M. D.,}

Jackson Professor of Clinical Medicine in Harvard University, Medical Department.

A Text-Book of Materia Medica and Therapeutics. In one octavo volume of about 600 pages, with illustrations. In press.

\section{STILLÉ, ALFRED, M. D., LL. D.,}

Professor of Theory and Practice of Med. and of Clinical Med. in the Univ. of Penna.

Therapeutics and Materia Medica. A Systematic Treatise on the Action and Uses of Medicinal Agents, including their Description and History. Fourth edition, revised and enlarged. In two large and handsome octavo volumes, containing 1936 pages. Cloth, $\$ 10.00$; leather, $\$ 1200$; very handsome half Russia, raised bands, $\$ 13.00$. 


\section{COATS, JOSEPH, M. D., F. F. P. S.,}

Pathologist to the Glasgow Western Infirmary.

A Treatise on Pathology. In one very handsome octavo volume of 829 pages, with 339 beautiful illustrations. Cloth, $\$ 5.50$; leather, $\$ 6.50$.

The work before us treats the subject of Path-| partment of medicine that is not as fully elucidated The logy more oxteasively than it is usually treated in similar works. Medical students as well as physicians, who desire a work for study or reference, that treats the subjects in the various departments in a very thorough manner, but without prolixity, will certainly give this one the preference to any with which we are acquainted. It sets forth the most recent discoveries, exhibits, in an interesting manner, the changes from a normal condition effected in structures by disesse, and points out the characteristics of various morbid agencies, so that they can be easily recognized. But, not limited to morbid anatomy, it explains fully how the functions of organs are disturbed by abnormal conditions. There is nothing belonging to its de-

\section{Medical News, Oct. 1883}

One of the best features of thls reatise consists in the judiclous admixture of foreign observation with private experience. Thus the subject is presented in a harmonious manner, facilitating the study of single toples and making the entire volume profitable and pleasant reading. The author includes in his descriptions, general pathology as well as the special pathological histology of the different systems and organs. He has succeeded in offering to students and practitioners a thoroughly acceptable work. - Medical Record, Dec. 22, 1883.

\section{GREEN, T. HENRY, M. D.,}

Lecturer on Pathology and Morbid Anatomy at Charing-Cross Hospital Medical School, London.

Pathology and Morbid Anatomy. Fifth American from the sixth revised and enlarged English edition. In one very handsome octavo volume of 482 pages, with 150 tine engravings. Cloth, $\$ 2.50$.

The fact that this well-known treatise has so rapidly reached its sixth edition is a strong evidence of its popularity. 'The suthor is to be congratulated upon the thoroughness with whlch he has prepared this work. It is thoroughly abreast with all the most recent adrances in pathology. No work in the English language is so admirably adapted to the wants of the student and practitioner as this, and we would recommend it most earnestly to every one.- Nashville Journal of Medi. cine and Surgery, Nov. 1884

An extended review of such a well-known book is unnecessary. We had already regarded the book as a model of its kind, and the author's as surance that he believes the present edition to be fully up to date will be received as sufficient proof that nothing of importance has been omitted. The book has been most carefully revised, and bears upon every page the marks of the care and accuracy that have won for it an international reputation.-New York Medical and Surgical Journal, July 18,1885 .

\section{WOODHEAD, G. SIMS, M. D., F. R. C. P. E., \\ Demonstrator of Pathology in the University of Edinburgh.}

Practical Pathology. A Manual for Students and Practitioners. In one beautiful octavo volume of 497 pages, with 136 exquisitely colored illustrations. Cloth, $\$ 6.00$.

It forms a real guide for the student and practl- themselves with this manual. The numerous tioner who is thoroughly in earnest in his en- drawings are not fancied pictures, or merely deavor to see for himself and do for himself. To schematic diagrams, but they represent faithfully the laboratory student it will be a helpful com- the actual images seen under the microscope. panion, and all those who may wish to familiarize The author merits all pralse for having produced themselves with modern methods of examining a valuable work.-Medical Record, May 31, 1884. morbid tissues are strongly urged to provide

\section{SCH HEWR, EDWARD A., F. R. S.,}

Assistant Professor of Physiology in University College, London.

The Essentials of Histology. In one octavo volume of 246 pages, with 281 illustrations. Cloth, $\$ 2.25$.

This admirable work was greatly needed. To an elementary text-book of histology, comprising those who are familiar with the author's former all the essential facts of the sclence, but omitting "Course of Practical Histology," the book needs unimportant details. The author has recomno recommendation. It has been written with the mended only those methods upon which long exobject of supplying the student with directions perience has proved that full dependence can be for the microscopical examination of the tissues, placed. The strlct observance of this plan perwhich are given in a clear and understandable mits of no doubt, and makes the work eminently way. Although especially adapted for laboratory satisfactory.-The Physician and Surgeon, July, 1887. work, at the same time it is intended to serve as

\section{KLEIN, E., M. D., F. R. S.,}

Joint Leeturer on General Anat. and Phys. in the Med. School of St. Bartholomew's Hosp., London. Flements of Histology. In one pocket-size $12 \mathrm{mo}$. volume of 360 pages, with 181 illus. Limp cloth, red edges, $\$ 1.50$. See Students' Series of Manuals, page 4.

This little volume, originally intended by its $/ \mathrm{man}$ body; and, while small in size, it is full to able author as a manual for medical students, overflowing with important facts in regard to these contains much valuable information, systematic- multiform and complex structures. We know of ally arranged, that will be acceptable to the no book of its size that will prove of greater value general practitioner. It gives a graphic and lucid to medical students and practitioners of medidescription of every tissue and organ in the hu- cine.- The Southern Practitioner, Nov. 1883.

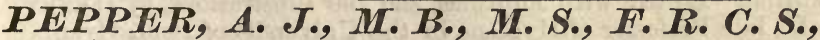

Surgeon and Lecturer at St. Mary's Hospital, London.

Surgical Pathology. In one pocket-size 12mo. volume of 511 pages, with 81 illustrations. Limp cloth, red edges, \$2.00. See Students' Series of Manuals, page 4.

It is not pretentious, but it will serve exceed- illustrated. The student will find in it nothing ingly well as a book of reference. It embodies a that is unnecessary. The list of subjects cover great deal of matter, extending over the whole the whole range of surgery. The book supplies a field of surgical pathology. Its form is practical, very manifest want and should meet with sucits language is clear, and the information set cess.-Nero York Medical Journal, May 31, 1884.

forth is well-arranged, well-indexed and well-

Cornil and Ranvier's Pathological Histology. - Translated by E. O. Shakespeare, M. D., and J. Henry C. Simes, M. D. Octavo, 800 pp., 360 illustrations. 


\section{FLINT, AUSTIN, M. D.,}

Prof. of the Principles and Practice of Med. and of Clin. Med. in Bellevue Hospital Medical College, N. $Y$.

A Treatise on the Principles and Practice of Medicine. Designed for the use of Students and Practitioners of Medicine. New (sixth) edition, thoroughly revised and rewritten by the Author, assisted by WILLIAM H. WELCH, M. D., Professor of Pathology, Johns Hopkins University, Baltimore, and A UstiN FLINT, JR., M. D., LL. D., Professor of Physiology, Bellevue Hospital Medical College, N. Y. In one very handsome octavo volume of about 1170 pages, with illustrations. Cloth, $\$ 5.50$; leather, $\$ 6.50$; very handsome half Russia, raised bands, $\$ 7.00$.

A new edition of a work of such established rep- / general approval by medical students and practi-

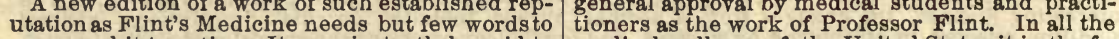
commend it to notice. It may in truth be said to medical colleges of the United States it is the faembody the frult of his labors in clinical medicine, vorite work upon Practice; and, as we have stated ripened by the experience of a long life devoted to before in alluding to it, there is no other medical its pursuit. America may well be proud of having work that can be so generally found in the libraproduced a man whose indefatigable industry and ries of physicians. In every state and territory gifts of genius have done so much to advance med- of this'vast country the book that will be most likely icine; and all English-reading students must be to be found in the office of a medical man, whether grateful for the work which he has left behind him. in city, town, village, or at some cross-roads, is It has few equals, either in point of literary excel- Flint's Practice. We make this statement to a lence, or of scientific learning, and no one can fail considerable extent from personal observation, and to study its pages without being struck by the lu- it is the testimony also of others. An examinacidity and accuracy which characterize them. It tion shows that very considerable changes have is qualities such as these which render it so valu- been made in the six th edition. The work may unable for its purpose, and give it a foremost place doubtedly be regarded as fairly representing the among the text-books of this generation. - The present state of the science of medicine, and as London Lancet, March 12, 1887.

No text-book on the principles and practice of medicine has ever met in this country with such

reflecting the views of those who exemplify in their prectice the present stage of progress of medical art.-Cincinnati Medical News, Oct. 1886.

\section{HARTSHORNE, HENRY, M. D., LL. D.,}

Lately Professor of Hygiene in the University of Pennsylvania.

Essentials of the Principles and Practice of Medicine. A Handbook for Students and Practitioners. Fifth edition, thoroughly revised and rewritten. In one royal $12 \mathrm{mo}$. volume of 669 pages, with 144 illustrations. Cloth, $\$ 2.75$; half bound, $\$ 3.00$.

Within the compass of 600 pages it treats of the / this one; and probably not one writer in our day history of medicine, general pathology, general symptomatology, and physical diagnosis (including laryngoscope, ophthalmoscope, etc.), general therapeutics, nosology, and special pathology and practice. There is a wonderful amount of information contained in this work, and it is one of the best of its kind that we have seen.-Glasgow Medical Journal, Nov. 1882.

An indispensable book. No work ever exhibited a better average of actual practical treatment than

had a better opportunity than Dr. Hartshorne for condensing all the views of eminent practitioners into $\mathrm{a} 12 \mathrm{mo}$. The numerous illustrations will be very useful to students especially. These essentials, as the name suggests, are not intended to supersede the text-books of Flint and Bartholow, but they are the most valuable in affording the means to see at a glance the whole literature of any disease, and the most valuable treatment.-Chicago Medical Journal and Examiner, April, 1882.

\section{BRISTOWE, JOHN SYER, M. D., F. R. C. P.,}

Physician and Joint Lecturer on Medicine at St. Thomas' Hospital, London.

A Treatise on the Practice of Medicine. Second American edition, revised by the Author. Edited, with additions, by JAMEs H. HuTCHINsoN, M.D., physician to the Pennsylvania Hospital. In one handsome octavo volume of 1085 pages, with illustrations. Cloth, $\$ 5.00$; leather, $\$ 6.00$; very handsome half Russia, raised bands, $\$ 6.50$.

The book is a model of conciseness, and com- and practice, as skin disesses, syphilis and Insanbines, as successfully as one could conceive it to ity, but they will not be objected to by readers, as be possible, an encyclopædic character with the he has studied them conscientiously, and drawn smallest dimensions. It differs from other admi- from the life.-Medical and Surgical Reporter, Derable text-books in the completeness with which cember 20,1879 .

it covers the whole field of medicine.-Michigan The reader will find every conceivable subject Medical New8, May 10, 1880.

His accuracy in the portraiture of disease, his care in stating subtle points of diagnosis, and the faithfully given pathology of abnormal processes have seldom been surpassed. He embraces many diseases not usually considered to belong to theory connected with the practice of medicine ably presented, in a style at once clear, Interesting and concise. The additions made by Dr. Hutchinson are appropriate and practical, and greatly add to its usefulness to American readers.-Buffalo Medical and Surgical Journal, March, 1880.

\section{WATSON, SIR THOMAS, M. D.,}

Late Physician in Ordinary to the Queen.

Lectures on the Principles and Practice of Physic. A new American from the fifth English edition. Edited, with additions, and 190 illustrations, by HENRY Hartshorne, A. M., M. D., late Professor of Hygiene in the University of Pennsylvania. In two large octavo volumes of 1840 pages. Cloth, $\$ 9.00$; leather, $\$ 11.00$.

LECTURES ON THE STUDY OF FEVER. By|A TREATISE ON FEVER. BY ROBERT D. LyoNs, A. Hudson, M. D., M. R. I. A. In one octavo K. C. C. In one 8 vo. vol. of 354 pp. Cloth, $\$ 2.25$. volume of 308 pages. Cloth, $\$ 2.50$.

STOKES' LECTURES ON FEVER. Edited by John William Moore, M. D., F. K. Q. C. P. In one octavo volume of 280 pages. Cloth, $\$ 2.00$.

LA ROCHE ON YELLOW FEVER, considered in its Historical, Pathological, Etiological and Therapeutical Relations. In two large and handsome octavo volumes of $1468 \mathrm{pp}$. Cloth, $\$ 7.00$.

A CENTURY OF AMERICAN MEDICLNE, 17\%6-1876. By Drs. E. H. CLARKE, H. J. Bigziow, 8. D. Gross, T. G. Thomas, and J. S. Bilitwas. In one $12 \mathrm{mo}$. volume of 370 pages. Cloth, $\$ 2.25$. 
For Sale by Subscription Only.

\title{
A System of Practical Medicine.
}

\author{
BY AMERICAN AUTHORS.
}

\author{
EDITED BY WILLIAM PEPPER, M. D., LL. D.,
}

PROVOST AND PROFESSOR OF THE THEORY AND PRACTICE OF MEDICINE AND OF CLINICAI, MEDICINE IN THE UNIVERSITY OF PENNSYLVANIA,

Assisted by Louis Starr, M. D., Clinical Professor of the Diseases of Children in the Hospital of the University of Pennsylvania.

The complete work, in five volumes, containing 5573 pages, with 198 illustrations, is just ready. Price per volume, cloth, \$5; leather, \$6; half Russia, raised bands and open back, \$7.

In this great work American medicine is for the first time reflected by its worthiest teachers, and presented in the full development of the practical utility which is its preëminent characteristic. The most able men-from the East and the West, from the North and the South, from all the prominent centres of education, and from all the hospitals which afford special opportunities for study and practice-have united in generous rivalry to bring together this vast aggregate of specialized experience.

The distinguished editor has so apportioned the work that to each author has been assigned the subject which he is peculiarly fitted to discuss, and in which his views will be accepted as the latest expression of scientific and practical knowledge. The practitioner will therefore find these volumes a complete, authoritative and unfailing work of reference, to which he may at all times turn with full certainty of finding what he needs in its most recent aspect, whether he seeks information on the general principles of medicine, or minute guidance in the treatment of special disease. So wide is the scope of the work that, with the exception of midwifery and matters strictly surgical, it embraces the whole domain of medicine, including the departments for which the physician is accustomed to rely on special treatises, such as diseases of women and children, of the genito-urinary organs, of the skin, of the nerves, hygiene and sanitary science, and medical ophthalmology and otology. Moreover, authors have inserted the formulas which they have found most efficient in the treatment of the various affections. It may thus be truly regarded as a Complete Library of Practical Medicine, and the general practitioner possessing it may feel secure that he will require little else in the daily round of professional duties.

In spite of every effort to condense the vast amount of practical information furnished, it has been impossible to present it in less than 5 large octavo volumes, containing about 5600 beautifully printed pages, and embodying the matter of about 15 ordinary octavos. Illustrations are introduced wherever requisite to elucidate the text.

A detailed prospectus will be sent to any address on application to the publishers.

These two volumes bring this admirable work / physicians who are acquainted with all the varieto a close, and fully sustain the high standard reached by the earlier volumes; we have only therefore to echo the eulogium pronounced upon them. We would warmly congratulate the editor and his collaborators at the conclusion of their laborious task on the admirable manner in which, from first to last, they have performed their several duties. They have succeeded in producing a work which will long remain a standard work of reference, to which practitioners will look for guidance, and authors will resort to for facts. From a literary point of view, the work is without any serious blemish, and in respect of production, it has the beautiful finish that Americans always give their works.-Edinburgh Medical Journal, Jan. 1887.

* The greatest distinctively American work on the practice of medicine, and, indeed, the superlative adjective would not be inappropriate were even all other productions placed in comparison. An examination of the five volumes is sufficient to convince one of the magnitude of the enterprise, and of the success which has attended its fulfilment.-The Medical Age, July 26, 1886.

This huge volume forms a fitting close to the great system of medicine which in so short a time has won so high a place in medical literature, and has done such credit to the profession in this country. Among the twenty-three contributors are the names of the leading neurologists in America, and most of the work in the volume is of the highest order.-Boston Medical and Surgical Journal, July 21, 1887.

We consider it one of the grandest works on Practical Medicine in the English language. It is a work of which the profession of this country can feel proud. Written exclusively by American

ties of climate in the United States, the character of the soil, the manners and customs of the people etc it is peculiarly adapted to the wants of American practitioners of medicine, and it seems to us that every one of them would desire to have it. It has been truly called a "Complete Library of Practical Medicine," and the gener practitioner will require little else in his round of professional duties.-Cincinnati Medical News, March, 1886.

Each of the volumes is provided with a most copious index, and the work altogether promises to be one which will add much to the medical literature of the present century, and reflect great credit upon the scholarship and practical acumen of its authors.-The London Lnncet, Oct. 3, 1885.

The feeling of proud satisfaction with which the American profession sees this, its representative system of practical medicine issued to the medical world, is fully justified by the character of the work. The entire caste of the system is in keeping with the best thoughts of the leaders and followers of our home school of medicine, and the combination of the scientific study of disease and the practical application of exact and experimental knowledge to the treatment of human maladies, makes every one of us share in the pride that has welcomed Dr. Pepper's labors. Sheared of the prolixity that wearies the readers of the German school, the articles glean these same fields for all that is valuable. It is the outcome of American brains, and is marked throughout by much of the sturdy independence of thought and originality that is a national characteristic. Yet nowhere is there lack of study of the most advanced views of the day. - North Carolina Medical Journal, Sept. 1886. 
FOTHERGILL, J. M., M. D., Edin,. M. R. C. P., Lond.,

Physician to the City of London Hospital for Diseases of the Chest.

The Practitioner's Handbook of Treatment; Or, The Principles of Therapeutics. New (third) edition. In one octavo volume of 661 pages. Cloth, $\$ 3.75$; leather, \$4.75. Just ready.

Dr. Fothergill is always interesting and instruct- / greatly increased by the introduction of many ive, and in this standard work he shows his prescriptions. That the profession appreciates peculiar power as a writer on therapeuties to the that the author has undertaken an important work best advantage. Everything he undertakes is and has accomplished it is shown by the demand done conscientiously. The book well sustains for this third edition.-New York Medical Journal, the favorable impression which it created at the June 11, 1887.

beginning of its eareer, and in its present im. This is a wonderful book. If there be such a proved form it will be welcomed more than ever thing as "medicine. made easy" this is the work by the busy practitioner and the scientific student to accomplish this result. It imparts information of medicine. - The Medical News, July 23, 1887.

To have a description of the normal physiologi- thinks as he lays the book down that he "knew cal processes of an organ and of the methods of that before," when in reality he did not, or else he treatment of its morbid conditions brought could before have reconciled facts which now together in a single chapter, and the relations become clear as daylight. The author deals with between the two clearly stated, cannot fail to prove the "Principles of Therapeutics" the study of a great convenience to many thoughtful but busy which will give great vantage to the physician.physicians. The practical value of the volume is Virginia Medical Monthly, June, 1887.

\section{REYNOLID, J. RUSSELT, M. D.,}

Professor of the Principles and Practice of Medicine in University College, London.

A System of Medicine. With notes and additions by HeNRx Hartshorne, A. M., M. D., late Professor of Hygiene in the University of Pennsylvania. In three large and handsome octavo volumes, containing 3056 double-columned pages, with 317 illustrations. Price per volume, cloth, $\$ 5.00$; sheep $\$ 6.00$; very handsome half Russia, raised bands, $\$ 6.50$. Per set, cloth, $\$ 15$; leather, $\$ 18$; half Russia, $\$ 19.50$. Sold only by subscription.

\section{STILLÉ, ALFRED, M. D., LL. D.,}

Professor Emeritus of the Theory and Practice of Med. and of Clinical Med. in the Univ. of Penna.

Cholera: Its Origin, History, Causation, Symptoms, Lesions, Prevention and Treatment. In one handsome $12 \mathrm{mo}$. volume of 163 pages, with a chart. Cloth, $\$ 1.25$. Just ready.

\section{FINLA YSON, JAIIES, M. D., Editor,}

Physician and Lecturer on Clinical Medicine in the Glasgow Western Infirmary, ete.

Clinical Manual for the Study of Medical Cases. With Chapters by Prof. Gairdner on the Physiognomy of Disease; Prof. Stephenson on Diseases of the Female Organs; Dr. Robertson on Insanity; Dr. Gemmell on Physical Diagnosis; Dr. Coats on Laryngoscopy and Post-Mortem Examinations, and by the Editor on Casetaking, Family History and Symptoms of Disorder in the Various Systems. New edition. In one 12mo. volume of 682 pages, with 158 illustrations. Cloth, \$2.50. Just ready.

The profession cannot but welcome the second / and a study of means to the end whish cannot edition of this very valuable work of Finlayson fail in laying an excellent foundation for the and his collaborators. The size of the book has student for future success as an able diagnostician. been increased and the number of illustrations nearly doubled. The manner in which the subject is treated is a most practical one. Symptoms alone and their diagnostic indications form the basis of discussion. The text explains clearly and thoroughly the methods of examination and the conclusions to be drawn from the physical signs.The Medical News, April 23, 1887.

This manual is one of the most complete and perfect of its kind. It goes thoroughly into the perfect of its kind. It goes thoroughly into the infornation, that it should be a part of the library It must lead to a thoroughness of observation, an July $23,1887$.

examination in detail of every scientific appliance,

\section{FENWICT, SAMUEL, M. U.,}

Assistant Physician to the London Hosprtal.

The Student's Guide to Medical Diagnosis. From the third revised and enlarged English edition. In one very handsome royal $12 \mathrm{mo}$. volume of 328 pages, with 87 illustrations on wood. Cloth, $\$ 2.25$.

\section{HABERSHON, S. O., M. D.,}

Senior Physician to and late Lect. on Principles and Practice of Med. at Guy's Hospital, London.

On the Diseases of the Abdomen; Comprising those of the Stomach, and other parts of the A limentary Canal, Esophagus, Cæcum, Intestines and Peritoneum. Second American from third enlarged and revised English edition. In one handsome octavo volume of 554 pages, with illustrations. Cloth, $\$ 3.50$.

\section{TANNER, THOMAS HAWKES, M. D.}

A Manual of Clinical Medicine and Physical Diagnosis. Third American from the second London edition. Revised and enlarged by Trubury Fox, M. D. In one small $12 \mathrm{mo}$. volume of 362 pages, with illustrations. Cloth, $\$ 1.50$. 


\section{BARTHOLOW, ROBERTS, A. M., M. D., LL. D.,}

Prof. of Materia Medica and General Therapeutics in the Jefferson Med. Coll. of Phila., etc.

Medical Electricity. A Practical Treatise on the Applications of Electricity to Medicine and Surgery. New (third) edition. In one very handsome octavo volume of 308 pages, with 110 illustrations. Cloth, \$2.50. Just ready.

The fact that this work has reached its third edi- | should read it, especially when it is recalled what (the should read it, especially abreast with the increasing use and knowledge of the therapeutics of electricity. Dr. Bartholow has electricity, demonstrates its claim to be considered here presented the profession with a concise work a practical treatise of tried value to the profession. that, beginning with elementary descriptions and The matter added to the present edition embraces principles, gradually grows, page by page, into a the most recent advances in electrical treatment. magnificently practical treatise, describing operathe most recent advances in electrical treatment. magnificently practical treatise, describing operawork constitutes a full, clear and concise manual that prove electricity to be marvellous as a curative well adapted to the needs of both student and agent in many forms of disease. The doctor canpractitioner-The Medical News, May 14, 1887. not now do better than to possess himself of Dr. This "practical treatise on the applications of Bartholow's treatise, just as it is.-Virginia Medielectricity to medicine and surgery" has grown to cal Monthly, June, 1887.

be so important a work that every practitioner

\section{RICHARDSON, B.W., M.A., M.D., LL.D., F.R.S., F.S.A.}

Fellow of the Roval College of Physicians, Iondon.

Preventive Medicine. In one octavo volume of 729 pages. Cloth, \$4; leather, $\$ 5$; very handsone half Russia, raised bands, $\$ 5.50$.

Dr. Richardson has succeeded in producing a tive collection of data upon the diseases common work which is elevaled in conception, comprehen- to the race, their origins, causes, and the measures sive in scope, scientific in character, systematic in for their prevention. The descriptions of diseases arrangement, and which is written in a clear, con- are clear, chaste and scholarly ; the discussion of cise and pleasant manner. He evinces the happy the question of disease is comprehensive, masterly faculty of extracting the pith of what is known on and fully abreast with the latest and best knowlthe subject, and of presenting it in a most simple, edge on the subject, and the preventive measures intelligent and practical form. There is perhaps advised are accurate, explicit and reliable.-The no similar work written for the general public American Journal of the Medical Sciences, A pril, 1884. thatcontains such a complete, reliable and instruc-

\section{HARTSHORNE, HENIRY, M. D., LL. D.,}

Formerly Professor of Hygiene in the University of Pennsylvania, and Professor of Physiology and Diseases of Children in the Woman's Medical College of Pennsylvania.

A Household Manual of Medicine, Surgery, Nursing and Hygiene: For Daily Use in the Preservation of Health and Care of the Sick and Injured, with an Introductory Outline of Anatomy and Physiology. In one very handsome royal octavo volume of 946 pages, with 8 plates and 283 engravings. Cloth, $\$ 4.00$; very handsome half Morocco, $\$ 5.00$.

\section{THE YEAR-BOOK OF TREATMENT FOR 1886.}

A Comprehensive and Critical Review for Practitioners of Medicine. In one $12 \mathrm{mo}$. volume of 309 pages, bound in limp cloth, $\$ 1.25$.

This "review" includes every department of every practitioner, whether he be ageneral one or medical and surgical as well as obstetrical practice. a specialist. It is a book to be kept on the office It attempts nothing in the way of etiology, diag- table for continuous reference. An excellent innosis or symptoms, but limits itself to the ad- dex to subjects, as well as to authors quoted, is vances made in the treatment of diseases, injuries, appended.-Virginia Medical Monthly, April, 1887. vances made in the treatment of diseases,injuries,

* * For special commutations with periodicals see page 3 .

THE YEAR-BOOK OF TREATMENT FOR 1885.

Similar to that of 1886 above. 12 mo., 320 pages. Limp cloth, $\$ 1.25$.

\section{SCHREIBER, DR. JOSEPH.}

A Manual of Treatment by Massage and Methodical Muscle Fxercise. Translated by Walter Mendelson, M. D., of New York. In one handsome octavo volume of 278 pages, with 117 fine engravings. Just ready. Cloth, \$2.75.

The present volume will do much to establish / science of mechanical therapeutics, adapted to mechanical therapeutics upon a scientific basis. the use of the general practitioner, this volume The work is a very welcomeaddition to the library, and we heartily recommend it to our readers as a step in the right direction.-New York Medica Journal, July 16, 1887 .

As a thorough and satisfactory exposition of the

illustrated by well-drawn woodcuts, leaving no room for obscurity in the description of the various manipulations recommended.-Atlanta Medical and Surgical Journal, Aug. 1887.

STURGES' INTRODUCTION TO THE STUDY OF CLINICAL MEDICINE. Being a Guide to the Investigation of Disease. In one handsome 12mo. volune of 127 pages. Cloth, $\$ 1.25$.

DAVIS' CLINICAL LECTURES ON VARIOUS IMPORTANT DISEASES. By N. S. DAVIE, M. D. Edited by FranK H. DAVIR, M. D. Second edition. 12mo. 287 pages. Cloth, $\$ 1.75$.

TODD'S CLINICAL LECTURES ON CERTAIN ACUTE DISEASES. In one octavo volume of 320 pages. Cloth, $\$ 2.50$.
PAVY'S TREATISE ON THE FUNCTION OF DIGESTION; its Disorders and their Treatment. From the second I,ondon edition. In one octavo volume of 238 pages. Cloth, $\$ 2.00$.

BARLOW'S MANUAL OF THE PRACTICE OF MEDICINE. With additions by D. F. CoNDIE, M. D. 1 vol. 8vo., pp. 603 . Cloth, $\$ 2.50$

CHAMBERS' MANUAL OF DIET AND REGIMEN IN HEALTH AND SICKNESS. In one handsome octavo volume of $302 \mathrm{pp}$. Cloth, $\$ 2.75$.

HOLLAND'S MEDICAL NOTES AND REFLECTIONS, 1 vol. 8vo., pp. 493 . Cloth, $\$ 3.50$. 


\section{FIINT, AUSTIN, M. D.,}

Professor of the Principles and Practice of Medicine in Bellevue Hospital Medical College, N. $Y$.

A Manual of Auscultation and Percussion; Of the Physical Diagnosis of Diseases of the Lungs and Heart, and of Thoracic Aneurism. Fourth edition. In one handsome royal $12 \mathrm{mo}$. volume of 278 pages, with 14 illustrations. Cloth, $\$ 1.75$.

This admirable little book is too well known to study. Dr. Flint's work is just such a book. It require any extended notice. That a third and contains the substance of the lessons which the large edition has been exhausted in little more author has for many years given in connection than two years, is evidence that the book is appre- with practical instruction in auscultation and ciated. We ourselves have used a former edition percussion to private classes, composed of medical as a text-book in teaching the physical examina- students and practitioners. The fact that within tion of the chest, and can consequently speak from a little more than two years a large edition of this experience.-Boston Medical and Surgical Journal, manual has been exhausted, is proof of the favor Feb. 11, 1886.

The student needs a first-class text-book in which the subject is fully explained for him to with which it has been regarded by the medica profession.-Cincinnati Medical News, Feb. 1886.

\section{BY THE SAME AUTHOR.}

Physical Fxploration of the Lungs by Means of Auscultation and Percussion. Three lectures delivered before the Philadelphia County Medical Society, 1882-83. In one handsome small $12 \mathrm{mo}$. volume of 83 pages. Cloth, $\$ 1.00$.

A Practical Treatise on the Physical Exploration of the Chest and the Diagnosis of Diseases Affecting the Respiratory Organs. Second and revised edition. In one handsome octavo volume of 591 pages. Cloth, $\$ 4.50$.

Phthisis: Its Morbid Anatomy, Etiology, Symptomatic Events and Complications, Fatality and Prognosis, Treatment and Physical Diagnosis; In a series of Clinical Studies. In one handsome octavo volume of 442 pages. Cloth, $\$ 3.50$.

A Practical Treatise on the Diagnosis, Pathology and Treatment of Diseases of the Heart. Second revised and enlarged edition. In one octavo volume of 550 pages, with a plate. Cloth, $\$ 4$.

Essays on Conservative Medicine and Kindred Topics. In one very hand. some royal $12 \mathrm{mo}$. volume of 210 pages. Cloth, $\$ 1.38$.

\section{GROSS, S. D., M.D., LL.D., D.C.L. Oxon., LL.D. Cantab.}

A Practical Treatise on Foreign Bodies in the Air-passages. In one octavo volume of 452 pages, with 59 illustrations. Cloth, $\$ 2.75$.

\section{COHEN, J. SOLIS, MY. D.,}

Lecturer on Laryngoscopy and Diseases of the Throat and Chest in the Jefferson Medical College.

Diseases of the Throat and Nasal Passages. A Guide to the Diagnosis and Treatment of Affections of the Pharynx, Esophagus, Trachea, Larynx and Nares. Third edition, thoroughly revised and rewritten, with a large number of new illustrations. In one very handsome octavo volume. Preparing.

\section{SEILER, CARL, M. D.,}

Lecturer on Laryngoscopy in the University of Pennsylvania.

A Handbook of Diagnosis and Treatment of Diseases of the Throat, Nose and Naso-Pharynx. Second edition. In one handsome royal $12 \mathrm{mo}$. volume of 294 pages, with 77 illustrations. Cloth, $\$ 1.75$.

It is one of the best of the practical text-books and there are a valuable bibliography and a good on this subject with which we are acquainted. The index of the whole. For any one who wishes to present edition has been increased in size, but its make himself familiar with the practical manageeminently practical character has been main- ment of cases of throat and nose disease, the book tained. Many new illustrations have also been will be found of great value.-New York Medrcal introduced, a case-record sheet has been added, Journal, June 9, 1883.

\section{BROADBENT, W. H., M. D., F. R. C.P.,}

Physician to and Lecturer on Medicine at St. Mary's Hospital.

The Pulse. In one 12mo. volume. Preparing. See Series of Clinical Manuals, page 4.

FULLER ON DISEASES OF THE LUNGS AND AIR-PASSAGES. Their Pathology, Physical Diagnosis, Symptoms and Treatment. From the second and revised English edition. In one octavo volume of 475 pages. Cloth, $\$ 3.50$.

SLADE ON DIPHTHERIA; its Nature and Treatment, with an account of the History of its Prevalence in various Countries. Second and revised edition. In one 12mo. vol. pp. 158. Cloth, \$1.25.

WALSHE ON THE DISEASES OF THE HEART AND GREAT VESSELS. Third American edition. In 1 vol. 8vo., 416 pp. Cloth, $\$ 3.00$.
SMITH ON CONSUMPTION ; its Early and Reme diable Stages. 1 vol. 8vo., pp. 253 . Cloth, $\$ 2.25$.

LA ROCHE ON PNEUMONIA. I vol. 8vo. of 490 pages. Cloth, $\$ 3.00$.

WILLIAMS ON PULMONARY CONSUMPTION its Nature, Varieties and Treatment. With an analysis of one thousand cases to exemplify its duration. In one 8vo. vol. of $303 \mathrm{pp}$. Cloth, $\$ 2.50$. JONES' CLINICAL OBSERVATIONS ON FUNC TIONAL NERVOUS DISORDERS. Second Am erican edition. In one handsome octavo volume of 340 pages. Cloth, $\$ 3.25$. 


\section{ROSS, JAMES, M. D., F.R. C.P., LL. D.,}

Senior Assistant Physician to the Manchester Royal Infirmary.

A Handbook on Diseases of the Nervous System. In one octavo volume of 725 pages, with 184 illustrations. Cloth, $\$ 4.50$; leather, $\$ 5.50$.

This admirable work is intended for students of / the department of medicine of which it treats. medicine and for such medical men as have notime Dr. Ross holds such a high scientific position that for lengthy treatises. In the present instance the any writings which bear his name are naturally duty of arranging the vast store of material at the expected to have the impress of a powerful inteldisposal of the author, and of abridging the de- lect. In every part this handbook merits the scription of the different aspects of nervous dis- highest praise, and will no doubt be found of the eases, has been performed with singular skill, and greatest value to the student as well as to the practhe result is a concise and philosophical guide to titioner.-Edinburgh Medical Journal, Jan. 1887.

\section{MITCHELL, S. WEIR, M. D.,}

Physician to Orthopuedic Hospital and the Infirmary for Diseases of the Nervous System, Phila., ete.

Lectures on Diseases of the Nervous System; Especially in Women. Second edition. In one $12 \mathrm{mo}$. volume of 288 pages. Cloth, $\$ 1.75$.

No work in our language develops or displays | teachings the stamp of authority all over the more features of that many-sided affection, hys- realm of medicine. The work, although written teria, or gives clearer directions for its differen- by a specialist, has no exclusive character, and tiation, or sounder suggestions relative to its the general practitioner above all others will find general management and treatment. The book its perussl profitable, since it deals with diseases is particularly valuable in that it represents in which he frequently encounters and must essay the main the author's own clinical studies, which to treat.-American Practitioner, August, 1885.

have been so extensive and fruitful as to give his

\section{HAMILTON, ALLAN MLLANE, M. D.,}

Attending Physician at the Hospital for Epileptics and Paralytics, Blackwell's Island, N. Y.

Nervous Diseases; Their Description and Treatment. Second edition, thoroughly revised and rewritten. In one octavo volume of 598 pages, with 72 illustrations. Cloth, $\$ 4$. When the first edition of this good book appeared | characterized this book as the best of its kind in we gave it our emphatic endorsement, and the any language, which is a handsome endorsement present edition enhances our appreciation of the from an exalted source. The improvements in the book and its author as a safe guide to students of new edition, and the additions to it, will justify its clinical neurology. One of the best and most purchase even by those who possess the old.critical of English neurological journals, Brain, has $\mid$ Alienist and Neurologist, April, 1882.

\section{TUKE, DANIEL HACK, M. D.,}

Joint Author of The Manual of Psychological Medicine, etc.

Illustrations of the Influence of the Mind upon the Body in Health and Disease. Designed to elucidate the Action of the Imagination. New edition. Thoroughly revised and rewritten. In one handsome octavo volume of 467 pages, with two colored plates. Cloth, $\$ 3.00$.

It is impossible to peruse these interesting chap- $\mid$ method of interpretation. Guided by an enlightters without being convinced of the author's per- ened deduction, the author has reclaimed for fect sincerity, impartiality, and thorough mental science a most interesting domain in psychology, grasp. Dr. Tuke has exhibited the requisite previously abandoned to charlatans and empirics. smount of sclentific address on all occasions, and This book, well conceived and well written, must the more intrioate the phenomena the more firmly commend itself to every thoughtful understandhas he adhered to a physiological and rational ing.-New York Medical Journal, September 6, 1884.

\section{CLOUSTON, THOMAS S., M. D., F. R. C. P., L. R. C. S.,} Lecturer on Mental Diseases in the University of Edinburgh.

Clinical Lectures on Mental Diseases. With an Appendix, containing an Abstract of the Statutes of the United States and of the Several States and Territories relating to the Custody of the Insane. By Charles F. Folsom, M. D., Assistant Professor of Mental Diseases, Med. Dep. of Harvard Univ. In one handsome octavo volume of 541 pages, with eight lithographic plates, four of which are beautifully colored. Cloth, $\$ 4$.

The practitioner as well as the student will ac- the general practitioner in guiding him to a diagcept the plain, practical teaching of the author as a nosis and indicating the treatment, especially in forward step in the literature of insanity. It is many obscure and doubtful cases of mental dis. refreshing to find a physician of Dr. Clouston's ease. To the American reader Dr. Folsom's $A p$ experience and high reputation giving the bed- pendix adds greatly to the value of the work, and side notes upon which his experience has been will make it a desirable addition to every library. founded and his mature judgment established. -American Psychological Journal, July, 1884.

Such elinical observations cannot but be useful to

Dr. Folsom's Abstract may also be obtained separately in one octavo volume of 108 pages. Cloth, $\$ 1.50$.

SAVAGE, GEORGE H., M. D., Lecturer on Mental Diseases at Guy's Hospital, London.

Insanity and Allied Neuroses, Practical and Clinical. In one 12mo. vol. of 551 pages, with 18 illus. Cloth, $\$ 2.00$. See Series of Clinical Manuals, page 4.

\section{PLAYFAIR, W. S., M. D., F. R. C. P.}

The Systematic Treatment of Nerve Prostration and Hysteria. In one handsome small $12 \mathrm{mo}$. volume of 97 pages. Cloth, $\$ 1.00$.

Blandford on Insanity and its Treatment: Lectures on the Treatment, Medical and Legal, of Insane Patients. In one very handsome octavo volume. 


\section{ASHHURST, JOHN, Jr., M. D.,}

Professor of Clinical Surgery, Unv. of Penna., Surgeon to the Episcopal Hospital, Philadelphia.

The Principles and Practice of Surgery. New (fourth) edition, enlarged and revised. In one large and handsome octavo volume of 1114 pages, with 597 illustrations. Cloth, $\$ 6$; leather, $\$ 7$; half Russia, $\$ 7.50$.

As with Erichsen so with Ashhurst, its position in professional favor is established, and one has now but to notice the changes, if any, in theory and practice, that are apparent in the present as compared with the preceding edition, published three years ago. The work has been brought well up to date, and is larger and better illustrated than before, and its author may rest assured that it will certainly have a "continuance of the favor with which it has heretofore been received."-The American Journal of the Medical Sciences, Jan. 1886.

Every advance in surgery worth notice, chronicled in recent literature, has been suitably recognized and noted in its proper place. Suffice it to say, we regard Ashhurst's Surgery, ss now presented in the fourth edition, as the best single volume on surgery published in the English language, valuable alike to the student and the practitioner, to the one as a text-book, to the other as a manual of practical surgery. With pleasure we give this volume our endorsement in full. - Nero Orleans Medical and Surgical Journal, Jan., 1886.

\section{GROSS, S. D., M. D., LL. D., D. C. L. Oxon., LL. D. Cantab.,}

Emeritus Professor of Surgery in the Jefferson Medical College of Philadelphia.

A System of Surgery: Pathological, Diagnostic, Therapeutic and Operative. Sixth edition, thoroughly revised and greatly improved. In two large and beautifullyprinted imperial octavo volumes containing 2382 pages, illustrated by 1623 engravings. Strongly bound in leather, raised bands, $\$ 15$; half Russia, raised bands, $\$ 16$.

Dr. Gross' System of Surgery has long been the His System of Surgery, which, since its first edistandard work on that subject for strdents and practitioners.-London Lancet, May 10, 1884.

The work as a whole needs no commendation. Many years ago it earned for itself the enviable reputation of the leading American work on surgery, and it is still capable of maintaining that standard. A considerable amount of new material has been introduced, and altogether the distinguished author has reason to be satisfied that he has placed the work fully abreast of the state of our knowledge.-Med. Record, Nov. 18, 1882.

\section{DRUITT, ROBERT, M. R. C. S., etc.}

Manual of Modern Surgery. Twelfth edition, thoroughly revised by STANLEY BoyD, M. B., B.S., F. R. C.S. In one 8 vo. volume of 965 pages, with 373 illustrations. Cloth, $\$ 4$; leather, $\$ 5$.

FROM THE EDITOR'S PREFACE TO THE TWELFTH EDITION.

Few works have been more widely known and appreciated than Druitt's "Surgery." In England I am informed that 50,000 copies have been sold, whilst in America it has been so highly appreciated that a copy was issued by the Government to each surgeon serving in the Federal Army during the great Civil War. The twelfth edition differs much from the eleventh; scarcely a paragraph of the latter remains unaltered. In spite of my utmost endeavors to compress, the book has increased considerably in size. This is due to many causes, especially to the greatly increased range of subjects with which I have had to deal, to the replacement of many old illustrations by a number of considerably larger ones, and to the addition of a copious index.

\section{GIBNEY, V. P., M. D.,}

Surgeon to the Orthopadic Hospital, New York, etc.

Orthopædic Surgery. For the use of Practitioners and Students. In one handsome octavo volume, profusely illustrated. Preparing.

\section{ROBERTS, JOHN B., A. M., M. D.,}

Lecturer on Anatomy and on Operative Surgery at the Philadelphia School of Anatomy.

The Principles and Practice of Modern Surgery. For the use of Students and Practitioners of Medicine and Surgery. In one very handsome octavo volume of about 500 pages, with many illustrations. Preparing.

\section{BELLAMY, EDWARD, F. R. C. S.,}

Surgeon and Lecturer on Surgery at Charing Cross Hospital, London.

Operative Surgery. Shortly. See Students' Series of Manuals, page 4.

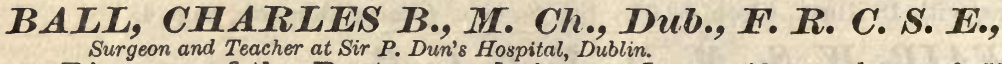

Diseases of the Rectum and Anus. In one $12 \mathrm{mo}$. volume of 550 pages. Preparing. See Series of Clinical Manuals, page 4.

MILLER'S PRACTICE OF SURGERY. Fourth and revised American from the last Edinburgh edition. In one large $8 \mathrm{ro}$. vol. of $682 \mathrm{pages}$, with 364 illustrations. Cloth, $\$ 3.70$.
MILLER'S PRINCIPLES OF SURGERY. Fourth American from the third Edinburgh edition. In one 8vo. vol. of 638 pages, with 340 illustrations. Floth, \$3.75. 


\section{ERICHSEN, JOHIN E., F. R. S., F. R. C. S.,}

Professor of Surgery in University College, London, etc.

The Science and Art of Surgery; Being a Treatise on Surgical Injuries, Diseases and Operations. From the eighth and enlarged English edition. In two large and beautiful octavo volumes of 2316 pages, illustrated with 984 engravings on wood. Cloth, $\$ 9$; leather, raised bands, $\$ 11$; half Russia, raised bands, $\$ 12$.

In noticing the eighth edition of this well- $/$ years and maintaining during that period a reknown work, it would appear superfluous to say putation as a leading work on surgery, there is not more than that it has, like its predecessors, been much to be said in the way of comment or critibrought fully up to the times, and is in conse- cism. That it still holds its own goes without sayquence one of the best treatises upon surgery that ing. The author infuses into it his large experihas ever been penned by one man. We have al- ence and ripe judgment. Wedded to no school, ways regarded "The Science and Art of Surgery" committed to no theory, biassed by no hobby, he as one of the best surgical text-books in the imparts an honest personality in his observations, English language, and this eighth edition only and his teachings are the rulings of an impartial confirms our previous opinion. We take great judge. Such men are always safe guides, and their pleasure in cordially commending it to our read- works stand the tests of time and experience.

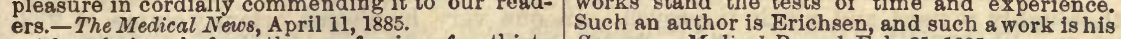
After being before the profession for thirty Surgery.-Medical Record, Feb. 21, 1885.

\section{BRYANT, THOMAS, F. R. C. S.,}

Surgeon and Lecturer on Surgery at Guy's Hospital, London.

The Practice of Surgery. Fourth American from the fourth and revised English edition. In one large and very handsome imperial octavo volume of 1040 pages, with 727 illustrations. Cloth, $\$ 6.50$; leather, $\$ 7.50$; half Russia, $\$ 8.00$.

The fourth edition of this work is fully abreast / books for the medical student. Almost every of the times. The author handles his subjects topic in surgery is presented in such a form as to with that degree of judgment and skill which is enable the busy practitioner to review any subject attained by. years of patient toil and varied ex- in every-day practice in a short time. No time is perience. The present edition is a thorough re- lost with useless theories or superfluous verbiage. vision of those which preceded it, with much new In short, the work is eminently clear, logical and matter added. His diction is so graceful and practical.-Chicago Medical Journal and Examiner, logical, and his explanations are so lucid, as to April, 1886.

place the work among the highest order of text-

By the same Author.

Diseases of the Breast. In one 12mo. volume. Preparing. See Series of Clinical Manuals, page 4.

\section{TREVES, FREDERICK, F.R.C.S.,}

Hunterian Professor at the Royal College of Surgeons of England.

A Manual of Surgery. In Treatises by Various Authors. In three $12 \mathrm{mo.}$ volumes, containing 1866 pages, with 213 engravings. Price per volume, cloth, \$2. See Students' Series of Manuals, page 4.

We have here the opinions of thirty-three in the choice, arrangement and logical sequence of authors, in an encyclopædic form for easy and the subjects. Every topic, as far as observed, is ready reference. The three volumes embrace treated with a fulness of essential detail, which is every variety of surgleal affections likely to be somewhat surprising. Another characteristic of the met with, the paragraphs are short and pithy, and work is the well-nigh unlversal acceptance of modthe salient points and the beginnings of new sub- ern and progressive views of pathology and treatjects are always printed in extra-heavy type, so ment. The entire work is conceived and executed that a person may find whatever information he in a scientific spirit. It contains the bone and marmay be in need of at a moment's glance.-Cin- row of modern surgery.-Annals of Surgery, Oct. cinnati Lancet-Clinic, August 21, 1886.

The hand of Mr. Treves is evident throughout

\section{BUTLIN, HENRY T., F. R. C. S., \\ Assistant Surgeon to St. Bartholomew's Hospital, London.}

Diseases of the Tongue. In one $12 \mathrm{mo}$. volume of 456 pages, with 8 colored plates and 3 woodcuts. Cloth, $\$ 3.50$. See Series of Clinical Manuals, page 4.

The language of the text is clear and concise. veniently scattered through general works on surThe author has aimed to state facts rather than to gery and the practice of medicine. The physician express opinions, and has compressed within the and surgeon will appreciate its value as an aid and compass of this small volume the pathology, etiol- guide.-Physician and Surgeon, Sept. 1886.

ogy, etc., of diseases of the tongue that are incon-

\section{TREVES, FREDERICK, F. R. C.S.,}

Surgeon to and Locturer on Surgery at the London Hospital.

Intestinal Obstruction. In one pocket-size $12 \mathrm{mo}$. volume of 522 pages, with 60 illustrations. Limp cloth, blue edges, $\$ 2.00$. See Series of Clinical Manuals, page 4.

A standard work on a subject that has not been | justice to the author in a few paragraphs. Intesso comprehensively treated by any contemporary tinal Obstruction is a work that will prove of English writer. Its completeness renders a full equal value to the practitioner, the student, the review difficult, since every chapter deserves mi- pathologist, the physician and the operating surnute attention, and it is impossible to do thorough geon.-British Medical Journal, Jan. 31, 1885.

\section{GOULD, A. PEARCE, M. S., M. B., F. R. C. S., \\ Assistant Surgeon to Middlesex Hospital.}

Elements of Surgical Diagnosis. In one pocket-size 12mo. volume of 589 pages. Cloth, \$2.00. See Students' Series of Manuals, page 4. 


\section{HOLMES, TIMOTHY, M. A.,}

Surgeon and Lecturer on Surgery at St. George's Hospital, London.

A System of Surgery; Theoretical and Practical. IN TREATISES BY VARIOUS AUTHORS. AMERICAN EDITION, THOROUGHLY REVISED AND RE-EDITED by JoHn H. Packard, M. D., Surgeon to the Episcopal and St. Joseph's Hospitals, Philadelphia, assisted by a corps of thirty-three of the most eminent American surgeons. In three large and very handsome imperial octavo volumes containing 3137 doublecolumned pages, with 979 illustrations on wood and 13 lithographic plates, beautifully colored. Price per set, cloth, $\$ 18.00$; leather, $\$ 21.00$; half Russia, $\$ 22.50$. Sold only by subscription.

\section{HAMTLTON, FRANK H., M. D., LL. D.,}

Surgeon to Bellevue Hospital, New York.

A Practical Treatise on Fractures and Dislocations. Seventh edition thoroughly revised and much improved. In one very handsome octavo volume of 998 pages, with 379 illustrations. Cloth, $\$ 5.50$; leather, $\$ 6.50$; very handsome half Russia, open back, $\$ 7.00$.

It is about twenty-five years ago since the first edition of this great work appeared. The edition now issued is the serenth, and this fact alone is enough to testify to the excellence of it in all particulars. Books upon special subjects do not usually command extended sale, but this one is without a rival in any language. It is essentially a practical treatise, and it gathers within its covers almost everything valuable that has been written about fractures and dislocations. The principles and methods of treatment are very fully given. The book is so well known that it does not require

any lengthened review. We can only say that it is still unapproached as a treatise, and that it is a proof of the zeal and industry and great ability of its distinguished author.-The Dublin Journal of Medical Science, Feb. 1886.

His famous treatise on Fractures and Disloca tions, published first in 1860 , is justly regarded as the best book on that subject in existence. It has now run through seven editions, and has been translated into French and German.-Medical Record, Aug. 14, 1886.

\section{SMITH, STEPHEN, M. D.,}

Professor of Clinical Surgery in the University of the City of New York.

The Principles and Practice of Operative Surgery. New (second) and thoroughly revised edition. In one very handsome octavo volume of 892 pages, with 1005 illustrations. Cloth, $\$ 4.00$; leather, $\$ 5.00$. Just ready.

This work is too well and too favorably known to | plete works in the English language, and is a fit require any words of commendation, and its mer- companion to Malgaigne's magnificent treatise. its effectually protect it from adverse criticism. The woodcuts are good, and are very numerous. It is a treatise upon the principles as well as the The descriptions of operative procedures are plain, practice of mechanical surgery. The subject mat- and the opinions expressed are conservative and ter is brought down to the very latest period, hence judicious. The work reflects great credit upon the we shall find the work to be a faithful exponent author and upon American surgical literature.of the art of surgery as practised now. Stephen The American Journal of the Medical Sciences, April, Smith's Operative Surgery is one of the most com- 1887.

\section{STIMSON, LEWIS A., B. A., M. D.,}

Professor of Pathological Anatomy at the University of the City of New York, Surgeon and Curator to Bellevue Hospital, Surgeon to the Presbyterian Hospital, New York, etc.

A Manual of Operative Surgery. New (second) edition. In one very handsome royal $12 \mathrm{mo}$. volume of 503 pages, with 342 illustrations. Cloth, $\$ 2.50$.

There is always room for a good book, so that / effected in operative methods and procedures by while many works on operative surgery must be the antiseptic system, and has added an account considered superfluous, that of Dr. Stimson has of many new operations and variations in the held its own. The author knows the difficult art steps of older operations. We do not desire to of condensation. Thus the manual serves as a extol this manual above many excellent standard work of reference, and at the same time as a British publications of the same class, still we behandy guide. It teaches what it professes, the lieve that it contains much that is worthy of imisteps of operations. In this edition Dr. Stimson tation.-British Medical Journal, Jan. 22, 1887.

has sought to indicate the changes that have been

By the same Author.

A Practical Treatise on Fractures. In one very handsome octavo volume of 598 pages, with 360 beautiful illustratıons. Cloth, $\$ 4.75$; leather, $\$ 5.75$.

The author has given to the medical profession / ough. The chapters on repair of fractures and their in this treatise on fractures what is likely to be- treatment show him not only to be a profound stucome a standard work on the subject. It is certainly dent, but likewise a practical surgeon and patholonot surpassed by any work written in the English, gist. His mode of treatment of the different fractor, for that matter, any other language. The au- ures is eminently sound and practical. We consider thor tells us in a short, concise and comprehensive this work one of the best on fractures; and it will manner, all that is known about his subject. There be welcomed not onlv as a text-book, but also by is nothing scanty or superficial about it, as in most the surgeon in full practice. $-N$. $O$. Medical and other treatises; on the contrary, everything is thor- Surgical Journal, March, 1883.

\section{MARSH, HOWARD, F. R. C. S.,}

Senior Assistant Surgeon to and Lecturer on Anatomy at St. Bartholomew's Hospital, London.

Diseases of the Joints. In one $12 \mathrm{mo}$. volume of 468 pages, with 64 woodcuts and a colored plate. Cloth, \$2.00. See Series of Clinical Manuals, page 4.

\section{PICK, T. PICKERING, F. R. C. S.,}

Surgeon to and Lecturer on Surgery at St. George's Hospital, London.

Fractures and Dislocations. In one 12mo. volume of 530 pages, with 93 illustrations. Limp cloth, 2.00. See Series of Clinical Manuals, page 4. 


\section{BURNETT, CHARLES H., A. M., IM. D.,}

Professor of Otology in the Philadelphia Polyclinic; President of the American Otological Society.

The Ear, Its Anatomy, Physiology and Diseases. A Practical Treatise for the use of Medical Students and Practitioners. New (second) edition. In one handsome octavo volume of 580 pages; with 107 illustrations. Cloth, $\$ 4.00$; leather, $\$ 5.00$.

We note with pleasure the appearance of a second / carried out, and much new matter added. Dr. edition of this valuable work. When it first came Burnett's work must be regarded as a very valua out it was accepted by the profession as one of the standard works on modern aural surgery in the English language; and in his second edition Dr. Burnett has fully maintained his reputation, for the book is replete with valuable information and suggestions. The revision has been carefully ble contribution to aural surgery, not only on account of its comprehensiveness, but because it contains the results of the careful personal observation and experience of this eminent aural surgeon. -London Lancet, Feb. 21, 1885.

\section{POLITZER, ADAM,}

Imperial-Royal Prof. of Aural Therap. in the Univ. of Vienna.

A Text-Book of the Ear and its Diseases. Translated, at the Author's request, by James Patterson Casselis, M. D., M. R. C. S. In one handsome octavo volume of 800 pages, with 257 original illustrations. Cloth, $\$ 5.50$.

The work itself we do not hesitate to pronounce | section, and this again by the pathological physithe best upon the subject of aural diseases which ology, an arrangement which serves to keep up the has ever appeared, systematic without being ton interest of the student by showing the direct apdiffuse on obsolete subjects, and eminently prac- plication of what has preceded to the study of distical in every sense. The anatomical descriptions ease. The whole work can be recommended as a of each separate division of the ear are admirable, reliable guide to the student, and an efficient aid and profusely illustrated by woodcuts. They are to the practitioner in his treatment.-Boston Medfollowed immediately by the physiolngy of the ical and Surgical Journal, June 7, 1883.

\section{JULER, HENRY E., F. R. C.S.,}

Senior Ass't Surgeon, Royal Westminster Ophthalmic Hosp. ; late Clinical Ass't, Moorfields, London.

A Handbook of Ophthalmic Science and Practice. In one handsome octavo volume of 460 pages, with 125 woodcuts, 27 colored plates, selections from the Test-types of Jaeger and Snellen, and Holmgren's Color-blindness Test. Cloth, $\$ 4.50$; leather, $\$ 5.50$.

This work is distinguished by the great number of colored plates which appear in it for illustrating various pathological conditions. They are very beautiful in appearance, and have been executed with great care as to accuracy. An exa mination of the work shows it to be one of high standing, one that will be regarded as an authority among ophthalmologists. The treatment recommended is such as the author has learned from actual experience to be the best.-Cincinnati Medical News, Dec. 1884.

It presents to the student concise descriptions

and typical illustrations of all important eye affections, placed in juxtaposition, so as to be grasped at a glance. Beyond a doubt it is the best illustrated handbook of ophthalmic science which has ever appeared. Then, what is still better, these illustrations are nearly all original. We have examined this entire work with great care, and it represents the commonly accepted views of advanced ophthalmologists. We can most heartily commend this book to all medical students, practitioners and specialists. - Detroit Lancet, $J_{\text {an. }} 1885$.

NORRIS, WM. F., M. D., and OLIVER, CHAS. A., M. D. Clin. Prof. of Ophthalmology in Univ. of Pa.

A Text-Book of Ophthalmology. In one octavo volume of about 500 pages, with illustrations. Preparing.

\section{CARTER, R. BRUDENELL, \& FROST, W.ADAMS,} H. R. C. S., H. R. C. S.,

Ophthalmic Surgeon to and Lecturer on Ophthalmic Surgery at St. George's Hospital, London.

Assistant Ophthalmic Surgeon to and Joint Lecturer on Ophthalmic Surgery at St. George's Hospital, London.

Ophthalmic Surgery. In one 12mo. volume of about 400 pages. Preparing. See Series of Clinical Manuals, page 4.

\section{WELLS, J. SOELBERG, F. R. C. S.,}

Professor of Ophthalmology in Kinq's College Hospital, London, etc.

A Treatise on Diseases of the Eye. New (fifth) American from the third London edition. In one large octavo volume. Preparing.

\section{NETTLESHIP, EDWARD, F. R. C. S.,}

Ophthalmic Surg. and Lect. on Ophth. Surg. at St. Thomas' Hospital, London.

The Student's Guide to Diseases of the Eye. New (third) edition, thoroughly revised. With a chapter on the Detection of Color-Blindness, by WILLIAM THomson, M. D., Professor of Ophthalmology in the Jefferson Medical College. In one royal $12 \mathrm{mo}$. volume of about 450 pages, with about 150 illustrations. Cloth, \$2. Shortly.

\section{BROWNE, EDGAR A.,}

Surgeon to the Liverpool Eye and Ear Infirmary and to the Dispensary for Skin Diseases.

How to Use the Ophthalmoscope. Being Elementary Instructions in Ophthalmoscopy, arranged for the use of Students. In one small royal $12 \mathrm{mo}$. volume of 116 pages, with 35 illustrations. Cloth, $\$ 1.00$. 


\section{Lea Brothers \& Co.'s Publications-Urin. Dis., Dentistry, etc.}

\section{ROBERTS, WILLIAM, M. D.,}

Lecturer on Medicine in the Manchester School of Medicine, etc.

A Practical Treatise on Urinary and Renal Diseases, including Urinary Deposits. Fourth American from the fourth London edition. In one handsome octavo volume of 609 pages, with 81 illustrations. Cloth, $\$ 3.50$.

The previous editions of this book have made it | The peculiar val ue and finish of the book are in so familiar to and so highly esteemed by the med. a measure derived from its resolute maintenance ical public, that little more is necessary than a of a clinical and practical character. It is an unmere announcement of the appearance of this, rivalled exposition of everything which relates their successor. But it is pleasant to be able to directly or indirectly to the diagnosia, prognosis say that, good as those were, this is still better. and treatment of urinary diseases, and possesses In fact, we think it may be said to be the best book a completeness not found elsewhere in our lanin print on the subject of which it treats.-The guage in its account of the different affections.American Journal of the Medical Sciences.-Jan. 1886. The Manchester Medical Chronicle, July, 1835.

\section{PURDY, CHARLES W., M. U.}

Bright's Disease and Allied Affections of the Kidneys. In one octavo volume of 288 pages, with illustrations. Cloth, \$2. Just ready.

The object of this work is to "furnish a system- | short space the theories, facts and treatments, and atic, practical and concise deseription of the going more fully into their later developments. pathology and treatment of the chief organic Un treatment the writer is particularly strong, diseases of the kidney associated with albuminu- steering clear of generalities, and seldom omitria, which shall represent the most recent ad- ting, what text-books usually do, the unimportant vances in our knowledge on these subjects;" and items which are all important to the general practhis definition of the object is a fair description of titioner.-The Manchester Medical Chronicle, Oct., the book. The work is a useful one, giving in a 1886.

\section{MORRIS, HENRY, M. B., F.R.C.S.,}

Surgeon to and Lecturer on Surgery at Middlesex Hospital, London.

Surgical Diseases of the Kidney. In one $12 \mathrm{mo}$. volume of 554 pages, with 40 woodcuts, and 6 colored plates. Limp cloth, \$2.2.5. See Series of Clinical Manuals, page 4.

In this manual we have a distinet addition to he took in hand. It is a full and trustworthy surgical literature, which gives information not book of reference, both for students and pracelsewhere to be met with in a single work. Such titioners in search of guidance. The illustrations a book was distinctly required, and Mr. Morris in the text and the chromo-lithographs are beauhas very diligently and ably performed the task tifully executed.-The London Lancet, Feb. 26, 1886.

LUCAS, CLEMLNT, M. B., B. S., F. R. C. S.,

Senior Assistant Surgeon to Guy's Hospital, London.

Diseases of the Urethra. In one 12mo. volume. Preparing. See Series of Clinical Manuals, page 4.

\section{THOMPSON, SIR HENRY,}

Surgeon and Professor of Clinical Surgery to University College Hospital, London.

Lectures on Diseases of the Urinary Organs. Second American from the third English edition. In one 8vo. volume of 203 pp., with 25 illustrations. Cloth, \$2.25.

\section{By the Same Author.}

On the Pathology and Treatment of Stricture of the Urethra and Urinary Fistulæ. From the third English edition. In one octavo volume of 359 pages, with 47 cuts and 3 plates. Cloth, $\$ 3.50$.

\section{THE AMERICAN SYSTEM OF DENTISTRY.}

In Treatises by,Various Authors. Edited by WrLbur F. LrTch, M. D., D. D. S., Professor of Prosthetic Dentistry, Materia Medica and Therapeutics in the Pennsylvania College of Dental Surgery. In three very handsome octavo volumes of about 1000 pages each, richly illustrated. Per volume, cloth, $\$ 6$; leather, $\$ 7$; half Morocco, gilt top, \$8. Volıme I., containing 1015 pages, with 6 plates and 537. woodcuts, just ready. For sale by subscription only.

As an encyclopædia of Dentistry it has no su. (doubtless it is), to mark an epoch in the history of perior. It should form a part of every dentist's dentistry. Dentists will be satisfied with it and fibrary, as the information it contains is of the proud of it-they must. It is sure to be precisely greatest value to all engaged in the practice of what the student needs to put him and keep him dentistry.-American Journal of Dental Science, in the right track, while the profession at large September, 1886.

A grand system, big enough and good enough and handsome enough for a monument (which will receive incalculable benefit from it.-Odontographic Journal, Jan. 1887.

\section{COLEMAN, A., L. R. C. P., F. R. C. S., Exam. L. D. S.,}

i Senior Dent. Surg. and Lect. on Dent. Surg. at St. Bartholomew's Hosp. and the Dent. Hosp., London.

A Manual of Dental Surgery and Pathology. Thoroughly revised and adapted to the use of American Students, by Thomas C. Stellwagen, M. A., M. D., D. D. S., Prof. of Physiology at the Philadelphia Dental College. In one handsome octavo volume of 412 pages, with 331 illustrations. Cloth, $\$ 3.25$.

\section{ESMARCH, Dr. FRIEDRICH,}

Professor of Surgery at the University of Kiel, etc.

Farly Aid in Injuries and Accidents. Five Ambulance Lectures. Translated by H. R. H. Princess Christian. In one handsome small $12 \mathrm{mo}$. volume of 109 pages, with 24 illustrations. Cloth, 75 cents.

BASHAM ON RENAL DISEASES : A Clinical | one 12mo. vol. of 304 pages, with 21 lllustrations. 


\section{GROSS, SAMUEL W., A. M., M. D., LL. D.,}

Professor of the Principles of Surgery and of Clinical Surgery in the Jefferson Medical College of Phila.

A Practical Treatise on Impotence, Sterility, and Allied Disorders of the Male Sexual Organs. New (third) edition, thoroughly revised. In one very handsome octavo volume of 163 pages, with 16 illustrations. Cloth, $\$ 1.50$. Just ready.

We must congratulate the author that another / that it has been translated into Russian may indiedition has been made necessary. The tone of cate that it filled a void even in foreign literature. the book is healthy, and a cheerful prognosis is $H$ is is a careful and physiological study of the given of many of the affections of which it treats. sexual act, so far as concerns the male, and all We feel confident that the book will continue to his conclusions are scientifically reached. The sell on its merits.- N. Y. Med. Journal, June 18, 1887. book has a place by itself in our literature, and

It must be gratifying to both author and pub- furnishes a large fund of information concerning lishers that large first and second editions of this important matters that are too often passed over little work were so soon exhausted, while the fact $\mid$ in silence.-The Medical Press, June, 1887.

\section{BUMSTEAD, E. J., and M. D., LL. D., Late Professor of Venereal Diseases at the College of Physicians and Surgeons, New York, etc.}

TAYLOR, R. W. A. M., M. D.,

Surgeon to Charity Hospital, .New York, Prof. of Venereat and Skin Diseases in the University of Vermont, Pres. of the Am. Dermatological Ass'n.

The Pathology and Treatment of Venereal Diseases. Including the results of recent investigations upon the subject. Fifth edition, revised and largely rewritten, by Dr. Taylor. In one large and handsome octavo volume of 898 pages with 139 illustrations, and thirteen chromo-lithographic figures. Cloth, $\$ 4.75$; leather, $\$ 5.75$; very handsome half Russia, $\$ 6.25$.

It is a splendid record of honest labor, wide $\mid$ known that it would be superfluous here to pass in research, just comparison, careful scrutiny and review its general or special points of excellence. original experience, which will always be held as The verdict of the profession has been passed; it a high credit to American medical literature. This has been accepted as the most thorough and comis not only the best work in the English language plete exposition of the pathology aud treatment of upon the subjects of which it treats, but also one venereal diseases in the language. Admirable as a which has no equa. in other tongues for its clear, model of clear description, an exponent of sound comprehensive and practical handling of its pathological doctrine, and a guide for rational and themes.-Am. Jour, of the Med. Sciences, Jan, 1884. successful treatment, it is an ornament to the medi-

It ls certainly the best single treatise on vene- cal literature of this country. 'The additions made real in our own, and probably the best in any lan. to the present edition are eminently judicious, guage.-Boston Med, and Surg. Journal, A pril 3, 1884. from the standpoint of practical utility.-Journal of

The character of this standard work is so weli Cutaneous and Venereal Diseases, Jan. 1884.

\section{CORNIL, V.,}

Professor to the Faculty of Medicine of Paris, and Physician to the Lourcine Hospital.

Syphilis, its Morbid Anatomy, Diagnosis and Treatment. Specially revised by the Author, and translated with notes and additions by J. HENRY C. Simes, M. D., Demonstrator of Pathological Histology in the University of Pennsylvania, and J. William White, M. D., Lecturer on Venereal Diseases and Demonstrator of Surgery in the University of Pennsylvania. In one handsome octavo volume of 461 pages, with 84 very beautiful illustrations. Cloth, $\$ 3.75$.

The anatomy, the histology, the pathology and perusal without the feeling that his grasp of the the clinical features of syphilis are represented in wide and important subject on which it treats is this work in their best, most practical and most a stronger and surer one.-The London Practiinstructive form, and no one will rise from its tioner, Jan. 1882.

\section{HUTCHINSON, JONATHAN, F. R. S., F. R. C. S.,} Consulting Surgeon to the London Hospital.

Syphilis. In one $12 \mathrm{mo}$. volume of 542 pages, with 8 chromo-lithographs. Cloth, \$2.25. Just ready. See Series of Clinical Manuals, page 4.

FROM THE PREFACE.

In the following pages I have aimed less at systematic completeness than at clinical exposition. To the latter subject I have devoted my best efforts, and my hope is that those who may honor this work by their attentive perusal will obtain from its pages clear impressions on the state of our knowledge on the topics which it concerns.

\section{GROSS, S. D., M. D., LL. D., D. C. L., etc.}

A Practical Treatise on the Diseases, Injuries and Malformations of the Urinary Bladder, the Prostate Gland and the Urethra. Third edition, thoroughly revised by SAMUEL W. GROSS, M. D. In one octavo volume of 574 pages, with 170 illustrations. Cloth, $\$ 4.50$.

\section{CULLERIER, A., \& BUMSTEAD, F. J., M.J., LL.D.,}

Surgeon to the Hopital du Midi.

Late Professor of Venereal Diseases in the College of Physiciane and Surgeons, New York.

An Atlas of Venereal Diseases. Translated and edited by Freeman J. BumSTEAD, M.D. In one imperial 4 to. volume of 328 pages, double-columns, with 26 plates, containing about 150 figures, beautifully colored, many of them the size of life. Strongly bound in cloth, $\$ 17.00$. A specimen of the plates and text sent by mail, on receipt of $25 \mathrm{cts}$. 


\section{KAPOSI, MORIZ,}

\section{of Vienna.}

The Pathology and Treatment of Diseases of the Skin. For the use of Practitioners and Students. Translated, with the author's permission, by W. XAVIER SuDDUTH, M. D., F.R. M. S. In one octavo volume of about 600 pages, with 74 engravings and 8 colored plates. Preparing.

\section{HYDE, J. NEVINS, A. M., M. D.,}

Professor of Dermatology and Venereal Diseases in Rush Medical College, Chicago.

A Practical Treatise on Diseases of the Skin. For the use of Students and Practitioners. In one handsome octavo volume of 570 pages, with 66 beautiful and elaborate illustrations. Cloth, $\$ 4.25$; leather, $\$ 5.25$.

The author has given the student and practi- Professor Hyde has iong been known as one of tioner a work admirably adapted to the wants of each. We can heartily commend the book as a valuable addition to our literature and a reliable guide to students and practitioners in their studies and practice.-Am. Journ. of Med. Sci., July, 1883.

The aim of the author has been to present to his readers a work not only expounding the most modern conceptions of his subject, but presenting what is of standard value. He has more especially devoted its pages to the treatment of disease, and by his detailed descriptions of therapeutic measures has adapted them to the needs of the physician in active practice. In dealing with these questions the author leaves nothing to the presumed knowledge of the reader, but enters thoroughly into the most minute description, so that one is not only told what should be done under given conditions but how to do it as well. It is therefore in the best sense "a practical treatise." That it is comprehensive, a glance at the index will show.-Maryland Medical Journal, July 7, 1883.

the most intelligent and enthusiastic representatives of dermatology in the west. His numerous contributions to the literature of this specialty have gained for him a favorable recognition as a careful, conscientious and original observer. He has attempted, as he informs us, the task of presenting in a condensed form the results of the latest observation and experience. A careful ex. amination of the work convinces us that he has accomplished his task with painstaking fidelity and with a creditable result.-Journal of Cutaneous and Venereal Diseases, June, 1883.

The several diseases are described very concisely, but at the same time with unusual clearness. The treatment is given in the simplest manner and apparently with great honesty. It is a good book, remarkably adapted to the needs of those for whom it was written. It should be at once placed in the library of every general practitioner.-Detroit Lancet, April, 1883.

\section{FOX, T., M.D., F.R.C.P., and FOX, T. C., B.A., M.R.C.S., \\ Physician to the Department for Skin Diseases,} University College Hospital, London.

Physician for Diseases of the Skin to the Westminster Hospital, London.

An Epitome of Skin Diseases. With Formulæ. For Students and Practitioners. Third edition, revised and enlarged. In one very handsome $12 \mathrm{mo}$. volume of 238 pages. Cloth, $\$ 1.25$.

The third edition of this convenient handbook $\mid$ manual to lie upon the table for instant reference. calls for notice owing to the revision and expansion Its alphabetical arrangement is suited to this use, which it has undergone. The arrangement of skin for all one has to know is the name of the disease, diseases in alphabetical order, which is the method and here are its description and the appropriate of classification adopted in this work, becomes a treatment at hand and ready for instant applicapositive advantage to the student. The book is tion. The present edition has been very carefully one which we can strongly recommend, not only revised and a number of new diseases are deto students but also to practitioners who require a scribed, while most of the recent additions to compendious summary of the present state of dermal therapeutics find mention, and the formudermatology.-British Medical Journal, July 2, 1883. lary at the end of the book has been considerably

We cordially recommend Fox's Epitome to those augmented.-The Medical News, December, 1883. whose time is limited and who wish a handy

\section{MORTIS, MALCOLM, F. R. C.S.,}

Joint Lecturer on Dermatology at St. Mary's Hospital Medical School, London.

Skin Diseases; Including their Definitions, Symptoms, Diagnosis, Prognosis, Morbid Anatomy and Treatment. A Manual for Students and Practitioners. In one $12 \mathrm{mo}$. volume of 316 pages, with illustrations. Cloth, $\$ 1.75$.

To physicians who would like to know something about skin diseases, so that when a patient presents himself for relief they can make a correct diagnosis and prescribe a rational treatment, we unhesitatingly recommend this little book of Dr. Morris. The affections of the skin are described in a terse, lucld manner, and their several characteristics so plainly set forth that diagnosis will be easy. The treatment in each case is such as the experience of the most eminent dermatologists advises.-Cincinnati Medical New8, April, 1880.

This is emphatically a learner's book; for we can safely say, that in the whole range of medical literature there is no book of a like scope which

for clearness of expression and methodical arrangement is better adapted to promote a rationa conception of dermatology $-\mathrm{a}$ branch confessedly difficult and perplexing to the beginner.-St. Louis Courier of Medicine, A pril, 1880.

The writer has certainly given in a small compass a large amount of well-compiled information, and his little book compares favorably with any other which has emanated from England, while in many points he has emancipated himself from the stubbornly adhered to errors of others of his countrymen. There is certainly excellent material in the book which will well repay perusal.-Boston Med. and Surg. Journ., March, 1880.

\section{WILSON, ERASMUS, $\boldsymbol{H}_{\text {. R. S. }}$}

The Student's Book of Cutaneous Medicine and Diseases of the Skin. In one handsome small octavo volume of 535 pages. Cloth, $\$ 3.50$.

\section{HILLIER, THOMAS, M. D.,}

Physician to the Skin Department of University College, London.

Handbook of Skin Diseases; for Students and Practitioners. Second American edition. In one $12 \mathrm{mo}$. volume of 353 pages, with plates. Cloth, $\$ 2.25$. 


\section{The American Systems of Gynecology and Obstetrics.}

Systems of Gynecology and Obstetrics, in Treatises by American Authors. Gynecology edited by MatThew D. Mann, A. M., M. D., Professor of Obstetrics and Gynecology in the Medical Department of the University of Buffalo; and Obstetrics edited by BARTon CoOKE HIRst, M. D., Obstetrician to the Philadelphia and to the Maternity Hospitals, Philadelphia. In four very handsome octavo volumes of about 800 pages each, fully illustrated by wood engravings and colored plates. Prices per volume: Cloth, $\$ 6.00$; leather, $\$ 7.00$; half Russia, $\$ 8.00$. Volume I. of the Gynecology, containing 784 pages, with 201 engravings on wood and 3 colored plates, is just ready. The subsequent volumes are to follow at regular intervals. For sale by subscription only. Address the Publishers. Full descriptive circular free on application.

\section{LIST OF CONTRIBUTORS.}

WILLIAM H. BAKER, M. D.

FORDYCE BARKER, M. D., LL. D., Edin.,

ROBERT BATTEY, M. D.,

BAMUEL C. BUSEY, M. D.

JAMES C. CAMERON, M. D.,

HENRY C. COE, A. M., M. D.,

E. C. DUDLEY, A. B., M. D.

EDWARD S. DUNSTER, M.'D.

B. MCE. EMME'T, M. D.,

GEORGE J. ENGELMANN, M. D.,

HAROLD C. ERNST, M. D.

HENRY J. GARRIGUES, A. M., M. D.,

WILLIAM GOOUELI, A. M., M. D.,

EGBERT H. GRANDIN, A. M., M. D.

CHARLES M. GREEN, M. D.,

SAMUEL W. GROSS, M. D.,

ROBERT P. HARRIS, M. D.

STEPHEN Y. HOWELL, M.'D.

JAMES B. HUNTER, M. D.

A. REEVES JACKSON, A. M., M. D.

EDWARD W. JENKS, M. D., LL. D.,

JOSEPH TABER JOHNSON, M. D.,

It is exceedingly fitting that a System of Gynecology should be given forth as the product of Amerlcan pens. America is the fountain of modern gynecology, and has contributed more than ny other country to its development. All things considered, the best treatise on gynecology must be expected from this country, and when the gynecologists of this country unite their efforts in
CHARLES CARROLL LEE, M. D.,

WILLIAM T. LUSK, M. D., LL. D.,

MATTHEW D. MANN, A. M. M. D.

H. NEWELL MARTIN, F. R. S., M. D., D. Sc., M.A.,

RICHARD B. MAURY, M. D.,

PAUL F. MUNDE, M. D.,

C. D. PALM ER, M. D.

ROSWELL PARK, M.'. D.

THEOPHILUS PARVIN,' M. D., LL. D.,

R. A. F. PENROSE, M. D., LL. D.,

THADDEUS A. REAMY, A. M., M.'D.

J. C. REEVE, M. D.

WILLIAM L. RICHẢRDSON, M. D.

A. D. ROCKWELL, A. M., M. D.

ALEX ANDER J. C. SKENE, M. D.,

J. LEWIS SMITH, M. D

R. STANSBURY SUTTOON, A. M., M. D. LL. D.,

T. GAILLARD THOMAS, M. D., LL. D., ELY VAN DE WARKER, M. D.,

W. GILL WYLIE, M. D.

a "system" the result would be disappointing were it not to excel anything of the kind heretofore attempted. An examination of the first volume of the work before us justifies every expectation formed of it. We must commend it to every reader interested in the division of medicine of which it treats. - The Medical Age, July 25, 1887.

\section{THOMAS, T. GATLLARD, M. D.,}

Professor of Diseases of Women in the College of Physicians and Surgeons, N. $\boldsymbol{Y}$.

A Practical Treatise on the Diseases of Women. Fifth edition, thoroughly revised and rewritten. In one large and handsome octavo volume of 810 pages, with 266 illustrations. Cloth, $\$ 5.00$; leather, $\$ 6.00$; very handsome half Russia, raised bands, $\$ 6.50$.

The words whlch follow "fifth edition" are in | book additionally interesting and gives it value as this case no mere formal announcement. The a work of reference.-London Medical Times and alterations and additions which have been made are Gazette, July 30,1881 .

both numerous and important. The attraction That the previous editions of the trestise of Dr. and the permanent character of this book lie in Thomas were thought worthy of translation into the clearness and truth of the clinical descriptions German, French, Italian and Spanish, is enough of diseases; the fertility of the author in thera- to give it the stamp of genuine merit. At home it peutic resources and the fulness with which the has made its way into the library of every obstetdetails of treatment are described; the definite rician and gynæcologist as a safe guide to practice. character of the teaching; and last, but not least, No small number of additions have been made to the evident candor which pervades it. We would the present edition to make it correspond to re also particularize the fulness with which the his- cent improvements in treatment.-Pacific Medical tory of the subject is gone into, which makes the and Surgical Journal, Jan. 1881.

\section{EDIS, ARTHUR W., M. D., Lond., F. R. C.P., M.R. C.S.,} Assist. Obstetric Physician to Middlesex Hospital, late Physician to British Lying-in Hospital.

The Diseases of Women. Including their Pathology, Causation, Symptoms, Diagnosis and Treatment. A Manual for Students and Practitioners. In one handsome octavo volume of 576 pages, with 148 illustrations. Cloth, $\$ 3.00$; leather, $\$ 4.00$.

It is a pleasure to read a book so thoroughly | are among the more common methods of treat good as this one. The special qualities which are ment, and yet very little is said about them in conspicuous are thoroughness in covering the many of the text-books. The book is one to be whole ground, clearness of description and con- warmly recommended especially to students and ciseness of statement. Another marked feature of general practitioners, who need a concise but comthe book is the attention paid to the details of plete résume of the whole subject. Specialists, too, many minor surgical operations and procedures, will find many useful hints in its pages. - Boston as, for instance, the use of tents, application of Med. and Surg. Journ., March 2, 1882.

leeches, and use of hot water injections. These

\section{BARNES, ROBERT, M. D., F. R. C.P.,}

Obstetric Physician to St. Thomas' Hospital, London, etc.

A Clinical Exposition of the Medical and Surgical Diseases of Women. In one handsome octavo volume, with numerous illustrations. New edition. Preparing. 


\section{EMIMET, THOMAS ADDIS, M. D., LL. D.,}

Surgeon to the Woman's Hospital, New York, etc.

The Principles and Practice of Gynæcology ; For the use of Students and Practitioners of Medicine. New (third) edition, thoroughly revised. In one large and very handsome octavo volume of 880 pages, with 150 illustrations. Cloth, $\$ 5$; leather, $\$ 6$; very handsome half Russia, raised bands, $\$ 6.50$.

We are in doubt whether to congratulate the / once a credit to its author and to American medauthor more than the profession upon the appear- ical literature. We repeat that it is a book to be ance of the third edition of this well-known work. studied, and one that is indispensable to every Embodying, as it does, the life-long experience of practitioner giving any attention to gynæcology.one who has conspicuously distinguished himself American Journal of the Medical Scienccs, April, 1885. as a bold and successful operator, and who has The time has passed when Emmet's Gyncecology devoted so much attention to the specialty, we was to be regarded as a book for a single country feel sure the profession will not fail to appreciate or for a single generation. It has always been his the privilege thus offered them of perusing the aim to popularize gynæcology, to bring it within views and practice of the author. His earnestness easy reach of the general practitioner. The origof purpose and conscientiousness are manifest. inality of the ideas, aside from the perfect conHe gives not only his individual experience but fidence which we feel in the author's statements, endeavors to represent the actual state of gyne- compels our admiration and respect. We may cological sclence and art.-British Medical Jour- well take an honest pride in Dr. Emmet's work nal, May 16, 1885.

No jot or tittle of the high praise bestowed upon the first edition is abated. It is still a book of marked personality, one based upon large clinical experience, containing large and valuable additions to our knowledge, evidently written not only with honesty of purpose, but with a conscienand feel that his book can hold its own against the criticism of two continents. It represents all that is most earnest and most thoughtful in American gynæcology. Emmet's work will continue to reflect the individuality, the sterling integrity and the kindly heart of its honored author long after smaller books have been forgotten.-American Journal of Obstetrics, May, 1885.

\section{DUNCAN, J. MATTHEWS, M.D., LL. D., F. R. S. E., etc.}

Clinical Lectures on the Diseases of Women; Delivered in Saint Bartholomew's Hospital. In one handsome octavo volume of 175 pages. Cloth, $\$ 1.50$.

They are in every way worthy of their author; rule, adequately handled in the text-books; others Indeed, we look upon them as among the most of them, while bearing upon topics that are usually valuable of hls contributions. They are all upon treated of at length in such works, yet bear such a matters of greatinterest to the general practitioner. stamp of individuality that they deserve to be Some of them deal with subjects that are not, as a $\mid$ widely read.-N. Y. Medical Journal, March, 1880.

\section{MAY, CHARLES H., M. D.}

Late House Surgeon to Mount Sinai Hospital, New York.

A Manual of the Diseases of Women. Being a concise and systematic exposition of the theory and practice of gynæcology. In one $12 \mathrm{mo}$. volume of 342 pages: Cloth, \$1.75.

Medical students will find this work adapted to / tions, and the presentation only of accepted views, their wants. Also practitioners of medicine will it constitutes a very satisfactory exposition of the find it exceedingly convenient to consult for the leading principles of gynæcology as they are unpurpose of refreshing their minds upon the lead- derstood at the present time.-Cincinnati Medical ing points of a gynæcological subject. By syste- News, Nov. 1885.

matic condensation, the omission of disputed ques-

\section{HODGE, HUGH L., M. D.,}

Emeritus Professor of Obstetrics, etc., in the University of Pennsylvania.

On Diseases Peculiar to Women; Including Displacements of the Uterus. Second edition, revised and enlarged. In one beautifully printed octavo volume of 519 pages, with original illustrations. Cloth, $\$ 4.50$.

\section{By the Same Author.}

The Principles and Practice of Obstetrics. Illustrated with large lithographic plates containing 159 figures from original photographs, and with numerous woodcuts. In one large quarto volume of 542 double-columned pages. Strongly bound in cloth, $\$ 14.00$. Specimens of the plates and letter-press will be forwarded to any address, free by mail, on receipt of six cents in postage stamps.

\section{RAMSBOTHAM, FRANCIS H., M. D.}

The Principles and Practice of Obstetric Medicine and Surgery; In reference to the Process of Parturition. A new and enlarged edition, thoroughly revised by the Author. With additions by W. V. KEATING, M. D., Professor of Obstetrics, etc., in the Jefferson Medical College of Philadelphia. In one large and handsome imperial octavo volume of 640 pages, with 64 full-page plates and 43 woodcuts in the text, containing in all nearly 200 beautiful figures. Strongly bound in leatler, with raised bands, $\$ 7$.

WINCKEL, $F$.

A Complete Treatise on the Pathology and Treatment of Childbed, For Students and Practitioners. Translated, with the consent of the Author, from the second German edition, by J. R. CHA Dwick, M. D. Octavo 484 pages. Cloth, \$4.00.

ASHWELL'S PRACTICAL TREATISE ON THE

DISEASES PECULIAR TO WOMEN. Third

American from the third and revised London

edition. In one 8vo, vol., pp. 520. Cloth. \$3.50.

CHURCHILL ON THE PUERPERAL FEVER
AND OTHER DISEASES PECULIAR TO WOMEN. In on $\theta$ 8vo. vol. of 464 pages. Cloth, $\$ 2.50$. MEIGS ON THE NATURE, SIGNS AND TREAT: MENT OF CHILDBED FEVER. In one 870. volume of 346 pages. Cloth, $\$ 2.00$. 


\section{PARVIN, THEOPHILUS, M. D., LL. D.,}

Prof. of Obstetrics and the Diseases of Women and Children in Jefferson Med. Coll., Phila.

The Science and Art of Obstetrics. In one handsome 8vo. volume of 697 pages, with 214 engravings and a colored plate. Cloth, $\$ 4.25$; leather, $\$ 5.25$.

It is a ripe harvest that Dr. Parvin offers to his farorable for an agreeable unfolding of the science readers. There is no book that can be more safely and art of obstetrics. This new book is the easy recommended to the student or that can be turned superior of any single work among its predecesto in moments of doubt with greater assurance of sors for the student or practitioner seeking the aid, as it is a liberal digest of safe counsel that has best thought of the day in this department of been patiently gathered.-The American Journal medicine.-The American Practitioner and Neus, of the Medical Sciences, July, 1887.

There is not in the language a treatise on the subject which so completely and intelligently gleans the whole field of obstetric literature, giving the reader the winnowed wheat in concise and well-jointed phrase, in language of exceptional purity and strength. The arrangement of the matter of this work is unique and exceedingly April 2, 1887

This treatise may be defined as exact, concise and scholarly. Parvin's distinguished position as a teacher, his scholarly attainments, and his honest endeavor to do his best by hoth the student and the physician, will secure for his treatise favorable recognition.-A merican Journal of Obstetrics, May, 1887.

\section{BARNES, ROBERT, M. D., and FANCOURT, M. D.,}

Phys. to the General Lying-in Hosp., Lond. $\quad$ Obstetric Phys. to St. Thomas' Hosp., Lond. A System of Obstetric Medicine and Surgery, Theoretical and Clinical. For the Student and the Practitioner. The Section on Embryology contributed by Prof. Milnes Marshall. In one handsome octavo volume of 872 pages, with 231 illustrations. Cloth, $\$ 5$; leather, $\$ 6$.

This system will be eagerly sought for, not only |ble teacher and trusted accoucheur, should embody on account of its intrinsic merit, but also because within a single treatise the system which he has the reputation which the elder Barnes, in particu- taught and in practice tested, and which is the outlar, has secured, carries with it the conviction that come of a lifetime of earnest labor, careful obserany book emanating from him is necessarily sound vation and deep study. 'The result of thls arrangein teaching and conservative in practice. It is in- ment is the production of a work which rises above deed eminently fitting that a man who has done so criticism and which in no respect need yield the much towards systematizing the obstetric art, who palm to any obstetrical treatise hitherto published. for so many years has been widely known as a capa- - A merican Journal of Obstetrics, Feb. 1886.

\section{PLAYFATR, W. S., M. D., F. R. C. P.,}

Professor of Obstetric Medicine in King's College, London, etc.

A Treatise on the Science and Practice of Midwifery. New (fourth) American, from the fifth English edition. Edited, with additions, by RoBERT P. HARRIs, M. D. In one handsome octavo volume of 654 pages, with 3 plates and 201 engravings. Cloth, $\$ 4$; leather, $\$ 5$; half Russia, $\$ 5.50$.

This still remains a favorite in America, not |supply.-American Journal of Obstetrics, Nov. 1885. only because the anthor is recognized as a safe guide and eminently progressive man, but also as sparing no effort to make each successive edition a faithful mirror of the latest and best practice. A work so frequently noticed as the present requires no further review. We believe that this edition is simply the forerunner of many others,
and that the demand will keep pace with the

Since its first publication, only eight years ago, it has rapidly become the favorite text-book, to the practical exclusion of all others. A large measure of its popularity is due to the clear and easy style in which it is written. Few text-books for students have very much to boast of in this respect.-Medical Record.

\section{KING, A. F. A., M. D.,}

Professor of Obstetrics and Irseases of Women in the Medical Department of the Columbran Univer. sity, Washington, D. C., and in the University of Vermont, etc.

A Manual of Obstetrics. New (third) edition. In one very handsome $12 \mathrm{mo.}$ volume of 376 pages, with 102 illustrations. Cloth, $\$ 2.25$.

This little manual, certainly the best of its kind, (bulky-it is concise. The chapters are divided with fully deserves the popularity which has made a sub-headings, which aid materially in the finding third edition necessary. Clear, practical, concise, of any particular subject, and the definitions are its teachings are so fully abreast with recent ad- clear and explicit. It fulfils its purpose admirably, vances in obstetric science that but few points and we know of no better work to place in the stucan be criticised.-American Journal of Obstetrics, dent's hands. The illustrations are good.-ArchMarch, 1887.

This volume deserves commendation. It is not

\section{BARKER, FORDYCE, A. M., M. D., LL. D. Edin.,}

Clinical Professor of Midwifery and the Diseases of Women in the Bellevue Hospital Medical College, New York, Honorary Fellow of the Obstetrical Societies of London and Edinburgh, etc., etc.

Obstetrical and Clinical Essays. In one handsome $12 \mathrm{mo}$. volume of about 300 pages. Preparing.

BARNES, FANCOURT, M. D.,

Obstetric Physician to St. Thomas' Hospital, London.

A Manual of Midwifery for Midwives and Medical Students. In one royal $12 \mathrm{mo}$. volume of 197 pages, with 50 illustrations. Cloth, $\$ 1.25$.

PARRY, JOHIN S., M. D.,

Obstetrician to the Philadelphia Hospital, Vice-President of the Obstet. Society of Philadelphia.

Extra-Uterine Pregnancy: Its Clinical History, Diagnosis, Prognosis and Treatment. In one handsome octavo volume of 272 pages. Cloth, $\$ 2.50$. 
LEISHMLAN, WILLIAM, M. D.,

Regius Professor of Midwifery in the University of Glasgow, etc.

A System of Midwifery, Including the Diseases of Pregnancy and the Puerperal State. Third American edition, revised by the Author, with additions by JoHN S. PARRY, M. D., Obstetrician to the Philadelphia Hospital, etc. In one large and very handsome octavo volume of 740 pages, with 205 illustrations. Cloth, $\$ 4.50$; leather, $\$ 5.50$; very handsome half Russia, raised bands, $\$ 6.00$.

The author is broad in his teachings, and dis- | must prove admirably adapted. Complete in all its cusses briefly the comparative anatomy of the pelvis and the mobility of the pelvic articulations. The second chapter is deroted especially to female oryans of generation are introduced. The structure and development of the ovum are admirably described. Then follow chapters upon the various subjects embraced in the study of midwifery. The descriptions throughout the work are plain aud pleasing. It is sufficient to state that in this, the last edition of this well-known work, every recent advancement in this field has been brought forward.-Physician and Surgeon, Jan. 1880.

To the American student the work before us Journal, Feb. 1880.

\section{LANDIS, HENRY G., A. M., M. D.,}

Professor of Obstetrics and the Diseases of Women in Starling Medical College, Columbus, 0.

The Management of Labor, and of the Lying-in Period. In one handsome $12 \mathrm{mo}$. volume of 334 pages, with 28 illustrations. Cloth, $\$ 1.75$.

The author has designed to place in the hands tempt any one who should happen to commence of the young practitioner a book in which he can find necessary information in an instant. As far as we can see, nothing is omitted. The advice is sound, and the proceedures are safe and practical. Centralblatt für Gynakologie, December 4, 1886.

This is a book we can heartily recommend. The author goes much more practically into the text-books, and is so readable throughout as to

the book to read it through. The author presupposes a theoretical knowledge of obstetrics, and has consistently excluded from this little work everything that is not of practical use in the lying-in room. We think that if it is as widely read as it deserves, it will do much to improve obstetric practice in general. - New Orleans Medical and Surgical Journal, Mar. 1886.

\section{SMITH, J. LEWIS, M. D.,}

Clinical Professor of Diseases of Children in the Bellevue Hospital Medical College, N. Y.

A Treatise on the Diseases of Infancy and Childhood. New (sixth) edition, thoroughly revised and rewritten. In one handsome octavo volume of 867 pages, with 40 illustrations. Cloth, $\$ 4.50$; leather, $\$ 5.50$; half Russia, $\$ 6.00$.

Rarely does a pleasanter task fall to the lot of / stands alone amongst other works on its subject, the bibliographer than to announce the appearance of recommending treatment in accordance with of a new edition of a medical classic like Prof. J. the most recent therapeutical views.-British and Lewis Smith's Treatise on the Diseases of Infancy Foreign Medico-Chirurgical Review.

and Childhood. For years it has stood high in the It is a pleasure to the busy practitioner-interconfidence of the profession, and with the addi- ested in the advancement of his profession-to tions and alterations now made it may be said to meet, fresh from the hands of its author, a medibe the best book in the language on the subject of cal classic such as Smith on Diseases of children. which it treats. An examination of the text fully Those familiar with former editions of the work sustains the claims made in the preface, that "in will readily recognize the painstaking with which preparing the sixth edition the author has revised the text to such an extent that a considerable part of the book may be considered new." If the young practitioner proposes to place in his library but one book on the diseases of children, we would unhesitatingly say, let that book be the one which is the subject of this notice.-The American Journal of the Medical Sciences, April, 1886.

No better work on children's diseases could be placed in the hands of the student, containing, as it does, a very complete sccount of the symptoms and pathology of the diseases of early life, and possessing the further advantage, in which it

this revision has been made. Many of the articles have been entirely rewritten. The whole work is enriched with a research and reasoning which plainly show that the author has spared nelther time nor labor in bringing it to its present approach towards perfection. The extended table of contents and the well-prepared index will enable the busy practitioner to reach readily and quickly for reference the various subjects treated of in the body of the work, and even those who are familiar with former editions will find the improvements in the present richly worth the cost of the work. Atlanta Medical and Surgical Journal, Dec. 1886.

\section{OWIEN, $\underset{\text { Surgeon to the Children's Hospital, Great Ormond St., London. }}{\boldsymbol{E} \text { M. }}$}

Surgical Diseases of Children. In one $12 \mathrm{mo}$. volume of 525 pages, with 4 chromo-lithographic plates and 85 woodcuts. Cloth, $\$ 2$. See Series of Clinical Manuals, page 4.

One is immediately struck on reading this book with its agreeable style and the evidence it everywhere presents of the practical familiarity of its
author with his subject. The book may be

honestly recommended to both students and practitioners. It is full of sound information, pleasantly given.-Annals of Surgery, May, 1886.

\section{WEST, CHARLES, M. D.,}

Physician to the Hospital for Sick Children, London, etc.

On Some Disorders of the Nervous System in Childhood. In one small $12 \mathrm{mo}$. volume of 127 pages. Cloth, $\$ 1.00$. 


\section{TIDY, CHARLES MEYMOTT, M. B., F. C. S.,}

Professor of Chemistry and of Forensic Medicine and Public Health at the London Hospital, etc.

Legal Medicine. Volume If. Legitimacy and Paternity, Pregnancy, Abortion, Rape, Indecent Exposure, Sodomy, Bestiality, Live Birth, Infanticide, Asphyxia, Drowning, Hanging, Strangulation, Suffocation. Making a very handsome imperial octavo volume of 529 pages. Cloth, $\$ 6.00$; leather, $\$ 7.00$.

VoLUME I. Containing 664 imperial octaro pages, with two beautiful colored plates. Cloth, $\$ 6.00$; leather, $\$ 7.00$.

The satisfaction expressed with the first portion tables of cases appended to each division of the of this work is in no wise lessened by a perusal of subject must have cost the author a prodigious the second volume. We find it characterized by smount of labor and research, but they constitute the same fulness of detail and clearness of ex- one of the most valuable features of the book, pression which we had occasion so highly to com- especialiy for reference in medico-legal trials. mend in our former notice, and which render it so American Journal of the Medical Sciences, April, 1884. valuable to the medical jurist. The copious

\section{TAYLOR, ALFRED S., M. D.,}

Lecturer on Medical Jurisprudence and Chemistry in Guy's Hospital, London.

A Manual of Medical Jurisprudence. Eighth American from the tenth London edition, thoroughly revised and rewritten. Edited by JoHN J. REESE, M. D., Professor of Medical Jurisprudence and Toxicology in the University of Pennsylvania. In one large octavo volume of 937 pages, with 70 illustrations. Cloth, $\$ 5.00$; leather, $\$ 6.00$; half Russia, raised bands, $\$ 6.50$.

The American editions of this standard manual have for a long time laid claim to the attention of the profession in this country; and the eighth comes before us as embodying the latest thoughts and emendations of Dr. Taylor upon the subject to which he devoted his life with an assiduity and success which made him facile princeps among English writers on medical jurisprudence. Both the author and the book have made a mark too deep to be affected by criticism, whether it be censure or praise. In this case, however, we should

only have to seek for laudatory terms.-American Journal of the Medical Sciences, Jan. 1881.

This celebrated work has been the standard authority in its department for thirty-seven years, both in England and America, in both the professions which it concerns, and it is improbable that it will be superseded in many years. The work is simply indispensable to every physician, and nearly so to every liberally-educated lawyer, and we heartily commend the present edition to both professions.-Albany Law Journal, March 26, 1881.

\section{By the Same Author.}

The Principles and Practice of Medical Jurisprudence. Third edition. In two handsome octavo volumes, containing 1416 pages, with 188 illustrations. Cloth, $\$ 10$; leather, $\$ 12$.

For years Dr. Taylor was the highest authority | matters connected with the subject," should be in England upon the subject to which he gave brought up to the present day and continued in especial attention. His experience was vast, his its authoritative position. To accomplish this rejudgment excellent, and his skili beyond cavil. It sult Dr. Stevenson has subjected it to most careful is therefore well that the work of one who, as Dr. editing, bringing it well up to the times.-AmeriStevenson says, had an "enormous grasp of ail can Journal of the Medical Scrences, Jan. 1884.

\section{By the Same Author.}

Poisons in Relation to Medical Jurisprudence and Medicine. Third A merican, from the third and revised English edition. In one large octavo volume of 788 pages. Cloth, $\$ 5.50$; leather, $\$ 6.50$.

\section{PEPPER, AUGUSTUS J., M. S., M. B., F. R. C. S.,}

Examiner in Forensic Medicine at the University of London.

Forensic Medicine. In one pocket-size 12mo. volume. Preparing. See Students' Series of Manuals, page 4.

\section{LEA, HENIYY C.}

Superstition and Force: Fssays on The Wager of Law, The Wager of Battle, The Ordeal and Torture. Third revised and enlarged edition. In one handsome royal $12 \mathrm{mo}$. volume of 552 pages. Cloth, $\$ 2.50$.

This valuable work is in reality a history of cir- $\mid$ should not be most carefully studied ; and however llization as interpreted by the progress of jurispru- well versed the reader may be in the science of dence. . In "Superstition and Force" we have a jurisprudence, he will find much in Mr. Lea's rolphilosophic survey of the long period intervening ume of which he was previously lgnorant. The between primitive barbarity and civilized enlight- book is a valuable addition to the litersture of soenment. There is not a chapter in the work that ciai science.-Westminster Review, Jan. 1880.

\section{By the Same Author.}

Studies in Church History. The Rise of the Temporal Power-Beneflt of Clergy-Excommunication. New edition. In one very handsome royal octavo volume of 605 pages. Cloth, $\$ 2.50$.

The author is pre-eminently a scholar. He takes | primitive church traced with so much clearness, up every topic allied with the leading theme, and traces it out to the minutest detail with a wealth of knowledge and impartiality of treatment that compel admiration. The amount of information compressed into the book is extraordinary. In no other single volume is the development of the and with so definite conflicting sources. The fifty pages on the growth of the papacy, for instance, are admirable for conciseness and freedom from prejudice.-Boston Traveller, May 3, 1883. 
Allen's Anatomy

al of the Medical Sciences American System of Practical Medicine :

An American System of Dentistry

*Ashhurst's Surgery

A shwell on Diseases of Women

Attield's Chemistry

Ball on the Rectum and Anus

Barker's Obstetrical and Clinical Essays,

Barlow's Practice of Medicine

Barnes' Midwifery

* Barnes on Diseases of Women

Barnes' System of Obstetric Medicine

Bartholow on Electricity

Bartholow's New Remedies and their Uses

Basham on Renal Diseases

Bell's Comparative Physiology and Anatomy

Bellamy's Operative Surgery

Bellamy's Surgical Anatomy

Blandford on Insanity

Bloxam's Chemistr

* Bristowe's Pruetice of Medicine

Broadbent on the Pulse

Browne on the Ophthalmoscope

Bruce's Materia Medica and Therapeutics

Brunton's Materia Medica and Therapeutics

Bryant on the Breas

*Bryant's Practice of Surgery

* Bumstead on Venereal Diseases.

* Burnett on the Ear

Butlin on the Tongue

Carpenter ou the Use and Abuse of Alcohol

* Carpenter's Human Phvsiology

Carter \& Frost's Oplithalmic Surgery

Century of American Medicine

Chamhers on Diet and Regimen

Chapman's Human Physiology

Charles' Physiological and Pathological Chem.

Churchill on Puerperal Fever

Clarke and Lock Wood's Dissectors' Manual

Classen's Quantitative Analysís

Cleland's Dissector

Clouston on Insanity

Clowes' Practical Chemistry

Coats' Pathology

Cohen on the Throat

Coleman's Dental Surgery

Condie on Diseases of Children

Cornil on Syphilis

*Cornil and Ranvier's Pathologicai Histology

Cullerier's Atlas of Venereal Diseases

Curnow's Medical Anatomy

Dalton on the Circulation

*Dalton's HumanPhysiology

Davis' Clinical Lectures

Draper's Medical Physics

Druitt's Moderl Surgery

Duncan on Diseases of Women

* Dunglison's Medical Dictionary

Edes' Materia Medica and Therapeutics

Edis on Diseases of Wonen

Ellis' Demonstrations of A natomy

Emmet's Gynæcology

* Erichsen's System of Surgery

Esmarch's Early Aid in Injuries and Accid'ts

Farquharson's 'Therapeutics and Mat. Med.

Fenwick's Medical Diagnosis

Finlayson's Clinical Diagnosis

Flint on Auscultation and Percussion

Fiint on Phthisis

Flint on Physical Exploration of the Lungs

Flint on Respiratory Organs

Flint on the Heart

Flint's Fssays

*Flint's Practice of Medicine

Folsom's Laws of U. S. on Custody of Insane

Foster's Physiology

* Fothergill's Handbook of Treatment

Fownes' Elementary Chemistry

Fox on Diseases of the Skin

Frankland and Japp's Inorganic Ciemistry

Fuller on the Lungs and Air Passages

Galloway's A nalysis

Gibney's Orthopædic Surgery

Gould's Surgical Diagnosis

* Gray's

Greene's Medical Chemistry

Griftith's Unjversal Formulary

Gross on Impotence and Sterility

Gross on Urinary Organs

Habershon on the Abdomen

*Hamilton on Fractures and Dislocations

Hamilton on Nervous Diseases

Hartshorne's A natomy and Physiology

Hartshorne's Conspectus of the Med. Sciences

Hartshorne's Essentials of Medicine

Hartshorne's Household Medicine

Hermann's Experimental Pharmacology

Hill on Syphilis

Filller's Handbook of Śkin Diseases

Hoblvu'k Medical Dictionary

Hodge on Women

Hoffmann and Power's Chemical Analysis

Holden's Landmarks

Books marked * are alsu buund in halt Russia.

6 *Holmes' System of Surgery

Horner's A natomy and Histology.

5 Hutchinson on Syphilis

Hyde on the Diseases of the Skin

20 Jones (C. Handfield) on Nervons Disorders

Juler's Ophthalmic science and Practice

Kaposi on Skin Diseases

4, 20 King's Manual of Obstetrics.

29 Kiein's Histology

17 Landis on I abor

29 La Roche on Pneumonja, Malaria, etc.

La Roche on Yellow Fever

29 Laurence and Moon's Ophthalmic Surgery

Lawsor on the Eye, Orbit and Eyelid

Lea's Studies in Church History

Lea's Superstition

4,7

Lehmann's Chemical Physiology

6 Heishman's Midwifery

Lucas on Diseases of the Urethra

4 Lyons on Fever

Maisch's Organic Materia Medica:

Marsh ou the Joints

1 May on I iseases of Women

Medical News

21

18

18

18 Tanner on figns and Diseases of Pregnancy

8 Tanner's Manual of Clinical Medicine

mas on Diseases of Women .

11 Thompson on Stricture

18 Tidy's Legal Medicine.

25 Todd on Acute Disease

25 Treves' Manual of Surgery

20 Treves' Surgical A pplied Anatomy 





\section{DAY USE RETURN TO DESK FROM WHICH BORROWED}

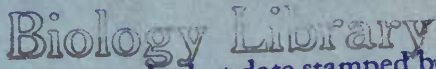
This book is due on the last date stamped below, or on the date to which renewed.

Renewed books are subject to immediate recall.

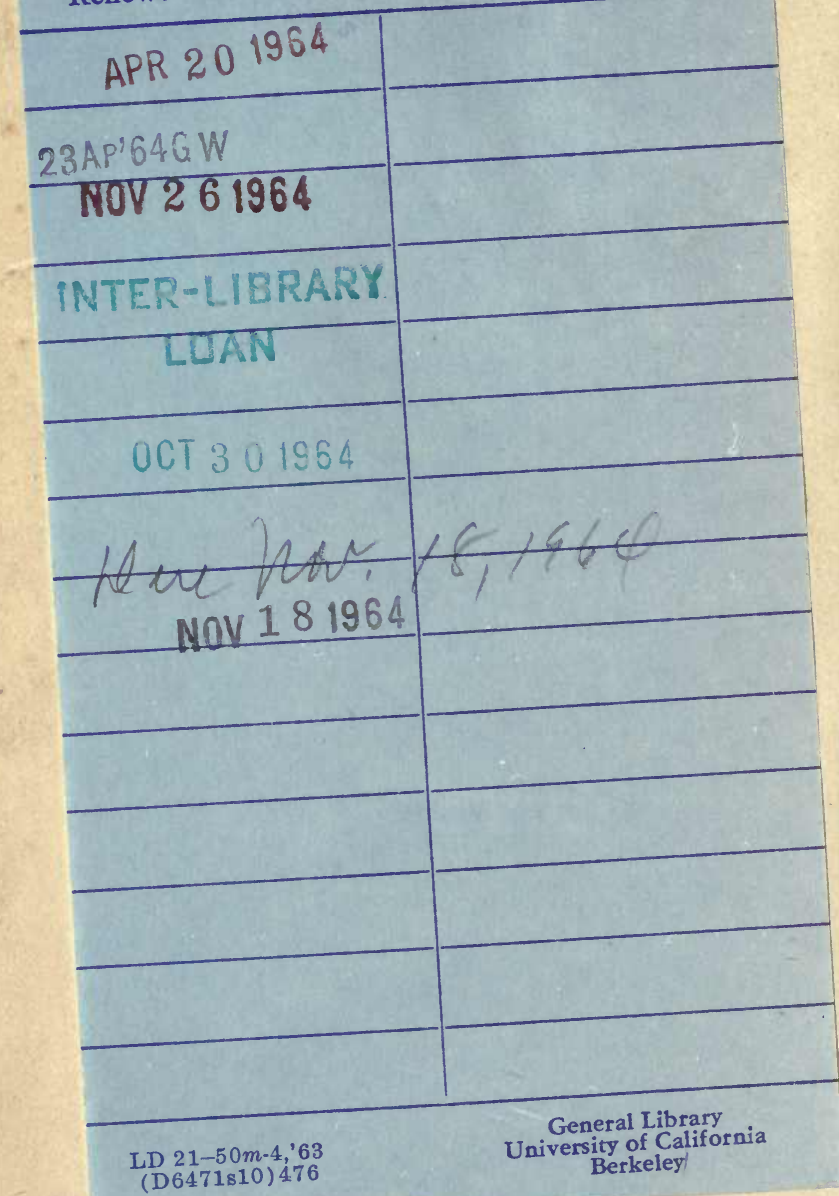

D $21-50 m-4,6$

Berkeley 
$\mathrm{C}_{2}$

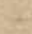

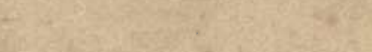

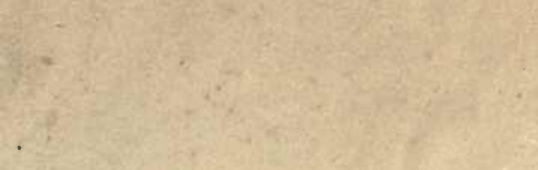

3.7. $=$

(

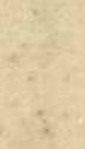

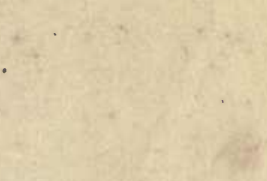

$+1$

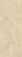

$+2+10=1$

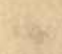

,

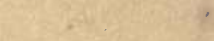

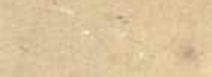
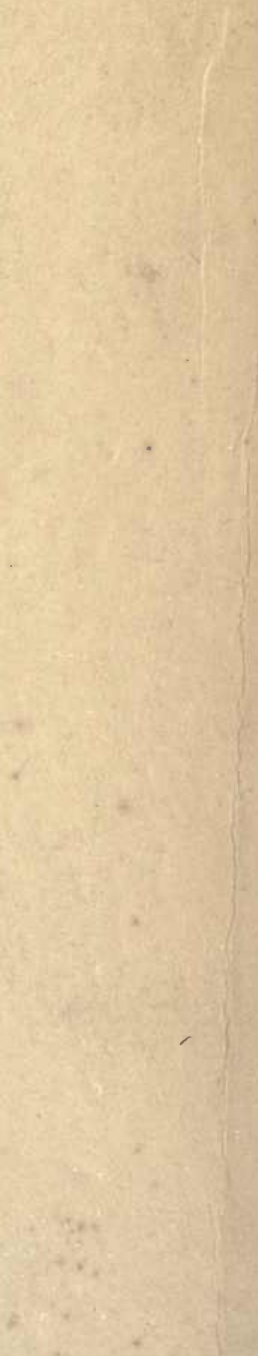
